Supporting Information for:

\title{
Tuning Supramolecular Selectivity for Hydrosulfide: Linear Free Energy Relationships Reveal Preferential C-H Hydrogen Bond Interactions
}

\author{
Hazel A. Fargher ${ }^{\dagger}$ Nathanael Lau, ${ }^{\dagger}$ H. Camille Richardson, ${ }^{\dagger}$ Paul Ha-Yeon Cheong, $* *$ \\ Michael M. Haley, ${ }^{* \dagger}$ Michael D. Pluth*,†, and Darren W. Johnson, ${ }^{*}, \dagger$ \\ ${ }^{\dagger}$ Department of Chemistry \& Biochemistry, Material Science Institute, and Knight Campus for Accelerating Scientific \\ Impact, University of Oregon, Eugene, Oregon 97403-1253, USA. \\ *Department of Chemistry, Oregon State University, Corvallis, Oregon 97331, USA. \\ E-mail: paulc@science.oregonstate.edu, haley@uoregon.edu,pluth@uoregon.edu,dwj@uoregon.edu
}

Table of Contents

Page

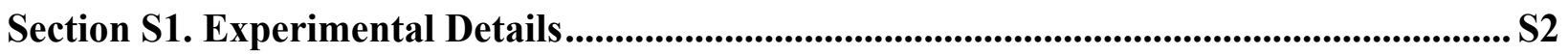

Receptor Synthesis and Characterization. ......................................................................... S2

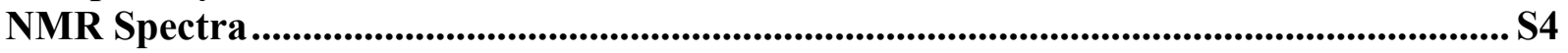

Section S2. Linear free energy relationship (LFER) analysis of substituent effect on ${ }^{1} \mathbf{H}$

NMR chemical shift $(\delta)$ of free host hydrogen bond (HB) donors at $0.8-2.2 \mathrm{mM}$ in 10\%

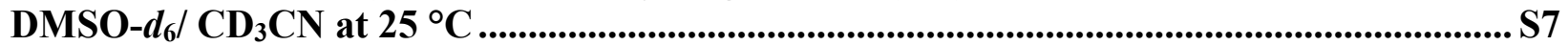

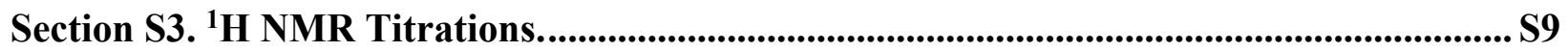

General Procedure for NMR Titrations ....................................................................... S9

${ }^{1}$ H NMR Titration Representative Data .......................................................................... S10

Section S4. Linear Regression Fitting and Statistics of $\Delta G_{\text {binding }}$ and $\sigma_{\mathrm{p}}$............................ S28

Section S5. Linear Regression Fitting and Statistics of $\log \left(K_{a}{ }^{R} / K_{a}{ }^{H}\right)$ and $\sigma_{p} \ldots \ldots \ldots \ldots \ldots . . . S 30$

Section S6. Analysis of Covariance on Hammett Plots................................................... S32

Section S7. Swain-Lupton Analysis ............................................................................................. S32

Section S8. Alternative Hammett parameters................................................................. S33

Section S9. Computational Details, Discussion, Geometries, and Energies ........................ S35

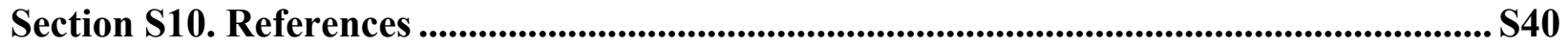

Section S11. Computed Geometries and Energies ............................................................... S41 


\section{Section S1. Experimental Details}

General Methods. All manipulations were performed under an inert atmosphere using an Innovative Atmospheres $\mathrm{N}_{2}$-filled glove box unless otherwise noted. NMR spectra were acquired at room temperature on a Bruker Avance III HD 600 equipped with a Prodigy multinuclear cryoprobe $\left({ }^{1} \mathrm{H}\right.$ : $\left.600 \mathrm{MHz},{ }^{13} \mathrm{C}: 151 \mathrm{MHz},{ }^{19} \mathrm{~F}: 565 \mathrm{MHz}\right) .{ }^{1} \mathrm{H}$ and ${ }^{13} \mathrm{C}$ chemical shifts $(\delta)$ are reported in ppm relative to residual $\mathrm{CHCl}_{3}\left({ }^{1} \mathrm{H}: 7.26 \mathrm{ppm},{ }^{13} \mathrm{C}: 77.16 \mathrm{ppm}\right)$ or DMSO $\left({ }^{1} \mathrm{H}: 2.50\right.$ ppm, $\left.{ }^{13} \mathrm{C}: 39.52 \mathrm{ppm}\right)$ shifts. ${ }^{19} \mathrm{~F}$ NMR shifts are referenced to $\mathrm{CFCl}_{3}(\delta 0 \mathrm{ppm})$ as an external standard. Mass spectra data were acquired on a Waters SYNAPT HDMS ToF in positive ion mode with a Shimadzu Prominence LC front end. $\mathrm{CD}_{3} \mathrm{CN}$ and DMSO- $d_{6}$ were distilled from calcium hydride then deoxygenated by purging with $\mathrm{N}_{2}$ and stored over $4 \AA$ molecular sieves in an inert atmosphere glove box. Tetrabutylammonium chloride $(\mathrm{NBu} 4 \mathrm{Cl})$ and tetrabutylammonium bromide $(\mathrm{NBu} 4 \mathrm{Br})$ were recrystallized by layering an anhydrous THF solution under anhydrous $\mathrm{Et}_{2} \mathrm{O}$. Tetrabutylammonium hydrosulfide $(\mathrm{NBu} 4 \mathrm{SH}),{ }^{1}$ tetrabutylammonium hydroselenide $\left(\mathrm{NBu}_{4} \mathrm{SeH}\right){ }^{2}$ 1,3-dibromo-5-(trifluoromethyl)benzene, ${ }^{3,4}$ 4-tert-butyl-2-((trimethylsilyl)ethynyl)aniline (4), ${ }^{5,6}$ and hosts $\mathbf{1}^{\mathrm{NMe} 2}, \mathbf{1}^{\mathrm{tBu}}, \mathbf{1}^{\mathrm{H}}, \mathbf{1}^{\mathrm{F}}$, and $\mathbf{1}^{\mathrm{Cl} 7}$ were all synthesized according to previous reports. All other reagents were purchased from commercial sources and used as received. Note: Hydrogen sulfide, hydrogen selenide, and related salts are highly toxic and should be handled carefully to avoid exposure.

Receptor Synthesis and Characterization.

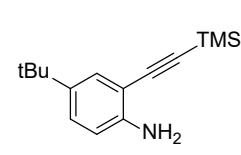

4

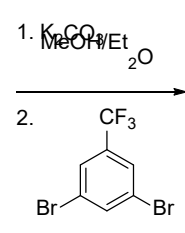

Pd(PPh3)4, Cul
THF/DIPA

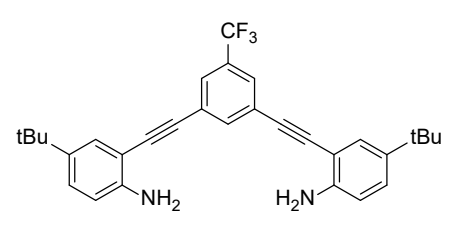

5
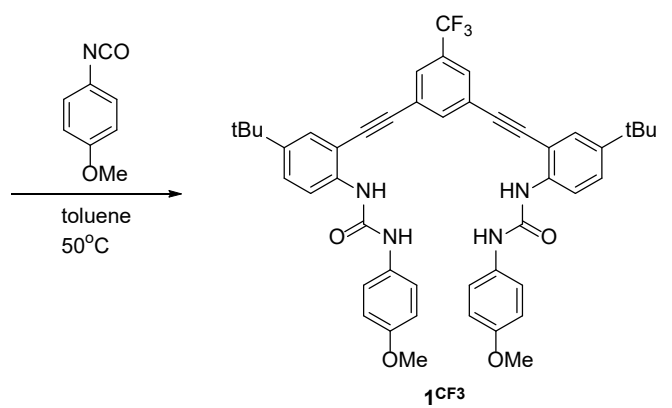

Scheme S1. Synthetic scheme for $\mathbf{1}^{\mathbf{C F} 3}$ starting from previously published intermediate $\mathbf{4}^{5,6}$ 
Dianiline 5. This preparation was adapted from previous reports. ${ }^{7}$ A suspension of 4-tertbutyl-2-((trimethylsilyl)ethynyl)aniline ${ }^{5,6}(4,2.40 \mathrm{~g}, 9.77 \mathrm{mmol}), \mathrm{K}_{2} \mathrm{CO}_{3}(6.04 \mathrm{~g}, 43.70 \mathrm{mmol})$, $\mathrm{MeOH}(20 \mathrm{~mL})$, and $\mathrm{Et}_{2} \mathrm{O}(10 \mathrm{~mL})$ was stirred at $25^{\circ} \mathrm{C}$ for $3 \mathrm{~h}$. The suspension was diluted with water and extracted with $\mathrm{CH}_{2} \mathrm{Cl}_{2}(3 \mathrm{x})$ and washed with brine (2x). The organic layer was dried $\left(\mathrm{Na}_{2} \mathrm{SO}_{4}\right)$ and concentrated in vacuo to afford a dark brown oil. The oil was dissolved in THF (10 $\mathrm{mL})$ and DIPA $(10 \mathrm{~mL})$ and purged with $\mathrm{N}_{2}$ for $1 \mathrm{~h}$. The solution was cannulated into an $\mathrm{N}_{2}$-purged solution of 1,3-dibromo-5-(trifluoromethyl)benzene ${ }^{3,4}$ (0.81 g, 2.7 mmol), $\mathrm{Pd}\left(\mathrm{PPh}_{3}\right)_{4}(0.15 \mathrm{~g}, 0.13$ mmol), $\mathrm{CuI}(0.02 \mathrm{~g}, 0.1 \mathrm{mmol})$, THF $(20 \mathrm{~mL})$, and $i-\mathrm{PrNH}_{2}(20 \mathrm{~mL})$. The solution was stirred for $12 \mathrm{~h}$ at $50{ }^{\circ} \mathrm{C}$, cooled, and concentrated in vacuo. The resulting oil was dissolved in $\mathrm{CH}_{2} \mathrm{Cl}_{2}$ and filtered through a $3 \mathrm{~cm}$ silica plug, which was washed with additional $\mathrm{CH}_{2} \mathrm{Cl}_{2}$. The filtrate was concentrated in vacuo and the resulting brown oil was purified by column chromatography (5:1 hexanes/EtOAc) to afford $5(0.48 \mathrm{~g}, 0.98 \mathrm{mmol}, 37 \%)$ as a beige solid. ${ }^{1} \mathrm{H} \mathrm{NMR}\left(600 \mathrm{MHz}, \mathrm{CDCl}_{3}\right)$ $\delta 7.84(\mathrm{~s}, 1 \mathrm{H}), 7.71(\mathrm{~s}, 2 \mathrm{H}), 7.39(\mathrm{~d}, J=2.1 \mathrm{~Hz}, 2 \mathrm{H}), 7.23(\mathrm{dd}, J=8.5,2.1 \mathrm{~Hz}, 2 \mathrm{H}), 6.70(\mathrm{~d}, J=$ $8.5 \mathrm{~Hz}, 2 \mathrm{H}), 4.18(\mathrm{~s}, 4 \mathrm{H}), 1.30(\mathrm{~s}, 18 \mathrm{H}) .{ }^{13} \mathrm{C} \mathrm{NMR}\left(151 \mathrm{MHz}, \mathrm{CDCl}_{3}\right) \delta 145.84,141.19,137.01$, 131.55 (q, $J=32.8 \mathrm{~Hz}), 129.05,128.03,127.37$ (q, $J=3.6 \mathrm{~Hz}), 124.99,123.52$ (q, $J=272.7 \mathrm{~Hz})$, 114.64, 106.61, 92.12, 89.07, 34.09, 31.52. ${ }^{19} \mathrm{~F}$ NMR (565 MHz, CDCl 3$) \delta-63.09$. HRMS (ESITOF-MS ${ }^{+}$) $m / z$ calcd for $\mathrm{C}_{31} \mathrm{H}_{32} \mathrm{~N}_{2} \mathrm{~F}_{3}[\mathrm{M}+\mathrm{H}]^{+} 489.2518$, found 489.2523 .

Receptor $1^{\text {CF3 }}$. This preparation was adapted from previous reports. ${ }^{7}$ All glassware was dried in a $150^{\circ} \mathrm{C}$ oven overnight. A round bottom flask was charged with dry toluene $(100 \mathrm{~mL})$ and dianiline 5 ( $0.41 \mathrm{~g}, 0.84 \mathrm{mmol})$. 4-Methoxyphenyl isocyanate $(0.40 \mathrm{~mL}, 3.1 \mathrm{mmol})$ was added dropwise and the solution was stirred for $12 \mathrm{~h}$ at $50{ }^{\circ} \mathrm{C}$. The reaction became cloudy upon completion, and the precipitate was collected, then recrystallized in toluene to afford $\mathbf{1}^{\mathbf{C F 3}}(0.15 \mathrm{~g}$, $0.20 \mathrm{mmol}, 24 \%)$ as a fine white powder. ${ }^{1} \mathrm{H}$ NMR (600 MHz, DMSO- $\left.d_{6}\right) \delta 9.31(\mathrm{~s}, 2 \mathrm{H}), 8.32(\mathrm{~s}$, $1 \mathrm{H}), 8.17$ (s, 2H), $8.11(\mathrm{~s}, 2 \mathrm{H}), 8.04$ (d, $J=8.8 \mathrm{~Hz}, 2 \mathrm{H}), 7.56$ (d, $J=2.4 \mathrm{~Hz}, 2 \mathrm{H}), 7.46$ (dd, $J=8.8$, $2.4 \mathrm{~Hz}, 2 \mathrm{H}), 7.38$ (d, $J=8.9 \mathrm{~Hz}, 4 \mathrm{H}), 6.85(\mathrm{~d}, J=8.9 \mathrm{~Hz}, 4 \mathrm{H}), 3.70(\mathrm{~s}, 6 \mathrm{H}), 1.30(\mathrm{~s}, 18 \mathrm{H}) .{ }^{13} \mathrm{C}$ NMR (151 MHz, DMSO- $d 6$ ) $\delta 154.65,152.40,144.47,138.33,137.79,132.41,130.36$ (q, $J=31.7$ $\mathrm{Hz}), 128.96,127.82(\mathrm{q}, J=3.9 \mathrm{~Hz}), 127.46,124.29,123.41$ (q, $J=273.3 \mathrm{~Hz}), 120.24,119.74$, $114.06,110.26,92.27,88.58,55.15,33.98,31.0 .{ }^{19} \mathrm{~F}$ NMR (565 MHz, DMSO- $d_{6}$ ) $\delta-61.47$. HRMS (ESI-TOF-MS ${ }^{+}$) $m / z$ calcd for $\mathrm{C}_{47} \mathrm{H}_{46} \mathrm{~N}_{4} \mathrm{O}_{4} \mathrm{~F}_{3}[\mathrm{M}+\mathrm{H}]^{+}$787.3471, found 787.3457 . 
NMR Spectra.

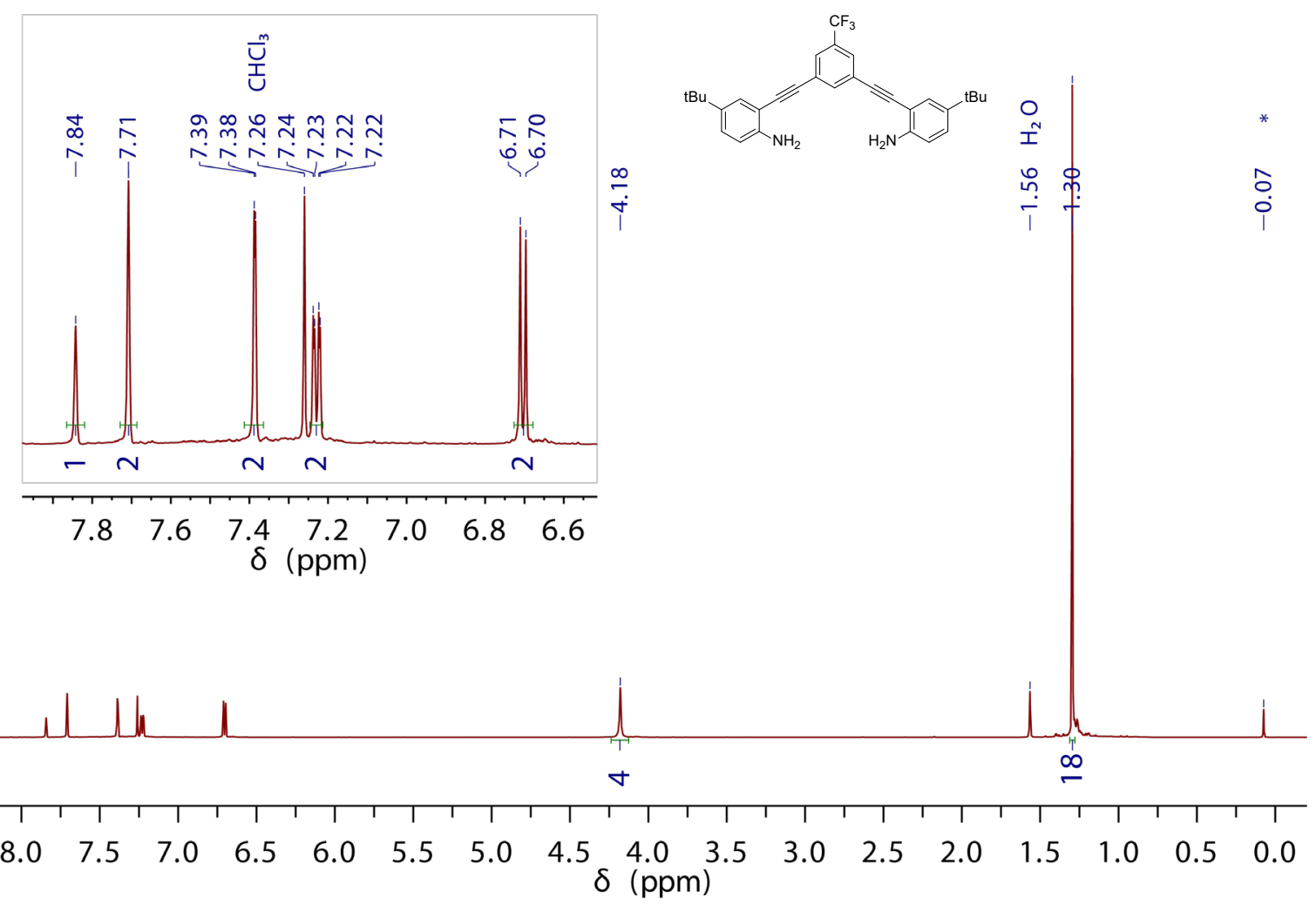

Figure S1. ${ }^{1} \mathrm{H}$ NMR spectrum of dianiline 5. * = silicon grease

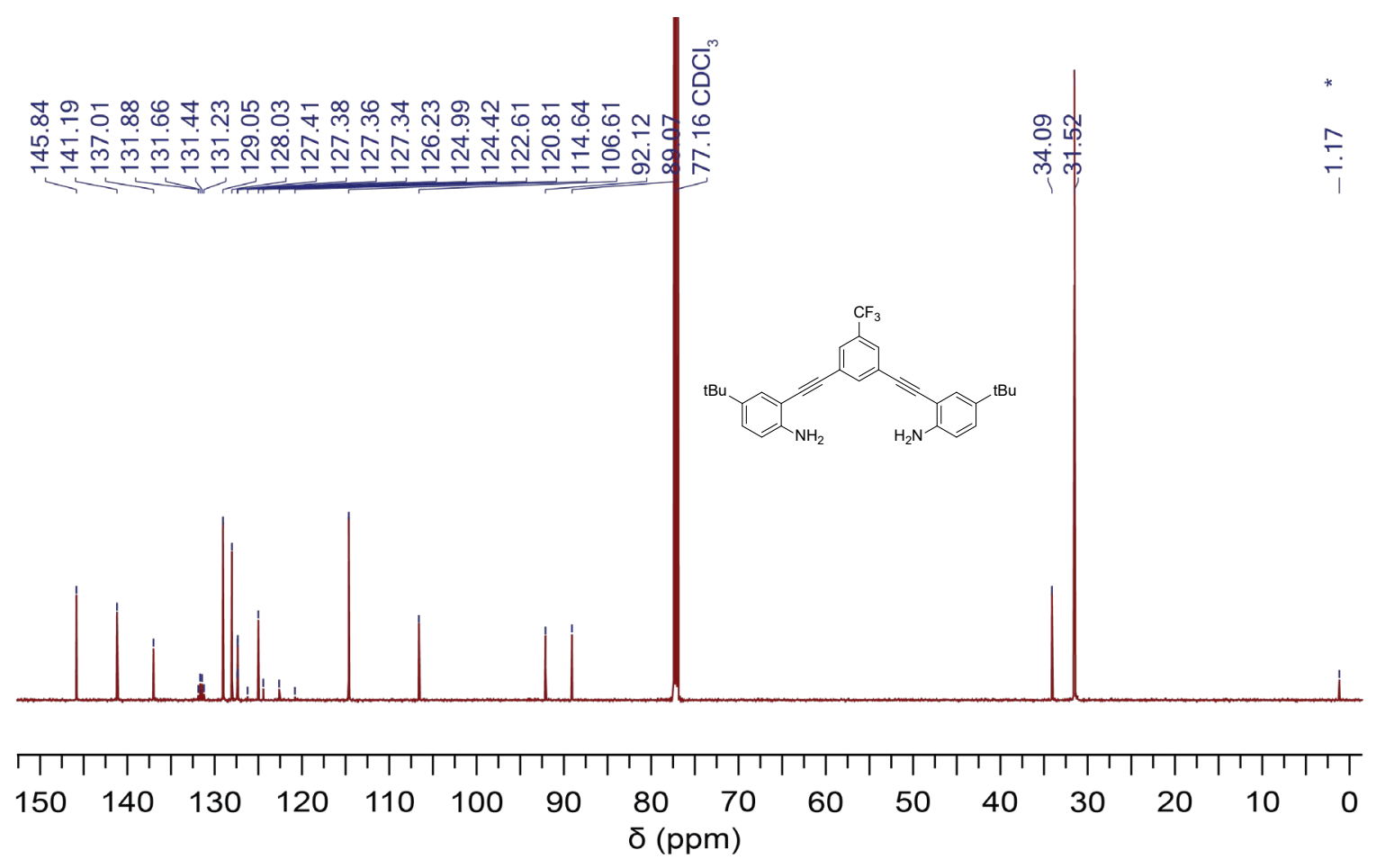

Figure S2. ${ }^{13} \mathrm{C}\left\{{ }^{1} \mathrm{H}\right\}$ NMR spectrum of dianiline 5. ${ }^{*}=$ silicon grease 


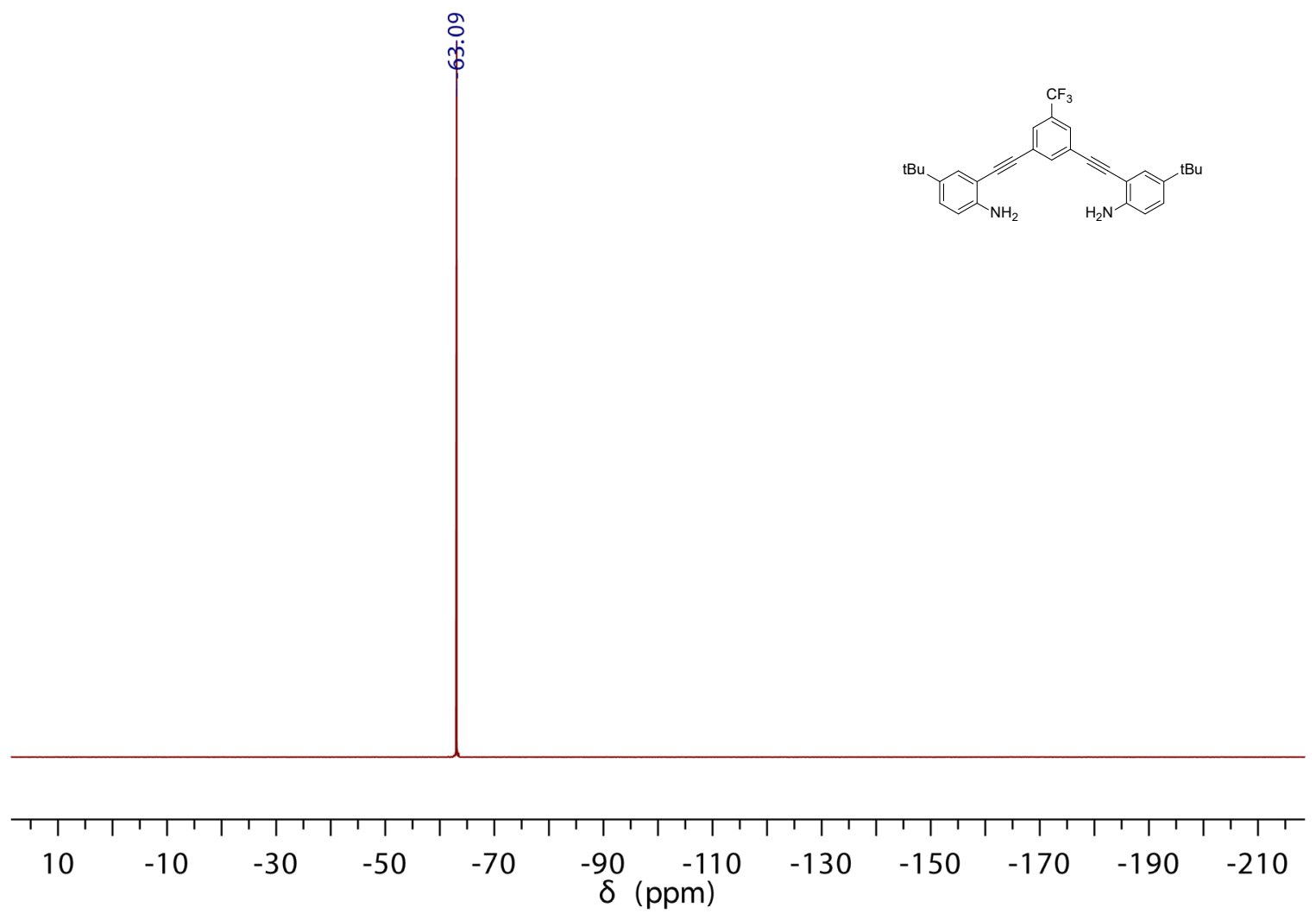

Figure S3. ${ }^{19} \mathrm{~F}$ NMR spectrum of dianiline $\mathbf{5}$.
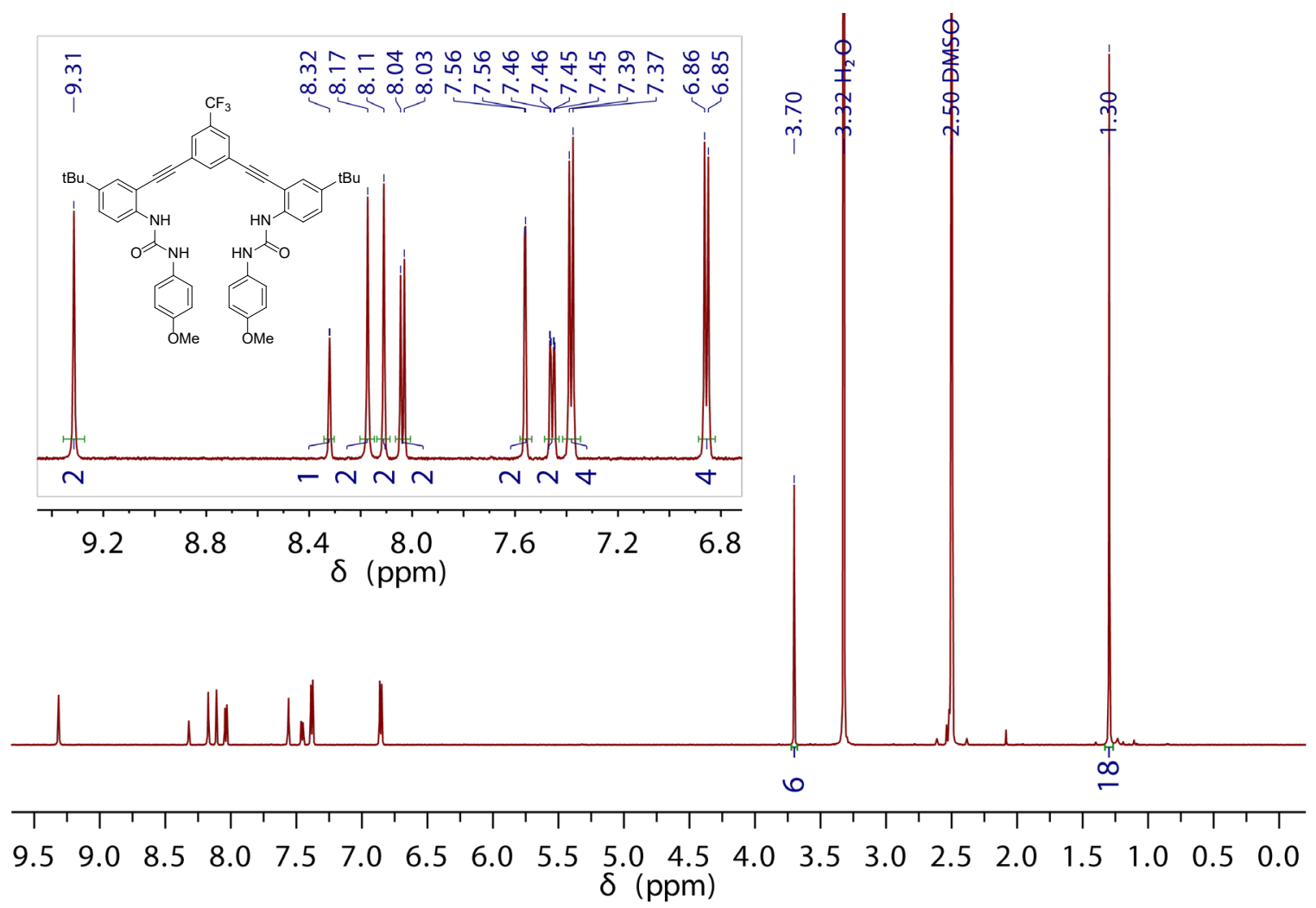

Figure S4. ${ }^{1} \mathrm{H}$ NMR spectrum of receptor $\mathbf{1}^{\text {CF3 }}$. 


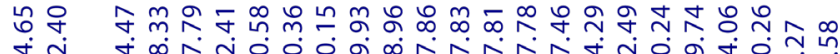

苩
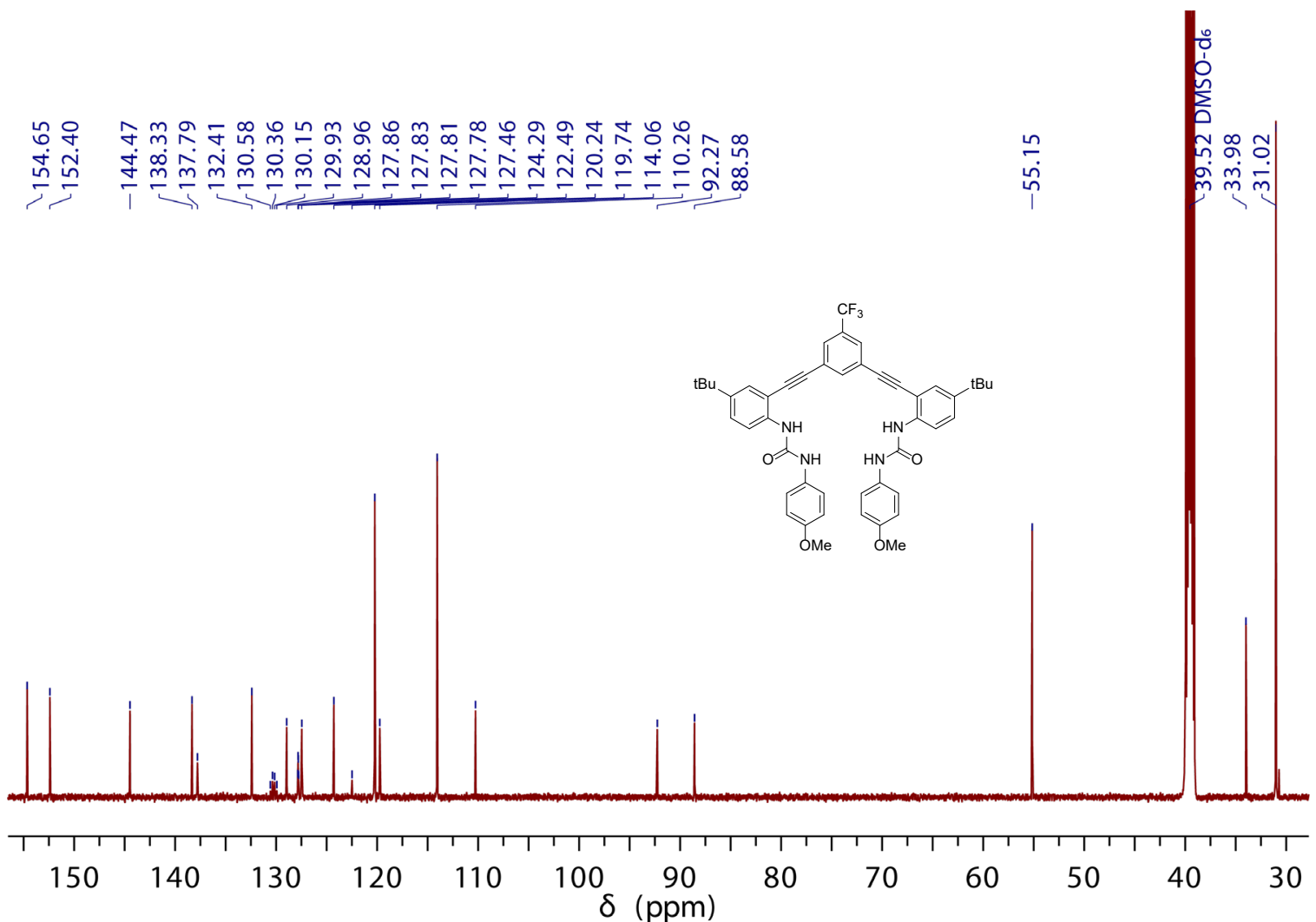

Figure S5. ${ }^{13} \mathrm{C}\left\{{ }^{1} \mathrm{H}\right\}$ NMR spectrum of receptor $\mathbf{1}^{\mathbf{C F} 3}$.

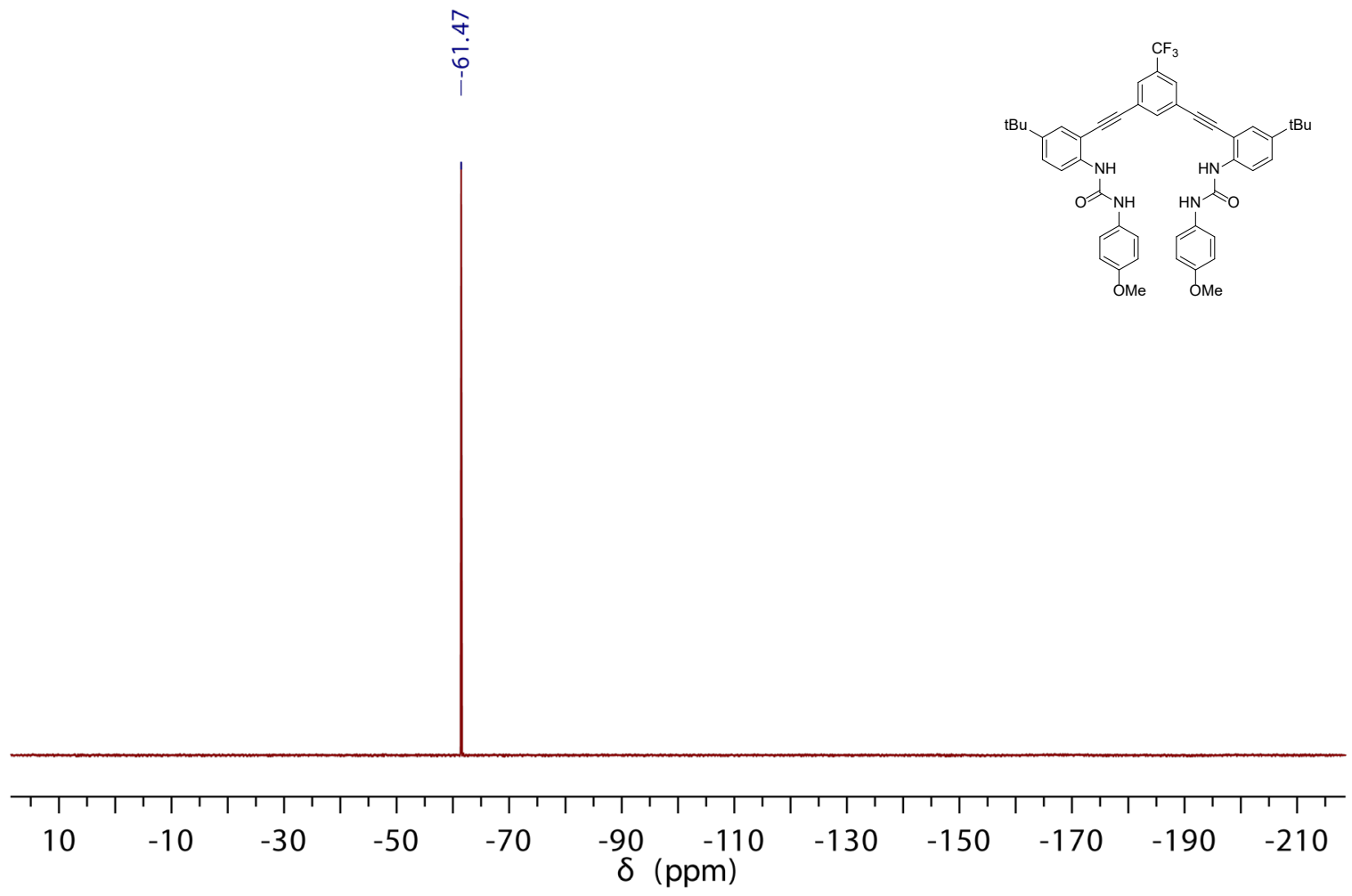

Figure S6. ${ }^{19} \mathrm{~F}$ NMR spectrum of receptor $\mathbf{1}^{\mathbf{C F} 3}$. 
Section S2. Linear free energy relationship (LFER) analysis of substituent effect on ${ }^{1} \mathrm{H}$ NMR chemical shift $(\delta)$ of free host hydrogen bond (HB) donors at $0.8-2.2 \mathrm{mM}$ in $10 \%$ DMSO- $d_{6} /$ $\mathrm{CD}_{3} \mathrm{CN}$ at $25^{\circ} \mathrm{C}$.

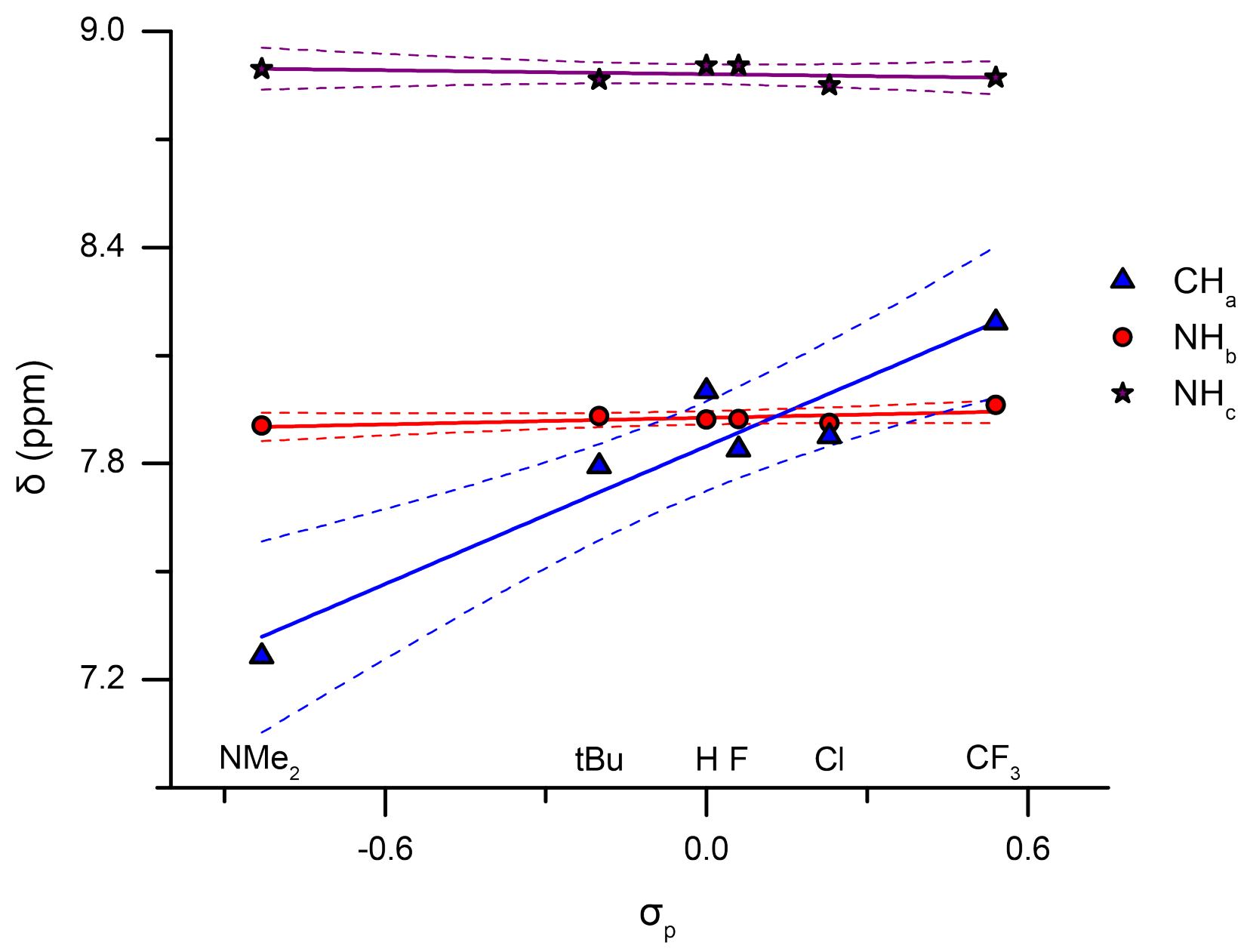

Figure S7. LFER between $\delta$ of HB donors in free host $\mathbf{1}^{\mathbf{R}}$ and $\sigma_{\mathrm{p}}$. Dashed lines represent the $95 \%$ confidence interval for each linear trend.

Table S1. Fitting statistics for the LFER between $\delta$ of HB donors in free host $\mathbf{1}^{\mathbf{R}}$ and $\sigma_{\mathrm{p}}$.

\begin{tabular}{|c|c|c|ccc|c|c|}
\hline \multirow{2}{*}{ HB Donor } & \multirow{2}{*}{ Slope } & \multirow{2}{*}{ Intercept } & \multicolumn{3}{|c|}{ p-value } & \multirow{2}{*}{$\mathbf{R}^{\mathbf{2}}$} & \multirow{2}{*}{$\mathbf{R}^{\mathbf{2}}{ }_{\text {adj. }}$} \\
\cline { 4 - 6 } & & & Slope & Intercept & Model & & \\
\hline $\mathbf{C H}_{\mathbf{a}}$ & $0.64 \pm 0.11$ & $7.85 \pm 0.04$ & $<0.01$ & $<0.01$ & $<0.01$ & 0.90 & 0.88 \\
$\mathbf{N H}_{\mathbf{b}}$ & $0.03 \pm 0.02$ & $7.93 \pm 0.01$ & 0.12 & $<0.01$ & 0.12 & 0.49 & 0.36 \\
$\mathbf{N H}_{\mathbf{c}}$ & $-0.02 \pm 0.02$ & $8.88 \pm 0.01$ & 0.48 & $<0.01$ & 0.48 & 0.13 & -0.09 \\
\hline
\end{tabular}




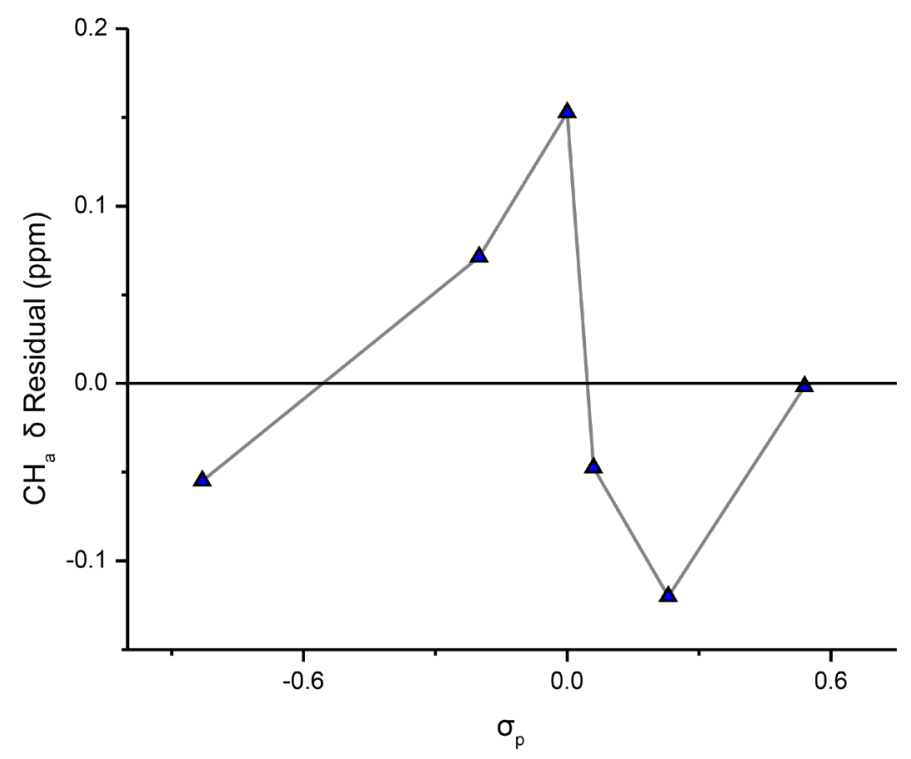

Figure S8. Linear regression residuals of $\delta$ of $\mathrm{CH}_{\mathrm{a}}$ in free host $\mathbf{1}^{\mathbf{R}}$ and $\sigma_{\mathrm{p}}$.

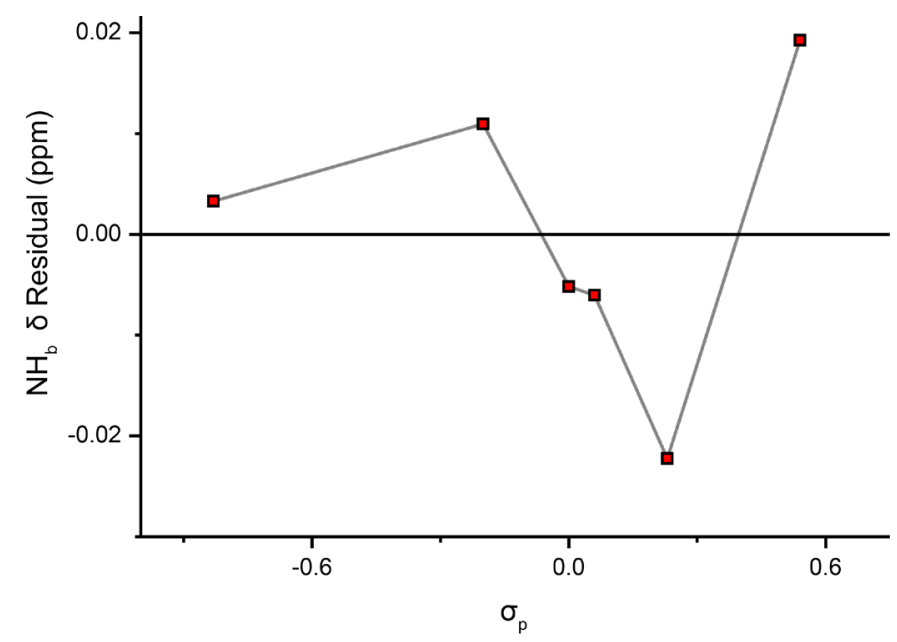

Figure S9. Linear regression residuals of $\delta$ of $\mathrm{NH}_{\mathrm{b}}$ in free host $\mathbf{1}^{\mathbf{R}}$ and $\sigma_{\mathrm{p}}$.

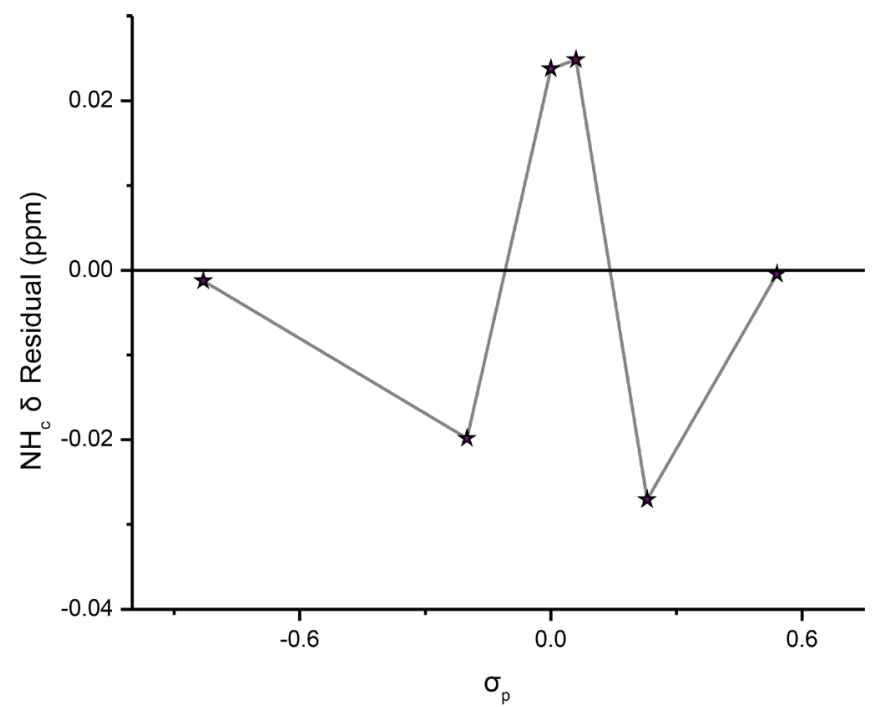

Figure S10. Linear regression residuals of $\delta$ of $\mathrm{NH}_{\mathrm{c}}$ in free host $\mathbf{1}^{\mathbf{R}}$ and $\sigma_{\mathrm{p}}$. 


\section{Section S3. ${ }^{1} \mathrm{H}$ NMR Titrations.}

General Methods. ${ }^{1} \mathrm{H}$ NMR spectra were acquired at room temperature on a Varian Inova $500 \mathrm{MHz}$ spectrometer $\left({ }^{1} \mathrm{H}: 500.11 \mathrm{MHz}\right) .{ }^{1} \mathrm{H}$ chemical shifts $(\delta)$ are expressed in ppm relative to residual $\mathrm{CH}_{3} \mathrm{CN}\left({ }^{1} \mathrm{H}: 1.94\right.$ ppm) shifts.

General Procedure for NMR Titrations. Method A. A solution of host in 10\% DMSO$d_{6} / \mathrm{CD}_{3} \mathrm{CN}(0.8-2.1 \mathrm{mM})$ was prepared and $500 \mu \mathrm{L}$ was added to a septum-sealed NMR tube. The remaining host solution $(2.5 \mathrm{~mL})$ was used to prepare a host/guest $(\mathrm{NBu} 4 \mathrm{Cl}$ or $\mathrm{NBu} 4 \mathrm{Br})(18.5-27.9$ $\mathrm{mM}$ ) stock solution. Aliquots of the host/guest solution were added to the NMR tube using Hamilton gas-tight syringes, and ${ }^{1} \mathrm{H}$ NMR spectra were recorded at $25{ }^{\circ} \mathrm{C}$ after each addition of guest. The $\Delta \delta$ of the various $\mathrm{NH}$ and aromatic $\mathrm{CH}$ protons were used to follow the progress of the titration, and association constants were determined using the Thordarson method. ${ }^{8}$

Method B. A solution of receptor host in $10 \%$ DMSO- $d_{6} / \mathrm{CD}_{3} \mathrm{CN}(1.8-2.2 \mathrm{mM})$ was prepared and $500 \mu \mathrm{L}$ was added to a septum-sealed NMR tube. A stock solution of guest $(\mathrm{NBu} 4 \mathrm{SH}$ or $\left.\mathrm{NBu}_{4} \mathrm{SeH}\right)$ was prepared in $10 \% \mathrm{DMSO}-d_{6} / \mathrm{CD}_{3} \mathrm{CN}(14.6-24.3 \mathrm{mM})$. Aliquots of the guest solution were added to the NMR tube using Hamilton gas-tight syringes, and ${ }^{1} \mathrm{H}$ NMR spectra were recorded at $25{ }^{\circ} \mathrm{C}$ after each addition of guest. The $\Delta \delta$ of the $\mathrm{NH}$ and the central aromatic $\mathrm{CH}$ proton was used to follow the progress of the titration, and association constants were determined using the Thordarson method. ${ }^{8}$ 
${ }^{1} H$ NMR Titration Representative Data

Table S2. Representative titration of receptor $1^{\mathrm{CF} 3}$ with $\mathrm{HS}^{-}$in $10 \% \mathrm{DMSO}-d_{6} / \mathrm{CD}_{3} \mathrm{CN}$ at $25{ }^{\circ} \mathrm{C}$.

\begin{tabular}{cccccccc} 
Entry & $\begin{array}{c}\mathrm{V}_{\text {Guest }} \\
(\mu \mathrm{L})\end{array}$ & $\begin{array}{c}{[\mathrm{Host}]} \\
(\mathrm{M})\end{array}$ & $\begin{array}{c}{\left[\mathrm{HS}^{-}\right]} \\
(\mathrm{M})\end{array}$ & $\begin{array}{c}\delta \mathrm{NH}_{\mathrm{c}} \\
(\mathrm{ppm})\end{array}$ & $\begin{array}{c}\delta \mathrm{NH}_{\mathrm{b}} \\
(\mathrm{ppm})\end{array}$ & $\begin{array}{c}\delta \mathrm{CH}_{\mathrm{a}} \\
(\mathrm{ppm})\end{array}$ \\
\hline 0 & 0 & $2.2 \mathrm{E}-03$ & $0.0 \mathrm{E}+00$ & 0.00 & 8.861 & 7.959 & 8.187 \\
1 & 5 & $2.2 \mathrm{E}-03$ & $2.4 \mathrm{E}-04$ & 0.11 & 9.12 & 8.013 & 8.351 \\
2 & 5 & $2.2 \mathrm{E}-03$ & $4.8 \mathrm{E}-04$ & 0.22 & 9.376 & 8.067 & 8.516 \\
3 & 5 & $2.2 \mathrm{E}-03$ & $7.1 \mathrm{E}-04$ & 0.33 & 9.628 & 8.119 & 8.68 \\
4 & 5 & $2.1 \mathrm{E}-03$ & $9.4 \mathrm{E}-04$ & 0.44 & 9.872 & 8.17 & 8.844 \\
5 & 5 & $2.1 \mathrm{E}-03$ & $1.2 \mathrm{E}-03$ & 0.55 & 10.118 & 8.22 & 8.996 \\
6 & 10 & $2.1 \mathrm{E}-03$ & $1.6 \mathrm{E}-03$ & 0.77 & 10.521 & 8.305 & 9.265 \\
7 & 10 & $2.0 \mathrm{E}-03$ & $2.0 \mathrm{E}-03$ & 0.98 & 10.827 & 8.366 & 9.471 \\
8 & 10 & $2.0 \mathrm{E}-03$ & $2.4 \mathrm{E}-03$ & 1.20 & 11 & 8.408 & 9.574 \\
9 & 10 & $2.0 \mathrm{E}-03$ & $2.8 \mathrm{E}-03$ & 1.42 & 11.093 & 8.438 & 9.618 \\
10 & 10 & $1.9 \mathrm{E}-03$ & $3.2 \mathrm{E}-03$ & 1.64 & 11.143 & 8.449 & 9.654 \\
11 & 10 & $1.9 \mathrm{E}-03$ & $3.5 \mathrm{E}-03$ & 1.86 & 11.174 & 8.469 & 9.667 \\
12 & 30 & $1.8 \mathrm{E}-03$ & $4.5 \mathrm{E}-03$ & 2.51 & 11.222 & 8.485 & 9.681 \\
13 & 70 & $1.6 \mathrm{E}-03$ & $6.6 \mathrm{E}-03$ & 4.05 & 11.268 & 8.522 & 9.681
\end{tabular}
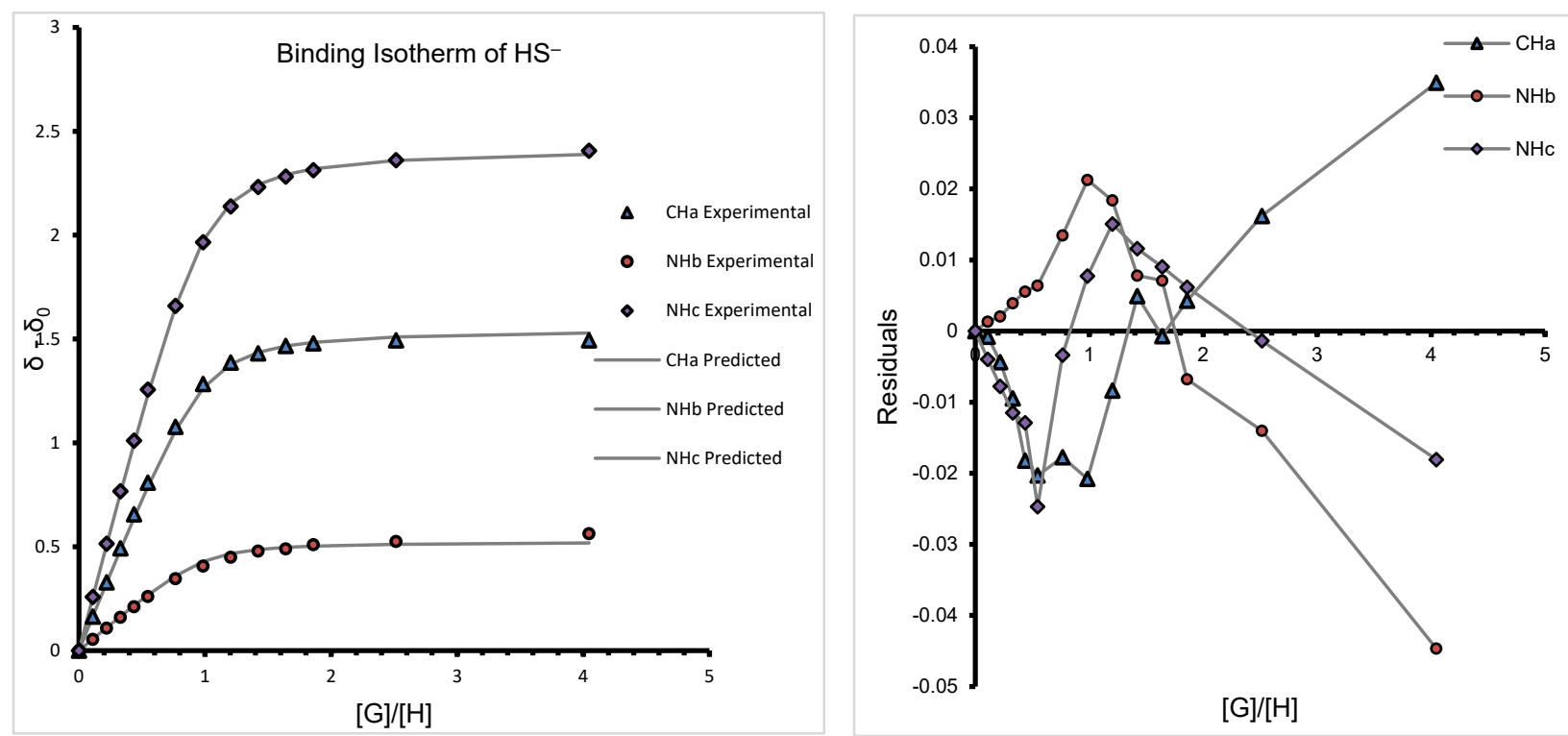

Figure S11. Representative binding isotherm and residuals for $\mathrm{HS}^{-}$titration of receptor $\mathbf{1}^{\mathrm{CF} 3}$ in $10 \%$ DMSO- $d_{6} / \mathrm{CD}_{3} \mathrm{CN}$ at $25{ }^{\circ} \mathrm{C}$ determined by ${ }^{1} \mathrm{H}$ NMR spectroscopy. 
Table S3. Representative titration of receptor $\mathbf{1}^{\mathrm{CF} 3}$ with $\mathrm{HSe}^{-}$in $10 \%$ DMSO- $d_{6} / \mathrm{CD}_{3} \mathrm{CN}$ at $25^{\circ} \mathrm{C}$.

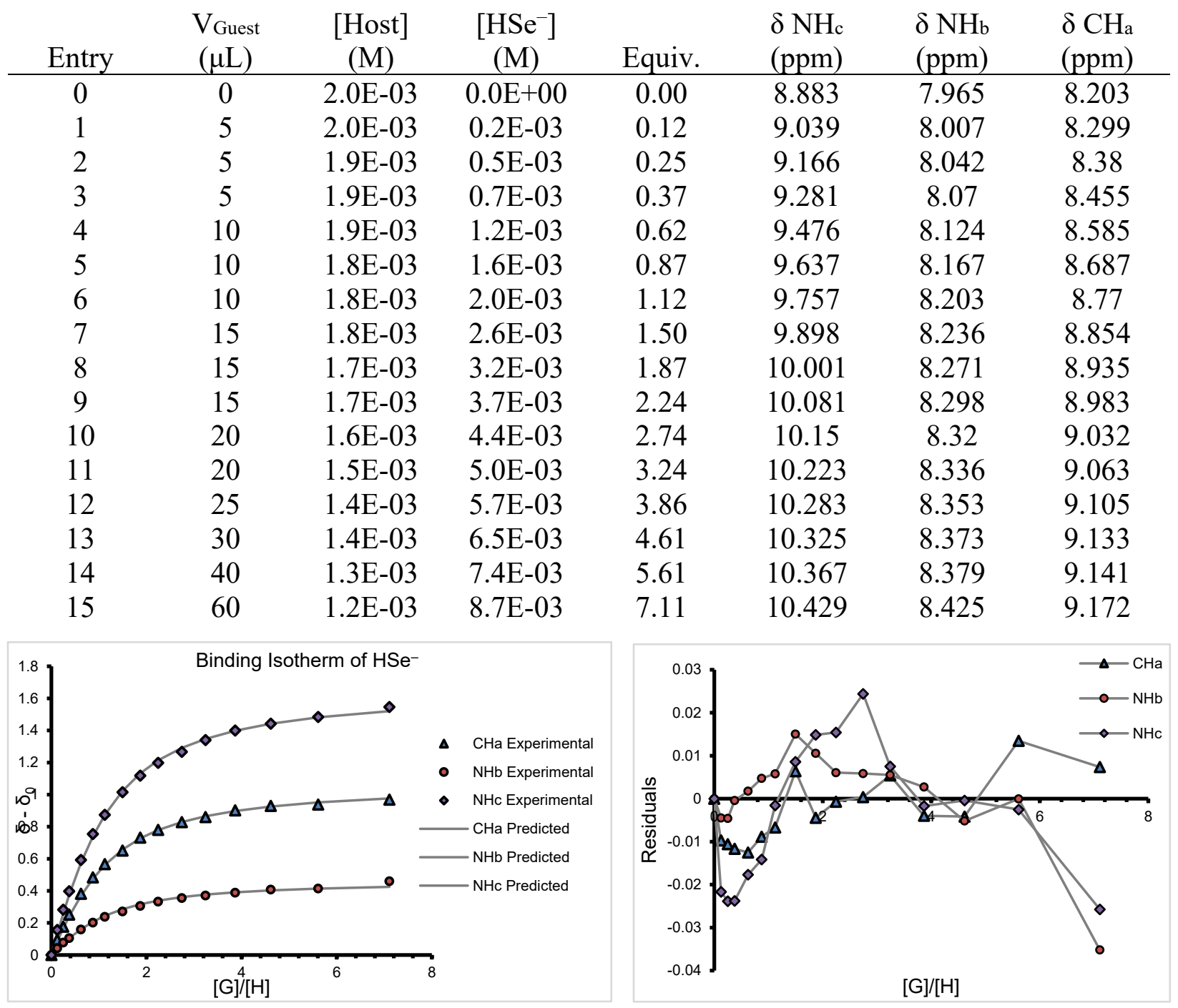

Figure S12. Representative binding isotherm and residuals for $\mathrm{HSe}^{-}$titration of receptor $\mathbf{1}^{\mathrm{CF} 3}$ in $10 \%$ DMSO- $d_{6} / \mathrm{CD}_{3} \mathrm{CN}$ at $25{ }^{\circ} \mathrm{C}$ determined by ${ }^{1} \mathrm{H}$ NMR spectroscopy. 
Table S4. Representative titration of receptor $\mathbf{1}^{\mathrm{CF} 3}$ with $\mathrm{Cl}^{-}$in $10 \%$ DMSO- $d_{6} / \mathrm{CD}_{3} \mathrm{CN}$ at $25{ }^{\circ} \mathrm{C}$.

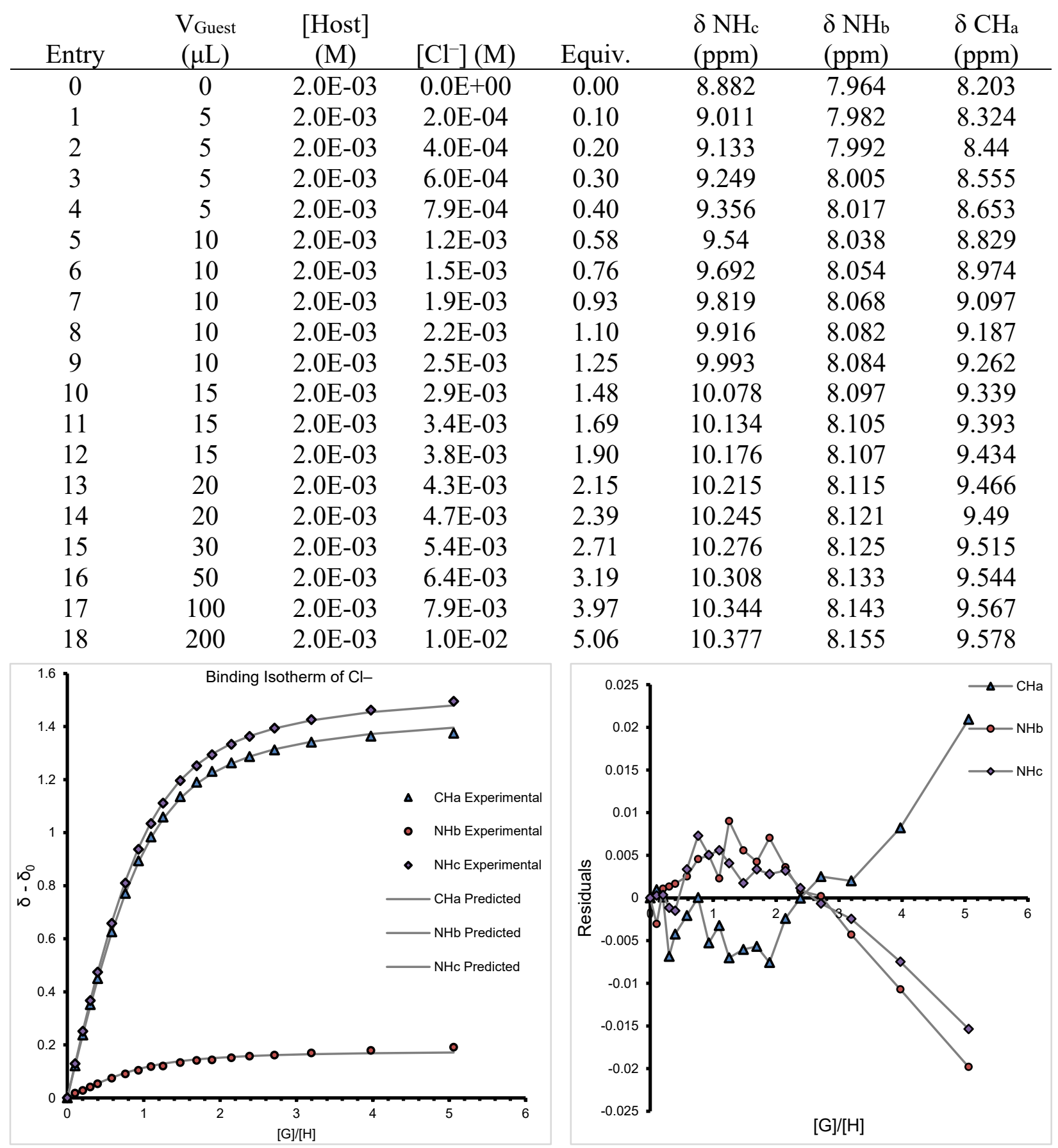

Figure S13. Representative binding isotherm and residuals for $\mathrm{Cl}^{-}$titration of receptor $\mathbf{1}^{\mathrm{CF} 3}$ in $10 \%$ DMSO- $d_{6} / \mathrm{CD}_{3} \mathrm{CN}$ at $25{ }^{\circ} \mathrm{C}$ determined by ${ }^{1} \mathrm{H}$ NMR spectroscopy. 
Table S5. Representative titration of receptor $1^{\mathrm{CF} 3}$ with $\mathrm{Br}^{-}$in $10 \%$ DMSO- $d_{6} / \mathrm{CD}_{3} \mathrm{CN}$ at $25^{\circ} \mathrm{C}$.

\begin{tabular}{cccccccc} 
Entry & $\begin{array}{c}\mathrm{V}_{\text {Guest }} \\
(\mu \mathrm{L})\end{array}$ & $\begin{array}{c}{[\mathrm{Host}]} \\
(\mathrm{M})\end{array}$ & $\begin{array}{c}\left.\mathrm{Br}^{-}\right](\mathrm{M}) \\
\text { Equiv. }\end{array}$ & $\begin{array}{c}\delta \mathrm{NH}_{\mathrm{c}} \\
(\mathrm{ppm})\end{array}$ & $\begin{array}{c}\delta \mathrm{NH}_{\mathrm{b}} \\
(\mathrm{ppm})\end{array}$ & $\begin{array}{c}\delta \mathrm{CH}_{\mathrm{a}} \\
(\mathrm{ppm})\end{array}$ \\
\hline 0 & 0 & $2.0 \mathrm{E}-03$ & $0.0 \mathrm{E}+00$ & 0.00 & 8.869 & 7.961 & 8.192 \\
1 & 5 & $2.0 \mathrm{E}-03$ & $2.8 \mathrm{E}-04$ & 0.14 & 8.921 & 7.969 & 8.247 \\
2 & 10 & $2.0 \mathrm{E}-03$ & $8.1 \mathrm{E}-04$ & 0.42 & 9.009 & 7.985 & 8.34 \\
3 & 15 & $2.0 \mathrm{E}-03$ & $1.6 \mathrm{E}-03$ & 0.81 & 9.119 & 7.996 & 8.457 \\
4 & 20 & $2.0 \mathrm{E}-03$ & $2.5 \mathrm{E}-03$ & 1.30 & 9.243 & 8.011 & 8.586 \\
5 & 20 & $2.0 \mathrm{E}-03$ & $3.4 \mathrm{E}-03$ & 1.75 & 9.34 & 8.025 & 8.689 \\
6 & 25 & $2.0 \mathrm{E}-03$ & $4.5 \mathrm{E}-03$ & 2.28 & 9.438 & 8.035 & 8.787 \\
7 & 30 & $2.0 \mathrm{E}-03$ & $5.6 \mathrm{E}-03$ & 2.85 & 9.526 & 8.047 & 8.877 \\
8 & 35 & $2.0 \mathrm{E}-03$ & $6.8 \mathrm{E}-03$ & 3.46 & 9.603 & 8.06 & 8.953 \\
9 & 40 & $2.0 \mathrm{E}-03$ & $8.0 \mathrm{E}-03$ & 4.08 & 9.669 & 8.064 & 9.017 \\
10 & 45 & $2.0 \mathrm{E}-03$ & $9.2 \mathrm{E}-03$ & 4.69 & 9.725 & 8.072 & 9.07 \\
11 & 50 & $2.0 \mathrm{E}-03$ & $1.0 \mathrm{E}-02$ & 5.29 & 9.772 & 8.079 & 9.114 \\
12 & 60 & $2.0 \mathrm{E}-03$ & $1.2 \mathrm{E}-02$ & 5.92 & 9.817 & 8.086 & 9.155 \\
13 & 70 & $2.0 \mathrm{E}-03$ & $1.3 \mathrm{E}-02$ & 6.55 & 9.856 & 8.093 & 9.191 \\
14 & 80 & $2.0 \mathrm{E}-03$ & $1.4 \mathrm{E}-02$ & 7.17 & 9.889 & 8.097 & 9.217 \\
15 & 100 & $2.0 \mathrm{E}-03$ & $1.5 \mathrm{E}-02$ & 7.81 & 9.913 & 8.102 & 9.239 \\
16 & 130 & $2.0 \mathrm{E}-03$ & $1.7 \mathrm{E}-02$ & 8.49 & 9.938 & 8.108 & 9.257 \\
17 & 140 & $2.0 \mathrm{E}-03$ & $1.8 \mathrm{E}-02$ & 9.08 & 9.956 & 8.11 & 9.273
\end{tabular}
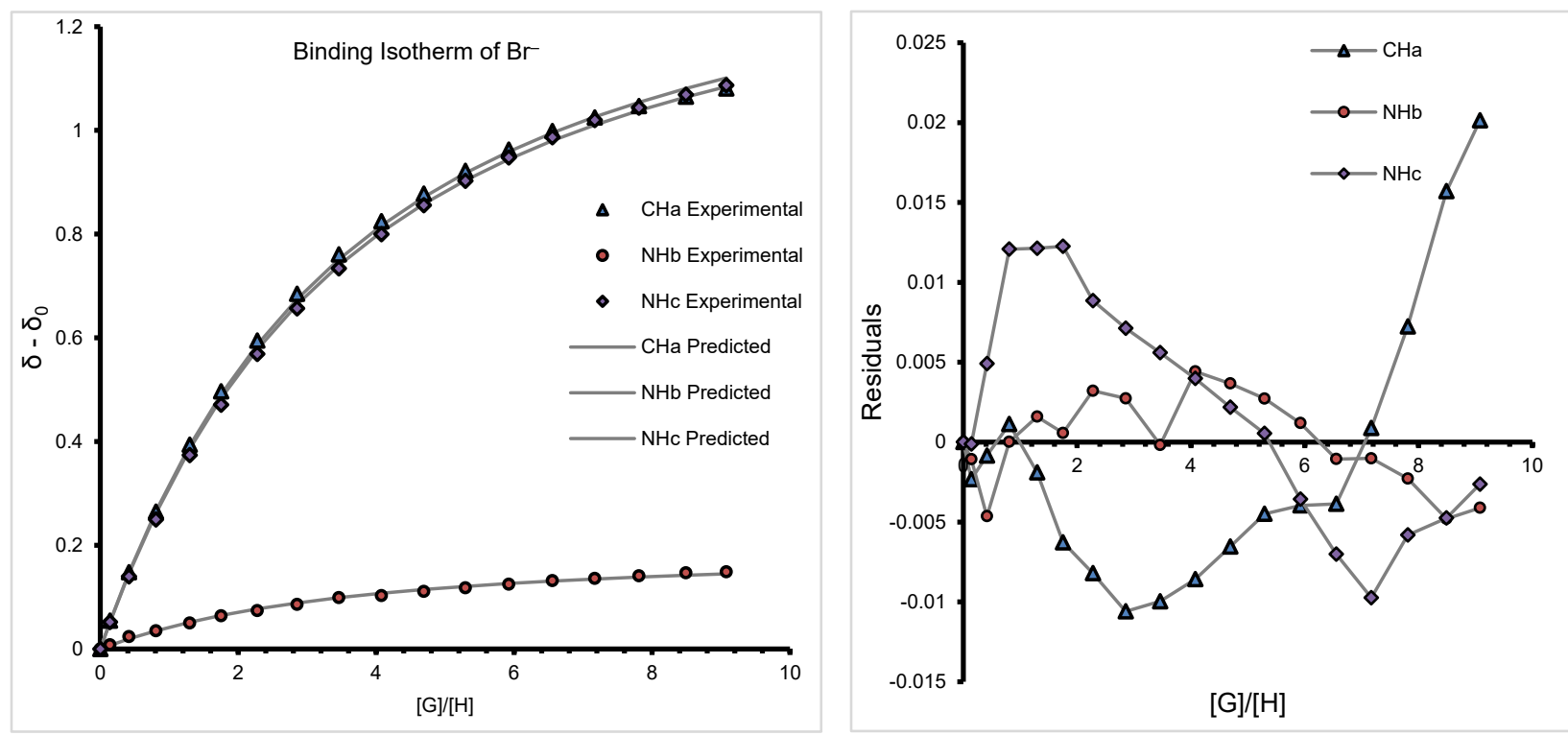

Figure S14. Representative binding isotherm and residuals for $\mathrm{Br}^{-}$titration of receptor $\mathbf{1}^{\mathrm{CF} 3}$ in $10 \%$ DMSO- $d_{6} / \mathrm{CD}_{3} \mathrm{CN}$ at $25{ }^{\circ} \mathrm{C}$ determined by ${ }^{1} \mathrm{H}$ NMR spectroscopy. 
Table S6. Representative titration of receptor $\mathbf{1}^{\mathrm{Cl}}$ with $\mathrm{HS}^{-}$in $10 \%$ DMSO- $d_{6} / \mathrm{CD}_{3} \mathrm{CN}$ at $25^{\circ} \mathrm{C}$.

\begin{tabular}{cccccccc} 
Entry & $\begin{array}{c}\mathrm{V}_{\text {Guest }} \\
(\mu \mathrm{L})\end{array}$ & $\begin{array}{c}{[\mathrm{Host}]} \\
(\mathrm{M})\end{array}$ & $\begin{array}{c}{\left[\mathrm{HS}^{-}\right]} \\
(\mathrm{M})\end{array}$ & $\begin{array}{c}\delta \mathrm{NH}_{\mathrm{c}} \\
\text { Equiv. }\end{array}$ & $\begin{array}{c}\delta \mathrm{NH}_{\mathrm{b}} \\
(\mathrm{ppm})\end{array}$ & $\begin{array}{c}\delta \mathrm{CH}_{\mathrm{a}} \\
(\mathrm{ppm})\end{array}$ \\
\hline 0 & 0 & $2.0 \mathrm{E}-03$ & $0.0 \mathrm{E}+00$ & 0.00 & 8.874 & 7.923 & 7.879 \\
1 & 5 & $2.0 \mathrm{E}-03$ & $2.0 \mathrm{E}-04$ & 0.10 & 9.086 & 7.972 & 8.011 \\
2 & 5 & $2.0 \mathrm{E}-03$ & $3.9 \mathrm{E}-04$ & 0.20 & 9.302 & 8.018 & 8.139 \\
3 & 5 & $2.0 \mathrm{E}-03$ & $5.8 \mathrm{E}-04$ & 0.29 & 9.514 & 8.063 & 8.269 \\
4 & 5 & $2.0 \mathrm{E}-03$ & $7.7 \mathrm{E}-04$ & 0.39 & 9.712 & 8.112 & 8.392 \\
5 & 5 & $1.9 \mathrm{E}-03$ & $9.5 \mathrm{E}-04$ & 0.49 & 9.903 & 8.158 & 8.51 \\
6 & 10 & $1.9 \mathrm{E}-03$ & $1.3 \mathrm{E}-03$ & 0.69 & 10.25 & 8.243 & 8.72 \\
7 & 10 & $1.9 \mathrm{E}-03$ & $1.6 \mathrm{E}-03$ & 0.88 & 10.534 & 8.301 & 8.901 \\
8 & 10 & $1.8 \mathrm{E}-03$ & $2.0 \mathrm{E}-03$ & 1.08 & 10.737 & 8.353 & 9.023 \\
9 & 10 & $1.8 \mathrm{E}-03$ & $2.3 \mathrm{E}-03$ & 1.27 & 10.878 & 8.393 & 9.103 \\
10 & 15 & $1.8 \mathrm{E}-03$ & $2.8 \mathrm{E}-03$ & 1.57 & 11.008 & 8.423 & 9.172 \\
11 & 15 & $1.7 \mathrm{E}-03$ & $3.2 \mathrm{E}-03$ & 1.86 & 11.048 & 8.457 & 9.209 \\
12 & 15 & $1.7 \mathrm{E}-03$ & $3.6 \mathrm{E}-03$ & 2.16 & 11.078 & 8.489 & 9.203 \\
13 & 20 & $1.6 \mathrm{E}-03$ & $4.1 \mathrm{E}-03$ & 2.55 & 11.114 & 8.531 & 9.209 \\
14 & 40 & $1.5 \mathrm{E}-03$ & $5.1 \mathrm{E}-03$ & 3.33 & 11.154 & 8.539 & 9.232 \\
15 & 80 & $1.4 \mathrm{E}-03$ & $6.7 \mathrm{E}-03$ & 4.90 & 11.188 & 8.573 & 9.219
\end{tabular}
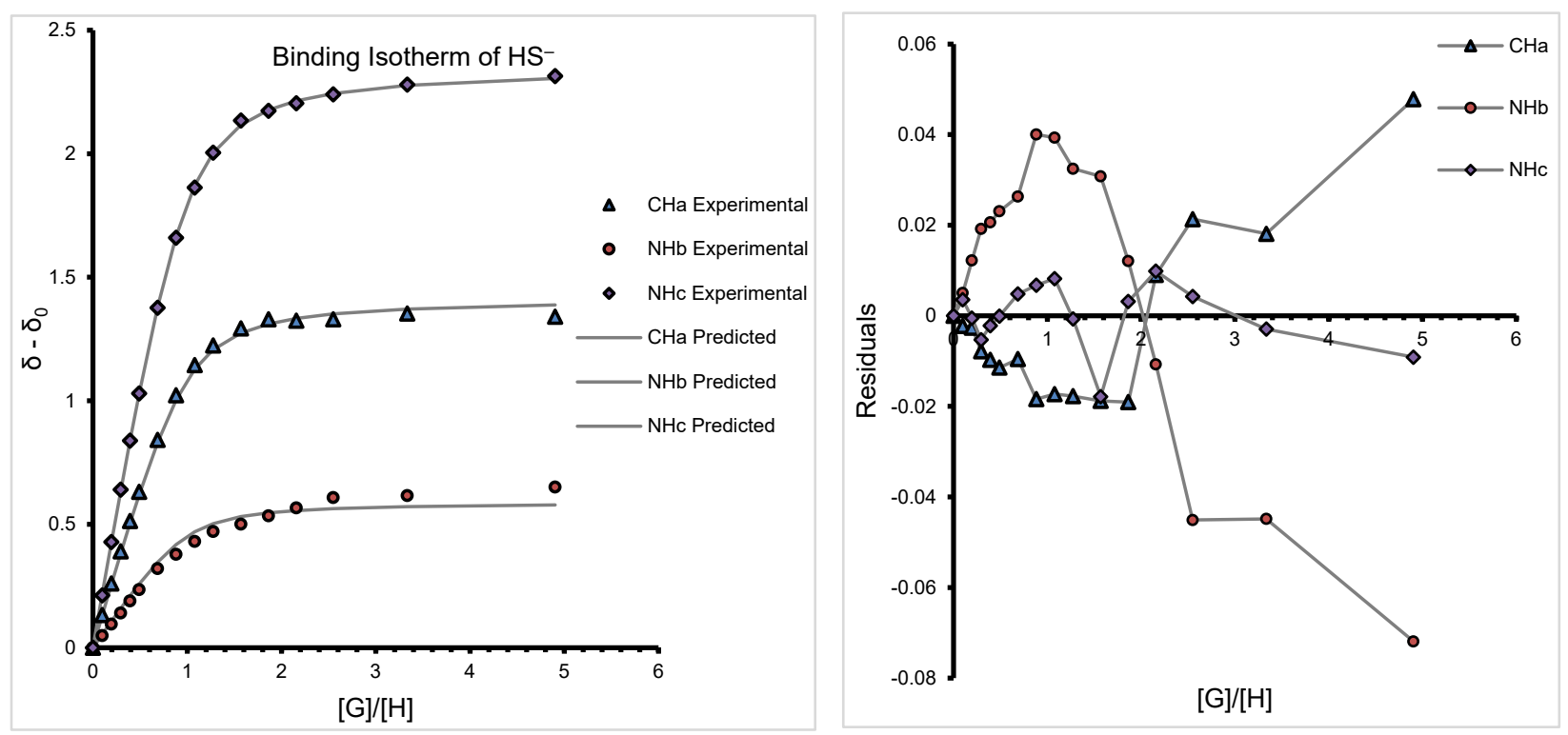

Figure S15. Representative binding isotherm and residuals for $\mathrm{HS}^{-}$titration of receptor $\mathbf{1}^{\mathrm{Cl}}$ in $10 \%$ DMSO- $d_{6} / \mathrm{CD}_{3} \mathrm{CN}$ at $25{ }^{\circ} \mathrm{C}$ determined by ${ }^{1} \mathrm{H}$ NMR spectroscopy. 
Table S7. Representative titration of receptor $1^{\mathrm{Cl}}$ with $\mathrm{HSe}^{-}$in $10 \%$ DMSO- $d_{6} / \mathrm{CD}_{3} \mathrm{CN}$ at $25{ }^{\circ} \mathrm{C}$.

\begin{tabular}{cccccccc} 
Entry & $\begin{array}{c}\mathrm{V}_{\text {Guest }} \\
(\mu \mathrm{L})\end{array}$ & $\begin{array}{c}{[\mathrm{Host}]} \\
(\mathrm{M})\end{array}$ & $\begin{array}{c}{\left[\mathrm{HSe}{ }^{-}\right]} \\
(\mathrm{M})\end{array}$ & Equiv. & $\begin{array}{c}\delta \mathrm{NH}_{\mathrm{c}} \\
(\mathrm{ppm})\end{array}$ & $\begin{array}{c}\delta \mathrm{NH}_{\mathrm{b}} \\
(\mathrm{ppm})\end{array}$ & $\begin{array}{c}\delta \mathrm{CH}_{\mathrm{a}} \\
(\mathrm{ppm})\end{array}$ \\
\hline 0 & 0 & $1.8 \mathrm{E}-03$ & $0.0 \mathrm{E}+00$ & 0.00 & 8.869 & 7.92 & 7.88 \\
1 & 5 & $1.8 \mathrm{E}-03$ & $1.7 \mathrm{E}-04$ & 0.10 & 8.957 & 7.943 & 7.934 \\
2 & 5 & $1.8 \mathrm{E}-03$ & $3.3 \mathrm{E}-04$ & 0.19 & 9.054 & 7.973 & 7.991 \\
3 & 5 & $1.7 \mathrm{E}-03$ & $5.0 \mathrm{E}-04$ & 0.29 & 9.125 & 7.993 & 8.033 \\
4 & 5 & $1.7 \mathrm{E}-03$ & $6.6 \mathrm{E}-04$ & 0.38 & 9.196 & 8.012 & 8.068 \\
5 & 5 & $1.7 \mathrm{E}-03$ & $8.1 \mathrm{E}-04$ & 0.48 & 9.257 & 8.036 & 8.112 \\
6 & 10 & $1.7 \mathrm{E}-03$ & $1.1 \mathrm{E}-03$ & 0.67 & 9.372 & 8.064 & 8.175 \\
7 & 10 & $1.6 \mathrm{E}-03$ & $1.4 \mathrm{E}-03$ & 0.86 & 9.456 & 8.092 & 8.227 \\
8 & 10 & $1.6 \mathrm{E}-03$ & $1.7 \mathrm{E}-03$ & 1.05 & 9.543 & 8.118 & 8.279 \\
9 & 10 & $1.6 \mathrm{E}-03$ & $2.0 \mathrm{E}-03$ & 1.24 & 9.615 & 8.14 & 8.32 \\
10 & 15 & $1.5 \mathrm{E}-03$ & $2.4 \mathrm{E}-03$ & 1.52 & 9.705 & 8.169 & 8.377 \\
11 & 15 & $1.5 \mathrm{E}-03$ & $2.7 \mathrm{E}-03$ & 1.81 & 9.789 & 8.194 & 8.418 \\
12 & 15 & $1.5 \mathrm{E}-03$ & $3.1 \mathrm{E}-03$ & 2.09 & 9.853 & 8.214 & 8.461 \\
13 & 20 & $1.4 \mathrm{E}-03$ & $3.5 \mathrm{E}-03$ & 2.47 & 9.925 & 8.239 & 8.503 \\
14 & 20 & $1.4 \mathrm{E}-03$ & $3.9 \mathrm{E}-03$ & 2.86 & 9.978 & 8.258 & 8.534 \\
15 & 25 & $1.3 \mathrm{E}-03$ & $4.4 \mathrm{E}-03$ & 3.33 & 10.036 & 8.281 & 8.568 \\
16 & 35 & $1.3 \mathrm{E}-03$ & $5.0 \mathrm{E}-03$ & 4.00 & 10.11 & 8.3 & 8.603 \\
17 & 60 & $1.2 \mathrm{E}-03$ & $6.0 \mathrm{E}-03$ & 5.14 & 10.182 & 8.336 & 8.642 \\
18 & 100 & $1.0 \mathrm{E}-03$ & $7.3 \mathrm{E}-03$ & 7.04 & 10.244 & 8.362 & 8.676
\end{tabular}
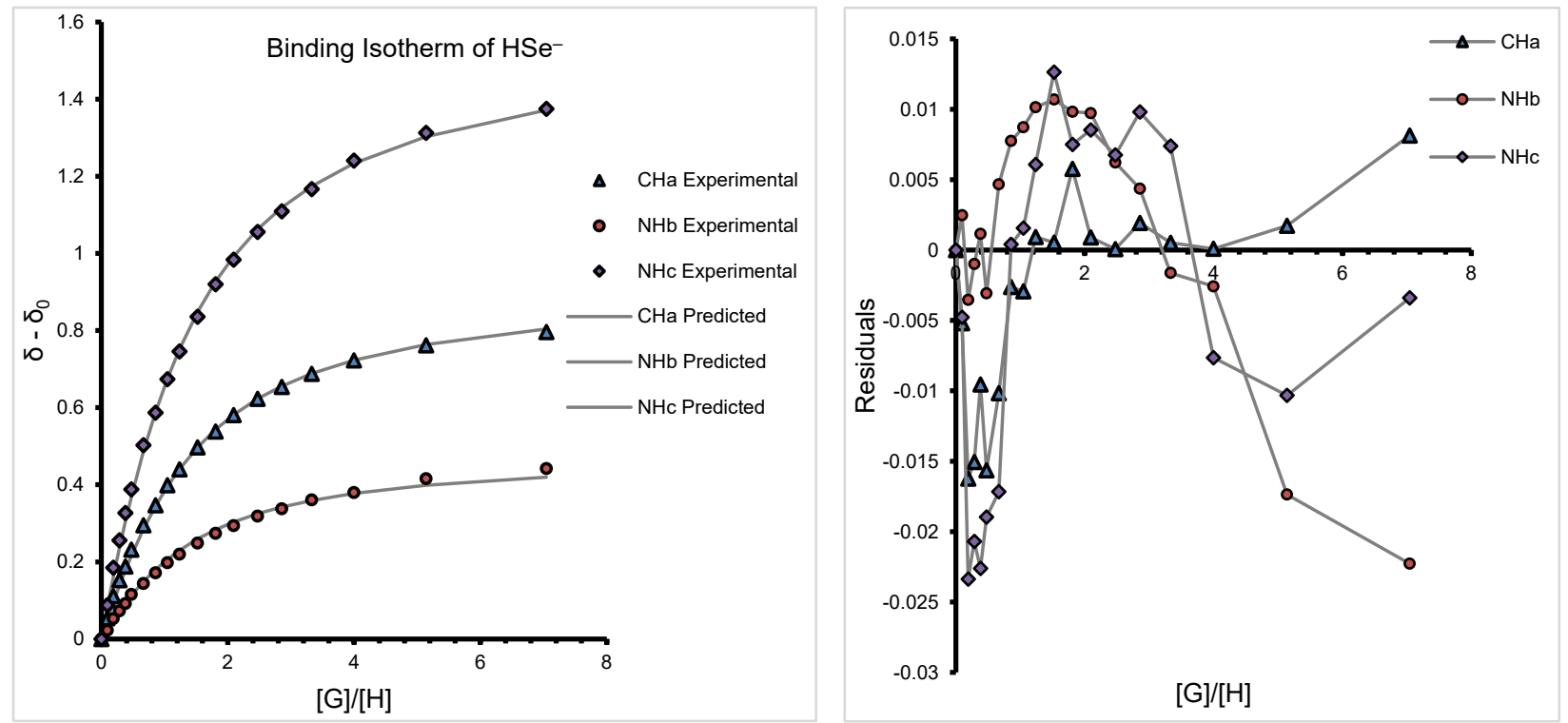

Figure S16. Representative binding isotherm and residuals for $\mathrm{HSe}^{-}$titration of receptor $\mathbf{1}^{\mathrm{Cl}}$ in $10 \%$ DMSO- $d_{6} / \mathrm{CD}_{3} \mathrm{CN}$ at $25{ }^{\circ} \mathrm{C}$ determined by ${ }^{1} \mathrm{H}$ NMR spectroscopy. 
Table S8. Representative titration of receptor $1^{\mathrm{Cl}}$ with $\mathrm{Cl}^{-}$in $10 \%$ DMSO- $d_{6} / \mathrm{CD}_{3} \mathrm{CN}$ at $25^{\circ} \mathrm{C}$.

\begin{tabular}{|c|c|c|c|c|c|c|c|}
\hline Entry & $\begin{array}{l}V_{\text {Guest }} \\
(\mu \mathrm{L})\end{array}$ & $\begin{array}{c}{[\text { Host }]} \\
(\mathrm{M})\end{array}$ & {$\left[\mathrm{Cl}^{-}\right](\mathrm{M})$} & Equiv. & $\begin{array}{l}\delta \mathrm{NH}_{\mathrm{c}} \\
(\mathrm{ppm})\end{array}$ & $\begin{array}{l}\delta \mathrm{NH}_{\mathrm{b}} \\
(\mathrm{ppm})\end{array}$ & $\begin{array}{l}\delta \mathrm{CH}_{\mathrm{a}} \\
(\mathrm{ppm})\end{array}$ \\
\hline 0 & 0 & $8.2 \mathrm{E}-04$ & $0.0 \mathrm{E}+00$ & 0.00 & 8.859 & 7.912 & 7.89 \\
\hline 1 & 5 & $8.2 \mathrm{E}-04$ & $2.3 \mathrm{E}-04$ & 0.28 & 9.166 & 7.952 & 8.174 \\
\hline 2 & 5 & $8.2 \mathrm{E}-04$ & 4.6E-04 & 0.56 & 9.37 & 7.977 & 8.363 \\
\hline 3 & 5 & $8.2 \mathrm{E}-04$ & $6.8 \mathrm{E}-04$ & 0.83 & 9.551 & 8.001 & 8.531 \\
\hline 4 & 10 & 8.2E-04 & $1.1 \mathrm{E}-03$ & 1.35 & 9.794 & 8.032 & 8.756 \\
\hline 5 & 10 & 8.2E-04 & $1.5 \mathrm{E}-03$ & 1.86 & 9.935 & 8.05 & 8.885 \\
\hline 6 & 10 & 8.2E-04 & $1.9 \mathrm{E}-03$ & 2.35 & 10.026 & 8.063 & 8.967 \\
\hline 7 & 15 & $8.2 \mathrm{E}-04$ & $2.5 \mathrm{E}-03$ & 3.04 & 10.112 & 8.075 & 9.044 \\
\hline 8 & 15 & 8.2E-04 & $3.1 \mathrm{E}-03$ & 3.71 & 10.167 & 8.086 & 9.089 \\
\hline 9 & 15 & 8.2E-04 & $3.6 \mathrm{E}-03$ & 4.33 & 10.204 & 8.09 & 9.121 \\
\hline 10 & 20 & 8.2E-04 & $4.2 \mathrm{E}-03$ & 5.12 & 10.24 & 8.098 & 9.15 \\
\hline 11 & 25 & $8.2 \mathrm{E}-04$ & $5.0 \mathrm{E}-03$ & 6.04 & 10.269 & 8.105 & 9.172 \\
\hline 12 & 30 & $8.2 \mathrm{E}-04$ & $5.8 \mathrm{E}-03$ & 7.05 & 10.294 & 8.112 & 9.187 \\
\hline 13 & 40 & 8.2E-04 & $6.8 \mathrm{E}-03$ & 8.26 & 10.316 & 8.119 & 9.201 \\
\hline 14 & 50 & $8.2 \mathrm{E}-04$ & 7.9E-03 & 9.60 & 10.339 & 8.128 & 9.21 \\
\hline 15 & 70 & 8.2E-04 & $9.2 \mathrm{E}-03$ & 11.19 & 10.357 & 8.136 & 9.216 \\
\hline 16 & 100 & $8.2 \mathrm{E}-04$ & $1.1 \mathrm{E}-02$ & 13.06 & 10.377 & 8.148 & 9.218 \\
\hline 17 & 150 & $8.2 \mathrm{E}-04$ & $1.3 \mathrm{E}-02$ & 15.20 & 10.39 & 8.155 & 9.217 \\
\hline 18 & 200 & $8.2 \mathrm{E}-04$ & $1.4 \mathrm{E}-02$ & 17.27 & 8.859 & 7.912 & 7.89 \\
\hline
\end{tabular}
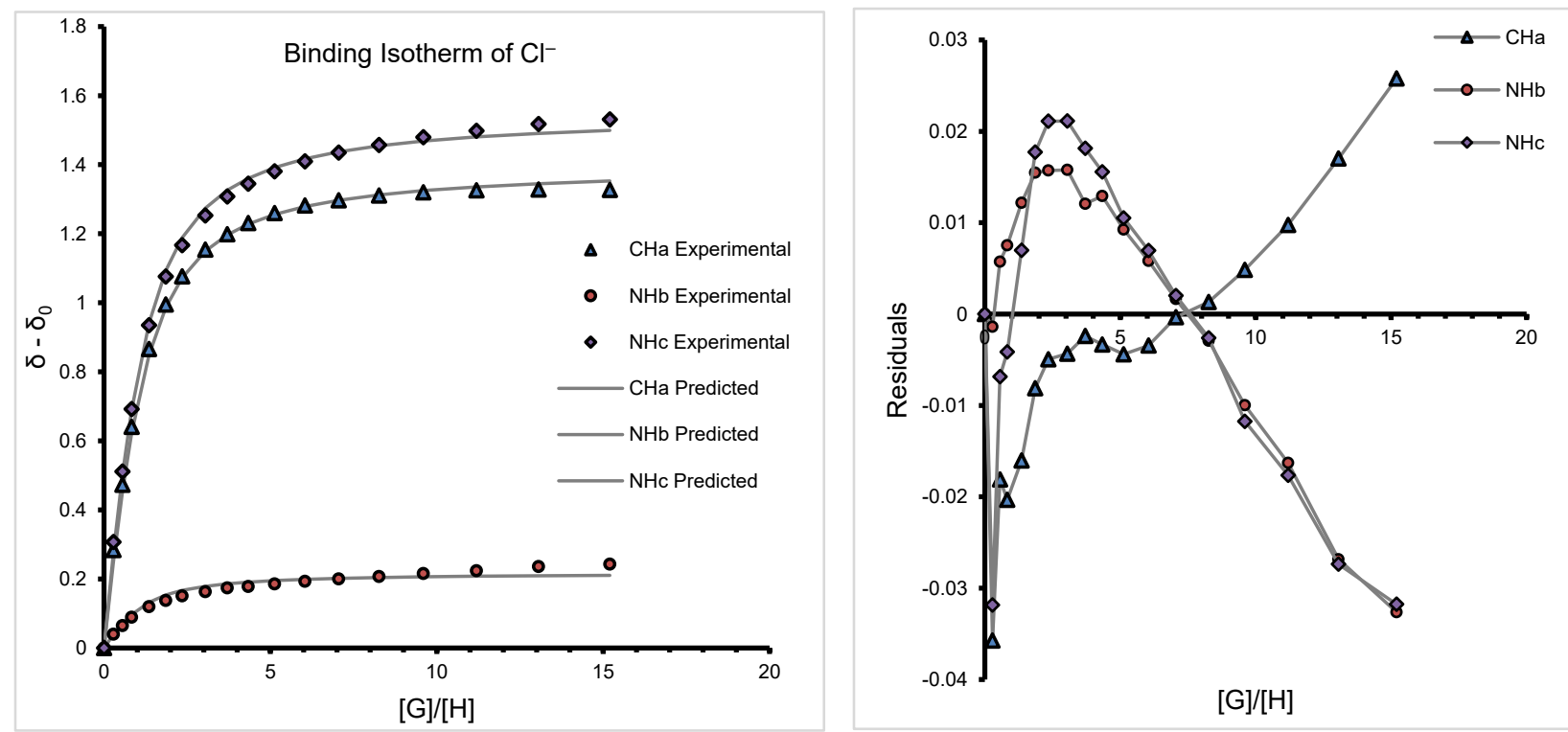

Figure S17. Representative binding isotherm and residuals for $\mathrm{Cl}^{-}$titration of receptor $\mathbf{1}^{\mathrm{Cl}}$ in $10 \%$ DMSO- $d_{6} / \mathrm{CD}_{3} \mathrm{CN}$ at $25{ }^{\circ} \mathrm{C}$ determined by ${ }^{1} \mathrm{H}$ NMR spectroscopy. 
Table S9. Representative titration of receptor $1^{\mathrm{Cl}}$ with $\mathrm{Br}^{-}$in $10 \%$ DMSO- $d_{6} / \mathrm{CD}_{3} \mathrm{CN}$ at $25{ }^{\circ} \mathrm{C}$.

\begin{tabular}{cccccccc} 
Entry & $\begin{array}{c}\mathrm{V}_{\text {Guest }}(\mu \mathrm{L}) \\
{[\mathrm{Host}]} \\
(\mathrm{M})\end{array}$ & $\begin{array}{c}\left.\mathrm{Br}^{-}\right](\mathrm{M}) \\
\text { Equiv. }\end{array}$ & $\begin{array}{c}\delta \mathrm{NH}_{\mathrm{c}} \\
(\mathrm{ppm})\end{array}$ & $\begin{array}{c}\delta \mathrm{NH}_{\mathrm{b}} \\
(\mathrm{ppm})\end{array}$ & $\begin{array}{c}\delta \mathrm{CH}_{\mathrm{a}} \\
(\mathrm{ppm})\end{array}$ \\
\hline 0 & 0 & $1.4 \mathrm{E}-03$ & $0.0 \mathrm{E}+00$ & 0.00 & 8.852 & 7.912 & 7.882 \\
1 & 10 & $1.4 \mathrm{E}-03$ & $5.9 \mathrm{E}-04$ & 0.42 & 8.936 & 7.926 & 7.966 \\
2 & 10 & $1.4 \mathrm{E}-03$ & $1.2 \mathrm{E}-03$ & 0.82 & 8.995 & 7.937 & 8.025 \\
3 & 10 & $1.4 \mathrm{E}-03$ & $1.7 \mathrm{E}-03$ & 1.21 & 9.044 & 7.946 & 8.073 \\
4 & 10 & $1.4 \mathrm{E}-03$ & $2.2 \mathrm{E}-03$ & 1.58 & 9.093 & 7.954 & 8.122 \\
5 & 15 & $1.4 \mathrm{E}-03$ & $3.0 \mathrm{E}-03$ & 2.11 & 9.16 & 7.963 & 8.193 \\
6 & 15 & $1.4 \mathrm{E}-03$ & $3.7 \mathrm{E}-03$ & 2.62 & 9.212 & 7.974 & 8.244 \\
7 & 20 & $1.4 \mathrm{E}-03$ & $4.6 \mathrm{E}-03$ & 3.25 & 9.273 & 7.983 & 8.306 \\
8 & 20 & $1.4 \mathrm{E}-03$ & $5.4 \mathrm{E}-03$ & 3.85 & 9.326 & 7.993 & 8.359 \\
9 & 25 & $1.4 \mathrm{E}-03$ & $6.4 \mathrm{E}-03$ & 4.53 & 9.383 & 8.003 & 8.415 \\
10 & 25 & $1.4 \mathrm{E}-03$ & $7.3 \mathrm{E}-03$ & 5.17 & 9.428 & 8.01 & 8.459 \\
11 & 30 & $1.4 \mathrm{E}-03$ & $8.3 \mathrm{E}-03$ & 5.87 & 9.471 & 8.018 & 8.501 \\
12 & 30 & $1.4 \mathrm{E}-03$ & $9.2 \mathrm{E}-03$ & 6.52 & 9.51 & 8.025 & 8.54 \\
13 & 40 & $1.4 \mathrm{E}-03$ & $1.0 \mathrm{E}-02$ & 7.30 & 9.545 & 8.03 & 8.571 \\
14 & 50 & $1.4 \mathrm{E}-03$ & $1.2 \mathrm{E}-02$ & 8.16 & 9.581 & 8.037 & 8.605 \\
15 & 60 & $1.4 \mathrm{E}-03$ & $1.3 \mathrm{E}-02$ & 9.07 & 9.616 & 8.045 & 8.637 \\
16 & 80 & $1.4 \mathrm{E}-03$ & $1.4 \mathrm{E}-02$ & 10.10 & 9.636 & 8.053 & 8.657 \\
17 & 100 & $1.4 \mathrm{E}-03$ & $1.6 \mathrm{E}-02$ & 11.17 & 9.674 & 8.052 & 8.692
\end{tabular}
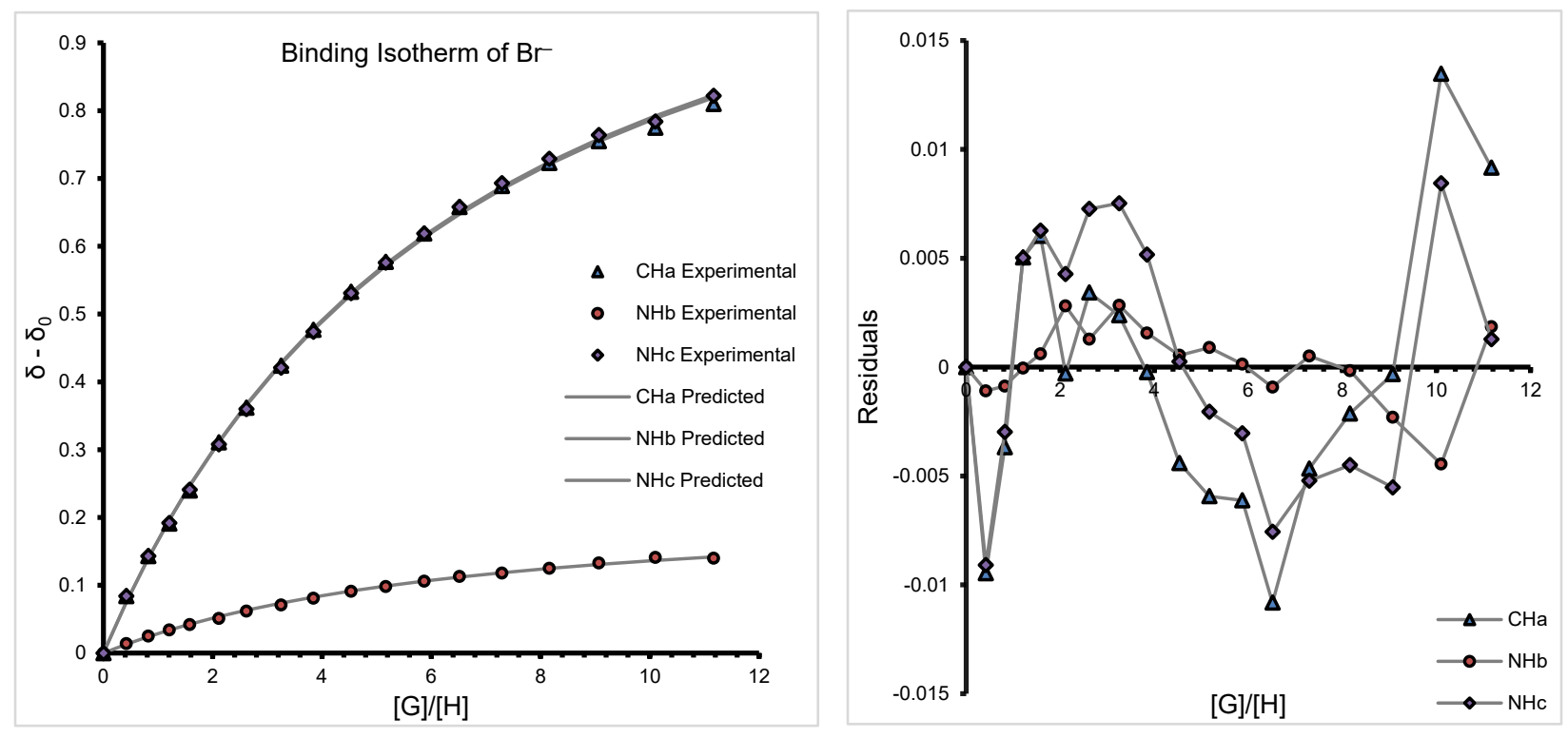

Figure S18. Representative binding isotherm and residuals for $\mathrm{Br}^{-}$titration of receptor $\mathbf{1}^{\mathrm{Cl}}$ in $10 \%$ DMSO- $d_{6} / \mathrm{CD}_{3} \mathrm{CN}$ at $25{ }^{\circ} \mathrm{C}$ determined by ${ }^{1} \mathrm{H}$ NMR spectroscopy. 
Table S10. Representative titration of receptor $1^{\mathrm{F}}$ with $\mathrm{HS}^{-}$in $10 \%$ DMSO- $d_{6} / \mathrm{CD}_{3} \mathrm{CN}$ at $25^{\circ} \mathrm{C}$.

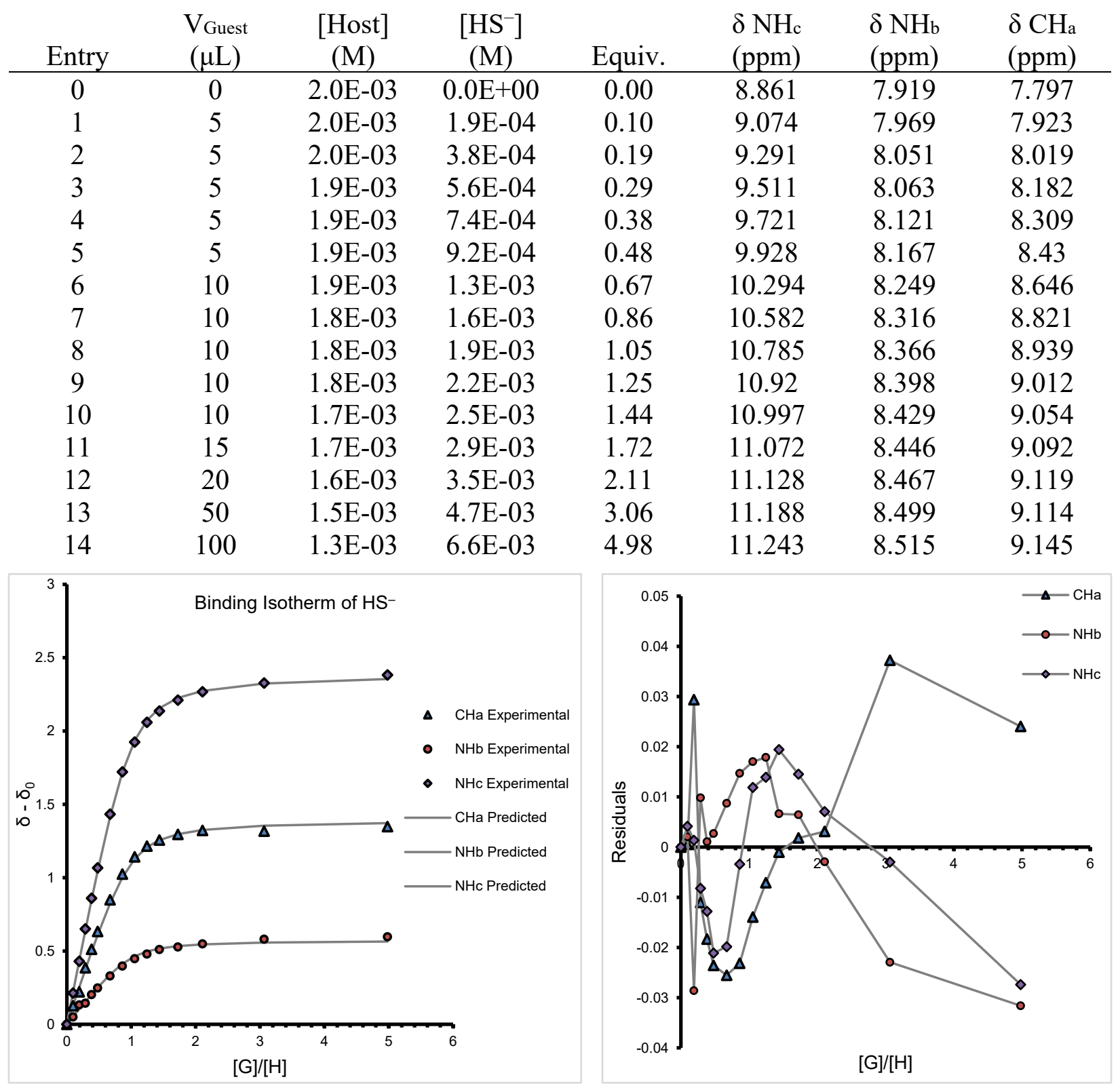

Figure S19. Representative binding isotherm and residuals for $\mathrm{HS}^{-}$titration of receptor $\mathbf{1}^{\mathrm{F}}$ in $10 \%$ DMSO- $d_{6} / \mathrm{CD}_{3} \mathrm{CN}$ at $25{ }^{\circ} \mathrm{C}$ determined by ${ }^{1} \mathrm{H}$ NMR spectroscopy. 
Table S11. Representative titration of receptor $\mathbf{1}^{\mathbf{F}}$ with $\mathrm{HSe}^{-}$in $10 \%$ DMSO- $d_{6} / \mathrm{CD}_{3} \mathrm{CN}$ at $25{ }^{\circ} \mathrm{C}$.

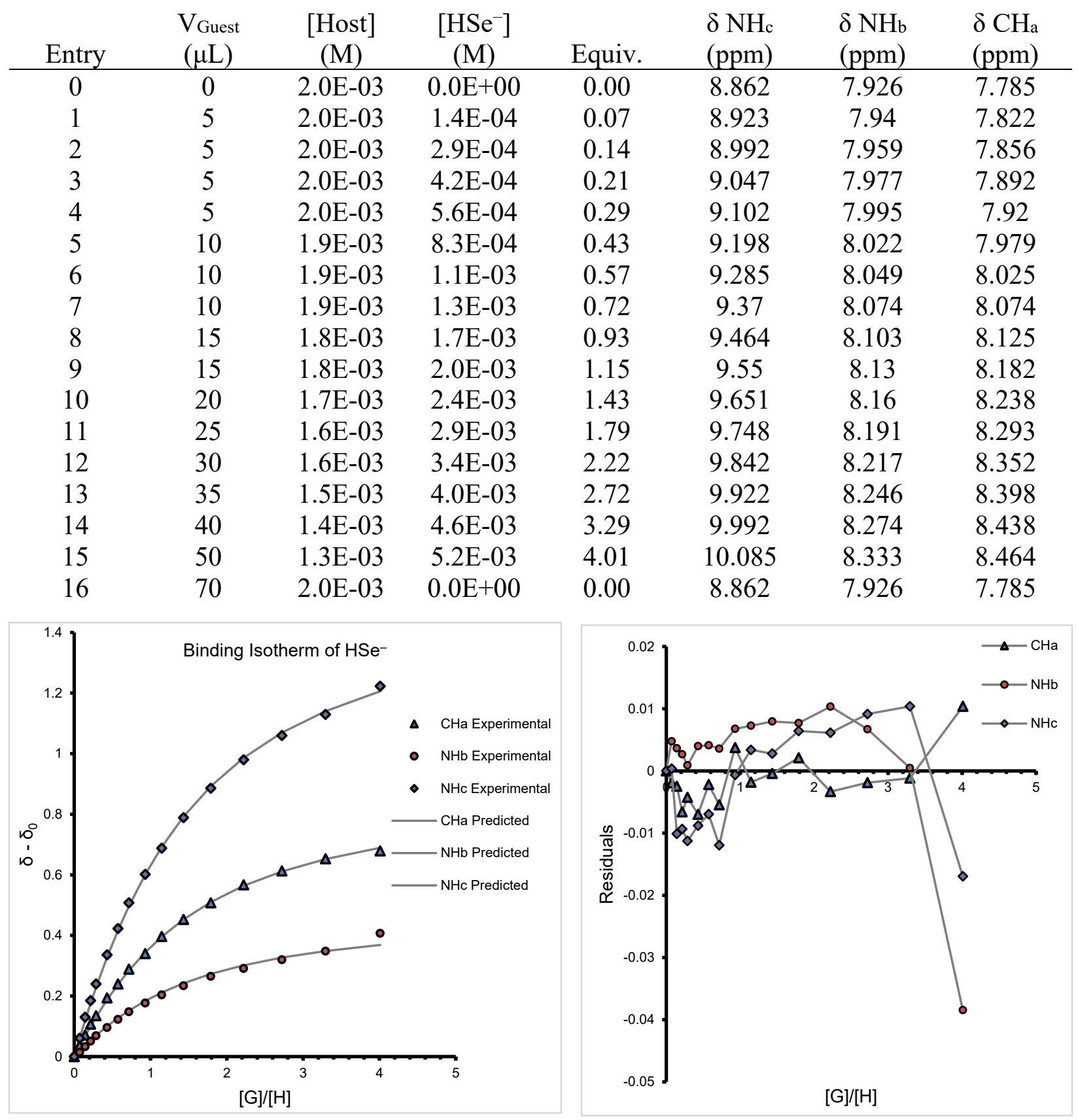

Figure S20. Representative binding isotherm and residuals for $\mathrm{HSe}^{-}$titration of receptor $\mathbf{1}^{\mathbf{F}}$ in $10 \%$ DMSO- $d_{6} / \mathrm{CD}_{3} \mathrm{CN}$ at $25{ }^{\circ} \mathrm{C}$ determined by ${ }^{1} \mathrm{H}$ NMR spectroscopy. 
Table S12. Representative titration of receptor $\mathbf{1}^{\mathrm{F}}$ with $\mathrm{Cl}^{-}$in $10 \%$ DMSO- $d_{6} / \mathrm{CD}_{3} \mathrm{CN}$ at $25^{\circ} \mathrm{C}$.

\begin{tabular}{cccccccc} 
Entry & $\begin{array}{c}\mathrm{V}_{\text {Guest }} \\
(\mu \mathrm{L})\end{array}$ & $\begin{array}{c}{[\mathrm{Host}]} \\
(\mathrm{M})\end{array}$ & $\begin{array}{c}\left.\mathrm{Cl}^{-}\right](\mathrm{M}) \\
\text { Equiv. }\end{array}$ & $\begin{array}{c}\delta \mathrm{NH}_{\mathrm{c}} \\
(\mathrm{ppm})\end{array}$ & $\begin{array}{c}\delta \mathrm{NH}_{\mathrm{b}} \\
(\mathrm{ppm})\end{array}$ & $\begin{array}{c}\delta \mathrm{CH}_{\mathrm{a}} \\
(\mathrm{ppm})\end{array}$ \\
\hline 0 & 0 & $1.1 \mathrm{E}-03$ & $0.0 \mathrm{E}+00$ & 0.00 & 8.849 & 7.916 & 7.79 \\
1 & 5 & $1.1 \mathrm{E}-03$ & $1.9 \mathrm{E}-04$ & 0.17 & 9.018 & 7.940 & 7.904 \\
2 & 5 & $1.1 \mathrm{E}-03$ & $3.7 \mathrm{E}-04$ & 0.34 & 9.177 & 7.96 & 8.07 \\
3 & 5 & $1.1 \mathrm{E}-03$ & $5.4 \mathrm{E}-04$ & 0.50 & 9.31 & 7.979 & 8.194 \\
4 & 10 & $1.1 \mathrm{E}-03$ & $8.9 \mathrm{E}-04$ & 0.82 & 9.538 & 8.009 & 8.398 \\
5 & 10 & $1.1 \mathrm{E}-03$ & $1.2 \mathrm{E}-03$ & 1.12 & 9.715 & 8.033 & 8.559 \\
6 & 15 & $1.1 \mathrm{E}-03$ & $1.7 \mathrm{E}-03$ & 1.56 & 9.875 & 8.064 & 8.695 \\
7 & 20 & $1.1 \mathrm{E}-03$ & $2.3 \mathrm{E}-03$ & 2.11 & 10.013 & 8.083 & 8.817 \\
8 & 25 & $1.1 \mathrm{E}-03$ & $3.0 \mathrm{E}-03$ & 2.74 & 10.116 & 8.091 & 8.903 \\
9 & 30 & $1.1 \mathrm{E}-03$ & $3.7 \mathrm{E}-03$ & 3.43 & 10.185 & 8.101 & 8.959 \\
10 & 40 & $1.1 \mathrm{E}-03$ & $4.6 \mathrm{E}-03$ & 4.26 & 10.239 & 8.115 & 9 \\
11 & 50 & $1.1 \mathrm{E}-03$ & $5.6 \mathrm{E}-03$ & 5.16 & 10.279 & 8.123 & 9.029 \\
12 & 70 & $1.1 \mathrm{E}-03$ & $6.8 \mathrm{E}-03$ & 6.23 & 10.312 & 8.133 & 9.045 \\
13 & 100 & $1.1 \mathrm{E}-03$ & $8.1 \mathrm{E}-03$ & 7.47 & 10.344 & 8.141 & 9.057 \\
14 & 150 & $1.1 \mathrm{E}-03$ & $9.7 \mathrm{E}-03$ & 8.88 & 10.374 & 8.157 & 9.064 \\
15 & 200 & $1.1 \mathrm{E}-03$ & $1.1 \mathrm{E}-02$ & 10.22 & 10.381 & 8.165 & 9.064
\end{tabular}
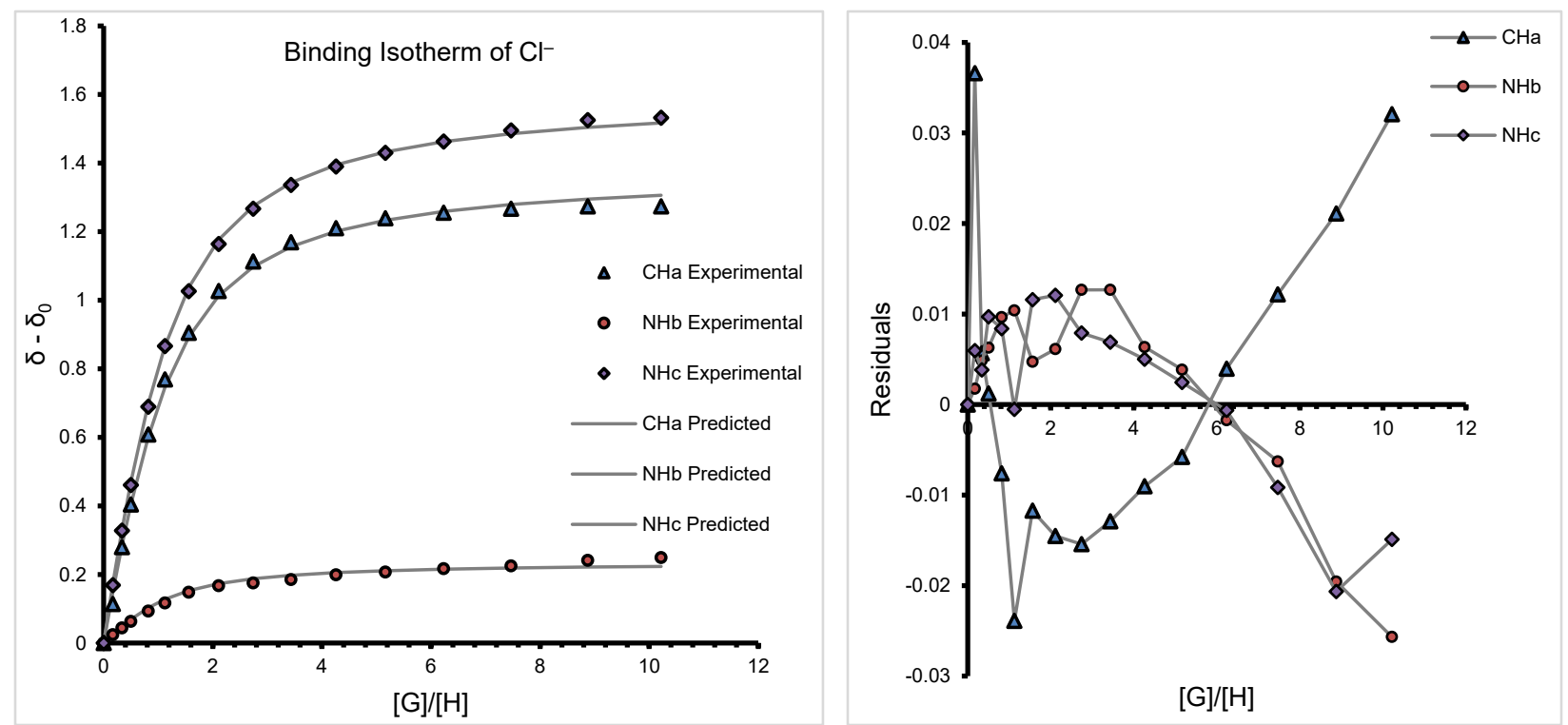

Figure S21. Representative binding isotherm and residuals for $\mathrm{Cl}^{-}$titration of receptor $\mathbf{1}^{\mathrm{F}}$ in $10 \%$ DMSO- $d_{6} / \mathrm{CD}_{3} \mathrm{CN}$ at $25{ }^{\circ} \mathrm{C}$ determined by ${ }^{1} \mathrm{H}$ NMR spectroscopy. 
Table S13. Representative titration of receptor $\mathbf{1}^{\mathbf{F}}$ with $\mathrm{Br}^{-}$in $10 \%$ DMSO- $d_{6} / \mathrm{CD}_{3} \mathrm{CN}$ at $25{ }^{\circ} \mathrm{C}$.

\begin{tabular}{cccccccc} 
Entry & $\begin{array}{c}\mathrm{V}_{\text {Guest }} \\
(\mu \mathrm{L})\end{array}$ & $\begin{array}{c}{[\mathrm{Host}]} \\
(\mathrm{M})\end{array}$ & $\begin{array}{c}\left.\mathrm{Br}^{-}\right](\mathrm{M}) \\
\text { Equiv. }\end{array}$ & $\begin{array}{c}\delta \mathrm{NH}_{\mathrm{c}} \\
(\mathrm{ppm})\end{array}$ & $\begin{array}{c}\delta \mathrm{NH}_{\mathrm{b}} \\
(\mathrm{ppm})\end{array}$ & $\begin{array}{c}\delta \mathrm{CH}_{\mathrm{a}} \\
(\mathrm{ppm})\end{array}$ \\
\hline 0 & 0 & $2.0 \mathrm{E}-03$ & $0.0 \mathrm{E}+00$ & 0.00 & 8.841 & 7.913 & 7.79 \\
1 & 10 & $2.0 \mathrm{E}-03$ & $8.2 \mathrm{E}-04$ & 0.41 & 8.96 & 7.935 & 7.898 \\
2 & 10 & $2.0 \mathrm{E}-03$ & $1.6 \mathrm{E}-03$ & 0.81 & 9.045 & 7.949 & 7.98 \\
3 & 10 & $2.0 \mathrm{E}-03$ & $2.4 \mathrm{E}-03$ & 1.19 & 9.114 & 7.962 & 8.051 \\
4 & 10 & $2.0 \mathrm{E}-03$ & $3.1 \mathrm{E}-03$ & 1.55 & 9.172 & 7.973 & 8.101 \\
5 & 15 & $2.0 \mathrm{E}-03$ & $4.1 \mathrm{E}-03$ & 2.08 & 9.25 & 7.986 & 8.175 \\
6 & 15 & $2.0 \mathrm{E}-03$ & $5.1 \mathrm{E}-03$ & 2.58 & 9.316 & 7.997 & 8.238 \\
7 & 20 & $2.0 \mathrm{E}-03$ & $6.4 \mathrm{E}-03$ & 3.20 & 9.381 & 8.009 & 8.298 \\
8 & 20 & $2.0 \mathrm{E}-03$ & $7.5 \mathrm{E}-03$ & 3.78 & 9.438 & 8.019 & 8.35 \\
9 & 25 & $2.0 \mathrm{E}-03$ & $8.9 \mathrm{E}-03$ & 4.46 & 9.497 & 8.029 & 8.404 \\
10 & 25 & $2.0 \mathrm{E}-03$ & $1.0 \mathrm{E}-02$ & 5.09 & 9.547 & 8.037 & 8.448 \\
11 & 30 & $2.0 \mathrm{E}-03$ & $1.2 \mathrm{E}-02$ & 5.78 & 9.595 & 8.047 & 8.488 \\
12 & 30 & $2.0 \mathrm{E}-03$ & $1.3 \mathrm{E}-02$ & 6.41 & 9.635 & 8.052 & 8.523 \\
13 & 40 & $2.0 \mathrm{E}-03$ & $1.4 \mathrm{E}-02$ & 7.18 & 9.663 & 8.06 & 8.546 \\
14 & 50 & $2.0 \mathrm{E}-03$ & $1.6 \mathrm{E}-02$ & 8.03 & 9.71 & 8.068 & 8.582 \\
15 & 60 & $2.0 \mathrm{E}-03$ & $1.8 \mathrm{E}-02$ & 8.93 & 9.732 & 8.074 & 8.597 \\
16 & 80 & $2.0 \mathrm{E}-03$ & $2.0 \mathrm{E}-02$ & 9.94 & 9.774 & 8.084 & 8.631 \\
17 & 100 & $2.0 \mathrm{E}-03$ & $2.2 \mathrm{E}-02$ & 10.99 & 9.797 & 8.09 & 8.647 \\
18 & 150 & $2.0 \mathrm{E}-03$ & $2.4 \mathrm{E}-02$ & 12.24 & 9.822 & 8.097 & 8.671
\end{tabular}
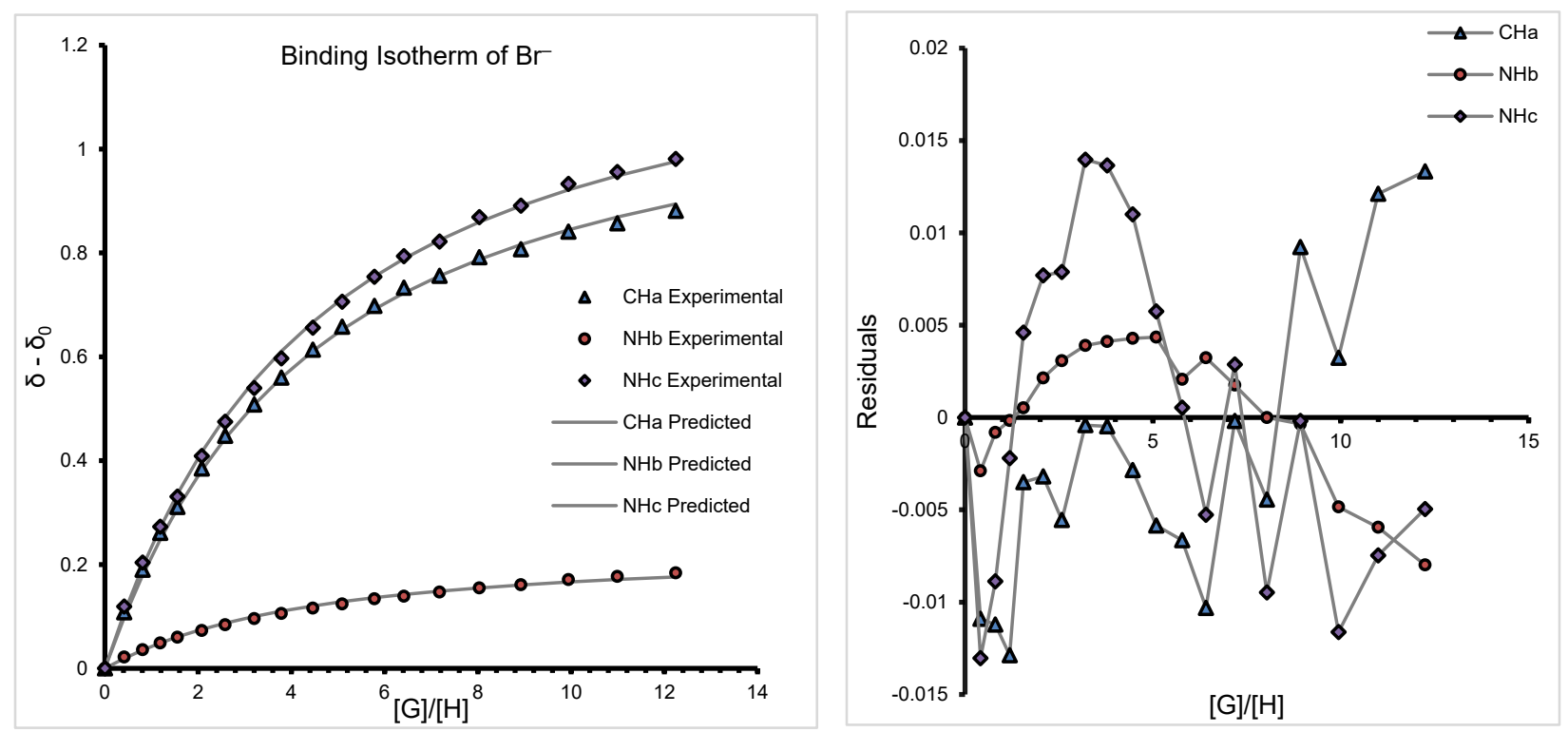

Figure S22. Representative binding isotherm and residuals for $\mathrm{Br}^{-}$titration of receptor $\mathbf{1}^{\mathrm{F}}$ in $10 \%$ DMSO- $d_{6} / \mathrm{CD}_{3} \mathrm{CN}$ at $25{ }^{\circ} \mathrm{C}$ determined by ${ }^{1} \mathrm{H}$ NMR spectroscopy. 
Table S14. Representative titration of receptor $\mathbf{1}^{\mathrm{H}}$ with $\mathrm{HSe}^{-}$in $10 \%$ DMSO- $d_{6} / \mathrm{CD}_{3} \mathrm{CN}$ at $25^{\circ} \mathrm{C}$.

\begin{tabular}{cccccccc} 
Entry & $\begin{array}{c}\mathrm{V}_{\text {Guest }} \\
(\mu \mathrm{L})\end{array}$ & $\begin{array}{c}{[\mathrm{Host}]} \\
(\mathrm{M})\end{array}$ & $\begin{array}{c}{\left[\mathrm{HSe}^{-}\right]} \\
(\mathrm{M})\end{array}$ & $\begin{array}{c}\delta \mathrm{NH}_{\mathrm{c}} \\
(\mathrm{ppm})\end{array}$ & $\begin{array}{c}\delta \mathrm{NH}_{\mathrm{b}} \\
(\mathrm{ppm})\end{array}$ & $\begin{array}{c}\delta \mathrm{CH}_{\mathrm{a}} \\
(\mathrm{ppm})\end{array}$ \\
\hline 0 & 0 & $2.0 \mathrm{E}-03$ & $0.0 \mathrm{E}+00$ & 0.00 & 8.907 & 7.922 & 8.002 \\
1 & 5 & $2.0 \mathrm{E}-03$ & $1.4 \mathrm{E}-04$ & 0.07 & 8.974 & 7.939 & 8.041 \\
2 & 5 & $2.0 \mathrm{E}-03$ & $2.9 \mathrm{E}-04$ & 0.15 & 9.039 & 7.958 & 8.079 \\
3 & 5 & $1.9 \mathrm{E}-03$ & $4.2 \mathrm{E}-04$ & 0.22 & 9.092 & 7.976 & 8.104 \\
4 & 5 & $1.9 \mathrm{E}-03$ & $5.6 \mathrm{E}-04$ & 0.29 & 9.144 & 7.991 & 8.129 \\
5 & 10 & $1.9 \mathrm{E}-03$ & $8.3 \mathrm{E}-04$ & 0.44 & 9.226 & 8.018 & 8.179 \\
6 & 10 & $1.8 \mathrm{E}-03$ & $1.1 \mathrm{E}-03$ & 0.58 & 9.304 & 8.039 & 8.222 \\
7 & 10 & $1.8 \mathrm{E}-03$ & $1.3 \mathrm{E}-03$ & 0.73 & 9.373 & 8.065 & 8.26 \\
8 & 15 & $1.8 \mathrm{E}-03$ & $1.7 \mathrm{E}-03$ & 0.95 & 9.472 & 8.092 & 8.312 \\
9 & 15 & $1.7 \mathrm{E}-03$ & $2.0 \mathrm{E}-03$ & 1.17 & 9.553 & 8.118 & 8.354 \\
10 & 20 & $1.7 \mathrm{E}-03$ & $2.4 \mathrm{E}-03$ & 1.46 & 9.638 & 8.143 & 8.411 \\
11 & 25 & $1.6 \mathrm{E}-03$ & $2.9 \mathrm{E}-03$ & 1.83 & 9.733 & 8.175 & 8.457 \\
12 & 30 & $1.5 \mathrm{E}-03$ & $3.4 \mathrm{E}-03$ & 2.27 & 9.83 & 8.199 & 8.508 \\
13 & 35 & $1.4 \mathrm{E}-03$ & $4.0 \mathrm{E}-03$ & 2.78 & 9.902 & 8.223 & 8.547 \\
14 & 40 & $1.4 \mathrm{E}-03$ & $4.6 \mathrm{E}-03$ & 3.36 & 9.977 & 8.265 & 8.58 \\
15 & 45 & $2.0 \mathrm{E}-03$ & $0.0 \mathrm{E}+00$ & 0.00 & 8.907 & 7.922 & 8.002
\end{tabular}
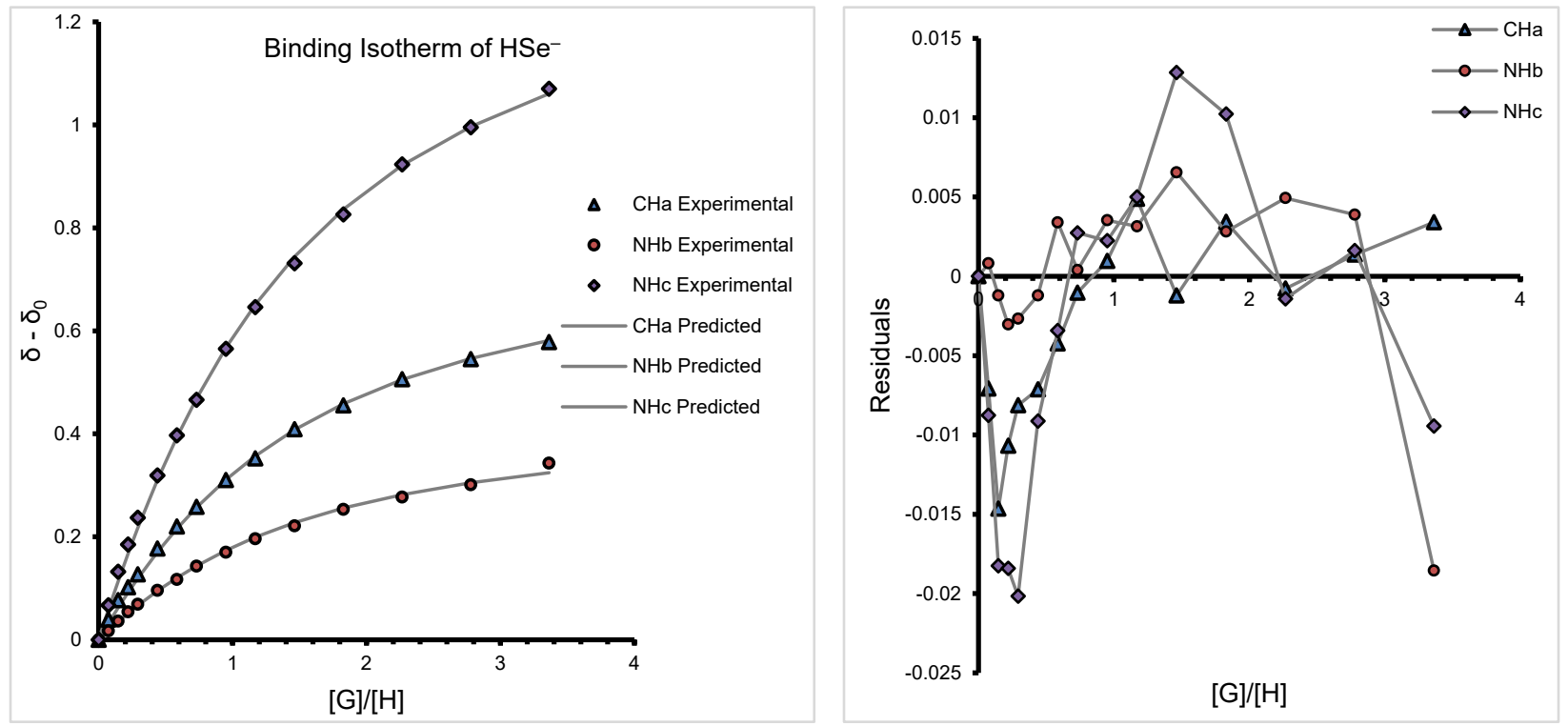

Figure S23. Representative binding isotherm and residuals for $\mathrm{HSe}^{-}$titration of receptor $\mathbf{1}^{\mathbf{H}}$ in $10 \%$ DMSO- $d_{6} / \mathrm{CD}_{3} \mathrm{CN}$ at $25{ }^{\circ} \mathrm{C}$ determined by ${ }^{1} \mathrm{H}$ NMR spectroscopy. 
Table S15. Representative titration of receptor $\mathbf{1}^{\mathrm{H}}$ with $\mathrm{Br}^{-}$in $10 \%$ DMSO- $d_{6} / \mathrm{CD}_{3} \mathrm{CN}$ at $25^{\circ} \mathrm{C}$.

\begin{tabular}{cccccccc} 
Entry & $\begin{array}{c}\mathrm{V}_{\text {Guest }}(\mu \mathrm{L}) \\
{[\text { Host }]} \\
(\mathrm{M})\end{array}$ & $\begin{array}{c}\left.\mathrm{Br}^{-}\right](\mathrm{M}) \\
\text { Equiv. }\end{array}$ & $\begin{array}{c}\delta \mathrm{NH}_{\mathrm{c}} \\
(\mathrm{ppm})\end{array}$ & $\begin{array}{c}\delta \mathrm{NH}_{\mathrm{b}} \\
(\mathrm{ppm})\end{array}$ & $\begin{array}{c}\delta \mathrm{CH}_{\mathrm{a}} \\
(\mathrm{ppm})\end{array}$ \\
\hline 0 & 0 & $1.0 \mathrm{E}-03$ & $0.0 \mathrm{E}+00$ & 0.00 & 8.843 & 7.905 & 7.974 \\
1 & 10 & $1.0 \mathrm{E}-03$ & $6.5 \mathrm{E}-04$ & 0.62 & 8.942 & 7.918 & 8.071 \\
2 & 10 & $1.0 \mathrm{E}-03$ & $1.3 \mathrm{E}-03$ & 1.22 & 9.021 & 7.932 & 8.144 \\
3 & 10 & $1.0 \mathrm{E}-03$ & $1.9 \mathrm{E}-03$ & 1.80 & 9.086 & 7.941 & 8.208 \\
4 & 10 & $1.0 \mathrm{E}-03$ & $2.5 \mathrm{E}-03$ & 2.35 & 9.15 & 7.95 & 8.27 \\
5 & 15 & $1.0 \mathrm{E}-03$ & $3.3 \mathrm{E}-03$ & 3.14 & 9.222 & 7.961 & 8.339 \\
6 & 15 & $1.0 \mathrm{E}-03$ & $4.1 \mathrm{E}-03$ & 3.89 & 9.286 & 7.97 & 8.4 \\
7 & 20 & $1.0 \mathrm{E}-03$ & $5.0 \mathrm{E}-03$ & 4.84 & 9.361 & 7.982 & 8.469 \\
8 & 20 & $1.0 \mathrm{E}-03$ & $6.0 \mathrm{E}-03$ & 5.72 & 9.419 & 7.989 & 8.526 \\
9 & 25 & $1.0 \mathrm{E}-03$ & $7.0 \mathrm{E}-03$ & 6.74 & 9.48 & 7.997 & 8.583 \\
10 & 25 & $1.0 \mathrm{E}-03$ & $8.0 \mathrm{E}-03$ & 7.69 & 9.532 & 8.005 & 8.63 \\
11 & 30 & $1.0 \mathrm{E}-03$ & $9.1 \mathrm{E}-03$ & 8.73 & 9.581 & 8.012 & 8.678 \\
12 & 30 & $1.0 \mathrm{E}-03$ & $1.0 \mathrm{E}-02$ & 9.69 & 9.62 & 8.017 & 8.712 \\
13 & 40 & $1.0 \mathrm{E}-03$ & $1.1 \mathrm{E}-02$ & 10.85 & 9.662 & 8.024 & 8.748 \\
14 & 50 & $1.0 \mathrm{E}-03$ & $1.3 \mathrm{E}-02$ & 12.14 & 9.701 & 8.031 & 8.786 \\
15 & 60 & $1.0 \mathrm{E}-03$ & $1.4 \mathrm{E}-02$ & 13.49 & 9.73 & 8.031 & 8.807 \\
16 & 80 & $1.0 \mathrm{E}-03$ & $1.6 \mathrm{E}-02$ & 15.02 & 9.775 & 8.039 & 8.851 \\
17 & 100 & $1.0 \mathrm{E}-03$ & $1.7 \mathrm{E}-02$ & 16.61 & 9.805 & 8.046 & 8.874
\end{tabular}
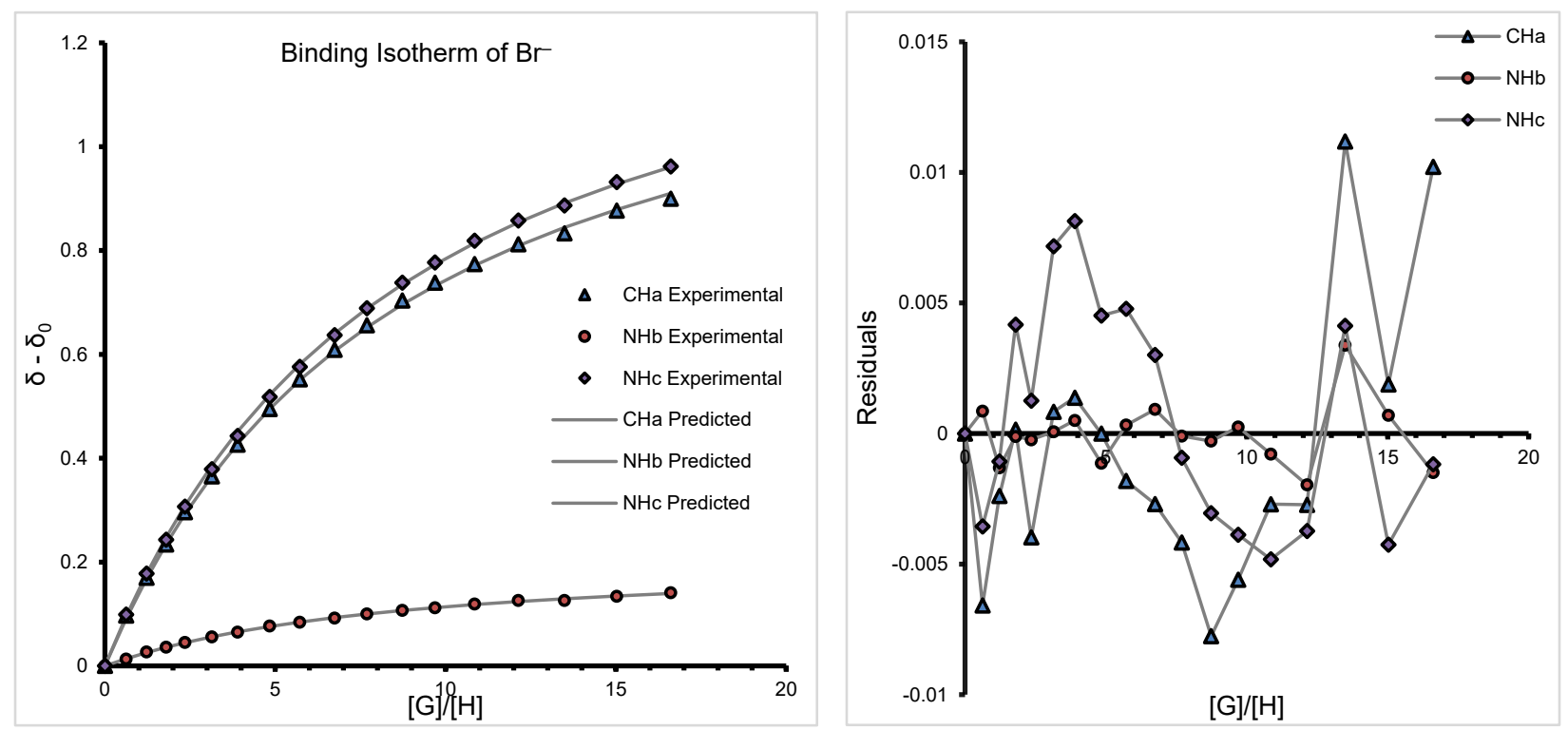

Figure S24. Representative binding isotherm and residuals for $\mathrm{Br}^{-}$titration of receptor $\mathbf{1}^{\mathbf{H}}$ in $10 \%$ DMSO- $d_{6} / \mathrm{CD}_{3} \mathrm{CN}$ at $25{ }^{\circ} \mathrm{C}$ determined by ${ }^{1} \mathrm{H}$ NMR spectroscopy. 
Table S16. Representative titration of receptor $1^{\mathrm{NMe} 2}$ with $\mathrm{HS}^{-}$in $10 \% \mathrm{DMSO}-d_{6} / \mathrm{CD}_{3} \mathrm{CN}$ at $25^{\circ} \mathrm{C}$.

\begin{tabular}{cccccccc} 
Entry & $\begin{array}{c}V_{\text {Guest }} \\
(\mu \mathrm{L})\end{array}$ & $\begin{array}{c}{[\mathrm{Host}]} \\
(\mathrm{M})\end{array}$ & $\begin{array}{c}{[\mathrm{HS}-} \\
(\mathrm{M})\end{array}$ & $\begin{array}{c}\delta \mathrm{NH}_{\mathrm{c}} \\
\text { Equiv. }\end{array}$ & $\begin{array}{c}\delta \mathrm{NH}_{\mathrm{b}} \\
(\mathrm{ppm})\end{array}$ & $\begin{array}{c}\delta \mathrm{CH}_{\mathrm{a}} \\
(\mathrm{ppm})\end{array}$ \\
\hline 0 & 0 & $2.1 \mathrm{E}-03$ & $0.0 \mathrm{E}+00$ & 0.00 & 8.885 & 7.903 & 7.265 \\
1 & 10 & $2.1 \mathrm{E}-03$ & $2.1 \mathrm{E}-04$ & 0.10 & 9.047 & 7.943 & 7.345 \\
2 & 20 & $2.0 \mathrm{E}-03$ & $4.0 \mathrm{E}-04$ & 0.20 & 9.221 & 7.981 & 7.431 \\
3 & 30 & $2.0 \mathrm{E}-03$ & $6.0 \mathrm{E}-04$ & 0.30 & 9.38 & 8.017 & 7.52 \\
4 & 40 & $1.9 \mathrm{E}-03$ & $7.8 \mathrm{E}-04$ & 0.40 & 9.55 & 8.051 & 7.601 \\
5 & 55 & $1.9 \mathrm{E}-03$ & $1.0 \mathrm{E}-03$ & 0.55 & 9.762 & 8.1 & 7.72 \\
6 & 70 & $1.8 \mathrm{E}-03$ & $1.3 \mathrm{E}-03$ & 0.70 & 9.979 & 8.148 & 7.828 \\
7 & 90 & $1.8 \mathrm{E}-03$ & $1.6 \mathrm{E}-03$ & 0.90 & 10.216 & 8.199 & 7.955 \\
8 & 110 & $1.7 \mathrm{E}-03$ & $1.9 \mathrm{E}-03$ & 1.10 & 10.412 & 8.244 & 8.061 \\
9 & 135 & $1.7 \mathrm{E}-03$ & $2.2 \mathrm{E}-03$ & 1.35 & 10.601 & 8.287 & 8.157 \\
10 & 160 & $1.6 \mathrm{E}-03$ & $2.6 \mathrm{E}-03$ & 1.60 & 10.746 & 8.321 & 8.23 \\
11 & 190 & $1.5 \mathrm{E}-03$ & $2.9 \mathrm{E}-03$ & 1.90 & 10.857 & 8.354 & 8.288 \\
12 & 220 & $1.5 \mathrm{E}-03$ & $3.2 \mathrm{E}-03$ & 2.21 & 10.92 & 8.37 & 8.339 \\
13 & 260 & $1.4 \mathrm{E}-03$ & $3.6 \mathrm{E}-03$ & 2.61 & 10.982 & 8.391 & 8.391 \\
14 & 310 & $1.3 \mathrm{E}-03$ & $4.0 \mathrm{E}-03$ & 3.11 & 11.041 & 8.411 & 8.411 \\
15 & 370 & $1.2 \mathrm{E}-03$ & $4.5 \mathrm{E}-03$ & 3.71 & 11.08 & 8.437 & 8.437 \\
16 & 450 & $1.1 \mathrm{E}-03$ & $5.0 \mathrm{E}-03$ & 4.51 & 11.094 & 8.451 & 8.451 \\
17 & 550 & $1.0 \mathrm{E}-03$ & $5.5 \mathrm{E}-03$ & 5.51 & 11.126 & 8.459 & 8.459
\end{tabular}
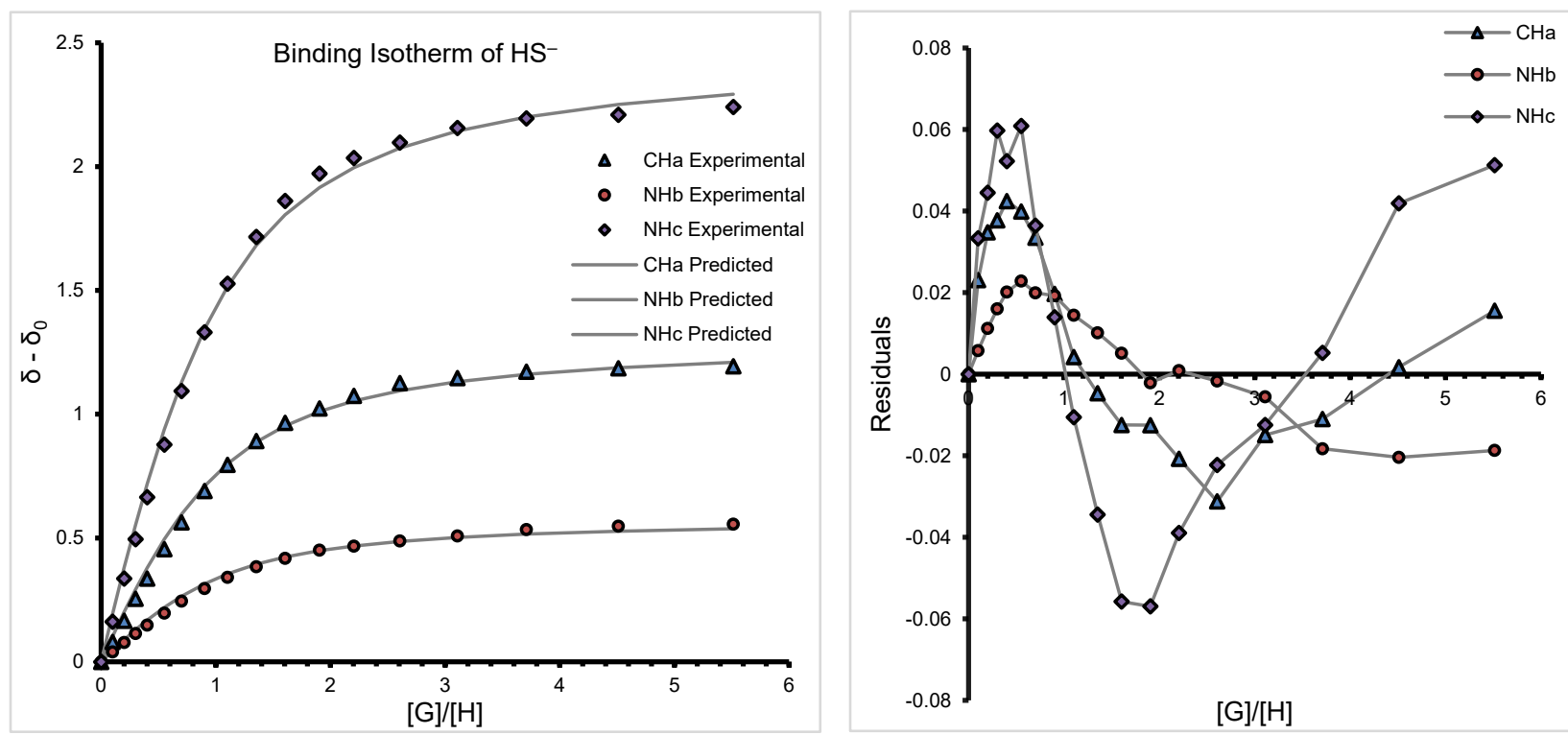

Figure S25. Representative binding isotherm and residuals for $\mathrm{HS}^{-}$titration of receptor $\mathbf{1}^{\mathbf{N M e} 2}$ in $10 \%$ DMSO- $d_{6} / \mathrm{CD}_{3} \mathrm{CN}$ at $25{ }^{\circ} \mathrm{C}$ determined by ${ }^{1} \mathrm{H}$ NMR spectroscopy. 
Table S17. Representative titration of receptor $\mathbf{1}^{\mathrm{NMe} 2}$ with $\mathrm{HSe}^{-}$in $10 \%$ DMSO- $d_{6} / \mathrm{CD}_{3} \mathrm{CN}$ at $25^{\circ} \mathrm{C}$.

\begin{tabular}{cccccccc} 
Entry & $\begin{array}{c}\mathrm{V}_{\text {Guest }} \\
(\mu \mathrm{L})\end{array}$ & $\begin{array}{c}{[\mathrm{Host}]} \\
(\mathrm{M})\end{array}$ & $\begin{array}{c}{\left[\mathrm{HSe}^{-}\right]} \\
(\mathrm{M})\end{array}$ & $\begin{array}{c}\delta \text { Equiv. } \\
(\mathrm{ppm})\end{array}$ & $\begin{array}{c}\delta \mathrm{NH}_{\mathrm{b}} \\
(\mathrm{ppm})\end{array}$ & $\begin{array}{c}\delta \mathrm{CH}_{\mathrm{a}} \\
(\mathrm{ppm})\end{array}$ \\
\hline 0 & 0 & $2.0 \mathrm{E}-03$ & $0.0 \mathrm{E}+00$ & 0.00 & 8.902 & 7.909 & 7.266 \\
1 & 5 & $2.0 \mathrm{E}-03$ & $1.7 \mathrm{E}-04$ & 0.09 & 8.991 & 7.935 & 7.307 \\
2 & 5 & $2.0 \mathrm{E}-03$ & $3.4 \mathrm{E}-04$ & 0.17 & 9.052 & 7.953 & 7.337 \\
3 & 5 & $1.9 \mathrm{E}-03$ & $5.1 \mathrm{E}-04$ & 0.26 & 9.109 & 7.97 & 7.363 \\
4 & 5 & $1.9 \mathrm{E}-03$ & $6.7 \mathrm{E}-04$ & 0.35 & 9.159 & 7.985 & 7.38 \\
5 & 10 & $1.9 \mathrm{E}-03$ & $9.8 \mathrm{E}-04$ & 0.52 & 9.242 & 8.02 & 7.417 \\
6 & 10 & $1.9 \mathrm{E}-03$ & $1.3 \mathrm{E}-03$ & 0.69 & 9.327 & 8.033 & 7.465 \\
7 & 10 & $1.8 \mathrm{E}-03$ & $1.6 \mathrm{E}-03$ & 0.86 & 9.397 & 8.062 & 7.498 \\
8 & 10 & $1.8 \mathrm{E}-03$ & $1.9 \mathrm{E}-03$ & 1.04 & 9.472 & 8.086 & 7.52 \\
9 & 15 & $1.7 \mathrm{E}-03$ & $2.3 \mathrm{E}-03$ & 1.30 & 9.556 & 8.113 & 7.566 \\
10 & 15 & $1.7 \mathrm{E}-03$ & $2.6 \mathrm{E}-03$ & 1.56 & 9.634 & 8.138 & 7.601 \\
11 & 20 & $1.6 \mathrm{E}-03$ & $3.1 \mathrm{E}-03$ & 1.90 & 9.71 & 8.168 & 7.638 \\
12 & 25 & $1.6 \mathrm{E}-03$ & $3.7 \mathrm{E}-03$ & 2.34 & 9.803 & 8.192 & 7.675 \\
13 & 30 & $1.5 \mathrm{E}-03$ & $4.3 \mathrm{E}-03$ & 2.85 & 9.872 & 8.222 & 7.71 \\
14 & 40 & $1.4 \mathrm{E}-03$ & $5.1 \mathrm{E}-03$ & 3.55 & 9.957 & 8.25 & 7.746 \\
15 & 50 & $1.3 \mathrm{E}-03$ & $5.9 \mathrm{E}-03$ & 4.41 & 10.025 & 8.275 & 7.778 \\
15 & 70 & $1.2 \mathrm{E}-03$ & $6.8 \mathrm{E}-03$ & 5.62 & 10.107 & 8.308 & 7.808
\end{tabular}
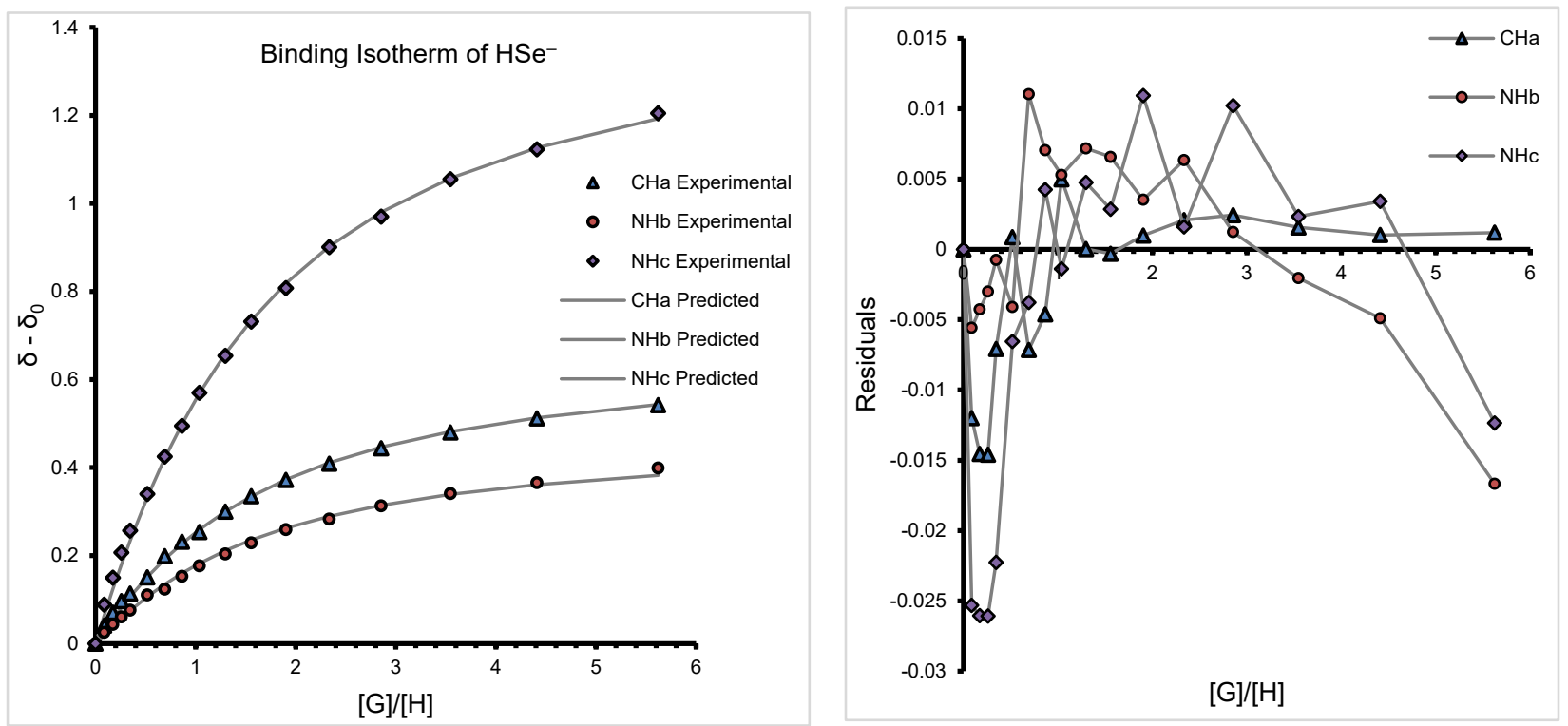

Figure S26. Representative binding isotherm and residuals for $\mathrm{HSe}^{-}$titration of receptor $\mathbf{1}^{\mathrm{NMe} 2}$ in $10 \%$ DMSO- $d_{6} / \mathrm{CD}_{3} \mathrm{CN}$ at $25{ }^{\circ} \mathrm{C}$ determined by ${ }^{1} \mathrm{H}$ NMR spectroscopy. 
Table S18. Representative titration of receptor $1^{\mathrm{NMe} 2}$ with $\mathrm{Cl}^{-}$in $10 \%$ DMSO- $d_{6} / \mathrm{CD}_{3} \mathrm{CN}$ at $25^{\circ} \mathrm{C}$.

\begin{tabular}{cccccccc} 
Entry & $\begin{array}{c}V_{\text {Guest }} \\
(\mu \mathrm{L})\end{array}$ & $\begin{array}{c}{[\mathrm{Host}]} \\
(\mathrm{M})\end{array}$ & $\begin{array}{c}\left.\mathrm{Cl}^{-}\right](\mathrm{M}) \\
\text { Equiv. }\end{array}$ & $\begin{array}{c}\delta \mathrm{NH}_{\mathrm{c}} \\
(\mathrm{ppm})\end{array}$ & $\begin{array}{c}\delta \mathrm{NH}_{\mathrm{b}} \\
(\mathrm{ppm})\end{array}$ & $\begin{array}{c}\delta \mathrm{CH}_{\mathrm{a}} \\
(\mathrm{ppm})\end{array}$ \\
\hline 0 & 0 & $2.1 \mathrm{E}-03$ & $0.0 \mathrm{E}+00$ & 0.00 & 8.875 & 7.901 & 7.257 \\
1 & 5 & $2.1 \mathrm{E}-03$ & $2.2 \mathrm{E}-04$ & 0.11 & 8.988 & 7.915 & 7.345 \\
2 & 5 & $2.1 \mathrm{E}-03$ & $4.4 \mathrm{E}-04$ & 0.21 & 9.092 & 7.928 & 7.428 \\
3 & 5 & $2.1 \mathrm{E}-03$ & $6.5 \mathrm{E}-04$ & 0.31 & 9.19 & 7.941 & 7.507 \\
4 & 10 & $2.1 \mathrm{E}-03$ & $1.1 \mathrm{E}-03$ & 0.51 & 9.364 & 7.963 & 7.648 \\
5 & 10 & $2.1 \mathrm{E}-03$ & $1.5 \mathrm{E}-03$ & 0.70 & 9.503 & 7.981 & 7.759 \\
6 & 10 & $2.1 \mathrm{E}-03$ & $1.8 \mathrm{E}-03$ & 0.88 & 9.621 & 7.996 & 7.853 \\
7 & 15 & $2.1 \mathrm{E}-03$ & $2.4 \mathrm{E}-03$ & 1.14 & 9.764 & 8.014 & 7.966 \\
8 & 15 & $2.1 \mathrm{E}-03$ & $2.9 \mathrm{E}-03$ & 1.39 & 9.868 & 8.028 & 8.05 \\
9 & 20 & $2.1 \mathrm{E}-03$ & $3.6 \mathrm{E}-03$ & 1.70 & 9.966 & 8.041 & 8.126 \\
10 & 20 & $2.1 \mathrm{E}-03$ & $4.2 \mathrm{E}-03$ & 1.99 & 10.04 & 8.053 & 8.181 \\
11 & 25 & $2.1 \mathrm{E}-03$ & $4.9 \mathrm{E}-03$ & 2.32 & 10.103 & 8.061 & 8.228 \\
12 & 30 & $2.1 \mathrm{E}-03$ & $5.7 \mathrm{E}-03$ & 2.70 & 10.157 & 8.071 & 8.266 \\
13 & 40 & $2.1 \mathrm{E}-03$ & $6.6 \mathrm{E}-03$ & 3.14 & 10.205 & 8.08 & 8.298 \\
14 & 60 & $2.1 \mathrm{E}-03$ & $7.8 \mathrm{E}-03$ & 3.73 & 10.254 & 8.091 & 8.327 \\
15 & 100 & $2.1 \mathrm{E}-03$ & $9.5 \mathrm{E}-03$ & 4.52 & 10.302 & 8.104 & 8.349 \\
16 & 180 & $2.1 \mathrm{E}-03$ & $1.2 \mathrm{E}-02$ & 5.56 & 10.346 & 8.12 & 8.366 \\
17 & 300 & $2.1 \mathrm{E}-03$ & $1.4 \mathrm{E}-02$ & 6.69 & 10.342 & 8.118 & 8.367
\end{tabular}
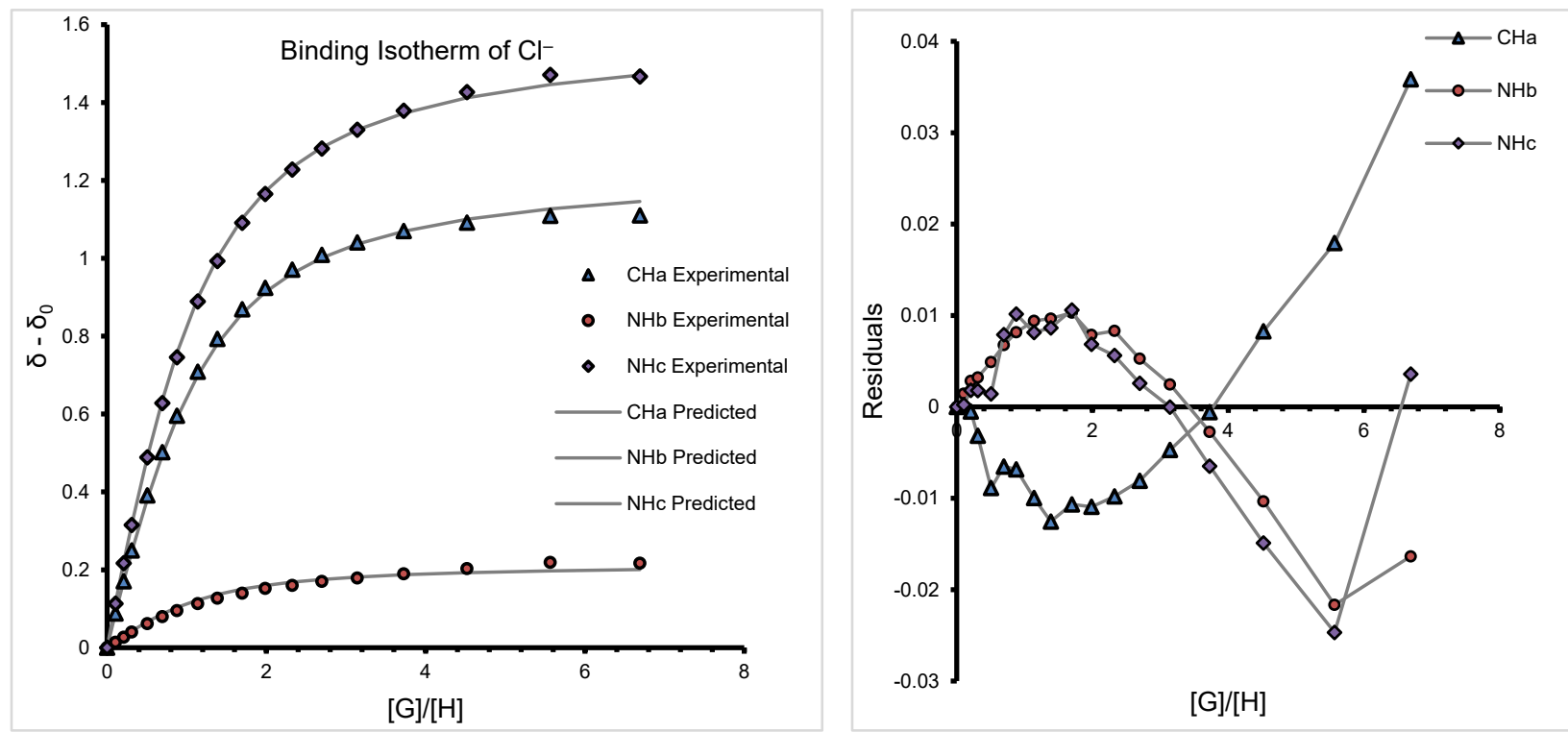

Figure S27. Representative binding isotherm and residuals for $\mathrm{Cl}^{-}$titration of receptor $\mathbf{1}^{\mathrm{NMe} 2}$ in $10 \%$ DMSO- $d_{6} / \mathrm{CD}_{3} \mathrm{CN}$ at $25{ }^{\circ} \mathrm{C}$ determined by ${ }^{1} \mathrm{H}$ NMR spectroscopy. 
Table S19. Representative titration of receptor $\mathbf{1}^{\mathrm{NMe} 2}$ with $\mathrm{Br}^{-}$in $10 \%$ DMSO- $d_{6} / \mathrm{CD}_{3} \mathrm{CN}$ at $25^{\circ} \mathrm{C}$.

\begin{tabular}{cccccccc} 
Entry & $\begin{array}{c}\mathrm{V}_{\text {Guest }} \\
(\mu \mathrm{L})\end{array}$ & $\begin{array}{c}{[\mathrm{Host}]} \\
(\mathrm{M})\end{array}$ & $\begin{array}{c}\left.\mathrm{Br}^{-}\right](\mathrm{M}) \\
\text { E }\end{array}$ & Equiv. & $\begin{array}{c}\delta \mathrm{NH}_{\mathrm{c}} \\
(\mathrm{ppm})\end{array}$ & $\begin{array}{c}\delta \mathrm{NH}_{\mathrm{b}} \\
(\mathrm{ppm})\end{array}$ & $\begin{array}{c}\delta \mathrm{CH}_{\mathrm{a}} \\
(\mathrm{ppm})\end{array}$ \\
\hline 0 & 0 & $2.0 \mathrm{E}-03$ & $0.0 \mathrm{E}+00$ & 0.00 & 8.895 & 7.906 & 7.264 \\
1 & 5 & $2.0 \mathrm{E}-03$ & $2.6 \mathrm{E}-04$ & 0.13 & 8.92 & 7.910 & 7.283 \\
2 & 10 & $2.0 \mathrm{E}-03$ & $7.6 \mathrm{E}-04$ & 0.39 & 8.961 & 7.919 & 7.315 \\
3 & 15 & $2.0 \mathrm{E}-03$ & $1.5 \mathrm{E}-03$ & 0.75 & 9.021 & 7.93 & 7.362 \\
4 & 20 & $2.0 \mathrm{E}-03$ & $2.4 \mathrm{E}-03$ & 1.20 & 9.089 & 7.942 & 7.396 \\
5 & 25 & $2.0 \mathrm{E}-03$ & $3.4 \mathrm{E}-03$ & 1.73 & 9.164 & 7.956 & 7.478 \\
6 & 30 & $2.0 \mathrm{E}-03$ & $4.5 \mathrm{E}-03$ & 2.30 & 9.233 & 7.967 & 7.535 \\
7 & 40 & $2.0 \mathrm{E}-03$ & $5.9 \mathrm{E}-03$ & 2.98 & 9.31 & 7.98 & 7.595 \\
8 & 50 & $2.0 \mathrm{E}-03$ & $7.3 \mathrm{E}-03$ & 3.71 & 9.387 & 7.993 & 7.657 \\
9 & 60 & $2.0 \mathrm{E}-03$ & $8.8 \mathrm{E}-03$ & 4.47 & 9.453 & 8.006 & 7.708 \\
10 & 80 & $2.0 \mathrm{E}-03$ & $1.0 \mathrm{E}-02$ & 5.31 & 9.512 & 8.016 & 7.754 \\
11 & 100 & $2.0 \mathrm{E}-03$ & $1.2 \mathrm{E}-02$ & 6.16 & 9.567 & 8.025 & 7.794 \\
12 & 130 & $2.0 \mathrm{E}-03$ & $1.4 \mathrm{E}-02$ & 7.02 & 9.612 & 8.034 & 7.829 \\
13 & 170 & $2.0 \mathrm{E}-03$ & $1.6 \mathrm{E}-02$ & 7.88 & 9.653 & 8.043 & 7.857 \\
14 & 120 & $2.0 \mathrm{E}-03$ & $1.6 \mathrm{E}-02$ & 8.35 & 9.671 & 8.047 & 7.871
\end{tabular}
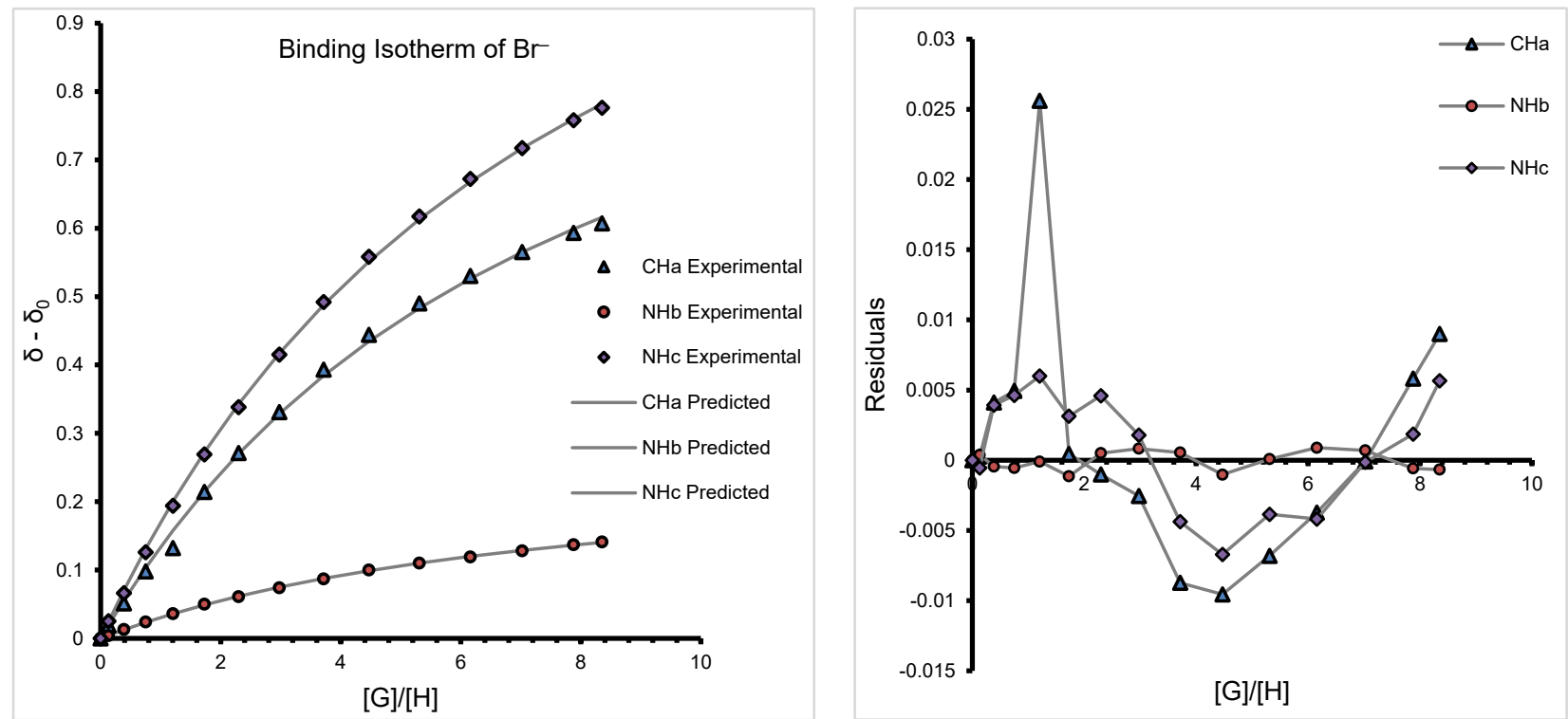

Figure S28. Representative binding isotherm and residuals for $\mathrm{Br}^{-}$titration of receptor $\mathbf{1}^{\mathrm{NMe} 2}$ in $10 \%$ DMSO- $d_{6} / \mathrm{CD}_{3} \mathrm{CN}$ at $25{ }^{\circ} \mathrm{C}$ determined by ${ }^{1} \mathrm{H}$ NMR spectroscopy. 
Section S4. Linear Regression Fitting and Statistics of $\Delta G_{b i n d i n g}$ and $\sigma_{p}$.

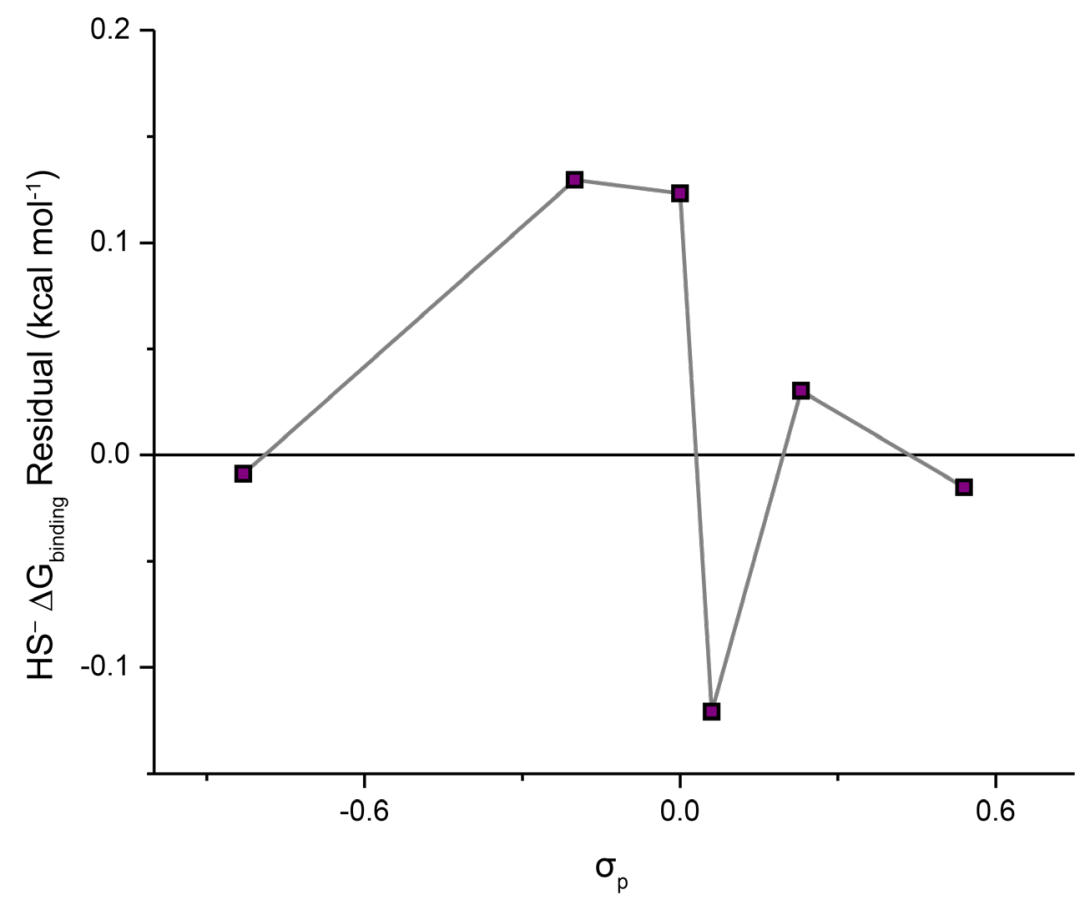

Figure S29. Linear regression residuals of $\Delta \mathrm{G}_{\text {binding }}$ of $\mathrm{HS}^{-}$and $\sigma_{\mathrm{p}}$.

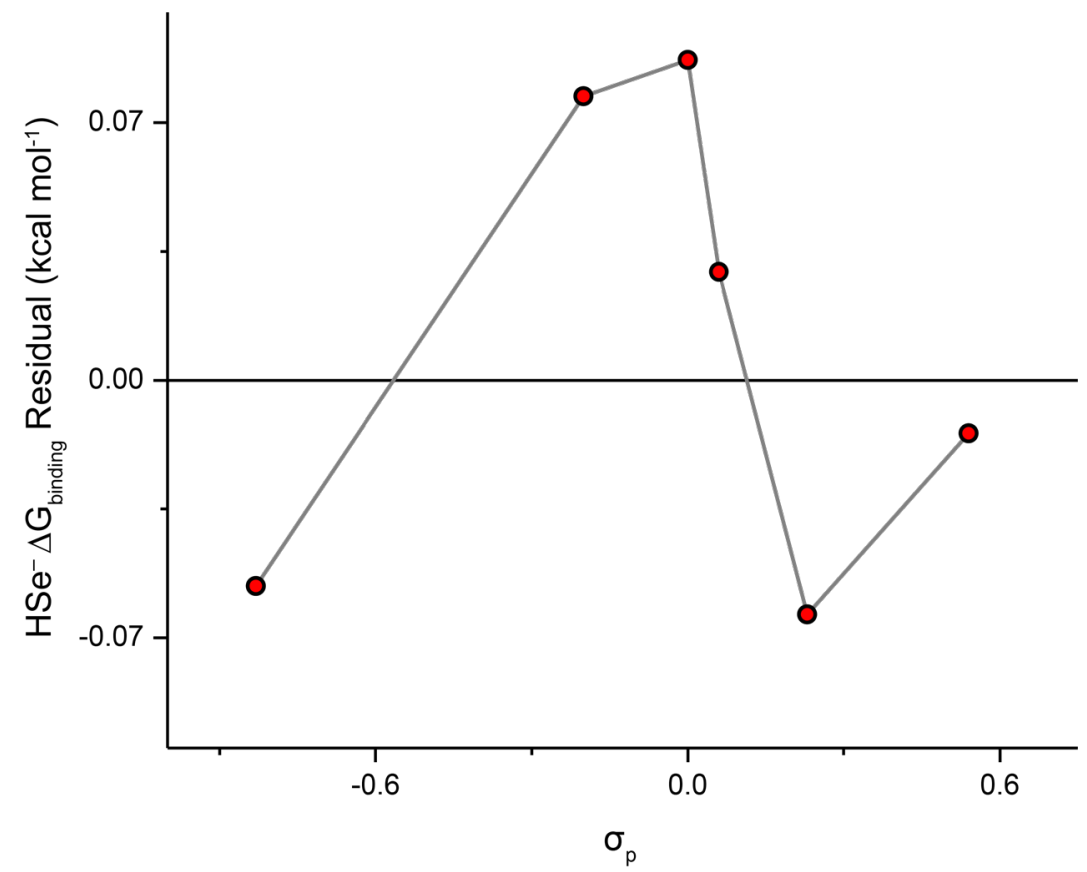

Figure S30. Linear regression residuals of $\Delta \mathrm{G}_{\text {binding }}$ of $\mathrm{HSe}^{-}$and $\sigma_{\mathrm{p}}$. 


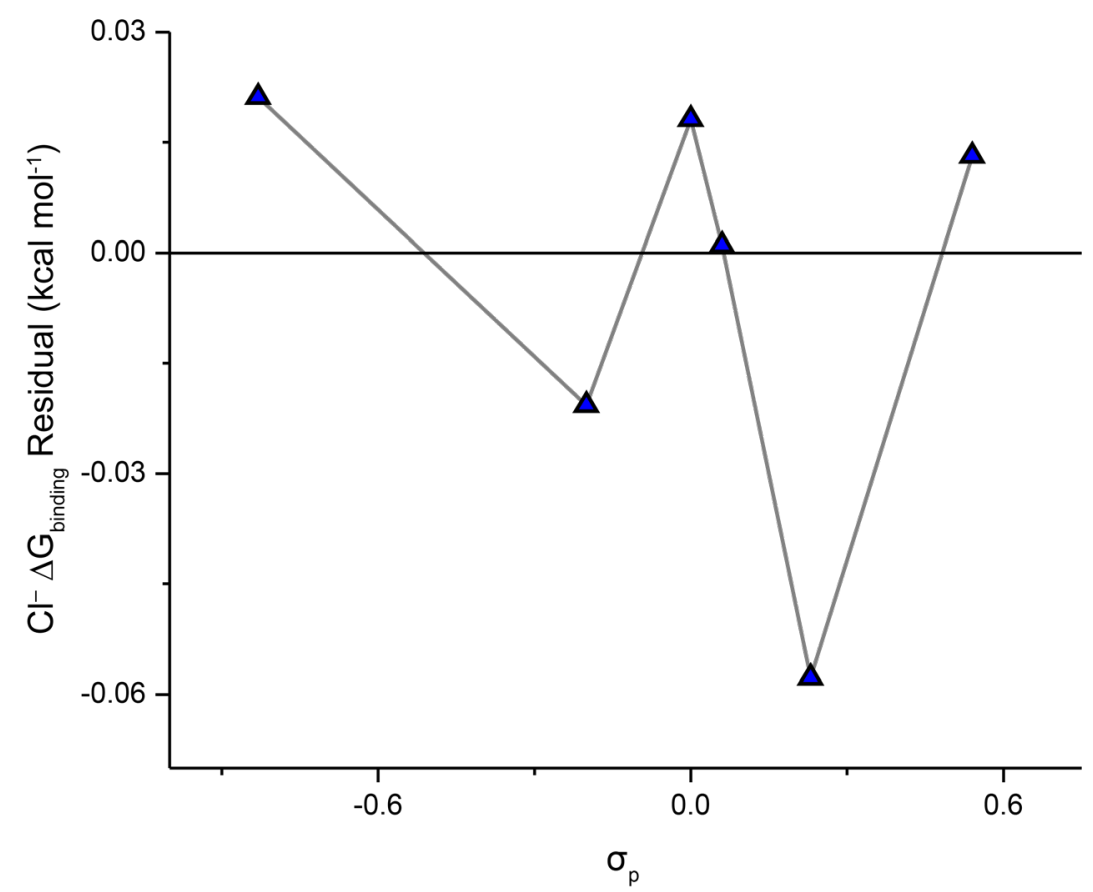

Figure S31. Linear regression residuals of $\Delta \mathrm{G}$ binding of $\mathrm{Cl}^{-}$and $\sigma_{\mathrm{p}}$.

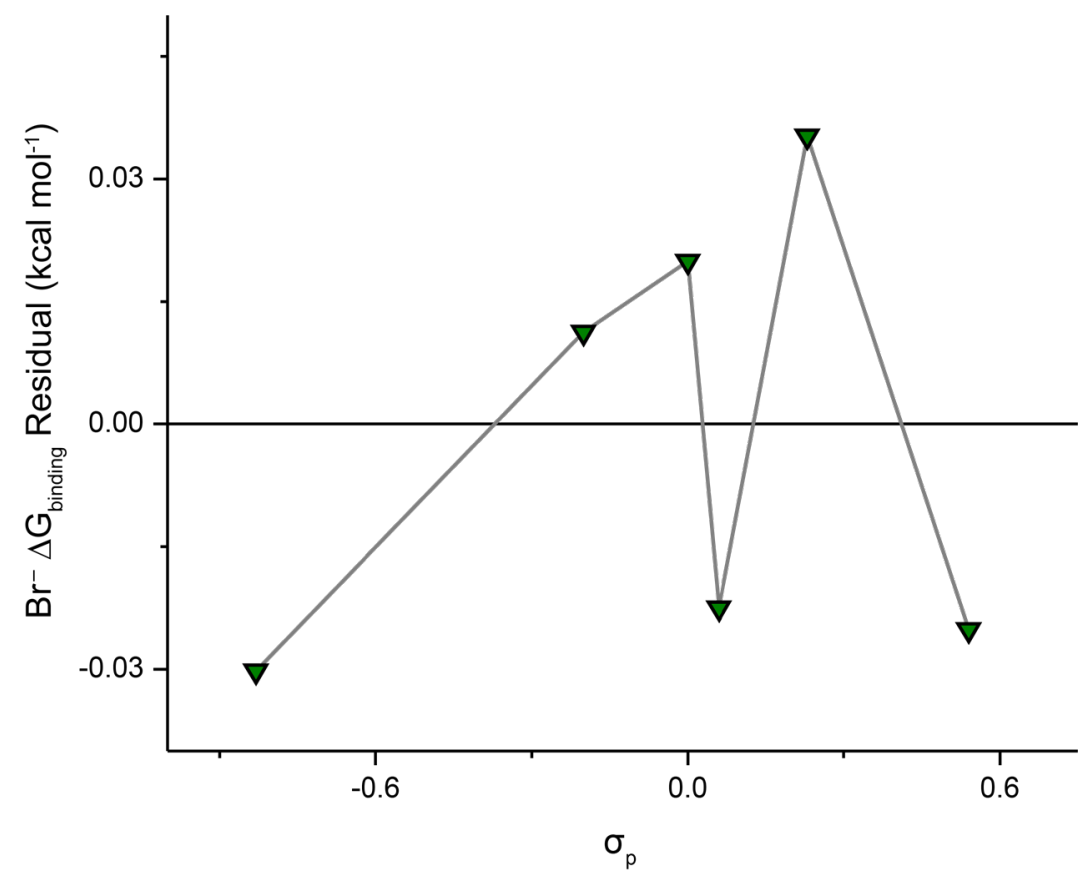

Figure S32. Linear regression residuals of $\Delta \mathrm{G}_{\text {binding }}$ of $\mathrm{Br}^{-}$and $\sigma_{\mathrm{p}}$. 
Section S5. Linear Regression Fitting and Statistics of $\log \left(K_{a}{ }^{R} / K_{a}{ }^{H}\right)$ and $\sigma_{p}$.

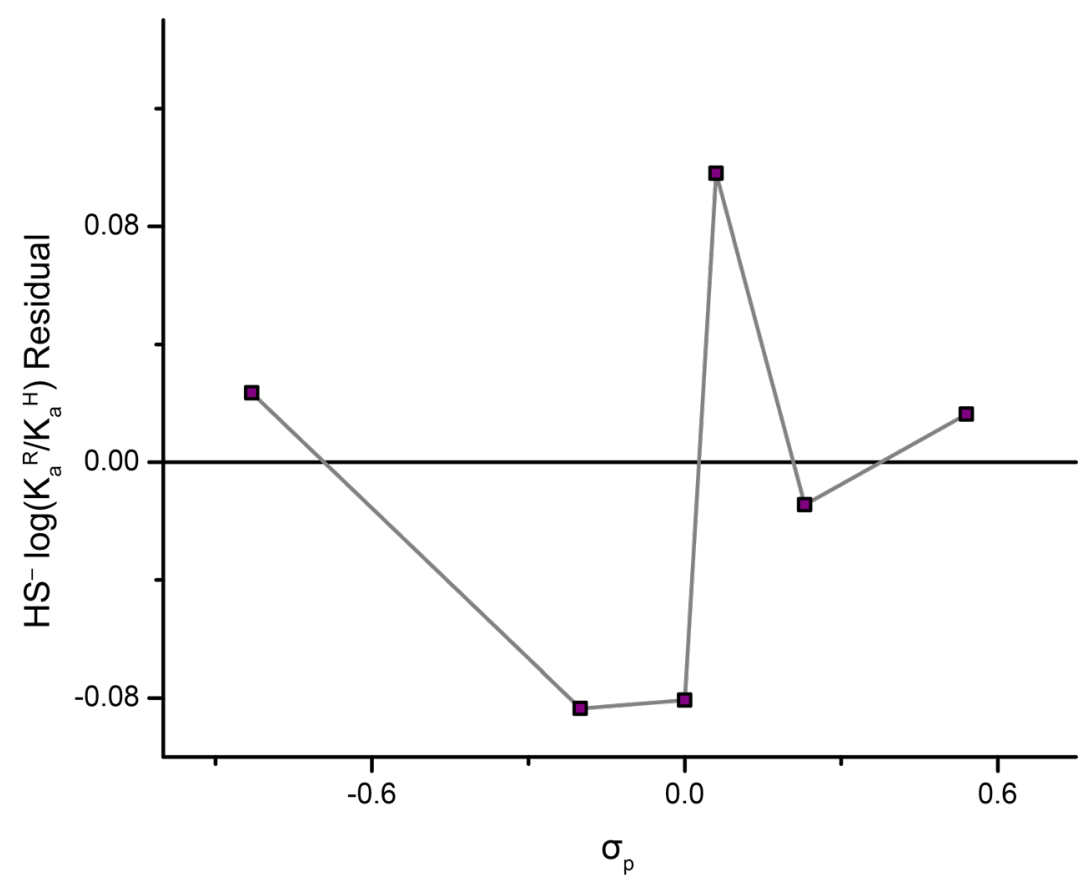

Figure S33. Linear regression residuals of $\log \left(\mathrm{K}_{\mathrm{a}}^{\mathrm{R}} / \mathrm{K}_{\mathrm{a}}{ }^{\mathrm{H}}\right)$ of $\mathrm{HS}^{-}$and $\sigma_{\mathrm{p}}$.

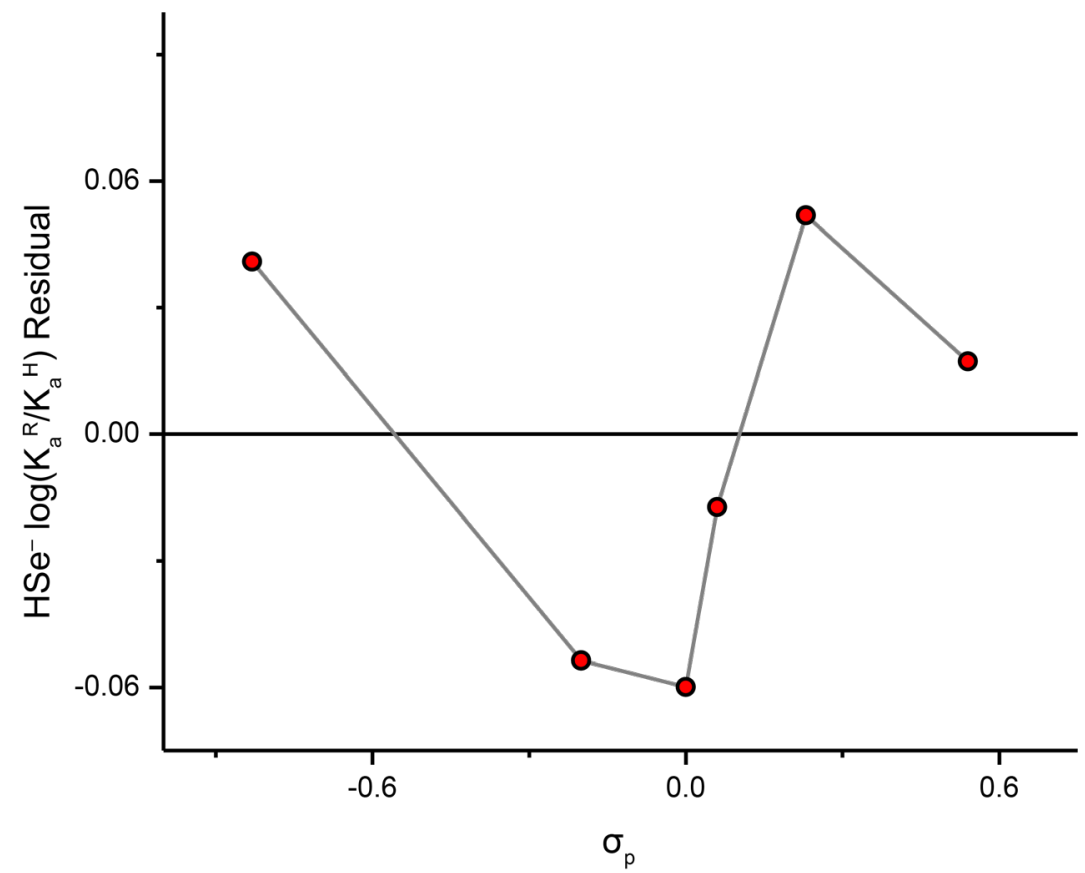

Figure S34. Linear regression residuals of $\log \left(\mathrm{K}_{\mathrm{a}}^{\mathrm{R}} / \mathrm{K}_{\mathrm{a}}{ }^{\mathrm{H}}\right)$ of $\mathrm{HS}^{-}$and $\sigma_{\mathrm{p}}$. 


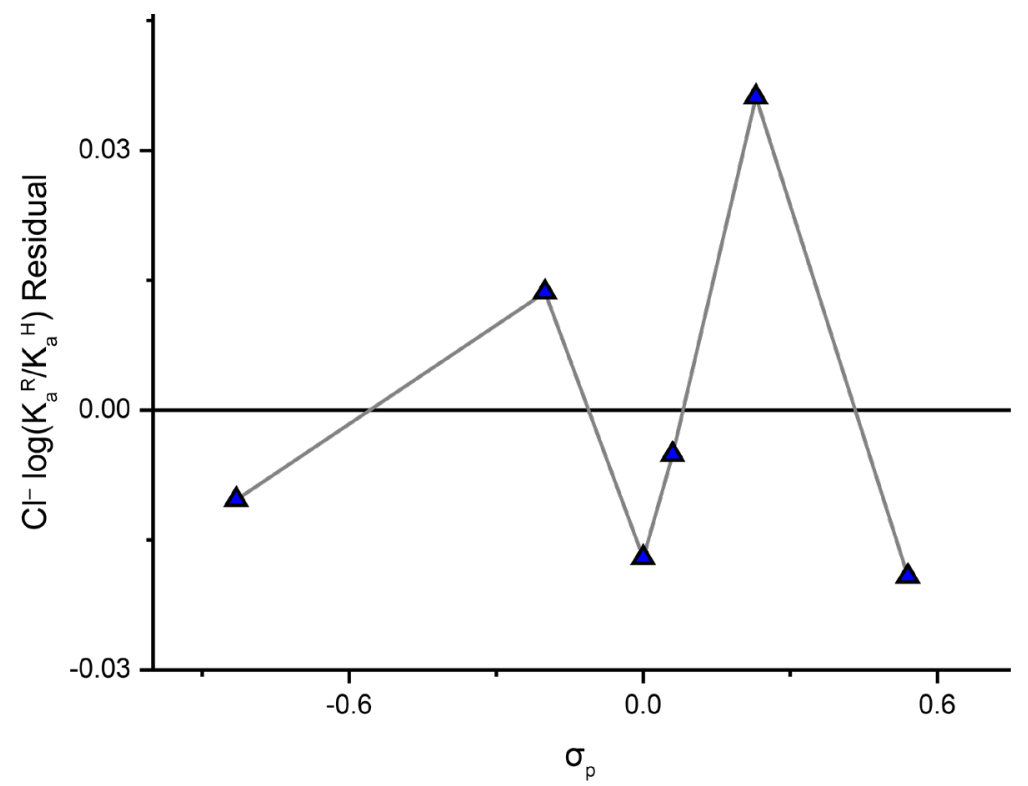

Figure S35. Linear regression residuals of $\log \left(\mathrm{K}_{\mathrm{a}}^{\mathrm{R}} / \mathrm{K}_{\mathrm{a}}{ }^{\mathrm{H}}\right)$ of $\mathrm{Cl}^{-}$and $\sigma_{\mathrm{p}}$.

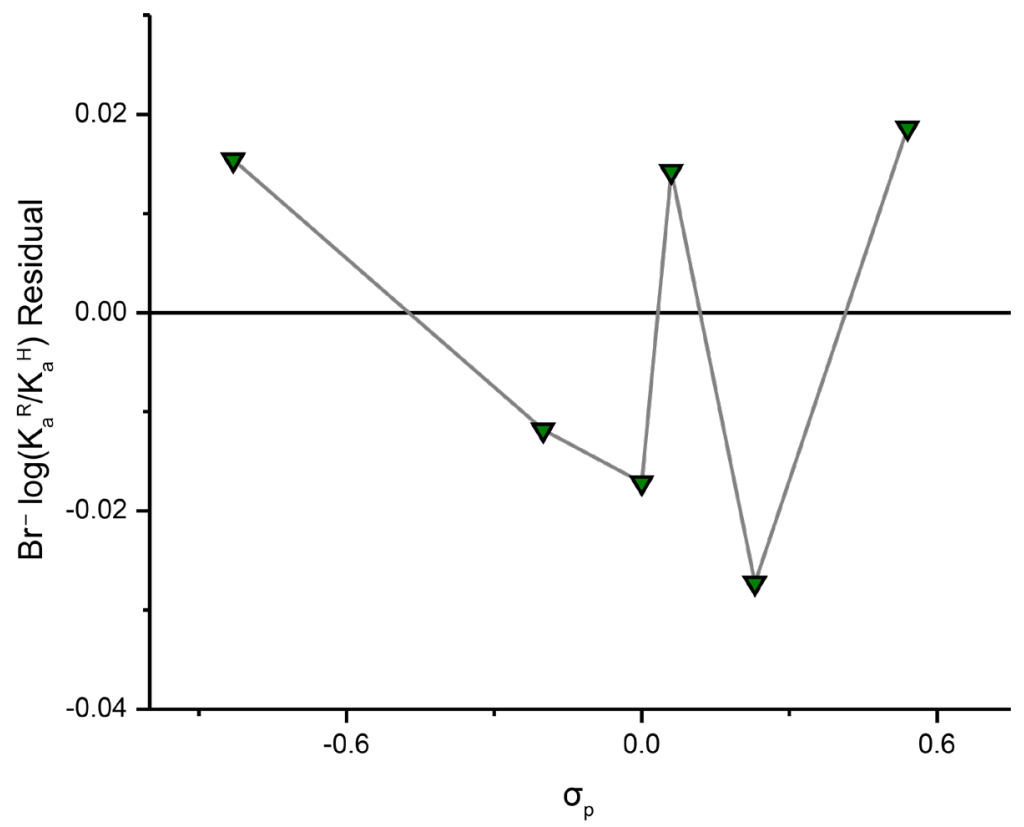

Figure S36. Linear regression residuals of $\log \left(\mathrm{K}_{\mathrm{a}}^{\mathrm{R}} / \mathrm{K}_{\mathrm{a}}{ }^{\mathrm{H}}\right)$ of $\mathrm{Br}^{-}$and $\sigma_{\mathrm{p}}$.

Table S20. Results of linear regression of $\log \left(\mathrm{K}_{\mathrm{a}}^{\mathrm{R}} / \mathrm{K}_{\mathrm{a}}^{\mathrm{H}}\right)$ vs. $\sigma_{\mathrm{p}}$ when the intercept is forced through the origin.

\begin{tabular}{|c|c|cc|c|}
\hline \multirow{2}{*}{ Guest } & \multirow{2}{*}{ Slope } & \multicolumn{2}{|c|}{ p-value } & \multirow{2}{*}{$\mathbf{R}^{2}$} \\
\cline { 3 - 4 } & & Slope & Model & \\
\hline $\mathrm{HS}^{-}$ & $0.70 \pm 0.10$ & $<0.01$ & $<0.01$ & 0.91 \\
$\mathrm{HSe}^{-}$ & $0.31 \pm 0.07$ & $<0.01$ & $<0.01$ & 0.80 \\
$\mathrm{Cl}^{-}$ & $0.25 \pm 0.03$ & $<0.01$ & $<0.01$ & 0.95 \\
$\mathrm{Br}^{-}$ & $0.22 \pm 0.03$ & $<0.01$ & $<0.01$ & 0.94 \\
\hline
\end{tabular}




\section{Section S6: Analysis of Covariance on Hammett Plots.}

Analysis of covariance of the linear regressions obtained for the Hammett plots for each anion was used to determine if the $K_{a}$ of each anion showed the same response to the changing $\sigma_{\mathrm{p}}$ of the $-\mathrm{R}$ substituent on the receptor. The $\mathrm{p}$-value represents the probability that the linear regressions for two anions would appear to have different slopes when they in fact have the same slope. Any p-value $>0.05$ is considered statistically insignificant at the $95 \%$ confidence interval, meaning we cannot confidently say that the linear regressions have different slopes.

Table S21. Results of analysis of covariance on Hammett plots.

\begin{tabular}{|c|c|}
\hline & p-value \\
\hline HSfferent models $^{-}$vs. $\mathbf{H S e}^{-}$ & $<0.01$ \\
HS$^{-}$vs. $\mathbf{C l}^{-}$ & $<0.01$ \\
$\mathbf{H S}^{-}$vs. $\mathbf{B r}^{-}$ & $<0.01$ \\
HSe $^{-}$vs. $\mathbf{C l}^{-}$ & 0.28 \\
HSe $^{-}$vs. $\mathbf{B r}^{-}$ & 0.11 \\
$\mathbf{C l}^{-}$vs. $\mathbf{B r}^{-}$ & 0.28 \\
\hline
\end{tabular}

\section{Section S7. Swain-Lupton Analysis}

Table 22. Results of multivariable linear regression for Swain-Lupton analysis when the intercept is forced through the origin.

\begin{tabular}{|c|c|c|ccc|c|c|c|}
\hline \multirow{2}{*}{ Guest } & \multirow{2}{*}{$\boldsymbol{\rho}_{\mathbf{f}}$} & \multirow{2}{*}{$\boldsymbol{\rho}_{\mathbf{r}}$} & \multicolumn{3}{|c|}{$\mathbf{p}$-value } & \multirow{2}{*}{$\mathbf{R}^{\mathbf{2}}$} & \multirow{2}{*}{$\mathbf{R}_{\mathbf{a d j}}$} & \multirow{2}{*}{$\% \mathbf{o R}$} \\
\cline { 5 - 6 } & & & $\boldsymbol{\rho}_{\mathbf{f}}$ & $\boldsymbol{\rho}_{\mathbf{r}}$ & $\mathbf{M o d e l}$ & & & \\
\hline $\mathbf{H S}^{-}$ & $0.95 \pm 0.05$ & $0.63 \pm 0.04$ & $>0.01$ & $>0.01$ & $>0.01$ & 0.99 & 0.99 & $40 \pm 3$ \\
$\mathbf{H S e}^{-}$ & $0.49 \pm 0.05$ & $0.26 \pm 0.04$ & $>0.01$ & $>0.01$ & $>0.01$ & 0.96 & 0.95 & $35 \pm 6$ \\
$\mathbf{C l}^{-}$ & $0.29 \pm 0.04$ & $0.24 \pm 0.03$ & $>0.01$ & $>0.01$ & $>0.01$ & 0.96 & 0.96 & $45 \pm 6$ \\
$\mathbf{B r}^{-}$ & $0.26 \pm 0.03$ & $0.20 \pm 0.02$ & $>0.01$ & $>0.01$ & $>0.01$ & 0.97 & 0.96 & $44 \pm 6$ \\
\hline
\end{tabular}




\section{Section S8. Alternative Hammett parameters}

Table S23 shows the fitting parameters and statistics the linear fit of the LFERs of the binding energy of $\mathrm{HS}^{-}, \mathrm{HSe}^{-}, \mathrm{Cl}^{-}$, and $\mathrm{Br}^{-}$with $\sigma_{\mathrm{m}}$. The Hammett parameter $\sigma_{\mathrm{m}}$ was developed for substituents in the meta position on benzoic acid. This parameter retains similar field and inductive effects of the para parameter while decreasing resonance contributions (the average importance of resonance in the $\sigma_{\mathrm{p}}$ value for each substituent is $53 \%$, while only $22 \%$ in $\sigma_{\mathrm{m}}{ }^{9}$ ). Although none of the resulting $R^{2}$ suggest a good fit of binding energies to $\sigma_{m}\left(R^{2}<0.90\right)$, the $R^{2}$ for the linear fit of the hydrochalcogenides is higher than that of the halides. This suggests that resonance contribution of the substituent is more important for the halides than the hydrochalcogenides.

Table S24 shows the fitting parameters and statistics the linear fit of the LFERs of the binding energy of $\mathrm{HS}^{-}, \mathrm{HSe}^{-}, \mathrm{Cl}^{-}$, and $\mathrm{Br}^{-}$with $\sigma_{\mathrm{p}}{ }^{+}$. The Hammett parameter $\sigma_{p}^{+}$was developed to give weight to substituents that can stabilize a build-up of charge through resonance effects (the average importance of resonance in the $\sigma_{p}^{+}$value for each substituent is $66 \%{ }^{9}$ ). We do not expect $\sigma_{p}^{+}$to bind well to our systems since the charged resonance structures in Figure S37 are informal. However, comparison of $\mathrm{R}^{2}$ shows that $\sigma_{p}^{+}$is a better fit for the halides than the hydrochalcogenides, yielding the same conclusion as for $\sigma_{\mathrm{m}}$; resonance contributions are more important to halide binding than hydrochalcogenide binding.

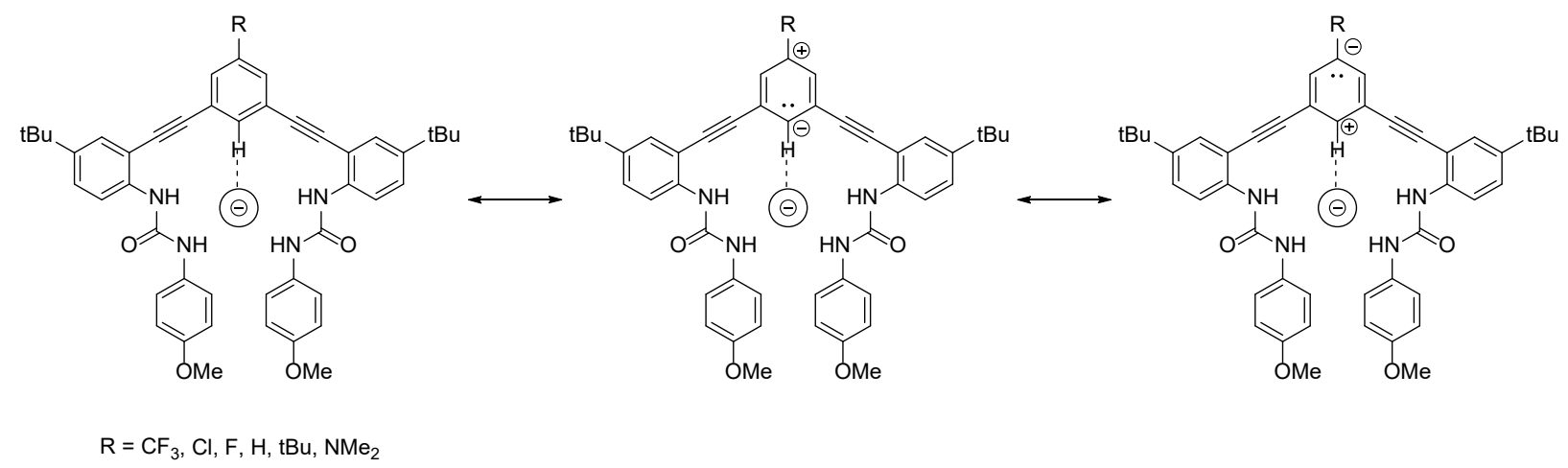

Figure S37. Informal resonance structures for receptors $\mathbf{1}^{\mathbf{R}}$ that may influence aryl C-H HB donor ability. 
Table S23. Fitting statistics for Hammett plots for all four anions using $\sigma_{\mathrm{m}}$.

\begin{tabular}{|c|c|c|ccc|c|c|}
\hline \multirow{2}{*}{ Guest } & \multirow{2}{*}{$\boldsymbol{\rho}$} & $\boldsymbol{\varepsilon}$ & \multicolumn{3}{|c|}{ p-value } & \multirow{2}{*}{$\mathbf{R}^{\mathbf{2}}$} & \multirow{2}{*}{$\mathbf{R}^{\mathbf{2}}$ adj. } \\
\cline { 4 - 6 } & & & $\boldsymbol{\rho}$ & $\boldsymbol{\varepsilon}$ & Model & & \\
\hline $\mathrm{HS}^{-}$ & $1.20 \pm 0.23$ & $-0.13 \pm 0.06$ & $<0.01$ & 0.12 & $<0.01$ & 0.87 & 0.84 \\
$\mathrm{HSe}^{-}$ & $0.55 \pm 0.10$ & $-0.04 \pm 0.02$ & $<0.01$ & 0.29 & $<0.01$ & 0.88 & 0.84 \\
$\mathrm{Cl}^{-}$ & $0.39 \pm 0.12$ & $-0.05 \pm 0.03$ & 0.03 & 0.21 & 0.03 & 0.74 & 0.67 \\
$\mathrm{Br}^{-}$ & $0.35 \pm 0.09$ & $-0.04 \pm 0.03$ & 0.02 & 0.18 & 0.02 & 0.78 & 0.72 \\
\hline
\end{tabular}

Table S24. Fitting statistics for Hammett plots for all four anions using $\sigma_{\mathrm{p}}{ }^{+}$.

\begin{tabular}{|c|c|c|ccc|c|c|}
\hline \multirow{2}{*}{ Guest } & \multirow{2}{*}{$\boldsymbol{\rho}$} & $\boldsymbol{\varepsilon}$ & \multicolumn{3}{|c|}{ p-value } & \multirow{2}{*}{$\mathbf{R}^{\mathbf{2}}$} & \multirow{2}{*}{$\mathbf{R}^{2}$ adj. } \\
\cline { 4 - 6 } & & & $\boldsymbol{\rho}$ & $\boldsymbol{\varepsilon}$ & Model & & \\
\hline $\mathrm{HS}^{-}$ & $0.40 \pm 0.08$ & $0.14 \pm 0.06$ & $<0.01$ & 0.08 & $<0.01$ & 0.86 & 0.83 \\
$\mathrm{HSe}^{-}$ & $0.17 \pm 0.05$ & $0.08 \pm 0.04$ & 0.02 & 0.08 & 0.02 & 0.76 & 0.70 \\
$\mathrm{Cl}^{-}$ & $0.15 \pm 0.02$ & $0.04 \pm 0.02$ & $<0.01$ & 0.06 & $<0.01$ & 0.93 & 0.91 \\
$\mathrm{Br}^{-}$ & $0.12 \pm 0.02$ & $0.04 \pm 0.02$ & $<0.01$ & 0.10 & $<0.01$ & 0.89 & 0.86 \\
\hline
\end{tabular}




\section{Section S9. Computational Details, Discussion, Geometries, and Energies}

We turned to computational investigations to better understand the preference of the $\mathrm{C}-\mathrm{H} \mathrm{HB}$ for $\mathrm{HS}^{-}$. All six receptors, four anions, $\mathrm{NBu}_{4}^{+}$and their respective complexes were optimized in gas phase at the PBE/6-31G* level of theory with final energy refinements at wB97X-D3/def2TZVPP. ${ }^{10}$ LFER models correlating $\sigma_{p}$ for $\mathbf{1}^{\mathbf{R}}$ to the computed gas phase binding energies agreed with experiments in that more electron-withdrawing para substituents favor the host/guest interaction for all four anions. Unlike the experimental data, however, the slopes for all four anions were indistinguishable (average slope $=-4$ ), and the receptor was seen to be most selective for $\mathrm{Cl}^{-}$ rather than $\mathrm{HS}^{-}$.

Exhaustive efforts to refine the energies with solvent corrections were unfruitful. We computed the solvation corrections using $\mathrm{SMD}^{11}$ at the wB97X-D3/def2-TZVPP level of theory in water, acetonitrile, DMSO, and chloroform. Trends in the binding energies computed in solvent did not match experimental trends. All solvent results showed either no statistically meaningful linear trend $\left(\mathrm{R}^{2}<0.6\right.$ and $\mathrm{p}$-value $\left.\sim 0.8\right)$, or the opposite trend in which more electron-donating para substituents favor the host-guest interaction. ${ }^{12}$ Collectively, these results reveal that the trends observed experimentally are the results of complex solvent-solute interaction or dynamic phenomena and also clearly reveal the limitations of current continuum solvent modeling methods for unique solvent systems as used in this work.

\section{Complete author list of Gaussian 09}

Gaussian 09, Revision D.01, Frisch, M. J.; Trucks, G. W.; Schlegel, H. B.; Scuseria, G. E.; Robb, M. A.; Cheeseman, J. R.; Scalmani, G.; Barone, V.; Mennucci, B.; Petersson, G. A.; Nakatsuji, H.; Caricato, M.; Li, X.; Hratchian, H. P.; Izmaylov, A. F.; Bloino, J.; Zheng, G.; Sonnenberg, J. L.; Hada, M.; Ehara, M.; Toyota, K.; Fukuda, R.; Hasegawa, J.; Ishida, M.; Nakajima, T.; Honda, Y.; Kitao, O.; Nakai, H.; Vreven, T.; Montgomery, J. A., Jr.; Peralta, J. E.; Ogliaro, F.; Bearpark, M.; Heyd, J. J.; Brothers, E.; Kudin, K. N.; Staroverov, V. N.; Kobayashi, R.; Normand, J.; Raghavachari, K.; Rendell, A.; Burant, J. C.; Iyengar, S. S.; Tomasi, J.; Cossi, M.; Rega, N.; Millam, J. M.; Klene, M.; Knox, J. E.; Cross, J. B.; Bakken, V.; Adamo, C.; Jaramillo, J.; Gomperts, R.; Stratmann, R. E.; Yazyev, O.; Austin, A. J.; Cammi, R.; Pomelli, C.; Ochterski, J. W.; Martin, R. L.; Morokuma, K.; Zakrzewski, V. G.; Voth, G. A.; Salvador, P.; Dannenberg, J. J.; Dapprich, S.; Daniels, A. D.; Farkas, Ö.; Foresman, J. B.; Ortiz, J. V.; Cioslowski, J.; Fox, D. J. Gaussian, Inc., Wallingford CT, 2009. 


\section{Complete author list of ORCA version 4.0}

ORCA version 4.0, Neese, F.; Wennmohs, F.; Aravena, D.; Atanasov, M.; Becker, U.; Bykov, D.; Chilkuri, V. G.; Datta, D.; Dutta, A. K.; Ganyushin, D.; Guo, Y.; Hansen, A.; Huntington, L.; Izsak, R.; Kollmar, C.; Kossmann, S.; Krupicka, M.; Lenk, D.; Liakos, D. G.; Manganas, D.; Pantazis, D. A.; Petrenko, T.; Pinski, P.; Reimann, C.; Retegan, M.; Riplinger, C.; Risthaus, T.; Roemelt, M.; Saitow, M.; Sandhofer, B.; Schapiro, I.; Sivalingam, K.; Stoychev, G.; Wezisla, B.; Kallay, M.; Grimme, S.; Valeev, E.; Chan, G.; Pittner, J.; Brehm, M.; Bistoni, G.; Schneider, W. WIREs: Comp. Mol. Sci. 8, e1327

\section{D3 corrections in ORCA}

S.Grimme, J.Antony, S.Ehrlich and H.Krieg, J.Chem.Phys., 2010, 132, 154104

\section{def2-TZVPP}

F. Weigend and R. Ahlrichs, Phys. Chem. Chem. Phys. 2005, 7, 3297

\section{$\operatorname{def} 2 / \mathbf{J}$}

F. Weigend, Phys. Chem. Chem. Phys. 2006, 8, 1057

\section{def2-TZVPP/C}

H-La, Hf-Rn : A. Hellweg, C. Hattig, S. Hofener and W. Klopper, Theor. Chem. Acc. 2007, 117, 587

Ce-Lu : J. Chmela and M. E. Harding, Mol. Phys. 2018

\section{General protocol for geometry optimization and energy calculations}

Geometry optimizations and single point energy refinements were completed using the Gaussian 09 computational package (see above reference) with the ultrafine integration grid to minimize errors. All pertinent ground state structures were located with geometry optimizations using PBE/6-31G(d). Higher level single point energy refinements were computed in ORCA version 4.0.1 with wB97X-D3/def2-TZVPP in SMD(chloroform). Final corrected energies were the sum of the single point energy refinement and the Gibbs thermal correction factor of the respective optimized structure. All reported computed thermodynamics and barriers are derived from differences in the final corrected energies. All energies are reported as $\mathrm{kcal} / \mathrm{mol}$ as converted from hartrees $(1 \mathrm{Ha}=627.5095 \mathrm{kcal} / \mathrm{mol})$. All 3D structure images were rendered in GaussView visualization software. $^{11}$ 
Figures S38-S42. Hammett Value $\left(\sigma_{\mathrm{p}}\right)$ for $1^{\mathrm{R}}$ vs computed binding energies $\left(\Delta \mathrm{G}_{\text {binding }}\right)$

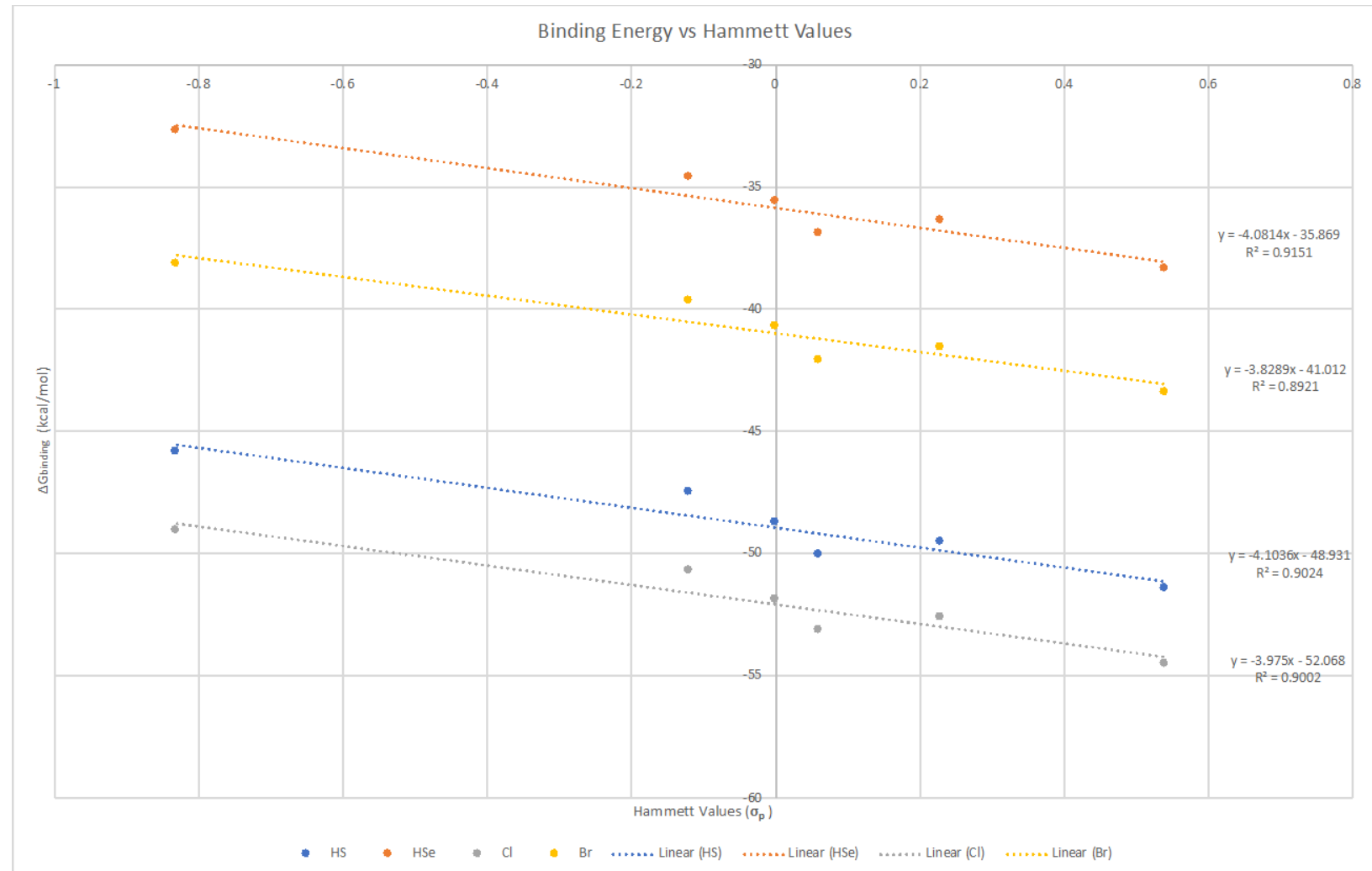

Figure S38. wB97X-D3/def2-TZVPP//PBE/6-31G(d)/

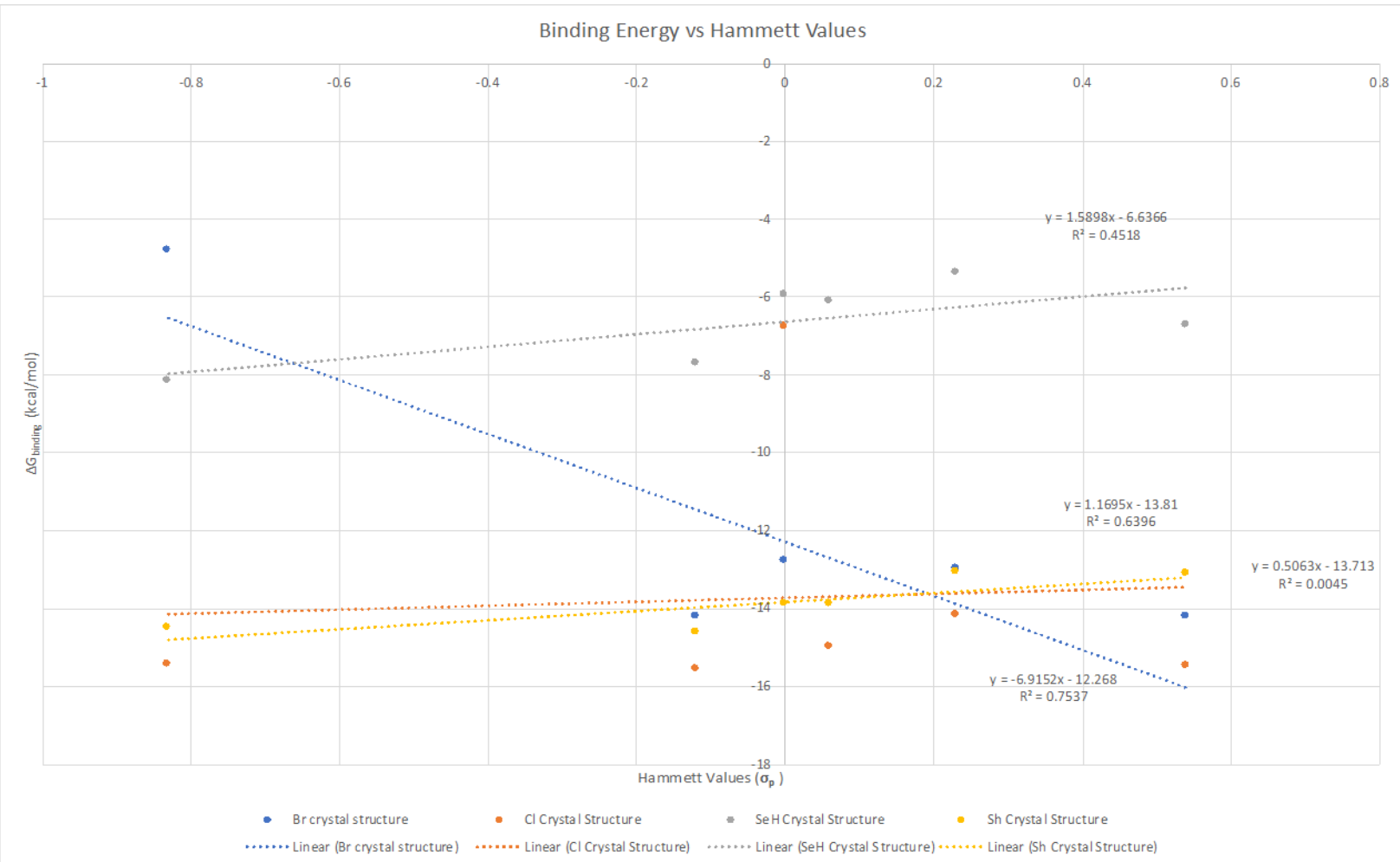

Figure S39. wb97x/def2-TZVPP/SMD/Chloroform//PBE/6-31G(d)/ 


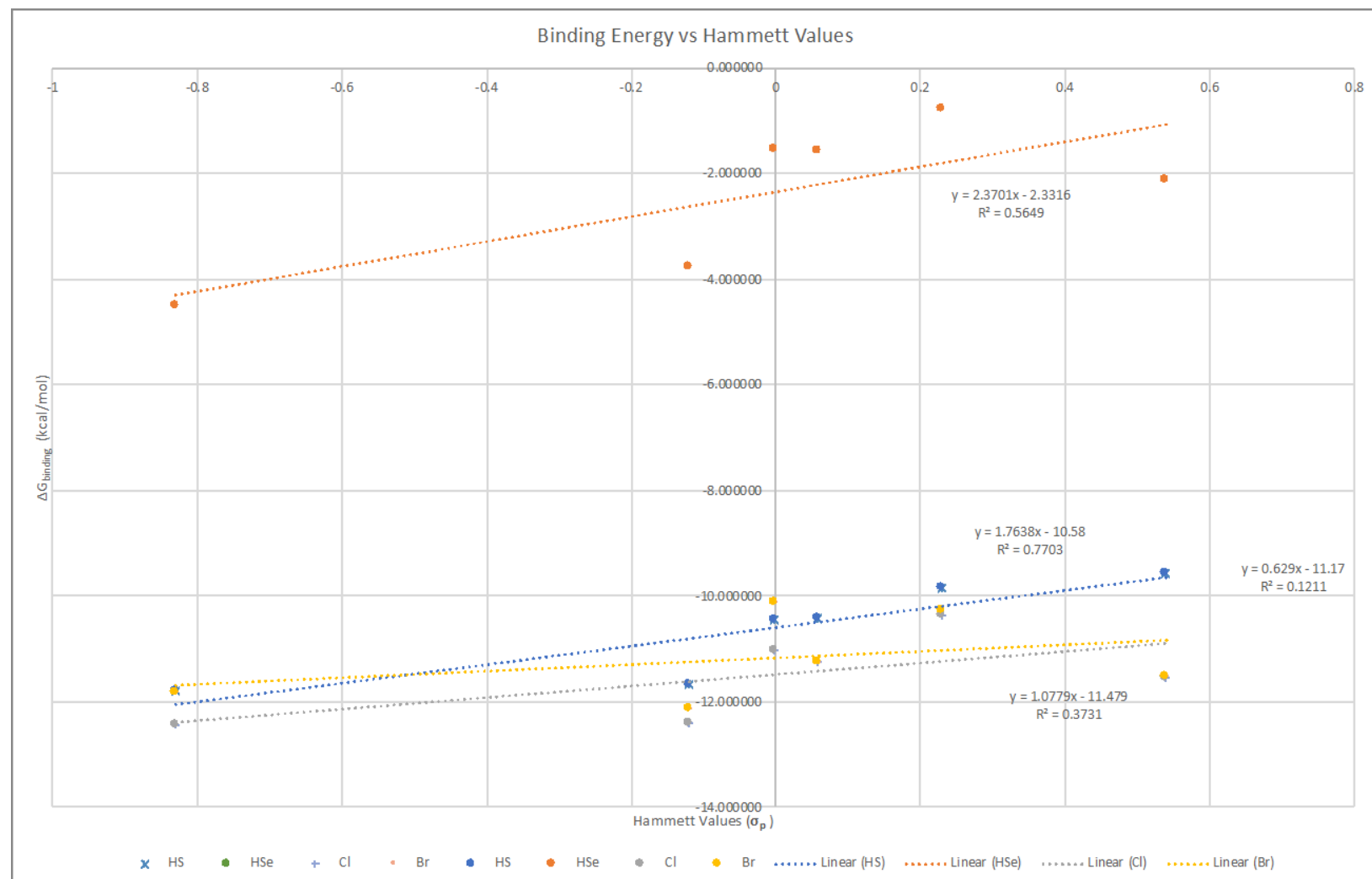

Figure S40. wb97x/def2-TZVPP/SMD/Acetonitrile//PBE/6-31G(d)

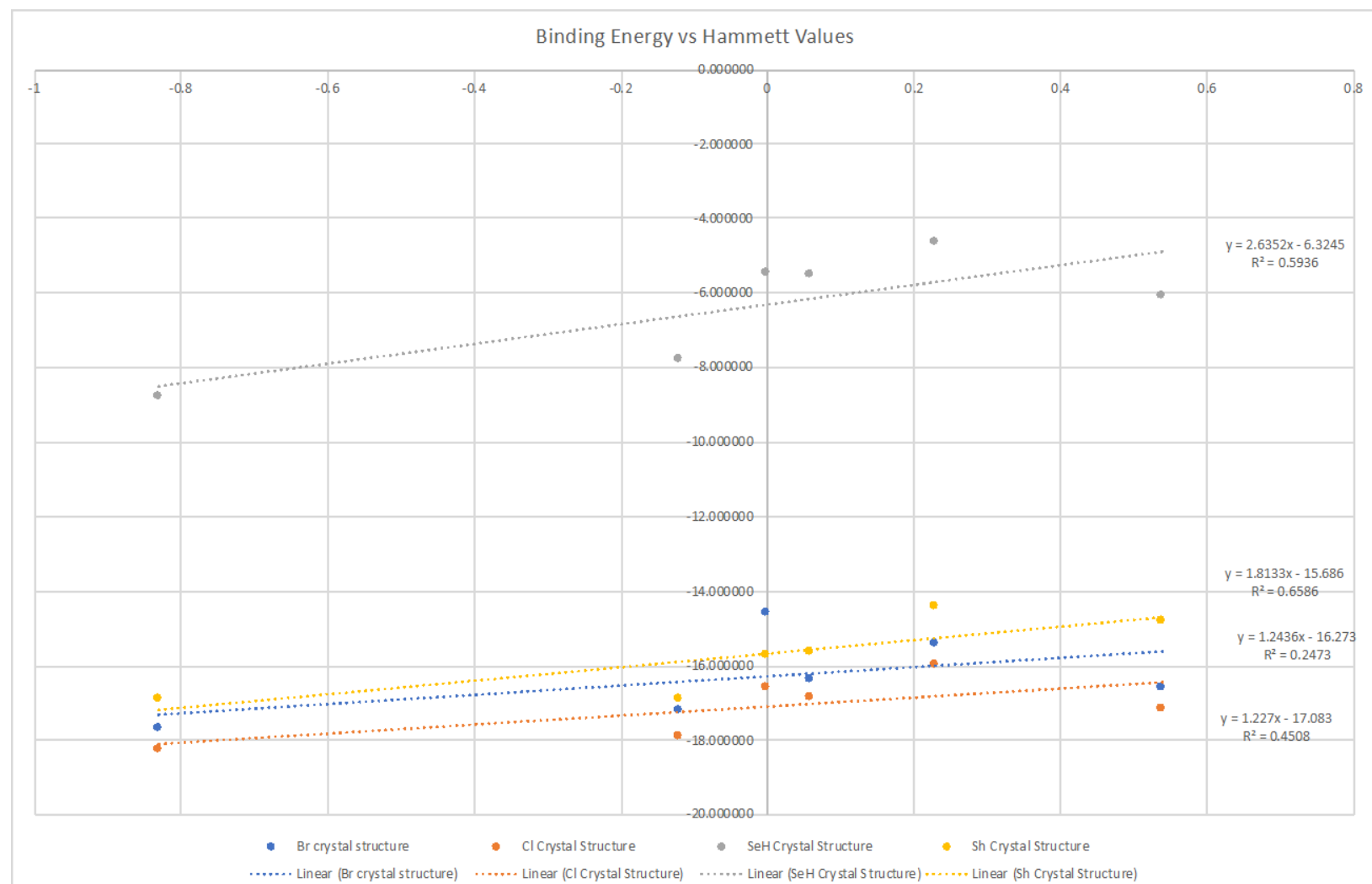

Figure S41. wb97x/def2-TZVPP/SMD/Water//PBE/6-31G(d) 


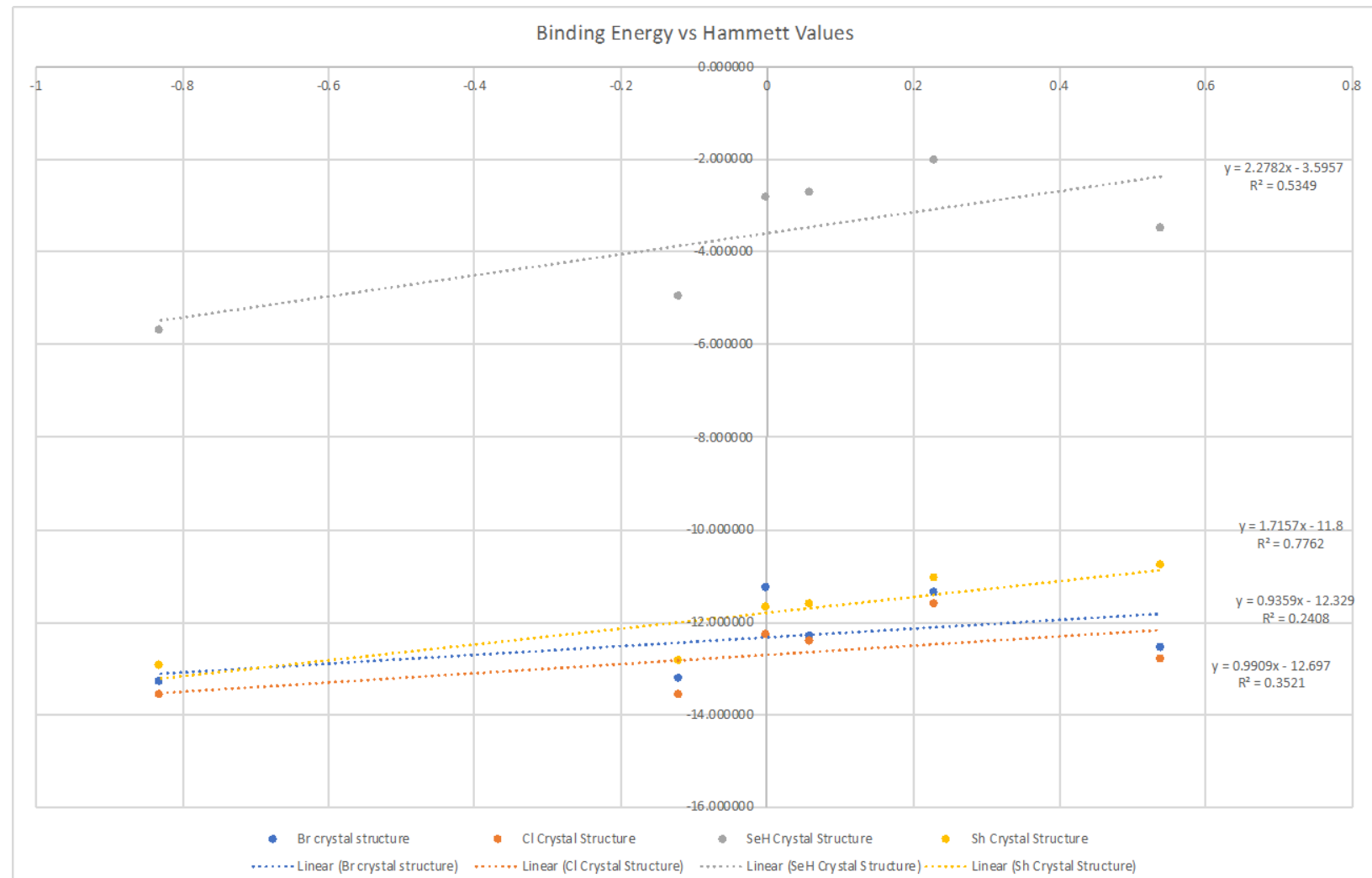

Figure S42. wb97x/def2-TZVPP/SMD/DMSO//PBE/6-31G(d) 


\section{Section S10. References}

(1) Hartle, M. D.; Meininger, D. J.; Zakharov, L. N.; Tonzetich, Z. J.; Pluth, M. D. NBu4SH Provides a Convenient Source of HS- Soluble in Organic Solution for H2S and AnionBinding Research. Dalton Trans. 2015, 44, 19782-19785.

(2) Fargher, H. A.; Lau, N.; Zakharov, L. N.; Haley, M. M.; Johnson, D. W.; Pluth, M. D. Expanding Reversible Chalcogenide Binding: Supramolecular Receptors for the Hydroselenide (HSe-) Anion. Chem. Sci. 2018, 10, 67-72.

(3) Rajca, A.; Rajca, S.; Wongsriratanakul, J.; Ross II, C. R. 4,6-Bis(Trifluoromethyl)-N,N'Di-Tert-Butyl-1,3-Phenylenebis(Aminoxyl) and Its Bis(Hexafluoroacetylacetonato)Manganese(II) Complex: Synthesis, X-Ray Crystallography, and Magnetism. Polyhedron 2001, 20, 1669-1675.

(4) Asmus, S.; Beckendorf, S.; Zurro, M.; Mück-Lichtenfeld, C.; Fröhlich, R.; García Mancheño, O. Influence of the Substitution and Conformation of C-H-Bond-Based BisTriazole Acceptors in Anion-Binding Catalysis. Chem. - Asian J. 2014, 9, 2178-2186.

(5) Kimball, D. B.; Weakley, T. J. R.; Haley, M. M. Cyclization of 1-(2-Alkynylphenyl)-3,3Dialkyltriazenes: A Convenient, High-Yield Synthesis of Substituted Cinnolines and Isoindazoles. J. Org. Chem. 2002, 67, 6395-6405.

(6) Carroll, C. N.; Berryman, O. B.; Johnson, C. A.; Zakharov, L. N.; Haley, M. M.; Johnson, D. W. Protonation Activates Anion Binding and Alters Binding Selectivity in New Inherently Fluorescent 2,6-Bis(2-Anilinoethynyl)Pyridine Bisureas. Chem. Commun. 2009, 0, 2520-2522.

(7) Tresca, B. W.; Hansen, R. J.; Chau, C. V.; Hay, B. P.; Zakharov, L. N.; Haley, M. M.; Johnson, D. W. Substituent Effects in CH Hydrogen Bond Interactions: Linear Free Energy Relationships and Influence of Anions. J. Am. Chem. Soc. 2015, 137, 1495914967.

(8) Thordarson, P. Determining Association Constants from Titration Experiments in Supramolecular Chemistry. Chem. Soc. Rev. 2011, 40, 1305-1323.

(9) Swain, C. G.; Lupton, E. C. Field and Resonance Components of Substituent Effects. J. Am. Chem. Soc. 1968, 90, 4328-4337.

(10) Neese, F. Software Update: The ORCA Program System, Version 4.0. Wiley Interdiscip. Rev. Comput. Mol. Sci. 2018, 8, e1327.

(11) Marenich, A. V.; Cramer, C. J.; Truhlar, D. G. Universal Solvation Model Based on Solute Electron Density and on a Continuum Model of the Solvent Defined by the Bulk Dielectric Constant and Atomic Surface Tensions. J. Phys. Chem. B 2009, 113, 63786396.

(12) Breneman, C. M.; Wiberg, K. B. Determining Atom-Centered Monopoles from Molecular Electrostatic Potentials. The Need for High Sampling Density in Formamide Conformational Analysis. J. Comput. Chem. 1990, 11, 361-373. 


\title{
Section S11. Computed Geometries and Energies
}

\section{Br- TBA+}

Supporting Information: TBA.log

Using Gaussian 09: EM64L-G09RevE.01 30-Nov-2015

\begin{abstract}
\#PBEPBE/6-31G(d)/auto scf=(direct,tight,maxcycle=300,xqc)
$\mathrm{opt}=($ maxcycle $=250)$ freq $=$ noraman $\mathrm{int}=(\mathrm{grid}=$ ultrafine $)$

\#P Geom=AllCheck Guess=TCheck SCRF=Check GenChk RPBEPBE/6-31G(d)/Auto Freq

Pointgroup $=\mathrm{C} 1 \quad$ Stoichiometry $=\mathrm{C} 16 \mathrm{H} 36 \mathrm{~N}(1+) \quad \mathrm{C} 1[\mathrm{X}(\mathrm{C} 16 \mathrm{H} 36 \mathrm{~N})]$ \#Atoms $=53$

Charge $=1 \quad$ Multiplicity $=1$
\end{abstract}

SCF Energy $=-684.962373697 \quad$ Predicted Change $=-2.587469 \mathrm{D}-07$

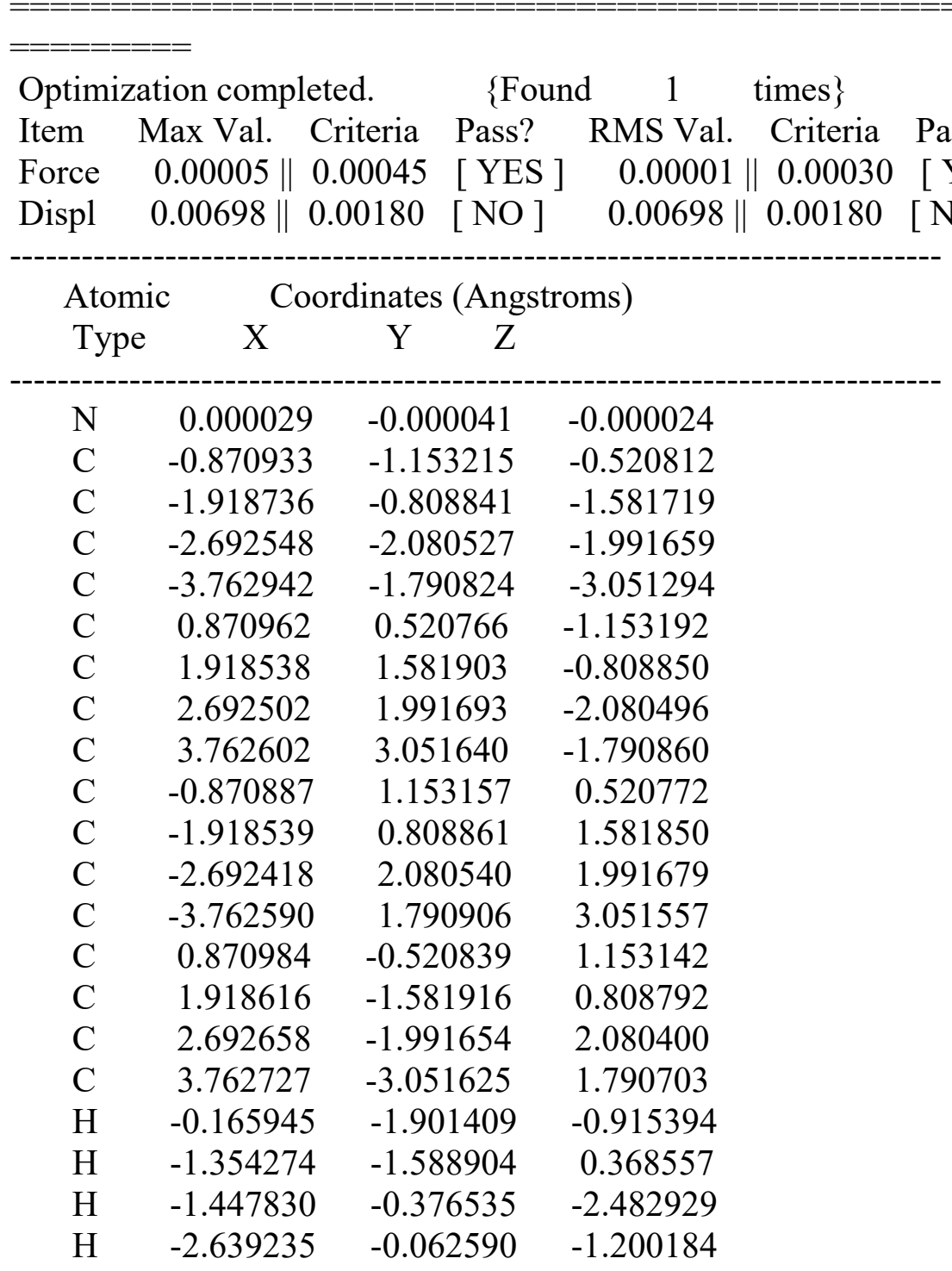




$\begin{array}{lrrr}\mathrm{H} & -3.165274 & -2.526408 & -1.095839 \\ \mathrm{H} & -1.980529 & -2.834793 & -2.377012 \\ \mathrm{H} & -4.299840 & -2.713354 & -3.324871 \\ \mathrm{H} & -4.509504 & -1.066203 & -2.682218 \\ \mathrm{H} & -3.316142 & -1.378177 & -3.972513 \\ \mathrm{H} & 1.354489 & -0.368560 & -1.588758 \\ \mathrm{H} & 0.165941 & 0.915147 & -1.901465 \\ \mathrm{H} & 1.447411 & 2.483130 & -0.376812 \\ \mathrm{H} & 2.638965 & 1.200637 & -0.062394 \\ \mathrm{H} & 1.980543 & 2.376689 & -2.835005 \\ \mathrm{H} & 3.165510 & 1.095858 & -2.526046 \\ \mathrm{H} & 4.299617 & 3.325091 & -2.713360 \\ \mathrm{H} & 3.315523 & 3.972870 & -1.378540 \\ \mathrm{H} & 4.509103 & 2.682913 & -1.065999 \\ \mathrm{H} & -0.165851 & 1.901399 & 0.915184 \\ \mathrm{H} & -1.354353 & 1.588748 & -0.368577 \\ \mathrm{H} & -2.639008 & 0.062477 & 1.200516 \\ \mathrm{H} & -1.447492 & 0.376753 & 2.483083 \\ \mathrm{H} & -3.165347 & 2.526206 & 1.095860 \\ \mathrm{H} & -1.980416 & 2.834958 & 2.376772 \\ \mathrm{H} & -4.299515 & 2.713431 & 3.325099 \\ \mathrm{H} & -4.509159 & 1.066165 & 2.682730 \\ \mathrm{H} & -3.315581 & 1.378436 & 3.972752 \\ \mathrm{H} & 0.165985 & -0.915272 & 1.901405 \\ \mathrm{H} & 1.354457 & 0.368511 & 1.588725 \\ \mathrm{H} & 1.447520 & -2.483177 & 0.376793 \\ \mathrm{H} & 2.639001 & -1.200623 & 0.062305 \\ \mathrm{H} & 1.980760 & -2.376629 & 2.834976 \\ \mathrm{H} & 3.165707 & -1.095811 & 2.525891 \\ \mathrm{H} & 4.299890 & -3.324999 & 2.713138 \\ \mathrm{H} & 3.315582 & -3.972889 & 1.378533 \\ \mathrm{H} & 4.509114 & -2.682965 & 1.065690\end{array}$

Statistical Thermodynamic Analysis

Temperature $=298.150$ Kelvin $\quad$ Pressure $=1.00000$ Atm

\footnotetext{
$======$

SCF Energy $=\quad-684.962373697 \quad$ Predicted Change $=-2.587469 \mathrm{D}-07$

Zero-point correction $(\mathrm{ZPE})=\quad-684.4672 \quad 0.49516$

Internal Energy $(\mathrm{U})=$

Enthalpy $(\mathrm{H})=$

$-684.44350 .51886$

Gibbs Free Energy $(\mathrm{G})=\quad-684.5221 \quad 0.44026$

Frequencies -- $37.3445 \quad 38.6743 \quad 39.4614$

Single points at WB97X-D3/def2-TZVPP: -3260.45555380036
} 
Cl- TBA+

Supporting Information: TBA_Cl.log

Using Gaussian 09: EM64L-G09RevE.01 30-Nov-2015

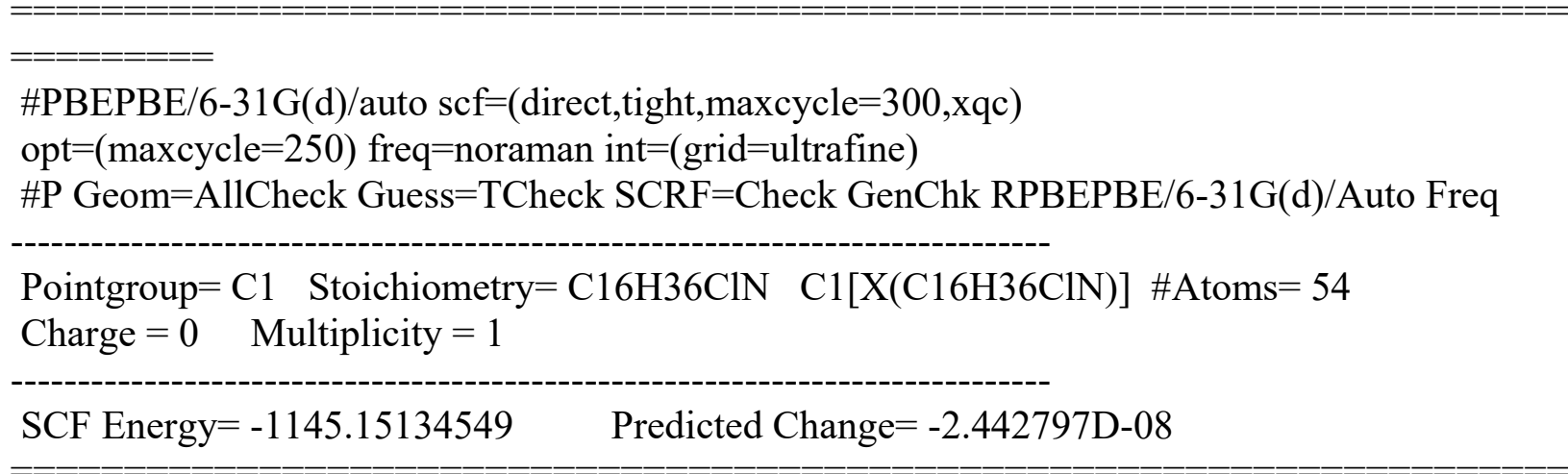

\begin{tabular}{|c|c|c|c|c|c|c|}
\hline \multicolumn{3}{|c|}{ Optimization completed. } & $\{$ Found & \multirow{2}{*}{\multicolumn{2}{|c|}{$\begin{array}{cc}1 & \text { times }\} \\
\text { RMS Val. } & \text { Criteria }\end{array}$}} & \multirow[b]{2}{*}{ Pass? } \\
\hline Item & Max Val. & Criteria & Pass? & & & \\
\hline Force & $0.00002 \|$ & 0.00045 & [ YES ] & 0.00000 & | 0.00030 & [YES ] \\
\hline Displ & $0.00249 \|$ & 0.00180 & {$[\mathrm{NO}]$} & $0.00249 \|$ & 0.00180 & [YES ] \\
\hline
\end{tabular}

\begin{tabular}{lrrr} 
Atomic & \multicolumn{3}{c}{ Coordinates (Angstroms) } \\
Type & $\mathrm{X}$ & $\mathrm{Y}$ & $\mathrm{Z}$ \\
$\mathrm{N}$ & 0.373767 & -0.443975 & 0.112532 \\
$\mathrm{C}$ & 1.692360 & -1.145594 & -0.197005 \\
$\mathrm{C}$ & 2.892758 & -0.806040 & 0.690466 \\
$\mathrm{C}$ & 4.157581 & -1.526530 & 0.183367 \\
$\mathrm{C}$ & 5.390024 & -1.222052 & 1.044160 \\
$\mathrm{C}$ & -0.069772 & -0.810098 & 1.534184 \\
$\mathrm{C}$ & -1.433622 & -0.246684 & 1.975223 \\
$\mathrm{C}$ & -1.336132 & 0.892875 & 3.006897 \\
$\mathrm{C}$ & -2.722862 & 1.437209 & 3.368259 \\
$\mathrm{C}$ & 0.504272 & 1.083811 & 0.026562 \\
$\mathrm{C}$ & 1.028828 & 1.641738 & -1.295473 \\
$\mathrm{C}$ & 0.916493 & 3.179511 & -1.269986 \\
$\mathrm{C}$ & 1.425659 & 3.823055 & -2.564861 \\
$\mathrm{C}$ & -0.669232 & -0.897567 & -0.928714 \\
$\mathrm{C}$ & -1.276058 & -2.285184 & -0.725820 \\
$\mathrm{C}$ & -2.232402 & -2.593936 & -1.895882 \\
$\mathrm{C}$ & -2.939706 & -3.944861 & -1.735080 \\
$\mathrm{H}$ & 1.477333 & -2.226570 & -0.155570 \\
$\mathrm{H}$ & 1.922921 & -0.893896 & -1.244458 \\
$\mathrm{H}$ & 2.709823 & -1.104229 & 1.738477 \\
$\mathrm{H}$ & 3.076825 & 0.283837 & 0.691299 \\
$\mathrm{H}$ & 4.353101 & -1.228201 & -0.864538 \\
$\mathrm{H}$ & 3.976991 & -2.618642 & 0.165596 \\
$\mathrm{H}$ & 6.281296 & -1.745600 & 0.659949
\end{tabular}




$\begin{array}{lrrr}\mathrm{H} & 5.613921 & -0.140779 & 1.053844 \\ \mathrm{H} & 5.237478 & -1.542364 & 2.089954 \\ \mathrm{H} & -0.043483 & -1.910955 & 1.567252 \\ \mathrm{H} & 0.733567 & -0.440506 & 2.192459 \\ \mathrm{H} & -2.018184 & 0.135423 & 1.110992 \\ \mathrm{H} & -2.020573 & -1.077903 & 2.410004 \\ \mathrm{H} & -0.728197 & 1.715023 & 2.584773 \\ \mathrm{H} & -0.805377 & 0.538665 & 3.913919 \\ \mathrm{H} & -2.651150 & 2.258016 & 4.102262 \\ \mathrm{H} & -3.220643 & 1.819954 & 2.460258 \\ \mathrm{H} & -3.360407 & 0.648057 & 3.807162 \\ \mathrm{H} & -0.527963 & 1.470329 & 0.171609 \\ \mathrm{H} & 1.147066 & 1.384656 & 0.870549 \\ \mathrm{H} & 2.078766 & 1.344689 & -1.490366 \\ \mathrm{H} & 0.409758 & 1.274500 & -2.132890 \\ \mathrm{H} & 1.483474 & 3.579259 & -0.406151 \\ \mathrm{H} & -0.147198 & 3.434980 & -1.108616 \\ \mathrm{H} & 1.329100 & 4.921157 & -2.524366 \\ \mathrm{H} & 2.489791 & 3.586039 & -2.748874 \\ \mathrm{H} & 0.845459 & 3.470772 & -3.435918 \\ \mathrm{H} & -0.146083 & -0.852574 & -1.897953 \\ \mathrm{H} & -1.461309 & -0.111792 & -0.932634 \\ \mathrm{H} & -0.506613 & -3.081266 & -0.664818 \\ \mathrm{H} & -1.856899 & -2.318317 & 0.213488 \\ \mathrm{H} & -1.666113 & -2.580172 & -2.847157 \\ \mathrm{H} & -2.978260 & -1.780800 & -1.964644 \\ \mathrm{H} & -3.616927 & -4.140212 & -2.583470 \\ \mathrm{H} & -2.215402 & -4.778109 & -1.685046 \\ \mathrm{H} & -3.545765 & -3.970329 & -0.812183 \\ \mathrm{Cl} & -2.719743 & 1.782810 & -0.611158 \\ ---------------------------------------------------------\end{array}$

Statistical Thermodynamic Analysis

Temperature $=298.150$ Kelvin Pressure $=1.00000$ Atm

\begin{tabular}{|c|c|}
\hline SCF Energy $=\quad-1145.15134549$ & Predicted Change $=-2.442797 \mathrm{D}-08$ \\
\hline Zero-point correction $(\mathrm{ZPE})=$ & -1144.65610 .49523 \\
\hline Internal Energy $(U)=$ & -1144.63050 .52075 \\
\hline Enthalpy $(\mathrm{H})=$ & $-1144.6296 \quad 0.52170$ \\
\hline Gibbs Free Energy $(G)=$ & $\begin{array}{ll}-1144.7133 & 0.43796\end{array}$ \\
\hline $\begin{array}{l}\text { Frequencies -- } 35.6843 \\
\text { Single points at WB97X-D3/def }\end{array}$ & $\begin{array}{cc}38.1890 & 44.1978 \\
\text { 2-TZVPP: }-1146.44269135176\end{array}$ \\
\hline
\end{tabular}


SeH- TBA+

Supporting Information: TBA_SeH.log

Using Gaussian 09: EM64L-G09RevE.01 30-Nov-2015

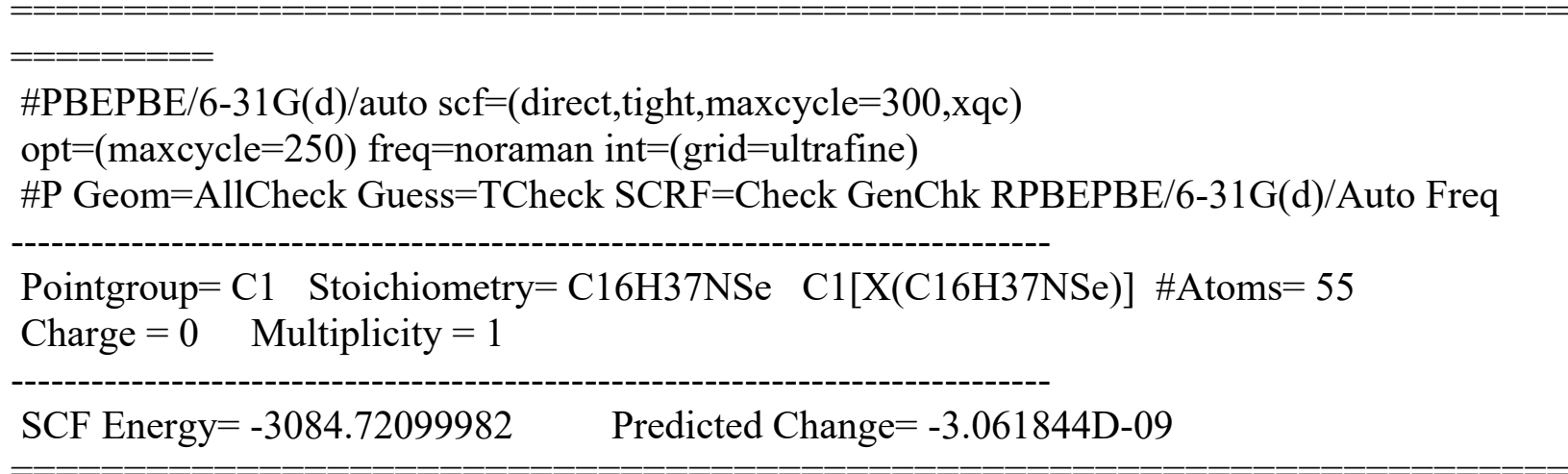

\begin{tabular}{|c|c|c|c|c|c|c|}
\hline \multicolumn{3}{|c|}{ Optimization completed. } & $\{$ Found & \multirow{2}{*}{$\begin{array}{c}2 \\
\text { RMS Val. }\end{array}$} & \multirow{2}{*}{$\begin{array}{l}\text { times }\} \\
\text { Criteria }\end{array}$} & \multirow[b]{2}{*}{ Pass? } \\
\hline Item & Max Val. & Criteria & Pass? & & & \\
\hline Force & 0.00000 & 0.00045 & [ YES ] & 0.00000 & 0.00030 & Y VES \\
\hline Displ & $0.00080 \|$ & 0.00180 & {$[\mathrm{YES}]$} & 0.00080 & 0.00180 & {$[\mathrm{YES}$} \\
\hline
\end{tabular}

\begin{tabular}{lrrr} 
Atomic & \multicolumn{3}{c}{ Coordinates (Angstroms) } \\
Type & $\mathrm{X}$ & $\mathrm{Y}$ & \\
$\mathrm{N}$ & -0.913885 & 0.496606 & 0.072998 \\
$\mathrm{C}$ & -2.321299 & 0.828332 & -0.415084 \\
$\mathrm{C}$ & -3.500002 & 0.312016 & 0.414645 \\
$\mathrm{C}$ & -4.833936 & 0.622528 & -0.292883 \\
$\mathrm{C}$ & -6.048667 & 0.133630 & 0.505834 \\
$\mathrm{C}$ & -0.721943 & 1.059264 & 1.488797 \\
$\mathrm{C}$ & 0.664139 & 0.809610 & 2.108095 \\
$\mathrm{C}$ & 0.690587 & -0.283356 & 3.197486 \\
$\mathrm{C}$ & 2.130870 & -0.691018 & 3.535915 \\
$\mathrm{C}$ & -0.660188 & -1.016029 & 0.125142 \\
$\mathrm{C}$ & -0.793427 & -1.773452 & -1.195326 \\
$\mathrm{C}$ & -0.257167 & -3.207796 & -1.005767 \\
$\mathrm{C}$ & -0.359579 & -4.044487 & -2.285960 \\
$\mathrm{C}$ & 0.089499 & 1.106850 & -0.927505 \\
$\mathrm{C}$ & 0.482089 & 2.569840 & -0.746099 \\
$\mathrm{C}$ & 1.574852 & 2.885013 & -1.791673 \\
$\mathrm{C}$ & 2.157787 & 4.293269 & -1.632159 \\
$\mathrm{H}$ & -2.355121 & 1.928489 & -0.496461 \\
$\mathrm{H}$ & -2.381341 & 0.419358 & -1.436407 \\
$\mathrm{H}$ & -3.513827 & 0.774802 & 1.417712 \\
$\mathrm{H}$ & -3.419685 & -0.780567 & 0.563360 \\
$\mathrm{H}$ & -4.834237 & 0.153038 & -1.295301 \\
$\mathrm{H}$ & -4.915027 & 1.713533 & -0.462307 \\
$\mathrm{H}$ & -6.989126 & 0.364236 & -0.021847
\end{tabular}




\begin{tabular}{lrrr}
$\mathrm{H}$ & -6.010954 & -0.958786 & 0.662846 \\
$\mathrm{H}$ & -6.094863 & 0.613846 & 1.499201 \\
$\mathrm{H}$ & -0.962654 & 2.131019 & 1.405512 \\
$\mathrm{H}$ & -1.510422 & 0.587589 & 2.097982 \\
$\mathrm{H}$ & 1.403102 & 0.512662 & 1.327264 \\
$\mathrm{H}$ & 0.14986460 & 1.762014 & 2.536037 \\
$\mathrm{H}$ & 0.143936 & -1.176985 & 2.831162 \\
$\mathrm{H}$ & 2.154277 & -1.490707 & 4.098114 \\
$\mathrm{H}$ & 2.625359 & -1.051370 & 2.612330 \\
$\mathrm{H}$ & 2.708667 & 0.165504 & 3.928885 \\
$\mathrm{H}$ & 0.393704 & -1.112962 & 0.461972 \\
$\mathrm{H}$ & -1.338178 & -1.421585 & 0.894057 \\
$\mathrm{H}$ & -1.840183 & -1.805610 & -1.557539 \\
$\mathrm{H}$ & -0.177951 & -1.288294 & -1.972887 \\
$\mathrm{H}$ & -0.807716 & -3.708496 & -0.185294 \\
$\mathrm{H}$ & 0.798888 & -3.133821 & -0.685186 \\
$\mathrm{H}$ & 0.041874 & -5.059448 & -2.127241 \\
$\mathrm{H}$ & -1.407344 & -4.146106 & -2.623274 \\
$\mathrm{H}$ & 0.217450 & -3.583093 & -3.106500 \\
$\mathrm{H}$ & -0.363227 & 0.956598 & -1.921373 \\
$\mathrm{H}$ & 1.015856 & 0.475404 & -0.864741 \\
$\mathrm{H}$ & -0.371013 & 3.269880 & -0.856652 \\
$\mathrm{H}$ & 0.916709 & 2.733374 & 0.256211 \\
$\mathrm{H}$ & 1.155934 & 2.766869 & -2.809830 \\
$\mathrm{H}$ & 2.368383 & 2.118743 & -1.689055 \\
$\mathrm{H}$ & 2.935570 & 4.487084 & -2.389957 \\
$\mathrm{H}$ & 1.380142 & 5.070915 & -1.742921 \\
$\mathrm{H}$ & 2.622417 & 4.421678 & -0.638363 \\
$\mathrm{H}$ & 3.697929 & 0.203374 & 0.240747 \\
$\mathrm{Se}$ & 2.887319 & -0.943387 & -0.272298 \\
--------------------------------------------------------------- \\
\hline
\end{tabular}

Statistical Thermodynamic Analysis

Temperature $=298.150$ Kelvin Pressure $=1.00000$ Atm

\footnotetext{
$========$
SCF Energy $=\quad-3084.72099982 \quad$ Predicted Change $=-3.061844 D-09$

Zero-point correction $(\mathrm{ZPE})=\quad-3084.21860 .50236$

Internal Energy $(\mathrm{U})=\quad-3084.19260 .52837$

Enthalpy $(\mathrm{H})=$

Gibbs Free Energy $(\mathrm{G})=\quad-3084.2765 \quad 0.44445$

Frequencies -- $19.1902 \quad 34.0754 \quad 40.4367$

Single points at WB97X-D3/def2-TZVPP: -3088.41159135713
} 
SH- TBA+

Supporting Information: TBA_SH.log

Using Gaussian 09: EM64L-G09RevE.01 30-Nov-2015

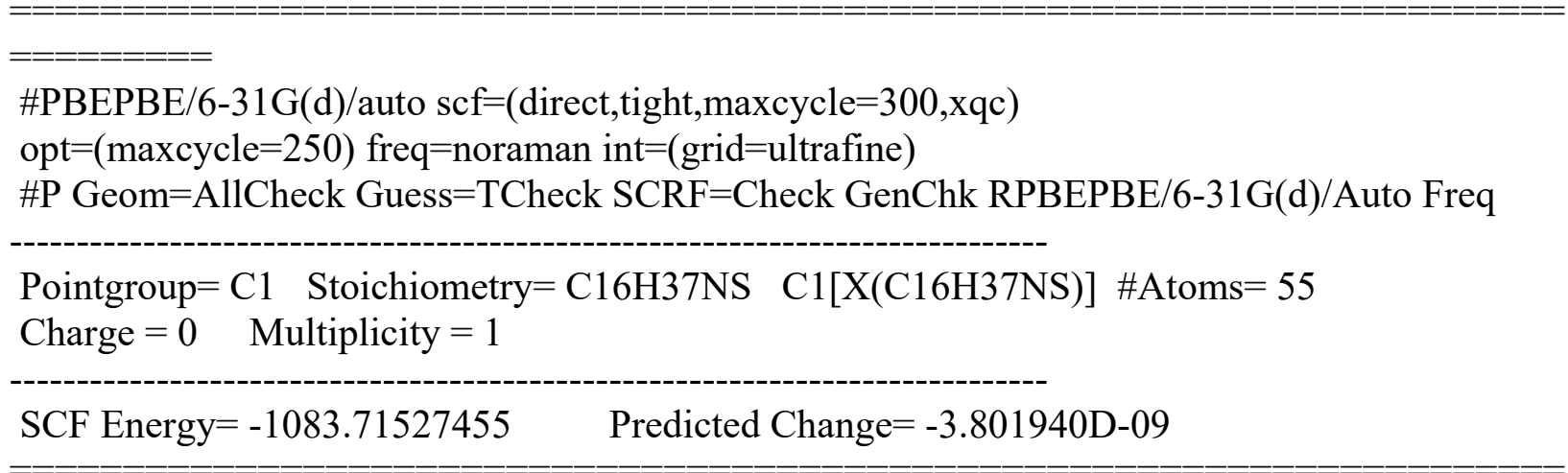

\begin{tabular}{|c|c|c|c|}
\hline \multicolumn{4}{|c|}{ Optimization completed on the basis of negligible forces. } \\
\hline \multirow{3}{*}{$\begin{array}{l}\text { Item } \\
\text { Force } \\
\text { Displ }\end{array}$} & & RMS Val. Criteria \\
\hline & \multicolumn{2}{|c|}{$0.00000 \| 0.00045 \quad[$ YES ] } & 0.00000|| 0.00030 \\
\hline & $0.00223 \|$ & $0180[\mathrm{NO}]$ & $0.00223 \| 0.00180$ \\
\hline \multicolumn{2}{|c|}{ Atomic } & \multicolumn{2}{|c|}{ Coordinates (Angstroms) } \\
\hline Type & $\mathrm{X}$ & $\mathrm{Y} \quad \mathrm{Z}$ & \\
\hline $\mathrm{N}$ & 0.375415 & -0.451929 & 0.115068 \\
\hline $\mathrm{C}$ & 1.693671 & -1.148495 & -0.208695 \\
\hline $\mathrm{C}$ & 2.906586 & -0.791977 & 0.654984 \\
\hline $\mathrm{C}$ & 4.165707 & -1.516964 & 0.140193 \\
\hline $\mathrm{C}$ & 5.410726 & -1.194194 & 0.975858 \\
\hline $\mathrm{C}$ & -0.054637 & -0.821706 & 1.538273 \\
\hline $\mathrm{C}$ & -1.408582 & -0.242275 & 1.988996 \\
\hline $\mathrm{C}$ & -1.279296 & 0.824939 & 3.091883 \\
\hline $\mathrm{C}$ & -2.644114 & 1.397092 & 3.490296 \\
\hline $\mathrm{C}$ & 0.498639 & 1.077344 & 0.036710 \\
\hline $\mathrm{C}$ & 1.017322 & 1.645048 & -1.283571 \\
\hline $\mathrm{C}$ & 0.948190 & 3.185176 & -1.231930 \\
\hline $\mathrm{C}$ & 1.437911 & 3.834080 & -2.531652 \\
\hline $\mathrm{C}$ & -0.678176 & -0.905102 & -0.913491 \\
\hline $\mathrm{C}$ & -1.260956 & -2.304629 & -0.726441 \\
\hline $\mathrm{C}$ & -2.230636 & -2.598847 & -1.889648 \\
\hline $\mathrm{C}$ & -2.940870 & -3.948869 & -1.736232 \\
\hline $\mathrm{H}$ & 1.485979 & -2.230137 & -0.151844 \\
\hline $\mathrm{H}$ & 1.904906 & -0.907432 & -1.262670 \\
\hline $\mathrm{H}$ & 2.740585 & -1.074093 & 1.710349 \\
\hline $\mathrm{H}$ & 3.087434 & 0.298267 & 0.636018 \\
\hline $\mathrm{H}$ & 4.343496 & -1.235758 & -0.915574 \\
\hline $\mathrm{H}$ & 3.988520 & -2.609764 & 0.143621 \\
\hline $\mathrm{H}$ & 6.297491 & -1.721518 & 0.586386 \\
\hline
\end{tabular}




\begin{tabular}{lrrr} 
H & 5.631229 & -0.112244 & 0.963615 \\
$\mathrm{H}$ & 5.275946 & -1.497132 & 2.029258 \\
$\mathrm{H}$ & -0.037985 & -1.922755 & 1.568724 \\
$\mathrm{H}$ & 0.757966 & -0.461013 & 2.189944 \\
$\mathrm{H}$ & -1.960984 & 0.213115 & 1.133525 \\
$\mathrm{H}$ & -2.037359 & -1.073900 & 2.359180 \\
$\mathrm{H}$ & -0.637624 & 1.648660 & 2.725020 \\
$\mathrm{H}$ & -0.767094 & 0.395443 & 3.976522 \\
$\mathrm{H}$ & -2.544272 & 2.155411 & 4.285498 \\
$\mathrm{H}$ & -3.122128 & 1.872654 & 2.616409 \\
$\mathrm{H}$ & -0.534895 & 1.464334 & 0.195050 \\
$\mathrm{H}$ & 1.146448 & 1.375362 & 0.878095 \\
$\mathrm{H}$ & 2.057108 & 1.326377 & -1.498106 \\
$\mathrm{H}$ & 0.382026 & 1.303813 & -2.120216 \\
$\mathrm{H}$ & 1.552913 & 3.553227 & -0.379535 \\
$\mathrm{H}$ & -0.100948 & 3.473837 & -1.033363 \\
$\mathrm{H}$ & 1.378756 & 4.933805 & -2.469944 \\
$\mathrm{H}$ & 2.487442 & 3.565751 & -2.753001 \\
$\mathrm{H}$ & 0.819197 & 3.516226 & -3.389205 \\
$\mathrm{H}$ & -0.176607 & -0.830135 & -1.892297 \\
$\mathrm{H}$ & -1.494875 & -0.137852 & -0.885161 \\
$\mathrm{H}$ & -0.483326 & -3.094384 & -0.688506 \\
$\mathrm{H}$ & -1.830473 & -2.360366 & 0.219361 \\
$\mathrm{H}$ & -1.673226 & -2.576889 & -2.845955 \\
$\mathrm{H}$ & -2.971988 & -1.779915 & -1.942515 \\
$\mathrm{H}$ & -3.625969 & -4.133511 & -2.580733 \\
$\mathrm{H}$ & -2.219353 & -4.785384 & -1.700455 \\
$\mathrm{H}$ & -3.539396 & -3.981764 & -0.808533 \\
$\mathrm{~S}$ & -2.784384 & 1.828279 & -0.753968 \\
$\mathrm{H}$ & -3.827382 & 1.792371 & 0.122988 \\
----------------------------------------------------------- \\
\hline
\end{tabular}

Statistical Thermodynamic Analysis

Temperature $=298.150$ Kelvin Pressure $=1.00000$ Atm

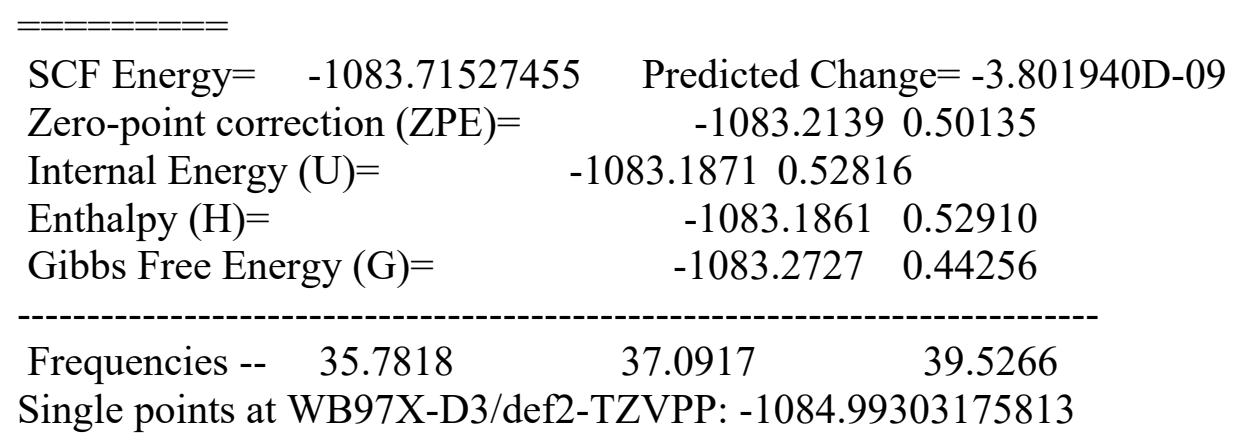


TBA

Supporting Information: TBA.log

Using Gaussian 09: EM64L-G09RevE.01 30-Nov-2015

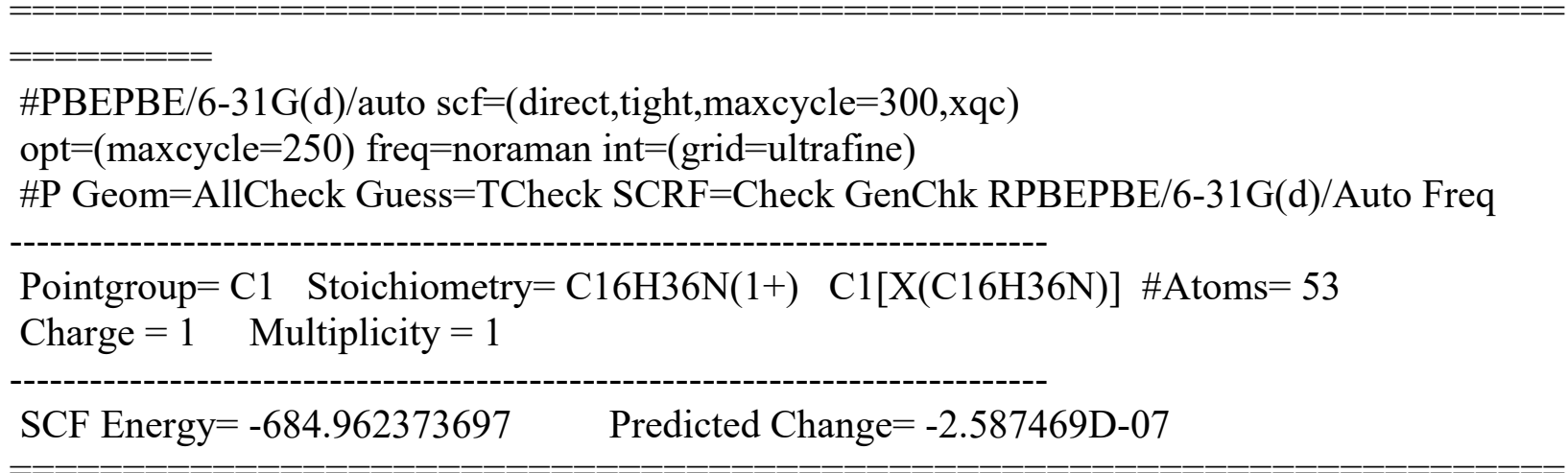

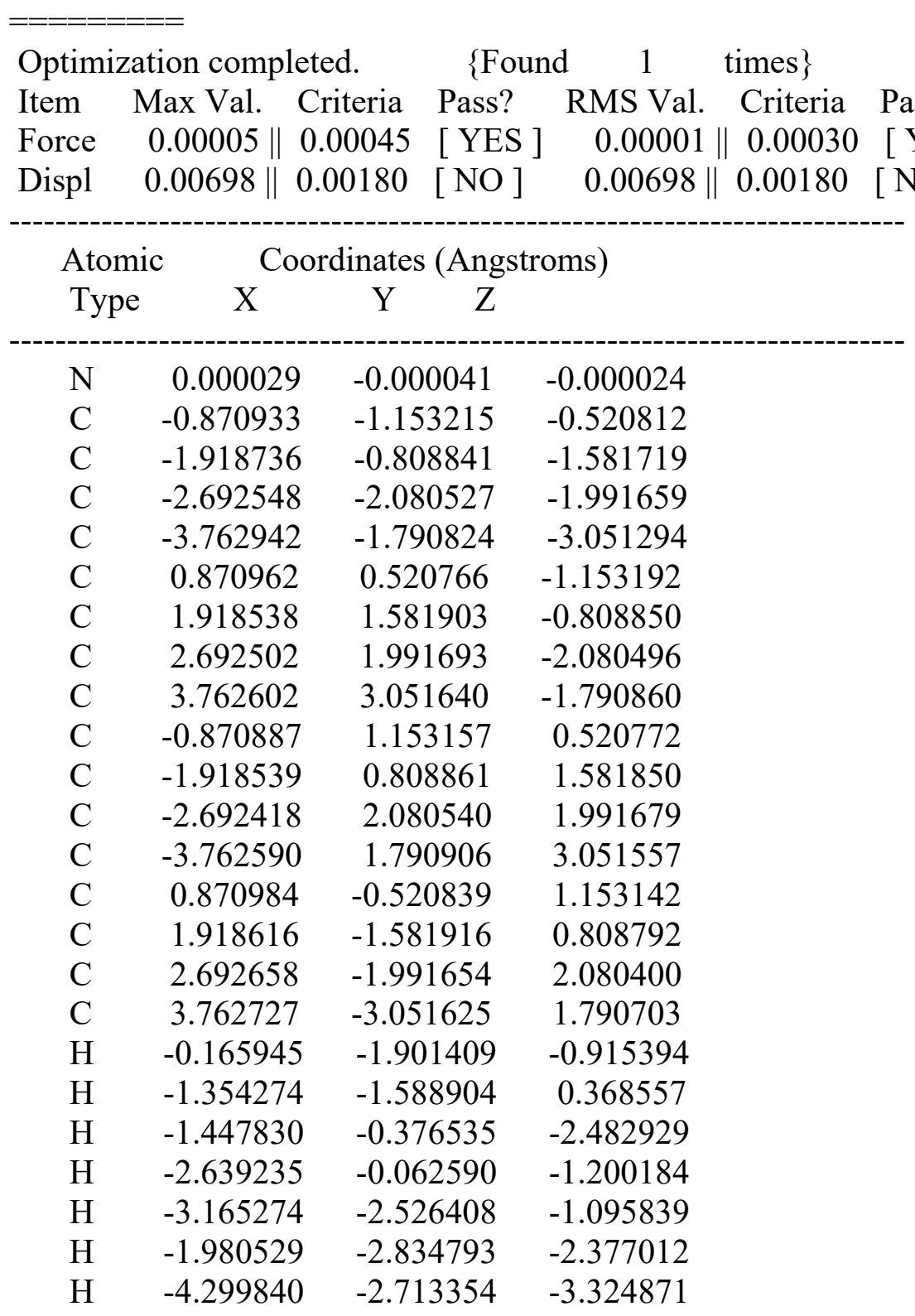




$\begin{array}{rrrr}\mathrm{H} & -4.509504 & -1.066203 & -2.682218 \\ \mathrm{H} & -3.316142 & -1.378177 & -3.972513 \\ \mathrm{H} & 1.354489 & -0.368560 & -1.588758 \\ \mathrm{H} & 0.165941 & 0.915147 & -1.901465 \\ \mathrm{H} & 1.447411 & 2.483130 & -0.376812 \\ \mathrm{H} & 2.638965 & 1.200637 & -0.062394 \\ \mathrm{H} & 1.980543 & 2.376689 & -2.835005 \\ \mathrm{H} & 3.165510 & 1.095858 & -2.526046 \\ \mathrm{H} & 4.299617 & 3.325091 & -2.713360 \\ \mathrm{H} & 3.315523 & 3.972870 & -1.378540 \\ \mathrm{H} & 4.509103 & 2.682913 & -1.065999 \\ \mathrm{H} & -0.165851 & 1.901399 & 0.915184 \\ \mathrm{H} & -1.354353 & 1.588748 & -0.368577 \\ \mathrm{H} & -2.639008 & 0.062477 & 1.200516 \\ \mathrm{H} & -1.447492 & 0.376753 & 2.483083 \\ \mathrm{H} & -3.165347 & 2.526206 & 1.095860 \\ \mathrm{H} & -1.980416 & 2.834958 & 2.376772 \\ \mathrm{H} & -4.299515 & 2.713431 & 3.325099 \\ \mathrm{H} & -4.509159 & 1.066165 & 2.682730 \\ \mathrm{H} & -3.315581 & 1.378436 & 3.972752 \\ \mathrm{H} & 0.165985 & -0.915272 & 1.901405 \\ \mathrm{H} & 1.354457 & 0.368511 & 1.588725 \\ \mathrm{H} & 1.447520 & -2.483177 & 0.376793 \\ \mathrm{H} & 2.639001 & -1.200623 & 0.062305 \\ \mathrm{H} & 1.980760 & -2.376629 & 2.834976 \\ \mathrm{H} & 3.165707 & -1.095811 & 2.525891 \\ \mathrm{H} & 4.299890 & -3.324999 & 2.713138 \\ \mathrm{H} & 3.315582 & -3.972889 & 1.378533 \\ \mathrm{H} & 4.509114 & -2.682965 & 1.065690\end{array}$

Statistical Thermodynamic Analysis

Temperature $=298.150$ Kelvin $\quad$ Pressure $=1.00000$ Atm

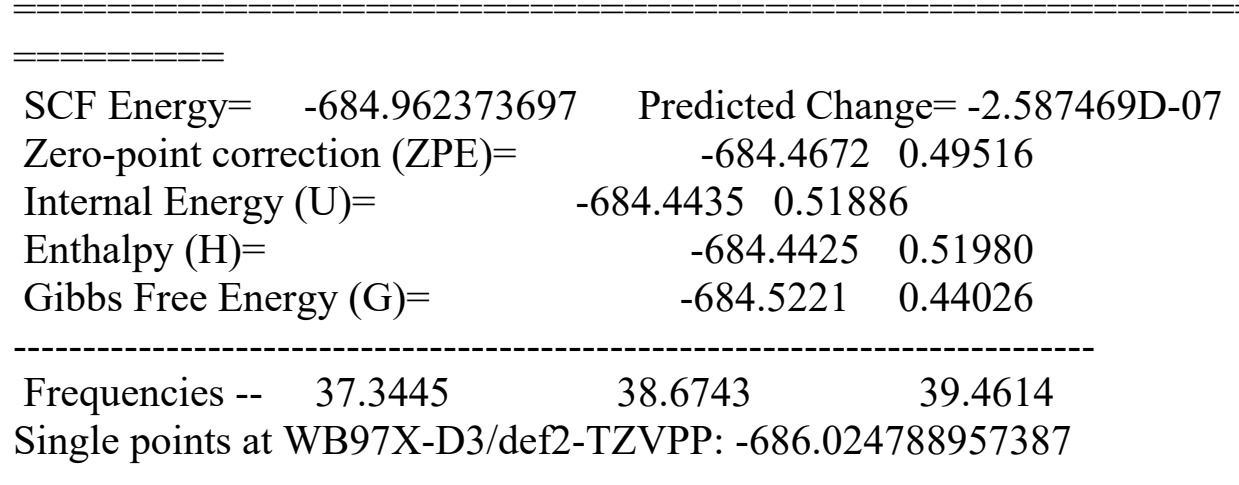


Br-

Supporting Information: Br.log

Using Gaussian 09: AM64L-G09RevD.01 24-Apr-2013

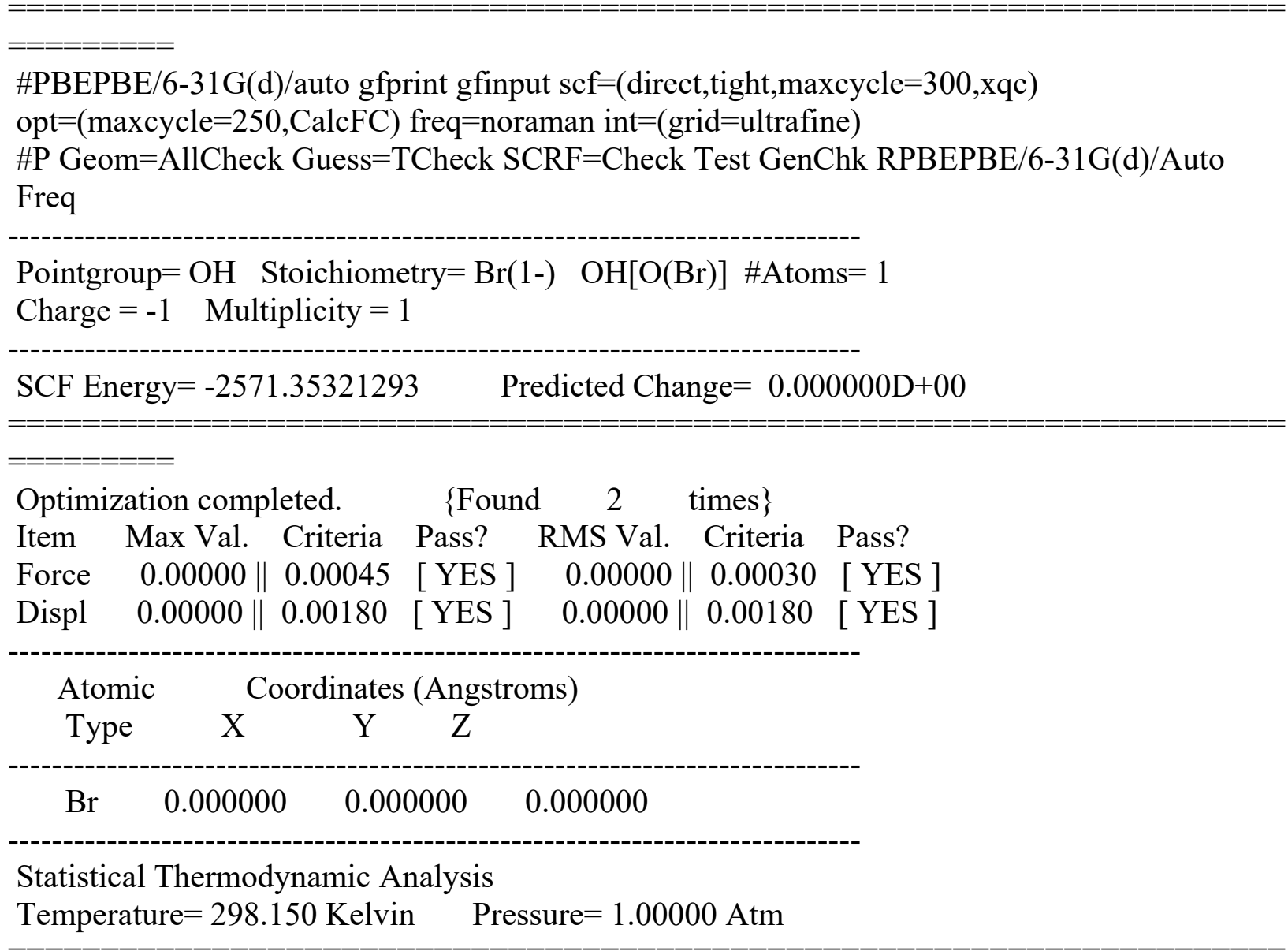

\begin{tabular}{|c|c|}
\hline-2571.35321293 & Predicted Change $=0.000000 \mathrm{D}+00$ \\
\hline Zero-point correction $(\mathrm{ZPE})=$ & -2571.35320 .00000 \\
\hline Internal Energy $(U)=$ & -2571.3517 0.00141 \\
\hline Enthalpy $(\mathrm{H})=$ & $\begin{array}{lll}-2571.3508 & 0.00236\end{array}$ \\
\hline Gibbs Free Energy $(G)=$ & $-2571.3693 \quad-0.01617$ \\
\hline
\end{tabular}

Single points at WB97X-D3/def2-TZVPP: -2574.29447076674 
Cl-

Supporting Information: Cl.log

Using Gaussian 09: AM64L-G09RevD.01 24-Apr-2013

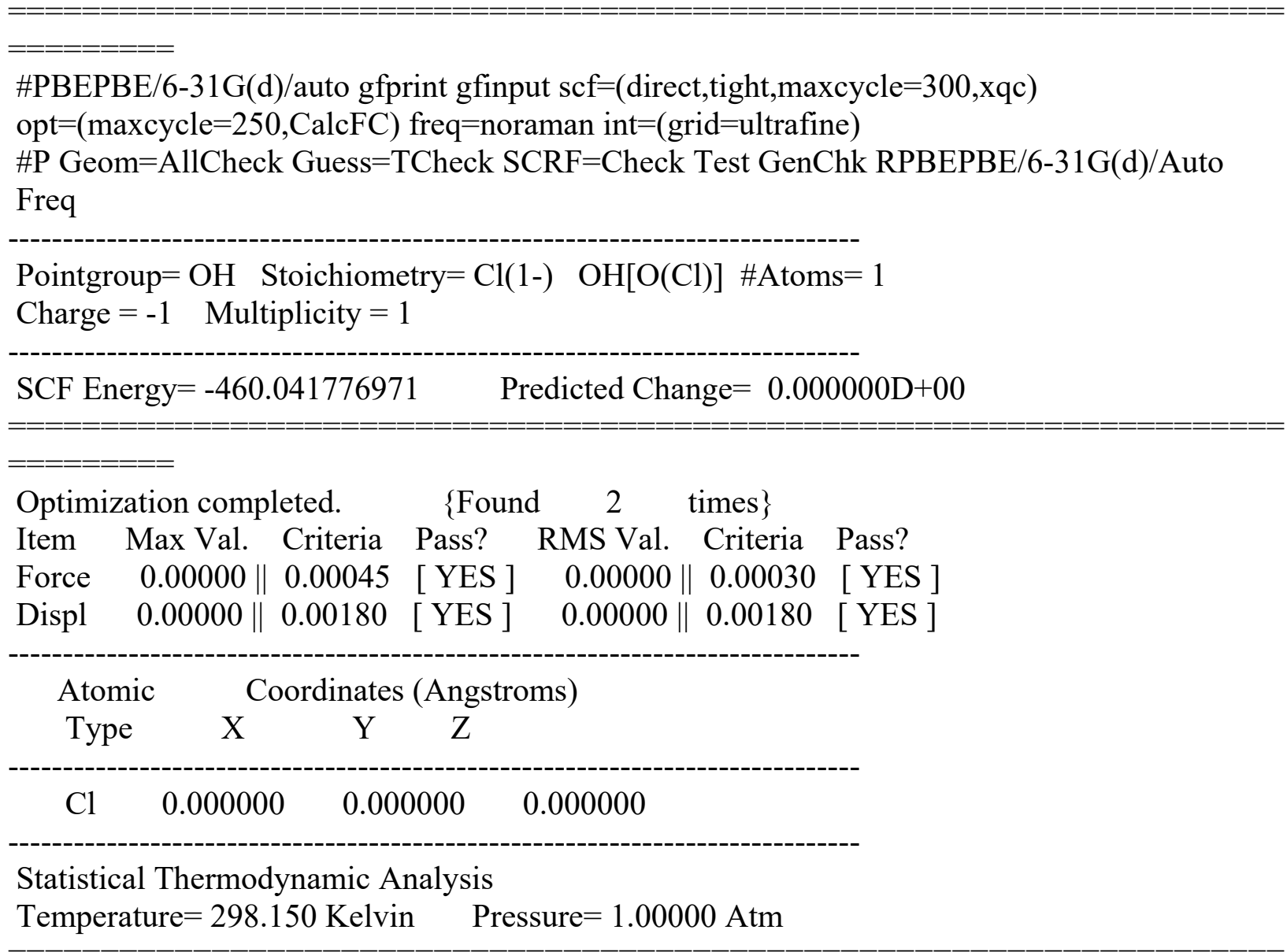

$\begin{array}{lcc}======= & & \\ \text { SCF Energy }= & -460.041776971 & \text { Predicted Change }=0.000000 \\ \text { Zero-point correction }(\mathrm{ZPE})= & -460.0417 & 0.00000 \\ \text { Internal Energy }(\mathrm{U})= & -460.0403 & 0.00141 \\ \text { Enthalpy }(\mathrm{H})= & -460.0394 & 0.00236 \\ \text { Gibbs Free Energy }(\mathrm{G})= & -460.0568 & -0.01502\end{array}$

Single points at WB97X-D3/def2-TZVPP: -460.269681586973 
SeH-

Supporting Information: SeH.log

Using Gaussian 09: AM64L-G09RevD.01 24-Apr-2013

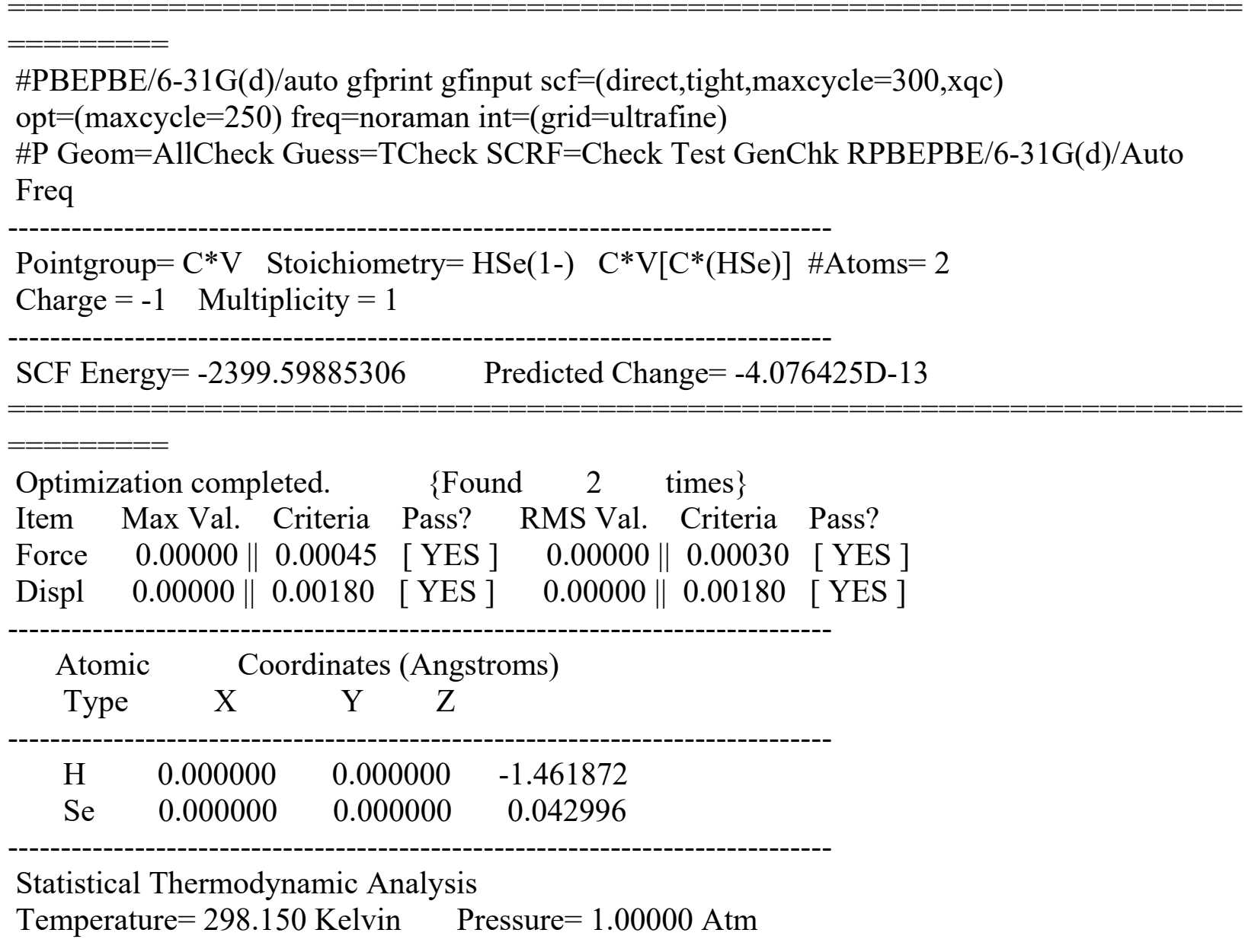

\begin{tabular}{|c|c|}
\hline SCF Energy $=\quad-2399.5988530$ & Predicted Change $=-4.076425 \mathrm{D}-13$ \\
\hline Zero-point correction $(\mathrm{ZPE})=$ & -2399.59370 .00513 \\
\hline Internal Energy $(U)=$ & -2399.59130 .00749 \\
\hline Enthalpy $(\mathrm{H})=$ & $-2399.5904 \quad 0.00843$ \\
\hline Gibbs Free Energy $(G)=$ & $-2399.6130 \quad-0.01421$ \\
\hline
\end{tabular}

Frequencies -- 2252.9165

Single points at WB97X-D3/def2-TZVPP: -2402.25409998737 
SH-

Supporting Information: SH.log

Using Gaussian 09: AM64L-G09RevD.01 24-Apr-2013

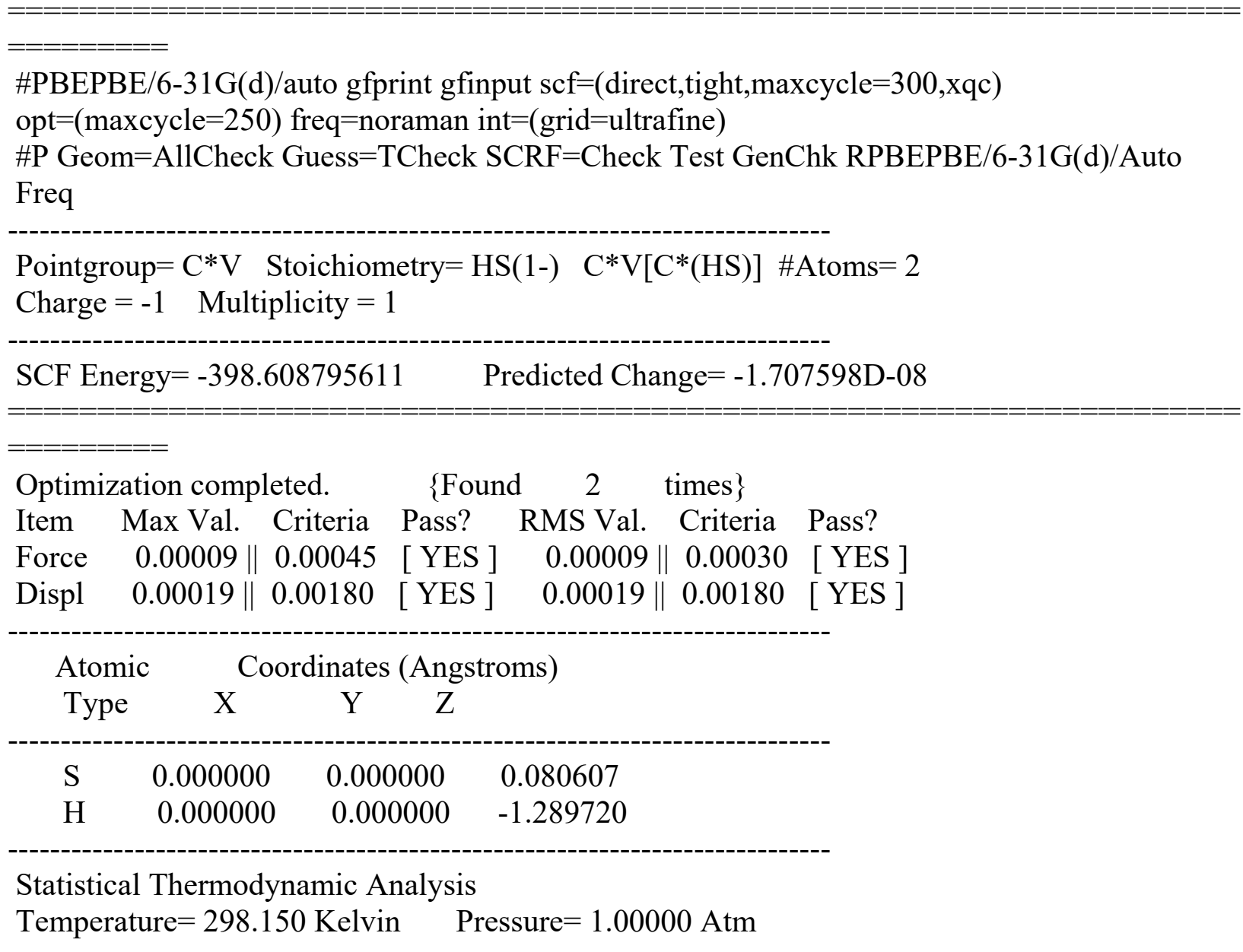

\begin{tabular}{|c|c|}
\hline-398.608795611 & Predicted Change $=-1.707598 \mathrm{D}-08$ \\
\hline Zero-point correction $(\mathrm{ZPE})=$ & $-398.6030 \quad 0.00575$ \\
\hline Internal Energy $(U)=$ & $-398.6006 \quad 0.00811$ \\
\hline Enthalpy $(\mathrm{H})=$ & $\begin{array}{lll}-398.5997 & 0.00905\end{array}$ \\
\hline Gibbs Free Energy $(G)=$ & $\begin{array}{ll}-398.6209 & -0.01213\end{array}$ \\
\hline
\end{tabular}

Frequencies -- 2524.1317

Single points at WB97X-D3/def2-TZVPP: -398.824000625576 
$\mathbf{R}=\mathbf{t B u}$ receptor unbound (from crystal structure)

Supporting Information: 000-unbound_crystal_structure_tBu.log

Using Gaussian 09: EM64L-G09RevE.01 30-Nov-2015

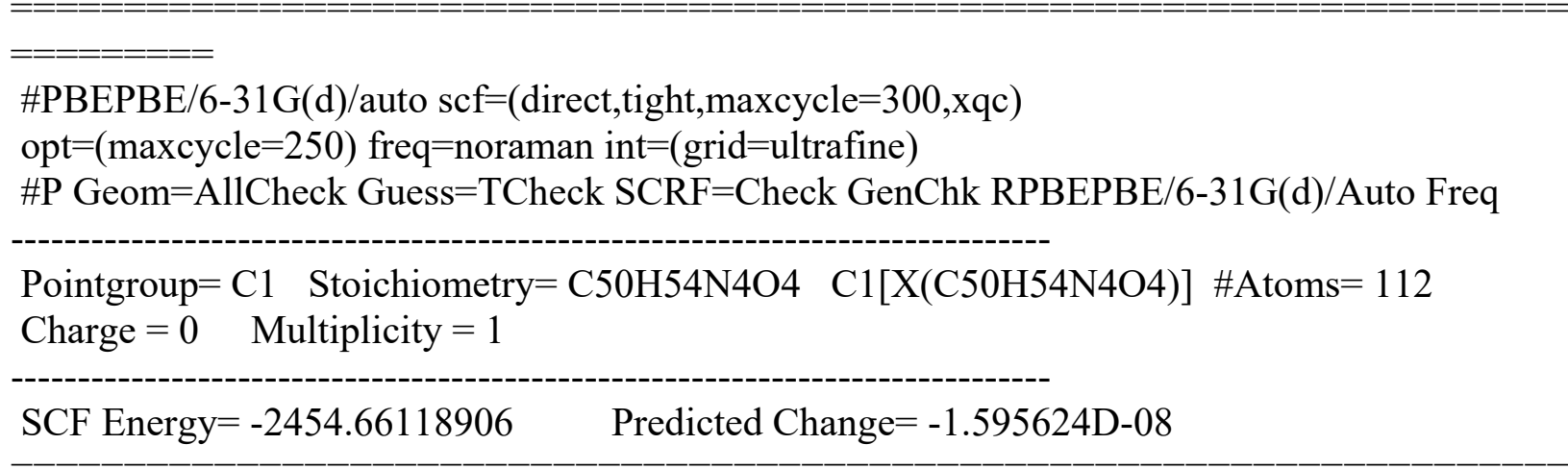

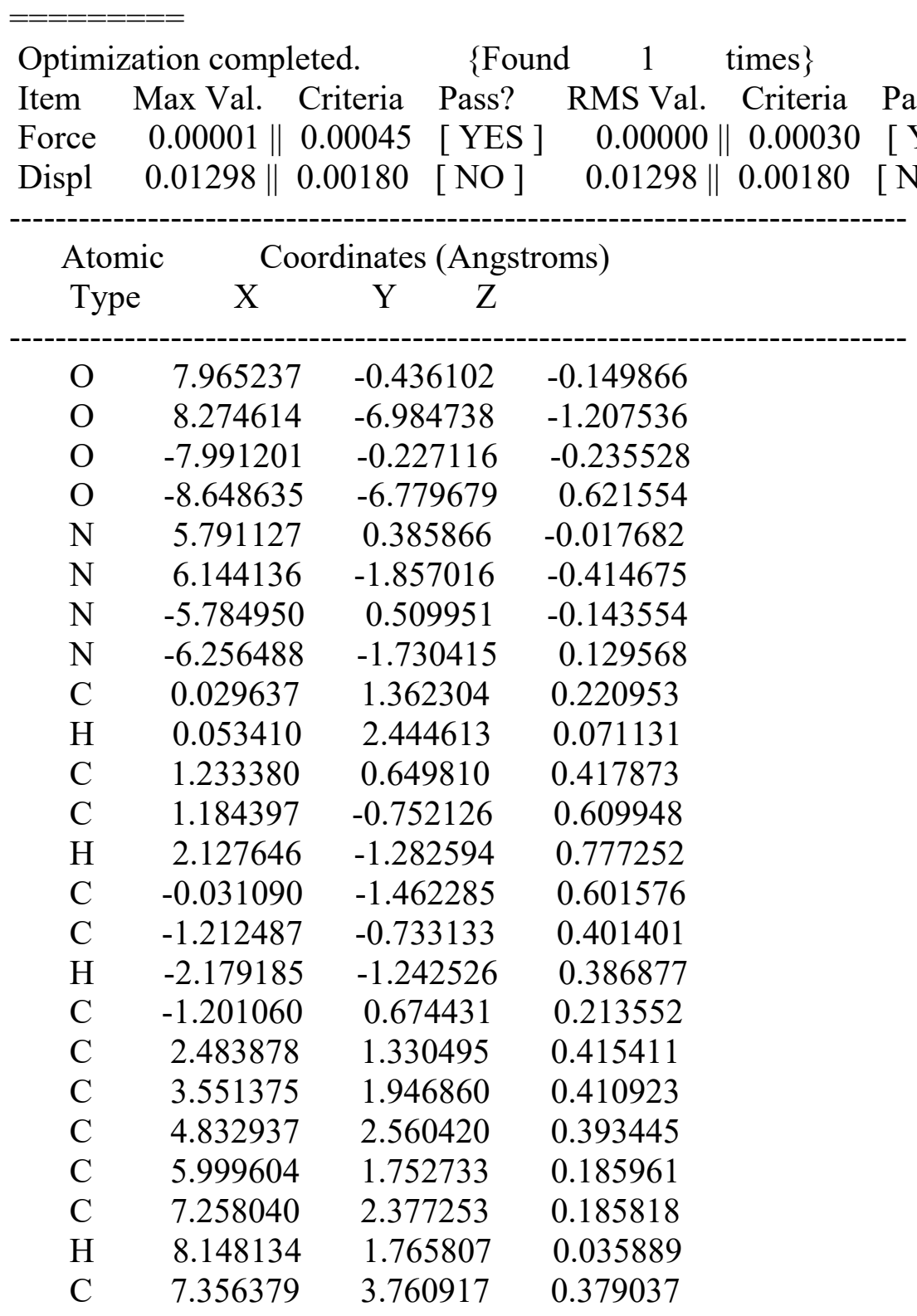




\begin{tabular}{|c|c|c|c|}
\hline $\mathrm{H}$ & 8.357715 & 4.200300 & 0.369901 \\
\hline $\mathrm{C}$ & 6.230041 & 4.585143 & 0.578628 \\
\hline $\mathrm{C}$ & 4.976419 & 3.952067 & 0.580897 \\
\hline $\mathrm{H}$ & 4.061737 & 4.533453 & 0.735158 \\
\hline $\mathrm{C}$ & 6.745951 & -0.621108 & -0.191528 \\
\hline $\mathrm{C}$ & 6.766820 & -3.109930 & -0.608255 \\
\hline $\mathrm{C}$ & 5.927351 & -4.220115 & -0.851568 \\
\hline $\mathrm{H}$ & 4.838469 & -4.083251 & -0.887787 \\
\hline $\mathrm{C}$ & 6.458181 & -5.492667 & -1.046869 \\
\hline $\mathrm{H}$ & 5.806726 & -6.350812 & -1.235879 \\
\hline $\mathrm{C}$ & 7.851857 & -5.693622 & -1.003413 \\
\hline $\mathrm{C}$ & 8.692611 & -4.593307 & -0.761597 \\
\hline $\mathrm{H}$ & 9.778056 & -4.714769 & -0.722790 \\
\hline $\mathrm{C}$ & 8.158683 & -3.310877 & -0.565531 \\
\hline $\mathrm{H}$ & 8.814615 & -2.459584 & -0.382502 \\
\hline $\mathrm{C}$ & 9.678841 & -7.218460 & -1.169114 \\
\hline $\mathrm{H}$ & 9.808678 & -8.295500 & -1.351493 \\
\hline $\mathrm{H}$ & 10.110164 & -6.960039 & -0.182390 \\
\hline $\mathrm{H}$ & 10.210737 & -6.647821 & -1.955163 \\
\hline $\mathrm{C}$ & -2.421875 & 1.377647 & 0.015297 \\
\hline $\mathrm{C}$ & -3.463399 & 2.013622 & -0.159929 \\
\hline $\mathrm{C}$ & -4.713008 & 2.659993 & -0.355298 \\
\hline $\mathrm{C}$ & -5.920235 & 1.885633 & -0.348991 \\
\hline $\mathrm{C}$ & -7.143910 & 2.547090 & -0.546264 \\
\hline $\mathrm{H}$ & -8.063646 & 1.961773 & -0.542517 \\
\hline $\mathrm{C}$ & -7.170269 & 3.933374 & -0.743727 \\
\hline $\mathrm{H}$ & -8.147027 & 4.401839 & -0.893097 \\
\hline $\mathrm{C}$ & -6.003240 & 4.724551 & -0.755423 \\
\hline $\mathrm{C}$ & -4.784670 & 4.055480 & -0.557303 \\
\hline $\mathrm{H}$ & -3.841081 & 4.610537 & -0.555003 \\
\hline $\mathrm{C}$ & -6.788077 & -0.463419 & -0.095671 \\
\hline $\mathrm{C}$ & -6.942809 & -2.959897 & 0.240167 \\
\hline $\mathrm{C}$ & -8.339187 & -3.093527 & 0.131800 \\
\hline $\mathrm{H}$ & -8.946893 & -2.206528 & -0.048068 \\
\hline $\mathrm{C}$ & -8.938563 & -4.356021 & 0.254586 \\
\hline $\mathrm{H}$ & -10.025642 & -4.424985 & 0.165334 \\
\hline $\mathrm{C}$ & -8.159635 & -5.502936 & 0.486665 \\
\hline $\mathrm{C}$ & -6.761420 & -5.369390 & 0.595194 \\
\hline $\mathrm{H}$ & -6.158240 & -6.263870 & 0.775818 \\
\hline $\mathrm{C}$ & -6.165559 & -4.116641 & 0.473440 \\
\hline $\mathrm{H}$ & -5.074241 & -4.032318 & 0.560766 \\
\hline $\mathrm{C}$ & -10.059103 & -6.945808 & 0.517030 \\
\hline $\mathrm{H}$ & -10.245105 & -8.021645 & 0.651646 \\
\hline $\mathrm{H}$ & -10.436259 & -6.630985 & -0.475482 \\
\hline $\mathrm{H}$ & -10.597471 & -6.380600 & 1.302606 \\
\hline $\mathrm{H}$ & 4.801570 & 0.125780 & 0.011507 \\
\hline $\mathrm{H}$ & 5.129266 & -1.876293 & -0.507244 \\
\hline $\mathrm{H}$ & -4.811013 & 0.220697 & -0.019494 \\
\hline
\end{tabular}




\begin{tabular}{|c|c|c|c|}
\hline $\mathrm{H}$ & -5.245367 & -1.806866 & 0.232505 \\
\hline $\mathrm{C}$ & -6.019699 & 6.251138 & -0.971402 \\
\hline $\mathrm{C}$ & -7.448572 & 6.795563 & -1.173632 \\
\hline $\mathrm{H}$ & -7.934693 & 6.354227 & -2.061335 \\
\hline $\mathrm{H}$ & -8.089900 & 6.603236 & -0.295675 \\
\hline $\mathrm{H}$ & -7.410187 & 7.888347 & -1.324326 \\
\hline $\mathrm{C}$ & -5.184717 & 6.602553 & -2.228031 \\
\hline $\mathrm{H}$ & -4.137819 & 6.267430 & -2.131928 \\
\hline $\mathrm{H}$ & -5.608997 & 6.127946 & -3.129774 \\
\hline $\mathrm{H}$ & -5.173910 & 7.696022 & -2.387362 \\
\hline $\mathrm{C}$ & -5.404079 & 6.952504 & 0.265103 \\
\hline $\mathrm{H}$ & -5.987610 & 6.731910 & 1.175728 \\
\hline $\mathrm{H}$ & -4.364464 & 6.629263 & 0.444665 \\
\hline $\mathrm{H}$ & -5.394813 & 8.047873 & 0.119202 \\
\hline $\mathrm{C}$ & 6.325597 & 6.109286 & 0.791180 \\
\hline $\mathrm{C}$ & 5.530509 & 6.835411 & -0.322559 \\
\hline $\mathrm{H}$ & 4.466815 & 6.541978 & -0.325121 \\
\hline $\mathrm{H}$ & 5.576536 & 7.929601 & -0.174862 \\
\hline $\mathrm{H}$ & 5.946010 & 6.605257 & -1.318958 \\
\hline $\mathrm{C}$ & 7.782879 & 6.612956 & 0.750376 \\
\hline $\mathrm{H}$ & 8.398629 & 6.154688 & 1.544054 \\
\hline $\mathrm{H}$ & 8.262058 & 6.406458 & -0.222696 \\
\hline $\mathrm{H}$ & 7.800992 & 7.705730 & 0.904853 \\
\hline $\mathrm{C}$ & 5.724399 & 6.475277 & 2.171201 \\
\hline $\mathrm{H}$ & 4.667009 & 6.170375 & 2.251819 \\
\hline $\mathrm{H}$ & 6.280333 & 5.982416 & 2.987537 \\
\hline $\mathrm{H}$ & 5.772275 & 7.567459 & 2.332629 \\
\hline $\mathrm{C}$ & -0.026218 & -2.990652 & 0.816558 \\
\hline $\mathrm{C}$ & 0.845414 & -3.661072 & -0.274720 \\
\hline $\mathrm{H}$ & 0.447715 & -3.456189 & -1.283629 \\
\hline $\mathrm{H}$ & 1.889209 & -3.302787 & -0.243821 \\
\hline $\mathrm{H}$ & 0.864575 & -4.755541 & -0.127260 \\
\hline $\mathrm{C}$ & 0.563394 & -3.306169 & 2.213822 \\
\hline $\mathrm{H}$ & 1.593673 & -2.926424 & 2.320286 \\
\hline $\mathrm{H}$ & -0.047082 & -2.851461 & 3.012890 \\
\hline $\mathrm{H}$ & 0.588752 & -4.398102 & 2.379135 \\
\hline $\mathrm{C}$ & -1.442103 & -3.599797 & 0.739917 \\
\hline $\mathrm{H}$ & -1.382831 & -4.691066 & 0.891997 \\
\hline $\mathrm{H}$ & -2.105831 & -3.192795 & 1.523566 \\
\hline $\mathrm{H}$ & -1.910651 & -3.426558 & -0.245306 \\
\hline
\end{tabular}

Statistical Thermodynamic Analysis

Temperature $=298.150$ Kelvin Pressure $=1.00000$ Atm

SCF Energy $=-2454.66118906 \quad$ Predicted Change $=-1.595624 \mathrm{D}-08$

Zero-point correction $(\mathrm{ZPE})=\quad-2453.74980 .91129$

Internal Energy $(U)=\quad-2453.68970 .97148$ 
$\begin{array}{lll}\text { Enthalpy }(\mathrm{H})= & -2453.6887 & 0.97242 \\ \text { Gibbs Free Energy }(\mathrm{G})= & -2453.8580 & 0.80311\end{array}$

Frequencies -- $2.2003 \quad 3.6565 \quad 5.6723$

Single points at WB97X-D3/def2-TZVPP: -2457.9395173635

$R=t B u$ receptor unbound (from conformational search)

Supporting Information: 000-unbound_conf_search_tBu.log

Using Gaussian 09: EM64L-G09RevE.01 30-Nov-2015

\#PBEPBE/6-31G(d)/auto scf=(direct,tight,maxcycle=300,xqc)

opt $=($ maxcycle $=250)$ freq $=$ noraman int $=($ grid $=$ ultrafine $)$

\#P Geom=AllCheck Guess=TCheck SCRF=Check GenChk RPBEPBE/6-31G(d)/Auto Freq

Pointgroup $=\mathrm{C} 1 \quad$ Stoichiometry $=\mathrm{C} 50 \mathrm{H} 54 \mathrm{~N} 4 \mathrm{O} 4 \mathrm{C} 1[\mathrm{X}(\mathrm{C} 50 \mathrm{H} 54 \mathrm{~N} 4 \mathrm{O} 4)] \quad \#$ Atoms $=112$

Charge $=0 \quad$ Multiplicity $=1$

SCF Energy $=-2454.67025479 \quad$ Predicted Change $=-3.149975 \mathrm{D}-08$

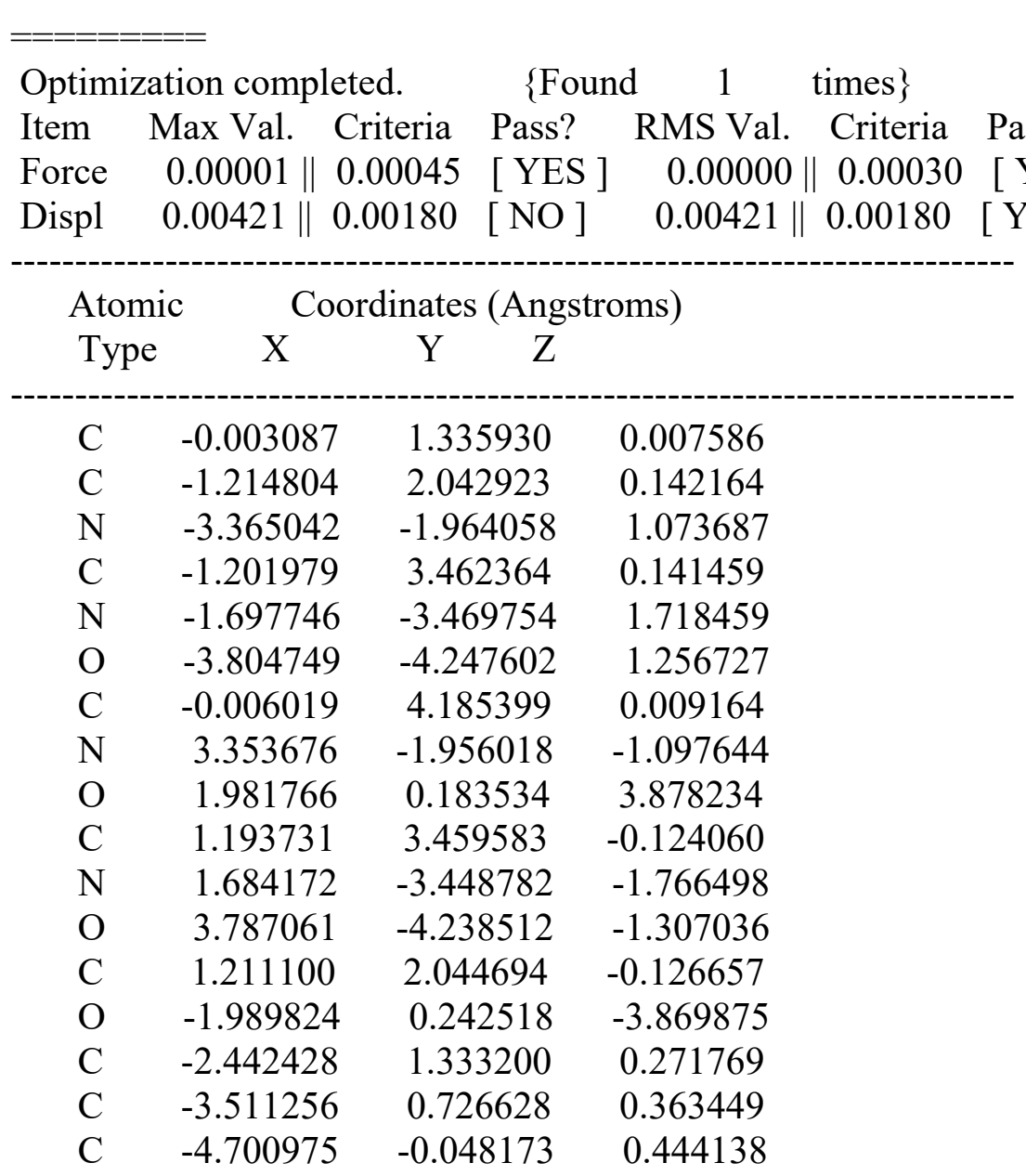




\begin{tabular}{|c|c|c|c|}
\hline $\mathrm{C}$ & -4.627882 & -1.440561 & 0.776797 \\
\hline $\mathrm{C}$ & -5.818432 & -2.187494 & 0.803844 \\
\hline $\mathrm{C}$ & -7.045308 & -1.572422 & 0.525361 \\
\hline $\mathrm{C}$ & -7.154604 & -0.203004 & 0.208636 \\
\hline $\mathrm{C}$ & -5.959499 & 0.533823 & 0.173987 \\
\hline $\mathrm{C}$ & -8.497955 & 0.489556 & -0.096829 \\
\hline $\mathrm{C}$ & -8.461703 & 1.075316 & -1.530566 \\
\hline $\mathrm{C}$ & -8.728448 & 1.636650 & 0.918458 \\
\hline $\mathrm{C}$ & -9.690129 & -0.484531 & -0.003607 \\
\hline $\mathrm{C}$ & -3.034085 & -3.287862 & 1.354738 \\
\hline $\mathrm{C}$ & -0.767336 & -2.518161 & 2.23199 \\
\hline $\mathrm{C}$ & -1.146623 & -1.546306 & 3.185310 \\
\hline $\mathrm{C}$ & -0.207580 & -0.650606 & 3.694370 \\
\hline $\mathrm{C}$ & 1.143299 & -0.723521 & \\
\hline $\mathrm{C}$ & 1.533405 & -1.698842 & 2.35527 \\
\hline $\mathrm{C}$ & 0.576597 & -2.575119 & 1.823 \\
\hline $\mathrm{C}$ & 3.361627 & 0.136280 & 3.507978 \\
\hline $\mathrm{C}$ & 2.438433 & 1.334075 & -0.25763 \\
\hline $\mathrm{C}$ & 3.505275 & 0.724557 & -0.35125 \\
\hline $\mathrm{C}$ & 4.692904 & -0.053161 & -0.436 \\
\hline $\mathrm{C}$ & 4.616766 & -1.440721 & -0.787847 \\
\hline $\mathrm{C}$ & 5.805044 & & \\
\hline $\mathrm{C}$ & 7.032567 & -1.583836 & -0.52701 \\
\hline $\mathrm{C}$ & 7.144853 & -0.219102 & -0.19170 \\
\hline $\mathrm{C}$ & 954 & 0.5 & -0.152751 \\
\hline $\mathrm{C}$ & 8.488991 & 0.464947 & 0.129056 \\
\hline $\mathrm{C}$ & 9.678538 & -0.511490 & 0.02730 \\
\hline $\mathrm{C}$ & 8.727398 & 1.625330 & -0.86916 \\
\hline $\mathrm{C}$ & 8.448364 & 1.030806 & 1.570652 \\
\hline $\mathrm{C}$ & 3.019561 & -3.275321 & -1.39577 \\
\hline $\mathrm{C}$ & 0.755265 & -2.488020 & -2.26502 \\
\hline $\mathrm{C}$ & 1.136237 & -1.501595 & -3.20258 \\
\hline $\mathrm{C}$ & 0.198523 & -0.596761 & -3.69779 \\
\hline $\mathrm{C}$ & -1.152675 & -0.674452 & -3.29958 \\
\hline $\mathrm{C}$ & -1.544413 & -1.664058 & -2.37643 \\
\hline $\mathrm{C}$ & -0.588964 & -2.549796 & -1.85794 \\
\hline $\mathrm{C}$ & -3.370075 & 0.190613 & -3.50163 \\
\hline $\mathrm{H}$ & -0.002202 & 0.242412 & 0.00689 \\
\hline $\mathrm{H}$ & -2.157625 & 3.980352 & 0.24916 \\
\hline $\mathrm{H}$ & -2.602762 & -1.279789 & 1.06827 \\
\hline $\mathrm{H}$ & -1.483797 & -4.459528 & 1.84152 \\
\hline $\mathrm{H}$ & 2.147785 & 3.984898 & -0.23147 \\
\hline $\mathrm{H}$ & 2.593462 & -1.269530 & -1.08635 \\
\hline $\mathrm{H}$ & 1.466864 & -4.436347 & -1.90057 \\
\hline $\mathrm{H}$ & -5.762903 & -3.250996 & 1.03872 \\
\hline $\mathrm{H}$ & -7.942185 & -2.197081 & 0.56187 \\
\hline $\mathrm{H}$ & -5.973344 & 1.600700 & -0.07202 \\
\hline $\mathrm{H}$ & -9.412961 & 1.589027 & -1.75957 \\
\hline
\end{tabular}




$\begin{array}{cccc}\mathrm{H} & -8.313629 & 0.277656 & -2.279240 \\ \mathrm{H} & -7.646636 & 1.809154 & -1.652900 \\ \mathrm{H} & -8.774422 & 1.247311 & 1.950279 \\ \mathrm{H} & -9.680993 & 2.153856 & 0.703038 \\ \mathrm{H} & -7.921722 & 2.388369 & 0.877858 \\ \mathrm{H} & -9.787975 & -0.917934 & 1.007113 \\ \mathrm{H} & -9.601922 & -1.312770 & -0.728394 \\ \mathrm{H} & -10.627173 & 0.054456 & -0.226835 \\ \mathrm{H} & -2.182149 & -1.505096 & 3.536868 \\ \mathrm{H} & -0.494590 & 0.106030 & 4.430423 \\ \mathrm{H} & 2.569161 & -1.774227 & 2.016107 \\ \mathrm{H} & 0.881404 & -3.313780 & 1.074080 \\ \mathrm{H} & 3.852491 & 0.922054 & 4.100654 \\ \mathrm{H} & 3.499123 & 0.345527 & 2.431262 \\ \mathrm{H} & 3.813612 & -0.843487 & 3.754290 \\ \mathrm{H} & 5.747161 & -3.251236 & -1.068548 \\ \mathrm{H} & 7.927590 & -2.210901 & -0.567671 \\ \mathrm{H} & 5.968111 & 1.584546 & 0.107553 \\ \mathrm{H} & 10.616324 & 0.021444 & 0.261665 \\ \mathrm{H} & 9.779125 & -0.931345 & -0.988849 \\ \mathrm{H} & 9.584838 & -1.349265 & 0.740340 \\ \mathrm{H} & 0.680629 & 2.136428 & -0.642505 \\ \mathrm{H} & 0.683 \\ \mathrm{H} & 7.922902 & 2.379026 & -0.821498 \\ \mathrm{H} & 8.776558 & 1.250277 & -1.906111 \\ \mathrm{H} & 9.400110 & 1.538501 & 1.810779 \\ \mathrm{H} & 8.294866 & 0.223247 & 2.307530 \\ \mathrm{H} & 7.634876 & 1.765243 & 1.699787 \\ \mathrm{H} & 2.171935 & -1.456279 & -3.553138 \\ \mathrm{H} & 0.486838 & 0.171081 & -4.421632 \\ \mathrm{H} & -2.580413 & -1.743531 & -2.038951 \\ \mathrm{H} & -0.895089 & -3.299712 & -1.120515 \\ \mathrm{H} & -3.822603 & -0.784804 & -3.763714 \\ \mathrm{H} & -3.859760 & 0.985939 & -4.082440 \\ \mathrm{H} & -3.508406 & 0.383166 & -2.421922 \\ \mathrm{C} & 0.032699 & 5.728170 & 0.004725 \\ \mathrm{H} & -1.369595 & 6.351133 & 0.159970 \\ \mathrm{H} & -1.845333 & 6.061868 & 1.113361 \\ \mathrm{H} & -1.286908 & 7.451621 & 0.150400 \\ \mathrm{H} & 0.915165 & 6.219809 & 1.179129 \\ \mathrm{H} & 0.501309 & 5.893216 & 2.148833 \\ \mathrm{H} & -6.219369 & 7.323061 & -1.347853 \\ \mathrm{H} & 5.891980 & -2.189946\end{array}$


Statistical Thermodynamic Analysis

Temperature $=298.150$ Kelvin $\quad$ Pressure $=1.00000$ Atm

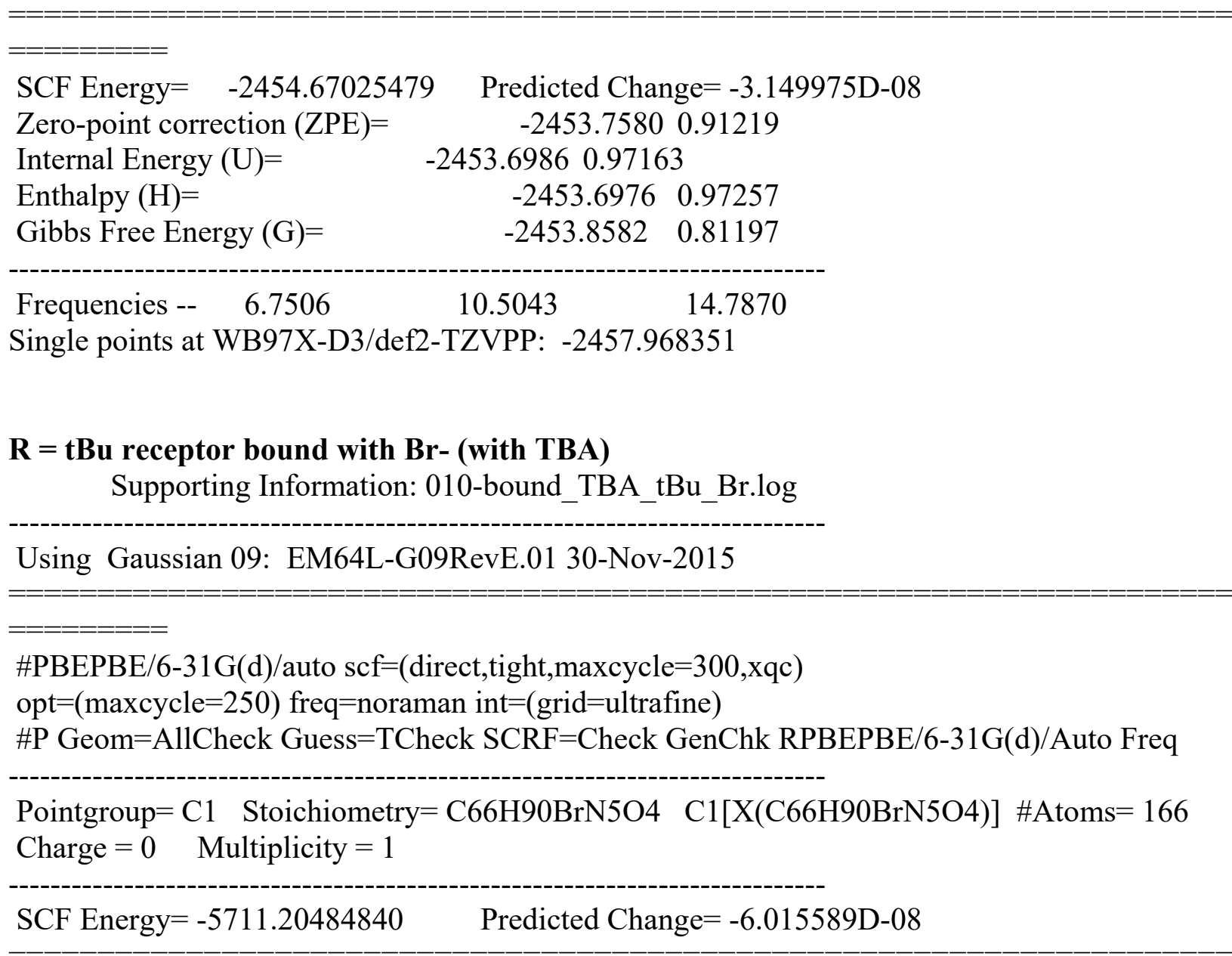

\begin{tabular}{|c|c|c|c|c|c|}
\hline \multicolumn{3}{|c|}{ Optimization completed. } & $\{$ Foul & nd & times $\}$ \\
\hline \multicolumn{3}{|c|}{ Item Max Val. Criteria } & Pass? & RMS Val. & Criteria \\
\hline Force & $0.00001 \|$ & 0.00045 & [ YES ] & 0.00000 & 0.00030 \\
\hline Displ & $0.00408 \|$ & 0.00180 & {$[\mathrm{NO}]$} & $0.00408 \|$ & 0.00180 \\
\hline \multicolumn{2}{|c|}{ Atomic } & \multicolumn{3}{|c|}{ Coordinates (Angstroms) } & \\
\hline Type & e & $\mathrm{Y}$ & Z & & \\
\hline $\mathrm{N}$ & -2.383068 & -1.06 & 9481 & -2.611581 & \\
\hline $\mathrm{C}$ & -1.057028 & -0.84 & 0490 & -3.355163 & \\
\hline $\mathrm{C}$ & -0.084289 & 0.18 & 3276 & -2.769080 & \\
\hline $\mathrm{C}$ & 1.199290 & 0.19 & 1864 & -3.627019 & \\
\hline $\mathrm{C}$ & 2.192662 & 1.27 & 3734 & -3.187849 & \\
\hline $\mathrm{C}$ & -2.081851 & -1.82 & 8715 & -1.310293 & \\
\hline $\mathrm{C}$ & -3.271173 & -2.26 & 2618 & -0.450380 & \\
\hline $\mathrm{C}$ & -2.741806 & -3.14 & 6163 & 0.701890 & \\
\hline $\mathrm{C}$ & -3.839000 & -3.56 & 9466 & 1.685455 & \\
\hline $\mathrm{C}$ & -3.058250 & 0.25 & 7760 & -2.259212 & \\
\hline
\end{tabular}




\begin{tabular}{|c|c|c|c|}
\hline $\mathrm{C}$ & -3.359693 & 1.202003 & -3.427052 \\
\hline $\mathrm{C}$ & -3.933438 & 2.533825 & -2.903890 \\
\hline $\mathrm{C}$ & -4.270252 & 3.508299 & -4.039017 \\
\hline $\mathrm{C}$ & -3.307440 & -1.888509 & -3.513537 \\
\hline $\mathrm{C}$ & -2.764824 & -3.244117 & -3.977478 \\
\hline $\mathrm{C}$ & -3.860085 & -4.070349 & -4.678857 \\
\hline $\mathrm{C}$ & -3.301509 & -5.379564 & -5.253277 \\
\hline $\mathrm{H}$ & -0.573936 & -1.829457 & -3.397722 \\
\hline $\mathrm{H}$ & -1.337613 & -0.558421 & -4.384762 \\
\hline $\mathrm{H}$ & 0.178009 & -0.048845 & -1.719245 \\
\hline $\mathrm{H}$ & -0.525202 & 1.197475 & -2.775579 \\
\hline $\mathrm{H}$ & 0.931151 & 0.344692 & -4.692090 \\
\hline $\mathrm{H}$ & 1.681596 & -0.802118 & -3.565213 \\
\hline $\mathrm{H}$ & 3.086361 & 1.268929 & -3.834222 \\
\hline $\mathrm{H}$ & 1.741845 & 2.280663 & -3.239954 \\
\hline $\mathrm{H}$ & 2.531817 & 1.113886 & -2.151161 \\
\hline $\mathrm{H}$ & -1.492516 & -2.706568 & -1.615028 \\
\hline $\mathrm{H}$ & -1.403886 & -1.190284 & -0.715886 \\
\hline $\mathrm{H}$ & -3.795470 & -1.390494 & -0.019040 \\
\hline $\mathrm{H}$ & -4.014536 & -2.844629 & -1.028264 \\
\hline $\mathrm{H}$ & -1.942010 & -2.597965 & 1.232459 \\
\hline $\mathrm{H}$ & -2.265741 & -4.046944 & 0.267817 \\
\hline $\mathrm{H}$ & -3.426323 & -4.239903 & 2.457591 \\
\hline $\mathrm{H}$ & -4.270649 & -2.700949 & 2.210779 \\
\hline $\mathrm{H}$ & -4.656125 & -4.107550 & 1.170692 \\
\hline $\mathrm{H}$ & -3.988317 & -0.007561 & -1.731942 \\
\hline $\mathrm{H}$ & -2.393908 & 0.744921 & -1.527229 \\
\hline $\mathrm{H}$ & -2.443818 & 1.412810 & -4.009067 \\
\hline $\mathrm{H}$ & -4.089922 & 0.752253 & -4.125251 \\
\hline $\mathrm{H}$ & -3.205214 & 2.995991 & -2.211406 \\
\hline $\mathrm{H}$ & -4.838883 & 2.332190 & -2.300990 \\
\hline $\mathrm{H}$ & -4.684894 & 4.449195 & -3.639241 \\
\hline $\mathrm{H}$ & -3.373056 & 3.763916 & -4.630394 \\
\hline $\mathrm{H}$ & -5.018348 & 3.080703 & -4.730500 \\
\hline $\mathrm{H}$ & -3.525819 & -1.253117 & -4.386112 \\
\hline $\mathrm{H}$ & -4.245922 & -2.014411 & -2.950244 \\
\hline $\mathrm{H}$ & -1.921462 & -3.100655 & -4.676643 \\
\hline $\mathrm{H}$ & -2.371585 & -3.832708 & -3.130117 \\
\hline $\mathrm{H}$ & -4.323565 & -3.473993 & -5.488794 \\
\hline $\mathrm{H}$ & -4.667638 & -4.291619 & -3.954898 \\
\hline $\mathrm{H}$ & -4.107377 & -6.006079 & -5.670663 \\
\hline $\mathrm{H}$ & -2.578577 & -5.178053 & -6.063792 \\
\hline $\mathrm{H}$ & -2.774512 & -5.963959 & -4.478467 \\
\hline $\mathrm{O}$ & -0.263618 & -6.715317 & -3.372444 \\
\hline $\mathrm{O}$ & -3.826839 & -0.744445 & 3.962502 \\
\hline $\mathrm{N}$ & 2.546193 & -2.476733 & -0.998631 \\
\hline $\mathrm{N}$ & -2.868952 & 0.470158 & 2.217126 \\
\hline $\mathrm{C}$ & 1.035052 & 3.011615 & 0.367763 \\
\hline
\end{tabular}




\begin{tabular}{|c|c|c|c|}
\hline $\mathrm{C}$ & 1.396156 & 5.790995 & -0.136377 \\
\hline $\mathrm{C}$ & 0.111790 & 5.254951 & 0.051429 \\
\hline $\mathrm{C}$ & -0.077689 & 3.871636 & 0.308071 \\
\hline $\mathrm{C}$ & 1.937784 & -3.571892 & -1.633327 \\
\hline $\mathrm{C}$ & 2.442521 & -4.203660 & -2.788159 \\
\hline $\mathrm{C}$ & 1.736044 & -5.258557 & -3.388398 \\
\hline $\mathrm{C}$ & 0.515667 & -5.702479 & -2.850305 \\
\hline $\mathrm{C}$ & 0.019306 & -5.087786 & -1.683549 \\
\hline $\mathrm{C}$ & 0.720557 & -4.040977 & -1.083352 \\
\hline $\mathrm{C}$ & 0.263117 & -7.416396 & -4.494693 \\
\hline $\mathrm{C}$ & -1.382404 & 3.341917 & 0.525125 \\
\hline $\mathrm{C}$ & -2.501877 & 2.881703 & 0.756829 \\
\hline $\mathrm{C}$ & -3.804202 & 2.391276 & 1.045150 \\
\hline $\mathrm{C}$ & -3.992107 & 1.193559 & 1.804931 \\
\hline $\mathrm{C}$ & -5.318104 & 0.786690 & 2.078490 \\
\hline $\mathrm{C}$ & -6.402937 & 1.534669 & 1.619450 \\
\hline $\mathrm{C}$ & -6.248318 & 2.720769 & 0.864932 \\
\hline $\mathrm{C}$ & -4.934921 & 3.121226 & 0.595076 \\
\hline $\mathrm{C}$ & -8.340742 & 2.606312 & -0.535242 \\
\hline $\mathrm{C}$ & -7.100526 & 4.778648 & -0.401349 \\
\hline $\mathrm{C}$ & -2.825762 & -0.437147 & 3.300594 \\
\hline $\mathrm{C}$ & 3.872479 & -2.083190 & -1.111417 \\
\hline $\mathrm{C}$ & 2.332866 & 3.529567 & 0.170655 \\
\hline $\mathrm{C}$ & 2.497499 & 4.913666 & -0.074495 \\
\hline $\mathrm{C}$ & -7.482598 & 3.508881 & 0.386174 \\
\hline $\mathrm{N}$ & -1.555223 & -0.927494 & 3.508509 \\
\hline $\mathrm{N}$ & 4.142412 & -0.936354 & -0.348385 \\
\hline $\mathrm{O}$ & 4.715422 & -2.665925 & -1.802558 \\
\hline $\mathrm{C}$ & 3.450276 & 2.647809 & 0.201332 \\
\hline $\mathrm{C}$ & -8.327543 & 3.936640 & 1.612247 \\
\hline $\mathrm{C}$ & -1.157047 & -1.907204 & 4.445468 \\
\hline $\mathrm{C}$ & 5.415088 & -0.377658 & -0.161068 \\
\hline $\mathrm{C}$ & 4.395882 & 1.859509 & 0.199595 \\
\hline $\mathrm{C}$ & 0.141060 & -2.448059 & 4.287689 \\
\hline $\mathrm{C}$ & -1.956033 & -2.350840 & 5.514990 \\
\hline $\mathrm{C}$ & 5.531700 & 1.007326 & 0.162734 \\
\hline $\mathrm{C}$ & 6.596983 & -1.142234 & -0.249485 \\
\hline $\mathrm{C}$ & 0.624934 & -3.401992 & 5.178881 \\
\hline $\mathrm{C}$ & -1.465670 & -3.315998 & 6.408956 \\
\hline $\mathrm{C}$ & 6.814241 & 1.561986 & 0.410405 \\
\hline $\mathrm{C}$ & 7.841292 & -0.557893 & -0.013958 \\
\hline $\mathrm{C}$ & -0.174204 & -3.846672 & 6.250877 \\
\hline $\mathrm{C}$ & 7.988108 & 0.805905 & 0.329763 \\
\hline $\mathrm{O}$ & 0.399063 & -4.792287 & 7.071538 \\
\hline $\mathrm{C}$ & 9.386447 & 1.396217 & 0.593752 \\
\hline $\mathrm{C}$ & -0.381626 & -5.250845 & 8.167731 \\
\hline $\mathrm{C}$ & 9.332328 & 2.895976 & 0.948539 \\
\hline $\mathrm{C}$ & 10.046068 & 0.641934 & 1.775321 \\
\hline
\end{tabular}




\begin{tabular}{|c|c|c|c|}
\hline $\mathrm{C}$ & 10.262524 & 1.228723 & -0.672849 \\
\hline $\mathrm{H}$ & 1.949848 & -1.984805 & -0.310977 \\
\hline $\mathrm{H}$ & -1.975516 & 0.745197 & 1.792014 \\
\hline $\mathrm{H}$ & 0.896174 & 1.944011 & 0.567608 \\
\hline $\mathrm{H}$ & -0.772017 & 5.896288 & 0.016224 \\
\hline $\mathrm{H}$ & 3.398266 & -3.874989 & -3.198669 \\
\hline $\mathrm{H}$ & 2.157885 & -5.725367 & -4.282496 \\
\hline $\mathrm{H}$ & -0.908598 & -5.467795 & -1.242161 \\
\hline $\mathrm{H}$ & 0.336125 & -3.561356 & -0.175547 \\
\hline $\mathrm{H}$ & -0.469875 & -8.202315 & -4.730351 \\
\hline $\mathrm{H}$ & 0.383123 & -6.754135 & -5.374928 \\
\hline $\mathrm{H}$ & 1.239472 & -7.883074 & -4.261504 \\
\hline $\mathrm{H}$ & -5.474504 & -0.105620 & 2.684149 \\
\hline $\mathrm{H}$ & -4.734977 & 4.036440 & 0.031916 \\
\hline $\mathrm{H}$ & -6.505198 & 5.477450 & 0.211843 \\
\hline $\mathrm{H}$ & -6.522223 & 4.540210 & -1.312321 \\
\hline $\mathrm{H}$ & -7.408820 & 1.174525 & 1.863936 \\
\hline $\mathrm{H}$ & -8.667874 & 1.687837 & -0.018733 \\
\hline $\mathrm{H}$ & -7.771361 & 2.304333 & -1.432591 \\
\hline $\mathrm{H}$ & -9.245804 & 3.145286 & -0.869593 \\
\hline $\mathrm{H}$ & -8.015262 & 5.308999 & -0.718382 \\
\hline $\mathrm{H}$ & 3.513879 & 5.292598 & -0.221656 \\
\hline $\mathrm{H}$ & -7.745958 & 4.594575 & 2.280661 \\
\hline $\mathrm{H}$ & -8.660551 & 3.067010 & 2.203621 \\
\hline $\mathrm{H}$ & -9.228724 & 4.486532 & 1.285291 \\
\hline $\mathrm{H}$ & 0.758840 & -2.113529 & 3.445580 \\
\hline $\mathrm{H}$ & -2.959573 & -1.942795 & 5.638478 \\
\hline $\mathrm{H}$ & 6.523175 & -2.196079 & -0.517969 \\
\hline $\mathrm{H}$ & 1.628264 & -3.821865 & 5.059425 \\
\hline $\mathrm{H}$ & -2.109815 & -3.640343 & 7.230491 \\
\hline $\mathrm{H}$ & 6.849497 & 2.624907 & 0.661843 \\
\hline $\mathrm{H}$ & 8.728417 & -1.196634 & -0.091152 \\
\hline $\mathrm{H}$ & 0.243486 & -5.986219 & 8.696372 \\
\hline $\mathrm{H}$ & -0.641225 & -4.426717 & 8.861215 \\
\hline $\mathrm{H}$ & -1.316241 & -5.741666 & 7.830898 \\
\hline $\mathrm{H}$ & 10.354427 & 3.272024 & 1.129290 \\
\hline $\mathrm{H}$ & 8.742562 & 3.079629 & 1.863711 \\
\hline $\mathrm{H}$ & 8.896301 & 3.495306 & 0.129990 \\
\hline $\mathrm{H}$ & 9.451031 & 0.757102 & 2.697830 \\
\hline $\mathrm{H}$ & 11.059536 & 1.039259 & 1.966850 \\
\hline $\mathrm{H}$ & 10.141800 & -0.437736 & 1.569236 \\
\hline $\mathrm{H}$ & 11.277081 & 1.629029 & -0.493636 \\
\hline $\mathrm{H}$ & 9.824949 & 1.770402 & -1.529394 \\
\hline $\mathrm{H}$ & 10.365752 & 0.169091 & -0.962284 \\
\hline $\mathrm{H}$ & 3.347586 & -0.426294 & 0.055626 \\
\hline $\mathrm{H}$ & -0.836155 & -0.702320 & 2.800881 \\
\hline $\mathrm{C}$ & 1.634179 & 7.292427 & -0.404758 \\
\hline $\mathrm{C}$ & 0.320665 & 8.100405 & -0.431813 \\
\hline
\end{tabular}


Atomic Coordinates (Angstroms)

\begin{tabular}{|c|c|c|c|}
\hline Type & X & Z & \\
\hline $\mathrm{N}$ & -2.334146 & -1.082144 & -2.510083 \\
\hline $\mathrm{C}$ & -0.988335 & -0.916481 & -3.237825 \\
\hline $\mathrm{C}$ & -0.008611 & 0.116483 & -2.682077 \\
\hline $\mathrm{C}$ & 1.289220 & 0.073075 & -3.516772 \\
\hline $\mathrm{C}$ & 2.289788 & 1.158215 & -3.102144 \\
\hline $\mathrm{C}$ & -2.087884 & -1.824340 & -1.183639 \\
\hline $\mathrm{C}$ & -3.329329 & -2.194529 & -0.364730 \\
\hline $\mathrm{C}$ & -2.943246 & -3.207397 & 0.735375 \\
\hline $\mathrm{C}$ & -4.122071 & -3.567657 & 1.648062 \\
\hline $\mathrm{C}$ & -2.967768 & 0.274918 & -2.196194 \\
\hline $\mathrm{C}$ & -3.250624 & 1.187743 & -3.393215 \\
\hline $\mathrm{C}$ & -3.753497 & 2.562950 & -2.911297 \\
\hline $\mathrm{C}$ & -4.074517 & 3.506553 & -4.076670 \\
\hline $\mathrm{C}$ & -3.270822 & -1.880444 & -3.417608 \\
\hline $\mathrm{C}$ & -2.817763 & -3.299468 & -3.771567 \\
\hline $\mathrm{C}$ & -3.901641 & -4.034967 & -4.583335 \\
\hline $\mathrm{C}$ & -3.423854 & -5.416108 & -5.051755 \\
\hline $\mathrm{H}$ & -0.523218 & -1.915097 & -3.224118 \\
\hline $\mathrm{H}$ & -1.243284 & -0.678111 & -4.284827 \\
\hline $\mathrm{H}$ & 0.227976 & -0.080459 & -1.619750 \\
\hline $\mathrm{H}$ & -0.433209 & 1.135799 & -2.739426 \\
\hline $\mathrm{H}$ & 1.041270 & 0.188830 & -4.591405 \\
\hline $\mathrm{H}$ & 1.755443 & -0.924346 & -3.408697 \\
\hline $\mathrm{H}$ & 3.195847 & 1.114927 & -3.729615 \\
\hline $\mathrm{H}$ & 1.854362 & 2.168121 & -3.204728 \\
\hline $\mathrm{H}$ & 2.606186 & 1.036586 & -2.053081 \\
\hline $\mathrm{H}$ & -1.521862 & -2.728381 & -1.455061 \\
\hline $\mathrm{H}$ & -1.404964 & -1.189844 & -0.589736 \\
\hline $\mathrm{H}$ & -3.773741 & -1.300180 & 0.107971 \\
\hline $\mathrm{H}$ & -4.114511 & -2.657187 & -0.992691 \\
\hline $\mathrm{H}$ & -2.117142 & -2.794113 & 1.341446 \\
\hline $\mathrm{H}$ & -2.551119 & -4.122211 & 0.250892 \\
\hline $\mathrm{H}$ & -3.821561 & -4.335669 & 2.380230 \\
\hline $\mathrm{H}$ & -4.466771 & -2.690471 & 2.221093 \\
\hline $\mathrm{H}$ & -4.974104 & -3.967287 & 1.067659 \\
\hline $\mathrm{H}$ & -3.901288 & 0.053474 & -1.654964 \\
\hline $\mathrm{H}$ & -2.284947 & 0.764470 & -1.483407 \\
\hline $\mathrm{H}$ & -2.338988 & 1.333821 & -4.001488 \\
\hline $\mathrm{H}$ & -4.014736 & 0.743678 & -4.057916 \\
\hline $\mathrm{H}$ & -2.990162 & 3.019714 & -2.253850 \\
\hline $\mathrm{H}$ & -4.652141 & 2.426185 & -2.280853 \\
\hline $\mathrm{H}$ & -4.437657 & 4.479916 & -3.705438 \\
\hline $\mathrm{H}$ & -3.181793 & 3.698570 & -4.698194 \\
\hline $\mathrm{H}$ & -4.857434 & 3.086301 & -4.733260 \\
\hline $\mathrm{H}$ & -3.385509 & -1.282597 & -4.335496 \\
\hline
\end{tabular}




\begin{tabular}{|c|c|c|c|}
\hline $\mathrm{H}$ & -4.248715 & -1.898230 & -2.910518 \\
\hline $\mathrm{H}$ & -1.883363 & -3.272059 & -4.360485 \\
\hline $\mathrm{H}$ & -2.598290 & -3.887960 & -2.863670 \\
\hline $\mathrm{H}$ & -4.196045 & -3.425029 & -5.459570 \\
\hline $\mathrm{H}$ & -4.810817 & -4.143390 & -3.961311 \\
\hline $\mathrm{H}$ & -4.237393 & -5.971201 & -5.547913 \\
\hline $\mathrm{H}$ & -2.592470 & -5.322099 & -5.773066 \\
\hline $\mathrm{H}$ & -3.058255 & -6.021458 & -4.203918 \\
\hline $\mathrm{O}$ & -0.523351 & -6.742669 & -3.056445 \\
\hline $\mathrm{O}$ & -3.943121 & -0.672880 & 3.709377 \\
\hline $\mathrm{N}$ & 2.390728 & -2.500585 & -0.802489 \\
\hline $\mathrm{N}$ & -2.860007 & 0.662124 & 2.136773 \\
\hline $\mathrm{C}$ & 1.218973 & 3.001957 & 0.467573 \\
\hline $\mathrm{C}$ & 1.735358 & 5.779113 & 0.077340 \\
\hline $\mathrm{C}$ & 0.422396 & 5.304943 & 0.227337 \\
\hline $\mathrm{C}$ & 0.155777 & 3.924652 & 0.426169 \\
\hline $\mathrm{C}$ & 1.734140 & -3.588243 & -1.407186 \\
\hline $\mathrm{C}$ & 2.117573 & -4.132616 & -2.650686 \\
\hline $\mathrm{C}$ & 1.391592 & -5.187401 & -3.226316 \\
\hline $\mathrm{C}$ & 0.264668 & -5.717913 & -2.573834 \\
\hline $\mathrm{C}$ & -0.115449 & -5.186952 & -1.325504 \\
\hline $\mathrm{C}$ & 0.610540 & -4.141834 & -0.749477 \\
\hline $\mathrm{C}$ & -0.079292 & -7.387775 & -4.246090 \\
\hline $\mathrm{C}$ & -1.182388 & 3.467144 & 0.597972 \\
\hline $\mathrm{C}$ & -2.340683 & 3.084587 & 0.772826 \\
\hline $\mathrm{C}$ & -3.674803 & 2.650717 & 0.998428 \\
\hline $\mathrm{C}$ & -3.941483 & 1.434701 & 1.704877 \\
\hline $\mathrm{C}$ & -5.292300 & 1.066366 & 1.899172 \\
\hline $\mathrm{C}$ & -6.325971 & 1.871490 & 1.418225 \\
\hline $\mathrm{C}$ & -6.093533 & 3.079478 & 0.720625 \\
\hline $\mathrm{C}$ & -4.754966 & 3.440468 & 0.527917 \\
\hline $\mathrm{C}$ & -8.126684 & 3.095495 & -0.769726 \\
\hline $\mathrm{C}$ & -6.809337 & 5.209759 & -0.508878 \\
\hline $\mathrm{C}$ & -2.898255 & -0.342567 & 3.128972 \\
\hline $\mathrm{C}$ & 3.739613 & -2.201008 & -0.956322 \\
\hline $\mathrm{C}$ & 2.545064 & 3.459904 & 0.307443 \\
\hline $\mathrm{C}$ & 2.786471 & 4.841782 & 0.118674 \\
\hline $\mathrm{C}$ & -7.274620 & 3.931513 & 0.217733 \\
\hline $\mathrm{N}$ & -1.656694 & -0.898949 & 3.347596 \\
\hline $\mathrm{N}$ & 4.111659 & -1.056910 & -0.237052 \\
\hline $\mathrm{O}$ & 4.521412 & -2.861894 & -1.648974 \\
\hline $\mathrm{C}$ & 3.619444 & 2.526140 & 0.314750 \\
\hline $\mathrm{C}$ & -8.154558 & 4.351852 & 1.421571 \\
\hline $\mathrm{C}$ & -1.340994 & -1.939459 & 4.251230 \\
\hline $\mathrm{C}$ & 5.421719 & -0.577585 & -0.099235 \\
\hline $\mathrm{C}$ & 4.540036 & 1.709165 & 0.287397 \\
\hline $\mathrm{C}$ & -0.037705 & -2.485077 & 4.168552 \\
\hline $\mathrm{C}$ & -2.227068 & -2.444435 & 5.221803 \\
\hline
\end{tabular}




\begin{tabular}{|c|c|c|c|}
\hline $\mathrm{C}$ & 5.628222 & 0.799763 & 0.214474 \\
\hline $\mathrm{C}$ & 6.554056 & -1.408664 & -0.225866 \\
\hline $\mathrm{C}$ & 0.363682 & -3.504027 & 5.028732 \\
\hline $\mathrm{C}$ & -1.818822 & -3.472035 & 6.086956 \\
\hline $\mathrm{C}$ & 6.947526 & 1.281878 & 0.413709 \\
\hline $\mathrm{C}$ & 7.836843 & -0.894300 & -0.036567 \\
\hline $\mathrm{C}$ & -0.523821 & -4.009838 & 5.998872 \\
\hline $\mathrm{C}$ & 8.073059 & 0.459938 & 0.295016 \\
\hline $\mathrm{O}$ & -0.027561 & -5.015874 & 6.797285 \\
\hline $\mathrm{C}$ & 9.511201 & 0.970742 & 0.505831 \\
\hline $\mathrm{C}$ & -0.896907 & -5.531543 & 7.797234 \\
\hline $\mathrm{C}$ & 9.554678 & 2.472406 & 0.853941 \\
\hline $\mathrm{C}$ & 10.168995 & 0.184443 & 1.667366 \\
\hline $\mathrm{C}$ & 10.330513 & 0.749439 & -0.790158 \\
\hline $\mathrm{H}$ & 1.837775 & -1.966300 & -0.106814 \\
\hline $\mathrm{H}$ & -1.936518 & 0.991628 & 1.834354 \\
\hline $\mathrm{H}$ & 1.021686 & 1.936210 & 0.629261 \\
\hline $\mathrm{H}$ & -0.426120 & 5.992848 & 0.205502 \\
\hline $\mathrm{H}$ & 3.005670 & -3.743336 & -3.150694 \\
\hline $\mathrm{H}$ & 1.724399 & -5.587129 & -4.187967 \\
\hline $\mathrm{H}$ & -0.967825 & -5.632139 & -0.801758 \\
\hline $\mathrm{H}$ & 0.318395 & -3.748036 & 0.230315 \\
\hline $\mathrm{H}$ & -0.790395 & -8.207367 & -4.427994 \\
\hline $\mathrm{H}$ & -0.086954 & -6.700959 & -5.115780 \\
\hline $\mathrm{H}$ & 0.939379 & -7.804109 & -4.126949 \\
\hline $\mathrm{H}$ & -5.508989 & 0.158911 & 2.462240 \\
\hline $\mathrm{H}$ & -4.495426 & 4.365397 & 0.006399 \\
\hline $\mathrm{H}$ & -6.215772 & 5.864346 & 0.152875 \\
\hline $\mathrm{H}$ & -6.200121 & 4.977414 & -1.401025 \\
\hline $\mathrm{H}$ & -7.354717 & 1.540864 & 1.601882 \\
\hline $\mathrm{H}$ & -8.511828 & 2.174532 & -0.299775 \\
\hline $\mathrm{H}$ & -7.531363 & 2.800708 & -1.652531 \\
\hline $\mathrm{H}$ & -8.994609 & 3.681610 & -1.122695 \\
\hline $\mathrm{H}$ & -7.687815 & 5.786101 & -0.847411 \\
\hline $\mathrm{H}$ & 3.823836 & 5.170316 & -0.000640 \\
\hline $\mathrm{H}$ & -7.577575 & 4.963998 & 2.135920 \\
\hline $\mathrm{H}$ & -8.545065 & 3.476773 & 1.968134 \\
\hline $\mathrm{H}$ & -9.019466 & 4.947285 & 1.076820 \\
\hline $\mathrm{H}$ & 0.658557 & -2.096572 & 3.416582 \\
\hline $\mathrm{H}$ & -3.233980 & -2.033063 & 5.292965 \\
\hline $\mathrm{H}$ & 6.410557 & -2.456973 & -0.487588 \\
\hline $\mathrm{H}$ & 1.371631 & -3.925208 & 4.965875 \\
\hline $\mathrm{H}$ & -2.530866 & -3.840001 & 6.830425 \\
\hline $\mathrm{H}$ & 7.051845 & 2.341836 & 0.658482 \\
\hline $\mathrm{H}$ & 8.683295 & -1.582268 & -0.142682 \\
\hline $\mathrm{H}$ & -0.322510 & -6.307726 & 8.325047 \\
\hline $\mathrm{H}$ & -1.202884 & -4.749142 & 8.519494 \\
\hline $\mathrm{H}$ & -1.806468 & -5.986793 & 7.357532 \\
\hline
\end{tabular}




$\begin{array}{lccc}\mathrm{H} & 10.602178 & 2.790585 & 0.996280 \\ \mathrm{H} & 9.009242 & 2.692424 & 1.788388 \\ \mathrm{H} & 9.124242 & 3.092490 & 0.047993 \\ \mathrm{H} & 9.615123 & 0.336890 & 2.609893 \\ \mathrm{H} & 11.209733 & 0.523823 & 1.820269 \\ \mathrm{H} & 10.195404 & -0.899690 & 1.464200 \\ \mathrm{H} & 11.371852 & 1.092302 & -0.649477 \\ \mathrm{H} & 9.893751 & 1.312000 & -1.633565 \\ \mathrm{H} & 10.363206 & -0.315372 & -1.077157 \\ \mathrm{H} & 3.367215 & -0.471666 & 0.158304 \\ \mathrm{H} & -0.899765 & -0.668731 & 2.677404 \\ \mathrm{C} & 2.056574 & 7.274876 & -0.128238 \\ \mathrm{C} & 0.788054 & 8.152117 & -0.140291 \\ \mathrm{H} & 0.103252 & 7.873844 & -0.960637 \\ \mathrm{H} & 0.233340 & 8.085458 & 0.811918 \\ \mathrm{H} & 1.071237 & 9.208772 & -0.287450 \\ \mathrm{C} & 2.972024 & 7.763312 & 1.021928 \\ \mathrm{H} & 2.470783 & 7.653822 & 1.999140 \\ \mathrm{H} & 3.917687 & 7.196912 & 1.063154 \\ \mathrm{H} & 3.224230 & 8.830016 & 0.881848 \\ \mathrm{C} & 2.787451 & 7.460611 & -1.481266 \\ \mathrm{H} & 2.151480 & 7.133333 & -2.322240 \\ \mathrm{H} & 3.040010 & 8.525269 & -1.635530 \\ \mathrm{H} & 3.726180 & 6.882882 & -1.523875 \\ \mathrm{Cl} & 0.519445 & -0.526846 & 0.964215 \\ -----------------------------------------------------------------\end{array}$

Statistical Thermodynamic Analysis

Temperature $=298.150$ Kelvin $\quad$ Pressure $=1.00000$ Atm

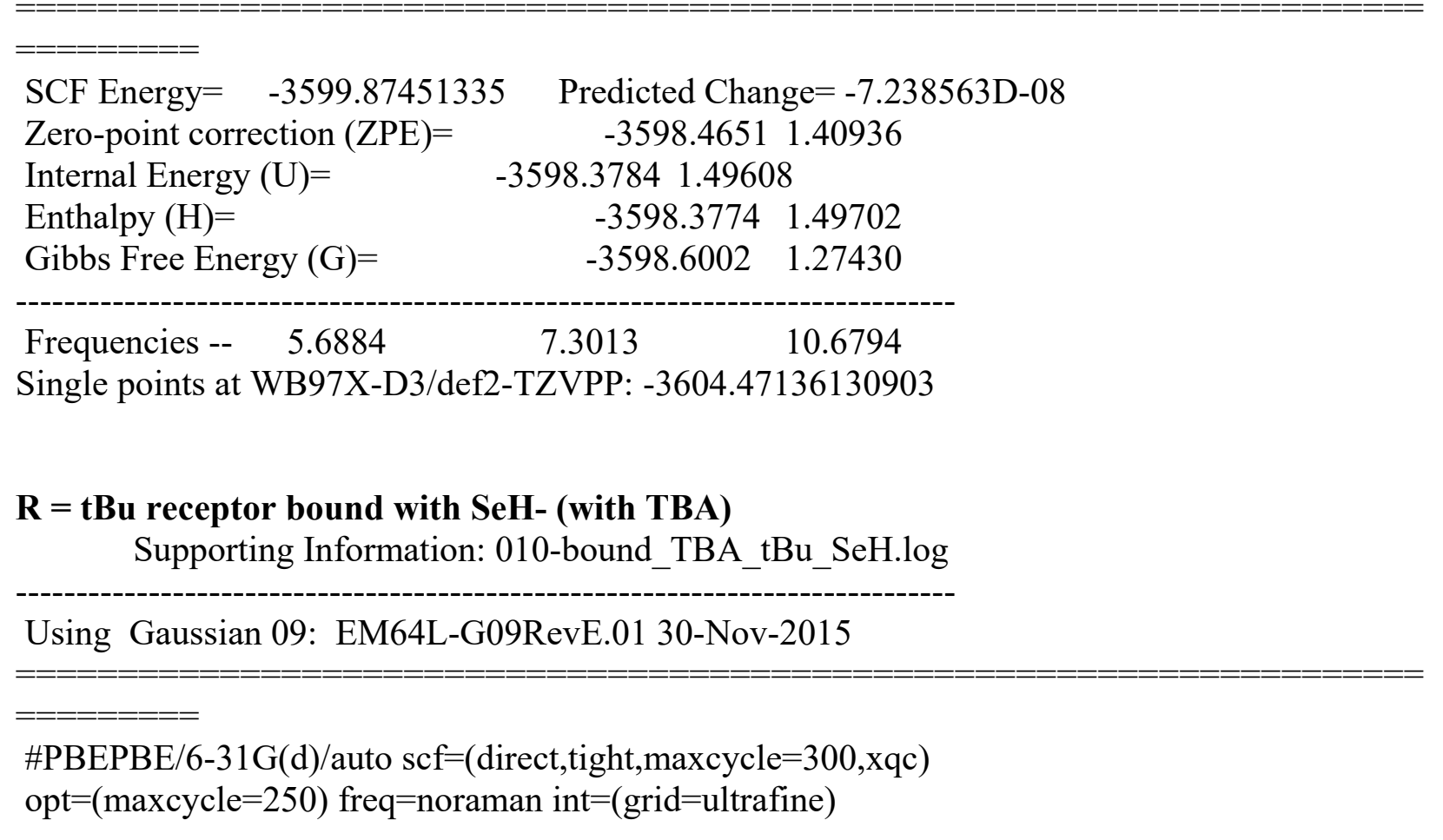


\#P Geom=AllCheck Guess=TCheck SCRF=Check GenChk RPBEPBE/6-31G(d)/Auto Freq

Pointgroup= $=$ 1 Stoichiometry= C66H91N5O4Se C1[X(C66H91N5O4Se)] \#Atoms=167 Charge $=0 \quad$ Multiplicity $=1$

SCF Energy $=-5539.46342934 \quad$ Predicted Change $=-9.530969 \mathrm{D}-09$

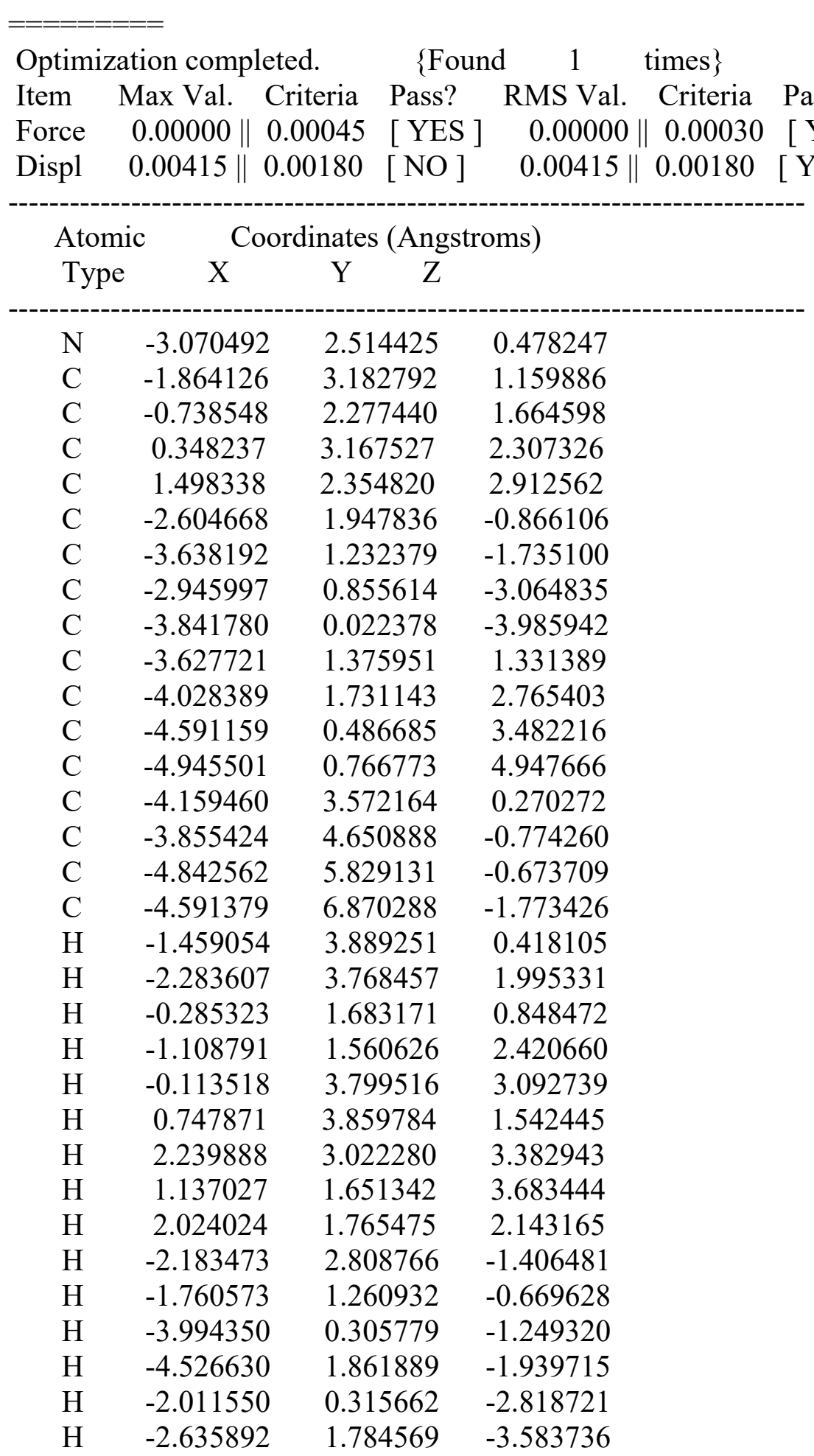




\begin{tabular}{|c|c|c|c|}
\hline $\mathrm{H}$ & -3.309262 & -0.243712 & -4.914225 \\
\hline $\mathrm{H}$ & -4.153361 & -0.916857 & -3.497635 \\
\hline $\mathrm{H}$ & -4.755315 & 0.576793 & -4.268959 \\
\hline $\mathrm{H}$ & -4.497397 & 0.986259 & 0.778795 \\
\hline $\mathrm{H}$ & -2.860909 & 0.584420 & 1.326159 \\
\hline $\mathrm{H}$ & -3.156349 & 2.103983 & 3.333130 \\
\hline $\mathrm{H}$ & -4.792958 & 2.530212 & 2.783981 \\
\hline $\mathrm{H}$ & -3.850129 & -0.332198 & 3.421779 \\
\hline $\mathrm{H}$ & -5.489165 & 0.129273 & 2.941756 \\
\hline $\mathrm{H}$ & -5.354599 & -0.134522 & 5.433894 \\
\hline $\mathrm{H}$ & -4.055467 & 1.081553 & 5.520949 \\
\hline $\mathrm{H}$ & -5.701995 & 1.567041 & 5.035258 \\
\hline $\mathrm{H}$ & -4.326628 & 4.032164 & 1.257508 \\
\hline $\mathrm{H}$ & -5.074719 & 3.020787 & -0.001148 \\
\hline $\mathrm{H}$ & -2.831116 & 5.046667 & -0.657281 \\
\hline $\mathrm{H}$ & -3.912548 & 4.220120 & -1.790453 \\
\hline $\mathrm{H}$ & -4.742602 & 6.302343 & 0.322119 \\
\hline $\mathrm{H}$ & -5.883546 & 5.457345 & -0.738788 \\
\hline $\mathrm{H}$ & -5.243524 & 7.750095 & -1.643650 \\
\hline $\mathrm{H}$ & -3.541588 & 7.214207 & -1.767763 \\
\hline $\mathrm{H}$ & -4.794345 & 6.447494 & -2.773164 \\
\hline $\mathrm{O}$ & -0.688989 & 7.292644 & -2.473543 \\
\hline $\mathrm{O}$ & -2.060571 & -4.207962 & -3.319148 \\
\hline $\mathrm{N}$ & 2.361043 & 2.806330 & -1.053532 \\
\hline $\mathrm{N}$ & -2.158323 & -2.598810 & -1.627096 \\
\hline $\mathrm{C}$ & 0.633012 & -1.512534 & 2.093123 \\
\hline $\mathrm{C}$ & 0.467178 & -2.057495 & 4.879792 \\
\hline $\mathrm{C}$ & -0.676577 & -2.114076 & 4.066689 \\
\hline $\mathrm{C}$ & -0.605135 & -1.851774 & 2.672961 \\
\hline $\mathrm{C}$ & 1.699493 & 3.987784 & -1.409854 \\
\hline $\mathrm{C}$ & 2.215582 & 5.287793 & -1.233080 \\
\hline $\mathrm{C}$ & 1.444828 & 6.409413 & -1.581248 \\
\hline $\mathrm{C}$ & 0.153851 & 6.258010 & -2.116126 \\
\hline $\mathrm{C}$ & -0.353846 & 4.958722 & -2.317605 \\
\hline $\mathrm{C}$ & 0.405878 & 3.842523 & -1.968776 \\
\hline $\mathrm{C}$ & -0.168604 & 8.612900 & -2.354736 \\
\hline $\mathrm{C}$ & -1.757393 & -1.969886 & 1.842695 \\
\hline $\mathrm{C}$ & -2.720016 & -2.154255 & 1.094068 \\
\hline $\mathrm{C}$ & -3.766975 & -2.484373 & 0.191208 \\
\hline $\mathrm{C}$ & -3.454666 & -2.807693 & -1.169162 \\
\hline $\mathrm{C}$ & -4.493333 & -3.306423 & -1.987220 \\
\hline $\mathrm{C}$ & -5.791453 & -3.425474 & -1.490304 \\
\hline $\mathrm{C}$ & -6.141424 & -3.057977 & -0.168549 \\
\hline $\mathrm{C}$ & -5.104693 & -2.596484 & 0.650713 \\
\hline $\mathrm{C}$ & -8.516871 & -2.316037 & -0.573770 \\
\hline $\mathrm{C}$ & -7.772986 & -2.738173 & 1.779845 \\
\hline $\mathrm{C}$ & -1.504924 & -3.329055 & -2.650052 \\
\hline $\mathrm{C}$ & 3.675784 & 2.651877 & -0.640819 \\
\hline
\end{tabular}




\begin{tabular}{|c|c|c|c|}
\hline $\mathrm{C}$ & 1.794356 & -1.448827 & 2.892036 \\
\hline $\mathrm{C}$ & 1.697923 & -1.729263 & 4.275301 \\
\hline $\mathrm{C}$ & -7.597938 & -3.190342 & 0.315847 \\
\hline $\mathrm{N}$ & -0.196405 & -2.920629 & -2.766878 \\
\hline $\mathrm{N}$ & 3.940590 & 1.317210 & -0.295771 \\
\hline $\mathrm{O}$ & 4.507355 & 3.563784 & -0.571787 \\
\hline $\mathrm{C}$ & 3.026309 & -1.068692 & 2.287488 \\
\hline $\mathrm{C}$ & -8.043973 & -4.670329 & 0.210878 \\
\hline $\mathrm{C}$ & 0.825717 & -3.443686 & -3.578950 \\
\hline $\mathrm{C}$ & 5.185272 & 0.843772 & 0.144669 \\
\hline $\mathrm{C}$ & 4.048276 & -0.693640 & 1.713849 \\
\hline $\mathrm{C}$ & 2.105349 & -2.857639 & -3.425207 \\
\hline $\mathrm{C}$ & 0.653310 & -4.488059 & -4.504223 \\
\hline $\mathrm{C}$ & 5.237672 & -0.208369 & 1.106336 \\
\hline $\mathrm{C}$ & 6.400735 & 1.363668 & -0.343001 \\
\hline $\mathrm{C}$ & 3.183407 & -3.307406 & -4.181505 \\
\hline $\mathrm{C}$ & 1.745008 & -4.940040 & -5.262779 \\
\hline $\mathrm{C}$ & 6.494620 & -0.728546 & 1.507056 \\
\hline $\mathrm{C}$ & 7.620273 & 0.842586 & 0.089791 \\
\hline $\mathrm{C}$ & 3.013861 & -4.355386 & -5.108964 \\
\hline $\mathrm{C}$ & 7.703655 & -0.223930 & 1.015092 \\
\hline $\mathrm{O}$ & 4.144897 & -4.725100 & -5.804130 \\
\hline $\mathrm{C}$ & 9.077369 & -0.775703 & 1.442449 \\
\hline $\mathrm{C}$ & 4.005197 & -5.783726 & -6.742050 \\
\hline $\mathrm{C}$ & 8.955700 & -1.930439 & 2.457286 \\
\hline $\mathrm{C}$ & 9.827496 & -1.304594 & 0.194276 \\
\hline $\mathrm{C}$ & 9.906273 & 0.358578 & 2.095227 \\
\hline $\mathrm{H}$ & 1.786706 & 1.943037 & -1.132705 \\
\hline $\mathrm{H}$ & -1.582998 & -1.937434 & -1.088715 \\
\hline $\mathrm{H}$ & 0.704652 & -1.278484 & 1.027225 \\
\hline $\mathrm{H}$ & -1.648360 & -2.381894 & 4.488542 \\
\hline $\mathrm{H}$ & 3.222083 & 5.406663 & -0.828760 \\
\hline $\mathrm{H}$ & 1.873841 & 7.403013 & -1.426259 \\
\hline $\mathrm{H}$ & -1.339677 & 4.846517 & -2.781991 \\
\hline $\mathrm{H}$ & 0.015515 & 2.825226 & -2.095971 \\
\hline $\mathrm{H}$ & -0.962417 & 9.283814 & -2.715853 \\
\hline $\mathrm{H}$ & 0.074412 & 8.866983 & -1.304068 \\
\hline $\mathrm{H}$ & 0.738045 & 8.750942 & -2.974926 \\
\hline $\mathrm{H}$ & -4.250260 & -3.610765 & -3.006307 \\
\hline $\mathrm{H}$ & -5.292198 & -2.341339 & 1.697315 \\
\hline $\mathrm{H}$ & -7.170445 & -3.350351 & 2.473503 \\
\hline $\mathrm{H}$ & -7.490059 & -1.678795 & 1.918703 \\
\hline $\mathrm{H}$ & -6.562878 & -3.812083 & -2.165964 \\
\hline $\mathrm{H}$ & -8.457632 & -2.609093 & -1.635661 \\
\hline $\mathrm{H}$ & -8.237024 & -1.250046 & -0.503004 \\
\hline $\mathrm{H}$ & -9.570460 & -2.416539 & -0.255456 \\
\hline $\mathrm{H}$ & -8.830900 & -2.841517 & 2.077564 \\
\hline $\mathrm{H}$ & 2.611375 & -1.674962 & 4.875790 \\
\hline
\end{tabular}




\begin{tabular}{lccc}
$\mathrm{H}$ & -7.415328 & -5.317078 & 0.846887 \\
$\mathrm{H}$ & -7.973067 & -5.046177 & -0.823781 \\
$\mathrm{H}$ & -9.094052 & -4.779968 & 0.537732 \\
$\mathrm{H}$ & 2.222042 & -2.040734 & -2.700767 \\
$\mathrm{H}$ & -0.332695 & -4.940775 & -4.620244 \\
$\mathrm{H}$ & 6.371633 & 2.187545 & -1.057089 \\
$\mathrm{H}$ & 4.175076 & -2.859528 & -4.066392 \\
$\mathrm{H}$ & 1.585202 & -5.754999 & -5.973728 \\
$\mathrm{H}$ & 6.483520 & -1.541503 & 2.237271 \\
$\mathrm{H}$ & 8.538539 & 1.275965 & -0.322272 \\
$\mathrm{H}$ & 5.002700 & -5.929270 & -7.183684 \\
$\mathrm{H}$ & 3.680738 & -6.724999 & -6.255612 \\
$\mathrm{H}$ & 3.284456 & -5.530724 & -7.544896 \\
$\mathrm{H}$ & 9.962254 & -2.293122 & 2.729384 \\
$\mathrm{H}$ & 8.394477 & -2.785012 & 2.040446 \\
$\mathrm{H}$ & 8.454917 & -1.608116 & 3.387161 \\
$\mathrm{H}$ & 9.267283 & -2.127003 & -0.283346 \\
$\mathrm{H}$ & 10.824726 & -1.686707 & 0.479166 \\
$\mathrm{H}$ & 9.973950 & -0.513899 & -0.561193 \\
$\mathrm{H}$ & 10.903514 & -0.015817 & 2.390167 \\
$\mathrm{H}$ & 9.402704 & 0.745493 & 2.998141 \\
$\mathrm{H}$ & 10.056169 & 1.205872 & 1.404734 \\
$\mathrm{H}$ & 3.149909 & 0.657305 & -0.296218 \\
$\mathrm{H}$ & 0.098756 & -2.117967 & -2.179141 \\
$\mathrm{H}$ & 0.705693 & 0.256027 & -2.703469 \\
$\mathrm{Se}$ & 0.480442 & 0.001466 & -1.252086 \\
$\mathrm{C}$ & 0.417005 & -2.341731 & 6.396096 \\
$\mathrm{C}$ & -1.007592 & -2.672189 & 6.886227 \\
$\mathrm{H}$ & -1.708778 & -1.838124 & 6.705382 \\
$\mathrm{H}$ & -1.411232 & -3.576425 & 6.398117 \\
$\mathrm{H}$ & -0.989309 & -2.861313 & 7.973473 \\
$\mathrm{C}$ & 1.333471 & -3.547798 & 6.720172 \\
$\mathrm{H}$ & 0.992580 & -4.455648 & 6.193232 \\
$\mathrm{H}$ & 2.379327 & -3.359463 & 6.424642 \\
$\mathrm{H}$ & 1.322093 & -3.754691 & 7.805536 \\
$\mathrm{C}$ & 0.917106 & -1.094184 & 7.166436 \\
$\mathrm{H}$ & 0.274166 & -0.219815 & 6.962967 \\
$\mathrm{H}$ & 0.901590 & -1.285914 & 8.254508 \\
----------------------------------------------------------------- \\
\hline
\end{tabular}

Statistical Thermodynamic Analysis

Temperature $=298.150$ Kelvin $\quad$ Pressure $=1.00000$ Atm

\begin{tabular}{|c|c|}
\hline-5539.46342934 & Predicted Change $=-9.530969 \mathrm{D}-09$ \\
\hline Zero-point correction $(\mathrm{ZPE})=$ & $-5538.0443 \quad 1.41905$ \\
\hline Internal Energy $(U)=$ & -5537.95781 .50557 \\
\hline Enthalpy $(H)=$ & $-5537.9569 \quad 1.50651$ \\
\hline
\end{tabular}


Gibbs Free Energy $(\mathrm{G})=$

$-5538.1768 \quad 1.28657$

Frequencies -- $6.1287 \quad 7.5295 \quad 11.1993$

Single points at WB97X-D3/def2-TZVPP: -5545.14712358432

$R=t B u$ receptor bound with $S H-$ (with $T B A$ )

Supporting Information: 010-bound_TBA_tBu_SH.log

Using Gaussian 09: EM64L-G09RevE.01 30-Nov-2015

\#PBEPBE/6-31G(d)/auto scf $=($ direct,tight, $\operatorname{maxcycle}=300, \mathrm{xqc})$

$\mathrm{opt}=($ maxcycle $=250)$ freq $=$ noraman $\mathrm{int}=($ grid $=$ ultrafine $)$

\#P Geom=AllCheck Guess=TCheck SCRF=Check GenChk RPBEPBE/6-31G(d)/Auto Freq

Pointgroup $=\mathrm{C} 1 \quad$ Stoichiometry $=\mathrm{C} 66 \mathrm{H} 91 \mathrm{~N} 5 \mathrm{O} 4 \mathrm{~S}$ C1[X(C66H91N5O4S $)]$ \#Atoms= 167

Charge $=0 \quad$ Multiplicity $=1$

SCF Energy $=-3538.43797594 \quad$ Predicted Change $=-1.126131 \mathrm{D}-07$

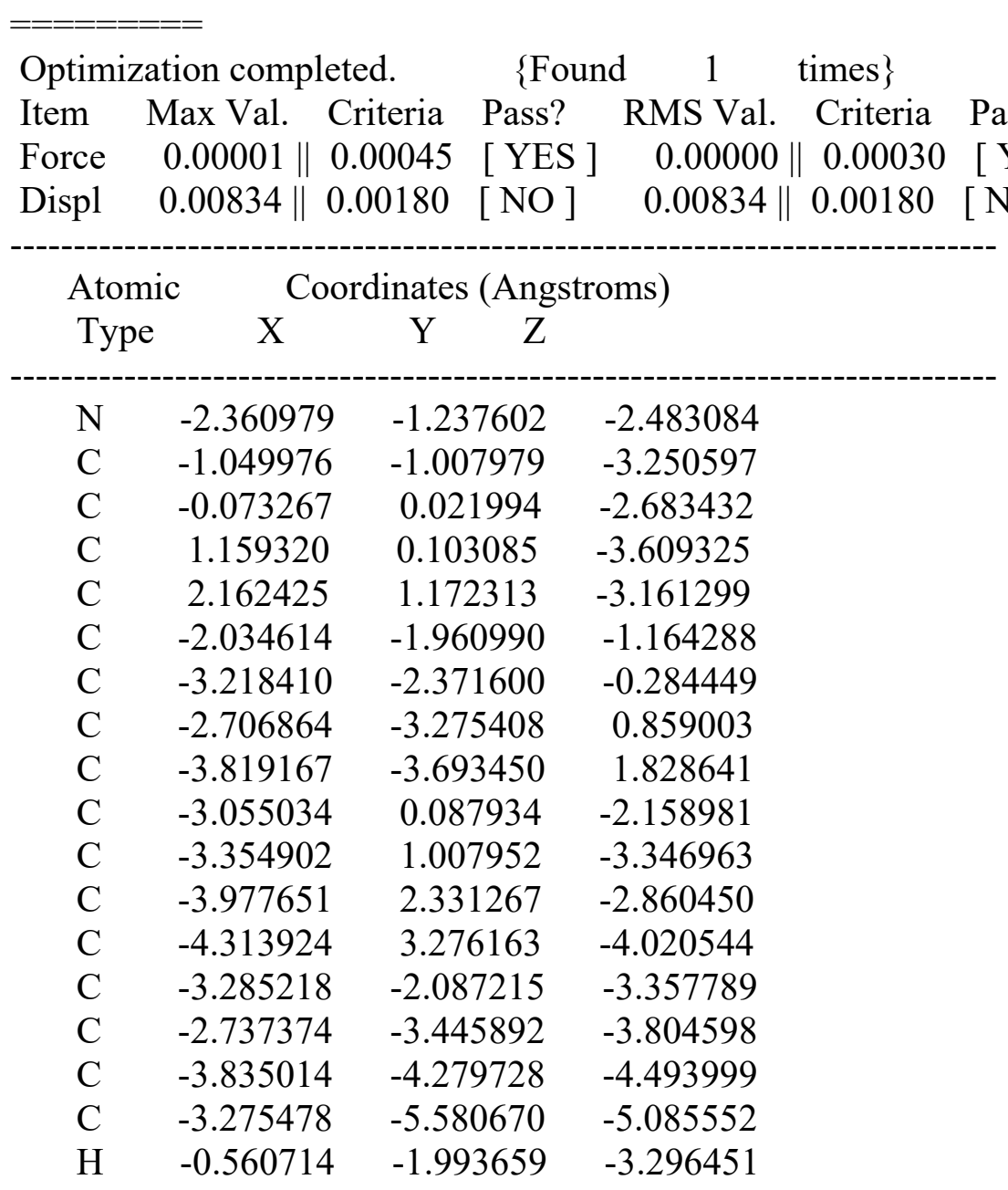




\begin{tabular}{|c|c|c|c|}
\hline $\mathrm{H}$ & -1.350596 & -0.731602 & -4.275994 \\
\hline $\mathrm{H}$ & 0.249418 & -0.257125 & -1.663234 \\
\hline $\mathrm{H}$ & -0.537223 & 1.023413 & -2.618665 \\
\hline $\mathrm{H}$ & 0.826191 & 0.316702 & -4.645213 \\
\hline $\mathrm{H}$ & 1.656946 & -0.884667 & -3.636712 \\
\hline $\mathrm{H}$ & 3.013887 & 1.225688 & -3.860441 \\
\hline $\mathrm{H}$ & 1.695505 & 2.172305 & -3.118285 \\
\hline $\mathrm{H}$ & 2.568283 & 0.950232 & -2.160657 \\
\hline $\mathrm{H}$ & -1.448602 & -2.844999 & -1.457773 \\
\hline $\mathrm{H}$ & -1.347032 & -1.305456 & -0.595568 \\
\hline $\mathrm{H}$ & -3.715701 & -1.487796 & 0.154541 \\
\hline $\mathrm{H}$ & -3.983840 & -2.933397 & -0.853536 \\
\hline $\mathrm{H}$ & -1.909093 & -2.743029 & 1.408511 \\
\hline $\mathrm{H}$ & -2.237493 & -4.176545 & 0.417488 \\
\hline $\mathrm{H}$ & -3.426936 & -4.389652 & 2.588886 \\
\hline $\mathrm{H}$ & -4.230460 & -2.823163 & 2.367403 \\
\hline $\mathrm{H}$ & -4.646219 & -4.202065 & 1.299866 \\
\hline $\mathrm{H}$ & -3.987482 & -0.180273 & -1.637297 \\
\hline $\mathrm{H}$ & -2.406264 & 0.596393 & -1.427768 \\
\hline $\mathrm{H}$ & -2.431354 & 1.235710 & -3.909907 \\
\hline $\mathrm{H}$ & -4.055219 & 0.528673 & -4.056046 \\
\hline $\mathrm{H}$ & -3.278868 & 2.825476 & -2.160054 \\
\hline $\mathrm{H}$ & -4.891368 & 2.114815 & -2.275539 \\
\hline $\mathrm{H}$ & -4.764039 & 4.211877 & -3.647749 \\
\hline $\mathrm{H}$ & -3.410149 & 3.546584 & -4.595086 \\
\hline $\mathrm{H}$ & -5.032963 & 2.815868 & -4.721826 \\
\hline $\mathrm{H}$ & -3.519438 & -1.471728 & -4.240399 \\
\hline $\mathrm{H}$ & -4.217100 & -2.211159 & -2.783573 \\
\hline $\mathrm{H}$ & -1.896398 & -3.309221 & -4.507860 \\
\hline $\mathrm{H}$ & -2.339602 & -4.022479 & -2.951282 \\
\hline $\mathrm{H}$ & -4.315710 & -3.683077 & -5.293783 \\
\hline $\mathrm{H}$ & -4.630197 & -4.511170 & -3.759630 \\
\hline $\mathrm{H}$ & -4.083603 & -6.213300 & -5.489208 \\
\hline $\mathrm{H}$ & -2.571650 & -5.367813 & -5.909920 \\
\hline $\mathrm{H}$ & -2.725602 & -6.162825 & -4.325546 \\
\hline $\mathrm{O}$ & -0.107794 & -6.715746 & -3.467551 \\
\hline $\mathrm{O}$ & -3.795938 & -0.812396 & 3.986063 \\
\hline $\mathrm{N}$ & 2.544685 & -2.394885 & -1.046969 \\
\hline $\mathrm{N}$ & -2.879381 & 0.485577 & 2.281762 \\
\hline $\mathrm{C}$ & 0.998381 & 2.929919 & 0.380189 \\
\hline $\mathrm{C}$ & 1.289862 & 5.648320 & -0.429281 \\
\hline $\mathrm{C}$ & 0.020769 & 5.104548 & -0.176412 \\
\hline $\mathrm{C}$ & -0.135476 & 3.752800 & 0.230400 \\
\hline $\mathrm{C}$ & 1.963136 & -3.499860 & -1.690711 \\
\hline $\mathrm{C}$ & 2.424620 & -4.023519 & -2.916846 \\
\hline $\mathrm{C}$ & 1.762898 & -5.098174 & -3.532193 \\
\hline $\mathrm{C}$ & 0.623123 & -5.670467 & -2.940070 \\
\hline $\mathrm{C}$ & 0.166599 & -5.163728 & -1.707422 \\
\hline
\end{tabular}




\begin{tabular}{|c|c|c|c|}
\hline $\mathrm{C}$ & 0.830382 & -4.100699 & -1.091150 \\
\hline $\mathrm{C}$ & 0.393168 & -7.311836 & -4.660042 \\
\hline $\mathrm{C}$ & -1.435846 & 3.233049 & 0.491854 \\
\hline $\mathrm{C}$ & -2.571144 & 2.816904 & 0.732608 \\
\hline $\mathrm{C}$ & -3.867656 & 2.325987 & 1.044242 \\
\hline $\mathrm{C}$ & -4.026929 & 1.149142 & 1.844134 \\
\hline $\mathrm{C}$ & -5.341510 & 0.714648 & 2.127490 \\
\hline $\mathrm{C}$ & -6.443027 & 1.421678 & 1.643075 \\
\hline $\mathrm{C}$ & -6.317365 & 2.590156 & 0.856124 \\
\hline $\mathrm{C}$ & -5.014124 & 3.016044 & 0.574445 \\
\hline $\mathrm{C}$ & -8.406995 & 2.384121 & -0.538524 \\
\hline $\mathrm{C}$ & -7.219990 & 4.588834 & -0.468159 \\
\hline $\mathrm{C}$ & -2.798234 & -0.414141 & 3.367267 \\
\hline $\mathrm{C}$ & 3.884156 & -2.031357 & -1.129751 \\
\hline $\mathrm{C}$ & 2.282145 & 3.453861 & 0.110866 \\
\hline $\mathrm{C}$ & 2.410681 & 4.807062 & -0.283727 \\
\hline $\mathrm{C}$ & -7.570899 & 3.333376 & 0.355918 \\
\hline $\mathrm{N}$ & -1.495639 & -0.779760 & 3.627514 \\
\hline $\mathrm{N}$ & 4.166050 & -0.908244 & -0.341213 \\
\hline $\mathrm{O}$ & 4.724748 & -2.619862 & -1.819587 \\
\hline $\mathrm{C}$ & 3.425946 & 2.611062 & 0.200436 \\
\hline $\mathrm{C}$ & -8.425791 & 3.775404 & 1.570000 \\
\hline $\mathrm{C}$ & -1.037743 & -1.617074 & 4.669439 \\
\hline $\mathrm{C}$ & 5.438617 & -0.353896 & -0.148723 \\
\hline $\mathrm{C}$ & 4.406780 & 1.868071 & 0.225010 \\
\hline $\mathrm{C}$ & 0.363260 & -1.757157 & 4.812155 \\
\hline $\mathrm{C}$ & -1.880420 & -2.306299 & 5.562446 \\
\hline $\mathrm{C}$ & 5.551288 & 1.027460 & 0.194221 \\
\hline $\mathrm{C}$ & 6.622830 & -1.112166 & -0.254744 \\
\hline $\mathrm{C}$ & 0.902204 & -2.568547 & 5.807513 \\
\hline $\mathrm{C}$ & -1.334118 & -3.117190 & 6.569607 \\
\hline $\mathrm{C}$ & 6.830923 & 1.583525 & 0.449467 \\
\hline $\mathrm{C}$ & 7.865302 & -0.526155 & -0.012088 \\
\hline $\mathrm{C}$ & 0.057852 & -3.258623 & 6.699162 \\
\hline $\mathrm{C}$ & 8.007298 & 0.832052 & 0.355379 \\
\hline $\mathrm{O}$ & 0.691202 & -4.029614 & 7.647588 \\
\hline $\mathrm{C}$ & 9.404060 & 1.422400 & 0.628108 \\
\hline $\mathrm{C}$ & -0.138173 & -4.727248 & 8.568123 \\
\hline $\mathrm{C}$ & 9.345646 & 2.916095 & 1.007065 \\
\hline $\mathrm{C}$ & 10.066571 & 0.651323 & 1.797155 \\
\hline $\mathrm{C}$ & 10.280350 & 1.278262 & -0.641207 \\
\hline $\mathrm{H}$ & 1.941209 & -1.888217 & -0.360491 \\
\hline $\mathrm{H}$ & -1.995607 & 0.867000 & 1.927255 \\
\hline $\mathrm{H}$ & 0.895823 & 1.892082 & 0.713753 \\
\hline $\mathrm{H}$ & -0.879429 & 5.715290 & -0.277607 \\
\hline $\mathrm{H}$ & 3.321269 & -3.598831 & -3.370788 \\
\hline $\mathrm{H}$ & 2.154409 & -5.478643 & -4.479470 \\
\hline $\mathrm{H}$ & -0.693435 & -5.642443 & -1.226342 \\
\hline
\end{tabular}




\begin{tabular}{|c|c|c|c|}
\hline $\mathrm{H}$ & 0.481956 & -3.726646 & -0.122363 \\
\hline $\mathrm{H}$ & -0.288598 & -8.144028 & -4.890498 \\
\hline $\mathrm{H}$ & 0.396331 & -6.598351 & -5.507906 \\
\hline $\mathrm{H}$ & 1.418264 & -7.705067 & -4.518950 \\
\hline $\mathrm{H}$ & -5.474761 & -0.162633 & 2.760136 \\
\hline $\mathrm{H}$ & -4.837319 & 3.917905 & -0.017312 \\
\hline $\mathrm{H}$ & -6.641998 & 5.319470 & 0.124194 \\
\hline $\mathrm{H}$ & -6.636047 & 4.338088 & -1.372199 \\
\hline $\mathrm{H}$ & -7.440293 & 1.044537 & 1.896947 \\
\hline $\mathrm{H}$ & -8.711349 & 1.472900 & 0.004138 \\
\hline $\mathrm{H}$ & -7.830861 & 2.070890 & -1.427652 \\
\hline $\mathrm{H}$ & -9.325187 & 2.890996 & -0.886954 \\
\hline $\mathrm{H}$ & -8.147335 & 5.087350 & -0.799785 \\
\hline $\mathrm{H}$ & 3.416992 & 5.188329 & -0.484046 \\
\hline $\mathrm{H}$ & -7.860480 & 4.466599 & 2.218527 \\
\hline $\mathrm{H}$ & -8.736991 & 2.915232 & 2.186580 \\
\hline $\mathrm{H}$ & -9.340424 & 4.293144 & 1.228273 \\
\hline $\mathrm{H}$ & 1.030126 & -1.214705 & 4.132406 \\
\hline $\mathrm{H}$ & -2.960923 & -2.197254 & 5.467779 \\
\hline $\mathrm{H}$ & 6.550490 & -2.161389 & -0.541785 \\
\hline $\mathrm{H}$ & 1.985564 & -2.676143 & 5.916594 \\
\hline $\mathrm{H}$ & -2.016919 & -3.635094 & 7.248376 \\
\hline $\mathrm{H}$ & 6.863615 & 2.642990 & 0.715637 \\
\hline $\mathrm{H}$ & 8.755294 & -1.159290 & -0.102269 \\
\hline $\mathrm{H}$ & 0.545348 & -5.272604 & 9.236175 \\
\hline $\mathrm{H}$ & -0.759871 & -4.034126 & 9.168549 \\
\hline $\mathrm{H}$ & -0.802799 & -5.451201 & 8.056392 \\
\hline $\mathrm{H}$ & 10.366678 & 3.292233 & 1.193718 \\
\hline $\mathrm{H}$ & 8.755425 & 3.083243 & 1.925099 \\
\hline $\mathrm{H}$ & 8.907666 & 3.527252 & 0.198358 \\
\hline $\mathrm{H}$ & 9.471648 & 0.750146 & 2.721643 \\
\hline $\mathrm{H}$ & 11.079102 & 1.048254 & 1.994562 \\
\hline $\mathrm{H}$ & 10.164901 & -0.424668 & 1.573776 \\
\hline $\mathrm{H}$ & 11.293711 & 1.678894 & -0.455903 \\
\hline $\mathrm{H}$ & 9.840671 & 1.832265 & -1.488756 \\
\hline $\mathrm{H}$ & 10.386870 & 0.223805 & -0.947802 \\
\hline $\mathrm{H}$ & 3.375082 & -0.427747 & 0.109277 \\
\hline $\mathrm{H}$ & -0.774180 & -0.515511 & 2.926212 \\
\hline $\mathrm{H}$ & 0.836278 & -1.844915 & 1.789337 \\
\hline $\mathrm{C}$ & 1.490477 & 7.117014 & -0.859405 \\
\hline $\mathrm{C}$ & 0.157560 & 7.885473 & -0.966867 \\
\hline $\mathrm{H}$ & -0.515332 & 7.438709 & -1.719912 \\
\hline $\mathrm{H}$ & -0.376458 & 7.919047 & -0.001185 \\
\hline $\mathrm{H}$ & 0.355573 & 8.926915 & -1.274249 \\
\hline $\mathrm{C}$ & 2.381845 & 7.836136 & 0.183588 \\
\hline $\mathrm{H}$ & 1.904683 & 7.834895 & 1.178817 \\
\hline $\mathrm{H}$ & 3.368740 & 7.353506 & 0.282625 \\
\hline $\mathrm{H}$ & 2.548990 & 8.886276 & -0.116899 \\
\hline
\end{tabular}




$\begin{array}{lrrr}\mathrm{C} & 2.185364 & 7.155318 & -2.243313 \\ \mathrm{H} & 1.564881 & 6.660855 & -3.011048 \\ \mathrm{H} & 2.351829 & 8.201481 & -2.557700 \\ \mathrm{H} & 3.165604 & 6.649843 & -2.225295 \\ \mathrm{~S} & 0.678146 & -0.692575 & 1.078549\end{array}$

Statistical Thermodynamic Analysis

Temperature $=298.150$ Kelvin $\quad$ Pressure $=1.00000$ Atm

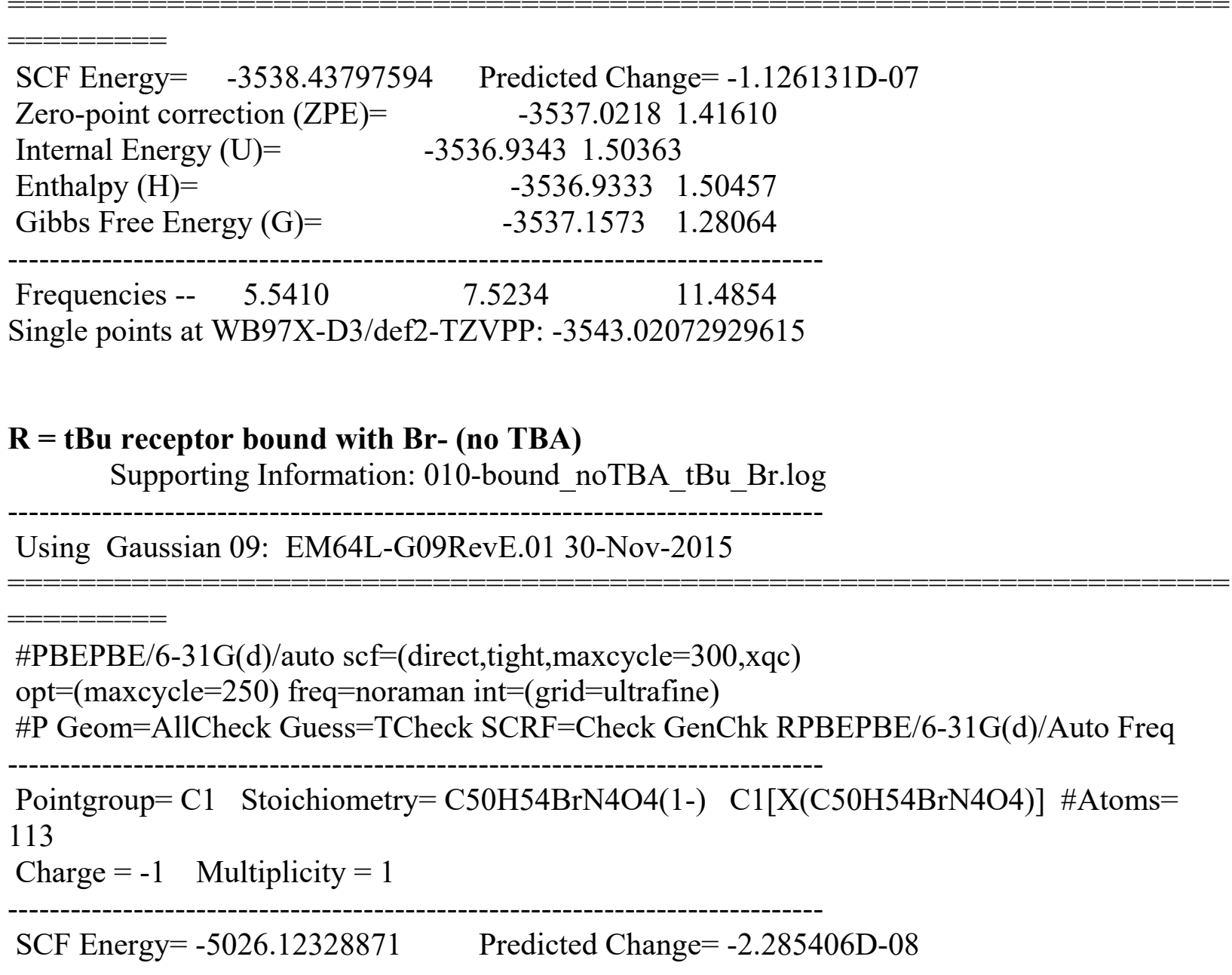

\begin{tabular}{|c|c|c|c|c|}
\hline \multicolumn{3}{|c|}{ Optimization completed. } & \multicolumn{2}{|c|}{ Found $\quad 1 \quad$ times } \\
\hline Item & Max Val. & Criteria & Pass? & RMS Val. Criteria \\
\hline Force & $0.00001 \|$ & 0.00045 & [YES ] & $0.00000 \| 0.00030$ \\
\hline Displ & $0.00439 \|$ & 0.00180 & [ NO ] & $0.00439 \| 0.00180$ \\
\hline \multicolumn{2}{|c|}{ Atomic } & \multicolumn{3}{|c|}{ Coordinates (Angstroms) } \\
\hline \multicolumn{2}{|c|}{ Type } & $\mathrm{Y}$ & Z & \\
\hline $\mathrm{O}$ & 4.54077 & -2.52 & 5406 & 1.990862 \\
\hline $\mathrm{O}$ & -0.15917 & -6.37 & 2650 & 4.683852 \\
\hline $\mathrm{O}$ & -4.96128 & -1.98 & 4627 & -1.800858 \\
\hline
\end{tabular}




\begin{tabular}{|c|c|c|c|}
\hline $\mathrm{O}$ & -1.187499 & -6.685438 & -4.575158 \\
\hline $\mathrm{N}$ & 3.690422 & -0.826229 & 0.636318 \\
\hline $\mathrm{H}$ & 2.823950 & -0.360281 & 0.336778 \\
\hline $\mathrm{N}$ & 2.254720 & -2.348714 & 1.609449 \\
\hline $\mathrm{H}$ & 1.536826 & -1.845716 & 1.050393 \\
\hline $\mathrm{N}$ & -3.748364 & -0.361453 & -0.641654 \\
\hline $\mathrm{H}$ & -2.798248 & -0.018484 & -0.446383 \\
\hline $\mathrm{N}$ & -2.650389 & -2.137905 & -1.630852 \\
\hline $\mathrm{H}$ & -1.811766 & -1.709760 & -1.190743 \\
\hline $\mathrm{C}$ & 0.262531 & 2.824226 & -0.047451 \\
\hline $\mathrm{H}$ & 0.195247 & 1.730473 & -0.021176 \\
\hline $\mathrm{C}$ & 1.522985 & 3.452837 & -0.130040 \\
\hline $\mathrm{C}$ & 1.594708 & 4.865830 & -0.174774 \\
\hline $\mathrm{H}$ & 2.584202 & 5.330778 & -0.238372 \\
\hline $\mathrm{C}$ & 0.433530 & 5.663306 & -0.134690 \\
\hline $\mathrm{C}$ & -0.812077 & 5.019617 & -0.052490 \\
\hline $\mathrm{H}$ & -1.739560 & 5.596746 & -0.022672 \\
\hline $\mathrm{C}$ & -0.909240 & 3.603450 & -0.012189 \\
\hline $\mathrm{C}$ & 2.698896 & 2.650210 & -0.162210 \\
\hline $\mathrm{C}$ & 3.690226 & 1.920793 & -0.178371 \\
\hline $\mathrm{C}$ & 4.877637 & 1.140805 & -0.187715 \\
\hline $\mathrm{C}$ & 4.889616 & -0.222102 & 0.258510 \\
\hline $\mathrm{C}$ & 6.128356 & -0.897335 & 0.273750 \\
\hline $\mathrm{H}$ & 6.153040 & -1.925132 & 0.634807 \\
\hline $\mathrm{C}$ & 7.301809 & -0.262360 & -0.144346 \\
\hline $\mathrm{H}$ & 8.229221 & -0.842220 & -0.111579 \\
\hline $\mathrm{C}$ & 7.316332 & 1.069580 & -0.606596 \\
\hline $\mathrm{C}$ & 6.085080 & 1.741910 & -0.614708 \\
\hline $\mathrm{H}$ & 6.023388 & 2.782574 & -0.951177 \\
\hline $\mathrm{C}$ & 3.569446 & -1.963492 & 1.463412 \\
\hline $\mathrm{C}$ & 1.746074 & -3.386610 & 2.409632 \\
\hline $\mathrm{C}$ & 2.531305 & -4.231999 & 3.216932 \\
\hline $\mathrm{H}$ & 3.613066 & -4.094989 & 3.231990 \\
\hline $\mathrm{C}$ & 1.924836 & -5.237337 & 3.988012 \\
\hline $\mathrm{H}$ & 2.562697 & -5.877112 & 4.604494 \\
\hline $\mathrm{C}$ & 0.532259 & -5.414273 & 3.963189 \\
\hline $\mathrm{C}$ & -0.254054 & -4.570749 & 3.154777 \\
\hline $\mathrm{H}$ & -1.338803 & -4.715090 & 3.137822 \\
\hline $\mathrm{C}$ & 0.341360 & -3.571266 & 2.390182 \\
\hline $\mathrm{H}$ & -0.266769 & -2.911527 & 1.758963 \\
\hline $\mathrm{C}$ & 0.615817 & -7.234010 & 5.500317 \\
\hline $\mathrm{H}$ & -0.098368 & -7.917641 & 5.985801 \\
\hline $\mathrm{H}$ & 1.173245 & -6.676646 & 6.280619 \\
\hline $\mathrm{H}$ & 1.341020 & -7.827454 & 4.907052 \\
\hline $\mathrm{C}$ & -2.176506 & 2.957964 & 0.060234 \\
\hline $\mathrm{C}$ & -3.256934 & 2.370924 & 0.118169 \\
\hline $\mathrm{C}$ & -4.549230 & 1.786171 & 0.203710 \\
\hline $\mathrm{C}$ & -4.806625 & 0.434470 & -0.200910 \\
\hline
\end{tabular}




\begin{tabular}{|c|c|c|c|}
\hline $\mathrm{C}$ & -6.135451 & -0.033571 & -0.112423 \\
\hline $\mathrm{H}$ & -6.346911 & -1.052099 & -0.435919 \\
\hline $\mathrm{C}$ & -7.162841 & 0.789668 & 0.357975 \\
\hline $\mathrm{H}$ & -8.169797 & 0.363745 & 0.403863 \\
\hline $\mathrm{C}$ & -6.936725 & 2.117712 & 0.773006 \\
\hline $\mathrm{C}$ & -5.616578 & 2.582731 & 0.682348 \\
\hline $\mathrm{H}$ & -5.367343 & 3.606629 & 0.981482 \\
\hline $\mathrm{C}$ & -3.873051 & -1.547041 & -1.398192 \\
\hline $\mathrm{C}$ & -2.384866 & -3.292446 & -2.388712 \\
\hline $\mathrm{C}$ & -3.364512 & -4.054757 & -3.054109 \\
\hline $\mathrm{H}$ & -4.409258 & -3.750192 & -2.985749 \\
\hline $\mathrm{C}$ & -2.996556 & -5.191802 & -3.792173 \\
\hline $\mathrm{H}$ & -3.781969 & -5.761570 & -4.296878 \\
\hline $\mathrm{C}$ & -1.651333 & -5.584629 & -3.875737 \\
\hline $\mathrm{C}$ & -0.670744 & -4.824482 & -3.209675 \\
\hline $\mathrm{H}$ & 0.376084 & -5.136834 & -3.276494 \\
\hline $\mathrm{C}$ & -1.029303 & -3.695586 & -2.477994 \\
\hline $\mathrm{H}$ & -0.267132 & -3.101270 & -1.958933 \\
\hline $\mathrm{C}$ & -2.161359 & -7.466852 & -5.246162 \\
\hline $\mathrm{H}$ & -1.610711 & -8.285953 & -5.735020 \\
\hline $\mathrm{H}$ & -2.703718 & -6.882578 & -6.017342 \\
\hline $\mathrm{H}$ & -2.904602 & -7.896735 & -4.544168 \\
\hline $\mathrm{C}$ & 8.594594 & 1.788697 & -1.082190 \\
\hline $\mathrm{C}$ & -8.050700 & 3.043890 & 1.300539 \\
\hline $\mathrm{C}$ & 8.422195 & 2.229647 & -2.557061 \\
\hline $\mathrm{H}$ & 7.558457 & 2.904714 & -2.681011 \\
\hline $\mathrm{H}$ & 9.323807 & 2.764216 & -2.910489 \\
\hline $\mathrm{H}$ & 8.260097 & 1.355685 & -3.211718 \\
\hline $\mathrm{C}$ & 9.840507 & 0.883736 & -0.993797 \\
\hline $\mathrm{H}$ & 10.035048 & 0.556009 & 0.042521 \\
\hline $\mathrm{H}$ & 9.737435 & -0.016153 & -1.625270 \\
\hline $\mathrm{H}$ & 10.729753 & 1.438357 & -1.342889 \\
\hline $\mathrm{C}$ & 8.844009 & 3.039315 & -0.202857 \\
\hline $\mathrm{H}$ & 8.988585 & 2.753754 & 0.853702 \\
\hline $\mathrm{H}$ & 9.748215 & 3.578540 & -0.542353 \\
\hline $\mathrm{H}$ & 7.995206 & 3.742757 & -0.246262 \\
\hline $\mathrm{C}$ & -7.711263 & 3.493549 & 2.743410 \\
\hline $\mathrm{H}$ & -6.746564 & 4.026998 & 2.786711 \\
\hline $\mathrm{H}$ & -8.492324 & 4.173658 & 3.131969 \\
\hline $\mathrm{H}$ & -7.643053 & 2.623819 & 3.419851 \\
\hline $\mathrm{C}$ & -8.160589 & 4.292679 & 0.390664 \\
\hline $\mathrm{H}$ & -8.420019 & 4.003600 & -0.642731 \\
\hline $\mathrm{H}$ & -8.943662 & 4.978391 & 0.765123 \\
\hline $\mathrm{H}$ & -7.210798 & 4.852758 & 0.353126 \\
\hline $\mathrm{C}$ & -9.425943 & 2.345812 & 1.326093 \\
\hline $\mathrm{H}$ & -9.740430 & 2.025061 & 0.317413 \\
\hline $\mathrm{H}$ & -9.421408 & 1.458372 & 1.983070 \\
\hline $\mathrm{H}$ & -10.191319 & 3.043639 & 1.710136 \\
\hline
\end{tabular}




$\begin{array}{lrrr}\mathrm{C} & 0.567249 & 7.201128 & -0.182611 \\ \mathrm{C} & 1.408594 & 7.681138 & 1.026005 \\ \mathrm{H} & 2.415297 & 7.230239 & 1.028128 \\ \mathrm{H} & 1.529546 & 8.779987 & 0.998604 \\ \mathrm{H} & 0.918456 & 7.411842 & 1.977758 \\ \mathrm{C} & 1.275669 & 7.614081 & -1.496627 \\ \mathrm{H} & 1.395394 & 8.712531 & -1.540434 \\ \mathrm{H} & 2.278063 & 7.161332 & -1.580177 \\ \mathrm{H} & 0.689296 & 7.295692 & -2.376028 \\ \mathrm{C} & -0.799582 & 7.913791 & -0.129659 \\ \mathrm{H} & -1.346674 & 7.681549 & 0.800745 \\ \mathrm{H} & -0.649128 & 9.007365 & -0.165827 \\ \mathrm{H} & -1.440213 & 7.635212 & -0.984520 \\ \mathrm{Br} & 0.006896 & -0.764524 & -0.232268\end{array}$

Statistical Thermodynamic Analysis

Temperature $=298.150$ Kelvin Pressure $=1.00000$ Atm

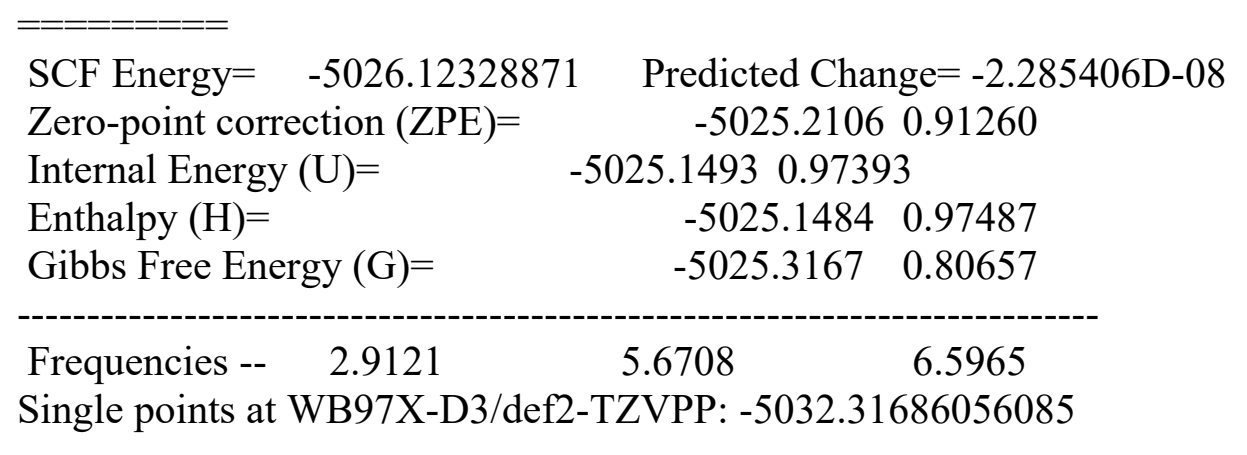

$\mathbf{R}=\mathbf{t B u}$ receptor bound with $\mathrm{Cl}-($ no TBA)

Supporting Information: 010-bound_noTBA_tBu_Cl_new.log

Using Gaussian 09: EM64L-G09RevE.01 30-Nov-2015

\#PBEPBE/6-31G(d)/auto scf $=($ direct,tight, $m a x c y c l e=300, x q c)$

$\mathrm{opt}=($ maxcycle $=250)$ freq $=$ noraman int $=($ grid $=$ ultrafine $)$

\#P Geom=AllCheck Guess=TCheck SCRF=Check GenChk RPBEPBE/6-31G(d)/Auto Freq

Pointgroup $=\mathrm{C} 1 \quad$ Stoichiometry $=\mathrm{C} 50 \mathrm{H} 54 \mathrm{ClN} 4 \mathrm{O} 4(1-) \quad \mathrm{C} 1[\mathrm{X}(\mathrm{C} 50 \mathrm{H} 54 \mathrm{ClN} 4 \mathrm{O} 4)]$ \#Atoms= 113 Charge $=-1 \quad$ Multiplicity $=1$

SCF Energy $=-2914.80117565 \quad$ Predicted Change $=-8.541415 \mathrm{D}-09$

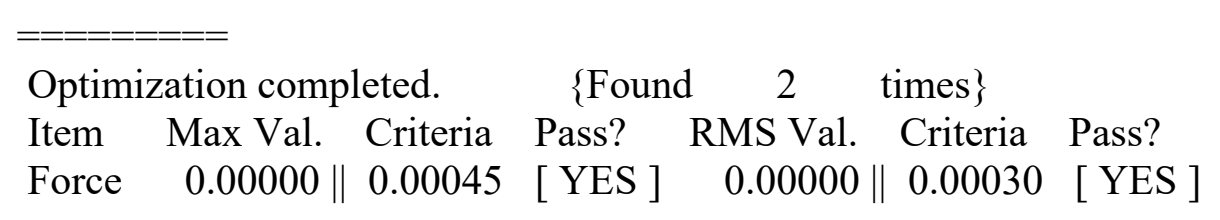


Displ $0.00178 \| 0.00180 \quad$ [YES ] $0.00178 \| 0.00180 \quad$ [ YES ]

\begin{tabular}{|c|c|c|c|}
\hline \multirow{2}{*}{$\begin{array}{l}\text { Atomic } \\
\text { Type }\end{array}$} & \multicolumn{3}{|c|}{ Coordinates (Angstroms) } \\
\hline & $\mathrm{X}$ & $\mathrm{Y} \quad \mathrm{Z}$ & \\
\hline $\mathrm{O}$ & -4.581630 & 2.411332 & 1.750358 \\
\hline $\mathrm{O}$ & -0.197046 & 6.759733 & 4.122713 \\
\hline $\mathrm{O}$ & 4.629249 & 2.346696 & -1.742474 \\
\hline $\mathrm{O}$ & 0.318991 & 6.765732 & -4.119149 \\
\hline $\mathrm{N}$ & -3.640006 & 0.596011 & 0.630834 \\
\hline $\mathrm{H}$ & -2.752667 & 0.123004 & 0.417759 \\
\hline $\mathrm{N}$ & -2.277655 & 2.256503 & 1.489462 \\
\hline $\mathrm{H}$ & -1.516790 & 1.713885 & 1.028702 \\
\hline $\mathrm{N}$ & 3.657211 & 0.542037 & -0.631812 \\
\hline $\mathrm{H}$ & 2.762373 & 0.081492 & -0.422908 \\
\hline $\mathrm{N}$ & 2.322343 & 2.224589 & -1.490951 \\
\hline $\mathrm{H}$ & 1.551641 & 1.691835 & -1.035157 \\
\hline $\mathrm{C}$ & -0.019425 & -2.874213 & -0.001853 \\
\hline $\mathrm{H}$ & -0.012699 & -1.777376 & -0.002589 \\
\hline $\mathrm{C}$ & -1.244046 & -3.574684 & -0.036168 \\
\hline $\mathrm{C}$ & -1.239125 & -4.990341 & -0.037545 \\
\hline $\mathrm{H}$ & -2.203036 & -5.509491 & -0.064490 \\
\hline $\mathrm{C}$ & -0.035784 & -5.722529 & -0.000741 \\
\hline $\mathrm{C}$ & 1.173614 & -5.009571 & 0.035548 \\
\hline $\mathrm{H}$ & 2.132125 & -5.533731 & 0.062904 \\
\hline $\mathrm{C}$ & 1.193052 & -3.589707 & 0.033002 \\
\hline $\mathrm{C}$ & -2.464038 & -2.841297 & -0.065720 \\
\hline $\mathrm{C}$ & -3.496766 & -2.172081 & -0.088549 \\
\hline $\mathrm{C}$ & -4.724231 & -1.458082 & -0.116249 \\
\hline $\mathrm{C}$ & -4.806437 & -0.078667 & 0.270789 \\
\hline $\mathrm{C}$ & -6.078616 & 0.532642 & 0.247071 \\
\hline $\mathrm{H}$ & -6.156654 & 1.573820 & 0.557822 \\
\hline $\mathrm{C}$ & -7.215591 & -0.180241 & -0.145464 \\
\hline $\mathrm{H}$ & -8.170818 & 0.353642 & -0.143687 \\
\hline $\mathrm{C}$ & -7.162084 & -1.532099 & -0.541341 \\
\hline $\mathrm{C}$ & -5.898032 & -2.139763 & -0.514790 \\
\hline $\mathrm{H}$ & -5.780960 & -3.189854 & -0.804155 \\
\hline $\mathrm{C}$ & -3.575464 & 1.819678 & 1.330508 \\
\hline $\mathrm{C}$ & -1.851585 & 3.411438 & 2.171871 \\
\hline $\mathrm{C}$ & -2.708377 & 4.299081 & 2.852874 \\
\hline $\mathrm{H}$ & -3.780482 & 4.102054 & 2.855846 \\
\hline $\mathrm{C}$ & -2.187309 & 5.423800 & 3.513213 \\
\hline $\mathrm{H}$ & -2.882204 & 6.091175 & 4.030971 \\
\hline $\mathrm{C}$ & -0.807581 & 5.682181 & 3.504718 \\
\hline $\mathrm{C}$ & 0.051208 & 4.798290 & 2.824222 \\
\hline $\mathrm{H}$ & 1.125686 & 5.006074 & 2.816248 \\
\hline $\mathrm{C}$ & -0.460777 & 3.681206 & 2.169131 \\
\hline $\mathrm{H}$ & 0.212728 & 2.997076 & 1.640587 \\
\hline
\end{tabular}




\begin{tabular}{|c|c|c|c|}
\hline $\mathrm{C}$ & -1.048591 & 7.667648 & 4.800713 \\
\hline $\mathrm{H}$ & -0.392289 & 8.448700 & 5.215899 \\
\hline $\mathrm{H}$ & -1.601516 & 7.179618 & 5.629237 \\
\hline $\mathrm{H}$ & -1.784240 & 8.136925 & 4.116187 \\
\hline $\mathrm{C}$ & 2.423442 & -2.874387 & 0.062105 \\
\hline $\mathrm{C}$ & 3.467899 & -2.223474 & 0.085247 \\
\hline $\mathrm{C}$ & 4.706988 & -1.530080 & 0.114273 \\
\hline $\mathrm{C}$ & 4.812229 & -0.151601 & -0.270720 \\
\hline $\mathrm{C}$ & 6.094049 & 0.439028 & -0.244119 \\
\hline $\mathrm{H}$ & 6.189319 & 1.479309 & -0.553046 \\
\hline $\mathrm{C}$ & 7.218766 & -0.292815 & 0.148952 \\
\hline $\mathrm{H}$ & 8.182503 & 0.225566 & 0.149535 \\
\hline $\mathrm{C}$ & 7.142780 & -1.644325 & 0.542336 \\
\hline $\mathrm{C}$ & 5.869096 & -2.231387 & 0.513065 \\
\hline $\mathrm{H}$ & 5.734597 & -3.279873 & 0.800642 \\
\hline $\mathrm{C}$ & 3.613060 & 1.768411 & -1.328146 \\
\hline $\mathrm{C}$ & 1.916066 & 3.387719 & -2.171565 \\
\hline $\mathrm{C}$ & 2.788618 & 4.264734 & -2.846308 \\
\hline $\mathrm{H}$ & 3.857728 & 4.052052 & -2.845460 \\
\hline $\mathrm{C}$ & 2.286877 & 5.398946 & -3.505376 \\
\hline $\mathrm{H}$ & 2.993658 & 6.057583 & -4.018213 \\
\hline $\mathrm{C}$ & 0.911067 & 5.677521 & -3.501847 \\
\hline $\mathrm{C}$ & 0.036580 & 4.804290 & -2.827625 \\
\hline $\mathrm{H}$ & -1.034772 & 5.027764 & -2.823540 \\
\hline $\mathrm{C}$ & 0.529356 & 3.677856 & -2.173796 \\
\hline $\mathrm{H}$ & -0.156283 & 3.002040 & -1.650160 \\
\hline $\mathrm{C}$ & 1.186570 & 7.662787 & -4.791248 \\
\hline $\mathrm{H}$ & 0.543544 & 8.454518 & -5.206986 \\
\hline $\mathrm{H}$ & 1.735631 & 7.168829 & -5.618830 \\
\hline $\mathrm{H}$ & 1.926257 & 8.119380 & -4.102508 \\
\hline $\mathrm{C}$ & -8.400205 & -2.336903 & -0.984425 \\
\hline $\mathrm{C}$ & 8.367043 & -2.469778 & 0.985918 \\
\hline $\mathrm{C}$ & -8.200029 & -2.837649 & -2.436502 \\
\hline $\mathrm{H}$ & -7.302221 & -3.472349 & -2.527141 \\
\hline $\mathrm{H}$ & -9.071629 & -3.434092 & -2.765465 \\
\hline $\mathrm{H}$ & -8.080579 & -1.988346 & -3.131545 \\
\hline $\mathrm{C}$ & -9.690645 & -1.493086 & -0.940827 \\
\hline $\mathrm{H}$ & -9.905085 & -1.126763 & 0.078504 \\
\hline $\mathrm{H}$ & -9.630870 & -0.620103 & -1.614242 \\
\hline $\mathrm{H}$ & -10.549493 & -2.107980 & -1.264226 \\
\hline $\mathrm{C}$ & -8.589654 & -3.555731 & -0.047403 \\
\hline $\mathrm{H}$ & -8.752813 & -3.228373 & 0.994231 \\
\hline $\mathrm{H}$ & -9.464020 & -4.155550 & -0.362588 \\
\hline $\mathrm{H}$ & -7.706084 & -4.216370 & -0.056017 \\
\hline $\mathrm{C}$ & 8.156270 & -2.970437 & 2.436527 \\
\hline $\mathrm{H}$ & 7.248399 & -3.591086 & 2.524171 \\
\hline $\mathrm{H}$ & 9.017738 & -3.581285 & 2.765757 \\
\hline $\mathrm{H}$ & 8.048996 & -2.120839 & 3.133201 \\
\hline
\end{tabular}




$\begin{array}{cccc}\mathrm{C} & 8.538833 & -3.689426 & 0.046561 \\ \mathrm{H} & 8.709041 & -3.362422 & -0.994056 \\ \mathrm{H} & 9.403021 & -4.303705 & 0.361970 \\ \mathrm{H} & 7.644868 & -4.335959 & 0.052168 \\ \mathrm{C} & 9.670832 & -1.646511 & 0.946629 \\ \mathrm{H} & 9.893079 & -1.281454 & -0.071484 \\ \mathrm{H} & 9.623575 & -0.774118 & 1.621804 \\ \mathrm{H} & 10.519168 & -2.275666 & 1.270356 \\ \mathrm{C} & -0.085758 & -7.265999 & -0.001515 \\ \mathrm{C} & -0.874514 & -7.755080 & 1.238497 \\ \mathrm{H} & -1.904379 & -7.360158 & 1.249760 \\ \mathrm{H} & -0.934892 & -8.859249 & 1.245351 \\ \mathrm{H} & -0.380818 & -7.430940 & 2.171113 \\ \mathrm{C} & -0.796757 & -7.755889 & -1.287363 \\ \mathrm{H} & -0.857085 & -8.860036 & -1.297076 \\ \mathrm{H} & -1.823861 & -7.360624 & -1.362106 \\ \mathrm{H} & -0.246467 & -7.432588 & -2.188050 \\ \mathrm{C} & 1.319087 & -7.901198 & 0.041920 \\ \mathrm{H} & 1.871277 & -7.611760 & 0.953076 \\ \mathrm{H} & 1.228619 & -9.001978 & 0.040328 \\ \mathrm{H} & 1.925863 & -7.613525 & -0.834386 \\ \mathrm{Cl} & 0.005455 & 0.615297 & -0.008780\end{array}$

Statistical Thermodynamic Analysis

Temperature $=298.150$ Kelvin Pressure $=1.00000$ Atm

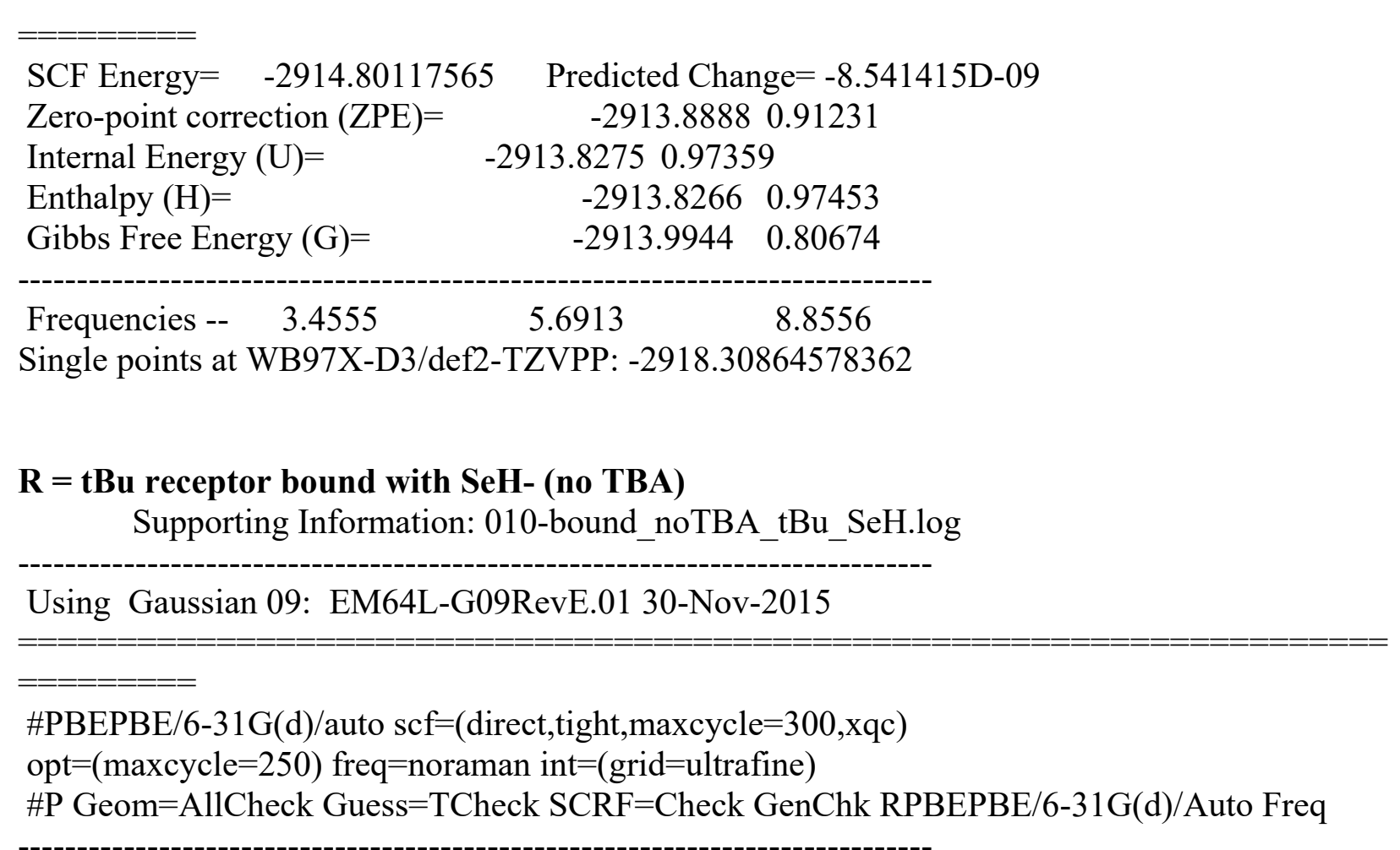


Pointgroup $=\mathrm{C} 1 \quad$ Stoichiometry $=\mathrm{C} 50 \mathrm{H} 55 \mathrm{~N} 4 \mathrm{O} 4 \mathrm{Se}(1-) \quad \mathrm{C} 1[\mathrm{X}(\mathrm{C} 50 \mathrm{H} 55 \mathrm{~N} 4 \mathrm{O} 4 \mathrm{Se})]$ \#Atoms= 114

Charge $=-1 \quad$ Multiplicity $=1$

SCF Energy $=-4854.38621499 \quad$ Predicted Change $=-1.787256 \mathrm{D}-08$

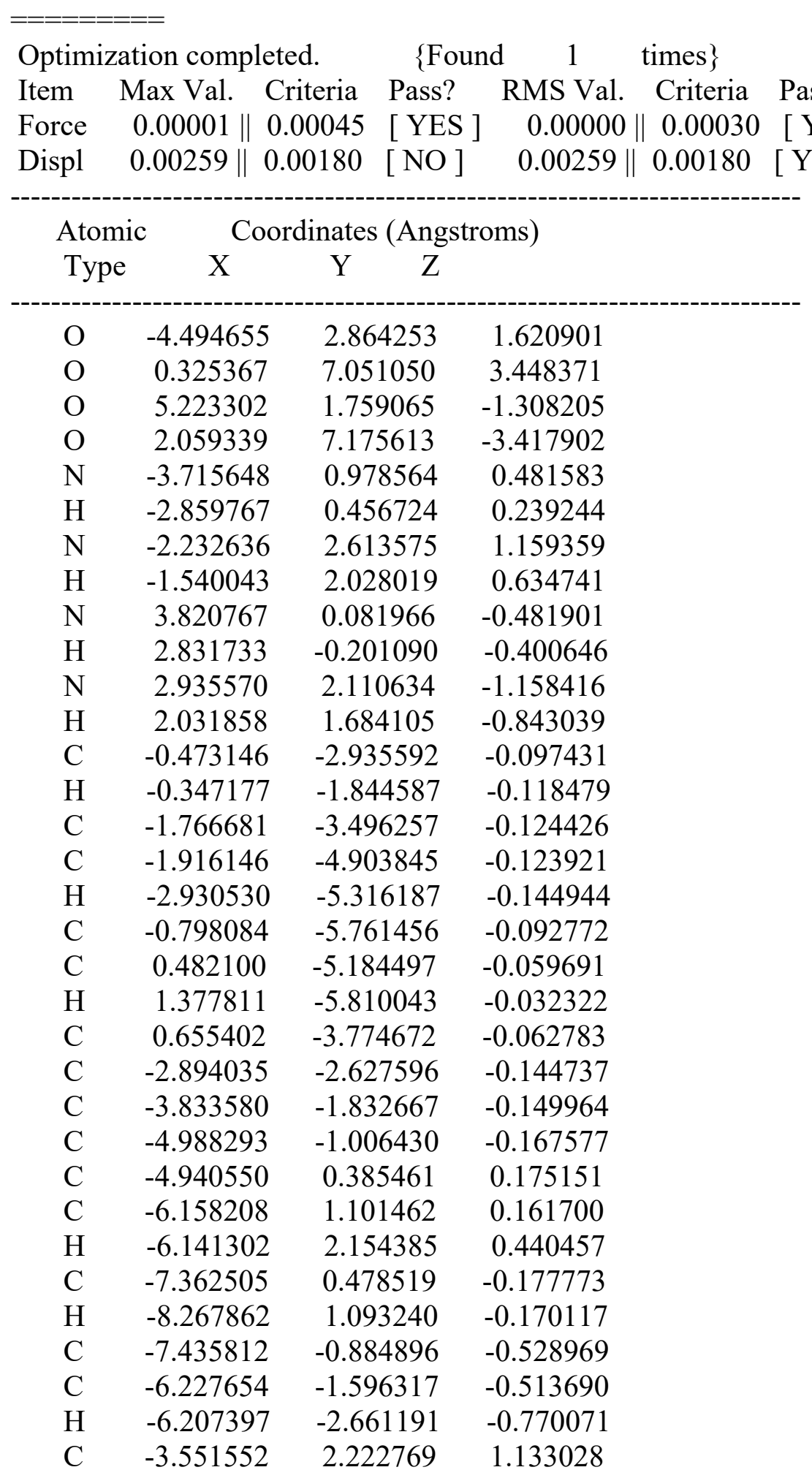




\begin{tabular}{|c|c|c|c|}
\hline $\mathrm{C}$ & -1.687882 & 3.758290 & 1.767666 \\
\hline $\mathrm{C}$ & -2.432339 & 4.722581 & 2.474385 \\
\hline $\mathrm{H}$ & -3.511640 & 4.596634 & 2.565824 \\
\hline $\mathrm{C}$ & -1.788125 & 5.831341 & 3.048047 \\
\hline $\mathrm{H}$ & -2.394238 & 6.562361 & 3.590824 \\
\hline $\mathrm{C}$ & -0.399227 & 5.995427 & 2.922523 \\
\hline $\mathrm{C}$ & 0.346409 & 5.032628 & 2.215021 \\
\hline $\mathrm{H}$ & 1.427738 & 5.167284 & 2.113560 \\
\hline $\mathrm{C}$ & -0.286106 & 3.929446 & 1.649410 \\
\hline $\mathrm{H}$ & 0.284526 & 3.170331 & 1.099499 \\
\hline $\mathrm{C}$ & -0.409754 & 8.036231 & 4.153818 \\
\hline $\mathrm{H}$ & 0.326100 & 8.785110 & 4.486746 \\
\hline $\mathrm{H}$ & -0.925651 & 7.614553 & 5.040656 \\
\hline $\mathrm{H}$ & -1.165704 & 8.530496 & 3.510125 \\
\hline $\mathrm{C}$ & 1.954916 & -3.196274 & -0.018955 \\
\hline $\mathrm{C}$ & 3.067012 & -2.671278 & 0.034858 \\
\hline $\mathrm{C}$ & 4.405961 & -2.210541 & 0.145942 \\
\hline $\mathrm{C}$ & 4.790673 & -0.854273 & -0.118731 \\
\hline $\mathrm{C}$ & 6.158387 & -0.528400 & 0.026959 \\
\hline $\mathrm{H}$ & 6.469370 & 0.493613 & -0.184009 \\
\hline $\mathrm{C}$ & 7.098917 & -1.488903 & 0.409167 \\
\hline $\mathrm{H}$ & 8.139954 & -1.164599 & 0.502926 \\
\hline $\mathrm{C}$ & 6.747723 & -2.827780 & 0.674711 \\
\hline $\mathrm{C}$ & 5.391080 & -3.151006 & 0.534240 \\
\hline $\mathrm{H}$ & 5.041182 & -4.171439 & 0.724791 \\
\hline $\mathrm{C}$ & 4.084021 & 1.365892 & -1.013479 \\
\hline $\mathrm{C}$ & 2.816992 & 3.386709 & -1.739433 \\
\hline $\mathrm{C}$ & 3.905341 & 4.186571 & -2.140914 \\
\hline $\mathrm{H}$ & 4.918357 & 3.805597 & -2.009880 \\
\hline $\mathrm{C}$ & 3.685211 & 5.453287 & -2.706268 \\
\hline $\mathrm{H}$ & 4.553191 & 6.046596 & -3.007823 \\
\hline $\mathrm{C}$ & 2.380643 & 5.943994 & -2.874588 \\
\hline $\mathrm{C}$ & 1.290954 & 5.147945 & -2.473221 \\
\hline $\mathrm{H}$ & 0.275753 & 5.535100 & -2.604344 \\
\hline $\mathrm{C}$ & 1.503539 & 3.888747 & -1.918403 \\
\hline $\mathrm{H}$ & 0.654271 & 3.264886 & -1.615511 \\
\hline $\mathrm{C}$ & 3.144716 & 7.996124 & -3.815451 \\
\hline $\mathrm{H}$ & 2.697772 & 8.921914 & -4.210741 \\
\hline $\mathrm{H}$ & 3.756628 & 7.520470 & -4.608959 \\
\hline $\mathrm{H}$ & 3.808535 & 8.247848 & -2.963479 \\
\hline $\mathrm{C}$ & -8.749822 & -1.594806 & -0.911338 \\
\hline $\mathrm{C}$ & 7.764159 & -3.906602 & 1.098334 \\
\hline $\mathrm{C}$ & -8.634500 & -2.159165 & -2.349247 \\
\hline $\mathrm{H}$ & -7.797082 & -2.871657 & -2.439965 \\
\hline $\mathrm{H}$ & -9.562827 & -2.688821 & -2.634425 \\
\hline $\mathrm{H}$ & -8.462828 & -1.347040 & -3.077039 \\
\hline $\mathrm{C}$ & -9.962052 & -0.642207 & -0.864796 \\
\hline $\mathrm{H}$ & -10.115235 & -0.225007 & 0.145985 \\
\hline
\end{tabular}




$\begin{array}{lccc}\mathrm{H} & -9.847468 & 0.199555 & -1.570143 \\ \mathrm{H} & -10.879065 & -1.191290 & -1.143903 \\ \mathrm{C} & -9.014866 & -2.760929 & 0.073172 \\ \mathrm{H} & -9.119457 & -2.386129 & 1.106296 \\ \mathrm{H} & -9.945565 & -3.294050 & -0.197501 \\ \mathrm{H} & -8.190784 & -3.494421 & 0.065203 \\ \mathrm{C} & 7.373077 & -4.470840 & 2.486855 \\ \mathrm{H} & 6.361064 & -4.910006 & 2.476450 \\ \mathrm{H} & 8.082118 & -5.260360 & 2.799244 \\ \mathrm{H} & 7.384299 & -3.674533 & 3.251385 \\ \mathrm{C} & 7.760920 & -5.057794 & 0.061991 \\ \mathrm{H} & 8.053863 & -4.688004 & -0.936110 \\ \mathrm{H} & 8.472433 & -5.850716 & 0.359822 \\ \mathrm{H} & 6.762328 & -5.517447 & -0.031364 \\ \mathrm{C} & 9.199373 & -3.349491 & 1.192864 \\ \mathrm{H} & 9.548659 & -2.953838 & 0.222985 \\ \mathrm{H} & 9.275239 & -2.540963 & 1.941017 \\ \mathrm{H} & 9.892755 & -4.153945 & 1.496807 \\ \mathrm{H} & 0.379691 & 0.473997 & 1.095265 \\ \mathrm{C} & -1.015229 & -7.290494 & -0.093193 \\ \mathrm{C} & -1.844024 & -7.691788 & 1.152229 \\ \mathrm{H} & -2.824275 & -7.186257 & 1.170990 \\ \mathrm{H} & -2.025085 & -8.782691 & 1.159209 \\ \mathrm{H} & -1.311134 & -7.424746 & 2.081387 \\ \mathrm{C} & -1.784383 & -7.699141 & -1.374000 \\ \mathrm{H} & -1.964703 & -8.790144 & -1.383239 \\ \mathrm{H} & -2.762817 & -7.194262 & -1.441557 \\ \mathrm{H} & -1.208480 & -7.437035 & -2.278557 \\ \mathrm{C} & 0.312356 & -8.074880 & -0.059754 \\ \mathrm{H} & 0.898188 & -7.849702 & 0.848536 \\ \mathrm{H} & 0.102563 & -9.159251 & -0.062887 \\ \mathrm{H} & 0.941641 & -7.852543 & -0.939179 \\ \mathrm{Se} & 0.035393 & 0.672133 & -0.334083\end{array}$

Statistical Thermodynamic Analysis

Temperature $=298.150$ Kelvin $\quad$ Pressure $=1.00000$ Atm

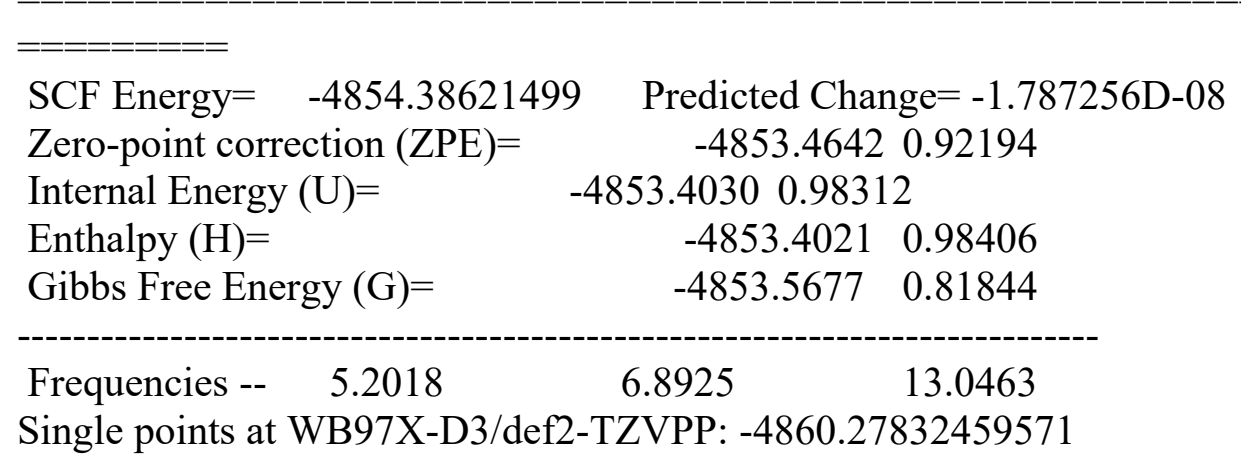


$\mathrm{R}=\mathrm{tBu}$ receptor bound with $\mathrm{SH}-($ no TBA)

Supporting Information: 010-bound_noTBA_tBu_SH.log

Using Gaussian 09: EM64L-G09RevE.01 30-Nov-2015

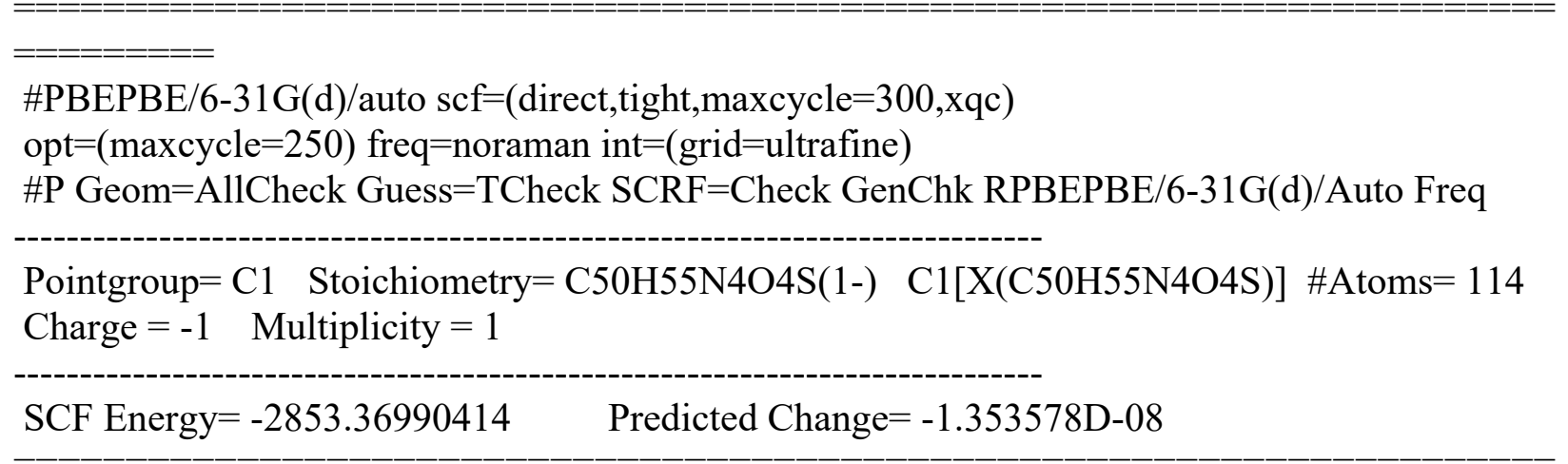

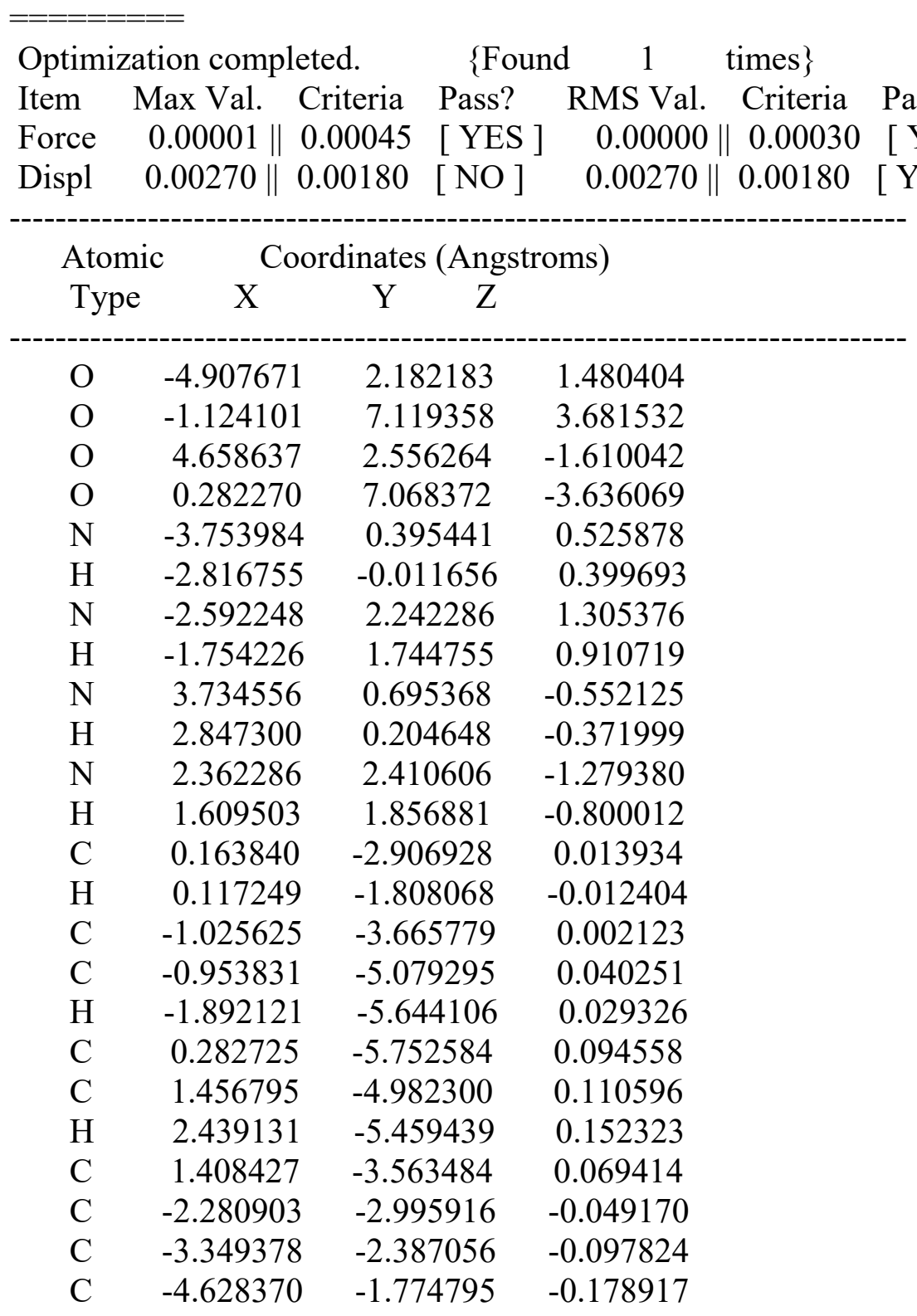




\begin{tabular}{|c|c|c|c|}
\hline $\mathrm{C}$ & -4.841060 & -0.394053 & 0.148831 \\
\hline $\mathrm{C}$ & -6.159766 & 0.102523 & 0.048913 \\
\hline $\mathrm{H}$ & -6.339161 & 1.144618 & 0.309669 \\
\hline $\mathrm{C}$ & -7.216077 & -0.720000 & -0.353507 \\
\hline $\mathrm{H}$ & -8.211620 & -0.269157 & -0.410770 \\
\hline C & -7.034321 & -2.077867 & -0.68488 \\
\hline $\mathrm{C}$ & -5.725233 & -2.570867 & -0.58666 \\
\hline $\mathrm{H}$ & -5.506856 & -3.617146 & -0.82687 \\
\hline $\mathrm{C}$ & -3.832798 & 1.665440 & 1.13767 \\
\hline $\mathrm{C}$ & -2.321974 & 3.480890 & 1.91845 \\
\hline $\mathrm{C}$ & -3.290553 & 4.293033 & 2.543332 \\
\hline $\mathrm{H}$ & -4.330126 & 3.965887 & 2.55102 \\
\hline $\mathrm{C}$ & -2.922760 & 5.510161 & 3.139750 \\
\hline $\mathrm{H}$ & -3.702607 & 6.112973 & 3.613979 \\
\hline $\mathrm{C}$ & -1.586525 & 5.939987 & 3.123822 \\
\hline $\mathrm{C}$ & -0.616134 & 5.134272 & \\
\hline $\mathrm{H}$ & 0.423572 & 5.475611 & 2.480744 \\
\hline $\mathrm{C}$ & -0.9767 & 3.926038 & 1.907679 \\
\hline $\mathrm{H}$ & -0.216433 & 3.305157 & 1.41978 \\
\hline $\mathrm{C}$ & -2.091464 & 7.949372 & \\
\hline $\mathrm{H}$ & -1.543880 & 8.829279 & 4.67440 \\
\hline $\mathrm{H}$ & -2.589018 & 7.443076 & \\
\hline $\mathrm{H}$ & -2.871680 & 8.282577 & 87184 \\
\hline $\mathrm{C}$ & 2.605065 & -2.792729 & 0.0 \\
\hline $\mathrm{C}$ & 3.622760 & -2.1004 & 0.0 \\
\hline $\mathrm{C}$ & 4.842079 & -1.373067 & 0.125067 \\
\hline $\mathrm{C}$ & 4.908321 & 0.017170 & -0.224963 \\
\hline $\mathrm{C}$ & 6.177017 & 0.637183 & 4727 \\
\hline $\mathrm{H}$ & 6.244734 & 1.687076 & -0.476940 \\
\hline $\mathrm{C}$ & 7.323550 & -0.076513 & 0.16644 \\
\hline $\mathrm{H}$ & 8.274110 & 0.465703 & 70428 \\
\hline $\mathrm{C}$ & 7.285655 & -1.439854 & 0.522907 \\
\hline $\mathrm{C}$ & 6.026171 & -2.055971 & 0.49157 \\
\hline $\mathrm{H}$ & 5.919618 & -3.114659 & 236 \\
\hline $\mathrm{C}$ & 3.659193 & 1.953840 & -1.188426 \\
\hline $\mathrm{C}$ & 1.934395 & 3.602117 & -1.895173 \\
\hline $\mathrm{C}$ & 2.779312 & 4.489420 & -2.59259 \\
\hline $\mathrm{H}$ & 3.843349 & 4.263173 & -2.66066 \\
\hline $\mathrm{C}$ & 2.258894 & 5.651888 & -3.18438 \\
\hline $\mathrm{H}$ & 2.945588 & & -3.71697 \\
\hline $\mathrm{C}$ & 0.890684 & 5.951381 & -3.09024 \\
\hline $\mathrm{C}$ & 0.043287 & 5.069883 & -2.39300 \\
\hline $\mathrm{H}$ & -1.021733 & 5.309282 & -2.31494 \\
\hline $\mathrm{C}$ & 0.555187 & 3.914757 & -1.80708 \\
\hline $\mathrm{H}$ & -0.110981 & 3.235811 & -1.26349 \\
\hline $\mathrm{C}$ & 1.123552 & 7.971958 & -4.33215 \\
\hline $\mathrm{H}$ & 0.470859 & 8.786419 & -4.68402 \\
\hline $\mathrm{H}$ & 1.613023 & 7.495416 & -5.20605 \\
\hline
\end{tabular}




$\begin{array}{lccc}\mathrm{H} & 1.911416 & 8.395177 & -3.676219 \\ \mathrm{C} & -8.181411 & -3.004455 & -1.134503 \\ \mathrm{C} & 8.534667 & -2.246892 & 0.929683 \\ \mathrm{C} & -7.882502 & -3.548571 & -2.553673 \\ \mathrm{H} & -6.932192 & -4.108242 & -2.582435 \\ \mathrm{H} & -8.687727 & -4.230403 & -2.885940 \\ \mathrm{H} & -7.806439 & -2.722951 & -3.282525 \\ \mathrm{C} & -9.538915 & -2.273287 & -1.176449 \\ \mathrm{H} & -9.823560 & -1.882918 & -0.183573 \\ \mathrm{H} & -9.525900 & -1.428234 & -1.887003 \\ \mathrm{H} & -10.329675 & -2.972825 & -1.501517 \\ \mathrm{C} & -8.304409 & -4.193465 & -0.149359 \\ \mathrm{H} & -8.536215 & -3.836713 & 0.869361 \\ \mathrm{H} & -9.111382 & -4.879919 & -0.467586 \\ \mathrm{H} & -7.368169 & -4.774637 & -0.095365 \\ \mathrm{C} & 8.354879 & -2.788773 & 2.369629 \\ \mathrm{H} & 7.462726 & -3.432441 & 2.452719 \\ \mathrm{H} & 9.234439 & -3.387467 & 2.672350 \\ \mathrm{H} & 8.237340 & -1.959712 & 3.089055 \\ \mathrm{C} & 8.722599 & -3.438251 & -0.042320 \\ \mathrm{H} & 8.871047 & -3.081340 & -1.076411 \\ \mathrm{H} & 9.605181 & -4.039421 & 0.246324 \\ \mathrm{H} & 7.844292 & -4.105909 & -0.041695 \\ \mathrm{C} & 9.818168 & -1.392186 & 0.894724 \\ \mathrm{H} & 10.017717 & -0.996076 & -0.116447 \\ \mathrm{H} & 9.759776 & -0.538648 & 1.592750 \\ \mathrm{H} & 10.685383 & -2.009037 & 1.190961 \\ \mathrm{~S} & 0.038419 & 0.649321 & 0.206392 \\ \mathrm{H} & 0.487981 & 0.607844 & 1.492428 \\ \mathrm{C} & 0.305880 & -7.296204 & 0.135132 \\ \mathrm{C} & -0.456610 & -7.788621 & 1.390157 \\ \mathrm{H} & -1.503942 & -7.442506 & 1.394000 \\ \mathrm{H} & -0.464749 & -8.893841 & 1.426813 \\ \mathrm{H} & 0.022936 & -7.416563 & 2.312222 \\ & -0.383640 & -7.853286 & -1.134960 \\ \mathrm{H} & -0.392430 & -8.958917 & -1.114958 \\ \mathrm{H} & 0.149435 & -7.528985 & -2.045594 \\ \mathrm{H} & 1.739232 & -7.863021 & 0.190957 \\ \mathrm{H} & -.700950 & -8.966540 & 0.217730 \\ \mathrm{H} & -7.569490 & -0.694068\end{array}$

Statistical Thermodynamic Analysis

Temperature $=298.150$ Kelvin $\quad$ Pressure $=1.00000$ Atm

$========$
SCF Energy $=\quad-2853.36990414 \quad$ Predicted Change $=-1.353578 D-08$ 
Zero-point correction $(\mathrm{ZPE})=$

Internal Energy $(\mathrm{U})=$

Enthalpy $(\mathrm{H})=$

Gibbs Free Energy $(\mathrm{G})=$
$-2852.45060 .91928$

$\begin{array}{lll}-2852.3887 & 0.98115\end{array}$

$\begin{array}{lll}-2852.3878 & 0.98210\end{array}$

$-2852.55520 .81463$

Frequencies -- $\quad 5.2430 \quad 6.3574 \quad 12.0917$

Single points at WB97X-D3/def2-TZVPP: -2856.86289323511

$\mathbf{R}=\mathrm{CF}_{3}$ receptor unbound (from crystal structure)

Supporting Information: 000-unbound_crystal_structure_CF3.log

Using Gaussian 09: EM64L-G09RevE.01 30-Nov-2015

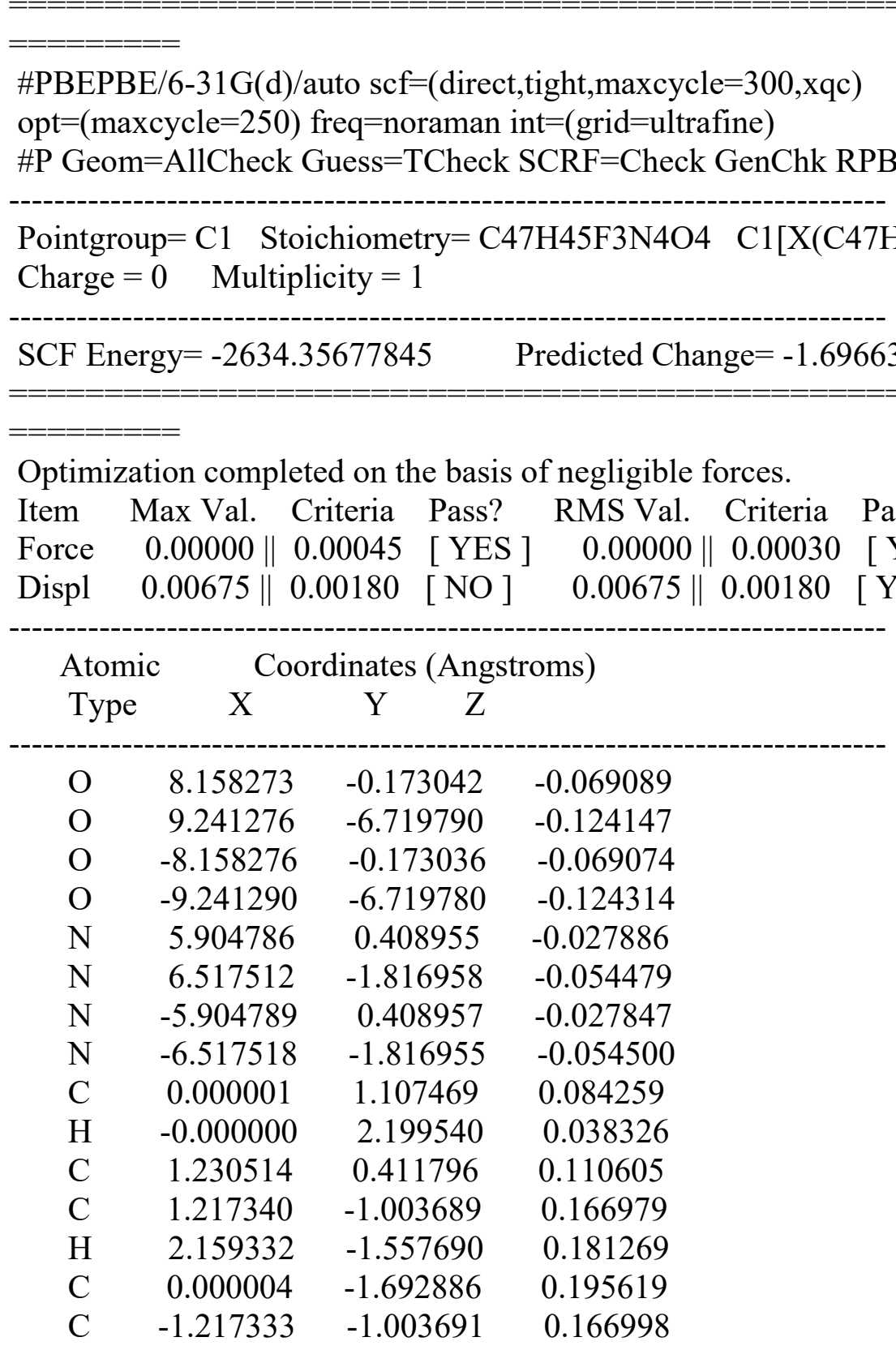




\begin{tabular}{|c|c|c|c|}
\hline $\mathrm{H}$ & -2.159324 & -1.557693 & 0.181303 \\
\hline $\mathrm{C}$ & -1.230510 & 0.411794 & 0.110623 \\
\hline $\mathrm{C}$ & 2.458703 & 1.126674 & 0.074528 \\
\hline $\mathrm{C}$ & 3.493998 & 1.795060 & 0.042890 \\
\hline $\mathrm{C}$ & 4.718563 & 2.511981 & 0.006518 \\
\hline $\mathrm{C}$ & 5.965455 & 1.804484 & -0.027739 \\
\hline $\mathrm{C}$ & 7.156603 & 2.550276 & -0.061146 \\
\hline $\mathrm{H}$ & 8.107697 & 2.018453 & -0.086116 \\
\hline $\mathrm{C}$ & 7.112150 & 3.949512 & -0.061431 \\
\hline $\mathrm{H}$ & 8.066702 & 4.482378 & -0.088063 \\
\hline $\mathrm{C}$ & 5.903749 & 4.676129 & -0.029371 \\
\hline $\mathrm{C}$ & 4.718884 & 3.924931 & 0.004254 \\
\hline $\mathrm{H}$ & 3.745395 & 4.425016 & 0.030909 \\
\hline $\mathrm{C}$ & 6.969189 & -0.502117 & -0.052228 \\
\hline $\mathrm{C}$ & 7.285514 & -3.003719 & -0.072916 \\
\hline $\mathrm{C}$ & 6.582283 & -4.228991 & -0.080312 \\
\hline $\mathrm{H}$ & 5.484771 & -4.228899 & -0.072549 \\
\hline $\mathrm{C}$ & 7.260182 & -5.445175 & -0.097304 \\
\hline $\mathrm{H}$ & 6.713935 & -6.392791 & -0.103045 \\
\hline $\mathrm{C}$ & 8.668717 & -5.471532 & -0.107381 \\
\hline $\mathrm{C}$ & 9.374139 & -4.255660 & -0.099994 \\
\hline $\mathrm{H}$ & 10.466905 & -4.241116 & -0.107573 \\
\hline $\mathrm{C}$ & 8.691974 & -3.029862 & -0.082911 \\
\hline $\mathrm{H}$ & 9.243432 & -2.089626 & -0.078029 \\
\hline $\mathrm{C}$ & 10.664136 & -6.778498 & -0.135081 \\
\hline $\mathrm{H}$ & 10.919901 & -7.848310 & -0.148022 \\
\hline $\mathrm{H}$ & 11.100324 & -6.309148 & 0.768162 \\
\hline $\mathrm{H}$ & 11.087188 & -6.290446 & -1.034652 \\
\hline $\mathrm{C}$ & -2.458701 & 1.126669 & 0.074564 \\
\hline $\mathrm{C}$ & -3.493998 & 1.795054 & 0.042942 \\
\hline $\mathrm{C}$ & -4.718561 & 2.511979 & 0.006592 \\
\hline $\mathrm{C}$ & -5.965455 & 1.804485 & -0.027673 \\
\hline $\mathrm{C}$ & -7.156602 & 2.550280 & -0.061057 \\
\hline $\mathrm{H}$ & -8.107697 & 2.018460 & -0.086033 \\
\hline $\mathrm{C}$ & -7.112146 & 3.949516 & -0.061313 \\
\hline $\mathrm{H}$ & -8.066697 & 4.482384 & -0.087929 \\
\hline $\mathrm{C}$ & -5.903743 & 4.676131 & -0.029246 \\
\hline $\mathrm{C}$ & -4.718880 & 3.924930 & 0.004357 \\
\hline $\mathrm{H}$ & -3.745389 & 4.425012 & 0.031017 \\
\hline $\mathrm{C}$ & -6.969193 & -0.502113 & -0.052216 \\
\hline $\mathrm{C}$ & -7.285522 & -3.003714 & -0.072972 \\
\hline $\mathrm{C}$ & -8.691982 & -3.029855 & -0.082968 \\
\hline $\mathrm{H}$ & -9.243438 & -2.089617 & -0.078058 \\
\hline $\mathrm{C}$ & -9.374149 & -4.255651 & -0.100088 \\
\hline $\mathrm{H}$ & -10.466915 & -4.241104 & -0.107666 \\
\hline $\mathrm{C}$ & -8.668729 & -5.471524 & -0.107511 \\
\hline $\mathrm{C}$ & -7.260194 & -5.445169 & -0.097433 \\
\hline $\mathrm{H}$ & -6.713948 & -6.392787 & -0.103202 \\
\hline
\end{tabular}




\begin{tabular}{lccc}
$\mathrm{C}$ & -6.582292 & -4.228988 & -0.080405 \\
$\mathrm{H}$ & -5.484781 & -4.228898 & -0.072642 \\
$\mathrm{C}$ & -10.664151 & -6.778486 & -0.135253 \\
$\mathrm{H}$ & -10.919917 & -7.848296 & -0.148229 \\
$\mathrm{H}$ & -11.087199 & -6.290404 & -1.034810 \\
$\mathrm{H}$ & -11.100341 & -6.309163 & 0.768003 \\
$\mathrm{H}$ & 4.947961 & 0.047743 & -0.001240 \\
$\mathrm{H}$ & 5.510301 & -1.972699 & -0.053329 \\
$\mathrm{H}$ & -4.947964 & 0.047742 & -0.001216 \\
$\mathrm{H}$ & -5.510307 & -1.972698 & -0.053348 \\
$\mathrm{C}$ & -5.843334 & 6.216731 & -0.029146 \\
$\mathrm{C}$ & -7.247231 & 6.854205 & -0.070151 \\
$\mathrm{H}$ & -7.802258 & 6.568269 & -0.980788 \\
$\mathrm{H}$ & -7.853094 & 6.571247 & 0.808446 \\
$\mathrm{H}$ & -7.154011 & 7.953995 & -0.069306 \\
$\mathrm{C}$ & -5.054218 & 6.701090 & -1.271118 \\
$\mathrm{H}$ & -4.024309 & 6.305180 & -1.283029 \\
$\mathrm{H}$ & -5.549456 & 6.380506 & -2.204023 \\
$\mathrm{H}$ & -4.989797 & 7.804209 & -1.277386 \\
$\mathrm{C}$ & -5.126755 & 6.703606 & 1.255101 \\
$\mathrm{H}$ & -5.674789 & 6.384869 & 2.158657 \\
$\mathrm{H}$ & -4.099335 & 6.307569 & 1.326953 \\
$\mathrm{H}$ & -5.062592 & 7.806733 & 1.262890 \\
$\mathrm{C}$ & 5.843342 & 6.216730 & -0.029303 \\
$\mathrm{C}$ & 5.054225 & 6.701065 & -1.271283 \\
$\mathrm{H}$ & 4.024316 & 6.305157 & -1.283183 \\
$\mathrm{H}$ & 4.989806 & 7.804184 & -1.277573 \\
$\mathrm{H}$ & 5.549460 & 6.380461 & -2.204182 \\
$\mathrm{C}$ & 7.247241 & 6.854201 & -0.070322 \\
$\mathrm{H}$ & 7.853103 & 6.571262 & 0.808280 \\
$\mathrm{H}$ & 7.802267 & 6.568243 & -0.980953 \\
$\mathrm{H}$ & 7.154023 & 7.953991 & -0.069503 \\
$\mathrm{C}$ & 5.126767 & 6.703632 & 1.254937 \\
$\mathrm{H}$ & 4.099346 & 6.307597 & 1.326799 \\
$\mathrm{H}$ & 5.674802 & 6.384913 & 2.158497 \\
----------------------------------------------------------- \\
\hline & 5.062606 & 7.806760 & 1.262703 \\
\hline & 0.000006 & -3.198047 & 0.325337 \\
$\mathrm{~F}$ & 1.000013 & -3.584323 & 1.631368 \\
\hline
\end{tabular}

Statistical Thermodynamic Analysis

Temperature $=298.150$ Kelvin $\quad$ Pressure $=1.00000$ Atm

SCF Energy $=\quad-2634.35677845 \quad$ Predicted Change $=-1.696635 \mathrm{D}-09$

Zero-point correction $(\mathrm{ZPE})=\quad-2633.55120 .80556$

Internal Energy $(U)=\quad-2633.49290 .86381$ 
Enthalpy $(\mathrm{H})=$

Gibbs Free Energy $(\mathrm{G})=$

Frequencies -- $\quad 1.6150$

Single points at WB97X-D3/def2-TZVPP: -2637.77445809497

\section{$\mathbf{R}=\mathrm{CF}_{3}$ receptor unbound (from conformational search)}

Supporting Information: 000-unbound_conf_search_CF3.log
$-2633.4920 \quad 0.86475$

$-2633.6602 \quad 0.69652$

$2.6410 \quad 4.6108$




\begin{tabular}{|c|c|c|c|}
\hline $\mathrm{C}$ & -4.609938 & -1.431854 & 0.789139 \\
\hline $\mathrm{C}$ & -5.797306 & -2.183931 & 0.821866 \\
\hline $\mathrm{C}$ & -7.026103 & -1.577061 & 0.535111 \\
\hline $\mathrm{C}$ & -7.142637 & -0.211186 & 0.204236 \\
\hline $\mathrm{C}$ & -5.951563 & 0.531012 & 0.164040 \\
\hline $\mathrm{C}$ & -8.489245 & 0.471008 & -0.109844 \\
\hline $\mathrm{C}$ & -8.453890 & 1.042187 & -1.549496 \\
\hline $\mathrm{C}$ & -8.726762 & 1.627077 & 0.893592 \\
\hline $\mathrm{C}$ & -9.675994 & -0.508832 & -0.008034 \\
\hline $\mathrm{C}$ & -3.009823 & -3.265169 & 1.393945 \\
\hline $\mathrm{C}$ & -0.745692 & -2.471564 & 2.257706 \\
\hline $\mathrm{C}$ & -1.126700 & -1.478462 & 3.188378 \\
\hline $\mathrm{C}$ & -0.188442 & -0.571816 & 3.679349 \\
\hline $\mathrm{C}$ & 1.163726 & -0.654953 & 3.285013 \\
\hline $\mathrm{C}$ & 1.555356 & -1.650871 & 2.368378 \\
\hline $\mathrm{C}$ & 0.599153 & -2.537820 & 1.853073 \\
\hline $\mathrm{C}$ & 3.385245 & 0.197202 & 3.501450 \\
\hline $\mathrm{C}$ & 2.441403 & 1.348370 & -0.282998 \\
\hline $\mathrm{C}$ & 3.505016 & 0.732813 & -0.371032 \\
\hline $\mathrm{C}$ & 4.689566 & -0.048949 & -0.448318 \\
\hline $\mathrm{C}$ & 4.608685 & -1.441617 & -0.778335 \\
\hline $\mathrm{C}$ & 5.795864 & -2.194414 & -0.800121 \\
\hline $\mathrm{C}$ & 7.024466 & -1.584389 & -0.519300 \\
\hline $\mathrm{C}$ & 7.141028 & -0.214533 & -0.205337 \\
\hline $\mathrm{C}$ & 5.950140 & 0.528491 & -0.176075 \\
\hline $\mathrm{C}$ & 8.487445 & 0.471091 & 0.102012 \\
\hline $\mathrm{C}$ & 9.673732 & -0.510877 & 0.016452 \\
\hline $\mathrm{C}$ & 8.727774 & 1.612788 & -0.917083 \\
\hline $\mathrm{C}$ & 8.449581 & 1.062434 & 1.533432 \\
\hline $\mathrm{C}$ & 3.009016 & -3.281646 & -1.363538 \\
\hline $\mathrm{C}$ & 0.745787 & -2.497507 & -2.238170 \\
\hline $\mathrm{C}$ & 1.127420 & -1.515164 & -3.179919 \\
\hline $\mathrm{C}$ & 0.189723 & -0.613532 & -3.681081 \\
\hline $\mathrm{C}$ & -1.162483 & -0.691461 & -3.285888 \\
\hline $\mathrm{C}$ & -1.554768 & -1.676893 & -2.358296 \\
\hline $\mathrm{C}$ & -0.599132 & -2.558544 & -1.833003 \\
\hline $\mathrm{C}$ & -3.383722 & 0.158695 & -3.512777 \\
\hline $\mathrm{H}$ & 0.001427 & 0.275641 & 0.008361 \\
\hline $\mathrm{H}$ & -2.153586 & 4.033763 & 0.186540 \\
\hline $\mathrm{H}$ & -2.585777 & -1.259772 & 1.083278 \\
\hline $\mathrm{H}$ & -1.454175 & -4.422085 & 1.899611 \\
\hline $\mathrm{H}$ & 2.145728 & 4.031346 & -0.300077 \\
\hline $\mathrm{H}$ & 2.584922 & -1.272632 & -1.076964 \\
\hline $\mathrm{H}$ & 1.453714 & -4.444101 & -1.857678 \\
\hline $\mathrm{H}$ & -5.737584 & -3.244615 & 1.068089 \\
\hline $\mathrm{H}$ & -7.920106 & -2.205478 & 0.576726 \\
\hline $\mathrm{H}$ & -5.970052 & 1.595299 & -0.092356 \\
\hline $\mathrm{H}$ & -9.407686 & 1.548105 & -1.784677 \\
\hline
\end{tabular}




$\begin{array}{cccc}\mathrm{H} & -8.300580 & 0.237921 & -2.290009 \\ \mathrm{H} & -7.643188 & 1.779750 & -1.678324 \\ \mathrm{H} & -8.771685 & 1.248338 & 1.929374 \\ \mathrm{H} & -9.681974 & 2.136381 & 0.671826 \\ \mathrm{H} & -7.924708 & 2.383351 & 0.845836 \\ \mathrm{H} & -9.773024 & -0.932349 & 1.006954 \\ \mathrm{H} & -9.582767 & -1.343764 & -0.724487 \\ \mathrm{H} & -10.615526 & 0.022869 & -0.237761 \\ \mathrm{H} & -2.162283 & -1.430407 & 3.538902 \\ \mathrm{H} & -0.476506 & 0.200106 & 4.398934 \\ \mathrm{H} & 2.592380 & -1.735689 & 2.035277 \\ \mathrm{H} & 0.905150 & -3.293368 & 1.121377 \\ \mathrm{H} & 3.874206 & 0.992979 & 4.082048 \\ \mathrm{H} & 3.539027 & 0.380411 & 2.422174 \\ \mathrm{H} & 3.825866 & -0.780092 & 3.776191 \\ \mathrm{H} & 5.736132 & -3.258052 & -1.033259 \\ \mathrm{H} & 7.918302 & -2.213581 & -0.551788 \\ \mathrm{H} & 5.968622 & 1.595899 & 0.067011 \\ \mathrm{H} & 10.613089 & 0.023273 & 0.241167 \\ \mathrm{H} & 9.772950 & -0.948258 & -0.992427 \\ \mathrm{H} & 9.578177 & -1.335911 & 0.743985 \\ \mathrm{H} & 9.682763 & 2.124717 & -0.700454 \\ \mathrm{H} & 7.925950 & 2.369984 & -0.881696 \\ \mathrm{H} & 8.774738 & 1.219452 & -1.947320 \\ \mathrm{H} & 9.403342 & 1.570761 & 1.763490 \\ \mathrm{H} & 8.294003 & 0.268768 & 2.284826 \\ \mathrm{H} & 7.639314 & 1.802459 & 1.650261 \\ \mathrm{H} & 2.163065 & -1.471653 & -3.530861 \\ \mathrm{H} & 0.478279 & 0.150059 & -4.409310 \\ \mathrm{H} & -2.591849 & -1.757301 & -2.024290 \\ \mathrm{H} & -0.905622 & -3.305632 & -1.092874 \\ \mathrm{H} & -3.824105 & -0.821547 & -3.777194 \\ \mathrm{H} & -3.872319 & 0.948081 & -4.102350 \\ \mathrm{H} & -3.538278 & 0.353629 & -2.435671 \\ \mathrm{~F} & 0.002757 & 5.682904 & -0.003683 \\ \mathrm{~F} & -1.155810 & 6.206538 & -0.489677 \\ & 0.143240 & 6.140837 & 1.272272 \\ & 1.028344 & 6.205704 & -0.729869\end{array}$

Statistical Thermodynamic Analysis

Temperature $=298.150$ Kelvin $\quad$ Pressure $=1.00000$ Atm

\begin{tabular}{|c|c|}
\hline-2634.3662940 & Predicted Change $=-7.418332 \mathrm{D}$ \\
\hline Zero-point correction $(\mathrm{ZPE})=$ & $-2633.5598 \quad 0.80644$ \\
\hline Internal Energy $(U)=$ & -2633.50230 .86391 \\
\hline Enthalpy $(\mathrm{H})=$ & $\begin{array}{lll}-2633.5014 & 0.86486\end{array}$ \\
\hline Gibbs Free Energy $(\mathrm{G})=$ & -2633.6598 \\
\hline
\end{tabular}


$\begin{array}{llll}\text { Frequencies -- } & 6.8859 & 10.1367 & 14.7901\end{array}$

Single points at WB97X-D3/def2-TZVPP: -2637.8050196599

$\mathrm{R}=\mathrm{CF}_{3}$ receptor bound with $\mathrm{Br}-$ (with $\left.\mathrm{TBA}\right)$

Supporting Information: 010-bound_TBA_CF3_Br.log

Using Gaussian 09: EM64L-G09RevE.01 30-Nov-2015

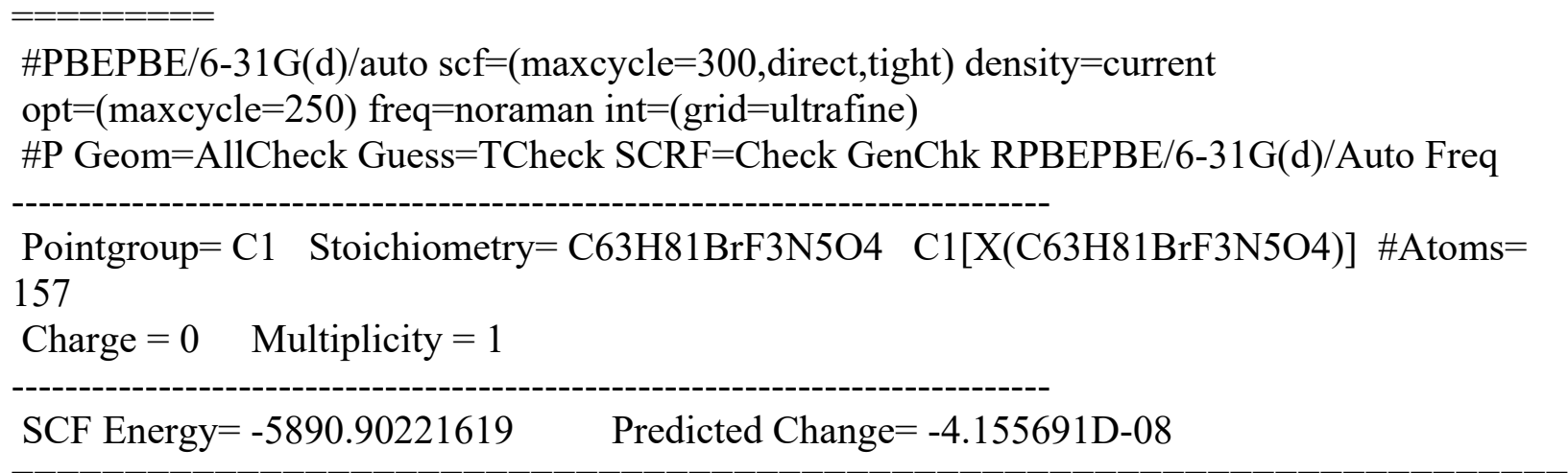

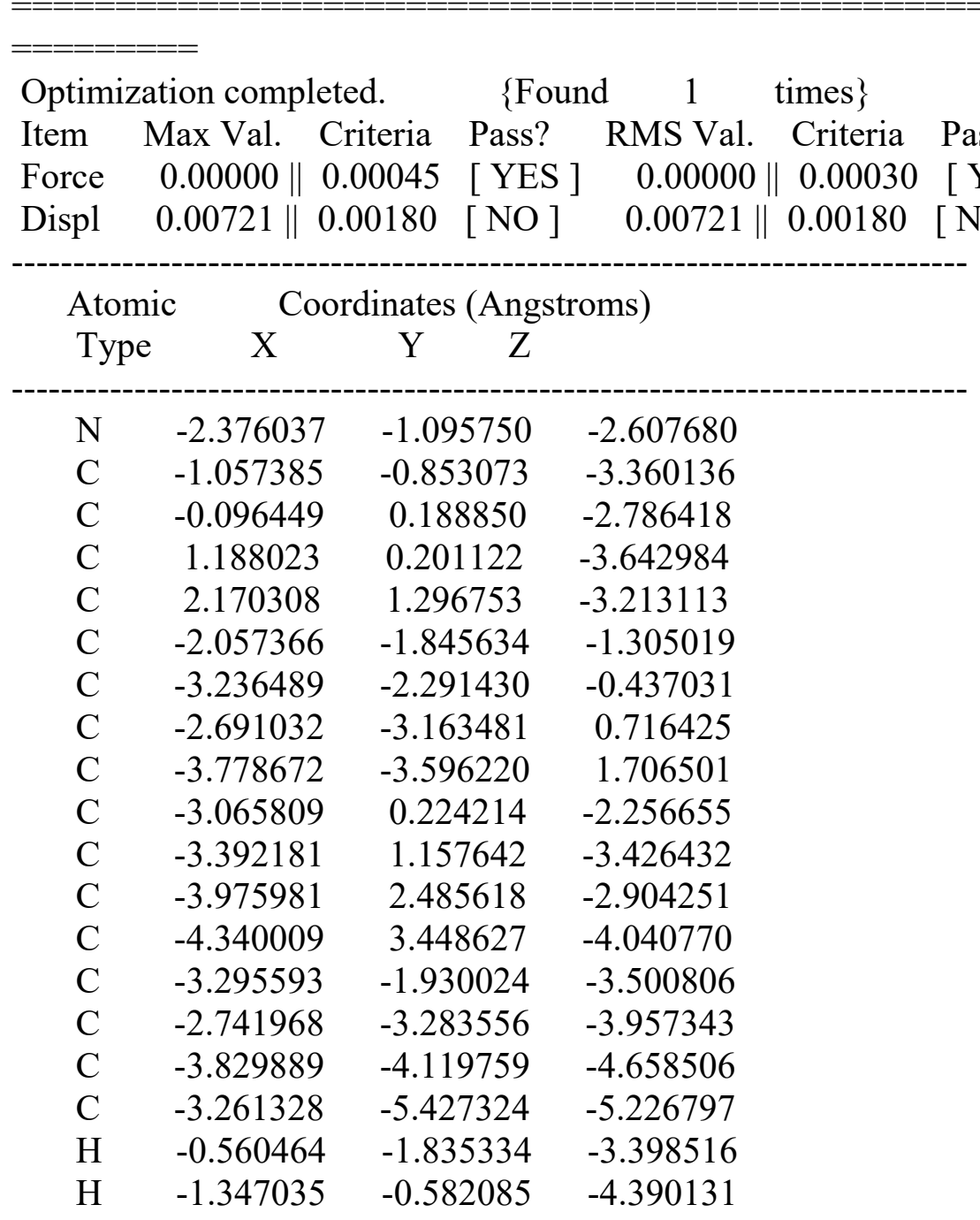




\begin{tabular}{|c|c|c|c|}
\hline $\mathrm{H}$ & 0.168009 & -0.025925 & -1.733420 \\
\hline $\mathrm{H}$ & -0.549332 & 1.197633 & -2.807417 \\
\hline $\mathrm{H}$ & 0.920119 & 0.340839 & -4.709743 \\
\hline $\mathrm{H}$ & 1.679752 & -0.787479 & -3.570398 \\
\hline $\mathrm{H}$ & 3.065579 & 1.293645 & -3.857177 \\
\hline $\mathrm{H}$ & 1.710531 & 2.298918 & -3.277955 \\
\hline $\mathrm{H}$ & 2.508425 & 1.150128 & -2.173998 \\
\hline $\mathrm{H}$ & -1.458483 & -2.717130 & -1.609487 \\
\hline $\mathrm{H}$ & -1.385040 & -1.195427 & -0.717119 \\
\hline $\mathrm{H}$ & -3.769997 & -1.424415 & -0.006646 \\
\hline $\mathrm{H}$ & -3.975049 & -2.885013 & -1.009136 \\
\hline $\mathrm{H}$ & -1.895899 & -2.603212 & 1.241493 \\
\hline $\mathrm{H}$ & -2.206108 & -4.060239 & 0.284034 \\
\hline $\mathrm{H}$ & -3.354067 & -4.256742 & 2.480683 \\
\hline $\mathrm{H}$ & -4.220221 & -2.730870 & 2.228769 \\
\hline $\mathrm{H}$ & -4.590215 & -4.148106 & 1.197673 \\
\hline $\mathrm{H}$ & -3.986898 & -0.050734 & -1.718709 \\
\hline $\mathrm{H}$ & -2.400739 & 0.724199 & -1.534076 \\
\hline $\mathrm{H}$ & -2.486208 & 1.375966 & -4.021096 \\
\hline $\mathrm{H}$ & -4.125391 & 0.694842 & -4.112762 \\
\hline $\mathrm{H}$ & -3.244571 & 2.961776 & -2.224642 \\
\hline $\mathrm{H}$ & -4.870816 & 2.276300 & -2.288152 \\
\hline $\mathrm{H}$ & -4.760844 & 4.386815 & -3.641290 \\
\hline $\mathrm{H}$ & -3.454092 & 3.711814 & -4.645525 \\
\hline $\mathrm{H}$ & -5.091866 & 3.007246 & -4.719308 \\
\hline $\mathrm{H}$ & -3.522674 & -1.302647 & -4.376940 \\
\hline $\mathrm{H}$ & -4.231152 & -2.060825 & -2.933718 \\
\hline $\mathrm{H}$ & -1.897691 & -3.137307 & -4.654831 \\
\hline $\mathrm{H}$ & -2.347328 & -3.865904 & -3.106301 \\
\hline $\mathrm{H}$ & -4.295055 & -3.529429 & -5.471845 \\
\hline $\mathrm{H}$ & -4.637924 & -4.343690 & -3.935914 \\
\hline $\mathrm{H}$ & -4.062093 & -6.060275 & -5.644210 \\
\hline $\mathrm{H}$ & -2.537544 & -5.223990 & -6.036093 \\
\hline $\mathrm{H}$ & -2.732869 & -6.005810 & -4.448589 \\
\hline $\mathrm{O}$ & -0.206590 & -6.724780 & -3.357712 \\
\hline $\mathrm{O}$ & -3.784780 & -0.773191 & 3.980478 \\
\hline $\mathrm{N}$ & 2.551056 & -2.445723 & -0.995491 \\
\hline $\mathrm{N}$ & -2.849909 & 0.456695 & 2.232651 \\
\hline $\mathrm{C}$ & 1.014323 & 3.059860 & 0.355805 \\
\hline $\mathrm{C}$ & 1.338188 & 5.788214 & -0.195198 \\
\hline $\mathrm{C}$ & 0.049896 & 5.275676 & 0.005568 \\
\hline $\mathrm{C}$ & -0.118546 & 3.898616 & 0.289222 \\
\hline $\mathrm{C}$ & 1.955095 & -3.549848 & -1.627492 \\
\hline $\mathrm{C}$ & 2.459703 & -4.168098 & -2.789575 \\
\hline $\mathrm{C}$ & 1.766520 & -5.233124 & -3.387197 \\
\hline $\mathrm{C}$ & 0.559154 & -5.700479 & -2.839363 \\
\hline $\mathrm{C}$ & 0.062982 & -5.098832 & -1.665705 \\
\hline $\mathrm{C}$ & 0.751870 & -4.042348 & -1.067988 \\
\hline
\end{tabular}




\begin{tabular}{|c|c|c|c|}
\hline $\mathrm{C}$ & 0.321243 & -7.412490 & -4.488046 \\
\hline $\mathrm{C}$ & -1.411697 & 3.348280 & 0.517574 \\
\hline $\mathrm{C}$ & -2.520545 & 2.870777 & 0.762787 \\
\hline $\mathrm{C}$ & -3.813758 & 2.364422 & 1.061091 \\
\hline $\mathrm{C}$ & -3.982329 & 1.167501 & 1.826854 \\
\hline $\mathrm{C}$ & -5.302650 & 0.749980 & 2.113285 \\
\hline $\mathrm{C}$ & -6.397546 & 1.485487 & 1.658990 \\
\hline $\mathrm{C}$ & -6.261427 & 2.669430 & 0.896856 \\
\hline $\mathrm{C}$ & -4.954673 & 3.081897 & 0.615666 \\
\hline $\mathrm{C}$ & -8.363407 & 2.525216 & -0.485597 \\
\hline $\mathrm{C}$ & -7.143879 & 4.710779 & -0.374505 \\
\hline $\mathrm{C}$ & -2.790872 & -0.453325 & 3.314184 \\
\hline $\mathrm{C}$ & 3.873406 & -2.040448 & -1.109736 \\
\hline $\mathrm{C}$ & 2.310411 & 3.577955 & 0.142725 \\
\hline $\mathrm{C}$ & 2.465880 & 4.959250 & -0.129580 \\
\hline $\mathrm{C}$ & -7.507290 & 3.442137 & 0.423509 \\
\hline $\mathrm{N}$ & -1.513954 & -0.929037 & 3.513551 \\
\hline $\mathrm{N}$ & 4.133990 & -0.888317 & -0.349989 \\
\hline $\mathrm{O}$ & 4.721264 & -2.617039 & -1.799463 \\
\hline $\mathrm{C}$ & 3.429565 & 2.701296 & 0.177379 \\
\hline $\mathrm{C}$ & -8.346393 & 3.868478 & 1.654110 \\
\hline $\mathrm{C}$ & -1.098463 & -1.909360 & 4.442541 \\
\hline $\mathrm{C}$ & 5.402788 & -0.321357 & -0.165099 \\
\hline $\mathrm{C}$ & 4.376292 & 1.914539 & 0.180454 \\
\hline $\mathrm{C}$ & 0.206574 & -2.430140 & 4.275529 \\
\hline $\mathrm{C}$ & -1.886988 & -2.372116 & 5.511587 \\
\hline $\mathrm{C}$ & 5.514223 & 1.066336 & 0.149427 \\
\hline $\mathrm{C}$ & 6.589060 & -1.080063 & -0.248602 \\
\hline $\mathrm{C}$ & 0.707666 & -3.383801 & 5.157354 \\
\hline $\mathrm{C}$ & -1.379324 & -3.336933 & 6.396140 \\
\hline $\mathrm{C}$ & 6.793974 & 1.630269 & 0.392725 \\
\hline $\mathrm{C}$ & 7.829963 & -0.487547 & -0.016881 \\
\hline $\mathrm{C}$ & -0.081001 & -3.847999 & 6.228972 \\
\hline $\mathrm{C}$ & 7.971090 & 0.879529 & 0.317382 \\
\hline $\mathrm{O}$ & 0.508730 & -4.791206 & 7.039876 \\
\hline $\mathrm{C}$ & 9.366634 & 1.478076 & 0.576748 \\
\hline $\mathrm{C}$ & -0.260212 & -5.268571 & 8.136714 \\
\hline $\mathrm{C}$ & 9.305217 & 2.979819 & 0.921708 \\
\hline $\mathrm{C}$ & 10.030163 & 0.734291 & 1.762805 \\
\hline $\mathrm{C}$ & 10.242392 & 1.306143 & -0.689513 \\
\hline $\mathrm{H}$ & 1.949788 & -1.956881 & -0.310332 \\
\hline $\mathrm{H}$ & -1.962152 & 0.733416 & 1.796722 \\
\hline $\mathrm{H}$ & 0.887384 & 1.992460 & 0.567635 \\
\hline $\mathrm{H}$ & -0.821577 & 5.932698 & -0.052967 \\
\hline $\mathrm{H}$ & 3.405646 & -3.821582 & -3.208045 \\
\hline $\mathrm{H}$ & 2.188130 & -5.689253 & -4.286854 \\
\hline $\mathrm{H}$ & -0.853971 & -5.496444 & -1.217172 \\
\hline $\mathrm{H}$ & 0.368055 & -3.575605 & -0.153331 \\
\hline
\end{tabular}




\begin{tabular}{|c|c|c|c|}
\hline $\mathrm{H}$ & -0.400299 & -8.210131 & -4.719343 \\
\hline $\mathrm{H}$ & 0.420697 & -6.744836 & -5.366686 \\
\hline $\mathrm{H}$ & 1.307741 & -7.862874 & -4.266109 \\
\hline $\mathrm{H}$ & -5.445824 & -0.141115 & 2.723925 \\
\hline $\mathrm{H}$ & -4.768057 & 3.996580 & 0.047119 \\
\hline $\mathrm{H}$ & -6.551433 & 5.419704 & 0.229761 \\
\hline $\mathrm{H}$ & -6.570199 & 4.472647 & -1.288483 \\
\hline $\mathrm{H}$ & -7.397936 & 1.116980 & 1.913157 \\
\hline $\mathrm{H}$ & -8.677614 & 1.606617 & 0.038729 \\
\hline $\mathrm{H}$ & -7.798415 & 2.223818 & -1.385909 \\
\hline $\mathrm{H}$ & -9.276300 & 3.053354 & -0.815626 \\
\hline $\mathrm{H}$ & -8.066243 & 5.229807 & -0.687854 \\
\hline $\mathrm{H}$ & 3.463563 & 5.370735 & -0.302495 \\
\hline $\mathrm{H}$ & -7.766225 & 4.536110 & 2.314036 \\
\hline $\mathrm{H}$ & -8.666541 & 2.999155 & 2.253039 \\
\hline $\mathrm{H}$ & -9.255308 & 4.407598 & 1.330961 \\
\hline $\mathrm{H}$ & 0.816230 & -2.079710 & 3.433960 \\
\hline $\mathrm{H}$ & -2.895485 & -1.978865 & 5.642580 \\
\hline $\mathrm{H}$ & 6.521229 & -2.136100 & -0.509730 \\
\hline $\mathrm{H}$ & 1.716469 & -3.788229 & 5.031132 \\
\hline $\mathrm{H}$ & -2.015297 & -3.676518 & 7.217839 \\
\hline $\mathrm{H}$ & 6.823966 & 2.695082 & 0.636548 \\
\hline $\mathrm{H}$ & 8.720180 & -1.122422 & -0.089533 \\
\hline $\mathrm{H}$ & 0.377960 & -5.998529 & 8.657072 \\
\hline $\mathrm{H}$ & -0.527955 & -4.452969 & 8.837018 \\
\hline $\mathrm{H}$ & -1.189181 & -5.770364 & 7.800645 \\
\hline $\mathrm{H}$ & 10.325528 & 3.361859 & 1.099422 \\
\hline $\mathrm{H}$ & 8.715376 & 3.166826 & 1.836153 \\
\hline $\mathrm{H}$ & 8.866257 & 3.571984 & 0.099557 \\
\hline $\mathrm{H}$ & 9.435300 & 0.852584 & 2.685018 \\
\hline $\mathrm{H}$ & 11.041619 & 1.138007 & 1.951013 \\
\hline $\mathrm{H}$ & 10.131559 & -0.346198 & 1.563678 \\
\hline $\mathrm{H}$ & 11.254957 & 1.712512 & -0.513320 \\
\hline $\mathrm{H}$ & 9.801992 & 1.840267 & -1.549305 \\
\hline $\mathrm{H}$ & 10.350881 & 0.245138 & -0.972017 \\
\hline $\mathrm{H}$ & 3.334535 & -0.383877 & 0.052267 \\
\hline $\mathrm{H}$ & -0.800364 & -0.688545 & 2.805723 \\
\hline $\mathrm{C}$ & 1.517510 & 7.269441 & -0.427194 \\
\hline $\mathrm{F}$ & 0.444242 & 7.814835 & -1.066715 \\
\hline $\mathrm{F}$ & 2.620522 & 7.535395 & -1.180299 \\
\hline $\mathrm{F}$ & 1.664951 & 7.946252 & 0.746541 \\
\hline $\mathrm{Br}$ & 0.530296 & -0.554313 & 0.883711 \\
\hline
\end{tabular}

Statistical Thermodynamic Analysis

Temperature $=298.150$ Kelvin $\quad$ Pressure $=1.00000$ Atm

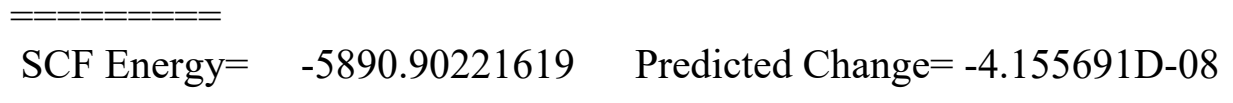


Zero-point correction $(\mathrm{ZPE})=$

Internal Energy $(\mathrm{U})=$

Enthalpy $(\mathrm{H})=$

Gibbs Free Energy $(\mathrm{G})=$
$-5889.59731 .30488$

$-5889.51281 .38939$

$-5889.51181 .39033$

$-5889.73121 .17093$

$\begin{array}{llll}\text { Frequencies -- } & 6.4756 & 7.7018 & 11.1890\end{array}$

Single points at WB97X-D3/def2-TZVPP: -5898.31675005218

$\mathrm{R}=\mathrm{CF}_{3}$ receptor bound with $\mathrm{Cl}-$ (with $\left.\mathrm{TBA}\right)$

Supporting Information: 010-bound_TBA_CF3_Cl.log

Using Gaussian 09: EM64L-G09RevE.01 30-Nov-2015

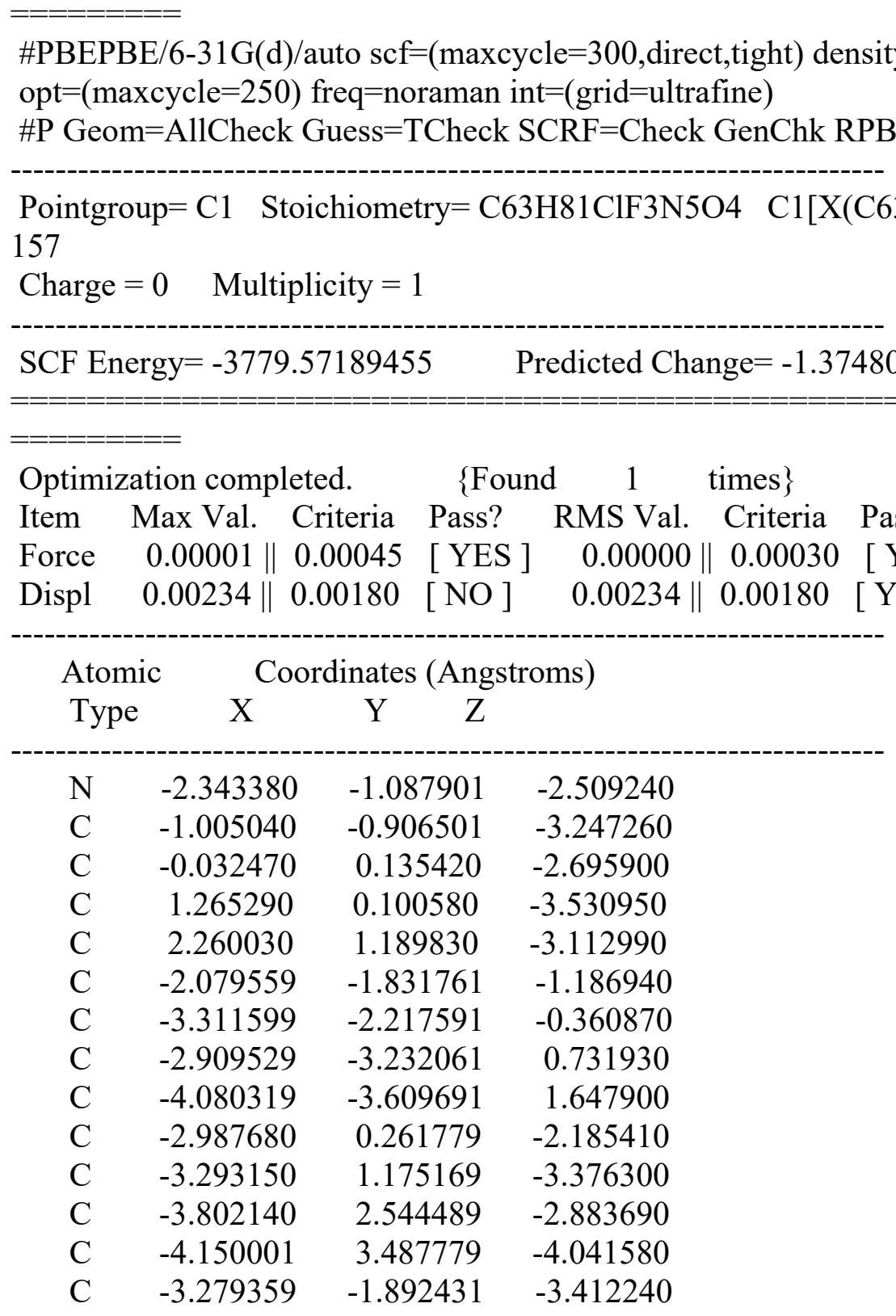




\begin{tabular}{|c|c|c|c|}
\hline $\mathrm{C}$ & -2.816689 & -3.307001 & -3.771380 \\
\hline $\mathrm{C}$ & -3.897929 & -4.048961 & -4.580870 \\
\hline $\mathrm{C}$ & -3.411308 & -5.425471 & -5.053780 \\
\hline $\mathrm{H}$ & -0.529499 & -1.900260 & -3.239440 \\
\hline $\mathrm{H}$ & -1.270380 & -0.668361 & -4.291680 \\
\hline $\mathrm{H}$ & 0.207230 & -0.056480 & -1.633380 \\
\hline $\mathrm{H}$ & -0.465660 & 1.151040 & -2.755760 \\
\hline $\mathrm{H}$ & 1.017180 & 0.217620 & -4.605260 \\
\hline $\mathrm{H}$ & 1.736740 & -0.894590 & -3.424800 \\
\hline $\mathrm{H}$ & 3.167180 & 1.152541 & -3.739130 \\
\hline $\mathrm{H}$ & 1.820060 & 2.197860 & -3.214770 \\
\hline $\mathrm{H}$ & 2.575300 & 1.066590 & -2.063650 \\
\hline $\mathrm{H}$ & -1.506099 & -2.729091 & -1.464980 \\
\hline $\mathrm{H}$ & -1.399850 & -1.191061 & -0.596260 \\
\hline $\mathrm{H}$ & -3.761649 & -1.329751 & 0.118790 \\
\hline $\mathrm{H}$ & -4.096379 & -2.684711 & -0.985880 \\
\hline $\mathrm{H}$ & -2.085349 & -2.813411 & 1.336970 \\
\hline $\mathrm{H}$ & -2.509799 & -4.139891 & 0.240790 \\
\hline $\mathrm{H}$ & -3.767799 & -4.377081 & 2.375620 \\
\hline $\mathrm{H}$ & -4.432369 & -2.738801 & 2.225970 \\
\hline $\mathrm{H}$ & -4.930029 & -4.016702 & 1.069300 \\
\hline $\mathrm{H}$ & -3.913070 & 0.029299 & -1.634930 \\
\hline $\mathrm{H}$ & -2.302110 & 0.756139 & -1.478630 \\
\hline $\mathrm{H}$ & -2.390710 & 1.331139 & -3.995720 \\
\hline $\mathrm{H}$ & -4.061720 & 0.726149 & -4.032400 \\
\hline $\mathrm{H}$ & -3.033171 & 3.007419 & -2.237140 \\
\hline $\mathrm{H}$ & -4.689730 & 2.397509 & -2.239890 \\
\hline $\mathrm{H}$ & -4.516171 & 4.456819 & -3.662280 \\
\hline $\mathrm{H}$ & -3.268931 & 3.689759 & -4.676300 \\
\hline $\mathrm{H}$ & -4.939141 & 3.061688 & -4.686760 \\
\hline $\mathrm{H}$ & -3.404509 & -1.293761 & -4.328230 \\
\hline $\mathrm{H}$ & -4.254019 & -1.919011 & -2.899340 \\
\hline $\mathrm{H}$ & -1.884819 & -3.271011 & -4.363840 \\
\hline $\mathrm{H}$ & -2.589379 & -3.895941 & -2.865670 \\
\hline $\mathrm{H}$ & -4.199869 & -3.439481 & -5.454810 \\
\hline $\mathrm{H}$ & -4.804079 & -4.165511 & -3.955930 \\
\hline $\mathrm{H}$ & -4.222298 & -5.985501 & -5.548460 \\
\hline $\mathrm{H}$ & -2.583068 & -5.323741 & -5.777640 \\
\hline $\mathrm{H}$ & -3.038628 & -6.029961 & -4.208410 \\
\hline $\mathrm{O}$ & -0.475518 & -6.716090 & -3.075760 \\
\hline $\mathrm{O}$ & -3.896490 & -0.730601 & 3.718550 \\
\hline $\mathrm{N}$ & 2.400581 & -2.458410 & -0.803100 \\
\hline $\mathrm{N}$ & -2.835120 & 0.638229 & 2.159470 \\
\hline $\mathrm{C}$ & 1.211509 & 3.053800 & 0.480600 \\
\hline $\mathrm{C}$ & 1.689389 & 5.781370 & 0.025420 \\
\hline $\mathrm{C}$ & 0.373679 & 5.332050 & 0.194960 \\
\hline $\mathrm{C}$ & 0.127669 & 3.957200 & 0.429170 \\
\hline $\mathrm{C}$ & 1.752381 & -3.549420 & -1.411980 \\
\hline
\end{tabular}




\begin{tabular}{|c|c|c|c|}
\hline $\mathrm{C}$ & 2.134531 & -4.077220 & -2.662830 \\
\hline $\mathrm{C}$ & 1.418522 & -5.135790 & -3.243750 \\
\hline $\mathrm{C}$ & 0.302562 & -5.686470 & -2.588870 \\
\hline $\mathrm{C}$ & -0.075898 & -5.172110 & -1.333030 \\
\hline $\mathrm{C}$ & 0.640781 & -4.123300 & -0.751890 \\
\hline $\mathrm{C}$ & -0.030978 & -7.345830 & -4.273770 \\
\hline $\mathrm{C}$ & -1.200251 & 3.479369 & 0.616410 \\
\hline $\mathrm{C}$ & -2.349251 & 3.078019 & 0.806580 \\
\hline $\mathrm{C}$ & -3.675840 & 2.629069 & 1.042580 \\
\hline $\mathrm{C}$ & -3.925130 & 1.406089 & 1.743540 \\
\hline $\mathrm{C}$ & -5.271870 & 1.027928 & 1.949570 \\
\hline $\mathrm{C}$ & -6.315270 & 1.828848 & 1.483790 \\
\hline $\mathrm{C}$ & -6.099371 & 3.042878 & 0.790610 \\
\hline $\mathrm{C}$ & -4.765921 & 3.414779 & 0.587300 \\
\hline $\mathrm{C}$ & -8.147671 & 3.051968 & -0.678250 \\
\hline $\mathrm{C}$ & -6.843091 & 5.174698 & -0.418840 \\
\hline $\mathrm{C}$ & -2.858380 & -0.381941 & 3.137220 \\
\hline $\mathrm{C}$ & 3.747941 & -2.152129 & -0.953690 \\
\hline $\mathrm{C}$ & 2.535779 & 3.510680 & 0.297420 \\
\hline $\mathrm{C}$ & 2.768769 & 4.889990 & 0.073720 \\
\hline $\mathrm{C}$ & -7.291711 & 3.888978 & 0.305010 \\
\hline $\mathrm{N}$ & -1.610330 & -0.928711 & 3.340180 \\
\hline $\mathrm{N}$ & 4.112720 & -1.005709 & -0.232750 \\
\hline $\mathrm{O}$ & 4.534731 & -2.808289 & -1.644540 \\
\hline $\mathrm{C}$ & 3.611380 & 2.580811 & 0.308680 \\
\hline $\mathrm{C}$ & -8.161831 & 4.295648 & 1.520650 \\
\hline $\mathrm{C}$ & -1.280139 & -1.986551 & 4.218360 \\
\hline $\mathrm{C}$ & 5.419890 & -0.520649 & -0.093760 \\
\hline $\mathrm{C}$ & 4.532810 & 1.764871 & 0.284730 \\
\hline $\mathrm{C}$ & 0.025621 & -2.521910 & 4.112000 \\
\hline $\mathrm{C}$ & -2.154419 & -2.518081 & 5.185340 \\
\hline $\mathrm{C}$ & 5.622590 & 0.858281 & 0.216190 \\
\hline $\mathrm{C}$ & 6.555961 & -1.347439 & -0.217140 \\
\hline $\mathrm{C}$ & 0.440941 & -3.557380 & 4.945390 \\
\hline $\mathrm{C}$ & -1.732389 & -3.562451 & 6.023270 \\
\hline $\mathrm{C}$ & 6.939880 & 1.347102 & 0.414830 \\
\hline $\mathrm{C}$ & 7.836160 & -0.827238 & -0.027820 \\
\hline $\mathrm{C}$ & -0.435049 & -4.090450 & 5.911540 \\
\hline $\mathrm{C}$ & 8.068070 & 0.529022 & 0.299900 \\
\hline $\mathrm{O}$ & 0.074052 & -5.110920 & 6.682190 \\
\hline $\mathrm{C}$ & 9.504190 & 1.045242 & 0.510320 \\
\hline $\mathrm{C}$ & -0.782578 & -5.653980 & 7.679030 \\
\hline $\mathrm{C}$ & 9.541930 & 2.548012 & 0.854150 \\
\hline $\mathrm{C}$ & 10.163440 & 0.264132 & 1.674550 \\
\hline $\mathrm{C}$ & 10.324770 & 0.822752 & -0.784700 \\
\hline $\mathrm{H}$ & 1.843011 & -1.925600 & -0.110630 \\
\hline $\mathrm{H}$ & -1.915850 & 0.970569 & 1.847360 \\
\hline $\mathrm{H}$ & 1.025590 & 1.988550 & 0.661800 \\
\hline
\end{tabular}




\begin{tabular}{|c|c|c|c|}
\hline $\mathrm{H}$ & -0.461121 & 6.035990 & 0.148930 \\
\hline $\mathrm{H}$ & 3.014241 & -3.672139 & -3.165220 \\
\hline $\mathrm{H}$ & 1.750112 & -5.522520 & -4.211080 \\
\hline $\mathrm{H}$ & -0.918698 & -5.633440 & -0.807870 \\
\hline $\mathrm{H}$ & 0.350801 & -3.744110 & 0.234300 \\
\hline $\mathrm{H}$ & -0.733278 & -8.172370 & -4.458120 \\
\hline $\mathrm{H}$ & -0.052488 & -6.651830 & -5.137430 \\
\hline $\mathrm{H}$ & 0.993292 & -7.750720 & -4.164180 \\
\hline $\mathrm{H}$ & -5.476670 & 0.115388 & 2.508760 \\
\hline $\mathrm{H}$ & -4.518061 & 4.344979 & 0.069560 \\
\hline $\mathrm{H}$ & -6.247941 & 5.830238 & 0.240500 \\
\hline $\mathrm{H}$ & -6.241541 & 4.952228 & -1.318680 \\
\hline $\mathrm{H}$ & -7.339730 & 1.489908 & 1.675920 \\
\hline $\mathrm{H}$ & -8.521360 & 2.125538 & -0.209740 \\
\hline $\mathrm{H}$ & -7.559701 & 2.766858 & -1.569120 \\
\hline $\mathrm{H}$ & -9.023431 & 3.633767 & -1.018500 \\
\hline $\mathrm{H}$ & -7.729191 & 5.746328 & -0.745020 \\
\hline $\mathrm{H}$ & 3.789469 & 5.251261 & -0.075350 \\
\hline $\mathrm{H}$ & -7.582001 & 4.907848 & 2.232600 \\
\hline $\mathrm{H}$ & -8.540871 & 3.414708 & 2.065930 \\
\hline $\mathrm{H}$ & -9.034251 & 4.886887 & 1.188160 \\
\hline $\mathrm{H}$ & 0.713371 & -2.111580 & 3.363790 \\
\hline $\mathrm{H}$ & -3.162829 & -2.114091 & 5.275290 \\
\hline $\mathrm{H}$ & 6.417251 & -2.397069 & -0.475920 \\
\hline $\mathrm{H}$ & 1.450881 & -3.970600 & 4.864630 \\
\hline $\mathrm{H}$ & -2.435279 & -3.951041 & 6.764910 \\
\hline $\mathrm{H}$ & 7.040010 & 2.408192 & 0.656260 \\
\hline $\mathrm{H}$ & 8.685251 & -1.512348 & -0.130840 \\
\hline $\mathrm{H}$ & -0.198368 & -6.438090 & 8.183730 \\
\hline $\mathrm{H}$ & -1.085779 & -4.889551 & 8.421300 \\
\hline $\mathrm{H}$ & -1.693558 & -6.104891 & 7.237950 \\
\hline $\mathrm{H}$ & 10.588139 & 2.870142 & 0.996380 \\
\hline $\mathrm{H}$ & 8.995349 & 2.769002 & 1.787710 \\
\hline $\mathrm{H}$ & 9.110349 & 3.164602 & 0.046170 \\
\hline $\mathrm{H}$ & 9.608570 & 0.417352 & 2.616350 \\
\hline $\mathrm{H}$ & 11.202740 & 0.607763 & 1.827100 \\
\hline $\mathrm{H}$ & 10.194230 & -0.820478 & 1.474450 \\
\hline $\mathrm{H}$ & 11.364640 & 1.169743 & -0.644090 \\
\hline $\mathrm{H}$ & 9.886870 & 1.381472 & -1.630030 \\
\hline $\mathrm{H}$ & 10.361770 & -0.242788 & -1.068560 \\
\hline $\mathrm{H}$ & 3.363610 & -0.424699 & 0.160090 \\
\hline $\mathrm{H}$ & -0.859040 & -0.675580 & 2.672150 \\
\hline $\mathrm{C}$ & 1.950898 & 7.257610 & -0.153370 \\
\hline $\mathrm{Cl}$ & 0.532410 & -0.460430 & 0.947150 \\
\hline $\mathrm{F}$ & 0.918068 & 7.881010 & -0.788070 \\
\hline $\mathrm{F}$ & 3.077908 & 7.489021 & -0.882000 \\
\hline $\mathrm{F}$ & 2.116278 & 7.885990 & 1.044780 \\
\hline
\end{tabular}


Statistical Thermodynamic Analysis

Temperature $=298.150$ Kelvin $\quad$ Pressure $=1.00000$ Atm

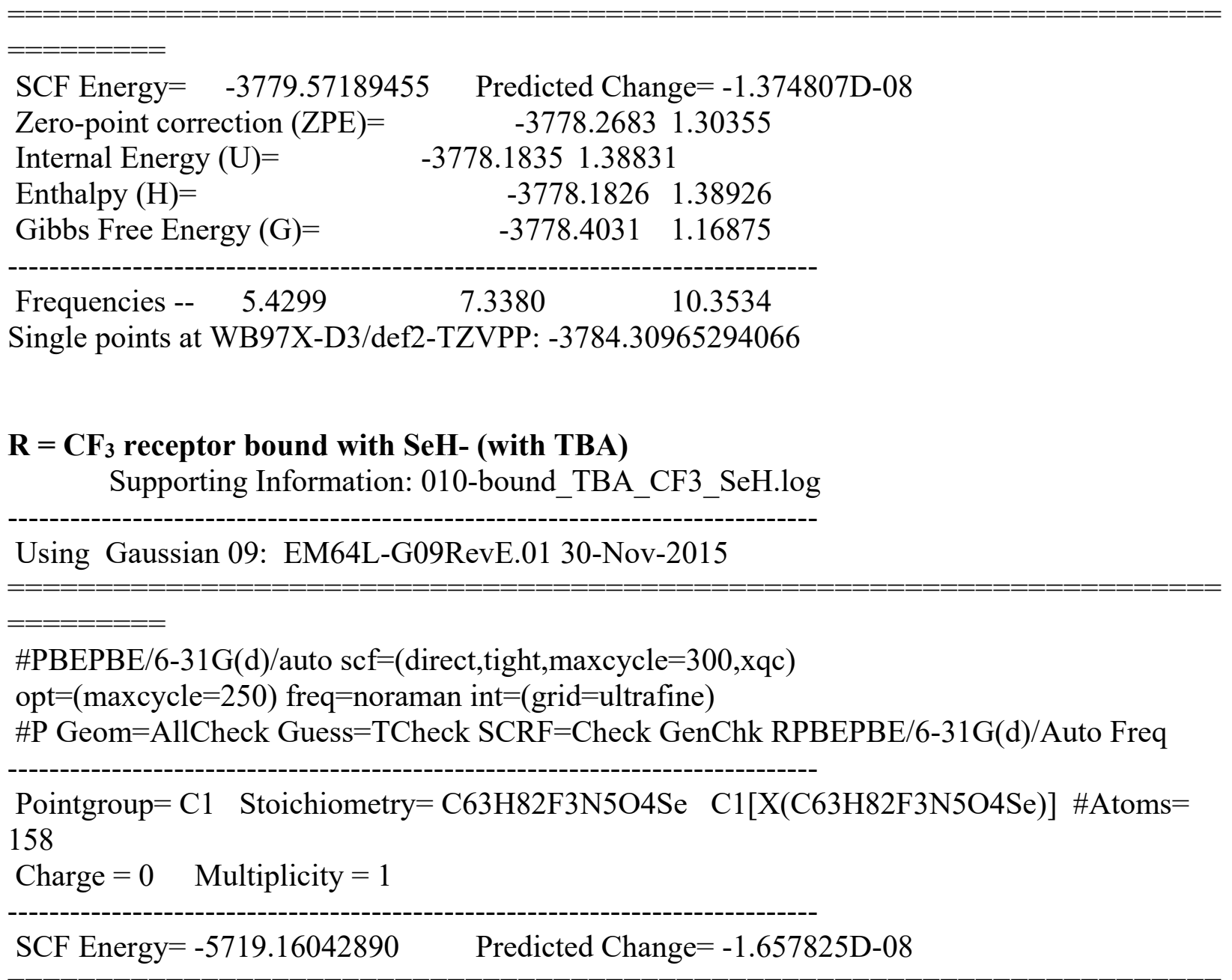

\begin{tabular}{|c|c|c|c|c|}
\hline \multicolumn{3}{|c|}{ Optimization completed. } & $\{$ Foun & nd $\quad 1 \quad$ times $\}$ \\
\hline \multicolumn{3}{|c|}{ Item Max Val. Criteria } & Pass? & RMS Val. Criteria \\
\hline Force & $0.00001 \|$ & 0.00045 & [ YES ] & 0.00000|| 0.00030 \\
\hline Displ & $0.00399 \|$ & 0.00180 & {$[\mathrm{NO}]$} & $0.00399 \| 0.00180$ \\
\hline \multicolumn{2}{|c|}{ Atomic } & \multicolumn{3}{|c|}{ Coordinates (Angstroms) } \\
\hline Type & X & $\mathrm{Y}$ & Z & \\
\hline $\mathrm{N}$ & -3.095285 & 2.49 & 8386 & 0.598679 \\
\hline $\mathrm{C}$ & -1.895949 & 3.13 & 5170 & 1.321483 \\
\hline $\mathrm{C}$ & -0.772090 & 2.20 & 7944 & 1.788857 \\
\hline $\mathrm{C}$ & 0.313830 & 3.06 & 5512 & 2.475579 \\
\hline $\mathrm{C}$ & 1.466267 & 2.22 & 3012 & 3.033858 \\
\hline $\mathrm{C}$ & -2.617262 & 1.99 & 7403 & -0.767120 \\
\hline $\mathrm{C}$ & -3.641668 & 1.32 & 2636 & -1.678523 \\
\hline $\mathrm{C}$ & -2.937438 & 1.01 & 2368 & -3.019122 \\
\hline $\mathrm{C}$ & -3.822163 & 0.21 & 9805 & -3.985700 \\
\hline
\end{tabular}




\begin{tabular}{|c|c|c|c|}
\hline $\mathrm{C}$ & -3.657992 & 1.319698 & 1.391995 \\
\hline $\mathrm{C}$ & -4.081103 & 1.608543 & 2.834357 \\
\hline $\mathrm{C}$ & -4.648591 & 0.331877 & 3.487936 \\
\hline $\mathrm{C}$ & -5.044606 & 0.552640 & 4.952870 \\
\hline $\mathrm{C}$ & -4.184789 & 3.563457 & 0.432147 \\
\hline $\mathrm{C}$ & -3.876099 & 4.688406 & -0.560833 \\
\hline $\mathrm{C}$ & -4.867635 & 5.858325 & -0.414788 \\
\hline $\mathrm{C}$ & -4.613780 & 6.947081 & -1.466726 \\
\hline $\mathrm{H}$ & -1.487377 & 3.878103 & 0.618275 \\
\hline $\mathrm{H}$ & -2.322538 & 3.677965 & 2.181796 \\
\hline $\mathrm{H}$ & -0.315039 & 1.651974 & 0.948281 \\
\hline $\mathrm{H}$ & -1.146464 & 1.458015 & 2.510166 \\
\hline $\mathrm{H}$ & -0.146920 & 3.654245 & 3.294149 \\
\hline $\mathrm{H}$ & 0.711101 & 3.797285 & 1.747159 \\
\hline $\mathrm{H}$ & 2.209065 & 2.864667 & 3.536743 \\
\hline $\mathrm{H}$ & 1.107409 & 1.480821 & 3.768677 \\
\hline $\mathrm{H}$ & 1.989830 & 1.675790 & 2.232370 \\
\hline $\mathrm{H}$ & -2.193174 & 2.883839 & -1.261966 \\
\hline $\mathrm{H}$ & -1.773717 & 1.303237 & -0.596455 \\
\hline $\mathrm{H}$ & -3.999320 & 0.372686 & -1.241397 \\
\hline $\mathrm{H}$ & -4.530114 & 1.959100 & -1.859772 \\
\hline $\mathrm{H}$ & -2.002466 & 0.465219 & -2.791622 \\
\hline $\mathrm{H}$ & -2.627676 & 1.966138 & -3.490987 \\
\hline $\mathrm{H}$ & -3.281370 & 0.000177 & -4.921284 \\
\hline $\mathrm{H}$ & -4.133101 & -0.742808 & -3.544767 \\
\hline $\mathrm{H}$ & -4.736057 & 0.782980 & -4.249364 \\
\hline $\mathrm{H}$ & -4.517752 & 0.951394 & 0.810092 \\
\hline $\mathrm{H}$ & -2.886261 & 0.533486 & 1.361742 \\
\hline $\mathrm{H}$ & -3.220190 & 1.960950 & 3.431469 \\
\hline $\mathrm{H}$ & -4.850626 & 2.401834 & 2.875857 \\
\hline $\mathrm{H}$ & -3.897437 & -0.476940 & 3.416442 \\
\hline $\mathrm{H}$ & -5.528245 & -0.013351 & 2.910528 \\
\hline $\mathrm{H}$ & -5.452859 & -0.371477 & 5.394611 \\
\hline $\mathrm{H}$ & -4.174839 & 0.857544 & 5.561074 \\
\hline $\mathrm{H}$ & -5.814841 & 1.338673 & 5.048691 \\
\hline $\mathrm{H}$ & -4.358423 & 3.978265 & 1.438026 \\
\hline $\mathrm{H}$ & -5.097590 & 3.023661 & 0.130836 \\
\hline $\mathrm{H}$ & -2.853804 & 5.081276 & -0.418821 \\
\hline $\mathrm{H}$ & -3.924854 & 4.303520 & -1.595669 \\
\hline $\mathrm{H}$ & -4.774867 & 6.287932 & 0.601268 \\
\hline $\mathrm{H}$ & -5.906995 & 5.486566 & -0.502062 \\
\hline $\mathrm{H}$ & -5.269137 & 7.818563 & -1.301929 \\
\hline $\mathrm{H}$ & -3.565076 & 7.293293 & -1.440689 \\
\hline $\mathrm{H}$ & -4.810606 & 6.567893 & -2.485014 \\
\hline $\mathrm{O}$ & -0.720085 & 7.411530 & -2.135606 \\
\hline $\mathrm{O}$ & -2.040006 & -4.024869 & -3.505544 \\
\hline $\mathrm{N}$ & 2.339451 & 2.879325 & -0.895157 \\
\hline $\mathrm{N}$ & -2.141927 & -2.502025 & -1.735030 \\
\hline
\end{tabular}




\begin{tabular}{|c|c|c|c|}
\hline $\mathrm{C}$ & 0.652500 & -1.625876 & 2.048874 \\
\hline $\mathrm{C}$ & 0.518463 & -2.377322 & 4.741774 \\
\hline $\mathrm{C}$ & -0.648594 & -2.386810 & 3.966548 \\
\hline $\mathrm{C}$ & -0.587406 & -2.016256 & 2.600908 \\
\hline $\mathrm{C}$ & 1.675083 & 4.073108 & -1.203745 \\
\hline $\mathrm{C}$ & 2.185554 & 5.365788 & -0.968179 \\
\hline $\mathrm{C}$ & 1.412447 & 6.498628 & -1.271670 \\
\hline $\mathrm{C}$ & 0.124599 & 6.365780 & -1.819092 \\
\hline $\mathrm{C}$ & -0.377216 & 5.074601 & -2.079263 \\
\hline C & 0.385182 & 3.947186 & -1.775251 \\
\hline $\mathrm{C}$ & -0.205816 & 8.727774 & -1.957062 \\
\hline $\mathrm{C}$ & -1.741164 & -2.070111 & 1.767409 \\
\hline $\mathrm{C}$ & -2.702111 & -2.213644 & 1.008178 \\
\hline $\mathrm{C}$ & -3.749182 & -2.491054 & 0.088925 \\
\hline $\mathrm{C}$ & -3.436254 & -2.738841 & -1.287252 \\
\hline $\mathrm{C}$ & -4.474327 & -3.197587 & -2.129521 \\
\hline $\mathrm{C}$ & -5.770841 & -3.347830 & -1.637866 \\
\hline $\mathrm{C}$ & -6.121301 & -3.053675 & -0.297501 \\
\hline C & -5.085729 & -2.633684 & 0.544436 \\
\hline $\mathrm{C}$ & -8.499197 & -2.299221 & -0.660010 \\
\hline $\mathrm{C}$ & -7.751563 & -2.846967 & 1.666670 \\
\hline $\mathrm{C}$ & -1.485282 & -3.182170 & -2.791231 \\
\hline $\mathrm{C}$ & 3.656305 & 2.711136 & -0.496831 \\
\hline $\mathrm{C}$ & 1.827064 & -1.613106 & 2.832358 \\
\hline $\mathrm{C}$ & 1.751591 & -2.001854 & 4.192079 \\
\hline $\mathrm{C}$ & -7.576752 & -3.217808 & 0.179998 \\
\hline $\mathrm{N}$ & -0.175652 & -2.771607 & -2.880193 \\
\hline $\mathrm{N}$ & 3.928388 & 1.361889 & -0.215879 \\
\hline $\mathrm{O}$ & 4.484779 & 3.621662 & -0.388377 \\
\hline $\mathrm{C}$ & 3.045630 & -1.176205 & 2.243845 \\
\hline $\mathrm{C}$ & -8.017138 & -4.691552 & -0.005757 \\
\hline $\mathrm{C}$ & 0.849644 & -3.251656 & -3.714857 \\
\hline $\mathrm{C}$ & 5.176523 & 0.875580 & 0.197826 \\
\hline $\mathrm{C}$ & 4.058124 & -0.752412 & 1.687625 \\
\hline $\mathrm{C}$ & 2.128132 & -2.674059 & -3.525238 \\
\hline $\mathrm{C}$ & 0.681076 & -4.246280 & -4.693871 \\
\hline $\mathrm{C}$ & 5.240234 & -0.223548 & 1.105075 \\
\hline $\mathrm{C}$ & 6.387745 & 1.429044 & -0.263468 \\
\hline $\mathrm{C}$ & 3.209576 & -3.083374 & -4.299351 \\
\hline $\mathrm{C}$ & 1.776063 & -4.657736 & -5.470414 \\
\hline $\mathrm{C}$ & 6.501949 & -0.752116 & 1.480601 \\
\hline $\mathrm{C}$ & 7.611524 & 0.896574 & 0.141978 \\
\hline $\mathrm{C}$ & 3.044135 & -4.081609 & -5.280990 \\
\hline $\mathrm{C}$ & 7.705779 & -0.213514 & 1.013878 \\
\hline $\mathrm{O}$ & 4.177793 & -4.414166 & -5.989508 \\
\hline $\mathrm{C}$ & 9.084503 & -0.774048 & 1.412279 \\
\hline $\mathrm{C}$ & 4.043141 & -5.423103 & -6.981826 \\
\hline $\mathrm{C}$ & 8.973392 & -1.972897 & 2.375856 \\
\hline
\end{tabular}




\begin{tabular}{|c|c|c|c|}
\hline $\mathrm{C}$ & 9.833164 & -1.242298 & 0.139232 \\
\hline $\mathrm{C}$ & 9.908221 & 0.335963 & 2.111707 \\
\hline $\mathrm{H}$ & 1.767481 & 2.018875 & -1.010779 \\
\hline $\mathrm{H}$ & -1.568446 & -1.863516 & -1.167572 \\
\hline $\mathrm{H}$ & 0.713222 & -1.308922 & 1.003433 \\
\hline $\mathrm{H}$ & -1.601955 & -2.688864 & 4.407858 \\
\hline $\mathrm{H}$ & 3.189548 & 5.470893 & -0.553987 \\
\hline $\mathrm{H}$ & 1.836857 & 7.486116 & -1.071729 \\
\hline $\mathrm{H}$ & -1.360069 & 4.979178 & -2.553517 \\
\hline $\mathrm{H}$ & 0.000063 & 2.934947 & -1.949664 \\
\hline $\mathrm{H}$ & -1.000698 & 9.410262 & -2.293095 \\
\hline $\mathrm{H}$ & 0.030520 & 8.936963 & -0.895122 \\
\hline $\mathrm{H}$ & 0.703271 & 8.895957 & -2.566024 \\
\hline $\mathrm{H}$ & -4.231664 & -3.445790 & -3.163805 \\
\hline $\mathrm{H}$ & -5.272599 & -2.436817 & 1.603586 \\
\hline $\mathrm{H}$ & -7.146297 & -3.493900 & 2.325504 \\
\hline $\mathrm{H}$ & -7.472712 & -1.795614 & 1.863034 \\
\hline $\mathrm{H}$ & -6.541833 & -3.700358 & -2.332352 \\
\hline $\mathrm{H}$ & -8.440390 & -2.533638 & -1.736404 \\
\hline $\mathrm{H}$ & -8.223028 & -1.237699 & -0.531210 \\
\hline $\mathrm{H}$ & -9.551969 & -2.420637 & -0.346657 \\
\hline $\mathrm{H}$ & -8.808668 & -2.970275 & 1.959325 \\
\hline $\mathrm{H}$ & 2.651735 & -1.989791 & 4.811707 \\
\hline $\mathrm{H}$ & -7.385685 & -5.369943 & 0.593375 \\
\hline $\mathrm{H}$ & -7.946292 & -5.009895 & -1.059551 \\
\hline $\mathrm{H}$ & -9.066375 & -4.822589 & 0.315579 \\
\hline $\mathrm{H}$ & 2.241066 & -1.896236 & -2.758426 \\
\hline $\mathrm{H}$ & -0.304175 & -4.692761 & -4.837337 \\
\hline $\mathrm{H}$ & 6.351644 & 2.287232 & -0.935436 \\
\hline $\mathrm{H}$ & 4.200615 & -2.642019 & -4.157066 \\
\hline $\mathrm{H}$ & 1.619730 & -5.434614 & -6.223464 \\
\hline $\mathrm{H}$ & 6.498339 & -1.600285 & 2.169656 \\
\hline $\mathrm{H}$ & 8.525621 & 1.357216 & -0.249065 \\
\hline $\mathrm{H}$ & 5.042740 & -5.544773 & -7.425790 \\
\hline $\mathrm{H}$ & 3.717487 & -6.388346 & -6.546003 \\
\hline $\mathrm{H}$ & 3.325830 & -5.129037 & -7.773571 \\
\hline $\mathrm{H}$ & 9.983141 & -2.339063 & 2.630503 \\
\hline $\mathrm{H}$ & 8.418621 & -2.813209 & 1.922948 \\
\hline $\mathrm{H}$ & 8.471774 & -1.695456 & 3.319628 \\
\hline $\mathrm{H}$ & 9.276634 & -2.046521 & -0.372446 \\
\hline $\mathrm{H}$ & 10.833699 & -1.630149 & 0.403757 \\
\hline $\mathrm{H}$ & 9.972047 & -0.417930 & -0.580862 \\
\hline $\mathrm{H}$ & 10.908373 & -0.045250 & 2.387025 \\
\hline $\mathrm{H}$ & 9.405442 & 0.679678 & 3.032325 \\
\hline $\mathrm{H}$ & 10.051658 & 1.213707 & 1.458851 \\
\hline $\mathrm{H}$ & 3.140873 & 0.698804 & -0.248192 \\
\hline $\mathrm{H}$ & 0.118132 & -2.002283 & -2.249389 \\
\hline $\mathrm{C}$ & 0.453441 & -2.840303 & 6.177907 \\
\hline
\end{tabular}




$\begin{array}{lrrc}\text { F } & -0.754440 & -2.556621 & 6.744438 \\ \text { F } & 1.412260 & -2.252370 & 6.944743 \\ \text { F } & 0.631493 & -4.186970 & 6.279103 \\ \text { H } & 0.717273 & 0.387181 & -2.655028 \\ \text { Se } & 0.477771 & 0.074652 & -1.217710\end{array}$

Statistical Thermodynamic Analysis

Temperature $=298.150$ Kelvin $\quad$ Pressure $=1.00000$ Atm

\begin{tabular}{|c|c|}
\hline $\begin{array}{l}\text { SCF Energy }=\quad-5719.16042890 \\
\text { Zero-point correction }(\mathrm{ZPE})=\end{array}$ & $0 \quad$ Predicted Change $=-1.657825 \mathrm{D}-08$ \\
\hline Zero-point correction $(\mathrm{ZPE})=$ & -5717.84701 .31333 \\
\hline Internal Energy $(U)=$ & -5717.76251 .39791 \\
\hline Enthalpy $(\mathrm{H})=$ & $-5717.7615 \quad 1.39885$ \\
\hline Gibbs Free Energy $(G)=$ & $-5717.9791 \quad 1.18123$ \\
\hline $\begin{array}{l}\text { Frequencies -- } 7.0750 \\
\text { Single points at WB97X-D3/def2 }\end{array}$ & $\begin{array}{cc}8.2158 & 11.2949 \\
\text { 2-TZVPP: }-5726.27072327374\end{array}$ \\
\hline $\begin{array}{r}\mathbf{R}=\mathbf{C F}_{3} \text { receptor bound with } \mathbf{S} \\
\text { Supporting Information: }\end{array}$ & $\begin{array}{l}\text { H- (with TBA) } \\
\text { 010-bound_TBA_CF3_SH_new-new.log }\end{array}$ \\
\hline
\end{tabular}

\#PBEPBE/6-31G(d)/auto scf $=($ direct,tight,maxcycle $=300, \mathrm{xqc})$

$\mathrm{opt}=($ maxcycle $=250)$ freq $=$ noraman int $=($ grid $=$ ultrafine $)$

\#P Geom=AllCheck Guess=TCheck SCRF $=$ Check GenChk RPBEPBE/6-31G(d)/Auto Freq

Pointgroup $=\mathrm{C} 1 \quad$ Stoichiometry $=\mathrm{C} 63 \mathrm{H} 82 \mathrm{~F} 3 \mathrm{~N} 5 \mathrm{O} 4 \mathrm{~S} \quad \mathrm{C} 1[\mathrm{X}(\mathrm{C} 63 \mathrm{H} 82 \mathrm{~F} 3 \mathrm{~N} 5 \mathrm{O} 4 \mathrm{~S})]$ \#Atoms $=158$ Charge $=0 \quad$ Multiplicity $=1$

SCF Energy $=-3718.13595790 \quad$ Predicted Change $=-1.125556 \mathrm{D}-07$

\begin{tabular}{|c|c|c|c|c|c|}
\hline \multicolumn{3}{|c|}{ Optimization completed. } & $\{$ Foun & nd & times\} \\
\hline Item & Max Val. & Criteria & Pass? & RMS Val. & Criteria \\
\hline Force & $0.00001 \|$ & 0.00045 & [YES & 0.00000 & 0.00030 \\
\hline Displ & $0.01429 \|$ & 0.00180 & {$[\mathrm{NO}]$} & 0.01429 & 0.00180 \\
\hline \multicolumn{2}{|c|}{ Atomic } & \multicolumn{3}{|c|}{ Coordinates (Angstroms) } & \\
\hline & $\mathrm{X}$ & $\mathrm{Y}$ & Z & & \\
\hline $\mathrm{N}$ & -2.57042 & -1.3 & 41138 & -2.336030 & \\
\hline $\mathrm{C}$ & -1.29887 & -1.1 & 88391 & -3.186759 & \\
\hline $\mathrm{C}$ & -0.27231 & -0.1 & 3077 & -2.705944 & \\
\hline $\mathrm{C}$ & 0.91182 & -0.1( & 15743 & -3.695448 & \\
\hline
\end{tabular}




\begin{tabular}{|c|c|c|c|}
\hline $\mathrm{C}$ & 1.965506 & 0.937244 & -3.303237 \\
\hline $\mathrm{C}$ & -2.184205 & -2.014539 & -1.010783 \\
\hline $\mathrm{C}$ & -3.328220 & -2.340056 & -0.046487 \\
\hline $\mathrm{C}$ & -2.804392 & -3.291313 & 1.051280 \\
\hline $\mathrm{C}$ & -3.868413 & -3.637485 & 2.100115 \\
\hline $\mathrm{C}$ & -3.220887 & 0.010140 & -2.036944 \\
\hline $\mathrm{C}$ & -3.607361 & 0.853558 & -3.255275 \\
\hline $\mathrm{C}$ & -4.216014 & 2.197993 & -2.810917 \\
\hline $\mathrm{C}$ & -4.633665 & 3.070197 & -4.001116 \\
\hline $\mathrm{C}$ & -3.556765 & -2.212902 & -3.121037 \\
\hline $\mathrm{C}$ & -3.188749 & -3.693582 & -3.252287 \\
\hline $\mathrm{C}$ & -4.082818 & -4.398006 & -4.290711 \\
\hline $\mathrm{C}$ & -3.806084 & -5.906601 & -4.345972 \\
\hline $\mathrm{H}$ & -0.834415 & -2.166354 & -3.230279 \\
\hline $\mathrm{H}$ & -1.651880 & -0.912596 & -4.199956 \\
\hline $\mathrm{H}$ & 0.100603 & -0.397378 & -1.696143 \\
\hline $\mathrm{H}$ & -0.715875 & 0.868170 & -2.647567 \\
\hline $\mathrm{H}$ & 0.533273 & 0.112751 & -4.714379 \\
\hline $\mathrm{H}$ & 1.379929 & -1.106996 & -3.741631 \\
\hline $\mathrm{H}$ & 2.785994 & 0.960418 & -4.039927 \\
\hline $\mathrm{H}$ & 1.530806 & 1.951186 & -3.249243 \\
\hline $\mathrm{H}$ & 2.408319 & 0.709766 & -2.319199 \\
\hline $\mathrm{H}$ & -1.654933 & -2.932773 & -1.307042 \\
\hline $\mathrm{H}$ & -1.437173 & -1.364494 & -0.515499 \\
\hline $\mathrm{H}$ & -3.718508 & -1.422967 & 0.429489 \\
\hline $\mathrm{H}$ & -4.177534 & -2.829085 & -0.561712 \\
\hline $\mathrm{H}$ & -1.931036 & -2.824469 & 1.543004 \\
\hline $\mathrm{H}$ & -2.434989 & -4.219800 & 0.571646 \\
\hline $\mathrm{H}$ & -3.475777 & -4.375339 & 2.819773 \\
\hline $\mathrm{H}$ & -4.167521 & -2.746673 & 2.677831 \\
\hline $\mathrm{H}$ & -4.770852 & -4.071197 & 1.631454 \\
\hline $\mathrm{H}$ & -4.111049 & -0.213204 & -1.427685 \\
\hline $\mathrm{H}$ & -2.511974 & 0.553531 & -1.391895 \\
\hline $\mathrm{H}$ & -2.723656 & 1.055322 & -3.888238 \\
\hline $\mathrm{H}$ & -4.342850 & 0.321294 & -3.886823 \\
\hline $\mathrm{H}$ & -3.481934 & 2.739026 & -2.184952 \\
\hline $\mathrm{H}$ & -5.089716 & 2.008023 & -2.159142 \\
\hline $\mathrm{H}$ & -5.071992 & 4.022175 & -3.656833 \\
\hline $\mathrm{H}$ & -3.769761 & 3.314485 & -4.644366 \\
\hline $\mathrm{H}$ & -5.387826 & 2.562572 & -4.628861 \\
\hline $\mathrm{H}$ & -3.639819 & -1.750740 & -4.117618 \\
\hline $\mathrm{H}$ & -4.533412 & -2.102114 & -2.622150 \\
\hline $\mathrm{H}$ & -2.133647 & -3.823236 & -3.551738 \\
\hline $\mathrm{H}$ & -3.301991 & -4.200881 & -2.277204 \\
\hline $\mathrm{H}$ & -3.908334 & -3.946121 & -5.286206 \\
\hline $\mathrm{H}$ & -5.149617 & -4.221029 & -4.051879 \\
\hline $\mathrm{H}$ & -4.382385 & -6.386962 & -5.154297 \\
\hline $\mathrm{H}$ & -2.733746 & -6.109272 & -4.515081 \\
\hline
\end{tabular}




\begin{tabular}{|c|c|c|c|}
\hline $\mathrm{H}$ & -4.088149 & -6.395713 & -3.396950 \\
\hline $\mathrm{O}$ & -0.026923 & -6.411341 & -3.959224 \\
\hline $\mathrm{O}$ & -3.558663 & -0.724673 & 4.200152 \\
\hline $\mathrm{N}$ & 2.642459 & -2.363674 & -1.123497 \\
\hline $\mathrm{N}$ & -2.758246 & 0.510871 & 2.393627 \\
\hline $\mathrm{C}$ & 0.997713 & 2.928018 & 0.230631 \\
\hline $\mathrm{C}$ & 1.205201 & 5.521247 & -0.822889 \\
\hline $\mathrm{C}$ & -0.056524 & 5.010106 & -0.495569 \\
\hline $\mathrm{C}$ & -0.167668 & 3.703567 & 0.040498 \\
\hline C & 2.065938 & -3.410115 & -1.860506 \\
\hline $\mathrm{C}$ & 2.584985 & -3.887033 & -3.082983 \\
\hline $\mathrm{C}$ & 1.917546 & -4.890571 & -3.802101 \\
\hline $\mathrm{C}$ & 0.716239 & -5.438782 & -3.319620 \\
\hline $\mathrm{C}$ & 0.206643 & -4.986998 & -2.086100 \\
\hline $\mathrm{C}$ & 0.874629 & -3.993038 & -1.366883 \\
\hline $\mathrm{C}$ & 0.533114 & -6.972007 & -5.143600 \\
\hline $\mathrm{C}$ & -1.443894 & 3.179449 & 0.390874 \\
\hline $\mathrm{C}$ & -2.558287 & 2.769380 & 0.721021 \\
\hline $\mathrm{C}$ & -3.829080 & 2.292455 & 1.137989 \\
\hline $\mathrm{C}$ & -3.931735 & 1.157462 & 2.005326 \\
\hline $\mathrm{C}$ & -5.224648 & 0.745418 & 2.401728 \\
\hline $\mathrm{C}$ & -6.355660 & 1.431679 & 1.958027 \\
\hline $\mathrm{C}$ & -6.284978 & 2.557257 & 1.103713 \\
\hline $\mathrm{C}$ & -5.004500 & 2.962816 & 0.712068 \\
\hline $\mathrm{C}$ & -8.463417 & 2.287742 & -0.133943 \\
\hline $\mathrm{C}$ & -7.275873 & 4.490766 & -0.253545 \\
\hline $\mathrm{C}$ & -2.604583 & -0.352536 & 3.501611 \\
\hline $\mathrm{C}$ & 3.981205 & -1.993285 & -1.158764 \\
\hline $\mathrm{C}$ & 2.267670 & 3.441977 & -0.116366 \\
\hline $\mathrm{C}$ & 2.363039 & 4.754565 & -0.640854 \\
\hline $\mathrm{C}$ & -7.569694 & 3.278437 & 0.653332 \\
\hline $\mathrm{N}$ & -1.288594 & -0.714690 & 3.683767 \\
\hline $\mathrm{N}$ & 4.230605 & -0.877719 & -0.348103 \\
\hline $\mathrm{O}$ & 4.848903 & -2.567928 & -1.825586 \\
\hline $\mathrm{C}$ & 3.426821 & 2.631120 & 0.027367 \\
\hline $\mathrm{C}$ & -8.340507 & 3.782328 & 1.899284 \\
\hline $\mathrm{C}$ & -0.760268 & -1.517852 & 4.719613 \\
\hline $\mathrm{C}$ & 5.490369 & -0.301804 & -0.141316 \\
\hline $\mathrm{C}$ & 4.424567 & 1.916061 & 0.112428 \\
\hline $\mathrm{C}$ & 0.646223 & -1.667818 & 4.761610 \\
\hline $\mathrm{C}$ & -1.539086 & -2.163118 & 5.699003 \\
\hline $\mathrm{C}$ & 5.580531 & 1.092789 & 0.149357 \\
\hline $\mathrm{C}$ & 6.684345 & -1.050108 & -0.187886 \\
\hline $\mathrm{C}$ & 1.252074 & -2.445990 & 5.744826 \\
\hline $\mathrm{C}$ & -0.925037 & -2.940753 & 6.693167 \\
\hline $\mathrm{C}$ & 6.846268 & 1.674738 & 0.415885 \\
\hline $\mathrm{C}$ & 7.913352 & -0.440382 & 0.063935 \\
\hline $\mathrm{C}$ & 0.471692 & -3.091864 & 6.723737 \\
\hline
\end{tabular}




\begin{tabular}{|c|c|c|c|}
\hline $\mathrm{C}$ & 8.031768 & 0.932859 & 0.382243 \\
\hline $\mathrm{O}$ & 1.168451 & -3.831925 & 7.651375 \\
\hline $\mathrm{C}$ & 9.414865 & 1.548538 & 0.668013 \\
\hline $\mathrm{C}$ & 0.406187 & -4.482043 & 8.660835 \\
\hline $\mathrm{C}$ & 9.330985 & 3.054656 & 0.988520 \\
\hline $\mathrm{C}$ & 10.054579 & 0.829126 & 1.881879 \\
\hline $\mathrm{C}$ & 10.324701 & 1.366477 & -0.572532 \\
\hline $\mathrm{H}$ & 2.014402 & -1.868356 & -0.452461 \\
\hline $\mathrm{H}$ & -1.899795 & 0.861675 & 1.955185 \\
\hline $\mathrm{H}$ & 0.925182 & 1.919912 & 0.653363 \\
\hline $\mathrm{H}$ & -0.954516 & 5.613868 & -0.649099 \\
\hline $\mathrm{H}$ & 3.525943 & -3.478697 & -3.454395 \\
\hline $\mathrm{H}$ & 2.352148 & -5.232966 & -4.745004 \\
\hline $\mathrm{H}$ & -0.699380 & -5.455896 & -1.687231 \\
\hline $\mathrm{H}$ & 0.485690 & -3.661363 & -0.397930 \\
\hline $\mathrm{H}$ & -0.169471 & -7.754155 & -5.467727 \\
\hline $\mathrm{H}$ & 0.635867 & -6.217865 & -5.948613 \\
\hline $\mathrm{H}$ & 1.523760 & -7.425693 & -4.949026 \\
\hline $\mathrm{H}$ & -5.314933 & -0.097367 & 3.086672 \\
\hline $\mathrm{H}$ & -4.867962 & 3.833642 & 0.065853 \\
\hline $\mathrm{H}$ & -6.658693 & 5.247745 & 0.261046 \\
\hline $\mathrm{H}$ & -6.756067 & 4.194760 & -1.182722 \\
\hline $\mathrm{H}$ & -7.333197 & 1.072622 & 2.299659 \\
\hline $\mathrm{H}$ & -8.730049 & 1.405886 & 0.473200 \\
\hline $\mathrm{H}$ & -7.948433 & 1.928627 & -1.043001 \\
\hline $\mathrm{H}$ & -9.403151 & 2.779187 & -0.444661 \\
\hline $\mathrm{H}$ & -8.223815 & 4.975632 & -0.544409 \\
\hline $\mathrm{H}$ & 3.340782 & 5.156917 & -0.917548 \\
\hline $\mathrm{H}$ & -7.733102 & 4.503025 & 2.473300 \\
\hline $\mathrm{H}$ & -8.609965 & 2.954817 & 2.577194 \\
\hline $\mathrm{H}$ & -9.275931 & 4.285487 & 1.594380 \\
\hline $\mathrm{H}$ & 1.264284 & -1.159830 & 4.012512 \\
\hline $\mathrm{H}$ & -2.622804 & -2.046685 & 5.681167 \\
\hline $\mathrm{H}$ & 6.631045 & -2.110525 & -0.435584 \\
\hline $\mathrm{H}$ & 2.339639 & -2.561205 & 5.776632 \\
\hline $\mathrm{H}$ & -1.558841 & -3.424886 & 7.440831 \\
\hline $\mathrm{H}$ & 6.861430 & 2.744121 & 0.640489 \\
\hline $\mathrm{H}$ & 8.812283 & -1.065640 & 0.021234 \\
\hline $\mathrm{H}$ & 1.134560 & -5.006773 & 9.297173 \\
\hline $\mathrm{H}$ & -0.162203 & -3.757815 & 9.277022 \\
\hline $\mathrm{H}$ & -0.301030 & -5.219210 & 8.231851 \\
\hline $\mathrm{H}$ & 10.342838 & 3.448846 & 1.187011 \\
\hline $\mathrm{H}$ & 8.715580 & 3.249748 & 1.884241 \\
\hline $\mathrm{H}$ & 8.908495 & 3.630353 & 0.146291 \\
\hline $\mathrm{H}$ & 9.434807 & 0.955171 & 2.786511 \\
\hline $\mathrm{H}$ & 11.056963 & 1.245544 & 2.090103 \\
\hline $\mathrm{H}$ & 10.171282 & -0.253153 & 1.701728 \\
\hline $\mathrm{H}$ & 11.328652 & 1.785003 & -0.376457 \\
\hline
\end{tabular}




$\begin{array}{cccc}\mathrm{H} & 9.901724 & 1.883691 & -1.451197 \\ \mathrm{H} & 10.450111 & 0.302578 & -0.836448 \\ \mathrm{H} & 3.421274 & -0.393077 & 0.062683 \\ \mathrm{H} & -0.614554 & -0.468541 & 2.931164 \\ \mathrm{C} & 1.321857 & 6.940000 & -1.326577 \\ \mathrm{~F} & 0.215910 & 7.319555 & -2.027832 \\ \mathrm{~F} & 2.399172 & 7.101830 & -2.143022 \\ \mathrm{~F} & 1.463146 & 7.825515 & -0.300432 \\ \mathrm{~S} & 0.699749 & -0.690097 & 0.983787 \\ \mathrm{H} & 0.858359 & -1.841885 & 1.695430\end{array}$

Statistical Thermodynamic Analysis

Temperature $=298.150$ Kelvin $\quad$ Pressure $=1.00000$ Atm

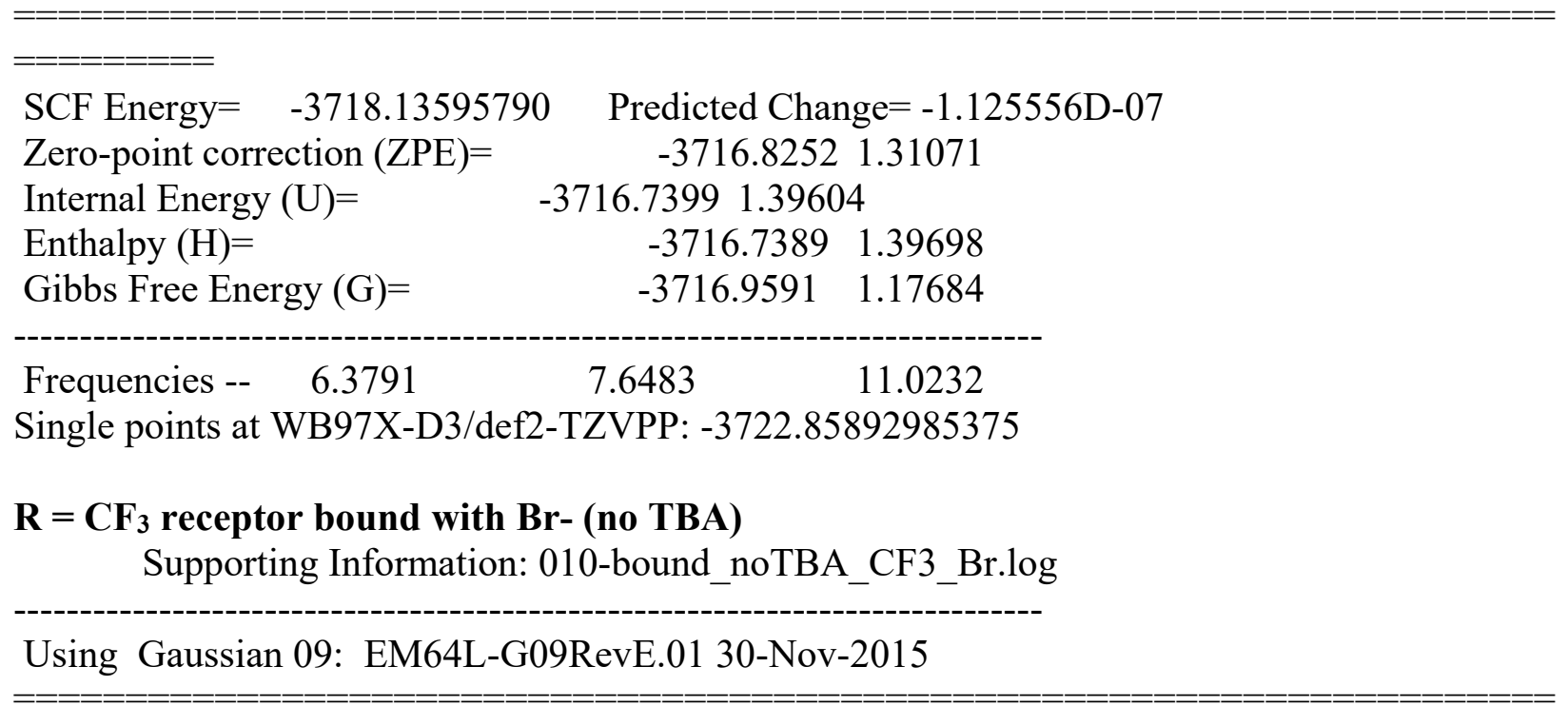

\#PBEPBE/6-31G(d)/auto scf $=($ direct,tight,maxcycle $=300, \mathrm{xqc})$

$\mathrm{opt}=($ maxcycle $=250)$ freq $=$ noraman int $=($ grid $=$ ultrafine $)$

\#P Geom=AllCheck Guess=TCheck SCRF $=$ Check GenChk RPBEPBE/6-31G(d)/Auto Freq

Pointgroup $=\mathrm{C} 1 \quad$ Stoichiometry $=\mathrm{C} 47 \mathrm{H} 45 \mathrm{BrF} 3 \mathrm{~N} 4 \mathrm{O} 4(1-) \quad \mathrm{C} 1[\mathrm{X}(\mathrm{C} 47 \mathrm{H} 45 \mathrm{BrF} 3 \mathrm{~N} 4 \mathrm{O} 4)]$

\#Atoms $=104$

Charge $=-1 \quad$ Multiplicity $=1$

SCF Energy $=-5205.82418606 \quad$ Predicted Change $=-6.881293 \mathrm{D}-09$

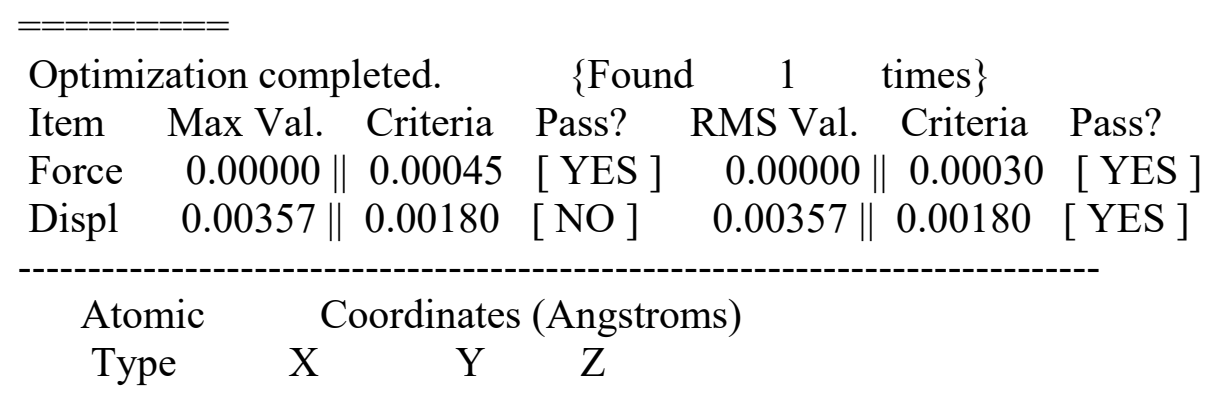




\begin{tabular}{|c|c|c|c|}
\hline $\mathrm{O}$ & 4.487034 & -2.535069 & 2.018535 \\
\hline $\mathrm{O}$ & -0.302504 & -6.290778 & 4.690616 \\
\hline $\mathrm{O}$ & -4.943088 & -1.983374 & -1.785116 \\
\hline $\mathrm{O}$ & -1.180467 & -6.707533 & -4.538263 \\
\hline $\mathrm{N}$ & 3.672250 & -0.834624 & 0.642976 \\
\hline $\mathrm{H}$ & 2.813901 & -0.363535 & 0.328084 \\
\hline $\mathrm{N}$ & 2.207298 & -2.329285 & 1.613711 \\
\hline $\mathrm{H}$ & 1.503270 & -1.818790 & 1.044594 \\
\hline $\mathrm{N}$ & -3.721841 & -0.345641 & -0.653905 \\
\hline $\mathrm{H}$ & -2.769596 & -0.003018 & -0.467834 \\
\hline $\mathrm{N}$ & -2.630702 & -2.130411 & -1.635178 \\
\hline $\mathrm{H}$ & -1.789130 & -1.692504 & -1.211595 \\
\hline $\mathrm{C}$ & 0.296921 & 2.854461 & -0.061920 \\
\hline $\mathrm{H}$ & 0.223787 & 1.760844 & -0.019415 \\
\hline $\mathrm{C}$ & 1.566161 & 3.465680 & -0.155762 \\
\hline $\mathrm{C}$ & 1.651903 & 4.877618 & -0.226785 \\
\hline $\mathrm{H}$ & 2.628795 & 5.361624 & -0.306448 \\
\hline $\mathrm{C}$ & 0.480158 & 5.646495 & -0.199910 \\
\hline $\mathrm{C}$ & -0.781623 & 5.042681 & -0.109765 \\
\hline $\mathrm{H}$ & -1.687610 & 5.654296 & -0.103495 \\
\hline $\mathrm{C}$ & -0.881375 & 3.631168 & -0.044258 \\
\hline $\mathrm{C}$ & 2.731752 & 2.650317 & -0.177231 \\
\hline $\mathrm{C}$ & 3.714089 & 1.908900 & -0.182318 \\
\hline $\mathrm{C}$ & 4.891219 & 1.114611 & -0.177040 \\
\hline $\mathrm{C}$ & 4.881632 & -0.246113 & 0.276221 \\
\hline $\mathrm{C}$ & 6.112982 & -0.934486 & 0.309681 \\
\hline $\mathrm{H}$ & 6.122451 & -1.960451 & 0.676562 \\
\hline $\mathrm{C}$ & 7.297239 & -0.314377 & -0.099185 \\
\hline $\mathrm{H}$ & 8.217835 & -0.904073 & -0.053177 \\
\hline $\mathrm{C}$ & 7.332469 & 1.014887 & -0.569172 \\
\hline $\mathrm{C}$ & 6.109611 & 1.701000 & -0.594163 \\
\hline $\mathrm{H}$ & 6.063357 & 2.740493 & -0.936390 \\
\hline $\mathrm{C}$ & 3.528520 & -1.964559 & 1.477802 \\
\hline $\mathrm{C}$ & 1.674175 & -3.353413 & 2.415826 \\
\hline $\mathrm{C}$ & 2.437692 & -4.206902 & 3.235020 \\
\hline $\mathrm{H}$ & 3.521505 & -4.088474 & 3.259113 \\
\hline $\mathrm{C}$ & 1.806949 & -5.196582 & 4.006758 \\
\hline $\mathrm{H}$ & 2.427964 & -5.843429 & 4.632913 \\
\hline $\mathrm{C}$ & 0.411665 & -5.349488 & 3.970575 \\
\hline $\mathrm{C}$ & -0.352830 & -4.497545 & 3.150016 \\
\hline $\mathrm{H}$ & -1.439685 & -4.623242 & 3.124284 \\
\hline $\mathrm{C}$ & 0.266849 & -3.513475 & 2.384836 \\
\hline $\mathrm{H}$ & -0.323876 & -2.847044 & 1.744312 \\
\hline $\mathrm{C}$ & 0.449829 & -7.161065 & 5.519217 \\
\hline $\mathrm{H}$ & -0.280881 & -7.828946 & 6.001877 \\
\hline $\mathrm{H}$ & 1.009729 & -6.608897 & 6.301324 \\
\hline $\mathrm{H}$ & 1.169536 & -7.770594 & 4.935797 \\
\hline
\end{tabular}




\begin{tabular}{|c|c|c|c|}
\hline $\mathrm{C}$ & -2.146194 & 2.983572 & 0.030151 \\
\hline $\mathrm{C}$ & -3.224143 & 2.392791 & 0.091464 \\
\hline $\mathrm{C}$ & -4.516074 & 1.808982 & 0.181440 \\
\hline $\mathrm{C}$ & -4.776031 & 0.455162 & -0.214716 \\
\hline $\mathrm{C}$ & -6.106379 & -0.008495 & -0.120246 \\
\hline $\mathrm{H}$ & -6.321281 & -1.028491 & -0.436555 \\
\hline $\mathrm{C}$ & -7.129871 & 0.820454 & 0.347549 \\
\hline $\mathrm{H}$ & -8.137688 & 0.397243 & 0.398655 \\
\hline $\mathrm{C}$ & -6.900707 & 2.151031 & 0.753783 \\
\hline $\mathrm{C}$ & -5.580200 & 2.612243 & 0.656987 \\
\hline $\mathrm{H}$ & -5.327733 & 3.637344 & 0.948917 \\
\hline $\mathrm{C}$ & -3.852063 & -1.540000 & -1.397526 \\
\hline $\mathrm{C}$ & -2.368686 & -3.293282 & -2.381875 \\
\hline $\mathrm{C}$ & -3.352316 & -4.067704 & -3.026725 \\
\hline $\mathrm{H}$ & -4.397582 & -3.766657 & -2.951113 \\
\hline $\mathrm{C}$ & -2.987408 & -5.212289 & -3.754440 \\
\hline $\mathrm{H}$ & -3.775690 & -5.791652 & -4.243413 \\
\hline $\mathrm{C}$ & -1.641390 & -5.600469 & -3.847856 \\
\hline $\mathrm{C}$ & -0.656843 & -4.827859 & -3.202154 \\
\hline $\mathrm{H}$ & 0.390500 & -5.136615 & -3.276620 \\
\hline $\mathrm{C}$ & -1.012525 & -3.691491 & -2.480819 \\
\hline $\mathrm{H}$ & -0.247271 & -3.087542 & -1.977808 \\
\hline $\mathrm{C}$ & -2.157939 & -7.501911 & -5.189119 \\
\hline $\mathrm{H}$ & -1.608495 & -8.324389 & -5.673495 \\
\hline $\mathrm{H}$ & -2.710986 & -6.929659 & -5.961599 \\
\hline $\mathrm{H}$ & -2.891359 & -7.926292 & -4.473702 \\
\hline $\mathrm{C}$ & 8.624128 & 1.716179 & -1.035031 \\
\hline $\mathrm{C}$ & -8.011738 & 3.082627 & 1.277847 \\
\hline $\mathrm{C}$ & 8.472697 & 2.149729 & -2.514401 \\
\hline $\mathrm{H}$ & 7.618941 & 2.834761 & -2.652091 \\
\hline $\mathrm{H}$ & 9.384496 & 2.671121 & -2.860983 \\
\hline $\mathrm{H}$ & 8.307301 & 1.273794 & -3.165592 \\
\hline $\mathrm{C}$ & 9.858078 & 0.797031 & -0.927333 \\
\hline $\mathrm{H}$ & 10.037829 & 0.473726 & 0.113044 \\
\hline $\mathrm{H}$ & 9.751538 & -0.105477 & -1.554505 \\
\hline $\mathrm{H}$ & 10.757373 & 1.339089 & -1.270098 \\
\hline $\mathrm{C}$ & 8.878286 & 2.969201 & -0.160492 \\
\hline $\mathrm{H}$ & 9.007226 & 2.688864 & 0.899461 \\
\hline $\mathrm{H}$ & 9.792867 & 3.494926 & -0.492926 \\
\hline $\mathrm{H}$ & 8.039151 & 3.683101 & -0.218330 \\
\hline $\mathrm{C}$ & -7.667674 & 3.541213 & 2.716799 \\
\hline $\mathrm{H}$ & -6.702496 & 4.074233 & 2.754233 \\
\hline $\mathrm{H}$ & -8.446924 & 4.224792 & 3.102565 \\
\hline $\mathrm{H}$ & -7.598862 & 2.676071 & 3.399032 \\
\hline $\mathrm{C}$ & -8.121458 & 4.325325 & 0.359646 \\
\hline $\mathrm{H}$ & -8.383606 & 4.029976 & -0.671267 \\
\hline $\mathrm{H}$ & -8.902691 & 5.014452 & 0.731328 \\
\hline $\mathrm{H}$ & -7.171219 & 4.884212 & 0.316505 \\
\hline
\end{tabular}




$\begin{array}{lccc}\mathrm{C} & -9.387916 & 2.386720 & 1.311330 \\ \mathrm{H} & -9.705683 & 2.059966 & 0.305601 \\ \mathrm{H} & -9.383520 & 1.503825 & 1.974431 \\ \mathrm{H} & -10.151032 & 3.088456 & 1.692504 \\ \mathrm{Br} & 0.024696 & -0.713355 & -0.279406 \\ \mathrm{C} & 0.582049 & 7.149445 & -0.202293 \\ \mathrm{~F} & -0.507609 & 7.738498 & -0.774179 \\ \mathrm{~F} & 1.681458 & 7.589750 & -0.879836 \\ \mathrm{~F} & 0.677521 & 7.655714 & 1.064087\end{array}$

Statistical Thermodynamic Analysis

Temperature $=298.150$ Kelvin $\quad$ Pressure $=1.00000$ Atm

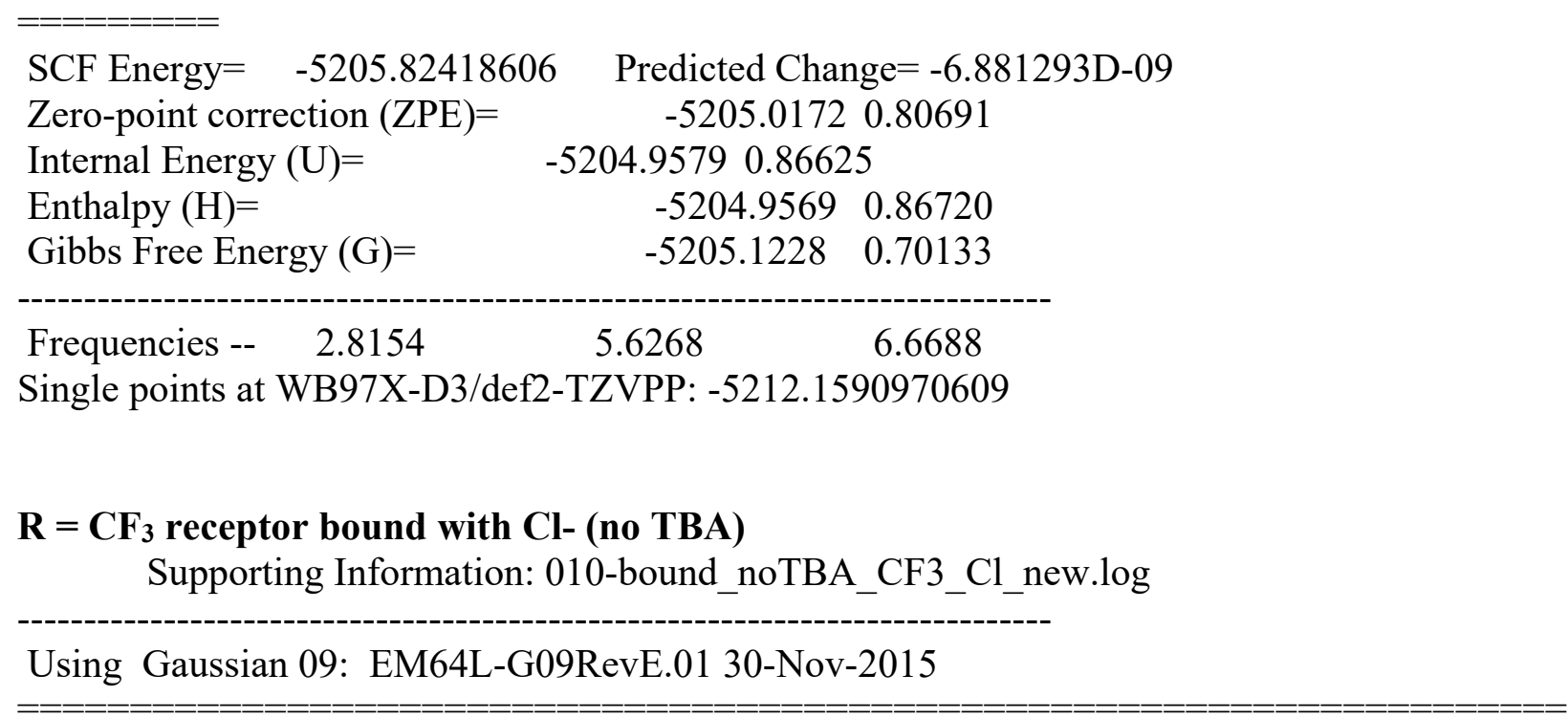

\#PBEPBE/6-31G(d)/auto scf $=($ direct,tight,maxcycle $=300, \mathrm{xqc})$

$\mathrm{opt}=($ maxcycle $=250)$ freq $=$ noraman $\mathrm{int}=($ grid $=$ ultrafine $)$

\#P Geom=AllCheck Guess=TCheck SCRF=Check GenChk RPBEPBE/6-31G(d)/Auto Freq

Pointgroup $=\mathrm{C} 1 \quad$ Stoichiometry $=\mathrm{C} 47 \mathrm{H} 45 \mathrm{ClF} 3 \mathrm{~N} 4 \mathrm{O} 4(1-) \quad \mathrm{C} 1[\mathrm{X}(\mathrm{C} 47 \mathrm{H} 45 \mathrm{ClF} 3 \mathrm{~N} 4 \mathrm{O} 4)]$

\#Atoms $=104$

Charge $=-1 \quad$ Multiplicity $=1$

SCF Energy $=-3094.50216251 \quad$ Predicted Change $=-1.490695 \mathrm{D}-08$

\begin{tabular}{|c|c|c|c|c|c|}
\hline \multicolumn{3}{|c|}{ Optimization completed. } & $\{$ Foun & times $\}$ & \\
\hline Item & Max Val. & Criteria & Pass? & \multirow{2}{*}{\multicolumn{2}{|c|}{$\begin{array}{l}\text { RMS Val. Criteria Pass? } \\
0.00000 \| 0.00030 \quad[\text { YES ] }\end{array}$}} \\
\hline Force & 0.00000 & 0.00045 & [ YES ] & & \\
\hline Displ & $0.00294 \mid$ & 0.00180 & [ NO ] & $0.00294 \| 0.00180$ & [ $\mathrm{YES}]$ \\
\hline & & ordinates & (Angst & & \\
\hline Ty & $X$ & $\mathrm{Y}$ & $\mathrm{Z}$ & & \\
\hline
\end{tabular}




\begin{tabular}{|c|c|c|c|}
\hline $\mathrm{O}$ & -4.575547 & 2.385070 & 1.715426 \\
\hline $\mathrm{O}$ & -0.201399 & 6.775863 & 4.030622 \\
\hline $\mathrm{O}$ & 4.572852 & 2.396223 & -1.714020 \\
\hline $\mathrm{O}$ & 0.192972 & 6.793478 & -4.005483 \\
\hline $\mathrm{N}$ & -3.627358 & 0.555626 & 0.623299 \\
\hline $\mathrm{H}$ & -2.737154 & 0.086231 & 0.413473 \\
\hline $\mathrm{N}$ & -2.270936 & 2.230455 & 1.463015 \\
\hline $\mathrm{H}$ & -1.508524 & 1.678082 & 1.017810 \\
\hline $\mathrm{N}$ & 3.627413 & 0.560703 & -0.629730 \\
\hline $\mathrm{H}$ & 2.737890 & 0.089320 & -0.421531 \\
\hline $\mathrm{N}$ & 2.268549 & 2.237547 & -1.461476 \\
\hline $\mathrm{H}$ & 1.507035 & 1.682643 & -1.017884 \\
\hline $\mathrm{C}$ & 0.001881 & -2.924457 & -0.002640 \\
\hline $\mathrm{H}$ & 0.001505 & -1.826877 & 0.005912 \\
\hline $\mathrm{C}$ & -1.224511 & -3.623243 & -0.040775 \\
\hline $\mathrm{C}$ & -1.217397 & -5.039580 & -0.057691 \\
\hline $\mathrm{H}$ & -2.161473 & -5.589362 & -0.094873 \\
\hline $\mathrm{C}$ & 0.003083 & -5.728266 & -0.032253 \\
\hline $\mathrm{C}$ & 1.223052 & -5.039204 & 0.006197 \\
\hline $\mathrm{H}$ & 2.168027 & -5.588644 & 0.014418 \\
\hline $\mathrm{C}$ & 1.228903 & -3.622822 & 0.018573 \\
\hline $\mathrm{C}$ & -2.443289 & -2.890376 & -0.064245 \\
\hline $\mathrm{C}$ & -3.474693 & -2.219194 & -0.082035 \\
\hline $\mathrm{C}$ & -4.703982 & -1.509446 & -0.106654 \\
\hline $\mathrm{C}$ & -4.790557 & -0.127786 & 0.271850 \\
\hline $\mathrm{C}$ & -6.066811 & 0.475848 & 0.249440 \\
\hline $\mathrm{H}$ & -6.149512 & 1.518706 & 0.553054 \\
\hline $\mathrm{C}$ & -7.200783 & -0.246198 & -0.133687 \\
\hline $\mathrm{H}$ & -8.158863 & 0.282495 & -0.131684 \\
\hline $\mathrm{C}$ & -7.142617 & -1.600790 & -0.520682 \\
\hline $\mathrm{C}$ & -5.875715 & -2.201246 & -0.495170 \\
\hline $\mathrm{H}$ & -5.753944 & -3.252576 & -0.777693 \\
\hline $\mathrm{C}$ & -3.567370 & 1.789650 & 1.306738 \\
\hline $\mathrm{C}$ & -1.847527 & 3.396431 & 2.128726 \\
\hline $\mathrm{C}$ & -2.707532 & 4.296240 & 2.789083 \\
\hline $\mathrm{H}$ & -3.779812 & 4.100189 & 2.789633 \\
\hline $\mathrm{C}$ & -2.189262 & 5.431707 & 3.432851 \\
\hline $\mathrm{H}$ & -2.886466 & 6.108509 & 3.934933 \\
\hline $\mathrm{C}$ & -0.809188 & 5.688779 & 3.428002 \\
\hline $\mathrm{C}$ & 0.052801 & 4.792485 & 2.768030 \\
\hline $\mathrm{H}$ & 1.127435 & 4.999277 & 2.762598 \\
\hline $\mathrm{C}$ & -0.456598 & 3.664721 & 2.129521 \\
\hline $\mathrm{H}$ & 0.219977 & 2.971310 & 1.617397 \\
\hline $\mathrm{C}$ & -1.055677 & 7.696477 & 4.688348 \\
\hline $\mathrm{H}$ & -0.400506 & 8.483570 & 5.093625 \\
\hline $\mathrm{H}$ & -1.613571 & 7.223215 & 5.521983 \\
\hline $\mathrm{H}$ & -1.786673 & 8.154770 & 3.991635 \\
\hline
\end{tabular}




\begin{tabular}{|c|c|c|c|}
\hline $\mathrm{C}$ & 2.447307 & -2.889284 & 0.043107 \\
\hline $\mathrm{C}$ & 3.478060 & -2.217194 & 0.063933 \\
\hline $\mathrm{C}$ & 4.706701 & -1.506323 & 0.090646 \\
\hline $\mathrm{C}$ & 4.791577 & -0.122963 & -0.281976 \\
\hline $\mathrm{C}$ & 6.067245 & 0.481858 & -0.257988 \\
\hline $\mathrm{H}$ & 6.148645 & 1.526103 & -0.557153 \\
\hline $\mathrm{C}$ & 7.202246 & -0.240722 & 0.121067 \\
\hline $\mathrm{H}$ & 8.159796 & 0.288937 & 0.120552 \\
\hline $\mathrm{C}$ & 7.145770 & -1.597031 & 0.502234 \\
\hline $\mathrm{C}$ & 5.879427 & -2.198615 & 0.475218 \\
\hline $\mathrm{H}$ & 5.758908 & -3.251245 & 0.753399 \\
\hline $\mathrm{C}$ & 3.565594 & 1.797710 & -1.307586 \\
\hline $\mathrm{C}$ & 1.843568 & 3.406367 & -2.121184 \\
\hline $\mathrm{C}$ & 2.702155 & 4.309590 & -2.778725 \\
\hline $\mathrm{H}$ & 3.774543 & 4.114157 & -2.781644 \\
\hline $\mathrm{C}$ & 2.182380 & 5.447690 & -3.416599 \\
\hline $\mathrm{H}$ & 2.878522 & 6.127130 & -3.916588 \\
\hline $\mathrm{C}$ & 0.802179 & 5.704004 & -3.408637 \\
\hline $\mathrm{C}$ & -0.058401 & 4.804267 & -2.751522 \\
\hline $\mathrm{H}$ & -1.133137 & 5.010451 & -2.743628 \\
\hline $\mathrm{C}$ & 0.452497 & 3.673910 & -2.118820 \\
\hline $\mathrm{H}$ & -0.222995 & 2.977835 & -1.608885 \\
\hline $\mathrm{C}$ & 1.045837 & 7.717515 & -4.660228 \\
\hline $\mathrm{H}$ & 0.389684 & 8.506096 & -5.060998 \\
\hline $\mathrm{H}$ & 1.602794 & 7.248337 & -5.496794 \\
\hline $\mathrm{H}$ & 1.777584 & 8.173025 & -3.962480 \\
\hline $\mathrm{C}$ & -8.378588 & -2.414603 & -0.953061 \\
\hline $\mathrm{C}$ & 8.382890 & -2.411409 & 0.930222 \\
\hline $\mathrm{C}$ & -8.181742 & -2.924055 & -2.402564 \\
\hline $\mathrm{H}$ & -7.281884 & -3.555906 & -2.492721 \\
\hline $\mathrm{H}$ & -9.052071 & -3.526400 & -2.723671 \\
\hline $\mathrm{H}$ & -8.068965 & -2.079078 & -3.103968 \\
\hline $\mathrm{C}$ & -9.672689 & -1.576393 & -0.909643 \\
\hline $\mathrm{H}$ & -9.884903 & -1.204345 & 0.108086 \\
\hline $\mathrm{H}$ & -9.620224 & -0.707842 & -1.589395 \\
\hline $\mathrm{H}$ & -10.529761 & -2.197662 & -1.225195 \\
\hline $\mathrm{C}$ & -8.557933 & -3.627854 & -0.006865 \\
\hline $\mathrm{H}$ & -8.717873 & -3.294485 & 1.033341 \\
\hline $\mathrm{H}$ & -9.431038 & -4.233365 & -0.314154 \\
\hline $\mathrm{H}$ & -7.671844 & -4.285084 & -0.015318 \\
\hline $\mathrm{C}$ & 8.187844 & -2.926736 & 2.377893 \\
\hline $\mathrm{H}$ & 7.288625 & -3.559731 & 2.466390 \\
\hline $\mathrm{H}$ & 9.059003 & -3.529564 & 2.695831 \\
\hline $\mathrm{H}$ & 8.074961 & -2.084627 & 3.082718 \\
\hline $\mathrm{C}$ & 8.562471 & -3.620772 & -0.020894 \\
\hline $\mathrm{H}$ & 8.721126 & -3.283183 & -1.059937 \\
\hline $\mathrm{H}$ & 9.436431 & -4.226656 & 0.283207 \\
\hline $\mathrm{H}$ & 7.677019 & -4.278880 & -0.014188 \\
\hline
\end{tabular}




$\begin{array}{lccc}\mathrm{C} & 9.676189 & -1.571858 & 0.888891 \\ \mathrm{H} & 9.887037 & -1.195513 & -0.127543 \\ \mathrm{H} & 9.623629 & -0.706110 & 1.572200 \\ \mathrm{H} & 10.534136 & -2.193617 & 1.201081 \\ \mathrm{C} & 0.001930 & -7.233562 & 0.021654 \\ \mathrm{~F} & 1.114940 & -7.770363 & -0.557032 \\ \mathrm{~F} & -1.081108 & -7.770591 & -0.611195 \\ \mathrm{~F} & -0.029604 & -7.697946 & 1.307157 \\ \mathrm{Cl} & -0.000014 & 0.542087 & -0.001438\end{array}$

Statistical Thermodynamic Analysis

Temperature $=298.150$ Kelvin $\quad$ Pressure $=1.00000$ Atm

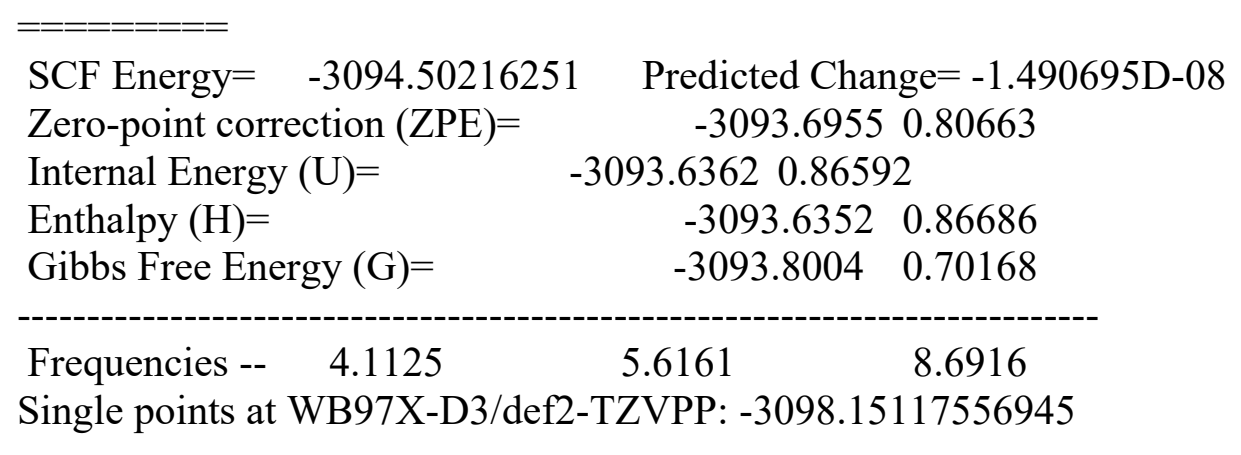

$\mathrm{R}=\mathrm{CF}_{3}$ receptor bound with SeH- (no TBA)

Supporting Information: 010-bound_noTBA_CF3_SeH.log

Using Gaussian 09: EM64L-G09RevE.01 30-Nov-2015

\#PBEPBE/6-31G(d)/auto scf $=($ direct,tight,maxcycle $=300, \mathrm{xqc})$

$\mathrm{opt}=($ maxcycle $=250)$ freq $=$ noraman int $=($ grid $=$ ultrafine $)$

\#P Geom=AllCheck Guess=TCheck SCRF $=$ Check GenChk RPBEPBE/6-31G(d)/Auto Freq

Pointgroup $=\mathrm{C} 1 \quad$ Stoichiometry $=\mathrm{C} 47 \mathrm{H} 46 \mathrm{~F} 3 \mathrm{~N} 4 \mathrm{O} 4 \mathrm{Se}(1-) \quad \mathrm{C} 1[\mathrm{X}(\mathrm{C} 47 \mathrm{H} 46 \mathrm{~F} 3 \mathrm{~N} 4 \mathrm{O} 4 \mathrm{Se})]$

\#Atoms $=105$

Charge $=-1 \quad$ Multiplicity $=1$

SCF Energy $=-5034.08726089 \quad$ Predicted Change $=-3.390430 \mathrm{D}-08$

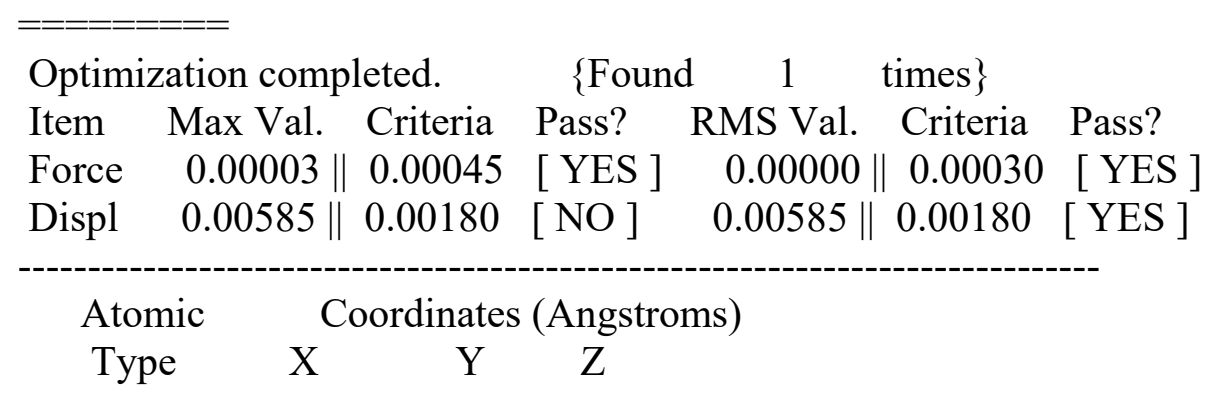




\begin{tabular}{|c|c|c|c|}
\hline $\mathrm{O}$ & 5.185079 & 1.797017 & -1.304508 \\
\hline $\mathrm{O}$ & 1.948551 & 7.152733 & -3.465473 \\
\hline $\mathrm{O}$ & -4.496367 & 2.833741 & 1.634054 \\
\hline $\mathrm{O}$ & 0.325263 & 7.039910 & 3.420253 \\
\hline $\mathrm{N}$ & 3.800475 & 0.106763 & -0.473437 \\
\hline $\mathrm{H}$ & 2.814191 & -0.186211 & -0.392589 \\
\hline $\mathrm{N}$ & 2.892136 & 2.118908 & -1.165994 \\
\hline $\mathrm{H}$ & 1.994363 & 1.680954 & -0.851116 \\
\hline $\mathrm{N}$ & -3.715096 & 0.946067 & 0.498236 \\
\hline $\mathrm{H}$ & -2.857203 & 0.428632 & 0.252828 \\
\hline $\mathrm{N}$ & -2.236439 & 2.591542 & 1.158636 \\
\hline $\mathrm{H}$ & -1.545335 & 2.004873 & 0.634292 \\
\hline $\mathrm{C}$ & -0.457867 & -2.966754 & -0.098755 \\
\hline $\mathrm{H}$ & -0.338522 & -1.873898 & -0.116409 \\
\hline $\mathrm{C}$ & 0.686286 & -3.790891 & -0.064219 \\
\hline $\mathrm{C}$ & 0.526151 & -5.198710 & -0.062826 \\
\hline $\mathrm{H}$ & 1.404935 & -5.848014 & -0.028322 \\
\hline $\mathrm{C}$ & -0.761896 & -5.750765 & -0.097510 \\
\hline $\mathrm{C}$ & -1.901133 & -4.934260 & -0.127666 \\
\hline $\mathrm{H}$ & -2.899736 & -5.378831 & -0.140289 \\
\hline $\mathrm{C}$ & -1.753148 & -3.525591 & -0.125742 \\
\hline $\mathrm{C}$ & 1.976553 & -3.195378 & -0.013592 \\
\hline $\mathrm{C}$ & 3.081102 & -2.655844 & 0.046249 \\
\hline $\mathrm{C}$ & 4.412532 & -2.176580 & 0.162266 \\
\hline $\mathrm{C}$ & 4.779787 & -0.815776 & -0.104086 \\
\hline $\mathrm{C}$ & 6.143046 & -0.472714 & 0.046036 \\
\hline $\mathrm{H}$ & 6.442322 & 0.552542 & -0.166067 \\
\hline $\mathrm{C}$ & 7.093167 & -1.420739 & 0.434428 \\
\hline $\mathrm{H}$ & 8.129645 & -1.083306 & 0.531892 \\
\hline $\mathrm{C}$ & 6.758577 & -2.763861 & 0.702088 \\
\hline $\mathrm{C}$ & 5.407256 & -3.104712 & 0.556718 \\
\hline $\mathrm{H}$ & 5.069605 & -4.129053 & 0.747964 \\
\hline $\mathrm{C}$ & 4.049952 & 1.391449 & -1.012052 \\
\hline $\mathrm{C}$ & 2.756651 & 3.389165 & -1.756434 \\
\hline $\mathrm{C}$ & 3.834521 & 4.202138 & -2.159167 \\
\hline $\mathrm{H}$ & 4.852703 & 3.837609 & -2.021360 \\
\hline $\mathrm{C}$ & 3.597499 & 5.461125 & -2.734684 \\
\hline $\mathrm{H}$ & 4.457511 & 6.065410 & -3.037093 \\
\hline $\mathrm{C}$ & 2.286233 & 5.930609 & -2.911997 \\
\hline $\mathrm{C}$ & 1.206979 & 5.121037 & -2.509255 \\
\hline $\mathrm{H}$ & 0.186655 & 5.491806 & -2.647485 \\
\hline $\mathrm{C}$ & 1.436515 & 3.869412 & -1.944279 \\
\hline $\mathrm{H}$ & 0.595573 & 3.235123 & -1.640030 \\
\hline $\mathrm{C}$ & 3.022838 & 7.987254 & -3.865006 \\
\hline $\mathrm{H}$ & 2.562960 & 8.902894 & -4.268829 \\
\hline $\mathrm{H}$ & 3.644951 & 7.515209 & -4.652598 \\
\hline $\mathrm{H}$ & 3.679015 & 8.255478 & -3.012252 \\
\hline
\end{tabular}




\begin{tabular}{|c|c|c|c|}
\hline $\mathrm{C}$ & -2.880287 & -2.658823 & -0.136751 \\
\hline $\mathrm{C}$ & -3.822341 & -1.867034 & -0.133800 \\
\hline $\mathrm{C}$ & -4.980726 & -1.046886 & -0.140768 \\
\hline $\mathrm{C}$ & -4.937809 & 0.345252 & 0.202160 \\
\hline $\mathrm{C}$ & -6.160898 & 1.052674 & 0.199905 \\
\hline $\mathrm{H}$ & -6.148979 & 2.105597 & 0.478761 \\
\hline $\mathrm{C}$ & -7.363188 & 0.421198 & -0.129397 \\
\hline $\mathrm{H}$ & -8.272592 & 1.029710 & -0.113689 \\
\hline $\mathrm{C}$ & -7.431470 & -0.942887 & -0.480490 \\
\hline $\mathrm{C}$ & -6.219008 & -1.646084 & -0.475980 \\
\hline $\mathrm{H}$ & -6.193753 & -2.710689 & -0.732674 \\
\hline $\mathrm{C}$ & -3.553341 & 2.194999 & 1.143219 \\
\hline $\mathrm{C}$ & -1.690790 & 3.739428 & 1.760594 \\
\hline $\mathrm{C}$ & -2.434212 & 4.707113 & 2.463413 \\
\hline $\mathrm{H}$ & -3.513323 & 4.581599 & 2.557742 \\
\hline $\mathrm{C}$ & -1.788913 & 5.818678 & 3.030231 \\
\hline $\mathrm{H}$ & -2.393931 & 6.552543 & 3.570294 \\
\hline $\mathrm{C}$ & -0.400142 & 5.982026 & 2.901400 \\
\hline $\mathrm{C}$ & 0.344340 & 5.015508 & 2.197643 \\
\hline $\mathrm{H}$ & 1.425477 & 5.149604 & 2.093808 \\
\hline $\mathrm{C}$ & -0.289351 & 3.909512 & 1.639027 \\
\hline $\mathrm{H}$ & 0.280319 & 3.147384 & 1.092540 \\
\hline $\mathrm{C}$ & -0.408160 & 8.029081 & 4.122450 \\
\hline $\mathrm{H}$ & 0.328791 & 8.779157 & 4.450002 \\
\hline $\mathrm{H}$ & -0.922159 & 7.611843 & 5.012382 \\
\hline $\mathrm{H}$ & -1.165062 & 8.520328 & 3.477699 \\
\hline $\mathrm{C}$ & 7.787292 & -3.828198 & 1.132542 \\
\hline $\mathrm{C}$ & -8.744338 & -1.660811 & -0.851553 \\
\hline $\mathrm{C}$ & 7.397858 & -4.393583 & 2.521058 \\
\hline $\mathrm{H}$ & 6.392114 & -4.846840 & 2.507989 \\
\hline $\mathrm{H}$ & 8.116187 & -5.172577 & 2.838172 \\
\hline $\mathrm{H}$ & 7.395443 & -3.595305 & 3.283608 \\
\hline $\mathrm{C}$ & 9.214607 & -3.251801 & 1.231049 \\
\hline $\mathrm{H}$ & 9.562720 & -2.854308 & 0.261488 \\
\hline $\mathrm{H}$ & 9.277348 & -2.440672 & 1.977627 \\
\hline $\mathrm{H}$ & 9.917053 & -4.046442 & 1.539640 \\
\hline $\mathrm{C}$ & 7.802941 & -4.981881 & 0.099099 \\
\hline $\mathrm{H}$ & 8.094472 & -4.611101 & -0.899030 \\
\hline $\mathrm{H}$ & 8.524044 & -5.764054 & 0.401777 \\
\hline $\mathrm{H}$ & 6.811285 & -5.455856 & 0.003636 \\
\hline $\mathrm{C}$ & -8.638519 & -2.221901 & -2.291472 \\
\hline $\mathrm{H}$ & -7.797959 & -2.929467 & -2.391233 \\
\hline $\mathrm{H}$ & -9.566161 & -2.756723 & -2.568680 \\
\hline $\mathrm{H}$ & -8.478745 & -1.407662 & -3.019612 \\
\hline $\mathrm{C}$ & -8.992123 & -2.830227 & 0.133550 \\
\hline $\mathrm{H}$ & -9.088925 & -2.458169 & 1.168411 \\
\hline $\mathrm{H}$ & -9.922150 & -3.368252 & -0.129104 \\
\hline $\mathrm{H}$ & -8.164188 & -3.559176 & 0.115973 \\
\hline
\end{tabular}




$\begin{array}{llll}\text { C } & -9.962053 & -0.715991 & -0.791778 \\ \text { H } & -10.108429 & -0.301355 & 0.221075 \\ \text { H } & -9.860191 & 0.127387 & -1.497172 \\ \text { H } & -10.877955 & -1.270809 & -1.062730 \\ \text { C } & -0.922560 & -7.246795 & -0.167866 \\ \text { F } & -2.089714 & -7.666864 & 0.400704 \\ \text { F } & 0.093193 & -7.904021 & 0.463315 \\ \text { F } & -0.934648 & -7.698436 & -1.458197 \\ \text { H } & 0.360991 & 0.443268 & 1.101355 \\ \text { Se } & 0.018734 & 0.632661 & -0.329697\end{array}$

Statistical Thermodynamic Analysis

Temperature $=298.150$ Kelvin $\quad$ Pressure $=1.00000$ Atm

\begin{tabular}{|c|c|}
\hline $\begin{array}{l}\text { SCF Energy }=\quad-5034.0872608 \\
\text { Zero-point correction }(\mathrm{ZPE})=\end{array}$ & 9 Predicted Change $=-3.390430 \mathrm{D}-08$ \\
\hline Zero-point correction $(\mathrm{ZPE})=$ & $-5033.2710 \quad 0.81625$ \\
\hline Internal Energy $(U)=$ & -5033.21180 .87545 \\
\hline Enthalpy $(\mathrm{H})=$ & $\begin{array}{lll}-5033.2108 & 0.87639\end{array}$ \\
\hline Gibbs Free Energy $(G)=$ & $\begin{array}{ll}-5033.3739 & 0.71327\end{array}$ \\
\hline $\begin{array}{l}\text { Frequencies -- } 5.4817 \\
\text { Single points at WB97X-D3/def2 }\end{array}$ & $\begin{array}{cc}7.0838 & 13.0974 \\
\text { 2-TZVPP: } & -5040.12069173254\end{array}$ \\
\hline $\begin{array}{r}\mathbf{R}=\mathbf{C F}_{3} \text { receptor bound with } \mathbf{S} \\
\text { Supporting Information: }\end{array}$ & $\begin{array}{l}\text { H- (no TBA) } \\
\text { 010-bound_noTBA_CF3_SH.log }\end{array}$ \\
\hline Using Gaussian 09: EM64L-GC & 09RevE.01 30-Nov-2015 \\
\hline
\end{tabular}

$========$
\#PBEPBE $/ 6-31 \mathrm{G}(\mathrm{d}) /$ auto $\mathrm{scf}=($ direct,tight, maxcycle $=300, \mathrm{xqc})$

$\mathrm{opt}=($ maxcycle $=250)$ freq $=$ noraman int $=($ grid $=$ ultrafine $)$

\#P Geom=AllCheck Guess=TCheck SCRF=Check GenChk RPBEPBE/6-31G(d)/Auto Freq

Pointgroup= $\mathrm{C} 1$ Stoichiometry $=\mathrm{C} 47 \mathrm{H} 46 \mathrm{~F} 3 \mathrm{~N} 4 \mathrm{O} 4 \mathrm{~S}(1-) \quad \mathrm{C} 1[\mathrm{X}(\mathrm{C} 47 \mathrm{H} 46 \mathrm{~F} 3 \mathrm{~N} 4 \mathrm{O} 4 \mathrm{~S})]$ \#Atoms= 105

Charge $=-1 \quad$ Multiplicity $=1$

SCF Energy $=-3033.07097655 \quad$ Predicted Change $=-1.257949 \mathrm{D}-08$

\begin{tabular}{|c|c|c|c|c|}
\hline \multicolumn{5}{|c|}{$========$} \\
\hline Optim & zation completed. & $\{$ Found & times $\}$ & \\
\hline Item & Max Val. Criteria & Pass? & RMS Val. Criteria & Pass? \\
\hline Force & $0.00001 \| 0.00045$ & [ YES ] & $0.00000 \| 0.00030$ & [YES ] \\
\hline Displ & $0.00313 \| 0.00180$ & {$[\mathrm{NO}]$} & $0.00313 \| 0.00180$ & [YES ] \\
\hline
\end{tabular}

Atomic Coordinates (Angstroms) 


\begin{tabular}{|c|c|c|c|}
\hline Type & $X$ & $\mathrm{Y}$ & \\
\hline $\mathrm{O}$ & 4.600514 & 2.583144 & -1.617483 \\
\hline $\mathrm{O}$ & 0.152063 & 7.026789 & -3.645624 \\
\hline $\mathrm{O}$ & -4.894474 & 2.158452 & 1.478842 \\
\hline $\mathrm{O}$ & -1.105795 & 7.104294 & 3.657708 \\
\hline $\mathrm{N}$ & 3.702077 & 0.712509 & -0.553250 \\
\hline $\mathrm{H}$ & 2.819866 & 0.214079 & -0.367947 \\
\hline $\mathrm{N}$ & 2.307199 & 2.409083 & -1.279245 \\
\hline $\mathrm{H}$ & 1.564883 & 1.843979 & -0.798201 \\
\hline $\mathrm{N}$ & -3.738305 & 0.365692 & 0.536962 \\
\hline $\mathrm{H}$ & -2.799451 & -0.038793 & 0.412923 \\
\hline $\mathrm{N}$ & -2.579142 & 2.218790 & 1.303753 \\
\hline $\mathrm{H}$ & -1.742054 & 1.715886 & 0.915566 \\
\hline $\mathrm{C}$ & 0.183255 & -2.944489 & 0.019830 \\
\hline $\mathrm{H}$ & 0.130458 & -1.844966 & -0.010899 \\
\hline $\mathrm{C}$ & 1.440776 & -3.583870 & 0.079687 \\
\hline $\mathrm{C}$ & 1.501181 & -4.998265 & 0.132292 \\
\hline $\mathrm{H}$ & 2.470307 & -5.501267 & 0.185573 \\
\hline $\mathrm{C}$ & 0.314988 & -5.744717 & 0.122088 \\
\hline $\mathrm{C}$ & -0.935967 & -5.115119 & 0.065319 \\
\hline $\mathrm{H}$ & -1.854026 & -5.708586 & 0.069999 \\
\hline $\mathrm{C}$ & -1.008623 & -3.701162 & 0.016556 \\
\hline $\mathrm{C}$ & 2.625335 & -2.796652 & 0.092621 \\
\hline $\mathrm{C}$ & 3.630199 & -2.086168 & 0.102621 \\
\hline $\mathrm{C}$ & 4.838236 & -1.341064 & 0.123981 \\
\hline $\mathrm{C}$ & 4.884160 & 0.049371 & -0.228729 \\
\hline $\mathrm{C}$ & 6.145637 & 0.684911 & -0.204124 \\
\hline $\mathrm{H}$ & 6.199612 & 1.735033 & -0.488339 \\
\hline $\mathrm{C}$ & 7.301540 & -0.013992 & 0.154663 \\
\hline $\mathrm{H}$ & 8.245198 & 0.540094 & 0.154580 \\
\hline $\mathrm{C}$ & 7.283036 & -1.377359 & 0.514065 \\
\hline $\mathrm{C}$ & 6.031887 & -2.009320 & 0.487808 \\
\hline $\mathrm{H}$ & 5.939510 & -3.068806 & 0.750434 \\
\hline $\mathrm{C}$ & 3.609936 & 1.969639 & -1.191896 \\
\hline $\mathrm{C}$ & 1.859866 & 3.592319 & -1.897612 \\
\hline $\mathrm{C}$ & 2.690913 & 4.492868 & -2.594414 \\
\hline $\mathrm{H}$ & 3.758738 & 4.284590 & -2.660671 \\
\hline $\mathrm{C}$ & 2.151769 & 5.645415 & -3.188651 \\
\hline $\mathrm{H}$ & 2.827878 & 6.320666 & -3.720852 \\
\hline $\mathrm{C}$ & 0.778375 & 5.921560 & -3.097400 \\
\hline $\mathrm{C}$ & -0.055174 & 5.026577 & -2.400555 \\
\hline $\mathrm{H}$ & -1.124221 & 5.247881 & -2.324786 \\
\hline $\mathrm{C}$ & 0.475466 & 3.881154 & -1.812367 \\
\hline $\mathrm{H}$ & -0.180579 & 3.191908 & -1.269572 \\
\hline $\mathrm{C}$ & 0.978734 & 7.944201 & -4.341719 \\
\hline $\mathrm{H}$ & 0.312316 & 8.746407 & -4.695803 \\
\hline $\mathrm{H}$ & 1.477786 & 7.474889 & -5.214005 \\
\hline
\end{tabular}




\begin{tabular}{|c|c|c|c|}
\hline $\mathrm{H}$ & 1.757667 & 8.382043 & -3.684859 \\
\hline $\mathrm{C}$ & -2.262525 & -3.031293 & -0.030624 \\
\hline $\mathrm{C}$ & -3.330081 & -2.420925 & -0.077400 \\
\hline $\mathrm{C}$ & -4.609519 & -1.810496 & -0.155206 \\
\hline $\mathrm{C}$ & -4.823416 & -0.428516 & 0.166976 \\
\hline $\mathrm{C}$ & -6.144349 & 0.063446 & 0.069555 \\
\hline $\mathrm{H}$ & -6.326040 & 1.106251 & 0.325690 \\
\hline $\mathrm{C}$ & -7.199085 & -0.764265 & -0.325113 \\
\hline $\mathrm{H}$ & -8.196064 & -0.316524 & -0.381100 \\
\hline $\mathrm{C}$ & -7.015748 & -2.123705 & -0.650597 \\
\hline $\mathrm{C}$ & -5.705485 & -2.612612 & -0.554685 \\
\hline $\mathrm{H}$ & -5.485037 & -3.659405 & -0.790354 \\
\hline $\mathrm{C}$ & -3.819224 & 1.640583 & 1.140155 \\
\hline $\mathrm{C}$ & -2.307449 & 3.460193 & 1.910979 \\
\hline $\mathrm{C}$ & -3.276470 & 4.279400 & 2.525528 \\
\hline $\mathrm{H}$ & -4.317202 & 3.955882 & 2.530120 \\
\hline $\mathrm{C}$ & -2.907426 & 5.498743 & 3.116446 \\
\hline $\mathrm{H}$ & -3.687493 & 6.107246 & 3.582871 \\
\hline $\mathrm{C}$ & -1.569506 & 5.923685 & 3.104975 \\
\hline $\mathrm{C}$ & -0.598682 & 5.110693 & 2.490340 \\
\hline $\mathrm{H}$ & 0.442264 & 5.448268 & 2.475455 \\
\hline $\mathrm{C}$ & -0.960640 & 3.900180 & 1.904536 \\
\hline $\mathrm{H}$ & -0.199638 & 3.274188 & 1.424600 \\
\hline $\mathrm{C}$ & -2.073128 & 7.942056 & 4.267870 \\
\hline $\mathrm{H}$ & -1.523742 & 8.821878 & 4.638138 \\
\hline $\mathrm{H}$ & -2.576725 & 7.442775 & 5.120687 \\
\hline $\mathrm{H}$ & -2.848160 & 8.273962 & 3.547473 \\
\hline $\mathrm{C}$ & 8.543898 & -2.167250 & 0.917846 \\
\hline $\mathrm{C}$ & -8.162409 & -3.055055 & -1.091306 \\
\hline $\mathrm{C}$ & 8.375981 & -2.708534 & 2.359442 \\
\hline $\mathrm{H}$ & 7.493246 & -3.364478 & 2.446929 \\
\hline $\mathrm{H}$ & 9.264583 & -3.294620 & 2.659971 \\
\hline $\mathrm{H}$ & 8.249834 & -1.879910 & 3.077908 \\
\hline $\mathrm{C}$ & 9.815804 & -1.295655 & 0.876521 \\
\hline $\mathrm{H}$ & 10.006889 & -0.899156 & -0.136140 \\
\hline $\mathrm{H}$ & 9.749152 & -0.441682 & 1.573301 \\
\hline $\mathrm{H}$ & 10.691867 & -1.900685 & 1.170772 \\
\hline $\mathrm{C}$ & 8.743475 & -3.357799 & -0.052815 \\
\hline $\mathrm{H}$ & 8.883114 & -3.001202 & -1.088221 \\
\hline $\mathrm{H}$ & 9.635107 & -3.946332 & 0.233586 \\
\hline $\mathrm{H}$ & 7.874554 & -4.037579 & -0.047211 \\
\hline $\mathrm{C}$ & -7.867254 & -3.605431 & -2.508843 \\
\hline $\mathrm{H}$ & -6.916370 & -4.164071 & -2.538361 \\
\hline $\mathrm{H}$ & -8.672489 & -4.290214 & -2.834560 \\
\hline $\mathrm{H}$ & -7.795373 & -2.783461 & -3.242233 \\
\hline $\mathrm{C}$ & -8.278814 & -4.239241 & -0.099597 \\
\hline $\mathrm{H}$ & -8.507318 & -3.878122 & 0.918315 \\
\hline $\mathrm{H}$ & -9.085714 & -4.928562 & -0.411331 \\
\hline
\end{tabular}




$\begin{array}{lccc}\mathrm{H} & -7.341564 & -4.818846 & -0.046657 \\ \mathrm{C} & -9.521486 & -2.326709 & -1.131760 \\ \mathrm{H} & -9.803568 & -1.932155 & -0.139798 \\ \mathrm{H} & -9.513258 & -1.485367 & -1.846803 \\ \mathrm{H} & -10.311752 & -3.029668 & -1.450295 \\ \mathrm{C} & 0.387615 & -7.249028 & 0.105615 \\ \mathrm{~F} & -0.700643 & -7.823782 & 0.694905 \\ \mathrm{~F} & 1.492707 & -7.718496 & 0.754062 \\ \mathrm{~F} & 0.445622 & -7.743177 & -1.167887 \\ \mathrm{~S} & 0.035553 & 0.591233 & 0.218585 \\ \mathrm{H} & 0.486093 & 0.562161 & 1.504546\end{array}$

Statistical Thermodynamic Analysis

Temperature $=298.150$ Kelvin Pressure $=1.00000$ Atm

\begin{tabular}{lrl}
\hline$=======$ & & \\
SCF Energy $=$ & -3033.07097655 & Predicted Change $=-1.2579$ \\
Zero-point correction $(\mathrm{ZPE})=$ & -3032.2573 & 0.81360 \\
Internal Energy $(\mathrm{U})=$ & -3032.1974 & 0.87349 \\
Enthalpy $(\mathrm{H})=$ & -3032.1965 & 0.87443 \\
Gibbs Free Energy $(\mathrm{G})=$ & -3032.3615 & 0.70938 \\
\hdashline Frequencies -- $\quad 5.1005$ & 6.5785 & 12.0437 \\
Single points at WB97X-D3/def2-TZVPP: -3036.70542929188
\end{tabular}

$\mathbf{R}=\mathbf{C l}$ receptor unbound (from crystal structure)

Supporting Information:000-unbound_crystal_structure_Cl.log

Using Gaussian 09: EM64L-G09RevE.01 30-Nov-2015

\begin{abstract}
\#PBEPBE/6-31G(d)/auto scf=(direct,tight,maxcycle=300,xqc)
$\mathrm{opt}=($ maxcycle $=250)$ freq $=$ noraman int $=($ grid $=$ ultrafine $)$

\#P Geom=AllCheck Guess=TCheck SCRF=Check GenChk RPBEPBE/6-31G(d)/Auto Freq

Pointgroup $=\mathrm{C} 1 \quad$ Stoichiometry $=\mathrm{C} 46 \mathrm{H} 45 \mathrm{ClN} 4 \mathrm{O} 4 \mathrm{C} 1[\mathrm{X}(\mathrm{C} 46 \mathrm{H} 45 \mathrm{ClN} 4 \mathrm{O} 4)]$ \#Atoms $=100$

Charge $=0 \quad$ Multiplicity $=1$
\end{abstract}

SCF Energy $=-2757.03199095 \quad$ Predicted Change $=-5.217529 \mathrm{D}-09$

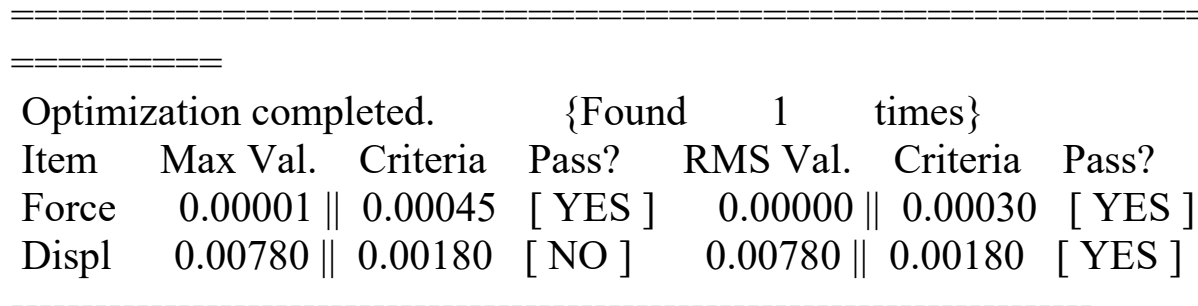

Atomic Coordinates (Angstroms) 


\begin{tabular}{|c|c|c|c|}
\hline Type & $X$ & Y & \\
\hline $\mathrm{O}$ & -8.180457 & -0.299966 & -0.000474 \\
\hline $\mathrm{O}$ & -9.356253 & -6.830114 & -0.000827 \\
\hline $\mathrm{O}$ & 8.180400 & -0.300208 & -0.000463 \\
\hline $\mathrm{O}$ & 9.355771 & -6.830433 & -0.000824 \\
\hline $\mathrm{N}$ & -5.919065 & 0.251561 & 0.000245 \\
\hline $\mathrm{N}$ & -6.562426 & -1.966119 & 0.000450 \\
\hline $\mathrm{N}$ & 5.919044 & 0.251466 & 0.000233 \\
\hline $\mathrm{N}$ & 6.562260 & -1.966256 & 0.000437 \\
\hline $\mathrm{C}$ & 0.000036 & 0.915142 & 0.001004 \\
\hline $\mathrm{H}$ & 0.000023 & 2.007694 & 0.000254 \\
\hline $\mathrm{C}$ & -1.228549 & 0.216609 & 0.001498 \\
\hline $\mathrm{C}$ & -1.221706 & -1.201599 & 0.002472 \\
\hline $\mathrm{H}$ & -2.161090 & -1.759206 & 0.002887 \\
\hline $\mathrm{C}$ & 0.000068 & -1.879346 & 0.002919 \\
\hline $\mathrm{C}$ & 1.221827 & -1.201571 & 0.002445 \\
\hline $\mathrm{H}$ & 2.161224 & -1.759155 & 0.002838 \\
\hline $\mathrm{C}$ & 1.228637 & 0.216637 & 0.001471 \\
\hline $\mathrm{C}$ & -2.457252 & 0.931828 & 0.001013 \\
\hline $\mathrm{C}$ & -3.488303 & 1.607461 & 0.000563 \\
\hline $\mathrm{C}$ & -4.705010 & 2.338895 & 0.000058 \\
\hline $\mathrm{C}$ & -5.961449 & 1.648086 & -0.000107 \\
\hline $\mathrm{C}$ & -7.143267 & 2.409204 & -0.000602 \\
\hline $\mathrm{H}$ & -8.101498 & 1.889792 & -0.000733 \\
\hline $\mathrm{C}$ & -7.080825 & 3.807805 & -0.000928 \\
\hline $\mathrm{H}$ & -8.028764 & 4.352990 & -0.001316 \\
\hline $\mathrm{C}$ & -5.862649 & 4.518412 & -0.000776 \\
\hline $\mathrm{C}$ & -4.687169 & 3.751728 & -0.000283 \\
\hline $\mathrm{H}$ & -3.706866 & 4.239045 & -0.000136 \\
\hline $\mathrm{C}$ & -6.995719 & -0.644860 & 0.000027 \\
\hline $\mathrm{C}$ & -7.347293 & -3.141826 & 0.000089 \\
\hline $\mathrm{C}$ & -6.661853 & -4.377162 & 0.000214 \\
\hline $\mathrm{H}$ & -5.564439 & -4.392641 & 0.000556 \\
\hline $\mathrm{C}$ & -7.357044 & -5.583686 & -0.000094 \\
\hline $\mathrm{H}$ & -6.824456 & -6.539078 & -0.000002 \\
\hline $\mathrm{C}$ & -8.765831 & -5.590048 & -0.000540 \\
\hline $\mathrm{C}$ & -9.453698 & -4.364147 & -0.000659 \\
\hline $\mathrm{H}$ & -10.546177 & -4.333959 & -0.000994 \\
\hline $\mathrm{C}$ & -8.754073 & -3.148112 & -0.000341 \\
\hline $\mathrm{H}$ & -9.292027 & -2.200084 & -0.000432 \\
\hline $\mathrm{C}$ & -10.779878 & -6.868356 & -0.001293 \\
\hline $\mathrm{H}$ & -11.051103 & -7.934431 & -0.001475 \\
\hline $\mathrm{H}$ & -11.202213 & -6.383402 & -0.902866 \\
\hline $\mathrm{H}$ & -11.202801 & -6.383561 & 0.900089 \\
\hline $\mathrm{C}$ & 2.457323 & 0.931886 & 0.000957 \\
\hline $\mathrm{C}$ & 3.488370 & 1.607523 & 0.000490 \\
\hline $\mathrm{C}$ & 4.705125 & 2.338878 & 0.000026 \\
\hline
\end{tabular}




\begin{tabular}{|c|c|c|c|}
\hline $\mathrm{C}$ & 5.961519 & 1.647988 & -0.000111 \\
\hline $\mathrm{C}$ & 7.143387 & 2.409029 & -0.000570 \\
\hline $\mathrm{H}$ & 8.101584 & 1.889556 & -0.000682 \\
\hline $\mathrm{C}$ & 7.081035 & 3.807634 & -0.000889 \\
\hline $\mathrm{H}$ & 8.029010 & 4.352758 & -0.001248 \\
\hline $\mathrm{C}$ & 5.862905 & 4.518320 & -0.000762 \\
\hline $\mathrm{C}$ & 4.687375 & 3.751713 & -0.000305 \\
\hline $\mathrm{H}$ & 3.707103 & 4.239093 & -0.000180 \\
\hline $\mathrm{C}$ & 6.995640 & -0.645025 & 0.000023 \\
\hline $\mathrm{C}$ & 7.347052 & -3.142014 & 0.000081 \\
\hline $\mathrm{C}$ & 8.753831 & -3.148392 & -0.000345 \\
\hline $\mathrm{H}$ & 9.291846 & -2.200399 & -0.000436 \\
\hline $\mathrm{C}$ & 9.453377 & -4.364472 & -0.000660 \\
\hline $\mathrm{H}$ & 10.545858 & -4.334355 & -0.000993 \\
\hline $\mathrm{C}$ & 8.765430 & -5.590328 & -0.000540 \\
\hline $\mathrm{C}$ & 7.356643 & -5.583875 & -0.000098 \\
\hline $\mathrm{H}$ & 6.823993 & -6.539232 & -0.000005 \\
\hline $\mathrm{C}$ & 6.661531 & -4.377306 & 0.000206 \\
\hline $\mathrm{H}$ & 5.564116 & -4.392714 & 0.000547 \\
\hline $\mathrm{C}$ & 10.779393 & -6.868768 & -0.001288 \\
\hline $\mathrm{H}$ & 11.050549 & -7.934861 & -0.001469 \\
\hline $\mathrm{H}$ & 11.202347 & -6.384000 & 0.900094 \\
\hline $\mathrm{H}$ & 11.201761 & -6.383842 & -0.902861 \\
\hline $\mathrm{H}$ & -4.966601 & -0.122016 & 0.000564 \\
\hline $\mathrm{H}$ & -5.557514 & -2.135889 & 0.000707 \\
\hline $\mathrm{H}$ & 4.966555 & -0.122049 & 0.000522 \\
\hline $\mathrm{H}$ & 5.557337 & -2.135960 & 0.000697 \\
\hline $\mathrm{C}$ & 5.782319 & 6.057999 & -0.001093 \\
\hline $\mathrm{C}$ & 7.178298 & 6.713889 & -0.001722 \\
\hline $\mathrm{H}$ & 7.762938 & 6.437259 & 0.893120 \\
\hline $\mathrm{H}$ & 7.762335 & 6.436825 & -0.896823 \\
\hline $\mathrm{H}$ & 7.070670 & 7.812370 & -0.001950 \\
\hline $\mathrm{C}$ & 5.023283 & 6.534023 & 1.262667 \\
\hline $\mathrm{H}$ & 3.999513 & 6.124544 & 1.305020 \\
\hline $\mathrm{H}$ & 5.549591 & 6.221477 & 2.181183 \\
\hline $\mathrm{H}$ & 4.944521 & 7.636219 & 1.269430 \\
\hline $\mathrm{C}$ & 5.022424 & 6.533454 & -1.264549 \\
\hline $\mathrm{H}$ & 5.548113 & 6.220509 & -2.183283 \\
\hline $\mathrm{H}$ & 3.998627 & 6.123954 & -1.306030 \\
\hline $\mathrm{H}$ & 4.943648 & 7.635646 & -1.271741 \\
\hline $\mathrm{C}$ & -5.781964 & 6.058086 & -0.001115 \\
\hline $\mathrm{C}$ & -5.022924 & 6.534069 & 1.262658 \\
\hline $\mathrm{H}$ & -3.999181 & 6.124524 & 1.305036 \\
\hline $\mathrm{H}$ & -4.944090 & 7.636260 & 1.269415 \\
\hline $\mathrm{H}$ & -5.549271 & 6.221563 & 2.181165 \\
\hline $\mathrm{C}$ & -7.177900 & 6.714066 & -0.001778 \\
\hline $\mathrm{H}$ & -7.761937 & 6.437032 & -0.896889 \\
\hline $\mathrm{H}$ & -7.762576 & 6.437480 & 0.893055 \\
\hline
\end{tabular}




$\begin{array}{lrrr}\mathrm{H} & -7.070201 & 7.812539 & -0.002012 \\ \mathrm{C} & -5.022011 & 6.533483 & -1.264557 \\ \mathrm{H} & -3.998240 & 6.123917 & -1.306014 \\ \mathrm{H} & -5.547700 & 6.220566 & -2.183301 \\ \mathrm{H} & -4.943164 & 7.635670 & -1.271756 \\ \mathrm{Cl} & 0.000088 & -3.635587 & 0.004138\end{array}$

Statistical Thermodynamic Analysis

Temperature $=298.150$ Kelvin $\quad$ Pressure $=1.00000$ Atm

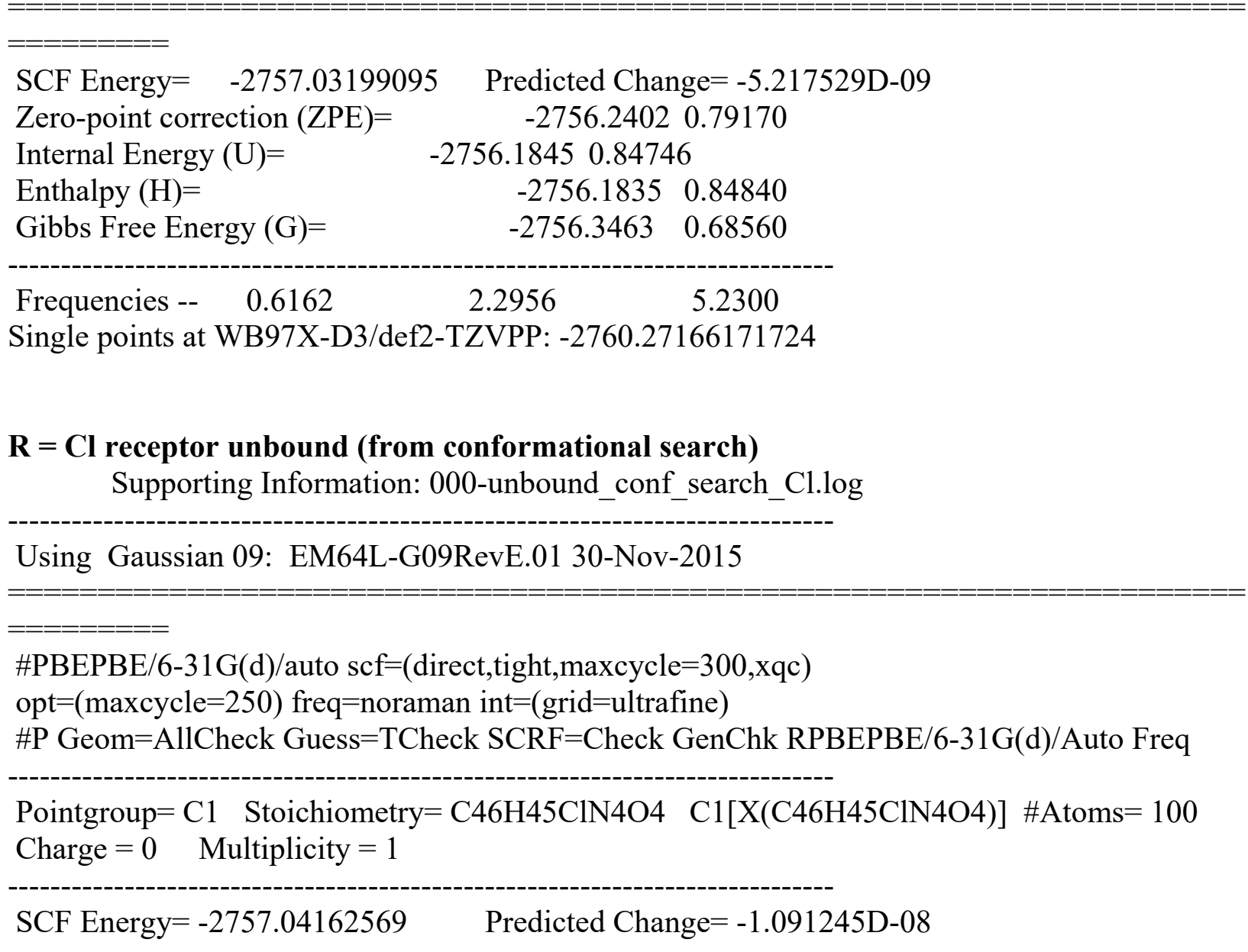

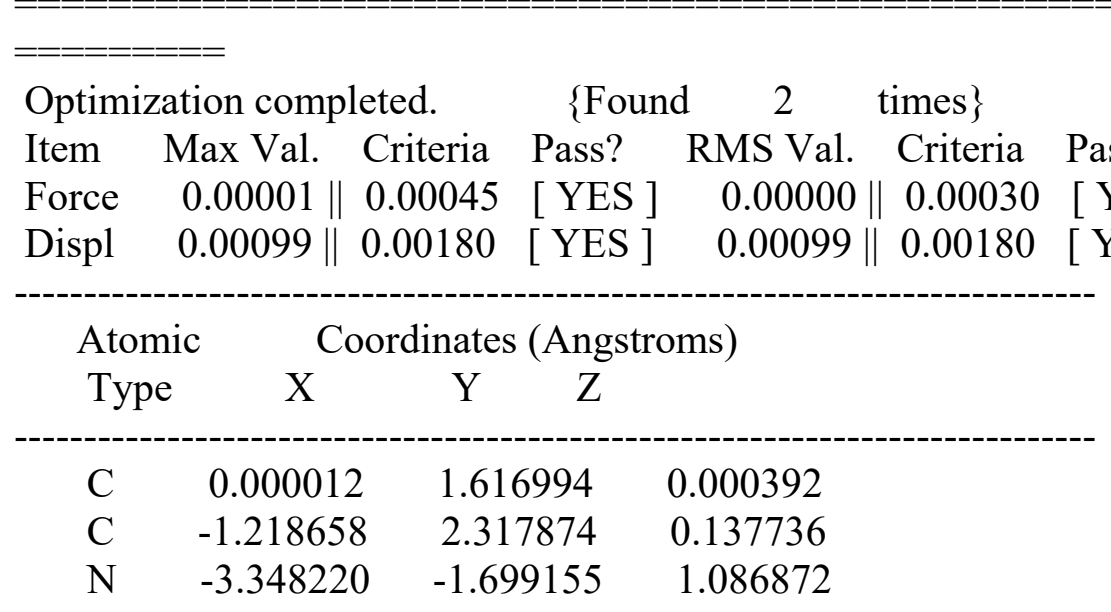




\begin{tabular}{|c|c|c|c|}
\hline $\mathrm{C}$ & -1.215185 & 3.735610 & 0.137982 \\
\hline $\mathrm{N}$ & -1.678705 & -3.195064 & 1.747936 \\
\hline $\mathrm{O}$ & -3.780835 & -3.983155 & 1.281473 \\
\hline $\mathrm{C}$ & 0.000003 & 4.412755 & 0.000581 \\
\hline $\mathrm{N}$ & 3.348082 & -1.698871 & -1.086787 \\
\hline $\mathrm{O}$ & 1.996792 & 0.486539 & 3.864811 \\
\hline $\mathrm{C}$ & 1.215194 & 3.735636 & -0.136908 \\
\hline $\mathrm{N}$ & 1.678508 & -3.194421 & -1.748560 \\
\hline $\mathrm{O}$ & 3.780743 & -3.982729 & -1.282946 \\
\hline $\mathrm{C}$ & 1.218679 & 2.317897 & -0.136855 \\
\hline $\mathrm{O}$ & -1.996779 & 0.488061 & -3.86429 \\
\hline $\mathrm{C}$ & -2.441558 & 1.602740 & 0.26966 \\
\hline $\mathrm{C}$ & -3.505639 & 0.988698 & 0.36272 \\
\hline $\mathrm{C}$ & -4.690959 & 0.208539 & 0.44 \\
\hline $\mathrm{C}$ & -4.611546 & -1.182063 & 0.78389 \\
\hline $\mathrm{C}$ & -5.799493 & -1.933478 & 0.81 \\
\hline $\mathrm{C}$ & -7.027619 & -1.324111 & 0.526346 \\
\hline $\mathrm{C}$ & -7.142732 & 0.043797 & 0.203690 \\
\hline $\mathrm{C}$ & -5.951029 & 0.785363 & 0.16 \\
\hline $\mathrm{C}$ & -8.488456 & 0.728904 & -0.10 \\
\hline $\mathrm{C}$ & -8.450797 & 1.309373 & -1.543713 \\
\hline $\mathrm{C}$ & -8.726660 & & 0.90270 \\
\hline $\mathrm{C}$ & -9.676030 & -0.250735 & -0.01 \\
\hline $\mathrm{C}$ & -3.013555 & -3.020821 & 1.37 \\
\hline $\mathrm{C}$ & -0.749650 & -2.235791 & 2.2 \\
\hline $\mathrm{C}$ & -1.130397 & -1.251491 & 3.188944 \\
\hline $\mathrm{C}$ & -0.192259 & -0.348794 & 3.68727 \\
\hline $\mathrm{C}$ & 1.159467 & -0.427362 & 3.29063 \\
\hline $\mathrm{C}$ & 1.550914 & -1.414776 & 2.36483 \\
\hline $\mathrm{C}$ & 0.594841 & -2.297707 & 1.84253 \\
\hline $\mathrm{C}$ & 3.379843 & 0.426982 & \\
\hline $\mathrm{C}$ & 2.441600 & 1.602804 & -0.26 \\
\hline $\mathrm{C}$ & 3.505686 & 0.988777 & -0.36 \\
\hline $\mathrm{C}$ & 4.690982 & 0.208643 & -0.44 \\
\hline $\mathrm{C}$ & 4.611493 & -1.181860 & -0.78400 \\
\hline $\mathrm{C}$ & 5.799440 & -1.933259 & -0.81134 \\
\hline $\mathrm{C}$ & 7.027633 & -1.323964 & -0.52709 \\
\hline $\mathrm{C}$ & 7.142817 & 0.043851 & -0.20406 \\
\hline $\mathrm{C}$ & 5.951118 & 0.785396 & -0.16915 \\
\hline $\mathrm{C}$ & 8.488612 & 0.728876 & 0.10733 \\
\hline $\mathrm{C}$ & 9.676179 & -0.250708 & 0.01285 \\
\hline $\mathrm{C}$ & 8.726517 & 1.878941 & -0.902867 \\
\hline $\mathrm{C}$ & 8.451337 & 1.308844 & 1.54342 \\
\hline $\mathrm{C}$ & 3.013423 & -3.020359 & -1.37796 \\
\hline $\mathrm{C}$ & 0.749512 & -2.234902 & -2.24921 \\
\hline $\mathrm{C}$ & 1.130305 & -1.250363 & -3.18898 \\
\hline $\mathrm{C}$ & 0.192219 & -0.347458 & -3.68702 \\
\hline $\mathrm{C}$ & -1.159503 & -0.426046 & -3.29037 \\
\hline
\end{tabular}




\begin{tabular}{|c|c|c|c|}
\hline $\mathrm{C}$ & -1.550994 & -1.413686 & -2.364835 \\
\hline $\mathrm{C}$ & -0.594965 & -2.296833 & -1.842818 \\
\hline $\mathrm{C}$ & -3.379840 & 0.428456 & -3.508251 \\
\hline $\mathrm{H}$ & 0.000017 & 0.523779 & 0.000339 \\
\hline $\mathrm{H}$ & -2.151141 & 4.288196 & 0.246464 \\
\hline $\mathrm{H}$ & -2.587542 & -1.013411 & 1.082033 \\
\hline $\mathrm{H}$ & -1.459782 & -4.183116 & 1.875919 \\
\hline $\mathrm{H}$ & 2.151148 & 4.288244 & -0.245292 \\
\hline $\mathrm{H}$ & 2.587416 & -1.013118 & -1.081596 \\
\hline $\mathrm{H}$ & 1.459665 & -4.182405 & -1.877232 \\
\hline $\mathrm{H}$ & -5.740728 & -2.995695 & 1.050484 \\
\hline $\mathrm{H}$ & -7.922073 & -1.952194 & 0.563100 \\
\hline $\mathrm{H}$ & -5.968508 & 1.851178 & -0.080793 \\
\hline $\mathrm{H}$ & -9.403948 & 1.817424 & -1.776979 \\
\hline $\mathrm{H}$ & -8.296931 & 0.509790 & -2.289161 \\
\hline $\mathrm{H}$ & -7.639392 & 2.047163 & -1.666654 \\
\hline $\mathrm{H}$ & -8.773357 & 1.493168 & 1.935932 \\
\hline $\mathrm{H}$ & -9.681175 & 2.390099 & 0.682875 \\
\hline $\mathrm{H}$ & -7.923911 & 2.634515 & 0.861040 \\
\hline $\mathrm{H}$ & -9.774727 & -0.680735 & 0.998076 \\
\hline $\mathrm{H}$ & -9.582312 & -1.081118 & -0.735675 \\
\hline $\mathrm{H}$ & -10.614902 & 0.283060 & -0.241641 \\
\hline $\mathrm{H}$ & -2.165852 & -1.207030 & 3.540354 \\
\hline $\mathrm{H}$ & -0.480142 & 0.416713 & 4.413738 \\
\hline $\mathrm{H}$ & 2.587587 & -1.495643 & 2.029680 \\
\hline $\mathrm{H}$ & 0.900644 & -3.046410 & 1.103758 \\
\hline $\mathrm{H}$ & 3.868978 & 1.218703 & 4.094723 \\
\hline $\mathrm{H}$ & 3.529206 & 0.620248 & 2.430572 \\
\hline $\mathrm{H}$ & 3.823973 & -0.551613 & 3.773072 \\
\hline $\mathrm{H}$ & 5.740625 & -2.995403 & -1.051408 \\
\hline $\mathrm{H}$ & 7.922081 & -1.952030 & -0.564261 \\
\hline $\mathrm{H}$ & 5.968648 & 1.851137 & 0.081244 \\
\hline $\mathrm{H}$ & 10.615096 & 0.283006 & 0.240479 \\
\hline $\mathrm{H}$ & 9.774656 & -0.680296 & -0.999439 \\
\hline $\mathrm{H}$ & 9.582632 & -1.081387 & 0.734191 \\
\hline $\mathrm{H}$ & 9.681076 & 2.390378 & -0.683119 \\
\hline $\mathrm{H}$ & 7.923756 & 2.634803 & -0.860733 \\
\hline $\mathrm{H}$ & 8.772950 & 1.493853 & -1.936244 \\
\hline $\mathrm{H}$ & 9.404548 & 1.816818 & 1.776609 \\
\hline $\mathrm{H}$ & 8.297676 & 0.509000 & 2.288634 \\
\hline $\mathrm{H}$ & 7.639962 & 2.046588 & 1.666841 \\
\hline $\mathrm{H}$ & 2.165752 & -1.205881 & -3.540413 \\
\hline $\mathrm{H}$ & 0.480129 & 0.418228 & -4.413294 \\
\hline $\mathrm{H}$ & -2.587665 & -1.494575 & -2.029678 \\
\hline $\mathrm{H}$ & -0.900799 & -3.045734 & -1.104252 \\
\hline $\mathrm{H}$ & -3.823993 & -0.550058 & -3.772863 \\
\hline $\mathrm{H}$ & -3.868935 & 1.220342 & -4.094065 \\
\hline $\mathrm{H}$ & -3.529224 & 0.621457 & -2.430058 \\
\hline
\end{tabular}


$\begin{array}{llll}\mathrm{Cl} & -0.000001 & 6.169180 & 0.000711\end{array}$

Statistical Thermodynamic Analysis

Temperature $=298.150$ Kelvin $\quad$ Pressure $=1.00000$ Atm

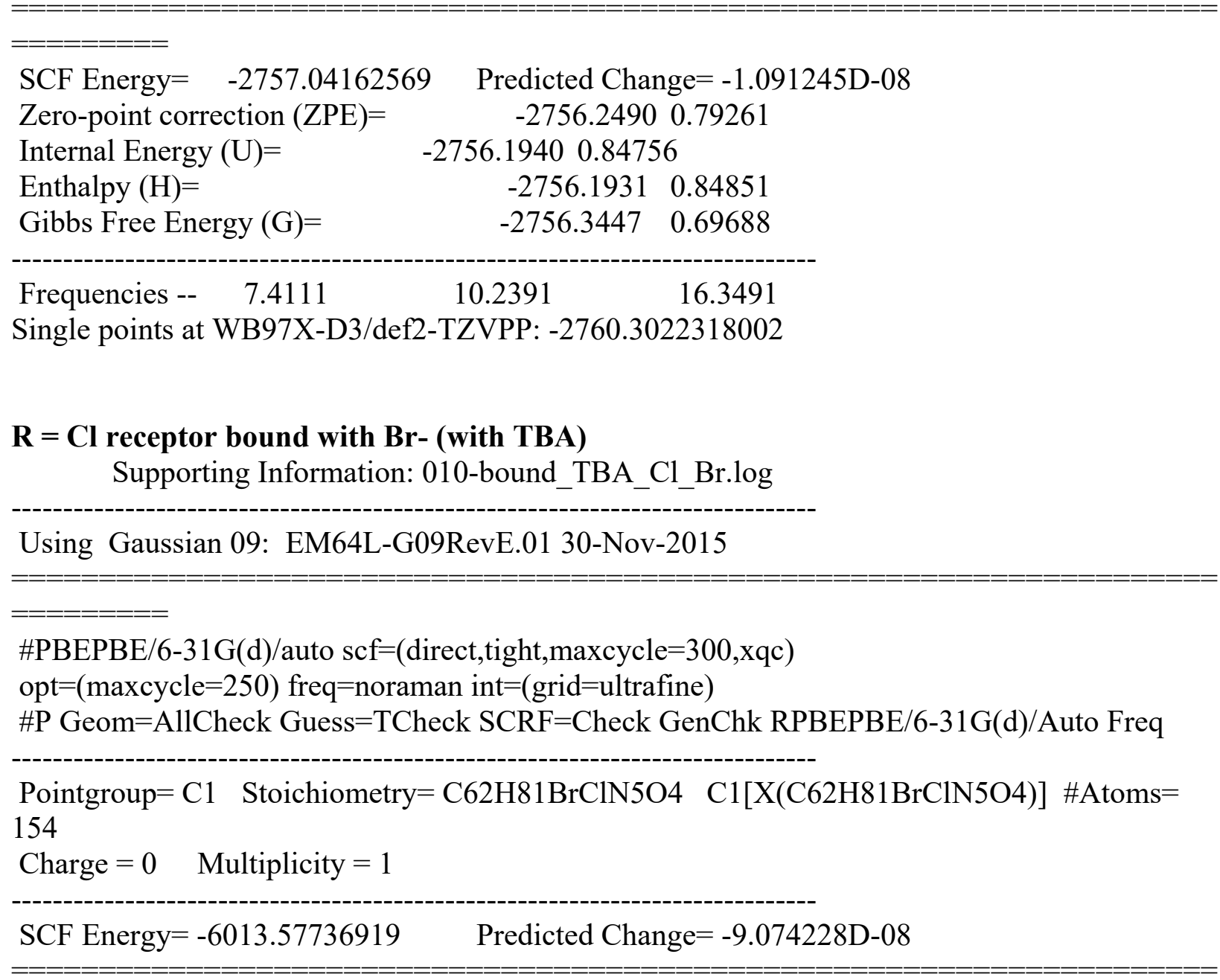

\begin{tabular}{|c|c|c|c|c|c|}
\hline \multicolumn{3}{|c|}{ Optimization completed. } & $\{$ Found & nd 1 & times $\}$ \\
\hline \multicolumn{3}{|c|}{ Item Max Val. Criteria } & Pass? & RMS Val. & Criteria \\
\hline Force & $0.00001 \|$ & 0.00045 & [ YES ] & 0.00000 & 0.00030 \\
\hline Displ & $0.00583 \|$ & 0.00180 & [ NO ] & $0.00583 \mid$ & 0.00180 \\
\hline \multicolumn{2}{|c|}{ Atomic } & oordinates & (Angstr & oms) & \\
\hline Type & e & $\mathrm{Y}$ & Z & & \\
\hline $\mathrm{N}$ & 2.28632 & 1.06 & 0167 & -2.590870 & \\
\hline $\mathrm{C}$ & 0.98130 & 0.77 & 3865 & -3.351382 & \\
\hline $\mathrm{C}$ & 0.07207 & -0.32 & 8994 & -2.808053 & \\
\hline $\mathrm{C}$ & -1.20961 & -0.37 & 9727 & -3.667408 & \\
\hline $\mathrm{C}$ & -2.13867 & -1.53 & 2350 & -3.269570 & \\
\hline $\mathrm{C}$ & 1.93173 & 1.75 & 5407 & -1.267364 & \\
\hline $\mathrm{C}$ & 3.08846 & 2.23 & 3503 & -0.386372 & \\
\hline
\end{tabular}




\begin{tabular}{|c|c|c|c|}
\hline $\mathrm{C}$ & 2.502487 & 3.043905 & 0.791809 \\
\hline $\mathrm{C}$ & 3.568889 & 3.498721 & 1.794960 \\
\hline $\mathrm{C}$ & 3.041133 & -0.233122 & -2.277059 \\
\hline $\mathrm{C}$ & 3.414291 & -1.114719 & -3.472681 \\
\hline $\mathrm{C}$ & 4.064275 & -2.426091 & -2.988113 \\
\hline $\mathrm{C}$ & 4.478139 & -3.335451 & -4.151434 \\
\hline $\mathrm{C}$ & 3.162784 & 1.964757 & -3.458540 \\
\hline $\mathrm{C}$ & 2.542291 & 3.301597 & -3.876836 \\
\hline $\mathrm{C}$ & 3.588760 & 4.212250 & -4.547664 \\
\hline $\mathrm{C}$ & 2.957408 & 5.506722 & -5.078285 \\
\hline $\mathrm{H}$ & 0.436849 & 1.731324 & -3.363440 \\
\hline $\mathrm{H}$ & 1.284064 & 0.545691 & -4.387972 \\
\hline $\mathrm{H}$ & -0.202972 & -0.156083 & -1.750079 \\
\hline $\mathrm{H}$ & 0.573086 & -1.313853 & -2.855679 \\
\hline $\mathrm{H}$ & -0.934125 & -0.477436 & -4.736928 \\
\hline $\mathrm{H}$ & -1.748329 & 0.581845 & -3.569370 \\
\hline $\mathrm{H}$ & -3.031643 & -1.555502 & -3.916451 \\
\hline $\mathrm{H}$ & -1.630858 & -2.509106 & -3.358516 \\
\hline $\mathrm{H}$ & -2.485606 & -1.429396 & -2.228160 \\
\hline $\mathrm{H}$ & 1.290136 & 2.604619 & -1.546744 \\
\hline $\mathrm{H}$ & 1.292802 & 1.056080 & -0.698939 \\
\hline $\mathrm{H}$ & 3.663917 & 1.381525 & 0.019240 \\
\hline $\mathrm{H}$ & 3.796785 & 2.878408 & -0.941015 \\
\hline $\mathrm{H}$ & 1.735757 & 2.430438 & 1.299249 \\
\hline $\mathrm{H}$ & 1.974504 & 3.928669 & 0.385929 \\
\hline $\mathrm{H}$ & 3.113304 & 4.112297 & 2.589894 \\
\hline $\mathrm{H}$ & 4.053969 & 2.640266 & 2.289312 \\
\hline $\mathrm{H}$ & 4.351171 & 4.106139 & 1.303861 \\
\hline $\mathrm{H}$ & 3.947071 & 0.072064 & -1.729802 \\
\hline $\mathrm{H}$ & 2.401876 & -0.786831 & -1.570477 \\
\hline $\mathrm{H}$ & 2.520623 & -1.361087 & -4.074995 \\
\hline $\mathrm{H}$ & 4.123451 & -0.595835 & -4.143966 \\
\hline $\mathrm{H}$ & 3.357224 & -2.958535 & -2.324717 \\
\hline $\mathrm{H}$ & 4.946585 & -2.189746 & -2.363768 \\
\hline $\mathrm{H}$ & 4.945467 & -4.262548 & -3.778592 \\
\hline $\mathrm{H}$ & 3.607458 & -3.625085 & -4.766148 \\
\hline $\mathrm{H}$ & 5.207570 & -2.836737 & -4.814567 \\
\hline $\mathrm{H}$ & 3.421031 & 1.375472 & -4.352337 \\
\hline $\mathrm{H}$ & 4.090545 & 2.125689 & -2.886457 \\
\hline $\mathrm{H}$ & 1.708661 & 3.133685 & -4.582234 \\
\hline $\mathrm{H}$ & 2.115868 & 3.837511 & -3.010755 \\
\hline $\mathrm{H}$ & 4.085172 & 3.671030 & -5.376567 \\
\hline $\mathrm{H}$ & 4.382602 & 4.453933 & -3.815111 \\
\hline $\mathrm{H}$ & 3.726859 & 6.191003 & -5.473013 \\
\hline $\mathrm{H}$ & 2.246645 & 5.292726 & -5.896329 \\
\hline $\mathrm{H}$ & 2.399033 & 6.034247 & -4.284938 \\
\hline $\mathrm{O}$ & -0.159253 & 6.598987 & -3.182617 \\
\hline $\mathrm{O}$ & 3.707346 & 0.616108 & 3.997267 \\
\hline
\end{tabular}




\begin{tabular}{|c|c|c|c|}
\hline $\mathrm{N}$ & -2.701388 & 2.122136 & -0.948491 \\
\hline $\mathrm{N}$ & 2.833894 & -0.605156 & 2.212003 \\
\hline $\mathrm{C}$ & -0.897613 & -3.328318 & 0.237514 \\
\hline $\mathrm{C}$ & -1.085174 & -6.043692 & -0.389863 \\
\hline $\mathrm{C}$ & 0.179213 & -5.487840 & -0.172289 \\
\hline $\mathrm{C}$ & 0.273134 & -4.110099 & 0.150085 \\
\hline $\mathrm{C}$ & -2.160226 & 3.271699 & -1.547874 \\
\hline $\mathrm{C}$ & -2.693556 & 3.895880 & -2.693809 \\
\hline $\mathrm{C}$ & -2.054145 & 5.010767 & -3.259508 \\
\hline $\mathrm{C}$ & -0.872718 & 5.522860 & -2.695352 \\
\hline $\mathrm{C}$ & -0.348282 & 4.914174 & -1.537707 \\
\hline $\mathrm{C}$ & -0.983968 & 3.808075 & -0.971545 \\
\hline $\mathrm{C}$ & -0.719879 & 7.290544 & -4.294590 \\
\hline $\mathrm{C}$ & 1.538229 & -3.507539 & 0.401840 \\
\hline $\mathrm{C}$ & 2.622154 & -2.984432 & 0.664994 \\
\hline $\mathrm{C}$ & 3.889190 & -2.426565 & 0.983289 \\
\hline $\mathrm{C}$ & 3.999508 & -1.247892 & 1.787083 \\
\hline $\mathrm{C}$ & 5.298059 & -0.777374 & 2.090522 \\
\hline $\mathrm{C}$ & 6.427472 & -1.445074 & 1.616418 \\
\hline $\mathrm{C}$ & 6.348980 & -2.609323 & 0.816840 \\
\hline $\mathrm{C}$ & 5.063727 & -3.074232 & 0.518719 \\
\hline $\mathrm{C}$ & 8.444626 & -2.322034 & -0.552972 \\
\hline $\mathrm{C}$ & 7.329269 & -4.565077 & -0.515829 \\
\hline $\mathrm{C}$ & 2.730668 & 0.269659 & 3.318871 \\
\hline $\mathrm{C}$ & -4.002727 & 1.657001 & -1.075582 \\
\hline $\mathrm{C}$ & -2.164700 & -3.906205 & 0.007436 \\
\hline $\mathrm{C}$ & -2.257621 & -5.286190 & -0.306255 \\
\hline $\mathrm{C}$ & 7.630871 & -3.306892 & 0.323413 \\
\hline $\mathrm{N}$ & 1.432598 & 0.679507 & 3.527987 \\
\hline $\mathrm{N}$ & -4.207335 & 0.472824 & -0.348802 \\
\hline $\mathrm{O}$ & -4.877555 & 2.211278 & -1.749787 \\
\hline $\mathrm{C}$ & -3.328174 & -3.090957 & 0.069717 \\
\hline $\mathrm{C}$ & 8.486783 & -3.732445 & 1.542635 \\
\hline $\mathrm{C}$ & 0.970448 & 1.611873 & 4.483810 \\
\hline $\mathrm{C}$ & -5.447153 & -0.160412 & -0.182917 \\
\hline $\mathrm{C}$ & -4.313031 & -2.352930 & 0.096410 \\
\hline $\mathrm{C}$ & -0.357577 & 2.075180 & 4.328859 \\
\hline $\mathrm{C}$ & 1.735151 & 2.080151 & 5.567687 \\
\hline $\mathrm{C}$ & -5.491086 & -1.560706 & 0.089617 \\
\hline $\mathrm{C}$ & -6.668737 & 0.542102 & -0.245638 \\
\hline $\mathrm{C}$ & -0.904052 & 2.978041 & 5.236828 \\
\hline $\mathrm{C}$ & 1.181525 & 2.993699 & 6.478725 \\
\hline $\mathrm{C}$ & -6.742073 & -2.192956 & 0.313543 \\
\hline $\mathrm{C}$ & -7.879611 & -0.116551 & -0.034445 \\
\hline $\mathrm{C}$ & -0.139351 & 3.447420 & 6.323430 \\
\hline $\mathrm{C}$ & -7.954285 & -1.498311 & 0.258208 \\
\hline $\mathrm{O}$ & -0.774001 & 4.337471 & 7.160243 \\
\hline $\mathrm{C}$ & -9.319310 & -2.171615 & 0.495890 \\
\hline
\end{tabular}




$\begin{array}{lccc}\mathrm{C} & -0.030008 & 4.817439 & 8.272963 \\ \mathrm{C} & -9.185213 & -3.678607 & 0.794221 \\ \mathrm{C} & -10.019083 & -1.498052 & 1.702898 \\ \mathrm{C} & -10.201499 & -2.003183 & -0.766361 \\ \mathrm{H} & -2.077115 & 1.644370 & -0.276095 \\ \mathrm{H} & 1.960768 & -0.910122 & 1.765487 \\ \mathrm{H} & -0.823942 & -2.263593 & 0.482638 \\ \mathrm{H} & 1.078315 & -6.104970 & -0.237250 \\ \mathrm{H} & -3.620235 & 3.513983 & -3.124413 \\ \mathrm{H} & -2.496996 & 5.470127 & -4.147244 \\ \mathrm{H} & 0.546961 & 5.344561 & -1.075756 \\ \mathrm{H} & -0.578583 & 3.335635 & -0.069217 \\ \mathrm{H} & -0.039380 & 8.129851 & -4.501610 \\ \mathrm{H} & -0.784399 & 6.643731 & -5.191896 \\ \mathrm{H} & -1.728205 & 7.684002 & -4.062683 \\ \mathrm{H} & 5.397568 & 0.099634 & 2.729601 \\ \mathrm{H} & 4.921800 & -3.978106 & -0.079317 \\ \mathrm{H} & 6.769776 & -5.319987 & 0.063654 \\ \mathrm{H} & 6.746620 & -4.325134 & -1.423639 \\ \mathrm{H} & 7.408780 & -1.037658 & 1.885161 \\ \mathrm{H} & 8.713479 & -1.406760 & 0.001494 \\ \mathrm{H} & 7.868066 & -2.019125 & -1.445408 \\ \mathrm{H} & 9.382385 & -2.795440 & -0.896103 \\ \mathrm{H} & -10.172011 & -0.417963 & 1.537289 \\ \mathrm{H} & 8.275634 & -5.029905 & -0.842446 \\ \mathrm{H} & -3.231663 & -5.746690 & -0.487035 \\ \mathrm{H} & 7.937410 & -4.447398 & 2.178938 \\ \mathrm{H} & 8.764392 & -2.868645 & 2.170108 \\ \mathrm{H} & 9.420736 & -4.217596 & 1.205598 \\ \mathrm{H} & -0.949084 & 1.721057 & 3.475959 \\ \mathrm{H} & 2.761038 & 1.731451 & 5.689461 \\ \mathrm{H} & -6.652021 & 1.607576 & -0.474802 \\ \mathrm{H} & -1.930724 & 3.337881 & 5.120002 \\ \mathrm{H} & 1.799907 & 3.338941 & 7.311432 \\ \mathrm{H} & -6.720456 & -3.264830 & 0.525253 \\ \mathrm{H} & -8.799504 & 0.476345 & -0.090004 \\ \mathrm{H} & -0.702697 & 5.500419 & 8.813267 \\ \mathrm{H} & -10.275178 & 3.994728 & 8.949247 \\ \mathrm{H} & -9.4201673969 & 5.372531 & 7.954764 \\ \mathrm{H} & -1.010203 & -1.955467 & 1.875926 \\ \mathrm{H} & -3.865114 & 1.703570 \\ \mathrm{H} & -0.003598 & 0.040097\end{array}$



$\mathrm{H} \quad 0.732315$
0.427186
2.810971
$\mathrm{Br} \quad-0.587549$
0.287826
0.882568
$\mathrm{Cl} \quad-1.203561 \quad-7.753841 \quad-0.786838$

Statistical Thermodynamic Analysis

Temperature $=298.150$ Kelvin $\quad$ Pressure $=1.00000$ Atm

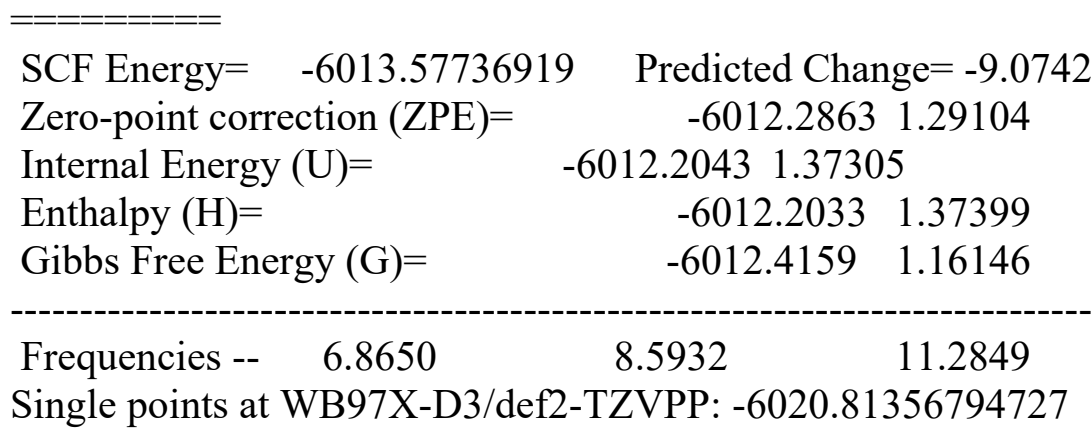

$\mathbf{R}=\mathbf{C l}$ receptor bound with $\mathrm{Cl}-$ (with $\mathbf{T B A}$ )

Supporting Information: 010-bound_TBA_Cl_Cl.log

Using Gaussian 09: EM64L-G09RevE.01 30-Nov-2015

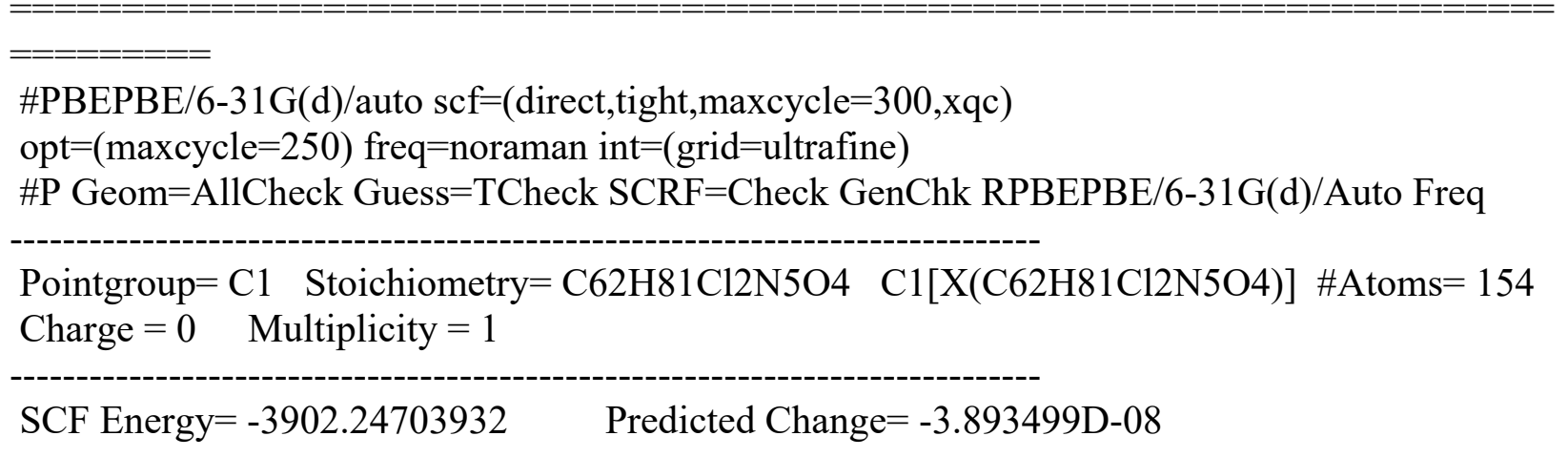

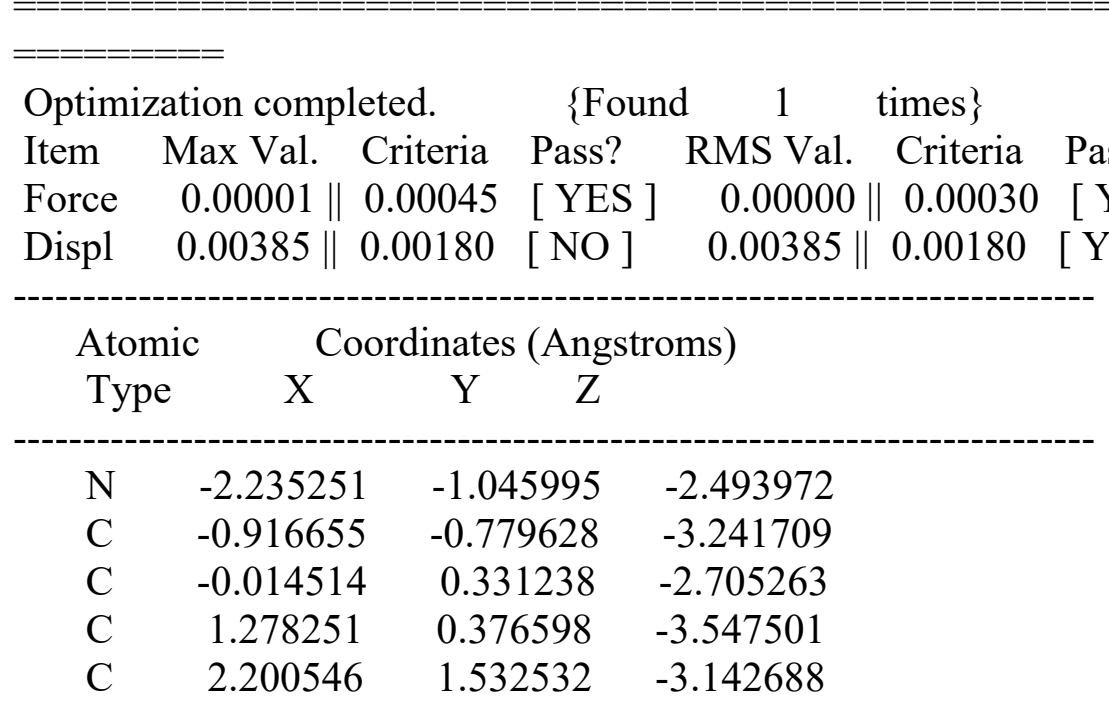




\begin{tabular}{|c|c|c|c|}
\hline $\mathrm{C}$ & -1.913741 & -1.755047 & -1.165555 \\
\hline $\mathrm{C}$ & -3.111324 & -2.215978 & -0.327647 \\
\hline $\mathrm{C}$ & -2.632582 & -3.183206 & 0.777078 \\
\hline $\mathrm{C}$ & -3.769842 & -3.630791 & 1.703669 \\
\hline $\mathrm{C}$ & -2.970425 & 0.259086 & -2.181740 \\
\hline $\mathrm{C}$ & -3.346025 & 1.133328 & -3.381963 \\
\hline $\mathrm{C}$ & -3.948560 & 2.469317 & -2.903892 \\
\hline $\mathrm{C}$ & -4.370386 & 3.369248 & -4.071664 \\
\hline $\mathrm{C}$ & -3.117175 & -1.923810 & -3.383204 \\
\hline $\mathrm{C}$ & -2.559927 & -3.307223 & -3.729201 \\
\hline $\mathrm{C}$ & -3.590432 & -4.129134 & -4.527600 \\
\hline $\mathrm{C}$ & -3.012080 & -5.473146 & -4.990482 \\
\hline $\mathrm{H}$ & -0.374112 & -1.738304 & -3.228342 \\
\hline $\mathrm{H}$ & -1.203696 & -0.569300 & -4.286400 \\
\hline $\mathrm{H}$ & 0.243435 & 0.166166 & -1.642510 \\
\hline $\mathrm{H}$ & -0.515843 & 1.314611 & -2.771727 \\
\hline $\mathrm{H}$ & 1.017153 & 0.468928 & -4.621149 \\
\hline $\mathrm{H}$ & 1.815480 & -0.584199 & -3.437086 \\
\hline $\mathrm{H}$ & 3.103825 & 1.552358 & -3.775202 \\
\hline $\mathrm{H}$ & 1.693288 & 2.508200 & -3.246349 \\
\hline $\mathrm{H}$ & 2.530393 & 1.436844 & -2.094977 \\
\hline $\mathrm{H}$ & -1.281157 & -2.613746 & -1.437430 \\
\hline $\mathrm{H}$ & -1.276660 & -1.062688 & -0.585822 \\
\hline $\mathrm{H}$ & -3.621265 & -1.355896 & 0.142491 \\
\hline $\mathrm{H}$ & -3.863522 & -2.746828 & -0.941724 \\
\hline $\mathrm{H}$ & -1.838639 & -2.697395 & 1.372131 \\
\hline $\mathrm{H}$ & -2.170041 & -4.066753 & 0.296805 \\
\hline $\mathrm{H}$ & -3.399571 & -4.359813 & 2.443614 \\
\hline $\mathrm{H}$ & -4.184347 & -2.778213 & 2.267475 \\
\hline $\mathrm{H}$ & -4.588393 & -4.109739 & 1.135302 \\
\hline $\mathrm{H}$ & -3.874030 & -0.030407 & -1.622157 \\
\hline $\mathrm{H}$ & -2.317045 & 0.809414 & -1.485885 \\
\hline $\mathrm{H}$ & -2.460105 & 1.344921 & -4.008782 \\
\hline $\mathrm{H}$ & -4.084064 & 0.622348 & -4.027701 \\
\hline $\mathrm{H}$ & -3.210661 & 2.994885 & -2.269171 \\
\hline $\mathrm{H}$ & -4.819293 & 2.268167 & -2.251831 \\
\hline $\mathrm{H}$ & -4.802589 & 4.314788 & -3.702661 \\
\hline $\mathrm{H}$ & -3.510104 & 3.624877 & -4.715506 \\
\hline $\mathrm{H}$ & -5.131130 & 2.878932 & -4.705266 \\
\hline $\mathrm{H}$ & -3.286851 & -1.346199 & -4.305493 \\
\hline $\mathrm{H}$ & -4.085848 & -2.011481 & -2.865689 \\
\hline $\mathrm{H}$ & -1.634371 & -3.214062 & -4.325322 \\
\hline $\mathrm{H}$ & -2.290461 & -3.870002 & -2.818473 \\
\hline $\mathrm{H}$ & -3.936548 & -3.549778 & -5.405657 \\
\hline $\mathrm{H}$ & -4.484286 & -4.301835 & -3.897967 \\
\hline $\mathrm{H}$ & -3.784481 & -6.092070 & -5.476693 \\
\hline $\mathrm{H}$ & -2.195470 & -5.321244 & -5.718744 \\
\hline $\mathrm{H}$ & -2.595408 & -6.042900 & -4.141578 \\
\hline
\end{tabular}




\begin{tabular}{|c|c|c|c|}
\hline $\mathrm{O}$ & 0.024483 & -6.531217 & -3.033826 \\
\hline $\mathrm{O}$ & -3.788475 & -0.707236 & 3.730897 \\
\hline $\mathrm{N}$ & 2.608077 & -2.069979 & -0.800848 \\
\hline $\mathrm{N}$ & -2.822821 & 0.705766 & 2.149367 \\
\hline $\mathrm{C}$ & 1.051555 & 3.354739 & 0.420396 \\
\hline $\mathrm{C}$ & 1.347043 & 6.095905 & -0.052519 \\
\hline $\mathrm{C}$ & 0.061475 & 5.576000 & 0.123244 \\
\hline $\mathrm{C}$ & -0.087134 & 4.186650 & 0.366351 \\
\hline $\mathrm{C}$ & 2.035115 & -3.207737 & -1.399300 \\
\hline $\mathrm{C}$ & 2.454478 & -3.722344 & -2.643680 \\
\hline $\mathrm{C}$ & 1.811179 & -4.831742 & -3.214658 \\
\hline $\mathrm{C}$ & 0.732022 & -5.447676 & -2.556162 \\
\hline $\mathrm{C}$ & 0.317405 & -4.946381 & -1.306486 \\
\hline $\mathrm{C}$ & 0.962144 & -3.846739 & -0.735110 \\
\hline $\mathrm{C}$ & 0.509972 & -7.139565 & -4.226897 \\
\hline $\mathrm{C}$ & -1.381103 & 3.627941 & 0.566179 \\
\hline $\mathrm{C}$ & -2.501031 & 3.155051 & 0.765337 \\
\hline $\mathrm{C}$ & -3.794907 & 2.623822 & 1.012044 \\
\hline $\mathrm{C}$ & -3.961969 & 1.395702 & 1.728173 \\
\hline $\mathrm{C}$ & -5.280584 & 0.932802 & 1.943034 \\
\hline $\mathrm{C}$ & -6.375407 & 1.658390 & 1.471655 \\
\hline $\mathrm{C}$ & -6.241031 & 2.875800 & 0.764056 \\
\hline $\mathrm{C}$ & -4.935197 & 3.331362 & 0.551796 \\
\hline $\mathrm{C}$ & -8.287883 & 2.734755 & -0.700102 \\
\hline $\mathrm{C}$ & -7.125503 & 4.941217 & -0.466036 \\
\hline $\mathrm{C}$ & -2.776671 & -0.299500 & 3.141631 \\
\hline $\mathrm{C}$ & 3.932716 & -1.677062 & -0.952164 \\
\hline $\mathrm{C}$ & 2.341041 & 3.899426 & 0.232670 \\
\hline $\mathrm{C}$ & 2.488496 & 5.290428 & -0.002549 \\
\hline $\mathrm{C}$ & -7.487206 & 3.636523 & 0.272413 \\
\hline $\mathrm{N}$ & -1.494955 & -0.759953 & 3.348923 \\
\hline $\mathrm{N}$ & 4.219401 & -0.501851 & -0.242680 \\
\hline $\mathrm{O}$ & 4.762795 & -2.286917 & -1.634798 \\
\hline $\mathrm{C}$ & 3.478475 & 3.046661 & 0.255842 \\
\hline $\mathrm{C}$ & -8.380429 & 3.998385 & 1.485443 \\
\hline $\mathrm{C}$ & -1.094544 & -1.780960 & 4.241410 \\
\hline $\mathrm{C}$ & 5.491408 & 0.069912 & -0.106797 \\
\hline $\mathrm{C}$ & 4.453490 & 2.295287 & 0.243085 \\
\hline $\mathrm{C}$ & 0.244392 & -2.228507 & 4.141871 \\
\hline $\mathrm{C}$ & -1.931532 & -2.356984 & 5.215861 \\
\hline $\mathrm{C}$ & 5.601498 & 1.462627 & 0.187676 \\
\hline $\mathrm{C}$ & 6.679807 & -0.681486 & -0.218258 \\
\hline $\mathrm{C}$ & 0.728080 & -3.222117 & 4.989246 \\
\hline $\mathrm{C}$ & -1.440517 & -3.358889 & 6.067947 \\
\hline $\mathrm{C}$ & 6.883023 & 2.039431 & 0.384114 \\
\hline $\mathrm{C}$ & 7.922332 & -0.075903 & -0.031816 \\
\hline $\mathrm{C}$ & -0.110467 & -3.799630 & 5.963007 \\
\hline $\mathrm{C}$ & 8.063076 & 1.296202 & 0.281266 \\
\hline
\end{tabular}




\begin{tabular}{|c|c|c|c|}
\hline $\mathrm{O}$ & 0.465740 & -4.772890 & 6.747518 \\
\hline $\mathrm{C}$ & 9.461423 & 1.908109 & 0.489507 \\
\hline $\mathrm{C}$ & -0.353491 & -5.359463 & 7.751047 \\
\hline $\mathrm{C}$ & 9.399187 & 3.413333 & 0.818802 \\
\hline $\mathrm{C}$ & 10.167992 & 1.183567 & 1.662538 \\
\hline $\mathrm{C}$ & 10.298311 & 1.727886 & -0.801658 \\
\hline $\mathrm{H}$ & 2.015381 & -1.570052 & -0.112842 \\
\hline $\mathrm{H}$ & -1.928224 & 1.094513 & 1.830787 \\
\hline $\mathrm{H}$ & 0.936872 & 2.281697 & 0.611584 \\
\hline $\mathrm{H}$ & -0.814046 & 6.228224 & 0.084985 \\
\hline $\mathrm{H}$ & 3.306719 & -3.265332 & -3.148832 \\
\hline $\mathrm{H}$ & 2.169867 & -5.205758 & -4.177312 \\
\hline $\mathrm{H}$ & -0.494349 & -5.457078 & -0.777981 \\
\hline $\mathrm{H}$ & 0.645973 & -3.476914 & 0.246564 \\
\hline $\mathrm{H}$ & -0.136795 & -8.011510 & -4.405194 \\
\hline $\mathrm{H}$ & 0.443948 & -6.454852 & -5.095727 \\
\hline $\mathrm{H}$ & 1.558407 & -7.475998 & -4.114121 \\
\hline $\mathrm{H}$ & -5.423921 & 0.015355 & 2.513196 \\
\hline $\mathrm{H}$ & -4.749870 & 4.269498 & 0.022383 \\
\hline $\mathrm{H}$ & -6.573007 & 5.641233 & 0.184676 \\
\hline $\mathrm{H}$ & -6.512907 & 4.749356 & -1.365463 \\
\hline $\mathrm{H}$ & -7.375032 & 1.255412 & 1.670930 \\
\hline $\mathrm{H}$ & -8.599174 & 1.790768 & -0.221130 \\
\hline $\mathrm{H}$ & -7.683781 & 2.479510 & -1.589268 \\
\hline $\mathrm{H}$ & -9.200477 & 3.254127 & -1.044555 \\
\hline $\mathrm{H}$ & -8.048022 & 5.450051 & -0.795314 \\
\hline $\mathrm{H}$ & 3.481231 & 5.722134 & -0.149490 \\
\hline $\mathrm{H}$ & -7.840738 & 4.654302 & 2.189985 \\
\hline $\mathrm{H}$ & -8.700991 & 3.100514 & 2.040490 \\
\hline $\mathrm{H}$ & -9.289715 & 4.528362 & 1.148396 \\
\hline $\mathrm{H}$ & 0.903105 & -1.783439 & 3.387436 \\
\hline $\mathrm{H}$ & -2.964956 & -2.020738 & 5.300352 \\
\hline $\mathrm{H}$ & 6.611279 & -1.740819 & -0.465417 \\
\hline $\mathrm{H}$ & 1.763566 & -3.567505 & 4.913621 \\
\hline $\mathrm{H}$ & -2.116066 & -3.783860 & 6.815005 \\
\hline $\mathrm{H}$ & 6.912351 & 3.107408 & 0.613861 \\
\hline $\mathrm{H}$ & 8.815078 & -0.704565 & -0.124923 \\
\hline $\mathrm{H}$ & 0.281714 & -6.095583 & 8.266322 \\
\hline $\mathrm{H}$ & -0.708697 & -4.607644 & 8.483080 \\
\hline $\mathrm{H}$ & -1.231326 & -5.876685 & 7.315556 \\
\hline $\mathrm{H}$ & 10.421528 & 3.804944 & 0.960389 \\
\hline $\mathrm{H}$ & 8.836367 & 3.606691 & 1.748830 \\
\hline $\mathrm{H}$ & 8.930414 & 3.992505 & 0.003871 \\
\hline $\mathrm{H}$ & 9.601885 & 1.308780 & 2.601779 \\
\hline $\mathrm{H}$ & 11.181940 & 1.596590 & 1.813623 \\
\hline $\mathrm{H}$ & 10.270906 & 0.101486 & 1.473120 \\
\hline $\mathrm{H}$ & 11.312616 & 2.144253 & -0.662508 \\
\hline $\mathrm{H}$ & 9.826555 & 2.248132 & -1.653301 \\
\hline
\end{tabular}




$\begin{array}{lccc}\mathrm{H} & 10.406452 & 0.664422 & -1.074950 \\ \mathrm{H} & 3.432900 & 0.032336 & 0.143413 \\ \mathrm{H} & -0.763011 & -0.467901 & 2.675193 \\ \mathrm{Cl} & 0.608747 & -0.196467 & 0.941834 \\ \mathrm{Cl} & 1.533195 & 7.819969 & -0.350893\end{array}$

Statistical Thermodynamic Analysis

Temperature $=298.150$ Kelvin $\quad$ Pressure $=1.00000$ Atm

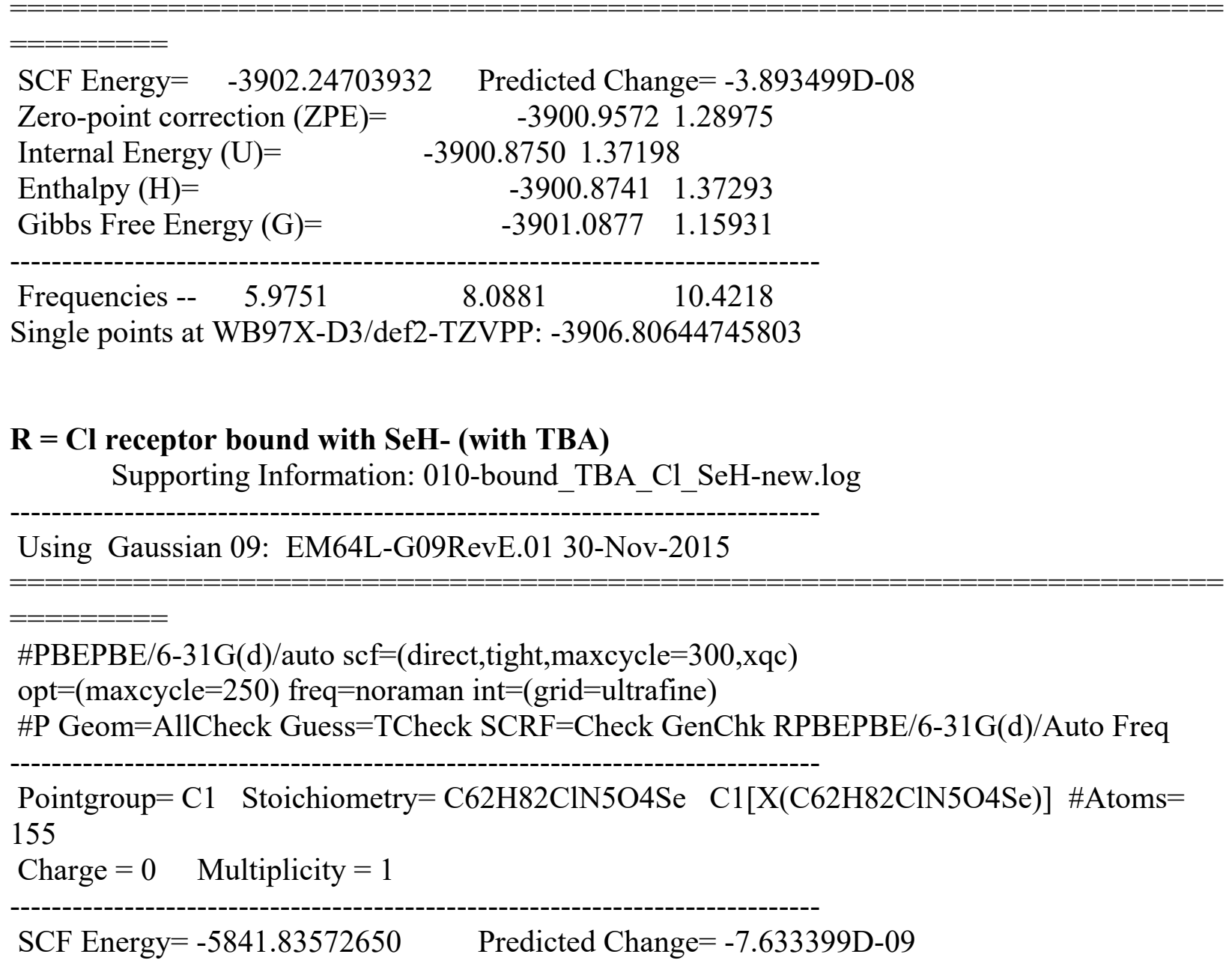

\begin{tabular}{|c|c|c|c|c|c|}
\hline & times\} \\
\hline Item & Max Val. & Criteria & Pass? & RMS Val. & Criteria \\
\hline Force & 0.00000 & 0.00045 & [ YES ] & 0.00000 & 0.00030 \\
\hline Displ & 0.00152 & 0.00180 & [YES ] & 0.00152 & 0.00180 \\
\hline \multicolumn{2}{|c|}{ Atomic } & \multicolumn{4}{|c|}{ Coordinates (Angstroms) } \\
\hline \multicolumn{2}{|c|}{ Type } & $\mathrm{Y}$ & Z & & \\
\hline $\mathrm{N}$ & -3.0605 & 2.5 & 4234 & 0.363013 & \\
\hline $\mathrm{C}$ & -1.8594 & 3.26 & 3542 & 0.978147 & \\
\hline $\mathrm{C}$ & -0.7456 & 2.4 & 7916 & 1.599034 & \\
\hline
\end{tabular}




\begin{tabular}{|c|c|c|c|}
\hline $\mathrm{C}$ & 0.341728 & 3.368944 & 2.146159 \\
\hline $\mathrm{C}$ & 1.482983 & 2.621297 & 2.844896 \\
\hline $\mathrm{C}$ & -2.581478 & 1.809101 & -0.903465 \\
\hline $\mathrm{C}$ & -3.606451 & 1.000444 & -1.697514 \\
\hline $\mathrm{C}$ & -2.900469 & 0.477705 & -2.969359 \\
\hline $\mathrm{C}$ & -3.787481 & -0.452489 & -3.801850 \\
\hline $\mathrm{C}$ & -3.630673 & 1.491078 & 1.333846 \\
\hline $\mathrm{C}$ & -4.057987 & 2.011280 & 2.708557 \\
\hline $\mathrm{C}$ & -4.625314 & 0.857937 & 3.561065 \\
\hline $\mathrm{C}$ & -5.027511 & 1.315653 & 4.968305 \\
\hline $\mathrm{C}$ & -4.144496 & 3.553553 & 0.024535 \\
\hline $\mathrm{C}$ & -3.826895 & 4.503732 & -1.134421 \\
\hline $\mathrm{C}$ & -4.809022 & 5.689704 & -1.177905 \\
\hline $\mathrm{C}$ & -4.546734 & 6.595069 & -2.389490 \\
\hline $\mathrm{H}$ & -1.441765 & 3.877728 & 0.164639 \\
\hline $\mathrm{H}$ & -2.286028 & 3.943658 & 1.734666 \\
\hline $\mathrm{H}$ & -0.287540 & 1.729650 & 0.863548 \\
\hline $\mathrm{H}$ & -1.129937 & 1.797703 & 2.430006 \\
\hline $\mathrm{H}$ & -0.120996 & 4.086053 & 2.853744 \\
\hline $\mathrm{H}$ & 0.750398 & 3.969904 & 1.312028 \\
\hline $\mathrm{H}$ & 2.226353 & 3.331727 & 3.243775 \\
\hline $\mathrm{H}$ & 1.112260 & 2.010557 & 3.686965 \\
\hline $\mathrm{H}$ & 2.009076 & 1.947726 & 2.148160 \\
\hline $\mathrm{H}$ & -2.153576 & 2.603577 & -1.532935 \\
\hline $\mathrm{H}$ & -1.740503 & 1.148879 & -0.621572 \\
\hline $\mathrm{H}$ & -3.968095 & 0.133577 & -1.115188 \\
\hline $\mathrm{H}$ & -4.492177 & 1.602941 & -1.979915 \\
\hline $\mathrm{H}$ & -1.969936 & -0.032167 & -2.654112 \\
\hline $\mathrm{H}$ & -2.583070 & 1.342773 & -3.585161 \\
\hline $\mathrm{H}$ & -3.245042 & -0.821590 & -4.688157 \\
\hline $\mathrm{H}$ & -4.106941 & -1.330644 & -3.214960 \\
\hline $\mathrm{H}$ & -4.696339 & 0.067711 & -4.155775 \\
\hline $\mathrm{H}$ & -4.489845 & 1.036286 & 0.815720 \\
\hline $\mathrm{H}$ & -2.862219 & 0.707625 & 1.434626 \\
\hline $\mathrm{H}$ & -3.199198 & 2.458521 & 3.241800 \\
\hline $\mathrm{H}$ & -4.828719 & 2.799255 & 2.617529 \\
\hline $\mathrm{H}$ & -3.872214 & 0.050413 & 3.625966 \\
\hline $\mathrm{H}$ & -5.501940 & 0.420054 & 3.045189 \\
\hline $\mathrm{H}$ & -5.435647 & 0.475757 & 5.554698 \\
\hline $\mathrm{H}$ & -4.160735 & 1.718947 & 5.521012 \\
\hline $\mathrm{H}$ & -5.799756 & 2.104680 & 4.930841 \\
\hline $\mathrm{H}$ & -4.318824 & 4.124535 & 0.950604 \\
\hline $\mathrm{H}$ & -5.059164 & 2.976600 & -0.189995 \\
\hline $\mathrm{H}$ & -2.801431 & 4.905919 & -1.055217 \\
\hline $\mathrm{H}$ & -3.878157 & 3.959340 & -2.094893 \\
\hline $\mathrm{H}$ & -4.712680 & 6.274907 & -0.243117 \\
\hline $\mathrm{H}$ & -5.851340 & 5.317011 & -1.205967 \\
\hline $\mathrm{H}$ & -5.194873 & 7.486989 & -2.366122 \\
\hline
\end{tabular}




\begin{tabular}{|c|c|c|c|}
\hline $\mathrm{H}$ & -3.495203 & 6.932418 & -2.417888 \\
\hline $\mathrm{H}$ & -4.747036 & 6.060243 & -3.334622 \\
\hline $\mathrm{O}$ & -0.649918 & 6.920366 & -3.112618 \\
\hline $\mathrm{O}$ & -2.057131 & -4.563962 & -2.631315 \\
\hline $\mathrm{N}$ & 2.371616 & 2.636218 & -1.123657 \\
\hline $\mathrm{N}$ & -2.148616 & -2.779409 & -1.124770 \\
\hline $\mathrm{C}$ & 0.641761 & -1.335024 & 2.485077 \\
\hline $\mathrm{C}$ & 0.499615 & -1.673527 & 5.251045 \\
\hline $\mathrm{C}$ & -0.669978 & -1.801063 & 4.495150 \\
\hline $\mathrm{C}$ & -0.598386 & -1.636243 & 3.088169 \\
\hline $\mathrm{C}$ & 1.717208 & 3.767239 & -1.627729 \\
\hline $\mathrm{C}$ & 2.229485 & 5.080023 & -1.591794 \\
\hline $\mathrm{C}$ & 1.465732 & 6.151881 & -2.082719 \\
\hline $\mathrm{C}$ & 0.185557 & 5.936279 & -2.621691 \\
\hline $\mathrm{C}$ & -0.317919 & 4.621107 & -2.681738 \\
\hline $\mathrm{C}$ & 0.435389 & 3.554691 & -2.191687 \\
\hline $\mathrm{C}$ & -0.131789 & 8.246867 & -3.139264 \\
\hline $\mathrm{C}$ & -1.750895 & -1.816958 & 2.271181 \\
\hline $\mathrm{C}$ & -2.711616 & -2.068207 & 1.540252 \\
\hline $\mathrm{C}$ & -3.759049 & -2.476857 & 0.671706 \\
\hline $\mathrm{C}$ & -3.445954 & -2.933882 & -0.649634 \\
\hline $\mathrm{C}$ & -4.486417 & -3.506606 & -1.415570 \\
\hline $\mathrm{C}$ & -5.784843 & -3.571134 & -0.910403 \\
\hline $\mathrm{C}$ & -6.134678 & -3.075375 & 0.369296 \\
\hline $\mathrm{C}$ & -5.097272 & -2.539337 & 1.140203 \\
\hline $\mathrm{C}$ & -8.506836 & -2.366988 & -0.106413 \\
\hline $\mathrm{C}$ & -7.765610 & -2.567438 & 2.277547 \\
\hline $\mathrm{C}$ & -1.495540 & -3.624329 & -2.057006 \\
\hline $\mathrm{C}$ & 3.684795 & 2.528377 & -0.693310 \\
\hline $\mathrm{C}$ & 1.812712 & -1.209533 & 3.263354 \\
\hline $\mathrm{C}$ & 1.738476 & -1.387765 & 4.667986 \\
\hline $\mathrm{C}$ & -7.591801 & -3.156501 & 0.862966 \\
\hline $\mathrm{N}$ & -0.181585 & -3.245888 & -2.204520 \\
\hline $\mathrm{N}$ & 3.948029 & 1.239981 & -0.199643 \\
\hline $\mathrm{O}$ & 4.516778 & 3.441699 & -0.724247 \\
\hline $\mathrm{C}$ & 3.037320 & -0.876631 & 2.621857 \\
\hline $\mathrm{C}$ & -8.042220 & -4.638482 & 0.897513 \\
\hline $\mathrm{C}$ & 0.841374 & -3.865005 & -2.945244 \\
\hline $\mathrm{C}$ & 5.191213 & 0.819360 & 0.293508 \\
\hline $\mathrm{C}$ & 4.055144 & -0.550873 & 2.011874 \\
\hline $\mathrm{C}$ & 2.126555 & -3.280476 & -2.841183 \\
\hline $\mathrm{C}$ & 0.664359 & -5.000565 & -3.754838 \\
\hline $\mathrm{C}$ & 5.243486 & -0.125329 & 1.361237 \\
\hline $\mathrm{C}$ & 6.408119 & 1.289445 & -0.239728 \\
\hline $\mathrm{C}$ & 3.206104 & -3.821104 & -3.533066 \\
\hline $\mathrm{C}$ & 1.757471 & -5.543878 & -4.448539 \\
\hline $\mathrm{C}$ & 6.500115 & -0.594734 & 1.822625 \\
\hline $\mathrm{C}$ & 7.626608 & 0.821708 & 0.252273 \\
\hline
\end{tabular}




\begin{tabular}{|c|c|c|c|}
\hline C & 3.032147 & -4.960702 & -4.344518 \\
\hline $\mathrm{C}$ & 7.709643 & -0.140103 & 1.286395 \\
\hline $\mathrm{O}$ & 4.164686 & -5.415883 & -4.983396 \\
\hline $\mathrm{C}$ & 9.083121 & -0.636399 & 1.777338 \\
\hline $\mathrm{C}$ & 4.021377 & -6.568703 & -5.802626 \\
\hline $\mathrm{C}$ & 8.960433 & -1.676223 & 2.909499 \\
\hline $\mathrm{C}$ & 9.842383 & -1.291802 & 0.596644 \\
\hline $\mathrm{C}$ & 9.902929 & 0.565730 & 2.309225 \\
\hline $\mathrm{H}$ & 1.796408 & 1.770171 & -1.107184 \\
\hline $\mathrm{H}$ & -1.569633 & -2.067862 & -0.659171 \\
\hline $\mathrm{H}$ & 0.706793 & -1.182659 & 1.404109 \\
\hline $\mathrm{H}$ & -1.620094 & -2.044898 & 4.976325 \\
\hline $\mathrm{H}$ & 3.227843 & 5.247591 & -1.184572 \\
\hline $\mathrm{H}$ & 1.891229 & 7.157843 & -2.035349 \\
\hline $\mathrm{H}$ & -1.294298 & 4.453584 & -3.149354 \\
\hline $\mathrm{H}$ & 0.049019 & 2.528190 & -2.209761 \\
\hline $\mathrm{H}$ & -0.918321 & 8.868794 & -3.592184 \\
\hline $\mathrm{H}$ & 0.090170 & 8.622914 & -2.121083 \\
\hline $\mathrm{H}$ & 0.786879 & 8.313001 & -3.753454 \\
\hline $\mathrm{H}$ & -4.244195 & -3.910880 & -2.399496 \\
\hline $\mathrm{H}$ & -5.284329 & -2.182522 & 2.156664 \\
\hline $\mathrm{H}$ & -7.164818 & -3.112789 & 3.026259 \\
\hline $\mathrm{H}$ & -7.480370 & -1.500292 & 2.315793 \\
\hline $\mathrm{H}$ & -6.557468 & -4.018856 & -1.545689 \\
\hline $\mathrm{H}$ & -8.447621 & -2.758899 & -1.135951 \\
\hline $\mathrm{H}$ & -8.223901 & -1.299855 & -0.135796 \\
\hline $\mathrm{H}$ & -9.560944 & -2.433914 & 0.218667 \\
\hline $\mathrm{H}$ & -8.823716 & -2.639492 & 2.583422 \\
\hline $\mathrm{H}$ & 2.635550 & -1.292632 & 5.284034 \\
\hline $\mathrm{H}$ & -7.416705 & -5.224225 & 1.592889 \\
\hline $\mathrm{H}$ & -7.971404 & -5.110679 & -0.096878 \\
\hline $\mathrm{H}$ & -9.093010 & -4.713297 & 1.231529 \\
\hline $\mathrm{H}$ & 2.246451 & -2.392089 & -2.207014 \\
\hline $\mathrm{H}$ & -0.325945 & -5.452164 & -3.832270 \\
\hline $\mathrm{H}$ & 6.380599 & 2.032942 & -1.037082 \\
\hline $\mathrm{H}$ & 4.202250 & -3.375071 & -3.455906 \\
\hline $\mathrm{H}$ & 1.594367 & -6.428624 & -5.069568 \\
\hline $\mathrm{H}$ & 6.487685 & -1.325598 & 2.634927 \\
\hline $\mathrm{H}$ & 8.545497 & 1.212764 & -0.198829 \\
\hline $\mathrm{H}$ & 5.021458 & -6.772388 & -6.214302 \\
\hline $\mathrm{H}$ & 3.680436 & -7.447691 & -5.220508 \\
\hline $\mathrm{H}$ & 3.312128 & -6.396129 & -6.636348 \\
\hline $\mathrm{H}$ & 9.966993 & -2.002176 & 3.224459 \\
\hline $\mathrm{H}$ & 8.406083 & -2.573844 & 2.583887 \\
\hline $\mathrm{H}$ & 8.452775 & -1.258690 & 3.796766 \\
\hline $\mathrm{H}$ & 9.288326 & -2.162961 & 0.206266 \\
\hline $\mathrm{H}$ & 10.839256 & -1.636849 & 0.926233 \\
\hline $\mathrm{H}$ & 9.990420 & -0.585321 & -0.237779 \\
\hline
\end{tabular}




$\begin{array}{cccc}\mathrm{H} & 10.900206 & 0.229979 & 2.647169 \\ \mathrm{H} & 9.392954 & 1.043986 & 3.163441 \\ \mathrm{H} & 10.052909 & 1.335440 & 1.533113 \\ \mathrm{H} & 3.157807 & 0.582426 & -0.134836 \\ \mathrm{H} & 0.118536 & -2.388585 & -1.703214 \\ \mathrm{H} & 0.754480 & -0.099645 & -2.483602 \\ \mathrm{Se} & 0.502868 & -0.178621 & -1.016716 \\ \mathrm{Cl} & 0.407606 & -1.880840 & 6.995572\end{array}$

Statistical Thermodynamic Analysis

Temperature $=298.150$ Kelvin $\quad$ Pressure $=1.00000$ Atm

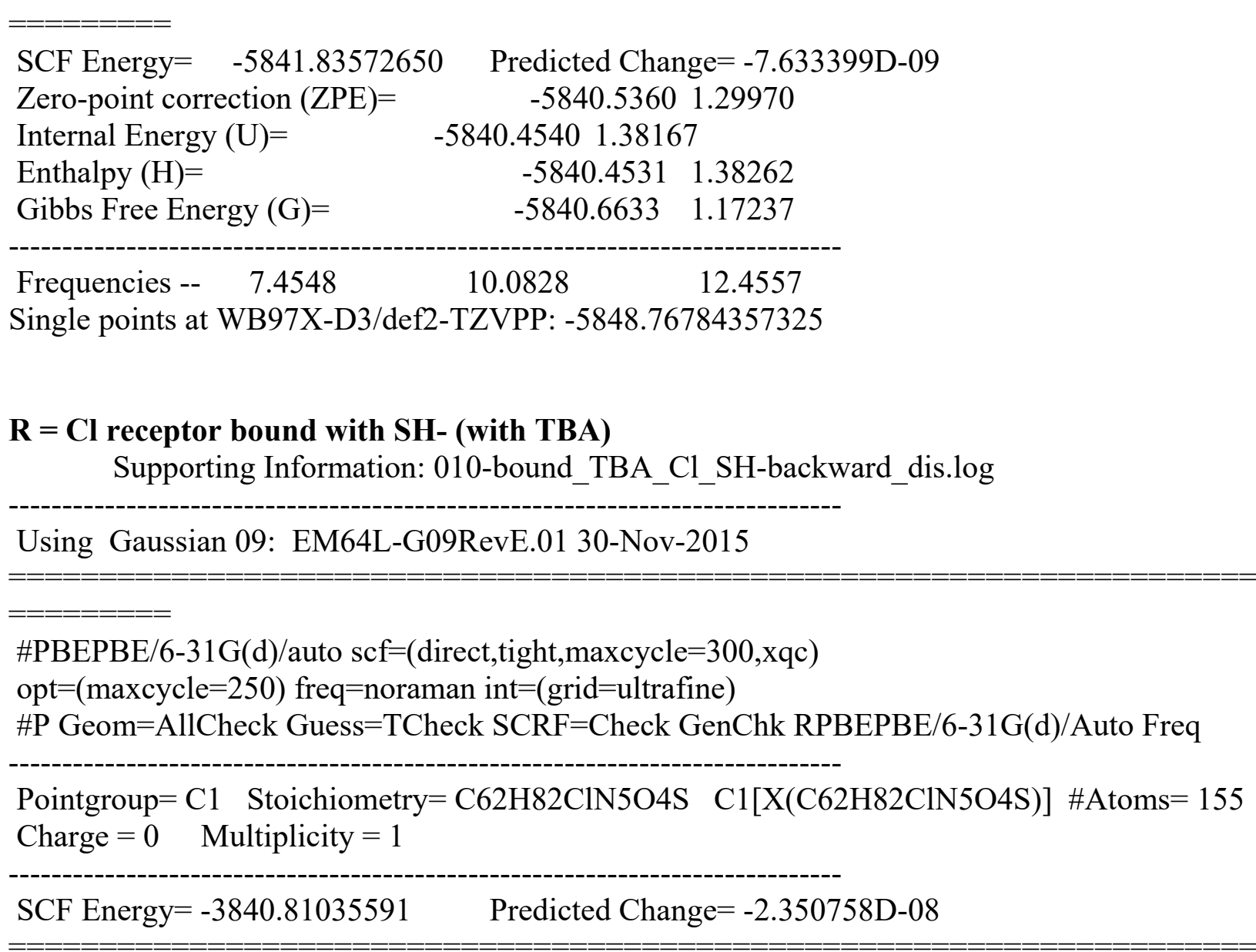

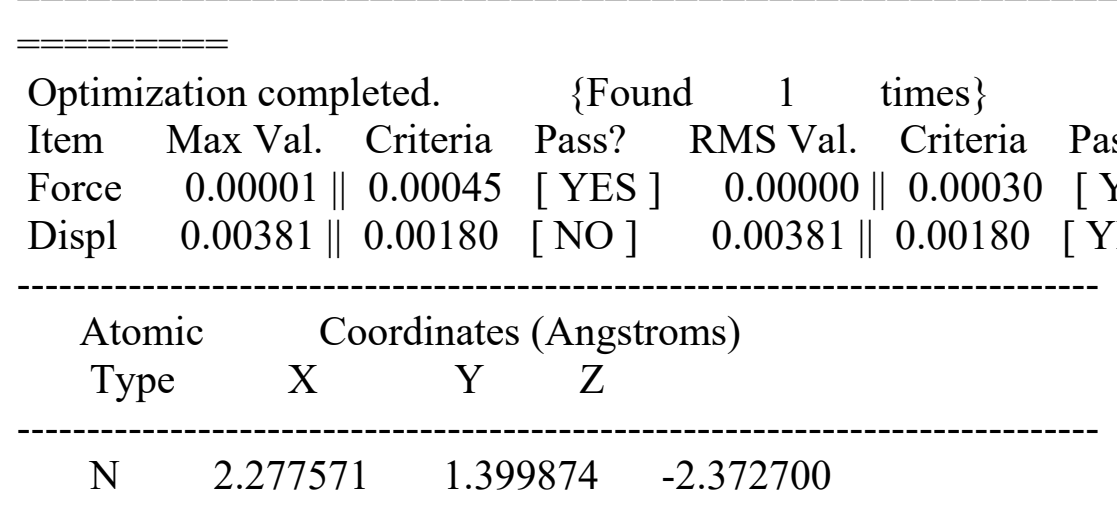




\begin{tabular}{|c|c|c|c|}
\hline $\mathrm{C}$ & 0.981360 & 1.200521 & -3.172824 \\
\hline $\mathrm{C}$ & 0.052101 & 0.067898 & -2.736674 \\
\hline $\mathrm{C}$ & -1.171022 & 0.033684 & -3.677890 \\
\hline $\mathrm{C}$ & -2.125602 & -1.122609 & -3.358562 \\
\hline $\mathrm{C}$ & 1.913796 & 1.951894 & -0.982741 \\
\hline $\mathrm{C}$ & 3.074538 & 2.301397 & -0.047358 \\
\hline $\mathrm{C}$ & 2.520726 & 3.048024 & 1.186253 \\
\hline $\mathrm{C}$ & 3.609362 & 3.393787 & 2.209732 \\
\hline $\mathrm{C}$ & 3.031546 & 0.079268 & -2.195867 \\
\hline $\mathrm{C}$ & 3.377587 & -0.686353 & -3.477135 \\
\hline $\mathrm{C}$ & 4.053512 & -2.028967 & -3.135868 \\
\hline $\mathrm{C}$ & 4.441223 & -2.820306 & -4.390964 \\
\hline $\mathrm{C}$ & 3.164768 & 2.385497 & -3.136036 \\
\hline $\mathrm{C}$ & 2.555165 & 3.758684 & -3.433704 \\
\hline $\mathrm{C}$ & 3.617540 & 4.716702 & -4.007322 \\
\hline $\mathrm{C}$ & 3.002385 & 6.051588 & -4.448857 \\
\hline $\mathrm{H}$ & 0.446773 & 2.161412 & -3.109153 \\
\hline $\mathrm{H}$ & 1.297913 & 1.058615 & -4.220544 \\
\hline $\mathrm{H}$ & -0.289035 & 0.213396 & -1.694912 \\
\hline $\mathrm{H}$ & 0.563107 & -0.911523 & -2.781348 \\
\hline $\mathrm{H}$ & -0.823970 & -0.050101 & -4.727617 \\
\hline $\mathrm{H}$ & -1.713362 & 0.994940 & -3.601792 \\
\hline $\mathrm{H}$ & -2.971801 & -1.137000 & -4.065803 \\
\hline $\mathrm{H}$ & -1.613483 & -2.099002 & -3.423523 \\
\hline $\mathrm{H}$ & -2.543693 & -1.030529 & -2.342573 \\
\hline $\mathrm{H}$ & 1.295759 & 2.841229 & -1.177867 \\
\hline $\mathrm{H}$ & 1.249157 & 1.208393 & -0.502353 \\
\hline $\mathrm{H}$ & 3.600813 & 1.391584 & 0.293686 \\
\hline $\mathrm{H}$ & 3.823301 & 2.951900 & -0.538897 \\
\hline $\mathrm{H}$ & 1.740591 & 2.425982 & 1.662016 \\
\hline $\mathrm{H}$ & 2.019786 & 3.975556 & 0.845914 \\
\hline $\mathrm{H}$ & 3.184988 & 3.981771 & 3.040806 \\
\hline $\mathrm{H}$ & 4.051779 & 2.483847 & 2.649201 \\
\hline $\mathrm{H}$ & 4.418779 & 3.991777 & 1.752064 \\
\hline $\mathrm{H}$ & 3.949111 & 0.329524 & -1.639971 \\
\hline $\mathrm{H}$ & 2.405190 & -0.537530 & -1.531590 \\
\hline $\mathrm{H}$ & 2.468484 & -0.887339 & -4.072716 \\
\hline $\mathrm{H}$ & 4.061227 & -0.100655 & -4.119352 \\
\hline $\mathrm{H}$ & 3.370568 & -2.629451 & -2.506417 \\
\hline $\mathrm{H}$ & 4.951537 & -1.842146 & -2.517264 \\
\hline $\mathrm{H}$ & 4.928045 & -3.772601 & -4.120518 \\
\hline $\mathrm{H}$ & 3.555483 & -3.061585 & -5.005042 \\
\hline $\mathrm{H}$ & 5.146011 & -2.252926 & -5.024940 \\
\hline $\mathrm{H}$ & 3.433931 & 1.884965 & -4.079300 \\
\hline $\mathrm{H}$ & 4.085759 & 2.487604 & -2.540443 \\
\hline $\mathrm{H}$ & 1.728621 & 3.663332 & -4.160541 \\
\hline $\mathrm{H}$ & 2.121832 & 4.214839 & -2.526482 \\
\hline $\mathrm{H}$ & 4.130825 & 4.240559 & -4.865493 \\
\hline
\end{tabular}




\begin{tabular}{|c|c|c|c|}
\hline $\mathrm{H}$ & 4.395984 & 4.896024 & -3.241139 \\
\hline $\mathrm{H}$ & 3.783009 & 6.762330 & -4.767666 \\
\hline $\mathrm{H}$ & 2.313113 & 5.905735 & -5.299679 \\
\hline $\mathrm{H}$ & 2.423410 & 6.515725 & -3.631366 \\
\hline $\mathrm{O}$ & -0.207063 & 6.845563 & -2.711237 \\
\hline $\mathrm{O}$ & 3.693827 & 0.294168 & 4.038746 \\
\hline $\mathrm{N}$ & -2.667083 & 2.151203 & -0.847517 \\
\hline $\mathrm{N}$ & 2.843308 & -0.843548 & 2.191201 \\
\hline $\mathrm{C}$ & -0.913035 & -3.245549 & -0.017394 \\
\hline $\mathrm{C}$ & -1.076115 & -5.809673 & -1.123486 \\
\hline $\mathrm{C}$ & 0.182150 & -5.288330 & -0.810218 \\
\hline $\mathrm{C}$ & 0.265067 & -3.988808 & -0.247327 \\
\hline $\mathrm{C}$ & -2.133125 & 3.350037 & -1.350471 \\
\hline $\mathrm{C}$ & -2.605048 & 3.986730 & -2.517535 \\
\hline $\mathrm{C}$ & -1.991403 & 5.155998 & -2.994634 \\
\hline $\mathrm{C}$ & -0.889995 & 5.712127 & -2.320089 \\
\hline $\mathrm{C}$ & -0.423674 & 5.090663 & -1.144840 \\
\hline $\mathrm{C}$ & -1.040342 & 3.932847 & -0.665561 \\
\hline $\mathrm{C}$ & -0.724968 & 7.552392 & -3.834301 \\
\hline $\mathrm{C}$ & 1.535589 & -3.439911 & 0.086014 \\
\hline $\mathrm{C}$ & 2.646638 & -2.998245 & 0.385181 \\
\hline $\mathrm{C}$ & 3.917084 & -2.487260 & 0.762033 \\
\hline $\mathrm{C}$ & 4.019715 & -1.404535 & 1.693356 \\
\hline $\mathrm{C}$ & 5.313416 & -0.952409 & 2.039849 \\
\hline $\mathrm{C}$ & 6.446314 & -1.551948 & 1.488303 \\
\hline $\mathrm{C}$ & 6.376140 & -2.624610 & 0.568489 \\
\hline $\mathrm{C}$ & 5.094409 & -3.069379 & 0.226204 \\
\hline $\mathrm{C}$ & 8.469080 & -2.168004 & -0.760027 \\
\hline $\mathrm{C}$ & 7.370515 & -4.408784 & -0.977400 \\
\hline $\mathrm{C}$ & 2.717750 & -0.074253 & 3.369550 \\
\hline $\mathrm{C}$ & -3.989193 & 1.744473 & -0.984135 \\
\hline $\mathrm{C}$ & -2.174419 & -3.783479 & -0.357591 \\
\hline $\mathrm{C}$ & -2.253684 & -5.086886 & -0.911616 \\
\hline $\mathrm{C}$ & 7.663110 & -3.251523 & -0.000822 \\
\hline $\mathrm{N}$ & 1.399326 & 0.204105 & 3.654751 \\
\hline $\mathrm{N}$ & -4.227004 & 0.525799 & -0.333565 \\
\hline $\mathrm{O}$ & -4.850951 & 2.373473 & -1.608536 \\
\hline $\mathrm{C}$ & -3.350299 & -3.003597 & -0.182716 \\
\hline $\mathrm{C}$ & 8.522850 & -3.805916 & 1.162749 \\
\hline $\mathrm{C}$ & 0.898510 & 0.902764 & 4.776200 \\
\hline $\mathrm{C}$ & -5.476025 & -0.097533 & -0.216986 \\
\hline $\mathrm{C}$ & -4.358894 & -2.305288 & -0.084560 \\
\hline $\mathrm{C}$ & -0.507877 & 0.959482 & 4.922222 \\
\hline $\mathrm{C}$ & 1.704699 & 1.533164 & 5.743177 \\
\hline $\mathrm{C}$ & -5.535049 & -1.512237 & -0.031868 \\
\hline $\mathrm{C}$ & -6.690625 & 0.618608 & -0.250701 \\
\hline $\mathrm{C}$ & -1.088109 & 1.634416 & 5.993313 \\
\hline $\mathrm{C}$ & 1.117276 & 2.205946 & 6.825991 \\
\hline
\end{tabular}




\begin{tabular}{|c|c|c|c|}
\hline $\mathrm{C}$ & -6.791900 & -2.145858 & 0.145408 \\
\hline $\mathrm{C}$ & -7.908686 & -0.041334 & -0.088228 \\
\hline $\mathrm{C}$ & -0.280333 & 2.266127 & 6.959118 \\
\hline $\mathrm{C}$ & -7.997437 & -1.437015 & 0.123618 \\
\hline $\mathrm{O}$ & -0.952615 & 2.900926 & 7.978377 \\
\hline $\mathrm{C}$ & -9.370184 & -2.110229 & 0.312931 \\
\hline $\mathrm{C}$ & -0.160899 & 3.534698 & 8.975457 \\
\hline $\mathrm{C}$ & -9.251695 & -3.633172 & 0.524295 \\
\hline $\mathrm{C}$ & -10.072292 & -1.501511 & 1.552568 \\
\hline $\mathrm{C}$ & -10.242313 & -1.861112 & -0.942957 \\
\hline $\mathrm{H}$ & -2.044649 & 1.593355 & -0.220786 \\
\hline $\mathrm{H}$ & 1.977133 & -1.210234 & 1.781859 \\
\hline $\mathrm{H}$ & -0.859051 & -2.249878 & 0.435197 \\
\hline $\mathrm{H}$ & 1.087919 & -5.873281 & -0.985594 \\
\hline $\mathrm{H}$ & -3.473082 & 3.573338 & -3.033376 \\
\hline $\mathrm{H}$ & -2.389724 & 5.622811 & -3.899457 \\
\hline $\mathrm{H}$ & 0.404662 & 5.553076 & -0.596935 \\
\hline $\mathrm{H}$ & -0.685893 & 3.468228 & 0.260971 \\
\hline $\mathrm{H}$ & -0.084746 & 8.439101 & -3.953564 \\
\hline $\mathrm{H}$ & -0.681239 & 6.944749 & -4.759861 \\
\hline $\mathrm{H}$ & -1.770545 & 7.874060 & -3.665470 \\
\hline $\mathrm{H}$ & 5.405718 & -0.149843 & 2.771261 \\
\hline $\mathrm{H}$ & 4.959501 & -3.903395 & -0.467215 \\
\hline $\mathrm{H}$ & 6.819294 & -5.229983 & -0.486972 \\
\hline $\mathrm{H}$ & 6.783715 & -4.072376 & -1.851303 \\
\hline $\mathrm{H}$ & 7.425028 & -1.165887 & 1.795433 \\
\hline $\mathrm{H}$ & 8.733114 & -1.319953 & -0.105462 \\
\hline $\mathrm{H}$ & 7.889391 & -1.769358 & -1.611841 \\
\hline $\mathrm{H}$ & 9.409358 & -2.593253 & -1.155278 \\
\hline $\mathrm{H}$ & 8.320227 & -4.824766 & -1.355999 \\
\hline $\mathrm{H}$ & -3.223888 & -5.512178 & -1.178496 \\
\hline $\mathrm{H}$ & 7.979338 & -4.592544 & 1.713831 \\
\hline $\mathrm{H}$ & 8.793677 & -3.016325 & 1.883964 \\
\hline $\mathrm{H}$ & 9.460492 & -4.242509 & 0.773558 \\
\hline $\mathrm{H}$ & -1.146245 & 0.459215 & 4.185193 \\
\hline $\mathrm{H}$ & 2.789487 & 1.485981 & 5.646219 \\
\hline $\mathrm{H}$ & -6.660723 & 1.695395 & -0.418058 \\
\hline $\mathrm{H}$ & -2.175626 & 1.677502 & 6.105257 \\
\hline $\mathrm{H}$ & 1.772421 & 2.680973 & 7.561012 \\
\hline $\mathrm{H}$ & -6.782129 & -3.228960 & 0.290637 \\
\hline $\mathrm{H}$ & -8.823567 & 0.561286 & -0.117021 \\
\hline $\mathrm{H}$ & -0.872362 & 3.971598 & 9.692220 \\
\hline $\mathrm{H}$ & 0.491613 & 2.811543 & 9.503228 \\
\hline $\mathrm{H}$ & 0.469232 & 4.340529 & 8.549822 \\
\hline $\mathrm{H}$ & -10.257234 & -4.068991 & 0.656902 \\
\hline $\mathrm{H}$ & -8.660982 & -3.877491 & 1.424538 \\
\hline $\mathrm{H}$ & -8.783829 & -4.132263 & -0.342479 \\
\hline $\mathrm{H}$ & -9.480931 & -1.679224 & 2.467489 \\
\hline
\end{tabular}




$\begin{array}{lccc}\mathrm{H} & -11.068920 & -1.958436 & 1.692222 \\ \mathrm{H} & -10.213625 & -0.412125 & 1.449347 \\ \mathrm{H} & -11.239155 & -2.321071 & -0.815185 \\ \mathrm{H} & -9.773860 & -2.298897 & -1.841515 \\ \mathrm{H} & -10.391010 & -0.784298 & -1.131782 \\ \mathrm{H} & -3.417750 & 0.029641 & 0.064851 \\ \mathrm{H} & 0.694586 & -0.018059 & 2.923221 \\ \mathrm{~S} & -0.743170 & 0.276687 & 1.075145 \\ \mathrm{H} & -0.984600 & 1.312350 & 1.927625 \\ \mathrm{Cl} & -1.178698 & -7.423332 & -1.816729\end{array}$

Statistical Thermodynamic Analysis

Temperature $=298.150$ Kelvin $\quad$ Pressure $=1.00000$ Atm

\begin{tabular}{|c|c|}
\hline $\begin{array}{l}\text { SCF Energy }=\quad-3840.81035591 \\
\text { Zero-point correction }(\mathrm{ZPE})=\end{array}$ & 1 Predicted Change $=-2.350758 \mathrm{D}-08$ \\
\hline Zero-point correction $(\mathrm{ZPE})=$ & -3839.51381 .29648 \\
\hline Internal Energy $(U)=$ & -3839.4307 1.37957 \\
\hline Enthalpy $(\mathrm{H})=$ & $-3839.4298 \quad 1.38052$ \\
\hline Gibbs Free Energy $(G)=$ & $-3839.6452 \quad 1.16513$ \\
\hline $\begin{array}{l}\text { Frequencies -- } 5.5859 \\
\text { Single points at WB97X-D3/def2 }\end{array}$ & $\begin{array}{cc}7.9934 & 10.9040 \\
\text { 2-TZVPP: } & -3845.35539842479\end{array}$ \\
\hline $\begin{array}{r}\mathbf{R}=\mathbf{C l} \text { receptor bound with } \mathbf{B r}- \\
\text { Supporting Information: }\end{array}$ & $\begin{array}{l}\text { (no TBA) } \\
\text { 010-bound noTBA } \mathrm{Cl} \text { Br.log }\end{array}$ \\
\hline
\end{tabular}

\#PBEPBE/6-31G(d)/auto scf=(direct,tight,maxcycle=300,xqc) $\mathrm{opt}=($ maxcycle $=250)$ freq $=$ noraman int $=($ grid $=$ ultrafine $)$ \#P Geom=AllCheck Guess=TCheck SCRF=Check GenChk RPBEPBE/6-31G(d)/Auto Freq

Pointgroup $=\mathrm{C} 1 \quad$ Stoichiometry $=\mathrm{C} 46 \mathrm{H} 45 \mathrm{BrClN} 4 \mathrm{O} 4(1-) \quad \mathrm{C} 1[\mathrm{X}(\mathrm{C} 46 \mathrm{H} 45 \mathrm{BrClN} 4 \mathrm{O} 4)]$ \#Atoms $=101$

Charge $=-1 \quad$ Multiplicity $=1$

SCF Energy $=-5328.49865680 \quad$ Predicted Change $=-6.098521 \mathrm{D}-09$

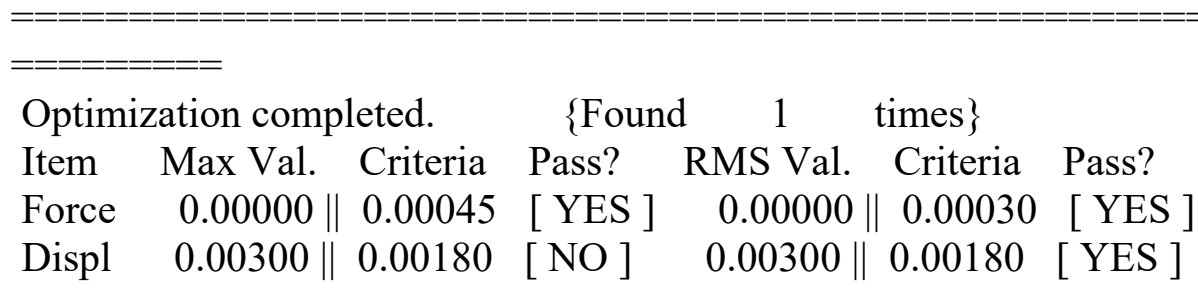

Atomic Coordinates (Angstroms) 


\begin{tabular}{|c|c|c|c|}
\hline Type & $X$ & Y & \\
\hline $\mathrm{O}$ & -4.593364 & 2.111365 & 2.004439 \\
\hline $\mathrm{O}$ & 0.041997 & 6.032565 & 4.706796 \\
\hline $\mathrm{O}$ & 4.872790 & 1.833226 & -1.795276 \\
\hline $\mathrm{O}$ & 0.965556 & 6.451206 & -4.527977 \\
\hline $\mathrm{N}$ & -3.712196 & 0.438067 & 0.636554 \\
\hline $\mathrm{H}$ & -2.836465 & -0.005588 & 0.329833 \\
\hline $\mathrm{N}$ & -2.305320 & 1.982487 & 1.616477 \\
\hline $\mathrm{H}$ & -1.579612 & 1.495399 & 1.053961 \\
\hline $\mathrm{N}$ & 3.703345 & 0.164671 & -0.654808 \\
\hline $\mathrm{H}$ & 2.761997 & -0.203760 & -0.462808 \\
\hline $\mathrm{N}$ & 2.558148 & 1.918484 & -1.629901 \\
\hline $\mathrm{H}$ & 1.731725 & 1.459976 & -1.198097 \\
\hline $\mathrm{C}$ & -0.216836 & -3.145927 & -0.082553 \\
\hline $\mathrm{H}$ & -0.174931 & -2.050920 & -0.047547 \\
\hline $\mathrm{C}$ & -1.466476 & -3.795697 & -0.167824 \\
\hline $\mathrm{C}$ & -1.518868 & -5.212504 & -0.224561 \\
\hline $\mathrm{H}$ & -2.479161 & -5.729564 & -0.288699 \\
\hline $\mathrm{C}$ & -0.320797 & -5.931157 & -0.191166 \\
\hline $\mathrm{C}$ & 0.927206 & -5.307976 & -0.107514 \\
\hline $\mathrm{H}$ & 1.846357 & -5.898254 & -0.088199 \\
\hline $\mathrm{C}$ & 0.980809 & -3.890822 & -0.055561 \\
\hline $\mathrm{C}$ & -2.656766 & -3.017069 & -0.189893 \\
\hline $\mathrm{C}$ & -3.660444 & -2.304738 & -0.196756 \\
\hline $\mathrm{C}$ & -4.862166 & -1.548015 & -0.196099 \\
\hline $\mathrm{C}$ & -4.899233 & -0.188539 & 0.259442 \\
\hline $\mathrm{C}$ & -6.152188 & 0.460174 & 0.284603 \\
\hline $\mathrm{H}$ & -6.197049 & 1.484823 & 0.652470 \\
\hline $\mathrm{C}$ & -7.313320 & -0.196675 & -0.133072 \\
\hline $\mathrm{H}$ & -8.252465 & 0.363434 & -0.092795 \\
\hline $\mathrm{C}$ & -7.302981 & -1.525807 & -0.604387 \\
\hline $\mathrm{C}$ & -6.058562 & -2.172319 & -0.621954 \\
\hline $\mathrm{H}$ & -5.976634 & -3.209272 & -0.965277 \\
\hline $\mathrm{C}$ & -3.612288 & 1.572853 & 1.471254 \\
\hline $\mathrm{C}$ & -1.813977 & 3.025250 & 2.421365 \\
\hline $\mathrm{C}$ & -2.612754 & 3.852169 & 3.234228 \\
\hline $\mathrm{H}$ & -3.691863 & 3.695741 & 3.250867 \\
\hline $\mathrm{C}$ & -2.022762 & 4.864195 & 4.009229 \\
\hline $\mathrm{H}$ & -2.670610 & 5.489332 & 4.630228 \\
\hline $\mathrm{C}$ & -0.633525 & 5.066039 & 3.982738 \\
\hline $\mathrm{C}$ & 0.166235 & 4.240701 & 3.168669 \\
\hline $\mathrm{H}$ & 1.248177 & 4.404425 & 3.150597 \\
\hline $\mathrm{C}$ & -0.412927 & 3.234706 & 2.400219 \\
\hline $\mathrm{H}$ & 0.205614 & 2.588834 & 1.764877 \\
\hline $\mathrm{C}$ & -0.746304 & 6.875932 & 5.529687 \\
\hline $\mathrm{H}$ & -0.043032 & 7.569294 & 6.017177 \\
\hline $\mathrm{H}$ & -1.291888 & 6.304522 & 6.308099 \\
\hline
\end{tabular}




\begin{tabular}{|c|c|c|c|}
\hline $\mathrm{H}$ & -1.482987 & 7.459621 & 4.941027 \\
\hline $\mathrm{C}$ & 2.227933 & -3.210381 & 0.020409 \\
\hline $\mathrm{C}$ & 3.288575 & -2.589081 & 0.082735 \\
\hline $\mathrm{C}$ & 4.562832 & -1.967732 & 0.172776 \\
\hline $\mathrm{C}$ & 4.781880 & -0.605888 & -0.220506 \\
\hline $\mathrm{C}$ & 6.098068 & -0.103266 & -0.128111 \\
\hline $\mathrm{H}$ & 6.282029 & 0.923315 & -0.442701 \\
\hline $\mathrm{C}$ & 7.146821 & -0.902572 & 0.335448 \\
\hline $\mathrm{H}$ & 8.141819 & -0.449863 & 0.385202 \\
\hline $\mathrm{C}$ & 6.957801 & -2.240153 & 0.739373 \\
\hline $\mathrm{C}$ & 5.651253 & -2.740013 & 0.644563 \\
\hline $\mathrm{H}$ & 5.429934 & -3.772766 & 0.934823 \\
\hline $\mathrm{C}$ & 3.796542 & 1.361353 & -1.399993 \\
\hline $\mathrm{C}$ & 2.259872 & 3.072826 & -2.376045 \\
\hline $\mathrm{C}$ & 3.217831 & 3.872243 & -3.029003 \\
\hline $\mathrm{H}$ & 4.271279 & 3.599272 & -2.960552 \\
\hline $\mathrm{C}$ & 2.817299 & 5.005688 & -3.755440 \\
\hline $\mathrm{H}$ & 3.586275 & 5.605182 & -4.250847 \\
\hline $\mathrm{C}$ & 1.460769 & 5.357710 & -3.839513 \\
\hline $\mathrm{C}$ & 0.501825 & 4.560090 & -3.185744 \\
\hline $\mathrm{H}$ & -0.553887 & 4.840717 & -3.253008 \\
\hline $\mathrm{C}$ & 0.892868 & 3.434593 & -2.465632 \\
\hline $\mathrm{H}$ & 0.147821 & 2.811066 & -1.956166 \\
\hline $\mathrm{C}$ & 1.916794 & 7.269942 & -5.187308 \\
\hline $\mathrm{H}$ & 1.342246 & 8.076630 & -5.669127 \\
\hline $\mathrm{H}$ & 2.479267 & 6.711065 & -5.962784 \\
\hline $\mathrm{H}$ & 2.643897 & 7.715059 & -4.478042 \\
\hline $\mathrm{C}$ & -8.568559 & -2.267767 & -1.078767 \\
\hline $\mathrm{C}$ & 8.096947 & -3.139649 & 1.259108 \\
\hline $\mathrm{C}$ & -8.392840 & -2.698565 & -2.556227 \\
\hline $\mathrm{H}$ & -7.517158 & -3.356808 & -2.686571 \\
\hline $\mathrm{H}$ & -9.285383 & -3.248652 & -2.908554 \\
\hline $\mathrm{H}$ & -8.250035 & -1.818861 & -3.207673 \\
\hline $\mathrm{C}$ & -9.831488 & -1.387566 & -0.981740 \\
\hline $\mathrm{H}$ & -10.029243 & -1.068924 & 0.056806 \\
\hline $\mathrm{H}$ & -9.748449 & -0.482819 & -1.609245 \\
\hline $\mathrm{H}$ & -10.710738 & -1.957908 & -1.330623 \\
\hline $\mathrm{C}$ & -8.789815 & -3.526786 & -0.203942 \\
\hline $\mathrm{H}$ & -8.936004 & -3.248874 & 0.854413 \\
\hline $\mathrm{H}$ & -9.684569 & -4.081922 & -0.542539 \\
\hline $\mathrm{H}$ & -7.927835 & -4.213554 & -0.253810 \\
\hline $\mathrm{C}$ & 7.770761 & -3.609232 & 2.698688 \\
\hline $\mathrm{H}$ & 6.821454 & -4.169880 & 2.738551 \\
\hline $\mathrm{H}$ & 8.570633 & -4.270331 & 3.081474 \\
\hline $\mathrm{H}$ & 7.679097 & -2.746990 & 3.381906 \\
\hline $\mathrm{C}$ & 8.239982 & -4.377921 & 0.339517 \\
\hline $\mathrm{H}$ & 8.489753 & -4.074253 & -0.692050 \\
\hline $\mathrm{H}$ & 9.042468 & -5.043985 & 0.707984 \\
\hline
\end{tabular}




$\begin{array}{lccc}\mathrm{H} & 7.306491 & -4.964560 & 0.299150 \\ \mathrm{C} & 9.452527 & -2.404302 & 1.289016 \\ \mathrm{H} & 9.757463 & -2.067528 & 0.282621 \\ \mathrm{H} & 9.424681 & -1.522525 & 1.953022 \\ \mathrm{H} & 10.236854 & -3.084021 & 1.667054 \\ \mathrm{Cl} & -0.386500 & -7.696094 & -0.258285 \\ \mathrm{Br} & -0.047023 & 0.437582 & -0.244806\end{array}$

Statistical Thermodynamic Analysis

Temperature $=298.150$ Kelvin $\quad$ Pressure $=1.00000$ Atm

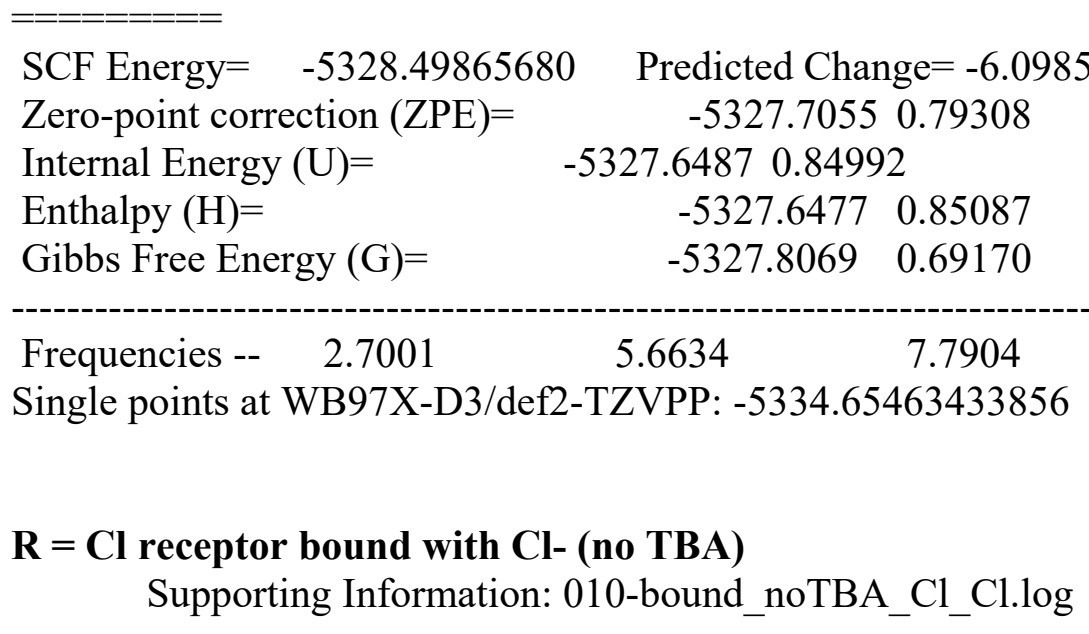

Using Gaussian 09: EM64L-G09RevE.01 30-Nov-2015

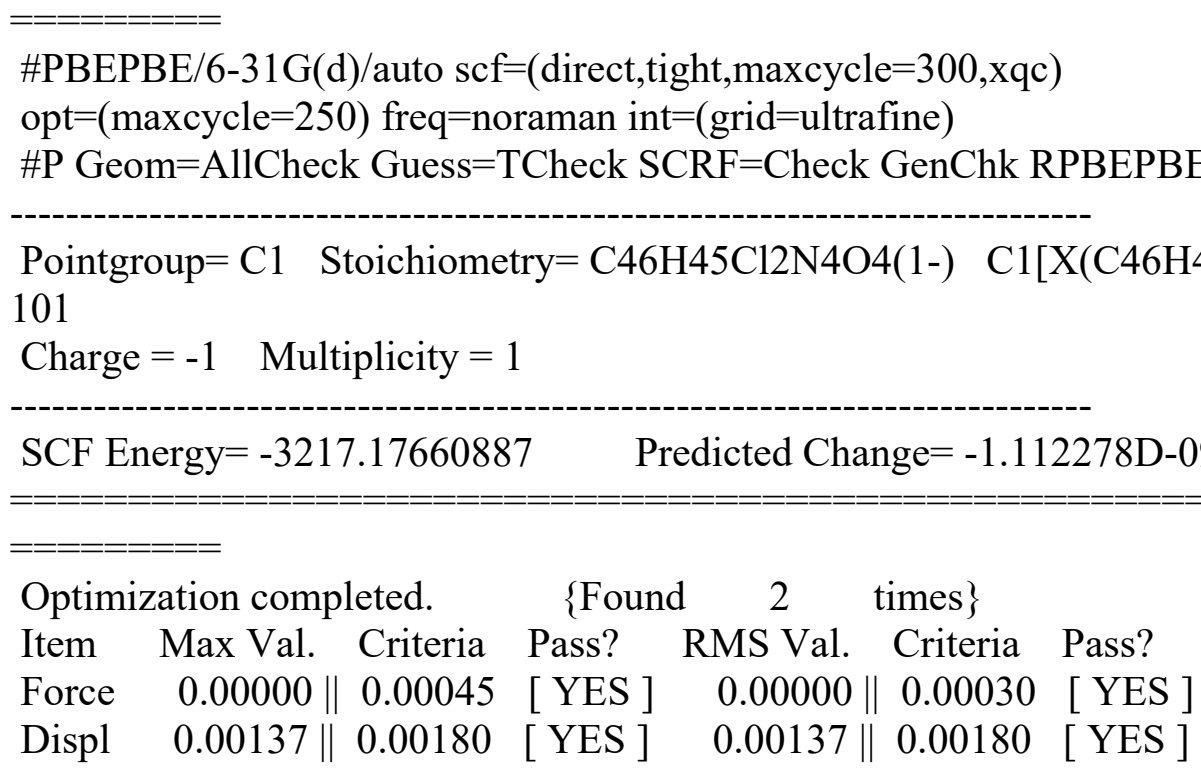

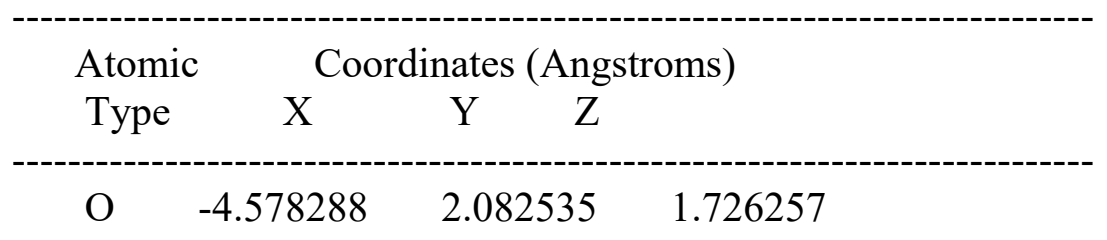




\begin{tabular}{|c|c|c|c|}
\hline $\mathrm{O}$ & -0.207147 & 6.473639 & 4.046139 \\
\hline $\mathrm{O}$ & 4.578272 & 2.082564 & -1.726254 \\
\hline $\mathrm{O}$ & 0.207085 & 6.473585 & -4.046193 \\
\hline $\mathrm{N}$ & -3.629250 & 0.256173 & 0.629915 \\
\hline $\mathrm{H}$ & -2.738955 & -0.212792 & 0.419639 \\
\hline $\mathrm{N}$ & -2.273672 & 1.930621 & 1.471784 \\
\hline $\mathrm{H}$ & -1.510997 & 1.380895 & 1.023435 \\
\hline $\mathrm{N}$ & 3.629256 & 0.256199 & -0.629895 \\
\hline $\mathrm{H}$ & 2.738966 & -0.212775 & -0.419621 \\
\hline $\mathrm{N}$ & 2.273659 & 1.930623 & -1.471779 \\
\hline $\mathrm{H}$ & 1.510991 & 1.380890 & -1.023427 \\
\hline $\mathrm{C}$ & 0.000013 & -3.218869 & -0.000000 \\
\hline $\mathrm{H}$ & 0.000008 & -2.121806 & 0.000012 \\
\hline $\mathrm{C}$ & -1.224706 & -3.919436 & -0.030313 \\
\hline $\mathrm{C}$ & -1.224593 & -5.338489 & -0.032226 \\
\hline $\mathrm{H}$ & -2.165918 & -5.892612 & -0.054151 \\
\hline $\mathrm{C}$ & 0.000025 & -6.010619 & -0.000027 \\
\hline $\mathrm{C}$ & 1.224638 & -5.338478 & 0.032185 \\
\hline $\mathrm{H}$ & 2.165967 & -5.892593 & 0.054101 \\
\hline $\mathrm{C}$ & 1.224738 & -3.919425 & 0.030299 \\
\hline $\mathrm{C}$ & -2.444969 & -3.189372 & -0.055194 \\
\hline $\mathrm{C}$ & -3.476560 & -2.518459 & -0.075163 \\
\hline $\mathrm{C}$ & -4.705732 & -1.808495 & -0.101426 \\
\hline $\mathrm{C}$ & -4.792348 & -0.426899 & 0.277250 \\
\hline $\mathrm{C}$ & -6.068318 & 0.177211 & 0.253701 \\
\hline $\mathrm{H}$ & -6.150963 & 1.220016 & 0.557545 \\
\hline $\mathrm{C}$ & -7.202253 & -0.544295 & -0.130666 \\
\hline $\mathrm{H}$ & -8.160152 & -0.015269 & -0.129455 \\
\hline $\mathrm{C}$ & -7.144041 & -1.898765 & -0.517982 \\
\hline $\mathrm{C}$ & -5.877303 & -2.499659 & -0.491457 \\
\hline $\mathrm{H}$ & -5.755633 & -3.550928 & -0.774291 \\
\hline $\mathrm{C}$ & -3.569792 & 1.488908 & 1.315611 \\
\hline $\mathrm{C}$ & -1.851078 & 3.095813 & 2.139282 \\
\hline $\mathrm{C}$ & -2.711356 & 3.992532 & 2.803522 \\
\hline $\mathrm{H}$ & -3.783293 & 3.794615 & 2.805619 \\
\hline $\mathrm{C}$ & -2.193831 & 5.127383 & 3.449013 \\
\hline $\mathrm{H}$ & -2.891224 & 5.801764 & 3.954089 \\
\hline $\mathrm{C}$ & -0.814232 & 5.386854 & 3.442061 \\
\hline $\mathrm{C}$ & 0.048042 & 4.493673 & 2.778256 \\
\hline $\mathrm{H}$ & 1.122312 & 4.702347 & 2.771324 \\
\hline $\mathrm{C}$ & -0.460594 & 3.366548 & 2.137992 \\
\hline $\mathrm{H}$ & 0.216022 & 2.675471 & 1.622744 \\
\hline $\mathrm{C}$ & -1.061778 & 7.391106 & 4.707664 \\
\hline $\mathrm{H}$ & -0.407294 & 8.178471 & 5.113553 \\
\hline $\mathrm{H}$ & -1.617237 & 6.914913 & 5.541277 \\
\hline $\mathrm{H}$ & -1.795004 & 7.849736 & 4.013496 \\
\hline $\mathrm{C}$ & 2.444994 & -3.189350 & 0.055203 \\
\hline $\mathrm{C}$ & 3.476578 & -2.518427 & 0.075194 \\
\hline
\end{tabular}




\begin{tabular}{|c|c|c|c|}
\hline $\mathrm{C}$ & 4.705749 & -1.808461 & 0.101451 \\
\hline $\mathrm{C}$ & 4.792359 & -0.426864 & -0.277229 \\
\hline $\mathrm{C}$ & 6.068327 & 0.177251 & -0.253675 \\
\hline $\mathrm{H}$ & 6.150969 & 1.220056 & -0.557518 \\
\hline $\mathrm{C}$ & 7.202264 & -0.544250 & 0.130696 \\
\hline $\mathrm{H}$ & 8.160161 & -0.015220 & 0.129488 \\
\hline $\mathrm{C}$ & 7.144058 & -1.898720 & 0.518011 \\
\hline $\mathrm{C}$ & 5.877321 & -2.499620 & 0.491484 \\
\hline $\mathrm{H}$ & 5.755655 & -3.550888 & 0.774322 \\
\hline $\mathrm{C}$ & 3.569785 & 1.488928 & -1.315600 \\
\hline $\mathrm{C}$ & 1.851052 & 3.095801 & -2.139291 \\
\hline $\mathrm{C}$ & 2.711321 & 3.992522 & -2.803542 \\
\hline $\mathrm{H}$ & 3.783260 & 3.794616 & -2.805635 \\
\hline $\mathrm{C}$ & 2.193784 & 5.127358 & -3.449048 \\
\hline $\mathrm{H}$ & 2.891170 & 5.801741 & -3.954132 \\
\hline $\mathrm{C}$ & 0.814182 & 5.386815 & -3.442100 \\
\hline $\mathrm{C}$ & -0.048083 & 4.493632 & -2.778285 \\
\hline $\mathrm{H}$ & -1.122355 & 4.702294 & -2.771356 \\
\hline $\mathrm{C}$ & 0.460565 & 3.366521 & -2.138006 \\
\hline $\mathrm{H}$ & -0.216044 & 2.675443 & -1.622750 \\
\hline $\mathrm{C}$ & 1.061707 & 7.391053 & -4.707728 \\
\hline $\mathrm{H}$ & 0.407215 & 8.178406 & -5.113629 \\
\hline $\mathrm{H}$ & 1.617171 & 6.914855 & -5.541335 \\
\hline $\mathrm{H}$ & 1.794928 & 7.849700 & -4.013566 \\
\hline $\mathrm{C}$ & -8.379802 & -2.712083 & -0.951879 \\
\hline $\mathrm{C}$ & 8.379820 & -2.712032 & 0.951915 \\
\hline $\mathrm{C}$ & -8.181751 & -3.220967 & -2.401416 \\
\hline $\mathrm{H}$ & -7.281877 & -3.852878 & -2.491008 \\
\hline $\mathrm{H}$ & -9.051892 & -3.823026 & -2.723619 \\
\hline $\mathrm{H}$ & -8.068160 & -2.375693 & -3.102328 \\
\hline $\mathrm{C}$ & -9.673781 & -1.873645 & -0.909318 \\
\hline $\mathrm{H}$ & -9.886789 & -1.501909 & 0.108359 \\
\hline $\mathrm{H}$ & -9.620482 & -1.004843 & -1.588679 \\
\hline $\mathrm{H}$ & -10.530720 & -2.494601 & -1.225882 \\
\hline $\mathrm{C}$ & -8.560367 & -3.925722 & -0.006413 \\
\hline $\mathrm{H}$ & -8.721241 & -3.592754 & 1.033780 \\
\hline $\mathrm{H}$ & -9.433305 & -4.530937 & -0.314811 \\
\hline $\mathrm{H}$ & -7.674368 & -4.583087 & -0.014280 \\
\hline $\mathrm{C}$ & 8.181755 & -3.220931 & 2.401446 \\
\hline $\mathrm{H}$ & 7.281884 & -3.852848 & 2.491021 \\
\hline $\mathrm{H}$ & 9.051896 & -3.822987 & 2.723655 \\
\hline $\mathrm{H}$ & 8.068150 & -2.375663 & 3.102364 \\
\hline $\mathrm{C}$ & 8.560408 & -3.925661 & 0.006442 \\
\hline $\mathrm{H}$ & 8.721290 & -3.592682 & -1.033747 \\
\hline $\mathrm{H}$ & 9.433349 & -4.530869 & 0.314844 \\
\hline $\mathrm{H}$ & 7.674416 & -4.583036 & 0.014293 \\
\hline $\mathrm{C}$ & 9.673792 & -1.873582 & 0.909378 \\
\hline $\mathrm{H}$ & 9.886805 & -1.501829 & -0.108292 \\
\hline
\end{tabular}




$\begin{array}{lccc}\mathrm{H} & 9.620481 & -1.004791 & 1.588752 \\ \mathrm{H} & 10.530734 & -2.494536 & 1.225939 \\ \mathrm{Cl} & 0.000034 & -7.778217 & -0.000043 \\ \mathrm{Cl} & 0.000008 & 0.253730 & 0.000023\end{array}$

Statistical Thermodynamic Analysis

Temperature $=298.150$ Kelvin $\quad$ Pressure $=1.00000$ Atm

\begin{tabular}{|c|c|}
\hline SCF Energy $=\quad-3217.17660887$ & $7 \quad$ Predicted Change $=-1.112278 \mathrm{D}-09$ \\
\hline Zero-point correction $(\mathrm{ZPE})=$ & $-3216.3838 \quad 0.79280$ \\
\hline Internal Energy $(U)=$ & -3216.32700 .84959 \\
\hline Enthalpy $(\mathrm{H})=$ & $-3216.3260 \quad 0.85053$ \\
\hline Gibbs Free Energy $(G)=$ & $\begin{array}{ll}-3216.4846 & 0.69199\end{array}$ \\
\hline $\begin{array}{l}\text { Frequencies -- } 3.9533 \\
\text { Single points at WB97X-D3/def2 }\end{array}$ & $\begin{array}{cc}6.2057 & 9.2986 \\
\text { 2-TZVPP: } & -3220.64662718719\end{array}$ \\
\hline $\begin{array}{r}\mathbf{R}=\mathbf{C l} \text { receptor bound with } \mathrm{SeH} \\
\text { Supporting Information: }\end{array}$ & $\begin{array}{l}\text { H- (no TBA) } \\
\text { 010-bound_noTBA_Cl_SeH.log }\end{array}$ \\
\hline
\end{tabular}

$======$

\#PBEPBE/6-31G(d)/auto scf=(direct,tight,maxcycle=300,xqc)

$\mathrm{opt}=($ maxcycle $=250)$ freq $=$ noraman int $=($ grid $=$ ultrafine $)$

\#P Geom=AllCheck Guess=TCheck SCRF=Check GenChk RPBEPBE/6-31G(d)/Auto Freq

Pointgroup= C1 Stoichiometry= C46H46ClN4O4Se(1-) C1[X(C46H46ClN4O4Se) $]$

\#Atoms $=102$

Charge $=-1 \quad$ Multiplicity $=1$

SCF Energy $=-5156.76170857 \quad$ Predicted Change $=-7.928147 \mathrm{D}-09$

\begin{tabular}{|c|c|c|c|c|c|}
\hline \multicolumn{3}{|c|}{ Optimization completed. } & $\{$ Four & 1 & times $\}$ \\
\hline \multirow{3}{*}{\multicolumn{3}{|c|}{$\begin{array}{lrc}\text { Item } & \text { Max Val. } & \text { Criteria } \\
\text { Force } & 0.00000 \| & 0.00045 \\
\text { Displ } & 0.00331 \| & 0.00180\end{array}$}} & Pass? & RMS Val. & Criteria \\
\hline & & & [YES ] & 0.00000 & 0.00030 \\
\hline & & & {$[\mathrm{NO}]$} & $0.00331 \|$ & 0.00180 \\
\hline \multirow{2}{*}{\multicolumn{2}{|c|}{$\begin{array}{l}\text { Atomic } \\
\text { Type }\end{array}$}} & \multicolumn{3}{|c|}{ Coordinates (Angstroms) } & \\
\hline & & $\mathrm{Y}$ & Z & & \\
\hline $\mathrm{O}$ & $5.0673 \xi$ & 1.77 & 0139 & -1.312408 & \\
\hline $\mathrm{O}$ & 1.5656 & 6.97 & 8545 & -3.417801 & \\
\hline $\mathrm{O}$ & -4.6607 & 2.25 & 6236 & 1.636304 & \\
\hline $\mathrm{O}$ & -0.0701 & 6.73 & 9548 & 3.454853 & \\
\hline
\end{tabular}




\begin{tabular}{|c|c|c|c|}
\hline $\mathrm{N}$ & 3.770869 & 0.008490 & -0.487480 \\
\hline $\mathrm{H}$ & 2.800456 & -0.333058 & -0.404847 \\
\hline $\mathrm{N}$ & 2.762253 & 1.978863 & -1.161719 \\
\hline $\mathrm{H}$ & 1.887809 & 1.495229 & -0.847434 \\
\hline $\mathrm{N}$ & -3.780444 & 0.458731 & 0.490966 \\
\hline $\mathrm{H}$ & -2.896534 & -0.012297 & 0.244562 \\
\hline $\mathrm{N}$ & -2.390040 & 2.173665 & 1.166387 \\
\hline $\mathrm{H}$ & -1.668251 & 1.627076 & 0.639789 \\
\hline $\mathrm{C}$ & -0.329310 & -3.274528 & -0.109942 \\
\hline $\mathrm{H}$ & -0.266326 & -2.177599 & -0.125566 \\
\hline $\mathrm{C}$ & 0.853693 & -4.040634 & -0.079131 \\
\hline $\mathrm{C}$ & 0.772342 & -5.457835 & -0.083659 \\
\hline $\mathrm{H}$ & 1.680087 & -6.065468 & -0.060950 \\
\hline $\mathrm{C}$ & -0.489060 & -6.058179 & -0.120370 \\
\hline $\mathrm{C}$ & -1.674140 & -5.317801 & -0.148228 \\
\hline $\mathrm{H}$ & -2.645375 & -5.817707 & -0.170742 \\
\hline $\mathrm{C}$ & -1.592256 & -3.901215 & -0.140895 \\
\hline $\mathrm{C}$ & 2.113368 & -3.383004 & -0.032009 \\
\hline $\mathrm{C}$ & 3.189489 & -2.788398 & 0.025059 \\
\hline $\mathrm{C}$ & 4.496375 & -2.244759 & 0.136648 \\
\hline $\mathrm{C}$ & 4.795821 & -0.866825 & -0.126777 \\
\hline $\mathrm{C}$ & 6.141714 & -0.458630 & 0.017149 \\
\hline $\mathrm{H}$ & 6.389673 & 0.580637 & -0.192910 \\
\hline $\mathrm{C}$ & 7.138949 & -1.360671 & 0.396865 \\
\hline $\mathrm{H}$ & 8.158435 & -0.973794 & 0.489581 \\
\hline $\mathrm{C}$ & 6.871267 & -2.719195 & 0.661778 \\
\hline $\mathrm{C}$ & 5.537168 & -3.124621 & 0.522853 \\
\hline $\mathrm{H}$ & 5.250673 & -4.164715 & 0.712703 \\
\hline $\mathrm{C}$ & 3.954740 & 1.307521 & -1.017968 \\
\hline $\mathrm{C}$ & 2.562813 & 3.245376 & -1.741980 \\
\hline $\mathrm{C}$ & 3.598203 & 4.113975 & -2.140087 \\
\hline $\mathrm{H}$ & 4.633501 & 3.799159 & -2.007428 \\
\hline $\mathrm{C}$ & 3.297872 & 5.364461 & -2.704523 \\
\hline $\mathrm{H}$ & 4.126203 & 6.013053 & -3.003578 \\
\hline $\mathrm{C}$ & 1.964590 & 5.769959 & -2.875265 \\
\hline $\mathrm{C}$ & 0.927619 & 4.904707 & -2.477304 \\
\hline $\mathrm{H}$ & -0.110113 & 5.225661 & -2.610474 \\
\hline $\mathrm{C}$ & 1.220098 & 3.661289 & -1.923322 \\
\hline $\mathrm{H}$ & 0.412364 & 2.983535 & -1.622765 \\
\hline $\mathrm{C}$ & 2.596470 & 7.868694 & -3.811456 \\
\hline $\mathrm{H}$ & 2.091096 & 8.763926 & -4.206608 \\
\hline $\mathrm{H}$ & 3.239858 & 7.434994 & -4.604017 \\
\hline $\mathrm{H}$ & 3.240112 & 8.161682 & -2.957281 \\
\hline $\mathrm{C}$ & -2.763932 & -3.095992 & -0.156176 \\
\hline $\mathrm{C}$ & -3.744675 & -2.352593 & -0.156218 \\
\hline $\mathrm{C}$ & -4.942265 & -1.590832 & -0.166592 \\
\hline $\mathrm{C}$ & -4.970264 & -0.200413 & 0.184701 \\
\hline $\mathrm{C}$ & -6.226784 & 0.445658 & 0.179721 \\
\hline
\end{tabular}




\begin{tabular}{|c|c|c|c|}
\hline $\mathrm{H}$ & -6.268567 & 1.496030 & 0.465269 \\
\hline $\mathrm{C}$ & -7.394519 & -0.242139 & -0.160939 \\
\hline $\mathrm{H}$ & -8.332970 & 0.320540 & -0.146782 \\
\hline $\mathrm{C}$ & -7.393091 & -1.605367 & -0.521582 \\
\hline $\mathrm{C}$ & -6.147404 & -2.248039 & -0.513706 \\
\hline $\mathrm{H}$ & -6.068103 & -3.308365 & -0.777158 \\
\hline $\mathrm{C}$ & -3.684755 & 1.710007 & 1.144371 \\
\hline $\mathrm{C}$ & -1.906566 & 3.345639 & 1.775007 \\
\hline $\mathrm{C}$ & -2.700916 & 4.268739 & 2.482113 \\
\hline $\mathrm{H}$ & -3.771965 & 4.085719 & 2.574274 \\
\hline $\mathrm{C}$ & -2.116089 & 5.409958 & 3.055595 \\
\hline $\mathrm{H}$ & -2.759713 & 6.107817 & 3.598741 \\
\hline $\mathrm{C}$ & -0.737828 & 5.647407 & 2.929352 \\
\hline $\mathrm{C}$ & 0.057535 & 4.725672 & 2.221227 \\
\hline $\mathrm{H}$ & 1.130097 & 4.917608 & 2.119297 \\
\hline $\mathrm{C}$ & -0.516030 & 3.590665 & 1.655842 \\
\hline $\mathrm{H}$ & 0.093749 & 2.863095 & 1.105504 \\
\hline $\mathrm{C}$ & -0.855772 & 7.684596 & 4.161118 \\
\hline $\mathrm{H}$ & -0.160105 & 8.471053 & 4.493631 \\
\hline $\mathrm{H}$ & -1.347927 & 7.236038 & 5.048180 \\
\hline $\mathrm{H}$ & -1.637010 & 8.138406 & 3.517924 \\
\hline $\mathrm{C}$ & 7.952833 & -3.733819 & 1.082507 \\
\hline $\mathrm{C}$ & -8.666586 & -2.384144 & -0.906800 \\
\hline $\mathrm{C}$ & 7.599660 & -4.321908 & 2.471289 \\
\hline $\mathrm{H}$ & 6.616651 & -4.822660 & 2.462684 \\
\hline $\mathrm{H}$ & 8.356436 & -5.066687 & 2.781171 \\
\hline $\mathrm{H}$ & 7.563813 & -3.527232 & 3.236753 \\
\hline $\mathrm{C}$ & 9.351230 & -3.089462 & 1.174660 \\
\hline $\mathrm{H}$ & 9.673945 & -2.672660 & 0.204430 \\
\hline $\mathrm{H}$ & 9.379159 & -2.278536 & 1.923556 \\
\hline $\mathrm{H}$ & 10.093056 & -3.850222 & 1.476489 \\
\hline $\mathrm{C}$ & 8.018017 & -4.882108 & 0.045013 \\
\hline $\mathrm{H}$ & 8.285310 & -4.494536 & -0.953521 \\
\hline $\mathrm{H}$ & 8.777807 & -5.629587 & 0.340738 \\
\hline $\mathrm{H}$ & 7.049762 & -5.402976 & -0.046319 \\
\hline $\mathrm{C}$ & -8.524207 & -2.927418 & -2.350381 \\
\hline $\mathrm{H}$ & -7.648593 & -3.591089 & -2.450209 \\
\hline $\mathrm{H}$ & -9.422206 & -3.505708 & -2.637832 \\
\hline $\mathrm{H}$ & -8.400987 & -2.100225 & -3.070989 \\
\hline $\mathrm{C}$ & -8.862645 & -3.572540 & 0.067219 \\
\hline $\mathrm{H}$ & -8.984000 & -3.214324 & 1.104376 \\
\hline $\mathrm{H}$ & -9.763555 & -4.153238 & -0.205887 \\
\hline $\mathrm{H}$ & -7.999910 & -4.259950 & 0.049288 \\
\hline $\mathrm{C}$ & -9.930015 & -1.501335 & -0.847534 \\
\hline $\mathrm{H}$ & -10.103080 & -1.102972 & 0.167635 \\
\hline $\mathrm{H}$ & -9.865748 & -0.648084 & -1.545427 \\
\hline $\mathrm{H}$ & -10.815542 & -2.098614 & -1.129048 \\
\hline $\mathrm{H}$ & 0.321084 & 0.169176 & 1.097263 \\
\hline
\end{tabular}




\begin{tabular}{|c|c|c|c|}
\hline $\mathrm{H}$ & 1.615051 & 1.512127 & -0.797855 \\
\hline $\mathrm{N}$ & -3.723678 & 0.134281 & 0.537094 \\
\hline $\mathrm{H}$ & -2.792255 & -0.287095 & 0.413435 \\
\hline $\mathrm{N}$ & -2.531776 & 1.967306 & 1.302288 \\
\hline $\mathrm{H}$ & -1.703405 & 1.449772 & 0.914218 \\
\hline $\mathrm{C}$ & 0.136249 & -3.246129 & 0.031945 \\
\hline $\mathrm{H}$ & 0.104758 & -2.146282 & 0.005253 \\
\hline $\mathrm{C}$ & 1.379313 & -3.912059 & 0.085466 \\
\hline $\mathrm{C}$ & 1.418870 & -5.330144 & 0.128695 \\
\hline $\mathrm{H}$ & 2.375244 & -5.856935 & 0.168435 \\
\hline $\mathrm{C}$ & 0.213514 & -6.036634 & 0.115198 \\
\hline $\mathrm{C}$ & -1.029002 & -5.399631 & 0.063585 \\
\hline $\mathrm{H}$ & -1.954814 & -5.979798 & 0.057915 \\
\hline $\mathrm{C}$ & -1.068013 & -3.981439 & 0.023452 \\
\hline $\mathrm{C}$ & 2.580722 & -3.151096 & 0.096721 \\
\hline $\mathrm{C}$ & 3.599443 & -2.460545 & 0.106843 \\
\hline $\mathrm{C}$ & 4.822264 & -1.739931 & 0.128821 \\
\hline $\mathrm{C}$ & 4.896624 & -0.350855 & -0.224468 \\
\hline $\mathrm{C}$ & 6.170508 & 0.259346 & -0.198332 \\
\hline $\mathrm{H}$ & 6.245776 & 1.308049 & -0.482929 \\
\hline $\mathrm{C}$ & 7.311803 & -0.462275 & 0.162309 \\
\hline $\mathrm{H}$ & 8.266340 & 0.072868 & 0.163450 \\
\hline $\mathrm{C}$ & 7.265421 & -1.824814 & 0.522192 \\
\hline $\mathrm{C}$ & 6.001881 & -2.431645 & 0.494542 \\
\hline $\mathrm{H}$ & 5.888034 & -3.488900 & 0.757746 \\
\hline $\mathrm{C}$ & 3.662791 & 1.593900 & -1.190893 \\
\hline $\mathrm{C}$ & 1.948091 & 3.252760 & -1.898630 \\
\hline $\mathrm{C}$ & 2.798484 & 4.134991 & -2.595584 \\
\hline $\mathrm{H}$ & 3.861597 & 3.903774 & -2.661484 \\
\hline $\mathrm{C}$ & 2.284456 & 5.298757 & -3.190248 \\
\hline $\mathrm{H}$ & 2.975118 & 5.959080 & -3.722523 \\
\hline $\mathrm{C}$ & 0.917355 & 5.604544 & -3.099301 \\
\hline $\mathrm{C}$ & 0.064553 & 4.727889 & -2.402460 \\
\hline $\mathrm{H}$ & -0.999498 & 4.972215 & -2.326960 \\
\hline $\mathrm{C}$ & 0.570188 & 3.571410 & -1.813872 \\
\hline $\mathrm{H}$ & -0.100578 & 2.896506 & -1.271022 \\
\hline $\mathrm{C}$ & 1.161615 & 7.622316 & -4.343694 \\
\hline $\mathrm{H}$ & 0.512844 & 8.438869 & -4.697784 \\
\hline $\mathrm{H}$ & 1.650515 & 7.142414 & -5.215983 \\
\hline $\mathrm{H}$ & 1.949817 & 8.043058 & -3.686700 \\
\hline $\mathrm{C}$ & -2.310147 & -3.290542 & -0.025674 \\
\hline $\mathrm{C}$ & -3.366117 & -2.660307 & -0.073283 \\
\hline $\mathrm{C}$ & -4.634047 & -2.026559 & -0.152926 \\
\hline $\mathrm{C}$ & -4.822930 & -0.640462 & 0.167282 \\
\hline $\mathrm{C}$ & -6.134502 & -0.124460 & 0.068011 \\
\hline $\mathrm{H}$ & -6.297157 & 0.921847 & 0.322672 \\
\hline $\mathrm{C}$ & -7.204045 & -0.933111 & -0.326459 \\
\hline $\mathrm{H}$ & -8.192568 & -0.467142 & -0.383890 \\
\hline
\end{tabular}




$\begin{array}{lccc}\mathrm{C} & -7.045298 & -2.296078 & -0.650020 \\ \mathrm{C} & -5.744264 & -2.808786 & -0.552430 \\ \mathrm{H} & -5.542870 & -3.859720 & -0.786737 \\ \mathrm{C} & -3.781869 & 1.411005 & 1.138906 \\ \mathrm{C} & -2.238649 & 3.204769 & 1.907516 \\ \mathrm{C} & -3.193071 & 4.041029 & 2.522024 \\ \mathrm{H} & -4.239101 & 3.735110 & 2.528122 \\ \mathrm{C} & -2.802922 & 5.254828 & 3.110821 \\ \mathrm{H} & -3.572151 & 5.876984 & 3.577264 \\ \mathrm{C} & -1.458083 & 5.657211 & 3.097288 \\ \mathrm{C} & -0.501738 & 4.827104 & 2.482835 \\ \mathrm{H} & 0.544737 & 5.147080 & 2.466366 \\ \mathrm{C} & -0.884612 & 3.622030 & 1.899121 \\ \mathrm{H} & -0.134831 & 2.982508 & 1.419327 \\ \mathrm{C} & -1.926491 & 7.685650 & 4.257403 \\ \mathrm{H} & -1.362100 & 8.556865 & 4.625475 \\ \mathrm{H} & -2.437464 & 7.196445 & 5.111687 \\ \mathrm{H} & -2.696688 & 8.029133 & 3.537237 \\ \mathrm{C} & 8.509629 & -2.639476 & 0.928358 \\ \mathrm{C} & -8.208309 & -3.206852 & -1.090841 \\ \mathrm{C} & 8.328345 & -3.177552 & 2.369532 \\ \mathrm{H} & 7.432644 & -3.815920 & 2.455282 \\ \mathrm{H} & 9.204642 & -3.781095 & 2.671691 \\ \mathrm{H} & 0.505148 & 0.262614 & 1.506032 \\ \mathrm{H} & 8.217190 & -2.346659 & 3.087856 \\ \mathrm{C} & 9.798545 & -1.793093 & 0.889767 \\ \mathrm{H} & 9.999454 & -1.400340 & -0.122453 \\ \mathrm{H} & 9.747135 & -0.937987 & 1.586446 \\ \mathrm{H} & 10.661962 & -2.415206 & 1.185810 \\ \mathrm{C} & 8.687774 & -3.833594 & -0.042094 \\ \mathrm{H} & 8.836557 & -3.479636 & -1.077138 \\ \mathrm{H} & 9.567048 & -4.439642 & 0.246025 \\ \mathrm{H} & 7.805550 & -4.496050 & -0.038462 \\ \mathrm{C} & -7.922708 & -3.762536 & -2.508272 \\ \mathrm{H} & -6.981870 & -4.337963 & -2.537487 \\ \mathrm{H} & -8.739937 & -4.432873 & -2.834227 \\ \mathrm{H} & -7.835946 & -2.941990 & -3.241650 \\ \mathrm{H} & -8.346206 & -4.388736 & -0.099124 \\ \mathrm{H} & -8.568359 & -4.023501 & 0.918730 \\ \mathrm{H} & -7.4195347 & -5.063408 & -0.411009 \\ \mathrm{H} & -9.534171 & -4.985141 & -0.045930 \\ \mathrm{H} & -2.454429 & -1.131629 \\ \mathrm{H} & -2.054558 & -0.139797 \\ \mathrm{H} & -297368 & 0.219453\end{array}$


Statistical Thermodynamic Analysis

Temperature $=298.150$ Kelvin $\quad$ Pressure $=1.00000$ Atm

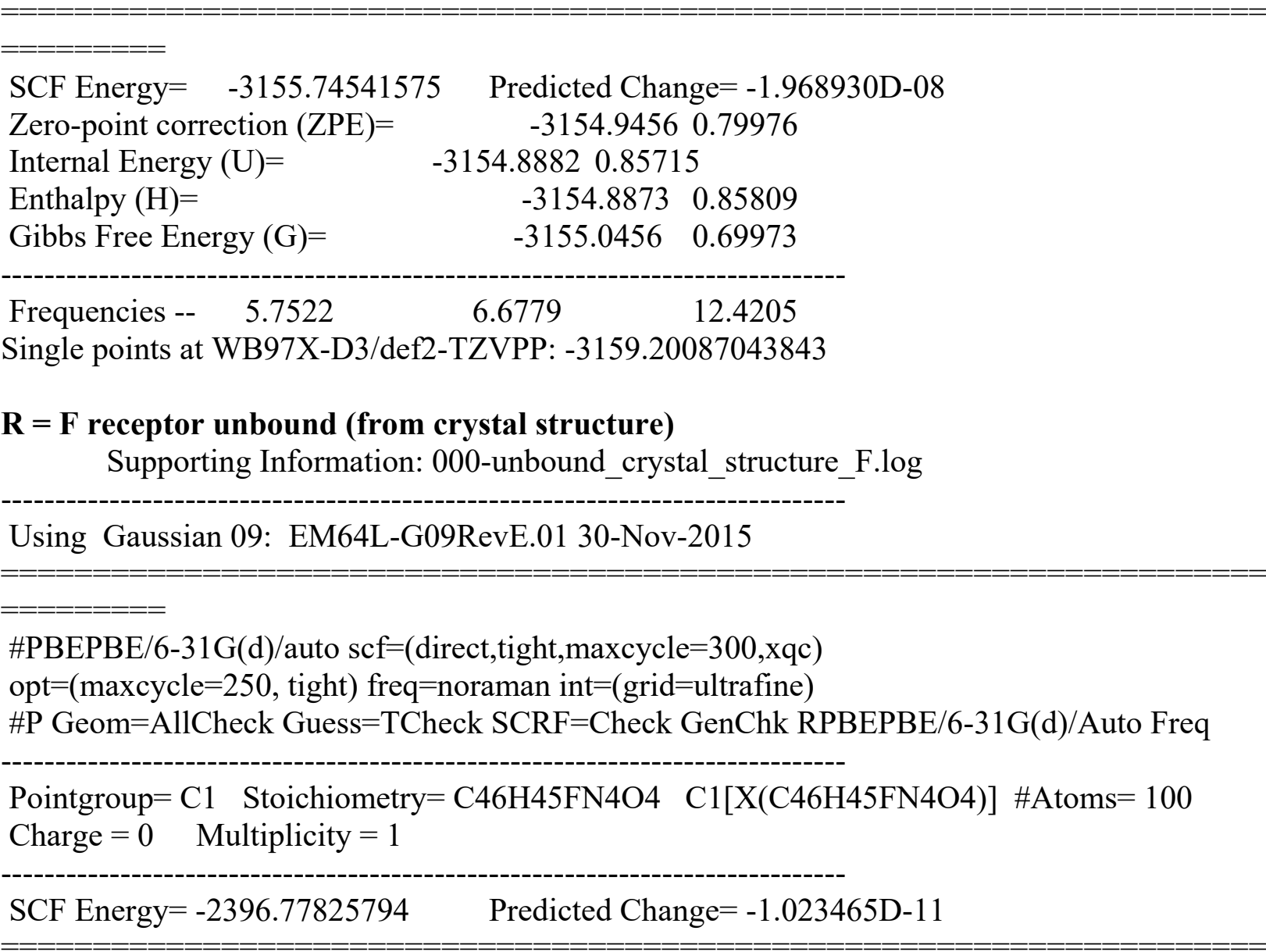

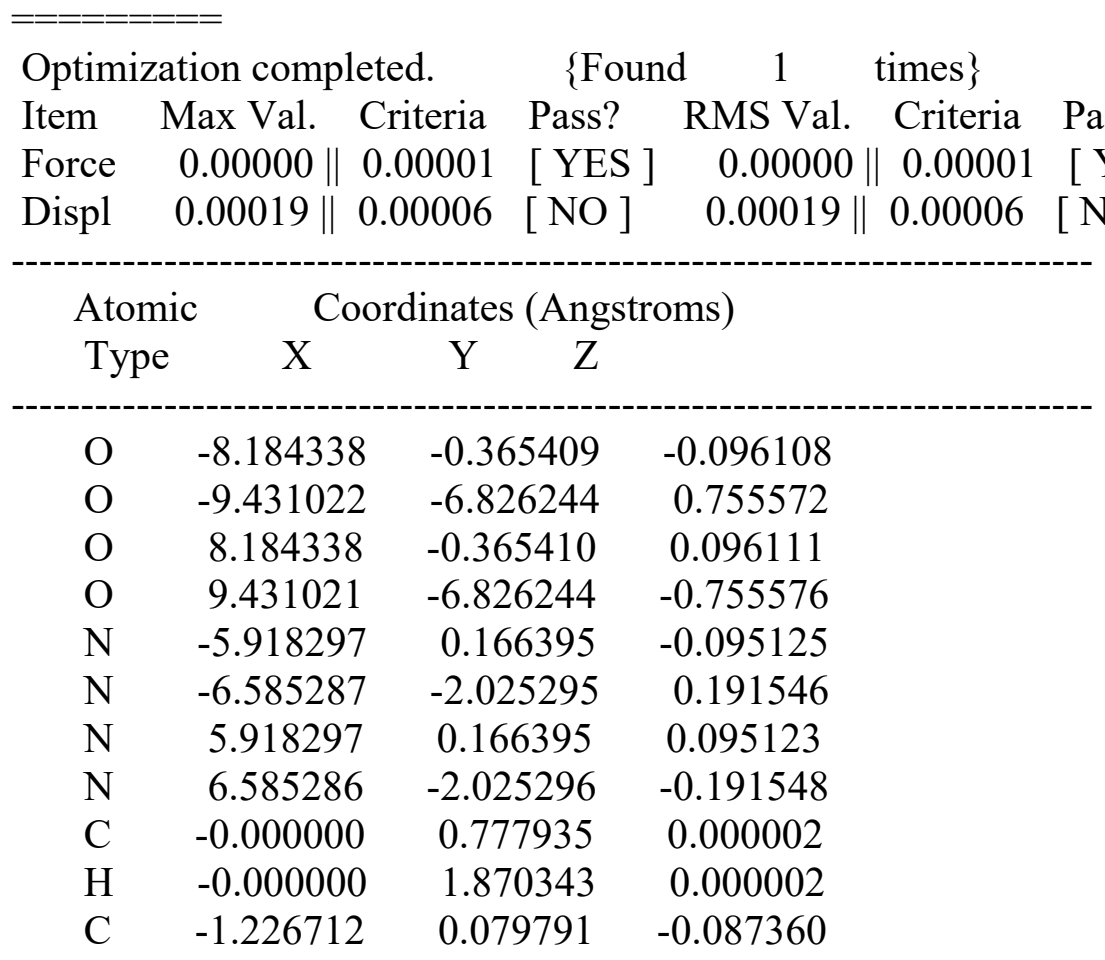




\begin{tabular}{|c|c|c|c|}
\hline $\mathrm{C}$ & -1.220601 & -1.337692 & -0.088391 \\
\hline $\mathrm{H}$ & -2.148495 & -1.909942 & -0.161995 \\
\hline $\mathrm{C}$ & -0.000000 & -2.005434 & 0.000004 \\
\hline $\mathrm{C}$ & 1.220600 & -1.337692 & 0.088399 \\
\hline $\mathrm{H}$ & 2.148494 & -1.909942 & 0.162004 \\
\hline $\mathrm{C}$ & 1.226712 & 0.079791 & 0.087365 \\
\hline $\mathrm{C}$ & -2.449415 & 0.800754 & -0.174926 \\
\hline $\mathrm{C}$ & -3.472644 & 1.483546 & -0.253487 \\
\hline $\mathrm{C}$ & -4.680766 & 2.224266 & -0.343517 \\
\hline $\mathrm{C}$ & -5.945181 & 1.552690 & -0.269347 \\
\hline $\mathrm{C}$ & -7.118180 & 2.320687 & -0.369125 \\
\hline $\mathrm{H}$ & -8.082405 & 1.815159 & -0.316540 \\
\hline $\mathrm{C}$ & -7.039655 & 3.708808 & -0.534384 \\
\hline $\mathrm{H}$ & -7.981090 & 4.260479 & -0.606604 \\
\hline $\mathrm{C}$ & -5.813264 & 4.400922 & -0.608199 \\
\hline $\mathrm{C}$ & -4.646688 & 3.626863 & -0.508895 \\
\hline $\mathrm{H}$ & -3.660921 & 4.100195 & -0.560378 \\
\hline $\mathrm{C}$ & -7.003959 & -0.713811 & -0.006988 \\
\hline $\mathrm{C}$ & -7.382441 & -3.184999 & 0.323445 \\
\hline $\mathrm{C}$ & -6.712600 & -4.409086 & 0.544510 \\
\hline $\mathrm{H}$ & -5.617109 & -4.427895 & 0.608737 \\
\hline $\mathrm{C}$ & -7.420593 & -5.600087 & 0.683557 \\
\hline $\mathrm{H}$ & -6.899958 & -6.546641 & 0.855084 \\
\hline $\mathrm{C}$ & -8.827160 & -5.601805 & 0.605000 \\
\hline $\mathrm{C}$ & -9.499665 & -4.387205 & 0.384836 \\
\hline $\mathrm{H}$ & -10.590061 & -4.353757 & 0.318976 \\
\hline $\mathrm{C}$ & -8.787085 & -3.186875 & 0.244682 \\
\hline $\mathrm{H}$ & -9.313277 & -2.247266 & 0.076052 \\
\hline $\mathrm{C}$ & -10.852899 & -6.859127 & 0.683225 \\
\hline $\mathrm{H}$ & -11.136081 & -7.912354 & 0.826817 \\
\hline $\mathrm{H}$ & -11.221063 & -6.512621 & -0.302069 \\
\hline $\mathrm{H}$ & -11.317651 & -6.243847 & 1.478103 \\
\hline $\mathrm{C}$ & 2.449415 & 0.800754 & 0.174929 \\
\hline $\mathrm{C}$ & 3.472644 & 1.483546 & 0.253490 \\
\hline $\mathrm{C}$ & 4.680766 & 2.224265 & 0.343518 \\
\hline $\mathrm{C}$ & 5.945181 & 1.552690 & 0.269346 \\
\hline $\mathrm{C}$ & 7.118180 & 2.320687 & 0.369122 \\
\hline $\mathrm{H}$ & 8.082405 & 1.815158 & 0.316537 \\
\hline $\mathrm{C}$ & 7.039655 & 3.708807 & 0.534381 \\
\hline $\mathrm{H}$ & 7.981091 & 4.260478 & 0.606600 \\
\hline $\mathrm{C}$ & 5.813265 & 4.400922 & 0.608198 \\
\hline $\mathrm{C}$ & 4.646689 & 3.626862 & 0.508895 \\
\hline $\mathrm{H}$ & 3.660922 & 4.100195 & 0.560380 \\
\hline $\mathrm{C}$ & 7.003959 & -0.713812 & 0.006988 \\
\hline $\mathrm{C}$ & 7.382440 & -3.185000 & -0.323446 \\
\hline $\mathrm{C}$ & 8.787084 & -3.186876 & -0.244678 \\
\hline $\mathrm{H}$ & 9.313275 & -2.247267 & -0.076045 \\
\hline $\mathrm{C}$ & 9.499664 & -4.387206 & -0.384833 \\
\hline
\end{tabular}




\begin{tabular}{lccc}
$\mathrm{H}$ & 10.590060 & -4.353759 & -0.318969 \\
$\mathrm{C}$ & 8.827159 & -5.601806 & -0.605002 \\
$\mathrm{C}$ & 7.420593 & -5.600087 & -0.683565 \\
$\mathrm{H}$ & 6.899959 & -6.546640 & -0.855096 \\
$\mathrm{C}$ & 6.712600 & -4.409086 & -0.544517 \\
$\mathrm{H}$ & 5.617109 & -4.427894 & -0.608748 \\
$\mathrm{C}$ & 10.852898 & -6.859128 & -0.683224 \\
$\mathrm{H}$ & 11.136080 & -7.912355 & -0.826818 \\
$\mathrm{H}$ & 11.221059 & -6.512624 & 0.302072 \\
$\mathrm{H}$ & 11.317654 & -6.243847 & -1.478100 \\
$\mathrm{H}$ & -4.969706 & -0.213524 & -0.042096 \\
$\mathrm{H}$ & -5.584501 & -2.194298 & 0.283776 \\
$\mathrm{H}$ & 4.969706 & -0.213524 & 0.042093 \\
$\mathrm{H}$ & 5.584500 & -2.194298 & -0.283783 \\
$\mathrm{C}$ & 5.713983 & 5.928745 & 0.790039 \\
$\mathrm{C}$ & 7.101823 & 6.596091 & 0.876879 \\
$\mathrm{H}$ & 7.684696 & 6.222290 & 1.736885 \\
$\mathrm{H}$ & 7.694309 & 6.433473 & -0.040509 \\
$\mathrm{H}$ & 6.980764 & 7.685587 & 1.005756 \\
$\mathrm{C}$ & 4.942199 & 6.242371 & 2.096104 \\
$\mathrm{H}$ & 3.923567 & 5.818309 & 2.082648 \\
$\mathrm{H}$ & 5.467588 & 5.829232 & 2.974529 \\
$\mathrm{H}$ & 4.849246 & 7.334929 & 2.233158 \\
$\mathrm{C}$ & 4.955247 & 6.542113 & -0.413248 \\
$\mathrm{H}$ & 5.490170 & 6.347139 & -1.358882 \\
$\mathrm{H}$ & 3.937072 & 6.127995 & -0.510546 \\
$\mathrm{H}$ & 4.862191 & 7.636291 & -0.289838 \\
$\mathrm{C}$ & -5.713982 & 5.928745 & -0.790041 \\
$\mathrm{C}$ & -4.955246 & 6.542113 & 0.413247 \\
$\mathrm{H}$ & -3.937072 & 6.127995 & 0.510546 \\
$\mathrm{H}$ & -4.862190 & 7.636291 & 0.289837 \\
$\mathrm{H}$ & -5.490170 & 6.347139 & 1.358881 \\
$\mathrm{C}$ & -7.101822 & 6.596091 & -0.876882 \\
$\mathrm{H}$ & -7.684694 & 6.222291 & -1.736888 \\
$\mathrm{H}$ & -7.694309 & 6.433474 & 0.040506 \\
$\mathrm{H}$ & -6.980763 & 7.685587 & -1.005758 \\
$\mathrm{C}$ & -4.942197 & 6.242371 & -2.096105 \\
$\mathrm{H}$ & -3.923565 & 5.818309 & -2.082648 \\
$\mathrm{H}$ & -5.467585 & 5.829232 & -2.974530 \\
------------------------------------------------------------------ \\
\hline
\end{tabular}

Statistical Thermodynamic Analysis

Temperature $=298.150$ Kelvin Pressure $=1.00000$ Atm

\begin{tabular}{|c|c|c|}
\hline SCF Energy= & -2396.77825794 & Predicted Change $=-1.023465 \mathrm{D}-11$ \\
\hline Zero-point corn & etion $(\mathrm{ZPE})=$ & -2395.98510 .79310 \\
\hline
\end{tabular}




\begin{tabular}{|c|c|c|}
\hline Internal Energy $(U)=$ & 2395.92980 .8484 & \\
\hline Enthalpy $(\mathrm{H})=$ & -2395.9288 & 0.84936 \\
\hline Gibbs Free Energy $(\mathrm{G})=$ & -2396.0889 & 0.68931 \\
\hline Frequencies -- $\quad 1.3718$ & 3.3166 & 5.7202 \\
\hline Single points at WB97X-D & TZVPP: -2399.91 & 74469530 \\
\hline
\end{tabular}

Using Gaussian 09: EM64L-G09RevE.01 30-Nov-2015

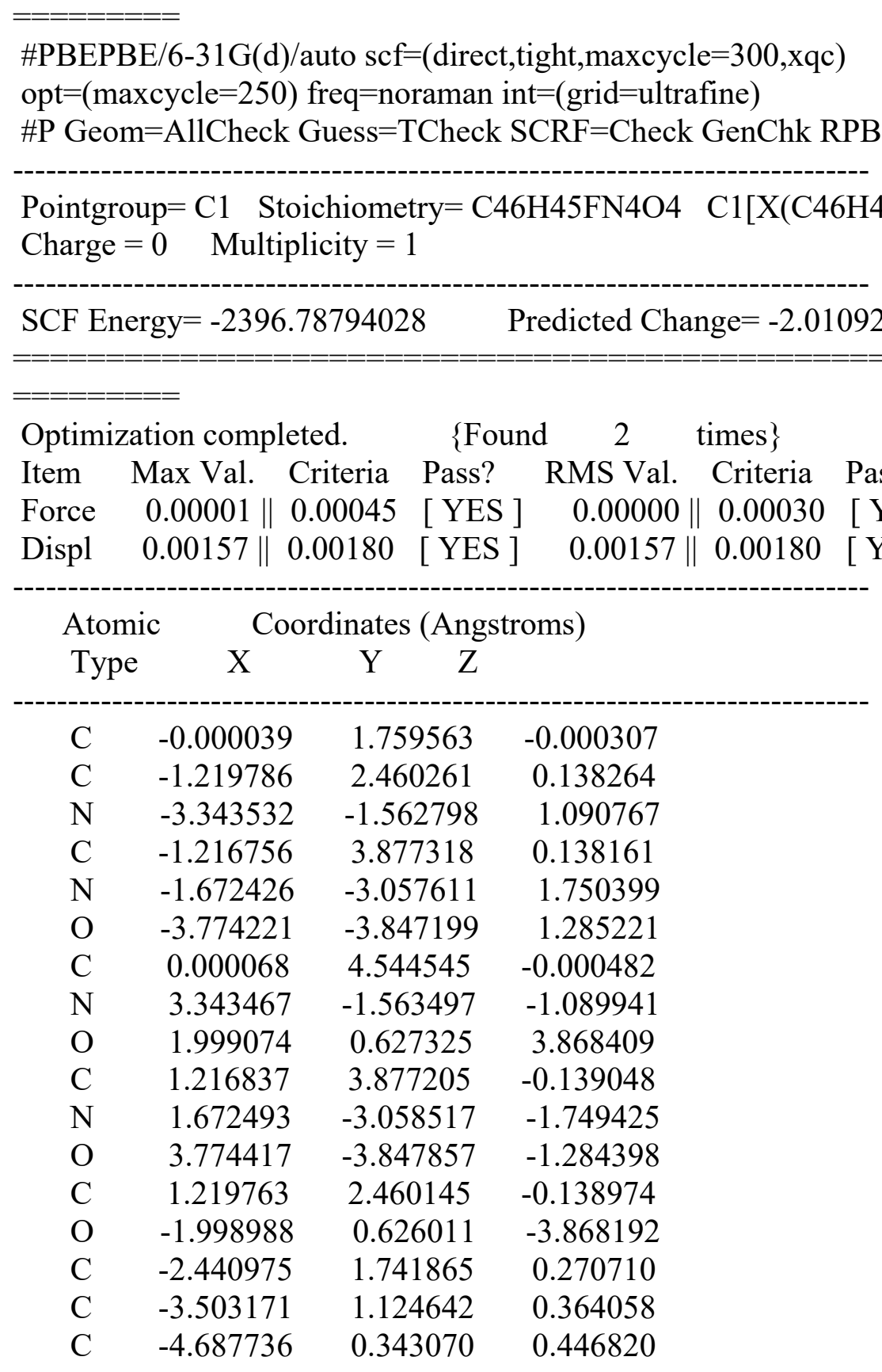




\begin{tabular}{|c|c|c|c|}
\hline C & -4.607378 & -1.047111 & 0.786998 \\
\hline $\mathrm{C}$ & -5.794540 & -1.799738 & 0.814322 \\
\hline 0 & -7.023319 & -1.192047 & 0.529026 \\
\hline $\mathrm{C}$ & -7.139494 & 0.175375 & 0.205053 \\
\hline $\mathrm{C}$ & -5.948406 & 0.918072 & 0.170321 \\
\hline $\mathrm{C}$ & -8.485735 & 0.858958 & -0.107678 \\
\hline $\mathrm{C}$ & -8.448136 & 1.437986 & -1.544140 \\
\hline $\mathrm{C}$ & -8.725488 & 2.009502 & 0.901522 \\
\hline $\mathrm{C}$ & -9.672506 & -0.121605 & -0.01333 \\
\hline $\mathrm{C}$ & -3.007659 & -2.884145 & 1.38051 \\
\hline $\mathrm{C}$ & -0.744319 & -2.097735 & 2.252032 \\
\hline $\mathrm{C}$ & -1.125541 & -1.116382 & 3.194900 \\
\hline $\mathrm{C}$ & -0.188450 & -0.212818 & 3.693577 \\
\hline $\mathrm{C}$ & 1.162609 & -0.287432 & 3.294059 \\
\hline $\mathrm{C}$ & 1.554587 & -1.272015 & 2.365542 \\
\hline $\mathrm{C}$ & 0.599 & -2.1 & 1.84316 \\
\hline $\mathrm{C}$ & 3.380815 & 0.573735 & 3.506371 \\
\hline $\mathrm{C}$ & 2.440892 & 1.7 & -0.27144 \\
\hline $\mathrm{C}$ & 3.503058 & 1.12 & -0.364792 \\
\hline $\mathrm{C}$ & 4.687621 & 0.34 & -0.44705 \\
\hline $\mathrm{C}$ & 4.607317 & -1.047589 & -0.78654 \\
\hline $\mathrm{C}$ & 5.794520 & -1.80 & -0.813539 \\
\hline $\mathrm{C}$ & 7.023280 & -1.192269 & -0.528602 \\
\hline $\mathrm{C}$ & 7.139402 & 0.175322 & -0.205313 \\
\hline $\mathrm{C}$ & 5.948 & 0.9 & -0.17088 \\
\hline $\mathrm{C}$ & 8.485622 & 0.85 & 0.107010 \\
\hline $\mathrm{C}$ & 9.672406 & -0.1 & 0. \\
\hline $\mathrm{C}$ & 8.725407 & 2.00 & -0.902957 \\
\hline $\mathrm{C}$ & 8.447959 & 1.439127 & 1.543079 \\
\hline $\mathrm{C}$ & 3.007747 & -2.88 & -1.379690 \\
\hline $\mathrm{C}$ & 0.744380 & & -2.251280 \\
\hline $\mathrm{C}$ & 1.125632 & -1.117515 & -3.194271 \\
\hline $\mathrm{C}$ & 0.188544 & -0.214051 & -3.693130 \\
\hline $\mathrm{C}$ & -1.162535 & -0.288643 & -3.29366 \\
\hline $\mathrm{C}$ & -1.554538 & -1.273091 & -2.36501 \\
\hline $\mathrm{C}$ & -0.599491 & -2.156932 & -1.84246 \\
\hline $\mathrm{C}$ & -3.380750 & 0.572437 & -3.50623 \\
\hline $\mathrm{H}$ & -0.000081 & 0.666511 & -0.00025 \\
\hline $\mathrm{H}$ & -2.144292 & 4.444320 & 0.24642 \\
\hline $\mathrm{H}$ & -2.583531 & -0.876227 & 1.08502 \\
\hline $\mathrm{H}$ & -1.452964 & -4.045549 & 1.87834 \\
\hline $\mathrm{H}$ & 2.144412 & 4.444126 & -0.24740 \\
\hline $\mathrm{H}$ & 2.583431 & -0.876965 & -1.08446 \\
\hline $\mathrm{H}$ & 1.453221 & -4.046478 & -1.87755 \\
\hline $\mathrm{H}$ & -5.734660 & -2.861667 & 1.05507 \\
\hline $\mathrm{H}$ & -7.917125 & -1.821042 & 0.56610 \\
\hline $\mathrm{H}$ & -5.966900 & 1.983639 & -0.08082 \\
\hline $\mathrm{H}$ & -9.401636 & 1.944993 & -1.77836 \\
\hline
\end{tabular}




$\begin{array}{cccc}\text { H } & -8.293262 & 0.637718 & -2.288645 \\ \mathrm{H} & -7.637222 & 2.176243 & -1.667526 \\ \mathrm{H} & -8.772365 & 1.625026 & 1.935112 \\ \mathrm{H} & -9.680317 & 2.520034 & 0.680729 \\ \mathrm{H} & -7.923244 & 2.765927 & 0.859543 \\ \mathrm{H} & -9.771160 & -0.550642 & 0.999175 \\ \mathrm{H} & -9.577649 & -0.952698 & -0.734013 \\ \mathrm{H} & -10.611800 & 0.411065 & -0.241929 \\ \mathrm{H} & -2.160637 & -1.074679 & 3.547717 \\ \mathrm{H} & -0.476726 & 0.550698 & 4.421989 \\ \mathrm{H} & 2.590698 & -1.349551 & 2.027912 \\ \mathrm{H} & 0.905463 & -2.902294 & 1.102042 \\ \mathrm{H} & 3.869527 & 1.365873 & 4.092211 \\ \mathrm{H} & 3.524828 & 0.769953 & 2.428031 \\ \mathrm{H} & 3.829805 & -0.403758 & 3.766629 \\ \mathrm{H} & 5.734688 & -2.862215 & -1.053762 \\ \mathrm{H} & 7.917117 & -1.821236 & -0.565420 \\ \mathrm{H} & 5.966728 & 1.983662 & 0.079715 \\ \mathrm{H} & 10.611702 & 0.411381 & 0.241512 \\ \mathrm{H} & 9.771019 & -0.551315 & -0.998798 \\ \mathrm{H} & 9.577597 & -0.952012 & 0.734715 \\ \mathrm{H} & 9.680247 & 2.519653 & -0.682500 \\ \mathrm{H} & 7.923182 & 2.765468 & -0.861492 \\ \mathrm{H} & 8.772284 & 1.623826 & -1.936288 \\ \mathrm{H} & 9.401422 & 1.946355 & 1.776971 \\ \mathrm{H} & 8.293123 & 0.639354 & 2.288122 \\ \mathrm{H} & 7.636994 & 2.177417 & 1.665950 \\ \mathrm{H} & 2.160754 & -1.075828 & -3.547016 \\ \mathrm{H} & 0.476833 & 0.549365 & -4.421641 \\ \mathrm{H} & -2.590666 & -1.350608 & -2.027434 \\ \mathrm{H} & -0.905429 & -2.903176 & -1.101256 \\ \mathrm{H} & -3.829686 & -0.405122 & -3.766334 \\ \mathrm{H} & -3.869455 & 1.364447 & -4.092253 \\ \mathrm{H} & -3.524842 & 0.768852 & -2.427940 \\ \mathrm{~F} & 0.000122 & 5.898651 & -0.000570 \\ ------------------------------------------------------------------\end{array}$

Statistical Thermodynamic Analysis

Temperature $=298.150$ Kelvin Pressure $=1.00000$ Atm

\footnotetext{
$======$

SCF Energy $=\quad-2396.78794028 \quad$ Predicted Change $=-2.010920 \mathrm{D}-08$

Zero-point correction $(\mathrm{ZPE})=\quad-2395.99390 .79396$

Internal Energy $(\mathrm{U})=\quad-2395.93940 .84851$

Enthalpy $(\mathrm{H})=\quad-2395.9384 \quad 0.84946$

Gibbs Free Energy $(\mathrm{G})=\quad-2396.0888 \quad 0.69913$

Frequencies -- $7.6855 \quad 10.2253 \quad 16.9228$

Single points at WB97X-D3/def2-TZVPP: -2399.94794805203
} 
$\mathrm{R}=\mathrm{F}$ receptor bound with $\mathrm{Br}-($ withTBA $)$

Supporting Information: 010-bound_TBA_F_Br.log

Using Gaussian 09: EM64L-G09RevE.01 30-Nov-2015

$=======$
$\#$ PBEPBE $/ 6-31 \mathrm{G}(\mathrm{d}) /$ auto $\mathrm{scf}=($ direct,tight, $\operatorname{maxcycle}=300, \mathrm{xqc})$

$\mathrm{opt}=($ maxcycle $=250)$ freq $=$ noraman $\mathrm{int}=($ grid $=$ ultrafine $)$

\#P Geom=AllCheck Guess=TCheck SCRF=Check GenChk RPBEPBE/6-31G(d)/Auto Freq

Pointgroup=C1 Stoichiometry= C62H81BrFN5O4 C1[X(C62H81BrFN5O4)] \#Atoms=154 Charge $=0 \quad$ Multiplicity $=1$

\section{SCF Energy $=-5653.32330045 \quad$ Predicted Change $=-5.880674 \mathrm{D}-08$}

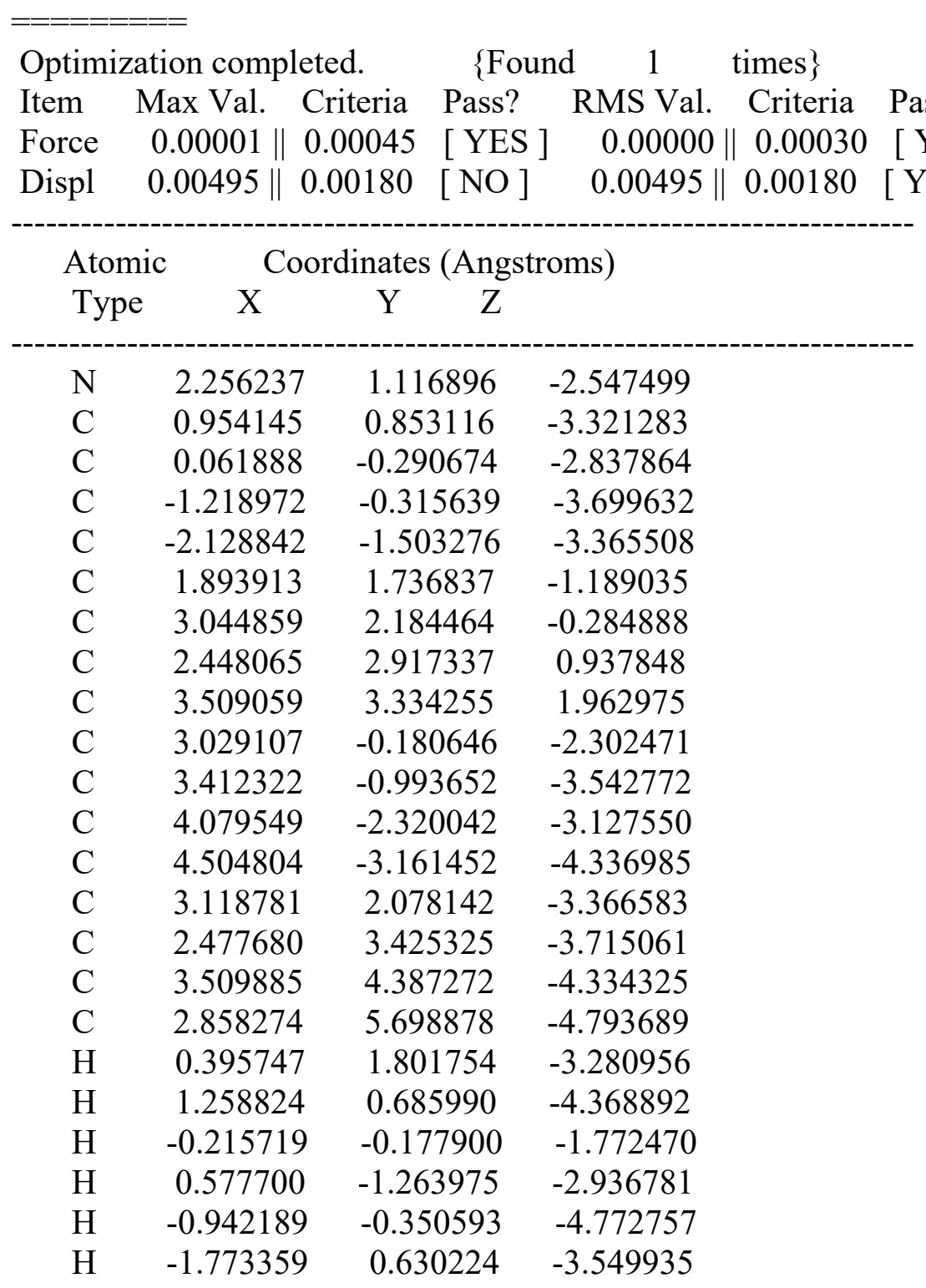




\begin{tabular}{|c|c|c|c|}
\hline $\mathrm{H}$ & -3.021939 & -1.505368 & -4.012655 \\
\hline $\mathrm{H}$ & -1.605198 & -2.465252 & -3.507813 \\
\hline $\mathrm{H}$ & -2.476326 & -1.463674 & -2.319989 \\
\hline $\mathrm{H}$ & 1.240225 & 2.590420 & -1.423202 \\
\hline $\mathrm{H}$ & 1.265340 & 1.000015 & -0.657676 \\
\hline $\mathrm{H}$ & 3.635807 & 1.321219 & 0.071602 \\
\hline $\mathrm{H}$ & 3.740647 & 2.871458 & -0.803790 \\
\hline $\mathrm{H}$ & 1.693694 & 2.263014 & 1.411613 \\
\hline $\mathrm{H}$ & 1.903367 & 3.813931 & 0.583332 \\
\hline $\mathrm{H}$ & 3.045017 & 3.895335 & 2.791107 \\
\hline $\mathrm{H}$ & 4.009393 & 2.458156 & 2.408744 \\
\hline $\mathrm{H}$ & 4.279885 & 3.980936 & 1.504992 \\
\hline $\mathrm{H}$ & 3.931872 & 0.107679 & -1.740973 \\
\hline $\mathrm{H}$ & 2.398705 & -0.779152 & -1.625073 \\
\hline $\mathrm{H}$ & 2.521193 & -1.219629 & -4.156663 \\
\hline $\mathrm{H}$ & 4.114064 & -0.431262 & -4.186261 \\
\hline $\mathrm{H}$ & 3.379816 & -2.895724 & -2.493175 \\
\hline $\mathrm{H}$ & 4.958924 & -2.105493 & -2.491319 \\
\hline $\mathrm{H}$ & 4.984085 & -4.100840 & -4.013286 \\
\hline $\mathrm{H}$ & 3.637783 & -3.429330 & -4.966599 \\
\hline $\mathrm{H}$ & 5.227661 & -2.619205 & -4.972590 \\
\hline $\mathrm{H}$ & 3.385849 & 1.539901 & -4.289540 \\
\hline $\mathrm{H}$ & 4.043855 & 2.223204 & -2.786016 \\
\hline $\mathrm{H}$ & 1.647667 & 3.281614 & -4.430001 \\
\hline $\mathrm{H}$ & 2.041604 & 3.907689 & -2.822706 \\
\hline $\mathrm{H}$ & 4.014601 & 3.899967 & -5.191185 \\
\hline $\mathrm{H}$ & 4.299871 & 4.600910 & -3.588996 \\
\hline $\mathrm{H}$ & 3.616782 & 6.415462 & -5.150470 \\
\hline $\mathrm{H}$ & 2.150239 & 5.518895 & -5.622208 \\
\hline $\mathrm{H}$ & 2.292507 & 6.173749 & -3.972746 \\
\hline $\mathrm{O}$ & -0.266677 & 6.649616 & -2.819039 \\
\hline $\mathrm{O}$ & 3.680260 & 0.344301 & 4.004871 \\
\hline $\mathrm{N}$ & -2.750529 & 2.014351 & -0.859462 \\
\hline $\mathrm{N}$ & 2.825761 & -0.788061 & 2.153116 \\
\hline $\mathrm{C}$ & -0.860938 & -3.469374 & 0.044166 \\
\hline $\mathrm{C}$ & -1.010768 & -6.148610 & -0.694976 \\
\hline $\mathrm{C}$ & 0.246914 & -5.593869 & -0.457025 \\
\hline $\mathrm{C}$ & 0.321507 & -4.230571 & -0.075756 \\
\hline $\mathrm{C}$ & -2.225086 & 3.204710 & -1.388885 \\
\hline $\mathrm{C}$ & -2.768739 & 3.892081 & -2.493086 \\
\hline $\mathrm{C}$ & -2.143681 & 5.047220 & -2.990081 \\
\hline $\mathrm{C}$ & -0.966771 & 5.537312 & -2.397737 \\
\hline $\mathrm{C}$ & -0.432391 & 4.864620 & -1.280798 \\
\hline $\mathrm{C}$ & -1.053583 & 3.718373 & -0.782762 \\
\hline $\mathrm{C}$ & -0.840099 & 7.404509 & -3.882174 \\
\hline $\mathrm{C}$ & 1.576707 & -3.619182 & 0.203750 \\
\hline $\mathrm{C}$ & 2.652178 & -3.090979 & 0.490400 \\
\hline $\mathrm{C}$ & 3.910258 & -2.527543 & 0.834333 \\
\hline
\end{tabular}




\begin{tabular}{|c|c|c|c|}
\hline $\mathrm{C}$ & 4.001688 & -1.388124 & 1.694915 \\
\hline $\mathrm{C}$ & 5.292078 & -0.909224 & 2.018920 \\
\hline $\mathrm{C}$ & 6.432464 & -1.532257 & 1.511286 \\
\hline $\mathrm{C}$ & 6.372865 & -2.657753 & 0.656568 \\
\hline $\mathrm{C}$ & 5.095335 & -3.130405 & 0.337554 \\
\hline $\mathrm{C}$ & 8.458934 & -2.269114 & -0.702716 \\
\hline $\mathrm{C}$ & 7.385681 & -4.532065 & -0.766285 \\
\hline $\mathrm{C}$ & 2.708984 & 0.020871 & 3.307552 \\
\hline $\mathrm{C}$ & -4.044136 & 1.537208 & -1.017058 \\
\hline $\mathrm{C}$ & -2.121178 & -4.053937 & -0.211360 \\
\hline $\mathrm{C}$ & -2.195234 & -5.419868 & -0.583061 \\
\hline $\mathrm{C}$ & 7.666007 & -3.308099 & 0.129096 \\
\hline $\mathrm{N}$ & 1.404441 & 0.397175 & 3.538797 \\
\hline $\mathrm{N}$ & -4.231851 & 0.311127 & -0.358710 \\
\hline $\mathrm{O}$ & -4.925719 & 2.115213 & -1.662107 \\
\hline $\mathrm{C}$ & -3.294606 & -3.256118 & -0.114049 \\
\hline $\mathrm{C}$ & 8.533156 & -3.772943 & 1.325841 \\
\hline $\mathrm{C}$ & 0.926852 & 1.264684 & 4.546484 \\
\hline $\mathrm{C}$ & -5.461568 & -0.350270 & -0.231469 \\
\hline $\mathrm{C}$ & -4.290556 & -2.534945 & -0.056443 \\
\hline $\mathrm{C}$ & -0.408285 & 1.715092 & 4.416600 \\
\hline $\mathrm{C}$ & 1.683139 & 1.680914 & 5.657160 \\
\hline $\mathrm{C}$ & -5.482026 & -1.763097 & -0.030268 \\
\hline $\mathrm{C}$ & -6.694819 & 0.333681 & -0.264592 \\
\hline $\mathrm{C}$ & -0.969912 & 2.554176 & 5.375075 \\
\hline $\mathrm{C}$ & 1.114175 & 2.530440 & 6.619315 \\
\hline $\mathrm{C}$ & -6.722960 & -2.426825 & 0.154686 \\
\hline $\mathrm{C}$ & -7.895118 & -0.355326 & -0.093292 \\
\hline $\mathrm{C}$ & -0.213767 & 2.971078 & 6.488708 \\
\hline $\mathrm{C}$ & -7.946917 & -1.751192 & 0.128258 \\
\hline $\mathrm{O}$ & -0.863615 & 3.800000 & 7.375325 \\
\hline $\mathrm{C}$ & -9.300998 & -2.459199 & 0.324308 \\
\hline $\mathrm{C}$ & -0.128680 & 4.225374 & 8.515855 \\
\hline $\mathrm{C}$ & -9.142009 & -3.977036 & 0.545396 \\
\hline $\mathrm{C}$ & -10.017135 & -1.860919 & 1.560986 \\
\hline $\mathrm{C}$ & -10.180996 & -2.241320 & -0.931890 \\
\hline $\mathrm{H}$ & -2.120613 & 1.508187 & -0.213491 \\
\hline $\mathrm{H}$ & 1.957417 & -1.084664 & 1.691742 \\
\hline $\mathrm{H}$ & -0.802112 & -2.416279 & 0.337531 \\
\hline $\mathrm{H}$ & 1.145385 & -6.208561 & -0.550999 \\
\hline $\mathrm{H}$ & -3.691877 & 3.526214 & -2.944691 \\
\hline $\mathrm{H}$ & -2.594017 & 5.555195 & -3.847048 \\
\hline $\mathrm{H}$ & 0.458937 & 5.276355 & -0.794872 \\
\hline $\mathrm{H}$ & -0.640312 & 3.195164 & 0.087458 \\
\hline $\mathrm{H}$ & -0.170649 & 8.263778 & -4.036785 \\
\hline $\mathrm{H}$ & -0.900518 & 6.816394 & -4.819292 \\
\hline $\mathrm{H}$ & -1.852173 & 7.769700 & -3.622036 \\
\hline $\mathrm{H}$ & 5.377314 & -0.063151 & 2.700385 \\
\hline
\end{tabular}




$\begin{array}{cccc}\mathrm{H} & 4.968256 & -4.006702 & -0.303270 \\ \mathrm{H} & 6.841038 & -5.322536 & -0.221079 \\ \mathrm{H} & 6.797050 & -4.261868 & -1.661650 \\ \mathrm{H} & 7.406846 & -1.120443 & 1.798085 \\ \mathrm{H} & 8.711972 & -1.375419 & -0.107180 \\ \mathrm{H} & 7.874349 & -1.936304 & -1.579138 \\ \mathrm{H} & 9.404471 & -2.708511 & -1.069014 \\ \mathrm{H} & 8.339781 & -4.964158 & -1.114717 \\ \mathrm{H} & -3.155414 & -5.899998 & -0.786858 \\ \mathrm{H} & 7.998948 & -4.526439 & 1.929761 \\ \mathrm{H} & 8.796149 & -2.933689 & 1.991708 \\ \mathrm{H} & 9.475168 & -4.224468 & 0.965250 \\ \mathrm{H} & -0.993217 & 1.402205 & 3.543275 \\ \mathrm{H} & 2.714523 & 1.342349 & 5.759775 \\ \mathrm{H} & -6.695427 & 1.409534 & -0.439374 \\ \mathrm{H} & -2.002136 & 2.903806 & 5.277756 \\ \mathrm{H} & 1.726331 & 2.836285 & 7.471802 \\ \mathrm{H} & -6.683575 & -3.507539 & 0.311975 \\ \mathrm{H} & -8.824912 & 0.223884 & -0.123279 \\ \mathrm{H} & -0.813066 & 4.863993 & 9.094400 \\ \mathrm{H} & 0.189504 & 3.369203 & 9.142848 \\ \mathrm{H} & 0.766985 & 4.813182 & 8.232902 \\ \mathrm{H} & -10.135552 & -4.438593 & 0.681355 \\ \mathrm{H} & -8.544713 & -4.199822 & 1.446890 \\ \mathrm{H} & -8.661290 & -4.468956 & -0.318438 \\ \mathrm{H} & -9.419976 & -2.016792 & 2.476110 \\ \mathrm{H} & -11.001135 & -2.343067 & 1.705242 \\ \mathrm{H} & -10.187382 & -0.776377 & 1.450733 \\ \mathrm{H} & -11.165329 & -2.726197 & -0.799497 \\ \mathrm{H} & -9.702557 & -2.672744 & -1.828270 \\ \mathrm{H} & -10.357936 & -1.170032 & -1.127616 \\ \mathrm{H} & -3.402764 & -0.172818 & 0.007019 \\ \mathrm{H} & 0.708384 & 0.174955 & 2.807782 \\ \mathrm{Br} & -0.608103 & 0.121170 & 0.874449 \\ \mathrm{~F} & -1.084428 & -7.454146 & -1.055271 \\ ------------------------------------------------------------------\end{array}$

Statistical Thermodynamic Analysis

Temperature $=298.150$ Kelvin Pressure $=1.00000$ Atm

\begin{tabular}{|c|c|}
\hline-5653.32330045 & Predicted Change $=-5.880674 \mathrm{D}-08$ \\
\hline Zero-point correction $(\mathrm{ZPE})=$ & -5652.03091 .29236 \\
\hline Internal Energy $(\mathrm{U})=$ & -5651.94931 .37397 \\
\hline Enthalpy $(\mathrm{H})=$ & $-5651.9483 \quad 1.37491$ \\
\hline Gibbs Free Energy $(G)=$ & $-5652.1597 \quad 1.16356$ \\
\hline Frequencies -- 6.8839 & $9.4243 \quad 10.5152$ \\
\hline \multicolumn{2}{|c|}{ Single points at WB97X-D3/def2-TZVPP: -5660.45898541473} \\
\hline
\end{tabular}


$\mathbf{R}=\mathbf{F}$ receptor bound with $\mathrm{Cl}-$ (with TBA)

Supporting Information: 010-bound_TBA_F_Cl.log

Using Gaussian 09: EM64L-G09RevE.01 30-Nov-2015

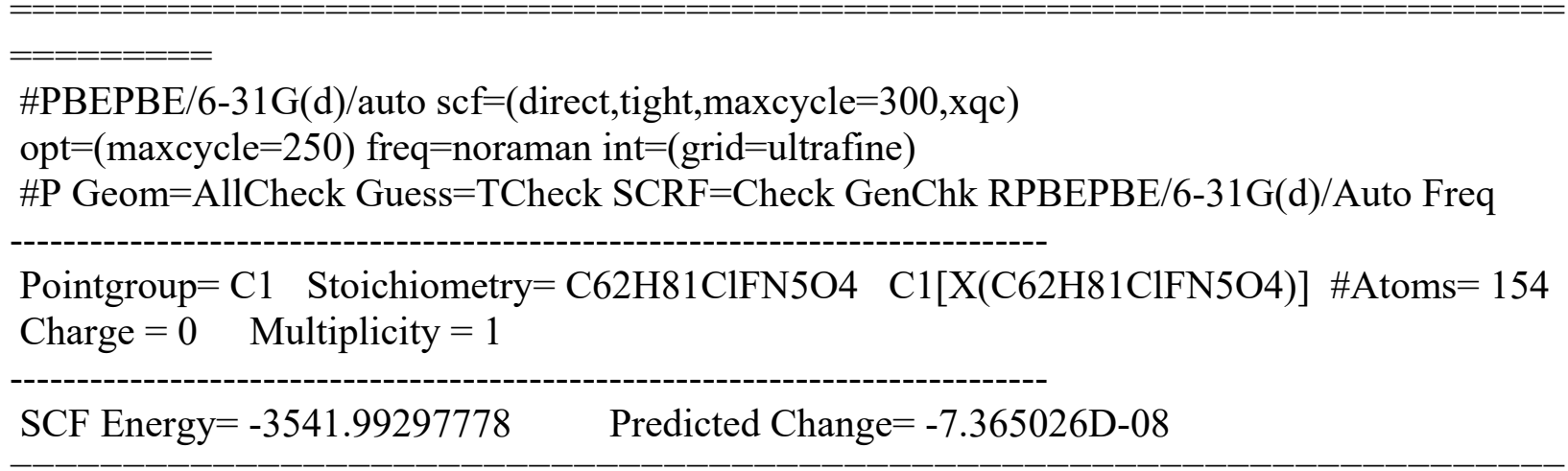

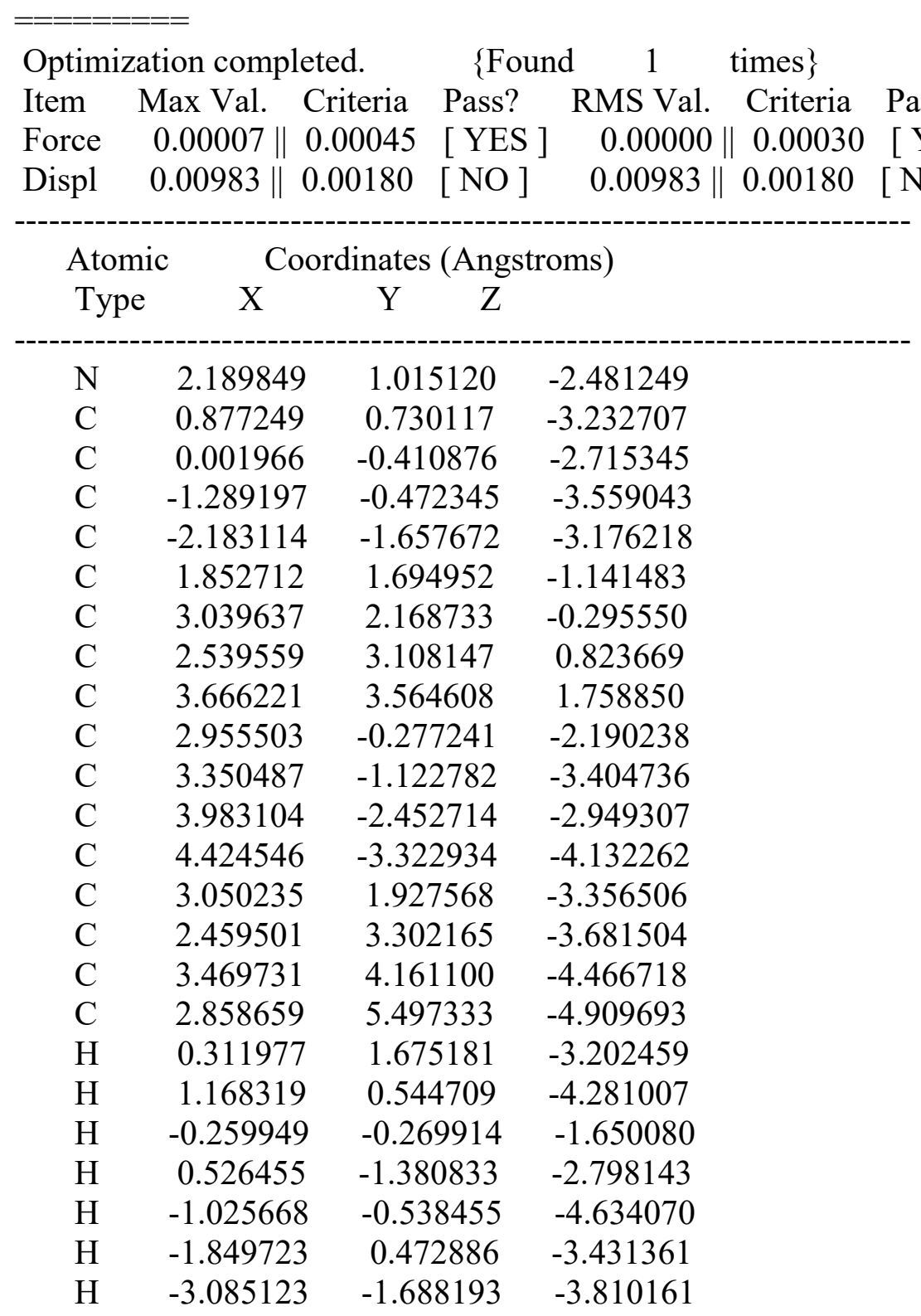




\begin{tabular}{|c|c|c|c|}
\hline $\mathrm{H}$ & -1.651787 & -2.618479 & -3.296693 \\
\hline $\mathrm{H}$ & -2.515979 & -1.589398 & -2.127350 \\
\hline $\mathrm{H}$ & 1.201127 & 2.543587 & -1.399567 \\
\hline $\mathrm{H}$ & 1.230992 & 0.979260 & -0.573460 \\
\hline $\mathrm{H}$ & 3.567749 & 1.312626 & 0.161613 \\
\hline $\mathrm{H}$ & 3.780506 & 2.725218 & -0.900682 \\
\hline $\mathrm{H}$ & 1.755225 & 2.596580 & 1.409842 \\
\hline $\mathrm{H}$ & 2.059258 & 3.989509 & 0.356934 \\
\hline $\mathrm{H}$ & 3.280176 & 4.274587 & 2.509197 \\
\hline $\mathrm{H}$ & 4.097262 & 2.712155 & 2.310314 \\
\hline $\mathrm{H}$ & 4.475588 & 4.068779 & 1.199219 \\
\hline $\mathrm{H}$ & 3.852465 & 0.024189 & -1.626277 \\
\hline $\mathrm{H}$ & 2.315631 & -0.853922 & -1.503220 \\
\hline $\mathrm{H}$ & 2.469393 & -1.343683 & -4.035183 \\
\hline $\mathrm{H}$ & 4.076711 & -0.584557 & -4.041648 \\
\hline $\mathrm{H}$ & 3.257581 & -3.005339 & -2.323410 \\
\hline $\mathrm{H}$ & 4.849393 & -2.243128 & -2.294045 \\
\hline $\mathrm{H}$ & 4.877935 & -4.264757 & -3.779468 \\
\hline $\mathrm{H}$ & 3.569963 & -3.586630 & -4.780464 \\
\hline $\mathrm{H}$ & 5.173924 & -2.805027 & -4.757414 \\
\hline $\mathrm{H}$ & 3.233772 & 1.368567 & -4.287589 \\
\hline $\mathrm{H}$ & 4.016510 & 2.030735 & -2.837375 \\
\hline $\mathrm{H}$ & 1.536587 & 3.195571 & -4.279446 \\
\hline $\mathrm{H}$ & 2.176180 & 3.844243 & -2.762475 \\
\hline $\mathrm{H}$ & 3.830247 & 3.603722 & -5.353174 \\
\hline $\mathrm{H}$ & 4.358881 & 4.346202 & -3.833944 \\
\hline $\mathrm{H}$ & 3.615703 & 6.142164 & -5.386330 \\
\hline $\mathrm{H}$ & 2.046032 & 5.336354 & -5.640461 \\
\hline $\mathrm{H}$ & 2.428101 & 6.043985 & -4.052564 \\
\hline $\mathrm{O}$ & -0.194910 & 6.463318 & -2.931218 \\
\hline $\mathrm{O}$ & 3.749375 & 0.608779 & 3.737030 \\
\hline $\mathrm{N}$ & -2.675338 & 1.903949 & -0.780636 \\
\hline $\mathrm{N}$ & 2.817223 & -0.795736 & 2.128205 \\
\hline $\mathrm{C}$ & -0.993000 & -3.507900 & 0.350748 \\
\hline $\mathrm{C}$ & -1.225690 & -6.240239 & -0.148970 \\
\hline $\mathrm{C}$ & 0.048599 & -5.702185 & 0.029058 \\
\hline $\mathrm{C}$ & 0.165339 & -4.313077 & 0.287571 \\
\hline $\mathrm{C}$ & -2.129151 & 3.065289 & -1.358218 \\
\hline $\mathrm{C}$ & -2.562229 & 3.594697 & -2.591691 \\
\hline $\mathrm{C}$ & -1.944455 & 4.728844 & -3.141939 \\
\hline $\mathrm{C}$ & -0.877666 & 5.355391 & -2.473481 \\
\hline $\mathrm{C}$ & -0.449625 & 4.839120 & -1.234512 \\
\hline $\mathrm{C}$ & -1.068950 & 3.714727 & -0.683580 \\
\hline $\mathrm{C}$ & -0.696030 & 7.083623 & -4.111540 \\
\hline $\mathrm{C}$ & 1.445067 & -3.724672 & 0.494937 \\
\hline $\mathrm{C}$ & 2.553049 & -3.227453 & 0.701688 \\
\hline $\mathrm{C}$ & 3.834217 & -2.670007 & 0.957776 \\
\hline $\mathrm{C}$ & 3.972535 & -1.451056 & 1.695308 \\
\hline
\end{tabular}




\begin{tabular}{|c|c|c|c|}
\hline $\mathrm{C}$ & 5.279723 & -0.960766 & 1.918741 \\
\hline $\mathrm{C}$ & 6.391630 & -1.651878 & 1.435484 \\
\hline $\mathrm{C}$ & 6.285900 & -2.859554 & 0.706929 \\
\hline $\mathrm{C}$ & 4.991083 & -3.342045 & 0.486032 \\
\hline $\mathrm{C}$ & 8.331074 & -2.645221 & -0.751297 \\
\hline $\mathrm{C}$ & 7.218502 & -4.881089 & -0.559790 \\
\hline $\mathrm{C}$ & 2.747403 & 0.188664 & 3.139593 \\
\hline $\mathrm{C}$ & -3.990264 & 1.482267 & -0.939781 \\
\hline $\mathrm{C}$ & -2.271158 & -4.079539 & 0.158079 \\
\hline $\mathrm{C}$ & -2.387218 & -5.470212 & -0.091725 \\
\hline $\mathrm{C}$ & 7.549691 & -3.582466 & 0.203250 \\
\hline $\mathrm{N}$ & 1.455181 & 0.614771 & 3.355488 \\
\hline $\mathrm{N}$ & -4.249474 & 0.289133 & -0.250318 \\
\hline $\mathrm{O}$ & -4.834128 & 2.083775 & -1.613015 \\
\hline $\mathrm{C}$ & -3.426127 & -3.250639 & 0.192776 \\
\hline $\mathrm{C}$ & 8.449465 & -3.946359 & 1.410802 \\
\hline $\mathrm{C}$ & 1.031390 & 1.608744 & 4.267391 \\
\hline $\mathrm{C}$ & -5.507775 & -0.314769 & -0.125057 \\
\hline $\mathrm{C}$ & -4.417171 & -2.520470 & 0.190776 \\
\hline $\mathrm{C}$ & -0.317593 & 2.027007 & 4.176288 \\
\hline $\mathrm{C}$ & 1.854951 & 2.184979 & 5.253138 \\
\hline $\mathrm{C}$ & -5.584596 & -1.714204 & 0.146990 \\
\hline $\mathrm{C}$ & -6.713532 & 0.409982 & -0.225788 \\
\hline $\mathrm{C}$ & -0.823974 & 2.992532 & 5.042676 \\
\hline $\mathrm{C}$ & 1.341029 & 3.158541 & 6.124356 \\
\hline $\mathrm{C}$ & -6.852326 & -2.323999 & 0.332674 \\
\hline $\mathrm{C}$ & -7.941598 & -0.227703 & -0.050734 \\
\hline $\mathrm{C}$ & 0.001155 & 3.570380 & 6.027607 \\
\hline $\mathrm{C}$ & -8.049765 & -1.607552 & 0.240148 \\
\hline $\mathrm{O}$ & -0.597201 & 4.514899 & 6.830671 \\
\hline $\mathrm{C}$ & -9.433336 & -2.256022 & 0.436497 \\
\hline $\mathrm{C}$ & 0.209079 & 5.101611 & 7.844469 \\
\hline $\mathrm{C}$ & -9.335465 & -3.764528 & 0.741213 \\
\hline $\mathrm{C}$ & -10.158278 & -1.567879 & 1.620183 \\
\hline $\mathrm{C}$ & -10.273077 & -2.074822 & -0.852667 \\
\hline $\mathrm{H}$ & -2.071386 & 1.406600 & -0.100387 \\
\hline $\mathrm{H}$ & 1.932216 & -1.200267 & 1.802439 \\
\hline $\mathrm{H}$ & -0.902819 & -2.435223 & 0.554865 \\
\hline $\mathrm{H}$ & 0.929354 & -6.347056 & -0.018741 \\
\hline $\mathrm{H}$ & -3.405252 & 3.129001 & -3.104280 \\
\hline $\mathrm{H}$ & -2.313230 & 5.113454 & -4.096587 \\
\hline $\mathrm{H}$ & 0.352170 & 5.356657 & -0.697550 \\
\hline $\mathrm{H}$ & -0.742651 & 3.332694 & 0.290085 \\
\hline $\mathrm{H}$ & -0.068727 & 7.972680 & -4.274259 \\
\hline $\mathrm{H}$ & -0.617105 & 6.417255 & -4.993460 \\
\hline $\mathrm{H}$ & -1.751289 & 7.394968 & -3.990515 \\
\hline $\mathrm{H}$ & 5.401285 & -0.050283 & 2.504897 \\
\hline $\mathrm{H}$ & 4.828073 & -4.274926 & -0.059817 \\
\hline
\end{tabular}




$\begin{array}{lccc}\mathrm{H} & 6.681853 & -5.605603 & 0.077222 \\ \mathrm{H} & 6.601909 & -4.686224 & -1.455848 \\ \mathrm{H} & 7.381441 & -1.228964 & 1.642223 \\ \mathrm{H} & 8.619894 & -1.703138 & -0.254816 \\ \mathrm{H} & 7.722744 & -2.387890 & -1.636972 \\ \mathrm{H} & 9.255920 & -3.137249 & -1.103237 \\ \mathrm{H} & 8.152560 & -5.362407 & -0.897821 \\ \mathrm{H} & -3.363032 & -5.937591 & -0.244459 \\ \mathrm{H} & 7.923831 & -4.627042 & 2.102370 \\ \mathrm{H} & 8.748830 & -3.051766 & 1.982731 \\ \mathrm{H} & 9.370951 & -4.449394 & 1.065641 \\ \mathrm{H} & -0.965915 & 1.581658 & 3.413066 \\ \mathrm{H} & 2.895914 & 1.871236 & 5.331255 \\ \mathrm{H} & -6.669860 & 1.474450 & -0.455939 \\ \mathrm{H} & -1.867180 & 3.315313 & 4.973366 \\ \mathrm{H} & 2.006743 & 3.584478 & 6.879675 \\ \mathrm{H} & -6.856373 & -3.395914 & 0.545333 \\ \mathrm{H} & -8.848899 & 0.381045 & -0.135146 \\ \mathrm{H} & -0.442430 & 5.813402 & 8.373413 \\ \mathrm{H} & 0.582077 & 4.344884 & 8.562485 \\ \mathrm{H} & 1.074504 & 5.646697 & 7.418190 \\ \mathrm{H} & -10.348280 & -4.182833 & 0.874923 \\ \mathrm{H} & -8.768998 & -3.959543 & 1.668670 \\ \mathrm{H} & -8.852009 & -4.318691 & -0.082481 \\ \mathrm{H} & -9.590277 & -1.694871 & 2.558052 \\ \mathrm{H} & -11.162296 & -2.007284 & 1.763131 \\ \mathrm{H} & -10.286663 & -0.485588 & 1.448303 \\ \mathrm{H} & -11.277419 & -2.517246 & -0.721736 \\ \mathrm{H} & -9.788233 & -2.569842 & -1.711975 \\ \mathrm{H} & -10.405981 & -1.009924 & -1.108853 \\ \mathrm{H} & -3.450983 & -0.232593 & 0.128251 \\ \mathrm{H} & 0.730343 & 0.319380 & 2.675484 \\ \mathrm{~F} & -1.339515 & -7.569730 & -0.392190 \\ \mathrm{Cl} & -0.634356 & 0.054013 & 0.936531 \\ -----------------------------------------------------------------\end{array}$

Statistical Thermodynamic Analysis

Temperature $=298.150$ Kelvin $\quad$ Pressure $=1.00000$ Atm

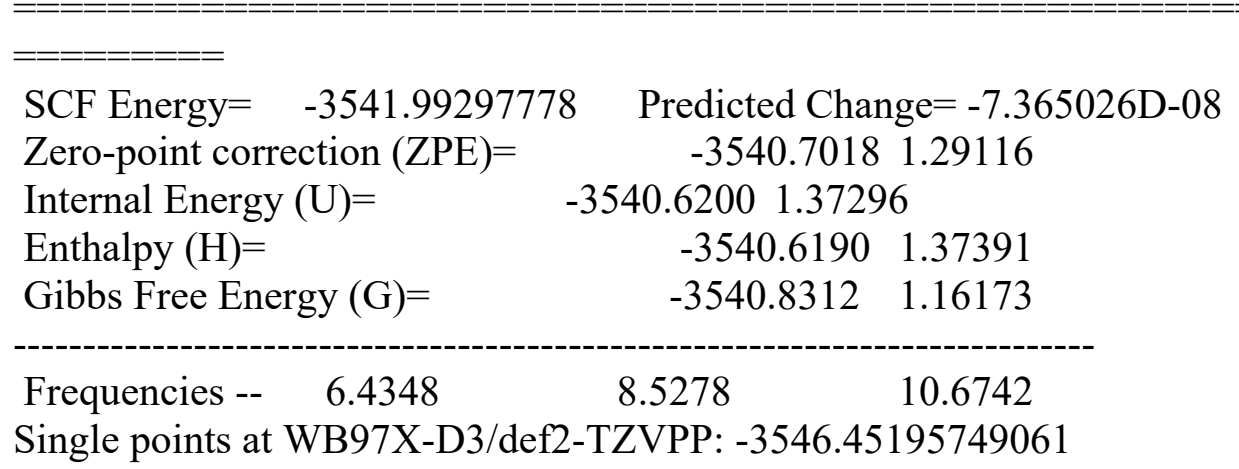


$R=F$ receptor bound with SeH- (with TBA)

Supporting Information: 010-bound_TBA_F_SeH.log

Using Gaussian 09: EM64L-G09RevE.01 30-Nov-2015

\#PBEPBE/6-31G(d)/auto scf=(direct,tight,maxcycle=300,xqc)

$\mathrm{opt}=($ maxcycle $=250)$ freq $=$ noraman int $=($ grid $=$ ultrafine $)$

\#P Geom=AllCheck Guess=TCheck SCRF=Check GenChk RPBEPBE/6-31G(d)/Auto Freq

Pointgroup= $\mathrm{C} 1 \quad$ Stoichiometry $=\mathrm{C} 62 \mathrm{H} 82 \mathrm{FN} 5 \mathrm{O} 4 \mathrm{Se} \quad \mathrm{C} 1[\mathrm{X}(\mathrm{C} 62 \mathrm{H} 82 \mathrm{FN} 5 \mathrm{O} 4 \mathrm{Se})]$ \#Atoms $=155$

Charge $=0 \quad$ Multiplicity $=1$

SCF Energy $=-5481.58175660 \quad$ Predicted Change $=-2.106502 \mathrm{D}-08$

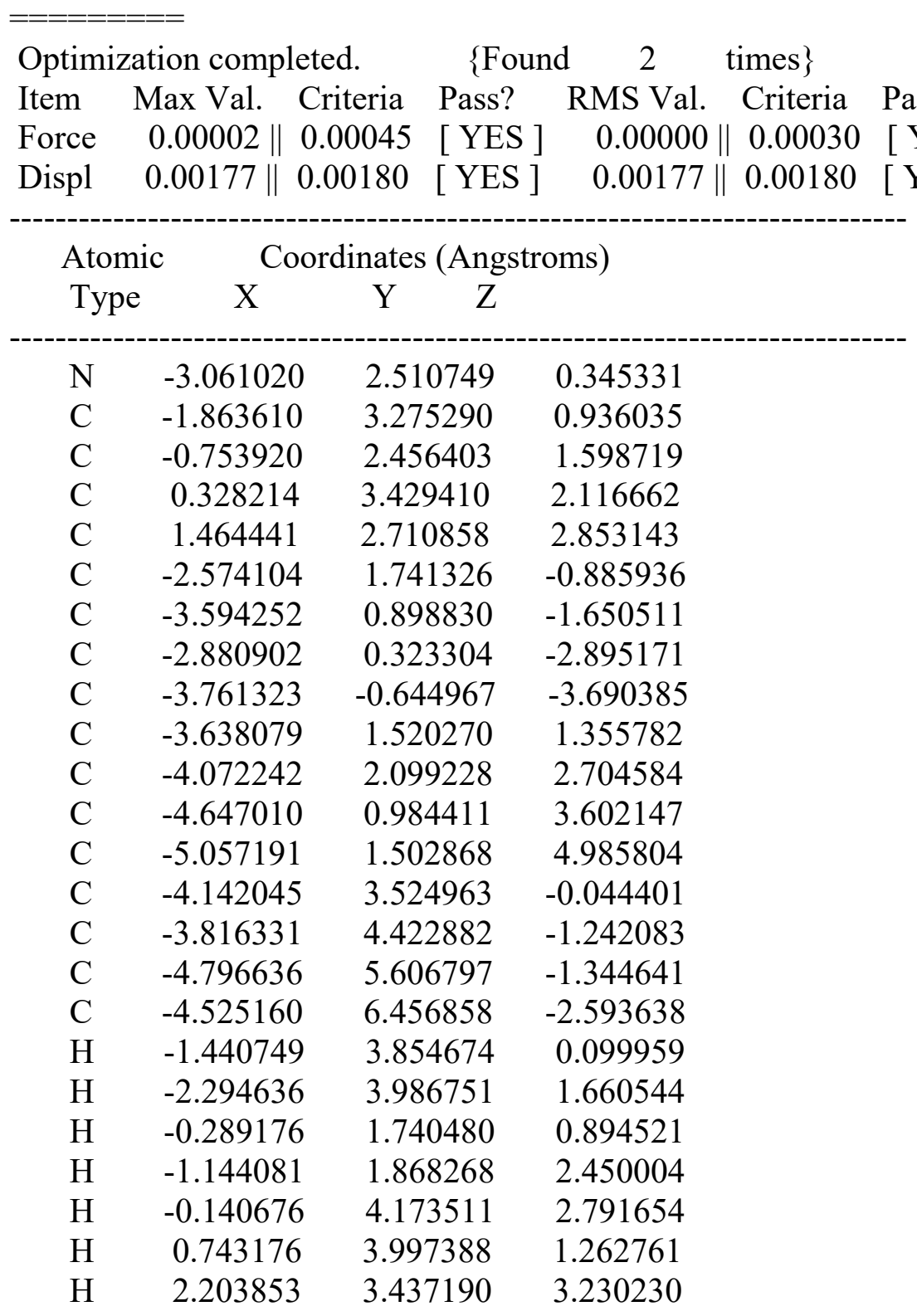




\begin{tabular}{|c|c|c|c|}
\hline $\mathrm{H}$ & 1.087506 & 2.132303 & 3.714957 \\
\hline $\mathrm{H}$ & 1.996713 & 2.011360 & 2.187423 \\
\hline $\mathrm{H}$ & -2.142131 & 2.507738 & -1.546627 \\
\hline $\mathrm{H}$ & -1.734553 & 1.094347 & -0.570700 \\
\hline $\mathrm{H}$ & -3.958480 & 0.057151 & -1.033831 \\
\hline $\mathrm{H}$ & -4.478960 & 1.487889 & -1.963008 \\
\hline $\mathrm{H}$ & -1.950518 & -0.169665 & -2.553687 \\
\hline $\mathrm{H}$ & -2.562882 & 1.161768 & -3.546452 \\
\hline $\mathrm{H}$ & -3.214039 & -1.049754 & -4.557936 \\
\hline $\mathrm{H}$ & -4.079922 & -1.498268 & -3.067481 \\
\hline $\mathrm{H}$ & -4.670636 & -0.143825 & -4.069784 \\
\hline $\mathrm{H}$ & -4.494931 & 1.044914 & 0.852507 \\
\hline $\mathrm{H}$ & -2.871343 & 0.740857 & 1.494335 \\
\hline $\mathrm{H}$ & -3.215415 & 2.566845 & 3.223332 \\
\hline $\mathrm{H}$ & -4.840619 & 2.884219 & 2.575757 \\
\hline $\mathrm{H}$ & -3.895869 & 0.179168 & 3.706294 \\
\hline $\mathrm{H}$ & -5.521302 & 0.526286 & 3.100129 \\
\hline $\mathrm{H}$ & -5.470426 & 0.689628 & 5.605330 \\
\hline $\mathrm{H}$ & -4.193219 & 1.928651 & 5.525985 \\
\hline $\mathrm{H}$ & -5.827874 & 2.290636 & 4.909622 \\
\hline $\mathrm{H}$ & -4.321438 & 4.136287 & 0.854566 \\
\hline $\mathrm{H}$ & -5.055945 & 2.939805 & -0.238896 \\
\hline $\mathrm{H}$ & -2.790892 & 4.827197 & -1.174317 \\
\hline $\mathrm{H}$ & -3.862207 & 3.836444 & -2.177731 \\
\hline $\mathrm{H}$ & -4.705563 & 6.232909 & -0.436204 \\
\hline $\mathrm{H}$ & -5.839261 & 5.234346 & -1.362941 \\
\hline $\mathrm{H}$ & -5.172666 & 7.349318 & -2.614688 \\
\hline $\mathrm{H}$ & -3.473158 & 6.791963 & -2.629959 \\
\hline $\mathrm{H}$ & -4.719535 & 5.880354 & -3.515202 \\
\hline $\mathrm{O}$ & -0.631740 & 6.761910 & -3.305899 \\
\hline $\mathrm{O}$ & -2.037320 & -4.697561 & -2.325011 \\
\hline $\mathrm{N}$ & 2.378198 & 2.569497 & -1.114061 \\
\hline $\mathrm{N}$ & -2.136204 & -2.848093 & -0.899538 \\
\hline $\mathrm{C}$ & 0.636618 & -1.247067 & 2.655631 \\
\hline $\mathrm{C}$ & 0.492191 & -1.484250 & 5.421872 \\
\hline $\mathrm{C}$ & -0.679005 & -1.638183 & 4.679266 \\
\hline $\mathrm{C}$ & -0.605332 & -1.525882 & 3.267728 \\
\hline $\mathrm{C}$ & 1.726757 & 3.677120 & -1.670842 \\
\hline $\mathrm{C}$ & 2.239806 & 4.989968 & -1.691630 \\
\hline $\mathrm{C}$ & 1.478909 & 6.039134 & -2.233344 \\
\hline $\mathrm{C}$ & 0.200919 & 5.800291 & -2.767637 \\
\hline $\mathrm{C}$ & -0.303224 & 4.484058 & -2.770887 \\
\hline $\mathrm{C}$ & 0.447177 & 3.440252 & -2.230325 \\
\hline $\mathrm{C}$ & -0.112915 & 8.085774 & -3.388456 \\
\hline $\mathrm{C}$ & -1.754459 & -1.738755 & 2.453285 \\
\hline $\mathrm{C}$ & -2.711884 & -2.019320 & 1.728710 \\
\hline $\mathrm{C}$ & -3.755325 & -2.465727 & 0.873566 \\
\hline $\mathrm{C}$ & -3.436130 & -2.980993 & -0.424632 \\
\hline
\end{tabular}




\begin{tabular}{|c|c|c|c|}
\hline $\mathrm{C}$ & -4.473045 & -3.586420 & -1.169910 \\
\hline $\mathrm{C}$ & -5.774320 & -3.627545 & -0.669458 \\
\hline $\mathrm{C}$ & -6.130190 & -3.075001 & 0.584949 \\
\hline $\mathrm{C}$ & -5.096081 & -2.506317 & 1.336877 \\
\hline $\mathrm{C}$ & -8.499702 & -2.389076 & 0.064356 \\
\hline $\mathrm{C}$ & -7.770843 & -2.477539 & 2.458710 \\
\hline $\mathrm{C}$ & -1.479054 & -3.732281 & -1.791265 \\
\hline $\mathrm{C}$ & 3.690338 & 2.479755 & -0.675979 \\
\hline $\mathrm{C}$ & 1.808050 & -1.093256 & 3.430007 \\
\hline $\mathrm{C}$ & 1.732502 & -1.220106 & 4.839305 \\
\hline $\mathrm{C}$ & -7.589950 & -3.132477 & 1.074294 \\
\hline $\mathrm{N}$ & -0.165220 & -3.358400 & -1.951781 \\
\hline $\mathrm{N}$ & 3.951834 & 1.213587 & -0.127781 \\
\hline $\mathrm{O}$ & 4.522434 & 3.391127 & -0.743241 \\
\hline $\mathrm{C}$ & 3.032497 & -0.785967 & 2.774937 \\
\hline $\mathrm{C}$ & -8.041448 & -4.610990 & 1.176274 \\
\hline $\mathrm{C}$ & 0.861146 & -4.008914 & -2.660016 \\
\hline $\mathrm{C}$ & 5.193782 & 0.813059 & 0.385145 \\
\hline $\mathrm{C}$ & 4.052015 & -0.485643 & 2.154911 \\
\hline $\mathrm{C}$ & 2.145334 & -3.418708 & -2.577495 \\
\hline $\mathrm{C}$ & 0.688508 & -5.179885 & -3.418539 \\
\hline $\mathrm{C}$ & 5.242425 & -0.087493 & 1.490432 \\
\hline $\mathrm{C}$ & 6.412135 & 1.260338 & -0.164075 \\
\hline $\mathrm{C}$ & 3.228127 & -3.988550 & -3.240248 \\
\hline $\mathrm{C}$ & 1.784914 & -5.752491 & -4.082911 \\
\hline $\mathrm{C}$ & 6.497711 & -0.538729 & 1.972951 \\
\hline $\mathrm{C}$ & 7.629238 & 0.811991 & 0.349043 \\
\hline $\mathrm{C}$ & 3.058538 & -5.163703 & -4.000274 \\
\hline $\mathrm{C}$ & 7.708898 & -0.107286 & 1.421273 \\
\hline $\mathrm{O}$ & 4.194116 & -5.645821 & -4.613794 \\
\hline $\mathrm{C}$ & 9.080783 & -0.585578 & 1.934154 \\
\hline $\mathrm{C}$ & 4.054654 & -6.833629 & -5.381991 \\
\hline $\mathrm{C}$ & 8.954409 & -1.577030 & 3.108483 \\
\hline $\mathrm{C}$ & 9.839619 & -1.291512 & 0.782594 \\
\hline $\mathrm{C}$ & 9.903269 & 0.635453 & 2.416440 \\
\hline $\mathrm{H}$ & 1.803192 & 1.704495 & -1.063593 \\
\hline $\mathrm{H}$ & -1.559429 & -2.116935 & -0.462540 \\
\hline $\mathrm{H}$ & 0.703316 & -1.136221 & 1.569960 \\
\hline $\mathrm{H}$ & -1.621845 & -1.865864 & 5.182621 \\
\hline $\mathrm{H}$ & 3.236559 & 5.174946 & -1.288048 \\
\hline $\mathrm{H}$ & 1.904946 & 7.045992 & -2.229285 \\
\hline $\mathrm{H}$ & -1.277502 & 4.296146 & -3.235107 \\
\hline $\mathrm{H}$ & 0.060318 & 2.414084 & -2.204339 \\
\hline $\mathrm{H}$ & -0.897091 & 8.687449 & -3.871816 \\
\hline $\mathrm{H}$ & 0.104674 & 8.506115 & -2.386773 \\
\hline $\mathrm{H}$ & 0.808578 & 8.124682 & -4.000768 \\
\hline $\mathrm{H}$ & -4.225923 & -4.034377 & -2.133497 \\
\hline $\mathrm{H}$ & -5.288051 & -2.104619 & 2.335516 \\
\hline
\end{tabular}




$\begin{array}{cccc}\text { H } & -7.174834 & -2.987734 & 3.235486 \\ \mathrm{H} & -7.484392 & -1.410073 & 2.448405 \\ \mathrm{H} & -6.543975 & -4.102492 & -1.288433 \\ \mathrm{H} & -8.435711 & -2.829103 & -0.945240 \\ \mathrm{H} & -8.215772 & -1.324670 & -0.013926 \\ \mathrm{H} & -9.555462 & -2.440017 & 0.387028 \\ \mathrm{H} & -8.830732 & -2.534016 & 2.761776 \\ \mathrm{H} & 2.620265 & -1.106598 & 5.466049 \\ \mathrm{H} & -7.419494 & -5.163659 & 1.901286 \\ \mathrm{H} & -7.966090 & -5.129297 & 0.205471 \\ \mathrm{H} & -9.093868 & -4.669812 & 1.508469 \\ \mathrm{H} & 2.261746 & -2.502477 & -1.983553 \\ \mathrm{H} & -0.301079 & -5.635570 & -3.479529 \\ \mathrm{H} & 6.386810 & 1.971073 & -0.990869 \\ \mathrm{H} & 4.223472 & -3.538213 & -3.179415 \\ \mathrm{H} & 1.625127 & -6.664379 & -4.664320 \\ \mathrm{H} & 6.482966 & -1.236192 & 2.814119 \\ \mathrm{H} & 8.549497 & 1.183584 & -0.115448 \\ \mathrm{H} & 5.056316 & -7.054249 & -5.780938 \\ \mathrm{H} & 3.712437 & -7.686584 & -4.763030 \\ \mathrm{H} & 3.348077 & -6.698585 & -6.224872 \\ \mathrm{H} & 9.959768 & -1.891547 & 3.438537 \\ \mathrm{H} & 8.398704 & -2.486297 & 2.819624 \\ \mathrm{H} & 8.445983 & -1.121770 & 3.976558 \\ \mathrm{H} & 9.283833 & -2.176988 & 0.428522 \\ \mathrm{H} & 10.835501 & -1.624773 & 1.127104 \\ \mathrm{H} & 9.989788 & -0.620925 & -0.080603 \\ \mathrm{H} & 10.899043 & 0.311326 & 2.769838 \\ \mathrm{H} & 9.393381 & 1.150585 & 3.249016 \\ \mathrm{H} & 10.056771 & 1.371507 & 1.609016 \\ \mathrm{H} & 3.161690 & 0.558723 & -0.038866 \\ \mathrm{H} & 0.131791 & -2.478377 & -1.489314 \\ \mathrm{~F} & 0.420520 & -1.596981 & 6.771204 \\ \mathrm{H} & 0.770927 & -0.221368 & -2.368404 \\ \mathrm{Se} & 0.512078 & -0.240282 & -0.900680 \\ ----------------------------------------------------------------- \\ -\end{array}$

Statistical Thermodynamic Analysis

Temperature $=298.150$ Kelvin Pressure $=1.00000$ Atm

\begin{tabular}{|c|c|}
\hline-5481.58175660 & Predicted Change $=-2.106502 \mathrm{D}-08$ \\
\hline Zero-point correction $(\mathrm{ZPE})=$ & -5480.28061 .30107 \\
\hline Internal Energy $(\mathrm{U})=$ & -5480.19911 .38263 \\
\hline Enthalpy $(\mathrm{H})=$ & $-5480.1981 \quad 1.38357$ \\
\hline Gibbs Free Energy $(G)=$ & $-5480.4070 \quad 1.17471$ \\
\hline Frequencies -- 7.5451 & $10.4487 \quad 12.6096$ \\
\hline \multicolumn{2}{|c|}{ Single points at WB97X-D3/def2-TZVPP: -5488.41334430156} \\
\hline
\end{tabular}


$\mathrm{R}=\mathrm{F}$ receptor bound with $\mathrm{SH}-$ (with TBA)

Supporting Information: 010-bound_TBA_F_SH.log

Using Gaussian 09: EM64L-G09RevE.01 30-Nov-2015

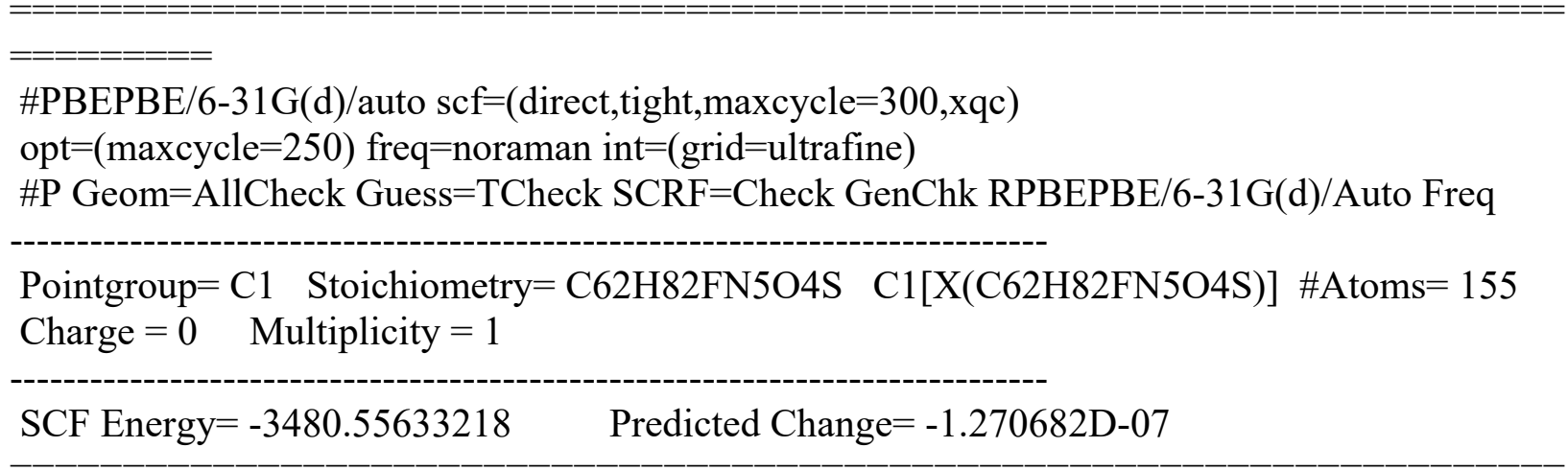

\begin{tabular}{|c|c|c|c|c|c|c|}
\hline \multicolumn{3}{|c|}{ Optimization completed. } & $\{$ Foun & 1 & times $\}$ & \\
\hline Item & Max Val. & Criteria & Pass? & RMS Val. & Criteria & Pass? \\
\hline Force & 0.00002 & 0.00045 & [ YES ] & 0.00000 & | 0.00030 & [ YES ] \\
\hline Displ & 0.00970 & 0.00180 & {$[\mathrm{NO}]$} & $0.00970 \|$ & 0.00180 & [YES ] \\
\hline
\end{tabular}

\begin{tabular}{lrrr} 
Atomic & \multicolumn{3}{c}{ Coordinates (Angstroms) } \\
Type & $\mathrm{X}$ & $\mathrm{Y}$ & $\mathrm{Z}$ \\
$\mathrm{N}$ & 2.254983 & 1.762042 & -2.076939 \\
$\mathrm{C}$ & 0.967302 & 1.713603 & -2.913588 \\
$\mathrm{C}$ & 0.048466 & 0.507133 & -2.721752 \\
$\mathrm{C}$ & -1.171083 & 0.653296 & -3.656803 \\
$\mathrm{C}$ & -2.112539 & -0.554540 & -3.586766 \\
$\mathrm{C}$ & 1.874115 & 2.021525 & -0.608285 \\
$\mathrm{C}$ & 3.023671 & 2.198501 & 0.387485 \\
$\mathrm{C}$ & 2.450711 & 2.676853 & 1.739834 \\
$\mathrm{C}$ & 3.527413 & 2.826483 & 2.821716 \\
$\mathrm{C}$ & 3.022191 & 0.439990 & -2.160230 \\
$\mathrm{C}$ & 3.391657 & -0.046786 & -3.565270 \\
$\mathrm{C}$ & 4.081786 & -1.423234 & -3.494526 \\
$\mathrm{C}$ & 4.490458 & -1.940339 & -4.879216 \\
$\mathrm{C}$ & 3.136695 & 2.888736 & -2.619501 \\
$\mathrm{C}$ & 2.517185 & 4.289265 & -2.633309 \\
$\mathrm{C}$ & 3.570497 & 5.350295 & -3.007729 \\
$\mathrm{C}$ & 2.944277 & 6.741561 & -3.173936 \\
$\mathrm{H}$ & 0.420704 & 2.636742 & -2.664167 \\
$\mathrm{H}$ & 1.293325 & 1.787601 & -3.965416 \\
$\mathrm{H}$ & -0.297455 & 0.434622 & -1.673923 \\
$\mathrm{H}$ & 0.570176 & -0.437672 & -2.961692 \\
$\mathrm{H}$ & -0.819710 & 0.790878 & -4.699421 \\
$\mathrm{H}$ & -1.725191 & 1.572286 & -3.387898 \\
$\mathrm{H}$ & -2.957297 & -0.432149 & -4.285262
\end{tabular}




\begin{tabular}{|c|c|c|c|}
\hline $\mathrm{H}$ & -1.588774 & -1.490800 & -3.849262 \\
\hline $\mathrm{H}$ & -2.533332 & -0.678785 & -2.575357 \\
\hline $\mathrm{H}$ & 1.241103 & 2.921474 & -0.629187 \\
\hline $\mathrm{H}$ & 1.220354 & 1.186695 & -0.289928 \\
\hline $\mathrm{H}$ & 3.564859 & 1.248204 & 0.545937 \\
\hline $\mathrm{H}$ & 3.762696 & 2.946008 & 0.040423 \\
\hline $\mathrm{H}$ & 1.676932 & 1.962258 & 2.075280 \\
\hline $\mathrm{H}$ & 1.937907 & 3.646697 & 1.586187 \\
\hline $\mathrm{H}$ & 3.089006 & 3.231668 & 3.749146 \\
\hline $\mathrm{H}$ & 3.980301 & 1.853158 & 3.075460 \\
\hline $\mathrm{H}$ & 4.330923 & 3.514201 & 2.499762 \\
\hline $\mathrm{H}$ & 3.930289 & 0.580792 & -1.552752 \\
\hline $\mathrm{H}$ & 2.395303 & -0.304242 & -1.643313 \\
\hline $\mathrm{H}$ & 2.491545 & -0.132858 & -4.201045 \\
\hline $\mathrm{H}$ & 4.074205 & 0.664108 & -4.066864 \\
\hline $\mathrm{H}$ & 3.401527 & -2.145955 & -3.006534 \\
\hline $\mathrm{H}$ & 4.972006 & -1.355232 & -2.841219 \\
\hline $\mathrm{H}$ & 4.987428 & -2.922183 & -4.801400 \\
\hline $\mathrm{H}$ & 3.613163 & -2.062259 & -5.539060 \\
\hline $\mathrm{H}$ & 5.193102 & -1.248796 & -5.377887 \\
\hline $\mathrm{H}$ & 3.411802 & 2.593144 & -3.644028 \\
\hline $\mathrm{H}$ & 4.055627 & 2.873786 & -2.012175 \\
\hline $\mathrm{H}$ & 1.687079 & 4.335956 & -3.360807 \\
\hline $\mathrm{H}$ & 2.086341 & 4.550882 & -1.651121 \\
\hline $\mathrm{H}$ & 4.083666 & 5.058175 & -3.944744 \\
\hline $\mathrm{H}$ & 4.350855 & 5.379790 & -2.223282 \\
\hline $\mathrm{H}$ & 3.718723 & 7.507623 & -3.345890 \\
\hline $\mathrm{H}$ & 2.253704 & 6.762472 & -4.035871 \\
\hline $\mathrm{H}$ & 2.363852 & 7.029412 & -2.280008 \\
\hline $\mathrm{O}$ & -0.298536 & 7.138482 & -1.363540 \\
\hline $\mathrm{O}$ & 3.643684 & -0.578415 & 3.994343 \\
\hline $\mathrm{N}$ & -2.708872 & 2.142088 & -0.477019 \\
\hline $\mathrm{N}$ & 2.823171 & -1.335278 & 1.948702 \\
\hline $\mathrm{C}$ & -0.879327 & -3.294379 & -0.731227 \\
\hline $\mathrm{C}$ & -1.004032 & -5.591890 & -2.304077 \\
\hline $\mathrm{C}$ & 0.247244 & -5.132684 & -1.894433 \\
\hline $\mathrm{C}$ & 0.310368 & -3.965695 & -1.091210 \\
\hline $\mathrm{C}$ & -2.188750 & 3.422694 & -0.730046 \\
\hline $\mathrm{C}$ & -2.663157 & 4.271570 & -1.752097 \\
\hline $\mathrm{C}$ & -2.061382 & 5.518482 & -1.985579 \\
\hline $\mathrm{C}$ & -0.969839 & 5.943026 & -1.207327 \\
\hline $\mathrm{C}$ & -0.501801 & 5.107812 & -0.173772 \\
\hline $\mathrm{C}$ & -1.106525 & 3.871597 & 0.063931 \\
\hline $\mathrm{C}$ & -0.817382 & 8.044771 & -2.332153 \\
\hline $\mathrm{C}$ & 1.570522 & -3.474681 & -0.646054 \\
\hline $\mathrm{C}$ & 2.672451 & -3.085068 & -0.254851 \\
\hline $\mathrm{C}$ & 3.932405 & -2.641144 & 0.228142 \\
\hline $\mathrm{C}$ & 4.011869 & -1.765882 & 1.358531 \\
\hline
\end{tabular}




\begin{tabular}{|c|c|c|c|}
\hline $\mathrm{C}$ & 5.295658 & -1.371893 & 1.799815 \\
\hline $\mathrm{C}$ & 6.441750 & -1.830225 & 1.148910 \\
\hline $\mathrm{C}$ & 6.394693 & -2.697502 & 0.032247 \\
\hline $\mathrm{C}$ & 5.122532 & -3.085171 & -0.403008 \\
\hline $\mathrm{C}$ & 8.481057 & -1.946824 & -1.165762 \\
\hline $\mathrm{C}$ & 7.428658 & -4.120956 & -1.829989 \\
\hline $\mathrm{C}$ & 2.677839 & -0.820141 & 3.255973 \\
\hline $\mathrm{C}$ & -4.023497 & 1.751722 & -0.703879 \\
\hline $\mathrm{C}$ & -2.133599 & -3.772011 & -1.175985 \\
\hline $\mathrm{C}$ & -2.193549 & -4.944691 & -1.970391 \\
\hline $\mathrm{C}$ & 7.695331 & -3.174873 & -0.641595 \\
\hline $\mathrm{N}$ & 1.353191 & -0.625340 & 3.579544 \\
\hline $\mathrm{N}$ & -4.245955 & 0.425567 & -0.308567 \\
\hline $\mathrm{O}$ & -4.890973 & 2.479430 & -1.200136 \\
\hline $\mathrm{C}$ & -3.319062 & -3.053617 & -0.858254 \\
\hline $\mathrm{C}$ & 8.563419 & -3.932205 & 0.394281 \\
\hline $\mathrm{C}$ & 0.834378 & -0.174541 & 4.814046 \\
\hline $\mathrm{C}$ & -5.486036 & -0.225790 & -0.327090 \\
\hline $\mathrm{C}$ & -4.336666 & -2.400584 & -0.629113 \\
\hline $\mathrm{C}$ & -0.573789 & -0.164566 & 4.953717 \\
\hline $\mathrm{C}$ & 1.624368 & 0.255087 & 5.897452 \\
\hline $\mathrm{C}$ & -5.524582 & -1.649644 & -0.427293 \\
\hline $\mathrm{C}$ & -6.710675 & 0.466389 & -0.225808 \\
\hline $\mathrm{C}$ & -1.171401 & 0.271323 & 6.133687 \\
\hline $\mathrm{C}$ & 1.019435 & 0.686504 & 7.088331 \\
\hline $\mathrm{C}$ & -6.772854 & -2.322566 & -0.386892 \\
\hline $\mathrm{C}$ & -7.919673 & -0.228922 & -0.205294 \\
\hline $\mathrm{C}$ & -0.379827 & 0.702147 & 7.216320 \\
\hline $\mathrm{C}$ & -7.988572 & -1.639929 & -0.274849 \\
\hline $\mathrm{O}$ & -1.068615 & 1.108180 & 8.336529 \\
\hline $\mathrm{C}$ & -9.352124 & -2.355608 & -0.230596 \\
\hline $\mathrm{C}$ & -0.293221 & 1.535223 & 9.449566 \\
\hline $\mathrm{C}$ & -9.212875 & -3.888611 & -0.323921 \\
\hline $\mathrm{C}$ & -10.066701 & -2.013777 & 1.100859 \\
\hline $\mathrm{C}$ & -10.223874 & -1.874611 & -1.417406 \\
\hline $\mathrm{H}$ & -2.081904 & 1.479267 & 0.032138 \\
\hline $\mathrm{H}$ & 1.966035 & -1.627220 & 1.466709 \\
\hline $\mathrm{H}$ & -0.840056 & -2.405097 & -0.094047 \\
\hline $\mathrm{H}$ & 1.152416 & -5.673646 & -2.180452 \\
\hline $\mathrm{H}$ & -3.523713 & 3.958180 & -2.345159 \\
\hline $\mathrm{H}$ & -2.461123 & 6.149811 & -2.783681 \\
\hline $\mathrm{H}$ & 0.318183 & 5.462427 & 0.460306 \\
\hline $\mathrm{H}$ & -0.751004 & 3.237546 & 0.883408 \\
\hline $\mathrm{H}$ & -0.186546 & 8.944100 & -2.271720 \\
\hline $\mathrm{H}$ & -0.761082 & 7.628873 & -3.357701 \\
\hline $\mathrm{H}$ & -1.867598 & 8.317297 & -2.112979 \\
\hline $\mathrm{H}$ & 5.370137 & -0.731141 & 2.678174 \\
\hline $\mathrm{H}$ & 5.005731 & -3.764956 & -1.250880 \\
\hline
\end{tabular}




$\begin{array}{cccc}\mathrm{H} & 6.891693 & -5.032919 & -1.515715 \\ \mathrm{H} & 6.838459 & -3.628996 & -2.624235 \\ \mathrm{H} & 7.412039 & -1.497902 & 1.535412 \\ \mathrm{H} & 8.725116 & -1.240157 & -0.354307 \\ \mathrm{H} & 7.895667 & -1.398929 & -1.925840 \\ \mathrm{H} & 9.431240 & -2.266533 & -1.630717 \\ \mathrm{H} & 8.387595 & -4.436201 & -2.276590 \\ \mathrm{H} & -3.149480 & -5.335027 & -2.327758 \\ \mathrm{H} & 8.034597 & -4.822851 & 0.775038 \\ \mathrm{H} & 8.816597 & -3.296327 & 1.259423 \\ \mathrm{H} & 9.510621 & -4.264702 & -0.067820 \\ \mathrm{H} & -1.199504 & -0.510756 & 4.123257 \\ \mathrm{H} & 2.710428 & 0.241522 & 5.804215 \\ \mathrm{H} & -6.695710 & 1.555285 & -0.175899 \\ \mathrm{H} & -2.260267 & 0.278474 & 6.240555 \\ \mathrm{H} & 1.662330 & 1.009658 & 7.911369 \\ \mathrm{H} & -6.747871 & -3.412730 & -0.459451 \\ \mathrm{H} & -8.843104 & 0.355020 & -0.119847 \\ \mathrm{H} & -1.015936 & 1.809005 & 10.232868 \\ \mathrm{H} & 0.365007 & 0.727589 & 9.826122 \\ \mathrm{H} & 0.329261 & 2.418037 & 9.202999 \\ \mathrm{H} & -10.212612 & -4.355297 & -0.285864 \\ \mathrm{H} & -8.621448 & -4.298356 & 0.513587 \\ \mathrm{H} & -8.735389 & -4.200237 & -1.269549 \\ \mathrm{H} & -9.475289 & -2.360552 & 1.965936 \\ \mathrm{H} & -11.056933 & -2.503042 & 1.141934 \\ \mathrm{H} & -10.223674 & -0.927547 & 1.214132 \\ \mathrm{H} & -11.214845 & -2.363249 & -1.388552 \\ \mathrm{H} & -9.746796 & -2.120380 & -2.382052 \\ \mathrm{H} & -10.386366 & -0.783669 & -1.390810 \\ \mathrm{H} & -3.431481 & -0.127687 & -0.007792 \\ \mathrm{H} & 0.656535 & -0.704415 & 2.811553 \\ \mathrm{~F} & -1.065221 & -6.710867 & -3.067677 \\ \mathrm{~S} & -0.769764 & -0.050378 & 1.049903 \\ \mathrm{H} & -1.015640 & 0.799561 & 2.086724\end{array}$

Statistical Thermodynamic Analysis

Temperature $=298.150$ Kelvin $\quad$ Pressure $=1.00000$ Atm

\begin{tabular}{|c|c|}
\hline-3480.55633218 & Predicted Change $=-1.270682 \mathrm{D}-07$ \\
\hline Zero-point correction $(\mathrm{ZPE})=$ & -3479.25851 .29782 \\
\hline Internal Energy $(U)=$ & -3479.17581 .38048 \\
\hline Enthalpy $(\mathrm{H})=$ & $\begin{array}{lll}-3479.1748 & 1.38143\end{array}$ \\
\hline Gibbs Free Energy $(G)=$ & $-3479.3885 \quad 1.16782$ \\
\hline Frequencies -- & 11.1668 \\
\hline
\end{tabular}


$R=F$ receptor bound with $B r-($ no $T B A)$

Supporting Information: 010-bound_noTBA_F_Br.log

Using Gaussian 09: EM64L-G09RevE.01 30-Nov-2015

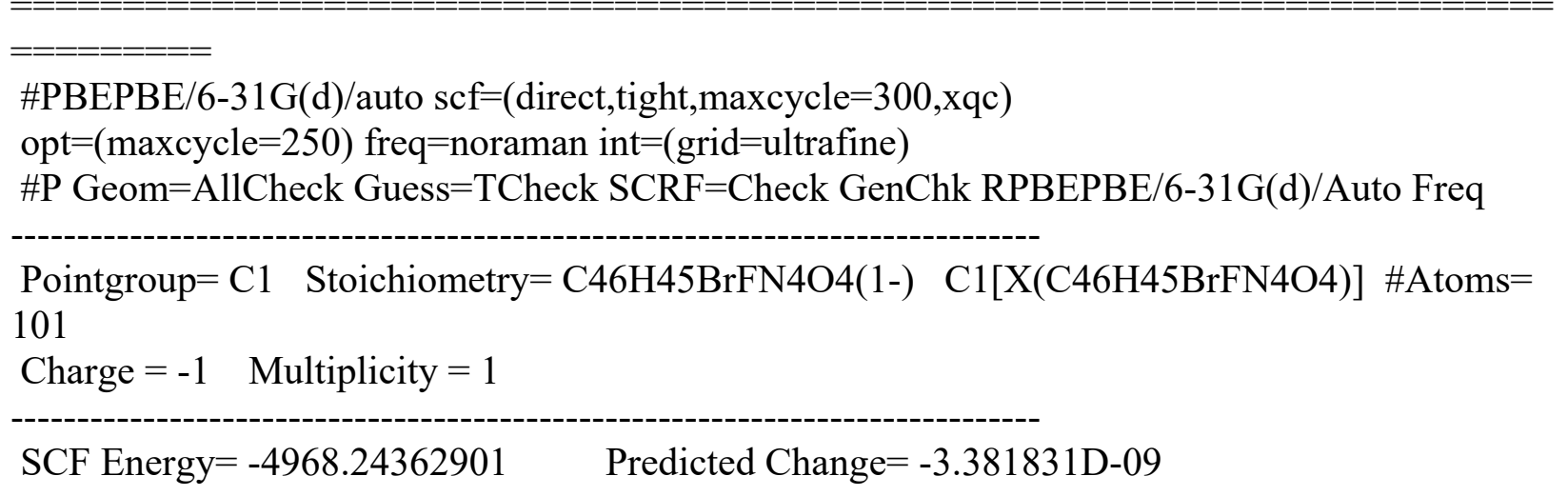

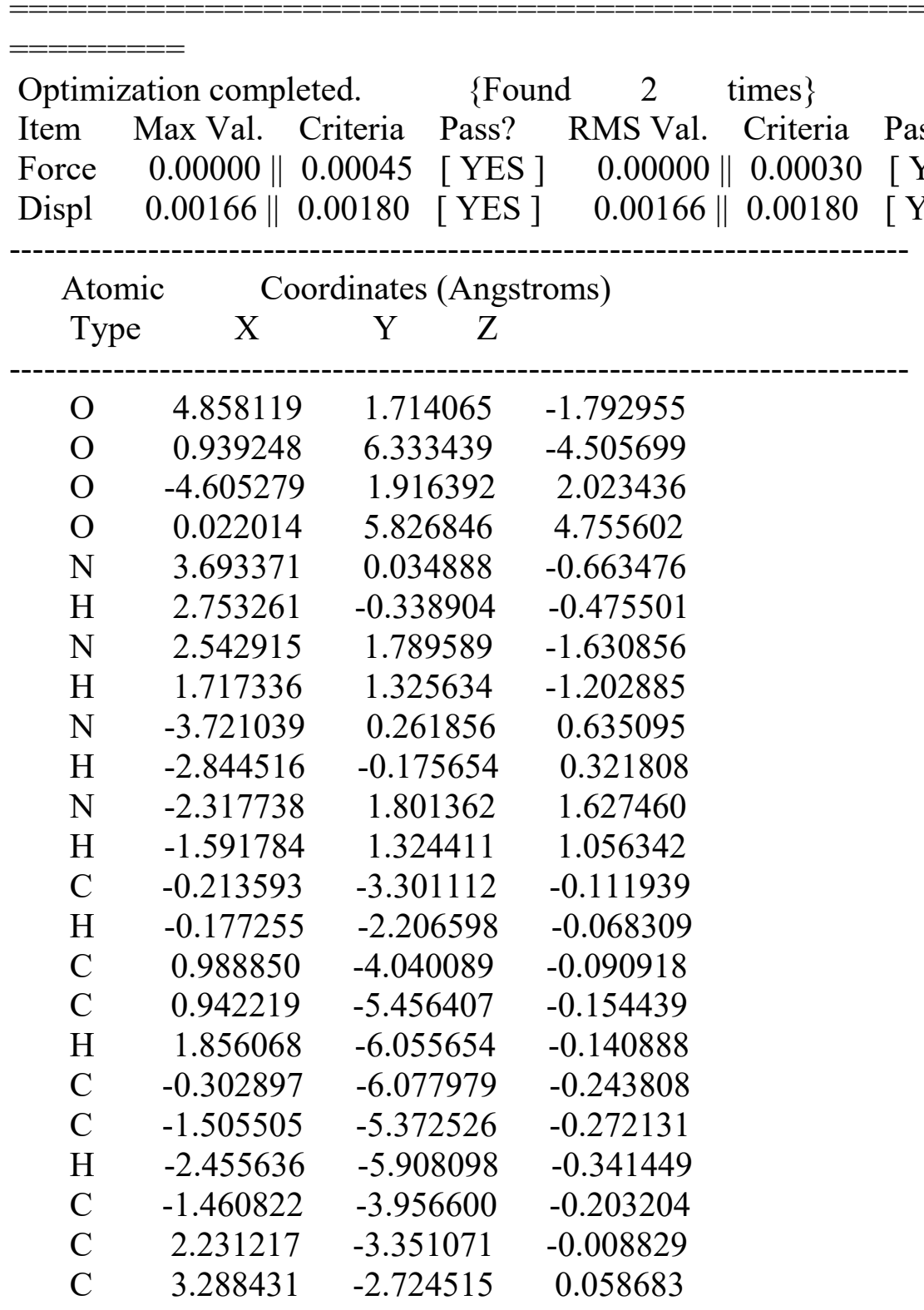




\begin{tabular}{|c|c|c|c|}
\hline $\mathrm{C}$ & 4.559989 & -2.098086 & 0.154855 \\
\hline $\mathrm{C}$ & 4.774444 & -0.733339 & -0.230676 \\
\hline $\mathrm{C}$ & 6.088076 & -0.225291 & -0.132491 \\
\hline $\mathrm{H}$ & 6.268100 & 0.803747 & -0.441303 \\
\hline $\mathrm{C}$ & 7.139531 & -1.022271 & 0.329207 \\
\hline $\mathrm{H}$ & 8.132399 & -0.565400 & 0.383598 \\
\hline $\mathrm{C}$ & 6.955204 & -2.362668 & 0.725484 \\
\hline $\mathrm{C}$ & 5.650912 & -2.867671 & 0.625023 \\
\hline $\mathrm{H}$ & 5.433432 & -3.902903 & 0.909395 \\
\hline $\mathrm{C}$ & 3.783074 & 1.235682 & -1.401895 \\
\hline $\mathrm{C}$ & 2.241848 & 2.947002 & -2.370846 \\
\hline $\mathrm{C}$ & 3.197933 & 3.752924 & -3.018661 \\
\hline $\mathrm{H}$ & 4.252120 & 3.482709 & -2.950650 \\
\hline $\mathrm{C}$ & 2.794639 & 4.889125 & -3.739278 \\
\hline $\mathrm{H}$ & 3.562296 & 5.493589 & -4.230722 \\
\hline $\mathrm{C}$ & 1.437169 & 5.237566 & -3.822646 \\
\hline $\mathrm{C}$ & 0.480098 & 4.433548 & -3.174023 \\
\hline $\mathrm{H}$ & -0.576408 & 4.711400 & -3.240615 \\
\hline $\mathrm{C}$ & 0.873816 & 3.305315 & -2.459654 \\
\hline $\mathrm{H}$ & 0.130225 & 2.676853 & -1.954088 \\
\hline $\mathrm{C}$ & 1.888778 & 7.158702 & -5.159167 \\
\hline $\mathrm{H}$ & 1.312456 & 7.966503 & -5.637036 \\
\hline $\mathrm{H}$ & 2.453861 & 6.605992 & -5.937197 \\
\hline $\mathrm{H}$ & 2.613830 & 7.601850 & -4.446543 \\
\hline $\mathrm{C}$ & -2.653556 & -3.181051 & -0.219168 \\
\hline $\mathrm{C}$ & -3.660417 & -2.473211 & -0.220214 \\
\hline $\mathrm{C}$ & -4.865153 & -1.720990 & -0.212505 \\
\hline $\mathrm{C}$ & -4.906703 & -0.365525 & 0.254337 \\
\hline $\mathrm{C}$ & -6.161527 & 0.279038 & 0.286407 \\
\hline $\mathrm{H}$ & -6.209156 & 1.300367 & 0.663126 \\
\hline $\mathrm{C}$ & -7.321203 & -0.377859 & -0.135541 \\
\hline $\mathrm{H}$ & -8.262122 & 0.178849 & -0.089537 \\
\hline $\mathrm{C}$ & -7.306693 & -1.702809 & -0.618093 \\
\hline $\mathrm{C}$ & -6.060064 & -2.345173 & -0.642356 \\
\hline $\mathrm{H}$ & -5.975221 & -3.378974 & -0.994447 \\
\hline $\mathrm{C}$ & -3.623509 & 1.387711 & 1.481543 \\
\hline $\mathrm{C}$ & -1.828437 & 2.837128 & 2.442272 \\
\hline $\mathrm{C}$ & -2.627974 & 3.650683 & 3.267861 \\
\hline $\mathrm{H}$ & -3.706248 & 3.488785 & 3.286722 \\
\hline $\mathrm{C}$ & -2.039828 & 4.656505 & 4.052320 \\
\hline $\mathrm{H}$ & -2.688273 & 5.271199 & 4.683079 \\
\hline $\mathrm{C}$ & -0.651722 & 4.865437 & 4.022797 \\
\hline $\mathrm{C}$ & 0.148781 & 4.053595 & 3.196000 \\
\hline $\mathrm{H}$ & 1.229846 & 4.222832 & 3.175569 \\
\hline $\mathrm{C}$ & -0.428471 & 3.053777 & 2.418104 \\
\hline $\mathrm{H}$ & 0.190511 & 2.418420 & 1.772628 \\
\hline $\mathrm{C}$ & -0.767233 & 6.656425 & 5.591356 \\
\hline $\mathrm{H}$ & -0.065566 & 7.347517 & 6.084397 \\
\hline
\end{tabular}




\begin{tabular}{lccc}
$\mathrm{H}$ & -1.307056 & 6.073162 & 6.365016 \\
$\mathrm{H}$ & -1.509133 & 7.243364 & 5.012511 \\
$\mathrm{C}$ & 8.096912 & -3.260101 & 1.243196 \\
$\mathrm{C}$ & -8.570242 & -2.444968 & -1.097607 \\
$\mathrm{C}$ & 7.769674 & -3.738101 & 2.679766 \\
$\mathrm{H}$ & 6.822347 & -4.302414 & 2.714795 \\
$\mathrm{H}$ & 8.571282 & -4.398007 & 3.061093 \\
$\mathrm{H}$ & 7.673190 & -2.879541 & 3.366945 \\
$\mathrm{C}$ & 9.449505 & -2.519572 & 1.279582 \\
$\mathrm{H}$ & 9.754988 & -2.176471 & 0.275495 \\
$\mathrm{H}$ & 9.416683 & -1.641207 & 1.947860 \\
$\mathrm{H}$ & 10.235868 & -3.197982 & 1.655823 \\
$\mathrm{C}$ & 8.246995 & -4.493327 & 0.317958 \\
$\mathrm{H}$ & 8.497817 & -4.183565 & -0.711548 \\
$\mathrm{H}$ & 9.051296 & -5.158098 & 0.684919 \\
$\mathrm{H}$ & 7.315823 & -5.083284 & 0.272560 \\
$\mathrm{C}$ & -8.394210 & -2.863551 & -2.578541 \\
$\mathrm{H}$ & -7.516425 & -3.517827 & -2.714604 \\
$\mathrm{H}$ & -9.285233 & -3.413666 & -2.934771 \\
$\mathrm{H}$ & -8.254546 & -1.978223 & -3.223011 \\
$\mathrm{C}$ & -8.787166 & -3.711549 & -0.232680 \\
$\mathrm{H}$ & -8.933756 & -3.442330 & 0.827870 \\
$\mathrm{H}$ & -9.680267 & -4.267024 & -0.575196 \\
$\mathrm{H}$ & -7.922826 & -4.394912 & -0.288217 \\
$\mathrm{C}$ & -9.835918 & -1.569576 & -0.992981 \\
$\mathrm{H}$ & -10.034067 & -1.259803 & 0.048169 \\
$\mathrm{H}$ & -9.755862 & -0.659545 & -1.613185 \\
$\mathrm{H}$ & -10.713629 & -2.139784 & -1.346022 \\
$\mathrm{~F}$ & -0.346472 & -7.437677 & -0.307357 \\
$\mathrm{Br}$ & -0.059677 & 0.292255 & -0.261748 \\
------------------------------------------------------------------ \\
\hline
\end{tabular}

Statistical Thermodynamic Analysis

Temperature $=298.150$ Kelvin Pressure $=1.00000 \mathrm{Atm}$

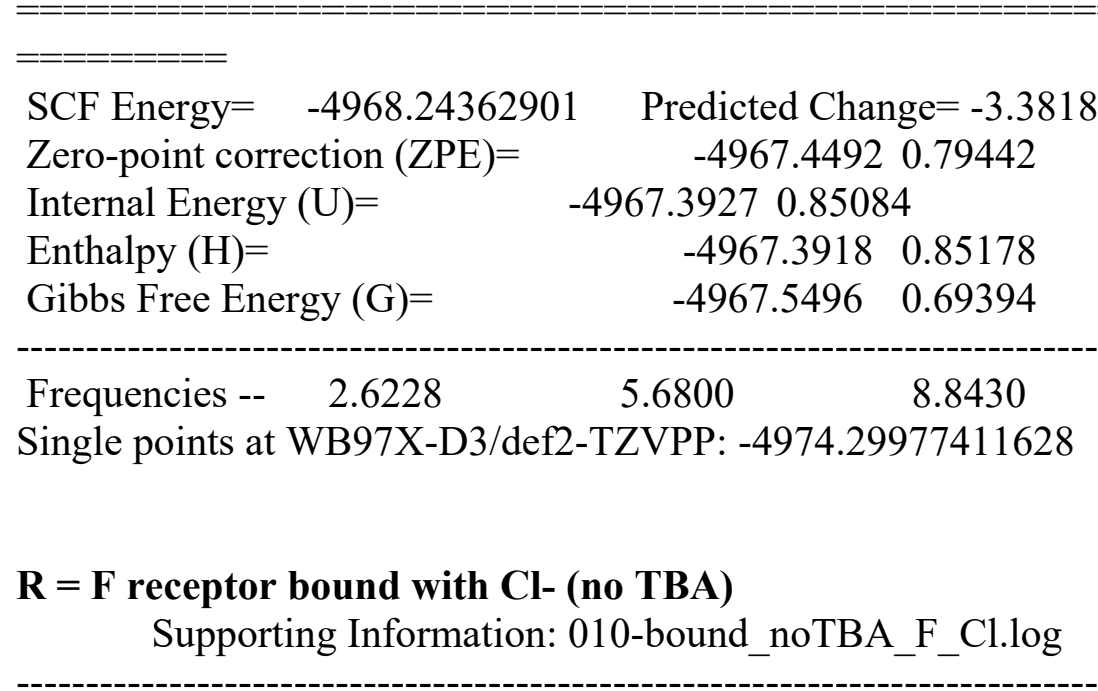


Using Gaussian 09: EM64L-G09RevE.01 30-Nov-2015

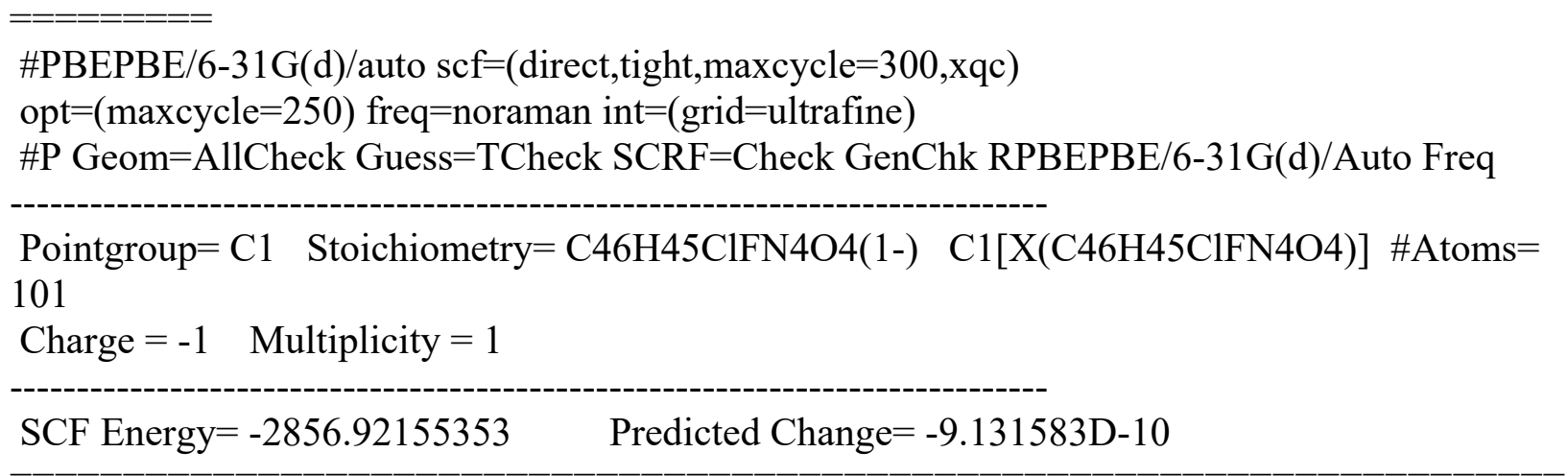

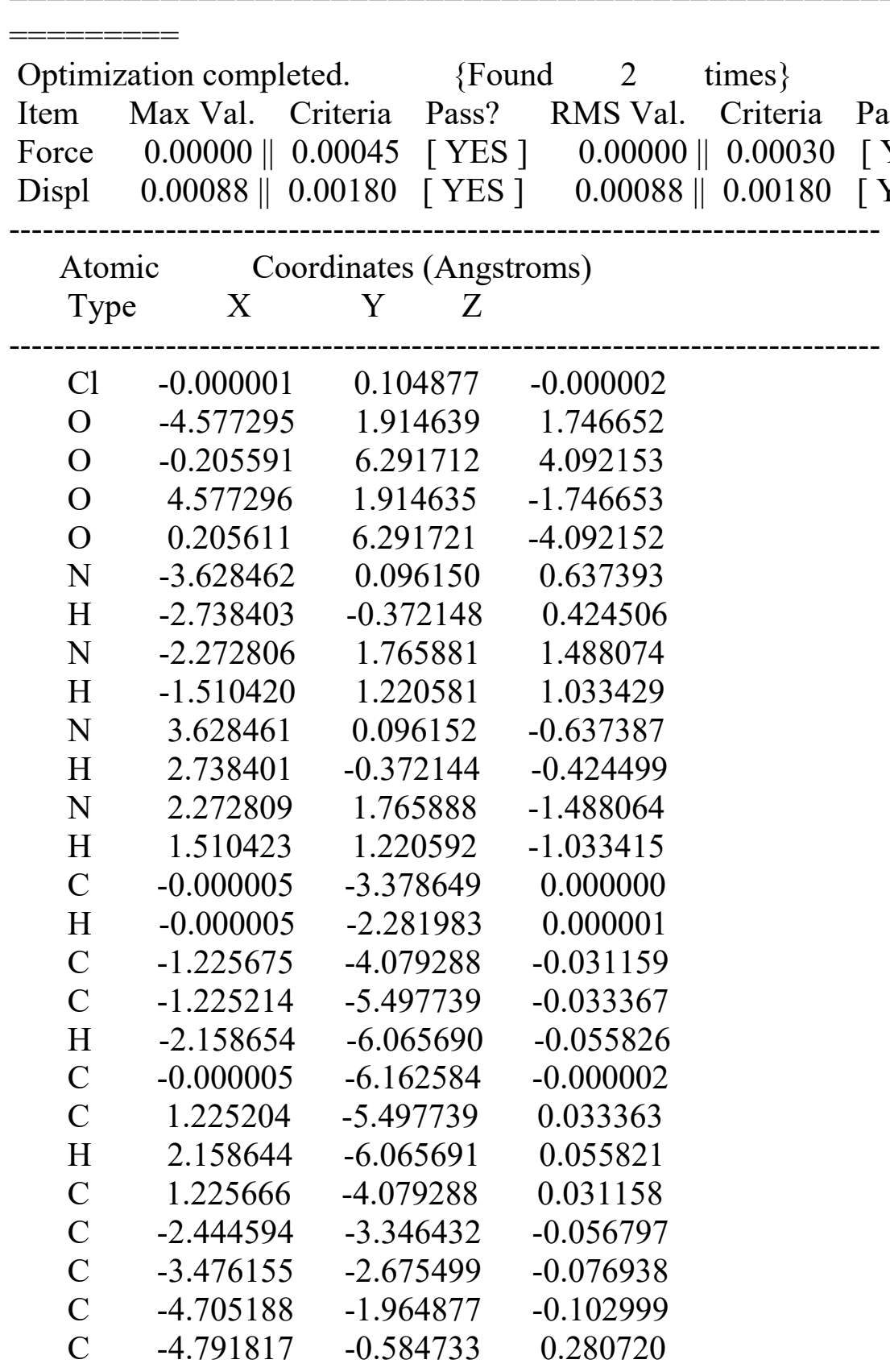




$\begin{array}{lrrr}\mathrm{C} & -6.067085 & 0.020656 & 0.257750 \\ \mathrm{H} & -6.149239 & 1.062315 & 0.565707 \\ \mathrm{C} & -7.201276 & -0.698287 & -0.130990 \\ \mathrm{H} & -8.158763 & -0.168495 & -0.129046 \\ \mathrm{C} & -7.143223 & -2.051164 & -0.523482 \\ \mathrm{C} & -5.876832 & -2.653197 & -0.497438 \\ \mathrm{H} & -5.755636 & -3.703500 & -0.784151 \\ \mathrm{C} & -3.568933 & 1.324233 & 1.330752 \\ \mathrm{C} & -1.850148 & 2.926441 & 2.163157 \\ \mathrm{C} & -2.709872 & 3.817065 & 2.836362 \\ \mathrm{H} & -3.781569 & 3.617801 & 2.839316 \\ \mathrm{C} & -2.192145 & 4.947558 & 3.489342 \\ \mathrm{H} & -2.889144 & 5.617213 & 4.001238 \\ \mathrm{C} & -0.812892 & 5.208715 & 3.481105 \\ \mathrm{C} & 0.048828 & 4.321722 & 2.808355 \\ \mathrm{H} & 1.122855 & 4.531711 & 2.800541 \\ \mathrm{C} & -0.459955 & 3.198941 & 2.160594 \\ \mathrm{H} & 0.216028 & 2.512580 & 1.638205 \\ \mathrm{C} & -1.059851 & 7.202908 & 4.762574 \\ \mathrm{H} & -0.405517 & 7.988063 & 5.173020 \\ \mathrm{H} & -1.612865 & 6.719676 & 5.593792 \\ \mathrm{H} & -0.216022 & 2.512598 & -1.638192 \\ \mathrm{H} & 0.405544 & 7.988068 & -5.173025 \\ \mathrm{H} & -1.795332 & 7.665881 & 4.073653 \\ \mathrm{C} & 2.444585 & -3.346431 & 0.056795 \\ \mathrm{C} & 3.476146 & -2.675498 & 0.076934 \\ \mathrm{C} & 4.705181 & -1.964880 & 0.102998 \\ \mathrm{C} & 4.791815 & -0.584736 & -0.280719 \\ \mathrm{C} & 6.067084 & 0.020648 & -0.257747 \\ \mathrm{H} & 6.149242 & 1.062307 & -0.565702 \\ \mathrm{C} & 7.201273 & -0.698299 & 0.130990 \\ \mathrm{H} & 8.158762 & -0.168510 & 0.129046 \\ \mathrm{C} & 7.143216 & -2.051178 & 0.523479 \\ \mathrm{C} & 5.876823 & -2.653206 & 0.497435 \\ \mathrm{H} & 5.755624 & -3.703509 & 0.784145 \\ \mathrm{C} & 3.568935 & 1.324234 & -1.330747 \\ \mathrm{C} & 1.850155 & 2.926448 & -2.163150 \\ \mathrm{H} & 2.709882 & 3.817065 & -2.836359 \\ \mathrm{H} & 2.192159 & 7.665882 & -4.073659\end{array}$




$\begin{array}{lccc}\text { C } & -8.378871 & -2.862042 & -0.962319 \\ \mathrm{C} & 8.378861 & -2.862061 & 0.962314 \\ \mathrm{C} & -8.178988 & -3.365820 & -2.413385 \\ \mathrm{H} & -7.279189 & -3.997712 & -2.503851 \\ \mathrm{H} & -9.048941 & -3.966278 & -2.739200 \\ \mathrm{H} & -8.063843 & -2.518018 & -3.110982 \\ \mathrm{C} & -9.672418 & -2.022984 & -0.918782 \\ \mathrm{H} & -9.886715 & -1.654784 & 0.099907 \\ \mathrm{H} & -9.617345 & -1.151694 & -1.594798 \\ \mathrm{H} & -10.529321 & -2.642163 & -1.239006 \\ \mathrm{C} & -8.561845 & -4.079026 & -0.021629 \\ \mathrm{H} & -8.724313 & -3.749650 & 1.019467 \\ \mathrm{H} & -9.434573 & -4.682752 & -0.333667 \\ \mathrm{H} & -7.676044 & -4.736655 & -0.030277 \\ \mathrm{C} & 8.178970 & -3.365854 & 2.413374 \\ \mathrm{H} & 7.279168 & -3.997744 & 2.503828 \\ \mathrm{H} & 9.048919 & -3.966316 & 2.739188 \\ \mathrm{H} & 8.063821 & -2.518058 & 3.110979 \\ \mathrm{C} & 8.561841 & -4.079035 & 0.021613 \\ \mathrm{H} & 8.724314 & -3.749649 & -1.019478 \\ \mathrm{H} & 9.434568 & -4.682764 & 0.333650 \\ \mathrm{H} & 7.676041 & -4.736665 & 0.030250 \\ \mathrm{C} & 9.672409 & -2.023003 & 0.918793 \\ \mathrm{H} & 9.886709 & -1.654787 & -0.099889 \\ \mathrm{H} & 9.617334 & -1.151722 & 1.594822 \\ \mathrm{H} & 10.529310 & -2.642186 & 1.239010 \\ \mathrm{~F} & -0.000005 & -7.524602 & -0.000003 \\ -------------------------------------------------------------------\end{array}$

Statistical Thermodynamic Analysis

Temperature $=298.150$ Kelvin $\quad$ Pressure $=1.00000$ Atm

\begin{tabular}{|c|c|}
\hline SCF Energy $=\quad-2856.92155353$ & Predicted Change $=-9.1315$ \\
\hline Zero-point correction $(\mathrm{ZPE})=$ & -2856.12740 .79413 \\
\hline Internal Energy $(U)=$ & -2856.07100 .85051 \\
\hline Enthalpy $(\mathrm{H})=$ & $-2856.0701 \quad 0.85145$ \\
\hline Gibbs Free Energy $(\mathrm{G})=$ & $-2856.2273 \quad 0.69417$ \\
\hline Frequencies -- 3.7057 & $6.4805 \quad 9.9776$ \\
\hline \multicolumn{2}{|c|}{ Single points at WB97X-D3/def2-TZVPP: -2860.2917178797 } \\
\hline
\end{tabular}

Using Gaussian 09: EM64L-G09RevE.01 30-Nov-2015 
\#PBEPBE/6-31G(d)/auto scf=(direct,tight,maxcycle=300,xqc)

$\mathrm{opt}=($ maxcycle $=250)$ freq $=$ noraman int $=($ grid $=$ ultrafine $)$

\#P Geom=AllCheck Guess=TCheck SCRF=Check GenChk RPBEPBE/6-31G(d)/Auto Freq

Pointgroup $=\mathrm{C} 1$ Stoichiometry $=\mathrm{C} 46 \mathrm{H} 46 \mathrm{FN} 4 \mathrm{O} 4 \mathrm{Se}(1-) \quad \mathrm{C} 1[\mathrm{X}(\mathrm{C} 46 \mathrm{H} 46 \mathrm{FN} 4 \mathrm{O} 4 \mathrm{Se})]$ \#Atoms= 102

Charge $=-1 \quad$ Multiplicity $=1$

SCF Energy $=-4796.50663819 \quad$ Predicted Change $=-8.598017 \mathrm{D}-09$

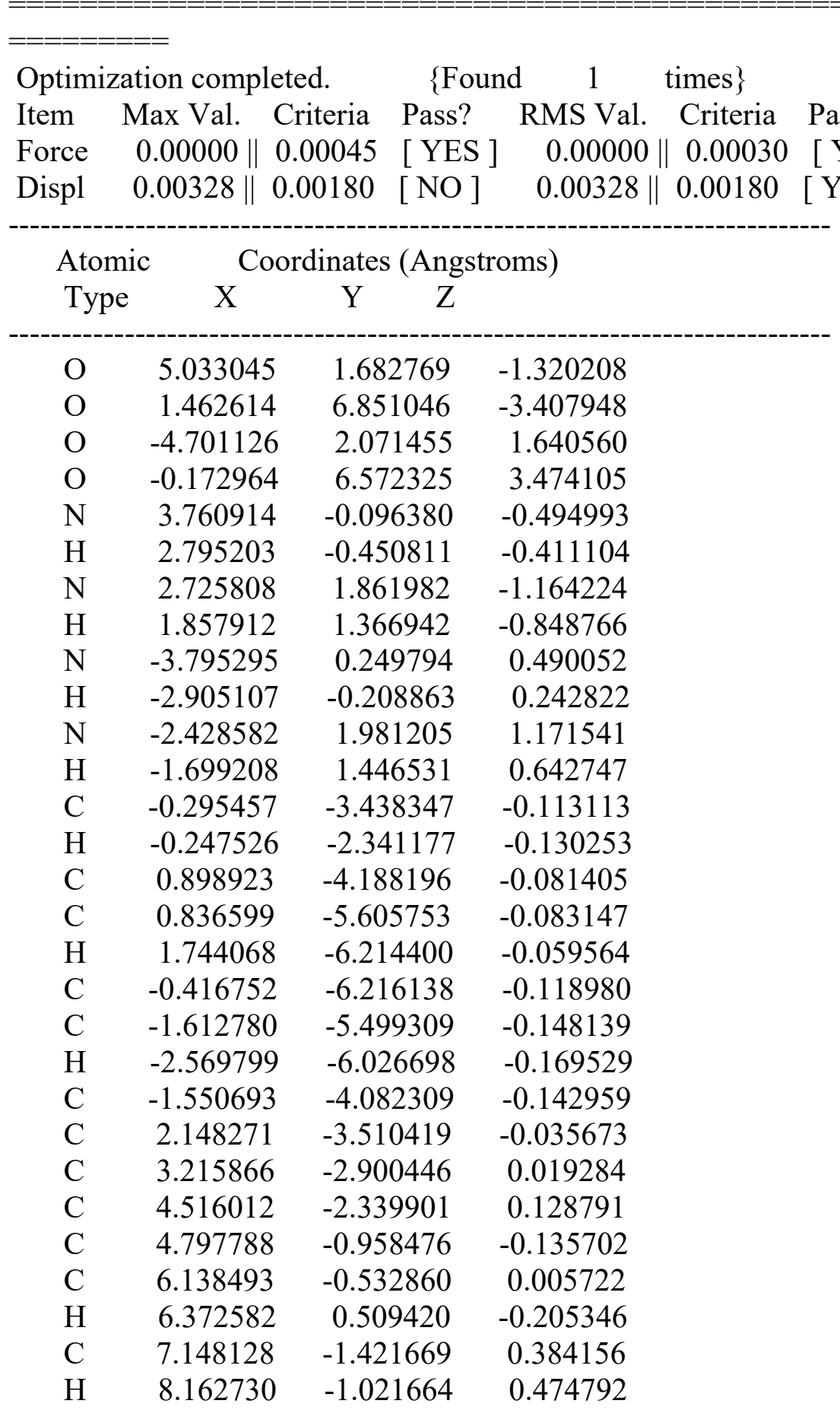




\begin{tabular}{|c|c|c|c|}
\hline $\mathrm{C}$ & 6.898088 & -2.783194 & 0.650199 \\
\hline $\mathrm{C}$ & 5.568892 & -3.205714 & 0.513732 \\
\hline $\mathrm{H}$ & 5.296065 & -4.249318 & 0.704625 \\
\hline $\mathrm{C}$ & 3.927006 & 1.205374 & -1.023965 \\
\hline $\mathrm{C}$ & 2.509514 & 3.126838 & -1.741750 \\
\hline $\mathrm{C}$ & 3.533178 & 4.008812 & -2.140973 \\
\hline $\mathrm{H}$ & 4.572553 & 3.706333 & -2.011529 \\
\hline $\mathrm{C}$ & 3.216192 & 5.256639 & -2.702236 \\
\hline $\mathrm{H}$ & 4.035817 & 5.915826 & -3.002179 \\
\hline $\mathrm{C}$ & 1.877664 & 5.646212 & -2.868728 \\
\hline $\mathrm{C}$ & 0.852365 & 4.767642 & -2.469773 \\
\hline $\mathrm{H}$ & -0.189558 & 5.076181 & -2.599617 \\
\hline $\mathrm{C}$ & 1.161344 & 3.526820 & -1.918905 \\
\hline $\mathrm{H}$ & 0.362739 & 2.838684 & -1.617472 \\
\hline $\mathrm{C}$ & 2.481579 & 7.754462 & -3.801915 \\
\hline $\mathrm{H}$ & 1.964526 & 8.644427 & -4.193900 \\
\hline $\mathrm{H}$ & 3.128441 & 7.330440 & -4.596923 \\
\hline $\mathrm{H}$ & 3.123669 & 8.053335 & -2.948579 \\
\hline $\mathrm{C}$ & -2.731637 & -3.290215 & -0.159762 \\
\hline $\mathrm{C}$ & -3.721899 & -2.559561 & -0.161269 \\
\hline $\mathrm{C}$ & -4.929343 & -1.813130 & -0.173703 \\
\hline $\mathrm{C}$ & -4.976177 & -0.423819 & 0.179788 \\
\hline $\mathrm{C}$ & -6.240470 & 0.206688 & 0.172695 \\
\hline $\mathrm{H}$ & -6.295946 & 1.255927 & 0.460136 \\
\hline $\mathrm{C}$ & -7.398855 & -0.494770 & -0.172333 \\
\hline $\mathrm{H}$ & -8.344288 & 0.056151 & -0.159687 \\
\hline $\mathrm{C}$ & -7.379103 & -1.857069 & -0.535504 \\
\hline $\mathrm{C}$ & -6.125385 & -2.484261 & -0.525276 \\
\hline $\mathrm{H}$ & -6.032238 & -3.543059 & -0.790457 \\
\hline $\mathrm{C}$ & -3.716948 & 1.499924 & 1.147280 \\
\hline $\mathrm{C}$ & -1.961659 & 3.157870 & 1.783692 \\
\hline $\mathrm{C}$ & -2.768698 & 4.067186 & 2.494380 \\
\hline $\mathrm{H}$ & -3.836973 & 3.868492 & 2.586346 \\
\hline $\mathrm{C}$ & -2.199964 & 5.214767 & 3.071398 \\
\hline $\mathrm{H}$ & -2.853243 & 5.901512 & 3.617210 \\
\hline $\mathrm{C}$ & -0.825344 & 5.472357 & 2.945218 \\
\hline $\mathrm{C}$ & -0.017294 & 4.564522 & 2.233538 \\
\hline $\mathrm{H}$ & 1.052353 & 4.772173 & 2.131650 \\
\hline $\mathrm{C}$ & -0.574804 & 3.423312 & 1.664575 \\
\hline $\mathrm{H}$ & 0.044910 & 2.706420 & 1.111256 \\
\hline $\mathrm{C}$ & -0.971808 & 7.503625 & 4.183649 \\
\hline $\mathrm{H}$ & -0.287456 & 8.299053 & 4.518370 \\
\hline $\mathrm{H}$ & -1.457076 & 7.045290 & 5.069544 \\
\hline $\mathrm{H}$ & -1.759904 & 7.948156 & 3.542299 \\
\hline $\mathrm{C}$ & 7.993182 & -3.783801 & 1.069562 \\
\hline $\mathrm{C}$ & -8.641642 & -2.650930 & -0.926032 \\
\hline $\mathrm{C}$ & 7.649885 & -4.375856 & 2.459138 \\
\hline $\mathrm{H}$ & 6.673097 & -4.888665 & 2.452317 \\
\hline
\end{tabular}




$\begin{array}{llll}\mathrm{H} & 8.416427 & -5.111044 & 2.768064 \\ \mathrm{H} & 7.605362 & -3.581371 & 3.224344 \\ \mathrm{C} & 9.383474 & -3.121777 & 1.159208 \\ \mathrm{H} & 9.699231 & -2.701323 & 0.188270 \\ \mathrm{H} & 9.402177 & -2.310134 & 1.907605 \\ \mathrm{H} & 10.135449 & -3.872872 & 1.460235 \\ \mathrm{C} & 8.071323 & -4.931656 & 0.032481 \\ \mathrm{H} & 8.332311 & -4.541108 & -0.966567 \\ \mathrm{H} & 8.840773 & -5.669605 & 0.327327 \\ \mathrm{H} & 7.109370 & -5.464348 & -0.057319 \\ \mathrm{C} & -8.488036 & -3.190223 & -2.369951 \\ \mathrm{H} & -7.603721 & -3.842525 & -2.467976 \\ \mathrm{H} & -9.377785 & -3.779344 & -2.661194 \\ \mathrm{H} & -8.372935 & -2.360384 & -3.088860 \\ \mathrm{C} & -8.826361 & -3.843171 & 0.045503 \\ \mathrm{H} & -8.955730 & -3.487976 & 1.082736 \\ \mathrm{H} & -9.719031 & -4.434709 & -0.231528 \\ \mathrm{H} & -7.954928 & -4.519584 & 0.029620 \\ \mathrm{C} & -9.916122 & -1.783937 & -0.869648 \\ \mathrm{H} & -10.097416 & -1.389380 & 0.145568 \\ \mathrm{H} & -9.859885 & -0.928760 & -1.565863 \\ \mathrm{H} & -10.793319 & -2.391574 & -1.155165 \\ \mathrm{~F} & -0.475763 & -7.576977 & -0.122724 \\ \mathrm{H} & 0.311581 & 0.017602 & 1.093683 \\ \mathrm{Se} & -0.046356 & 0.196199 & -0.334861\end{array}$

Statistical Thermodynamic Analysis

Temperature $=298.150$ Kelvin $\quad$ Pressure $=1.00000$ Atm

\begin{tabular}{|c|c|c|}
\hline \multicolumn{3}{|c|}{$\begin{array}{l}======= \\
\text { SCF Fnerove }=-479650663819 \quad \text { Predicted Chanoe }=-85988\end{array}$} \\
\hline \multicolumn{3}{|c|}{ 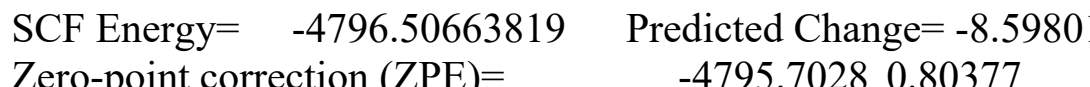 } \\
\hline Internal Energy $(U)=$ & -4795.64650 .8600 & \\
\hline Enthalpy $(\mathrm{H})=$ & -4795.6456 & 0.86098 \\
\hline Gibbs Free Energy $(G)=$ & -4795.8006 & 0.7 \\
\hline Frequencies -- 6.5624 & 7.6336 & \\
\hline \multicolumn{3}{|c|}{ Single points at WB97X-D3/def2-TZVPP: -4802.26130039795 } \\
\hline \multicolumn{3}{|c|}{$\begin{array}{l}\mathbf{R}=\mathbf{F} \text { receptor bound with SH- }(\text { no TBA) } \\
\quad \text { Supporting Information: } 010 \text {-bound noTBA F SH.log }\end{array}$} \\
\hline
\end{tabular}

Using Gaussian 09: EM64L-G09RevE.01 30-Nov-2015

\#PBEPBE/6-31G(d)/auto scf=(direct,tight, $m a x c y c l e=300, x q c)$ $\mathrm{opt}=($ maxcycle $=250)$ freq $=$ noraman int $=($ grid $=$ ultrafine $)$ 
\#P Geom=AllCheck Guess=TCheck SCRF=Check GenChk RPBEPBE/6-31G(d)/Auto Freq

Pointgroup= C1 Stoichiometry= C46H46FN4O4S(1-) C1[X(C46H46FN4O4S)] \#Atoms= 102

Charge $=-1 \quad$ Multiplicity $=1$

SCF Energy $=-2795.49032126 \quad$ Predicted Change $=-9.574982 \mathrm{D}-09$

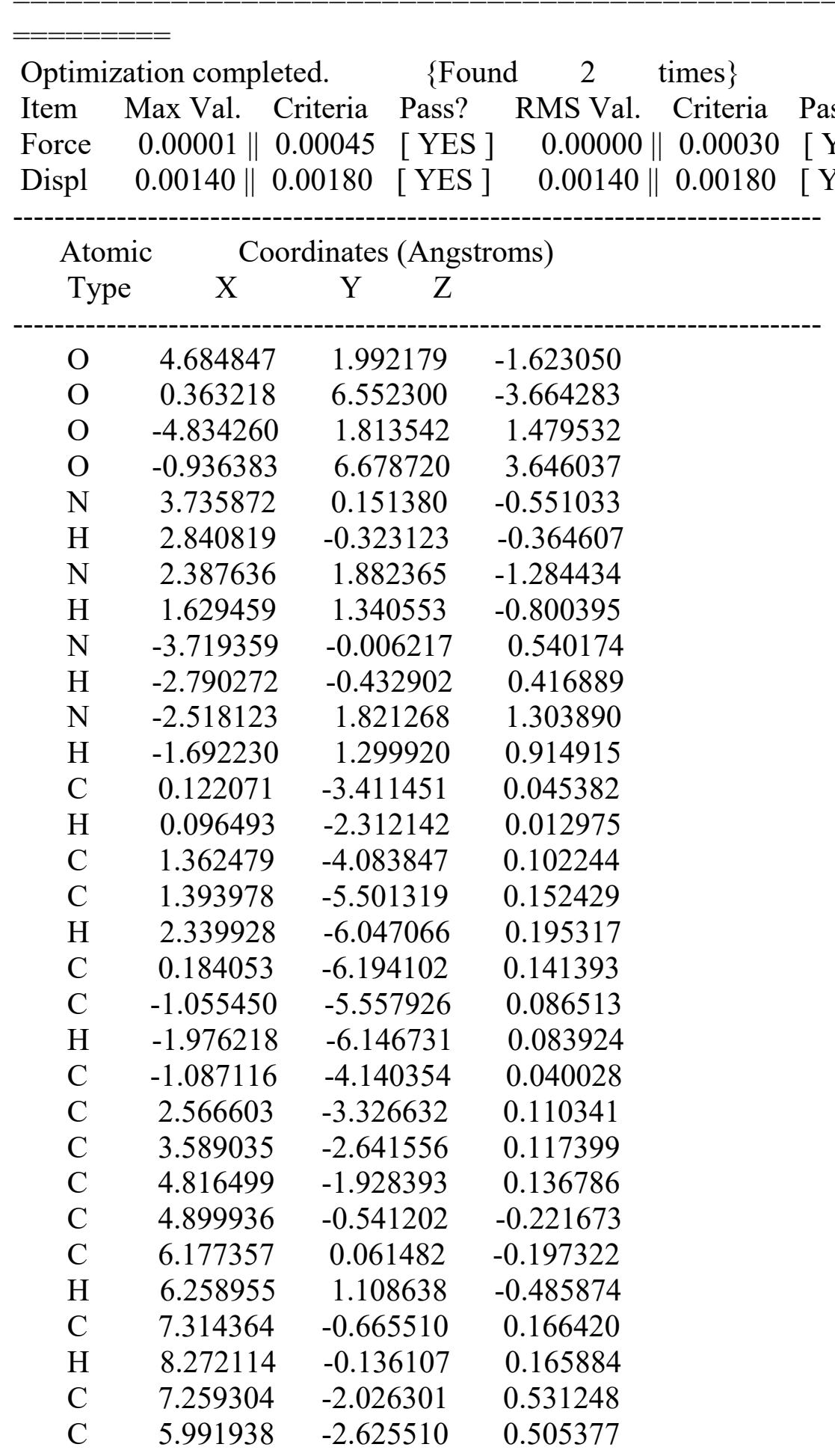




\begin{tabular}{|c|c|c|c|}
\hline $\mathrm{H}$ & 5.871593 & -3.681090 & 0.772494 \\
\hline $\mathrm{C}$ & 3.677843 & 1.407549 & -1.194893 \\
\hline $\mathrm{C}$ & 1.973850 & 3.075591 & -1.906596 \\
\hline $\mathrm{C}$ & 2.829400 & 3.949327 & -2.608016 \\
\hline $\mathrm{H}$ & 3.890671 & 3.710072 & -2.674936 \\
\hline $\mathrm{C}$ & 2.322876 & 5.114934 & -3.205570 \\
\hline $\mathrm{H}$ & 3.017407 & 5.768443 & -3.741233 \\
\hline $\mathrm{C}$ & 0.958248 & 5.431044 & -3.113164 \\
\hline $\mathrm{C}$ & 0.100295 & 4.562970 & -2.411952 \\
\hline $\mathrm{H}$ & -0.961803 & 4.815363 & -2.335359 \\
\hline $\mathrm{C}$ & 0.598449 & 3.404728 & -1.820428 \\
\hline $\mathrm{H}$ & -0.076123 & 2.736512 & -1.273999 \\
\hline $\mathrm{C}$ & 1.215118 & 7.443047 & -4.364301 \\
\hline $\mathrm{H}$ & 0.571893 & 8.263386 & -4.719832 \\
\hline $\mathrm{H}$ & 1.698998 & 6.956914 & -5.235978 \\
\hline $\mathrm{H}$ & 2.007644 & 7.859941 & -3.710013 \\
\hline $\mathrm{C}$ & -2.324147 & -3.440037 & -0.012840 \\
\hline $\mathrm{C}$ & -3.376325 & -2.803746 & -0.063427 \\
\hline $\mathrm{C}$ & -4.640829 & -2.163171 & -0.146537 \\
\hline $\mathrm{C}$ & -4.822754 & -0.775489 & 0.170580 \\
\hline $\mathrm{C}$ & -6.131081 & -0.252051 & 0.068550 \\
\hline $\mathrm{H}$ & -6.288009 & 0.795658 & 0.321078 \\
\hline $\mathrm{C}$ & -7.204944 & -1.055203 & -0.325752 \\
\hline $\mathrm{H}$ & -8.190723 & -0.583690 & -0.385352 \\
\hline $\mathrm{C}$ & -7.053214 & -2.419548 & -0.646428 \\
\hline $\mathrm{C}$ & -5.755088 & -2.939446 & -0.546061 \\
\hline $\mathrm{H}$ & -5.559376 & -3.991985 & -0.778099 \\
\hline $\mathrm{C}$ & -3.771011 & 1.270772 & 1.141358 \\
\hline $\mathrm{C}$ & -2.218978 & 3.057533 & 1.908293 \\
\hline $\mathrm{C}$ & -3.169049 & 3.898392 & 2.523370 \\
\hline $\mathrm{H}$ & -4.216435 & 3.597145 & 2.530415 \\
\hline $\mathrm{C}$ & -2.772912 & 5.110679 & 3.111350 \\
\hline $\mathrm{H}$ & -3.538908 & 5.736497 & 3.578242 \\
\hline $\mathrm{C}$ & -1.426306 & 5.506986 & 3.096411 \\
\hline $\mathrm{C}$ & -0.474270 & 4.672367 & 2.481415 \\
\hline $\mathrm{H}$ & 0.573624 & 4.987639 & 2.463859 \\
\hline $\mathrm{C}$ & -0.863054 & 3.468782 & 1.898505 \\
\hline $\mathrm{H}$ & -0.116746 & 2.825561 & 1.418173 \\
\hline $\mathrm{C}$ & -1.884578 & 7.538097 & 4.255856 \\
\hline $\mathrm{H}$ & -1.316122 & 8.407092 & 4.622994 \\
\hline $\mathrm{H}$ & -2.397116 & 7.051727 & 5.110862 \\
\hline $\mathrm{H}$ & -2.653900 & 7.884566 & 3.536143 \\
\hline $\mathrm{C}$ & 8.498286 & -2.847216 & 0.940845 \\
\hline $\mathrm{C}$ & -8.220629 & -3.324719 & -1.087215 \\
\hline $\mathrm{C}$ & 8.312952 & -3.379748 & 2.383562 \\
\hline $\mathrm{H}$ & 7.413165 & -4.012147 & 2.470717 \\
\hline $\mathrm{H}$ & 9.185322 & -3.987792 & 2.688209 \\
\hline $\mathrm{H}$ & 8.206495 & -2.545905 & 3.099172 \\
\hline
\end{tabular}




$\begin{array}{cccc}\mathrm{C} & 9.792382 & -2.008853 & 0.900562 \\ \mathrm{H} & 9.996312 & -1.620504 & -0.112748 \\ \mathrm{H} & 9.745485 & -1.151188 & 1.594396 \\ \mathrm{H} & 10.651869 & -2.635180 & 1.199259 \\ \mathrm{C} & 8.669967 & -4.045443 & -0.025703 \\ \mathrm{H} & 8.821805 & -3.695532 & -1.061682 \\ \mathrm{H} & 9.545218 & -4.656149 & 0.264992 \\ \mathrm{H} & 7.783484 & -4.702187 & -0.020844 \\ \mathrm{C} & -7.936438 & -3.884187 & -2.503444 \\ \mathrm{H} & -6.998449 & -4.464347 & -2.530608 \\ \mathrm{H} & -8.756750 & -4.550828 & -2.829391 \\ \mathrm{H} & -7.844549 & -3.065203 & -3.237940 \\ \mathrm{C} & -8.366231 & -4.504369 & -0.093928 \\ \mathrm{H} & -8.587748 & -4.136304 & 0.923049 \\ \mathrm{H} & -9.188568 & -5.175215 & -0.405813 \\ \mathrm{H} & -7.442753 & -5.105473 & -0.038543 \\ \mathrm{C} & -9.562417 & -2.565233 & -1.130791 \\ \mathrm{H} & -9.836185 & -2.162140 & -0.139938 \\ \mathrm{H} & -9.533650 & -1.725681 & -1.847389 \\ \mathrm{H} & -10.368539 & -3.250199 & -1.448933 \\ \mathrm{~F} & 0.214314 & -7.555153 & 0.187618 \\ \mathrm{~S} & 0.061753 & 0.142353 & 0.218455 \\ \mathrm{H} & 0.511224 & 0.104106 & 1.504582\end{array}$

Statistical Thermodynamic Analysis

Temperature $=298.150$ Kelvin Pressure $=1.00000 \mathrm{Atm}$

\begin{tabular}{|c|c|}
\hline SCF Energy $=\quad-2795.49032126$ & Predicted Change $=-9.574982 \mathrm{D}-09$ \\
\hline Zero-point correction $(\mathrm{ZPE})=$ & -2794.68920 .80109 \\
\hline Internal Energy $(U)=$ & 2794.63220 .85806 \\
\hline Enthalpy $(\mathrm{H})=$ & $-2794.6313 \quad 0.85901$ \\
\hline Gibbs Free Energy $(\mathrm{G})=$ & $-2794.7883 \quad 0.70196$ \\
\hline $\begin{array}{l}\text { Frequencies -- } 6.0998 \\
\text { Single points at WB97X-D3/def2- }\end{array}$ & $\begin{array}{cc}6.8846 & 12.7408 \\
\text {-TZVPP: } & -2798.84596446817\end{array}$ \\
\hline \multicolumn{2}{|c|}{$\begin{array}{l}\mathbf{R}=\mathbf{H} \text { receptor unbound (from crystal structure) } \\
\quad \text { Supporting Information: } 000 \text {-unbound_crystal_structure_H-back.log }\end{array}$} \\
\hline
\end{tabular}

\#PBEPBE/6-31G(d)/auto scf=(direct,tight,maxcycle=300,xqc) opt $=($ maxcycle $=250$, tight $)$ freq $=$ noraman int $=($ grid $=$ ultrafine $)$ \#P Geom=AllCheck Guess=TCheck SCRF=Check GenChk RPBEPBE/6-31G(d)/Auto Freq 
Pointgroup $=\mathrm{C} 1 \quad$ Stoichiometry= C46H46N4O4 C1[X(C46H46N4O4) $]$ \#Atoms $=100$ Charge $=0 \quad$ Multiplicity $=1$

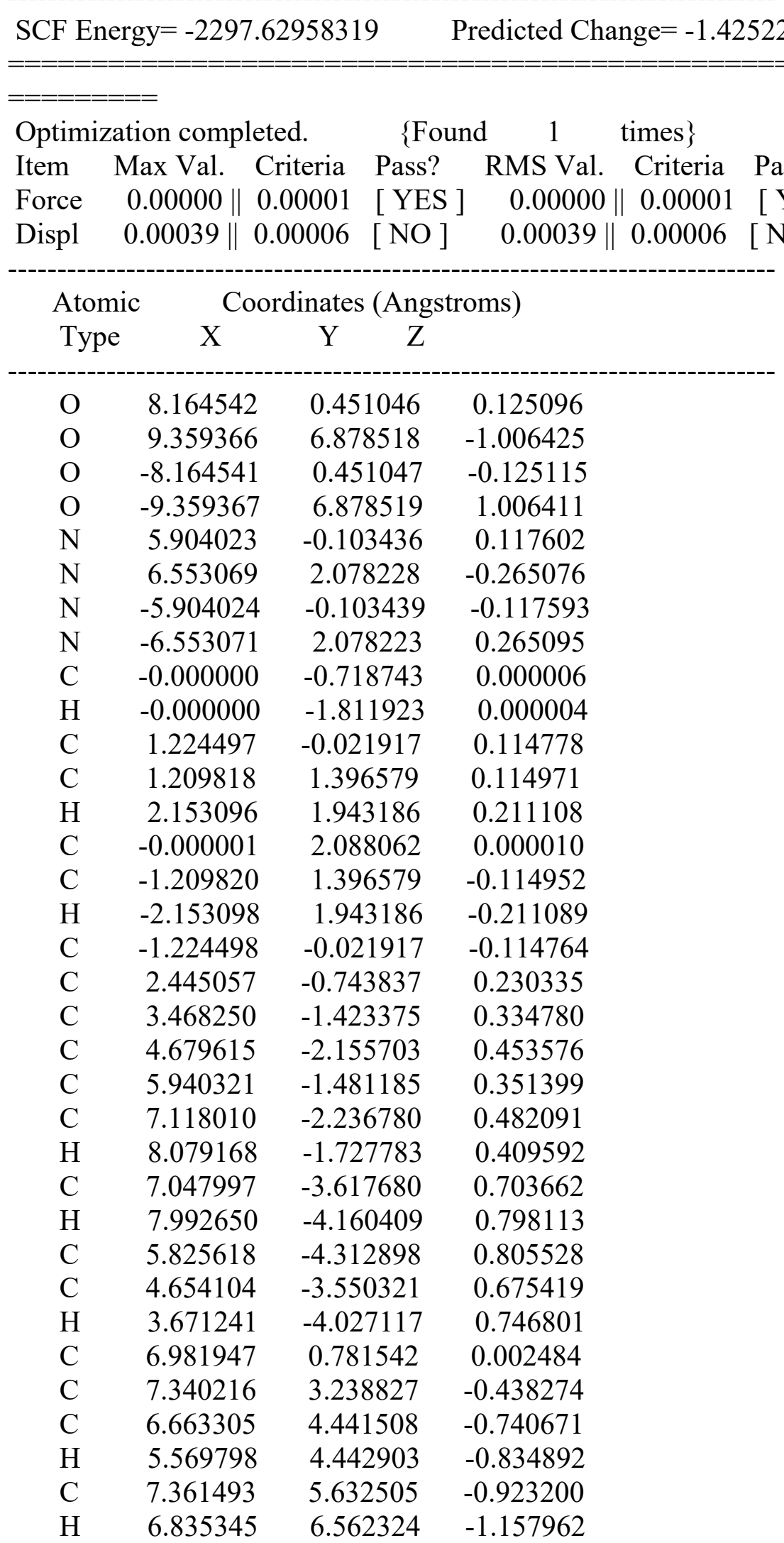




\begin{tabular}{|c|c|c|c|}
\hline $\mathrm{C}$ & 8.765277 & 5.655951 & -0.807853 \\
\hline $\mathrm{C}$ & 9.444955 & 4.462869 & -0.506957 \\
\hline $\mathrm{H}$ & 10.533522 & 4.446426 & -0.411076 \\
\hline $\mathrm{C}$ & 8.742245 & 3.262651 & -0.322946 \\
\hline $\mathrm{H}$ & 9.274062 & 2.339412 & -0.092691 \\
\hline $\mathrm{C}$ & 10.778358 & 6.932578 & -0.899140 \\
\hline $\mathrm{H}$ & 11.053814 & 7.980242 & -1.090580 \\
\hline $\mathrm{H}$ & 11.270711 & 6.282286 & -1.648215 \\
\hline $\mathrm{H}$ & 11.124138 & 6.642639 & 0.112206 \\
\hline $\mathrm{C}$ & -2.445058 & -0.743838 & -0.230324 \\
\hline $\mathrm{C}$ & -3.468250 & -1.423376 & -0.334772 \\
\hline $\mathrm{C}$ & -4.679615 & -2.155704 & -0.453571 \\
\hline $\mathrm{C}$ & -5.940321 & -1.481187 & -0.351396 \\
\hline $\mathrm{C}$ & -7.118010 & -2.236782 & -0.482093 \\
\hline $\mathrm{H}$ & -8.079167 & -1.727784 & -0.409595 \\
\hline $\mathrm{C}$ & -7.047996 & -3.617681 & -0.703667 \\
\hline $\mathrm{H}$ & -7.992648 & -4.160410 & -0.798122 \\
\hline $\mathrm{C}$ & -5.825616 & -4.312899 & -0.805532 \\
\hline $\mathrm{C}$ & -4.654102 & -3.550322 & -0.675418 \\
\hline $\mathrm{H}$ & -3.671239 & -4.027117 & -0.746800 \\
\hline $\mathrm{C}$ & -6.981948 & 0.781540 & -0.002483 \\
\hline $\mathrm{C}$ & -7.340219 & 3.238824 & 0.438282 \\
\hline $\mathrm{C}$ & -8.742246 & 3.262651 & 0.322934 \\
\hline $\mathrm{H}$ & -9.274061 & 2.339414 & 0.092669 \\
\hline $\mathrm{C}$ & -9.444955 & 4.462871 & 0.506937 \\
\hline $\mathrm{H}$ & -10.533521 & 4.446431 & 0.411040 \\
\hline $\mathrm{C}$ & -8.765278 & 5.655950 & 0.807846 \\
\hline $\mathrm{C}$ & -7.361496 & 5.632500 & 0.923214 \\
\hline $\mathrm{H}$ & -6.835349 & 6.562318 & 1.157985 \\
\hline $\mathrm{C}$ & -6.663309 & 4.441502 & 0.740692 \\
\hline $\mathrm{H}$ & -5.569803 & 4.442893 & 0.834929 \\
\hline $\mathrm{C}$ & -10.778358 & 6.932581 & 0.899114 \\
\hline $\mathrm{H}$ & -11.053814 & 7.980244 & 1.090557 \\
\hline $\mathrm{H}$ & -11.270718 & 6.282286 & 1.648183 \\
\hline $\mathrm{H}$ & -11.124130 & 6.642647 & -0.112236 \\
\hline $\mathrm{H}$ & 4.952160 & 0.266273 & 0.049402 \\
\hline $\mathrm{H}$ & 5.553506 & 2.227926 & -0.396022 \\
\hline $\mathrm{H}$ & -4.952161 & 0.266269 & -0.049384 \\
\hline $\mathrm{H}$ & -5.553510 & 2.227917 & 0.396063 \\
\hline $\mathrm{C}$ & -5.735030 & -5.832583 & -1.049581 \\
\hline $\mathrm{C}$ & -7.126582 & -6.488559 & -1.160363 \\
\hline $\mathrm{H}$ & -7.716509 & -6.359727 & -0.235975 \\
\hline $\mathrm{H}$ & -7.709017 & -6.077242 & -2.003376 \\
\hline $\mathrm{H}$ & -7.011705 & -7.572636 & -1.333282 \\
\hline $\mathrm{C}$ & -4.977184 & -6.498657 & 0.125927 \\
\hline $\mathrm{H}$ & -3.956666 & -6.094148 & 0.237824 \\
\hline $\mathrm{H}$ & -5.509199 & -6.339651 & 1.079910 \\
\hline $\mathrm{H}$ & -4.890186 & -7.587376 & -0.042275 \\
\hline
\end{tabular}




$\begin{array}{cccc}\mathrm{C} & -4.967629 & -6.096976 & -2.369054 \\ \mathrm{H} & -5.492673 & -5.645642 & -3.228703 \\ \mathrm{H} & -3.946821 & -5.678985 & -2.340639 \\ \mathrm{H} & -4.880718 & -7.183541 & -2.550705 \\ \mathrm{C} & 5.735033 & -5.832583 & 1.049575 \\ \mathrm{C} & 4.967638 & -6.096976 & 2.369052 \\ \mathrm{H} & 3.946832 & -5.678984 & 2.340643 \\ \mathrm{H} & 4.880727 & -7.183542 & 2.550702 \\ \mathrm{H} & 5.492688 & -5.645645 & 3.228699 \\ \mathrm{C} & 7.126584 & -6.488559 & 1.160347 \\ \mathrm{H} & 7.716507 & -6.359724 & 0.235957 \\ \mathrm{H} & 7.709025 & -6.077246 & 2.003358 \\ \mathrm{H} & 7.011709 & -7.572637 & 1.333263 \\ \mathrm{C} & 4.977180 & -6.498655 & -0.125930 \\ \mathrm{H} & 3.956661 & -6.094145 & -0.237821 \\ \mathrm{H} & 5.509189 & -6.339647 & -1.079915 \\ \mathrm{H} & 4.890183 & -7.587374 & 0.042270 \\ \mathrm{H} & -0.000001 & 3.182893 & 0.000012\end{array}$

Statistical Thermodynamic Analysis

Temperature $=298.150$ Kelvin $\quad$ Pressure $=1.00000$ Atm

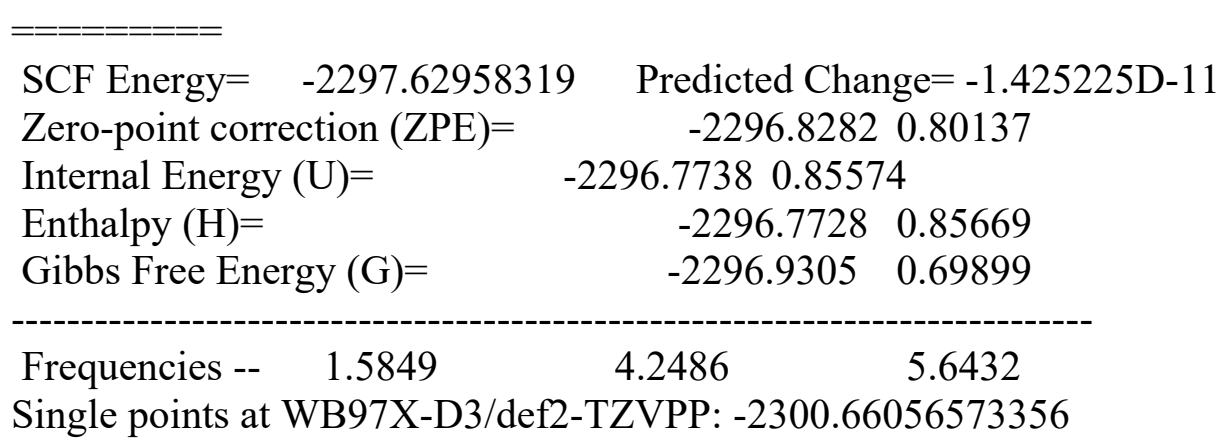

\section{$R=H$ receptor unbound (from conformational search)}

Supporting Information: 000-unbound_conf_search_H.log

Using Gaussian 09: EM64L-G09RevE.01 30-Nov-2015

\footnotetext{
$======$

\#PBEPBE/6-31G(d)/auto scf=(direct,tight, $\operatorname{maxcycle=300,xqc)}$

$\mathrm{opt}=($ maxcycle $=250)$ freq $=$ noraman int $=($ grid $=$ ultrafine $)$

\#P Geom=AllCheck Guess=TCheck SCRF=Check GenChk RPBEPBE/6-31G(d)/Auto Freq

Pointgroup $=\mathrm{C} 1 \quad$ Stoichiometry $=\mathrm{C} 46 \mathrm{H} 46 \mathrm{~N} 4 \mathrm{O} 4 \mathrm{C} 1[\mathrm{X}(\mathrm{C} 46 \mathrm{H} 46 \mathrm{~N} 4 \mathrm{O} 4)]$ \#Atoms $=100$

Charge $=0 \quad$ Multiplicity $=1$

SCF Energy $=-2297.63926138 \quad$ Predicted Change $=-9.397471 \mathrm{D}-09$
} 


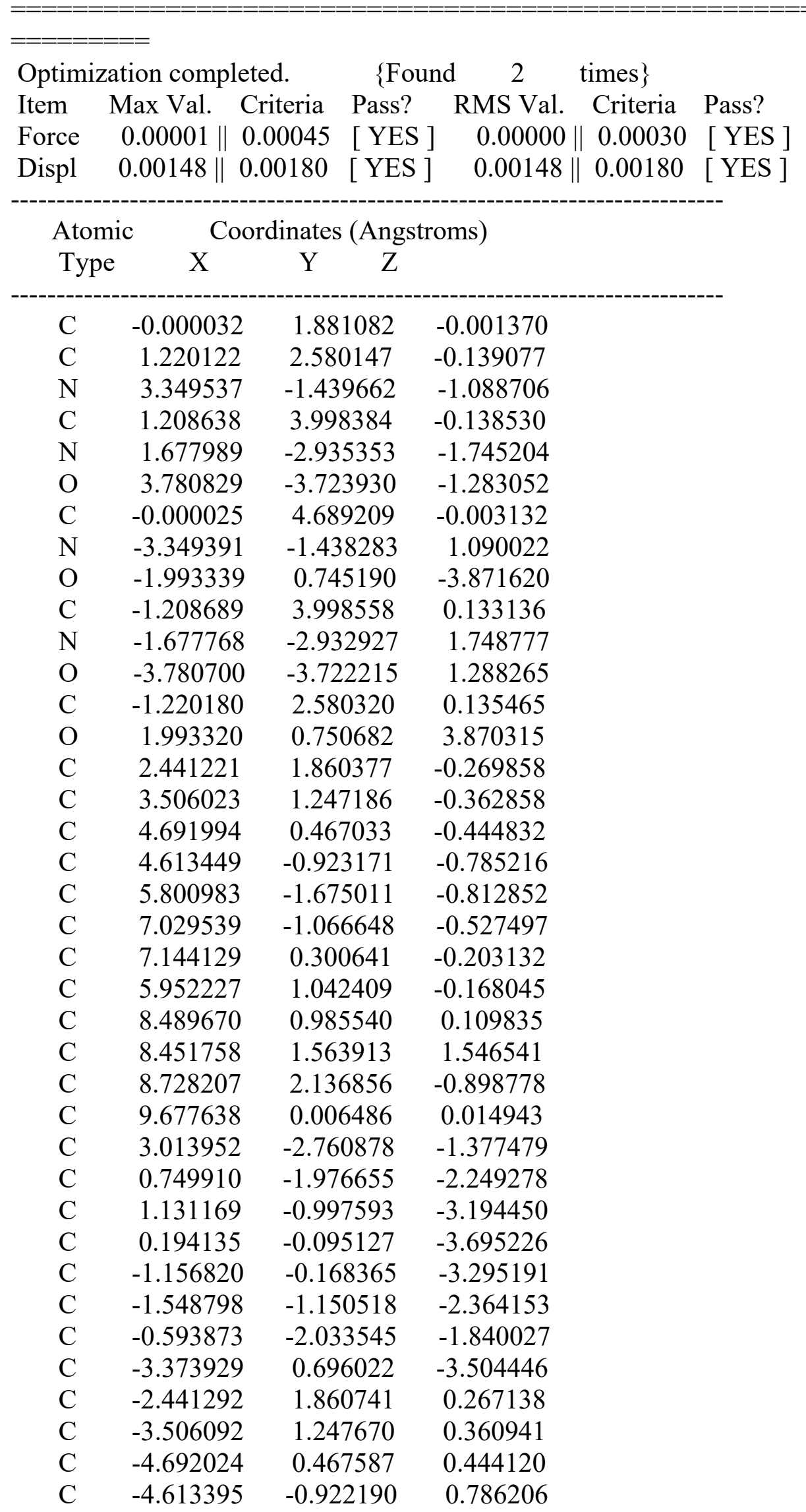




\begin{tabular}{|c|c|c|c|}
\hline $\mathrm{C}$ & -5.800920 & -1.674005 & 0.814992 \\
\hline $\mathrm{C}$ & -7.029538 & -1.066003 & 0.529136 \\
\hline $\mathrm{C}$ & -7.144209 & 0.300885 & 0.203107 \\
\hline $\mathrm{C}$ & -5.952318 & 1.042611 & 0.166863 \\
\hline $\mathrm{C}$ & -8.489819 & 0.985392 & -0.110420 \\
\hline $\mathrm{C}$ & -9.677757 & 0.006436 & -0.014136 \\
\hline $\mathrm{C}$ & -8.728166 & 2.137904 & 0.896869 \\
\hline $\mathrm{C}$ & -8.452206 & 1.562054 & -1.547821 \\
\hline $\mathrm{C}$ & -3.013799 & -2.759018 & 1.381005 \\
\hline $\mathrm{C}$ & -0.749760 & -1.973459 & 2.251560 \\
\hline $\mathrm{C}$ & -1.131091 & -0.993135 & 3.195394 \\
\hline $\mathrm{C}$ & -0.194114 & -0.089946 & 3.694968 \\
\hline $\mathrm{C}$ & 1.156857 & -0.163666 & 3.295070 \\
\hline $\mathrm{C}$ & 1.548905 & -1.147052 & 2.365363 \\
\hline $\mathrm{C}$ & 0.594030 & -2.030830 & 1.842411 \\
\hline $\mathrm{C}$ & 3.373916 & 0.701109 & 3.503224 \\
\hline $\mathrm{H}$ & -0.000036 & 0.787283 & -0.000697 \\
\hline $\mathrm{H}$ & 2.152670 & 4.540423 & -0.246670 \\
\hline $\mathrm{H}$ & 2.589612 & -0.752875 & -1.081457 \\
\hline $\mathrm{H}$ & 1.459414 & -3.923513 & -1.872857 \\
\hline $\mathrm{H}$ & -2.152720 & 4.540736 & 0.240591 \\
\hline $\mathrm{H}$ & -2.589501 & -0.751470 & 1.081800 \\
\hline $\mathrm{H}$ & -1.459278 & -3.920885 & 1.878153 \\
\hline $\mathrm{H}$ & 5.741665 & -2.736954 & -1.053740 \\
\hline $\mathrm{H}$ & 7.923827 & -1.694960 & -0.564770 \\
\hline $\mathrm{H}$ & 5.969950 & 2.107905 & 0.083583 \\
\hline $\mathrm{H}$ & 9.404652 & 2.072076 & 1.780942 \\
\hline $\mathrm{H}$ & 8.297960 & 0.763049 & 2.290628 \\
\hline $\mathrm{H}$ & 7.639751 & 2.300889 & 1.670387 \\
\hline $\mathrm{H}$ & 8.775489 & 1.752798 & -1.932512 \\
\hline $\mathrm{H}$ & 9.682431 & 2.648550 & -0.677824 \\
\hline $\mathrm{H}$ & 7.924864 & 2.892100 & -0.856537 \\
\hline $\mathrm{H}$ & 9.776507 & -0.422067 & -0.997745 \\
\hline $\mathrm{H}$ & 9.583662 & -0.825068 & 0.735199 \\
\hline $\mathrm{H}$ & 10.616424 & 0.540068 & 0.243665 \\
\hline $\mathrm{H}$ & 2.166448 & -0.956397 & -3.546761 \\
\hline $\mathrm{H}$ & 0.482563 & 0.666920 & -4.425115 \\
\hline $\mathrm{H}$ & -2.584663 & -1.226480 & -2.025441 \\
\hline $\mathrm{H}$ & -0.899880 & -2.777764 & -1.096814 \\
\hline $\mathrm{H}$ & -3.862874 & 1.487409 & -4.091170 \\
\hline $\mathrm{H}$ & -3.513431 & 0.896020 & -2.426229 \\
\hline $\mathrm{H}$ & -3.826259 & -0.281183 & -3.760085 \\
\hline $\mathrm{H}$ & -5.741549 & -2.735645 & 1.057191 \\
\hline $\mathrm{H}$ & -7.923809 & -1.694283 & 0.567353 \\
\hline $\mathrm{H}$ & -5.970096 & 2.107798 & -0.086070 \\
\hline $\mathrm{H}$ & -10.616590 & 0.539723 & -0.243357 \\
\hline $\mathrm{H}$ & -9.776461 & -0.420874 & 0.999093 \\
\hline $\mathrm{H}$ & -9.583881 & -0.825999 & -0.733385 \\
\hline
\end{tabular}




$\begin{array}{cccc}\mathrm{H} & -9.682439 & 2.649328 & 0.675500 \\ \mathrm{H} & -7.924829 & 2.893095 & 0.853566 \\ \mathrm{H} & -8.775235 & 1.755072 & 1.931068 \\ \mathrm{H} & -9.405151 & 2.069933 & -1.782624 \\ \mathrm{H} & -8.298561 & 0.760306 & -2.290987 \\ \mathrm{H} & -7.640233 & 2.298890 & -1.672721 \\ \mathrm{H} & -2.166380 & -0.951519 & 3.547630 \\ \mathrm{H} & -0.482592 & 0.673066 & 4.423828 \\ \mathrm{H} & 2.584786 & -1.223435 & 2.026793 \\ \mathrm{H} & 0.900094 & -2.776060 & 1.100236 \\ \mathrm{H} & 3.826305 & -0.275723 & 3.760182 \\ \mathrm{H} & 3.862802 & 1.493313 & 4.088893 \\ \mathrm{H} & 3.513422 & 0.899671 & 2.424743 \\ \mathrm{H} & -0.000026 & 5.784076 & -0.003826\end{array}$

Statistical Thermodynamic Analysis

Temperature $=298.150$ Kelvin $\quad$ Pressure $=1.00000$ Atm

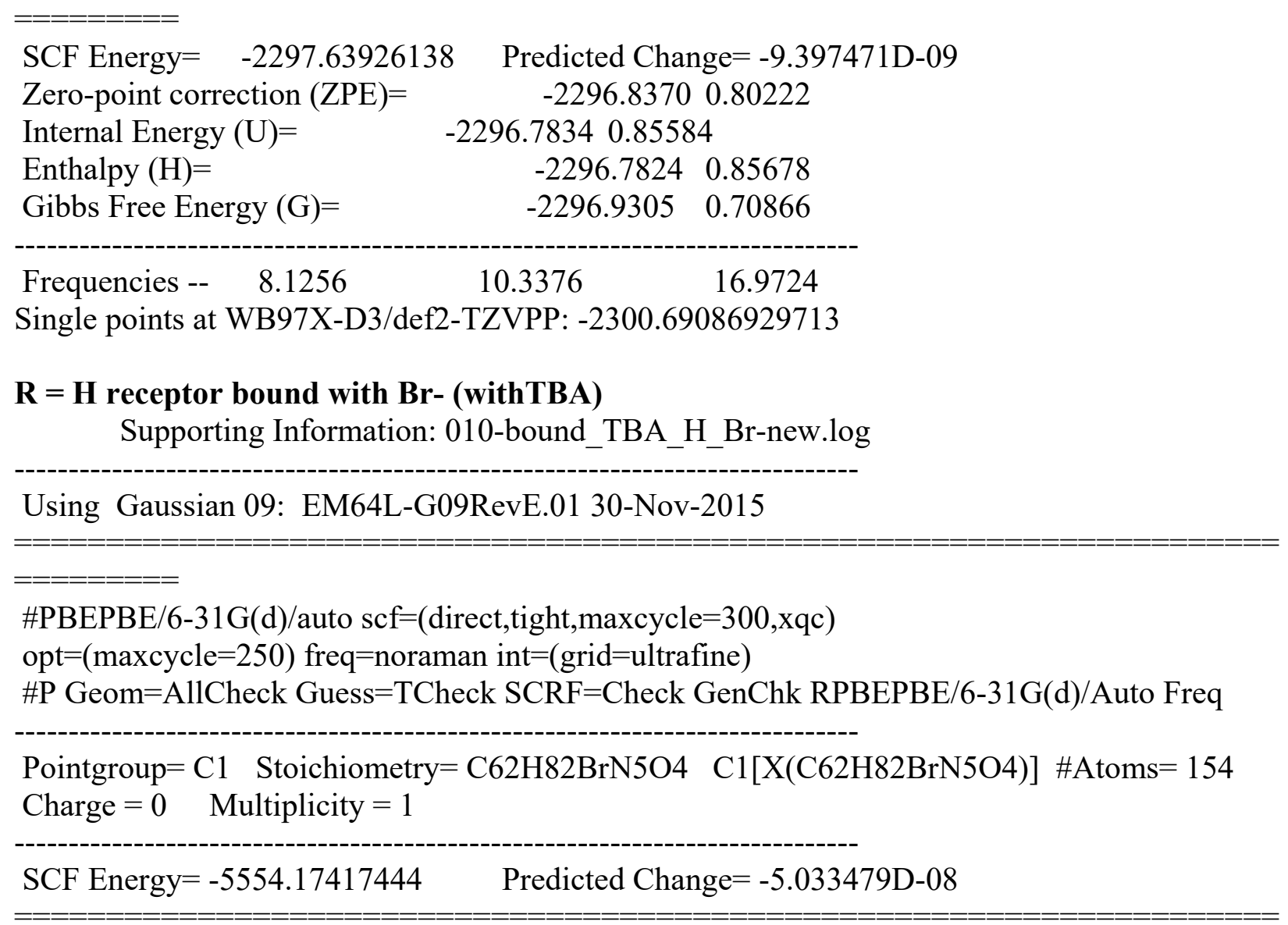

\begin{tabular}{|c|c|c|c|c|c|}
\hline \multicolumn{6}{|c|}{ Optimization completed. } \\
\hline Item & Max Val. Criteria & Pass? & RMS Val. & Criteria & Pass? \\
\hline Force & $0.00001 \| 0.00045$ & [YES ] & 0.00000 & $\| 0.00030$ & [YES ] \\
\hline Displ & $0.00457 \| 0.00180$ & [ NO ] & $0.00457 \|$ & 0.00180 & [YES ] \\
\hline
\end{tabular}




\begin{tabular}{|c|c|c|c|}
\hline \multirow{2}{*}{$\begin{array}{l}\text { Atomic } \\
\text { Type }\end{array}$} & \multicolumn{3}{|c|}{ Coordinates (Angstroms) } \\
\hline & $\mathrm{X}$ & $\mathrm{Y} \quad \mathrm{Z}$ & \\
\hline $\mathrm{N}$ & 2.228904 & 1.253628 & -2.456318 \\
\hline $\mathrm{C}$ & 0.929362 & 1.050068 & -3.251916 \\
\hline $\mathrm{C}$ & 0.046085 & -0.139324 & -2.873561 \\
\hline $\mathrm{C}$ & -1.230652 & -0.101055 & -3.741000 \\
\hline $\mathrm{C}$ & -2.130549 & -1.321743 & -3.517074 \\
\hline $\mathrm{C}$ & 1.859268 & 1.742122 & -1.047446 \\
\hline $\mathrm{C}$ & 3.003813 & 2.113196 & -0.101659 \\
\hline $\mathrm{C}$ & 2.396054 & 2.734470 & 1.176356 \\
\hline $\mathrm{C}$ & 3.447932 & 3.065315 & 2.241446 \\
\hline $\mathrm{C}$ & 3.014893 & -0.053275 & -2.331186 \\
\hline $\mathrm{C}$ & 3.399788 & -0.747894 & -3.640990 \\
\hline $\mathrm{C}$ & 4.085935 & -2.097167 & -3.348891 \\
\hline $\mathrm{C}$ & 4.507179 & -2.825697 & -4.630848 \\
\hline $\mathrm{C}$ & 3.082232 & 2.294288 & -3.182706 \\
\hline $\mathrm{C}$ & 2.428236 & 3.661381 & -3.407528 \\
\hline $\mathrm{C}$ & 3.451427 & 4.686012 & -3.934256 \\
\hline $\mathrm{C}$ & 2.786545 & 6.026237 & -4.277354 \\
\hline $\mathrm{H}$ & 0.363459 & 1.986866 & -3.127370 \\
\hline $\mathrm{H}$ & 1.235310 & 0.980122 & -4.310108 \\
\hline $\mathrm{H}$ & -0.236954 & -0.121397 & -1.803770 \\
\hline $\mathrm{H}$ & 0.571306 & -1.095850 & -3.053180 \\
\hline $\mathrm{H}$ & -0.948565 & -0.039554 & -4.811619 \\
\hline $\mathrm{H}$ & -1.794847 & 0.822985 & -3.512384 \\
\hline $\mathrm{H}$ & -3.020318 & -1.275516 & -4.167205 \\
\hline $\mathrm{H}$ & -1.596831 & -2.262805 & -3.739340 \\
\hline $\mathrm{H}$ & -2.483794 & -1.376993 & -2.474243 \\
\hline $\mathrm{H}$ & 1.198882 & 2.608371 & -1.203433 \\
\hline $\mathrm{H}$ & 1.235576 & 0.954428 & -0.588111 \\
\hline $\mathrm{H}$ & 3.596394 & 1.224286 & 0.181484 \\
\hline $\mathrm{H}$ & 3.699566 & 2.845820 & -0.553904 \\
\hline $\mathrm{H}$ & 1.640932 & 2.038665 & 1.585650 \\
\hline $\mathrm{H}$ & 1.849975 & 3.656450 & 0.896868 \\
\hline $\mathrm{H}$ & 2.975861 & 3.553166 & 3.110443 \\
\hline $\mathrm{H}$ & 3.947893 & 2.156068 & 2.615422 \\
\hline $\mathrm{H}$ & 4.220099 & 3.750515 & 1.845957 \\
\hline $\mathrm{H}$ & 3.917269 & 0.192528 & -1.749110 \\
\hline $\mathrm{H}$ & 2.393494 & -0.716466 & -1.707944 \\
\hline $\mathrm{H}$ & 2.506969 & -0.929948 & -4.266832 \\
\hline $\mathrm{H}$ & 4.089827 & -0.121805 & -4.236587 \\
\hline $\mathrm{H}$ & 3.399558 & -2.733653 & -2.759625 \\
\hline $\mathrm{H}$ & 4.969858 & -1.927674 & -2.705420 \\
\hline $\mathrm{H}$ & 5.001474 & -3.783187 & -4.394502 \\
\hline $\mathrm{H}$ & 3.636102 & -3.049472 & -5.272037 \\
\hline $\mathrm{H}$ & 5.215994 & -2.221435 & -5.225030 \\
\hline
\end{tabular}




\begin{tabular}{|c|c|c|c|}
\hline $\mathrm{H}$ & 3.355126 & 1.845246 & -4.150563 \\
\hline $\mathrm{H}$ & 4.005600 & 2.394743 & -2.590072 \\
\hline $\mathrm{H}$ & 1.600314 & 3.575638 & -4.134088 \\
\hline $\mathrm{H}$ & 1.986638 & 4.055457 & -2.475430 \\
\hline $\mathrm{H}$ & 3.964148 & 4.282772 & -4.829226 \\
\hline $\mathrm{H}$ & 4.236739 & 4.840647 & -3.169677 \\
\hline $\mathrm{H}$ & 3.538148 & 6.780565 & -4.564122 \\
\hline $\mathrm{H}$ & 2.084383 & 5.913456 & -5.122597 \\
\hline $\mathrm{H}$ & 2.211408 & 6.418862 & -3.420262 \\
\hline $\mathrm{O}$ & -0.350979 & 6.760159 & -2.247581 \\
\hline $\mathrm{O}$ & 3.651691 & -0.084842 & 4.021663 \\
\hline $\mathrm{N}$ & -2.790430 & 1.945998 & -0.708443 \\
\hline $\mathrm{N}$ & 2.821919 & -1.054426 & 2.069249 \\
\hline $\mathrm{C}$ & -0.824839 & -3.563751 & -0.302118 \\
\hline $\mathrm{C}$ & -0.936623 & -6.184821 & -1.300323 \\
\hline $\mathrm{C}$ & 0.305692 & -5.613675 & -1.002196 \\
\hline $\mathrm{C}$ & 0.369164 & -4.291463 & -0.492754 \\
\hline $\mathrm{C}$ & -2.276819 & 3.183378 & -1.129927 \\
\hline $\mathrm{C}$ & -2.825456 & 3.959608 & -2.171022 \\
\hline $\mathrm{C}$ & -2.211087 & 5.159998 & -2.563158 \\
\hline $\mathrm{C}$ & -1.040517 & 5.608144 & -1.926789 \\
\hline $\mathrm{C}$ & -0.501528 & 4.845676 & -0.871462 \\
\hline $\mathrm{C}$ & -1.111769 & 3.653858 & -0.477746 \\
\hline $\mathrm{C}$ & -0.928799 & 7.597257 & -3.244565 \\
\hline $\mathrm{C}$ & 1.614960 & -3.689915 & -0.153478 \\
\hline $\mathrm{C}$ & 2.684393 & -3.179217 & 0.184135 \\
\hline $\mathrm{C}$ & 3.934446 & -2.637278 & 0.588024 \\
\hline $\mathrm{C}$ & 4.008330 & -1.590237 & 1.560501 \\
\hline $\mathrm{C}$ & 5.290943 & -1.131664 & 1.939617 \\
\hline $\mathrm{C}$ & 6.441550 & -1.685885 & 1.377720 \\
\hline $\mathrm{C}$ & 6.399519 & -2.717398 & 0.411104 \\
\hline $\mathrm{C}$ & 5.129254 & -3.170634 & 0.038412 \\
\hline $\mathrm{C}$ & 8.496858 & -2.164235 & -0.873731 \\
\hline $\mathrm{C}$ & 7.440430 & -4.411064 & -1.204715 \\
\hline $\mathrm{C}$ & 2.688459 & -0.356257 & 3.291491 \\
\hline $\mathrm{C}$ & -4.077483 & 1.468372 & -0.913636 \\
\hline $\mathrm{C}$ & -2.076498 & -4.138250 & -0.613005 \\
\hline $\mathrm{C}$ & -2.122417 & -5.465544 & -1.111254 \\
\hline $\mathrm{C}$ & 7.702801 & -3.295009 & -0.173179 \\
\hline $\mathrm{N}$ & 1.377629 & -0.019438 & 3.548344 \\
\hline $\mathrm{N}$ & -4.251404 & 0.187224 & -0.366847 \\
\hline $\mathrm{O}$ & -4.964131 & 2.090268 & -1.509271 \\
\hline $\mathrm{C}$ & -3.261241 & -3.368692 & -0.442500 \\
\hline $\mathrm{C}$ & 8.561252 & -3.887396 & 0.972494 \\
\hline $\mathrm{C}$ & 0.884130 & 0.746416 & 4.628190 \\
\hline $\mathrm{C}$ & -5.472674 & -0.499198 & -0.303325 \\
\hline $\mathrm{C}$ & -4.269846 & -2.673216 & -0.321500 \\
\hline $\mathrm{C}$ & -0.455928 & 1.190872 & 4.532337 \\
\hline
\end{tabular}




\begin{tabular}{|c|c|c|c|}
\hline $\mathrm{C}$ & 1.629824 & 1.068568 & 5.776726 \\
\hline $\mathrm{C}$ & -5.473080 & -1.924397 & -0.228724 \\
\hline $\mathrm{C}$ & -6.714865 & 0.168450 & -0.279063 \\
\hline $\mathrm{C}$ & -1.032236 & 1.932553 & 5.559965 \\
\hline $\mathrm{C}$ & 1.045990 & 1.820214 & 6.808914 \\
\hline $\mathrm{C}$ & -6.704929 & -2.618324 & -0.105748 \\
\hline $\mathrm{C}$ & -7.906001 & -0.549180 & -0.172545 \\
\hline $\mathrm{C}$ & -0.286519 & 2.255390 & 6.711174 \\
\hline $\mathrm{C}$ & -7.938232 & -1.959603 & -0.075023 \\
\hline $\mathrm{O}$ & -0.950597 & 2.992938 & 7.665660 \\
\hline $\mathrm{C}$ & -9.282575 & -2.700740 & 0.055141 \\
\hline $\mathrm{C}$ & -0.225555 & 3.323179 & 8.843284 \\
\hline $\mathrm{C}$ & -9.102733 & -4.229643 & 0.145188 \\
\hline $\mathrm{C}$ & -10.009632 & -2.221621 & 1.336531 \\
\hline $\mathrm{C}$ & -10.163393 & -2.387719 & -1.180194 \\
\hline $\mathrm{H}$ & -2.156847 & 1.392842 & -0.105941 \\
\hline $\mathrm{H}$ & 1.960351 & -1.319603 & 1.577242 \\
\hline $\mathrm{H}$ & -0.781178 & -2.542044 & 0.090175 \\
\hline $\mathrm{H}$ & 1.230684 & -6.180247 & -1.146306 \\
\hline $\mathrm{H}$ & -3.744069 & 3.625451 & -2.655284 \\
\hline $\mathrm{H}$ & -2.664971 & 5.736485 & -3.373651 \\
\hline $\mathrm{H}$ & 0.384568 & 5.222083 & -0.348691 \\
\hline $\mathrm{H}$ & -0.694470 & 3.060289 & 0.344064 \\
\hline $\mathrm{H}$ & -0.267575 & 8.473086 & -3.323007 \\
\hline $\mathrm{H}$ & -0.980684 & 7.090503 & -4.228573 \\
\hline $\mathrm{H}$ & -1.945273 & 7.929180 & -2.958203 \\
\hline $\mathrm{H}$ & 5.362193 & -0.358570 & 2.704296 \\
\hline $\mathrm{H}$ & 5.016190 & -3.978132 & -0.689553 \\
\hline $\mathrm{H}$ & 6.900094 & -5.263687 & -0.757701 \\
\hline $\mathrm{H}$ & 6.854612 & -4.045200 & -2.067371 \\
\hline $\mathrm{H}$ & 7.409444 & -1.294705 & 1.711498 \\
\hline $\mathrm{H}$ & 8.739218 & -1.342462 & -0.178529 \\
\hline $\mathrm{H}$ & 7.918362 & -1.737224 & -1.712512 \\
\hline $\mathrm{H}$ & 9.448452 & -2.554517 & -1.278074 \\
\hline $\mathrm{H}$ & 8.400909 & -4.791887 & -1.593177 \\
\hline $\mathrm{H}$ & -3.091079 & -5.914805 & -1.350189 \\
\hline $\mathrm{H}$ & 8.026314 & -4.707666 & 1.481323 \\
\hline $\mathrm{H}$ & 8.810699 & -3.127225 & 1.731988 \\
\hline $\mathrm{H}$ & 9.510315 & -4.289447 & 0.573659 \\
\hline $\mathrm{H}$ & -1.032911 & 0.952551 & 3.630662 \\
\hline $\mathrm{H}$ & 2.664823 & 0.734413 & 5.854062 \\
\hline $\mathrm{H}$ & -6.729765 & 1.255495 & -0.357989 \\
\hline $\mathrm{H}$ & -2.068175 & 2.277489 & 5.488732 \\
\hline $\mathrm{H}$ & 1.650409 & 2.054352 & 7.689184 \\
\hline $\mathrm{H}$ & -6.650867 & -3.708106 & -0.044738 \\
\hline $\mathrm{H}$ & -8.843789 & 0.017505 & -0.153772 \\
\hline $\mathrm{H}$ & -0.919992 & 3.899305 & 9.473221 \\
\hline $\mathrm{H}$ & 0.100685 & 2.418222 & 9.392900 \\
\hline
\end{tabular}




$\begin{array}{cccc}\mathrm{H} & 0.664007 & 3.944529 & 8.618656 \\ \mathrm{H} & -10.089909 & -4.714825 & 0.238987 \\ \mathrm{H} & -8.502963 & -4.520622 & 1.025342 \\ \mathrm{H} & -8.614437 & -4.638772 & -0.756751 \\ \mathrm{H} & -9.411578 & -2.446514 & 2.236587 \\ \mathrm{H} & -10.986539 & -2.728933 & 1.436135 \\ \mathrm{H} & -10.195956 & -1.134275 & 1.319252 \\ \mathrm{H} & -11.141005 & -2.896036 & -1.093310 \\ \mathrm{H} & -9.677014 & -2.733229 & -2.108964 \\ \mathrm{H} & -10.355065 & -1.306078 & -1.283168 \\ \mathrm{H} & -3.417453 & -0.317150 & -0.042073 \\ \mathrm{H} & 0.688511 & -0.180455 & 2.795057 \\ \mathrm{H} & -0.981794 & -7.208675 & -1.686313 \\ \mathrm{Br} & -0.628294 & -0.061734 & 0.861863\end{array}$

Statistical Thermodynamic Analysis

Temperature $=298.150$ Kelvin $\quad$ Pressure $=1.00000$ Atm

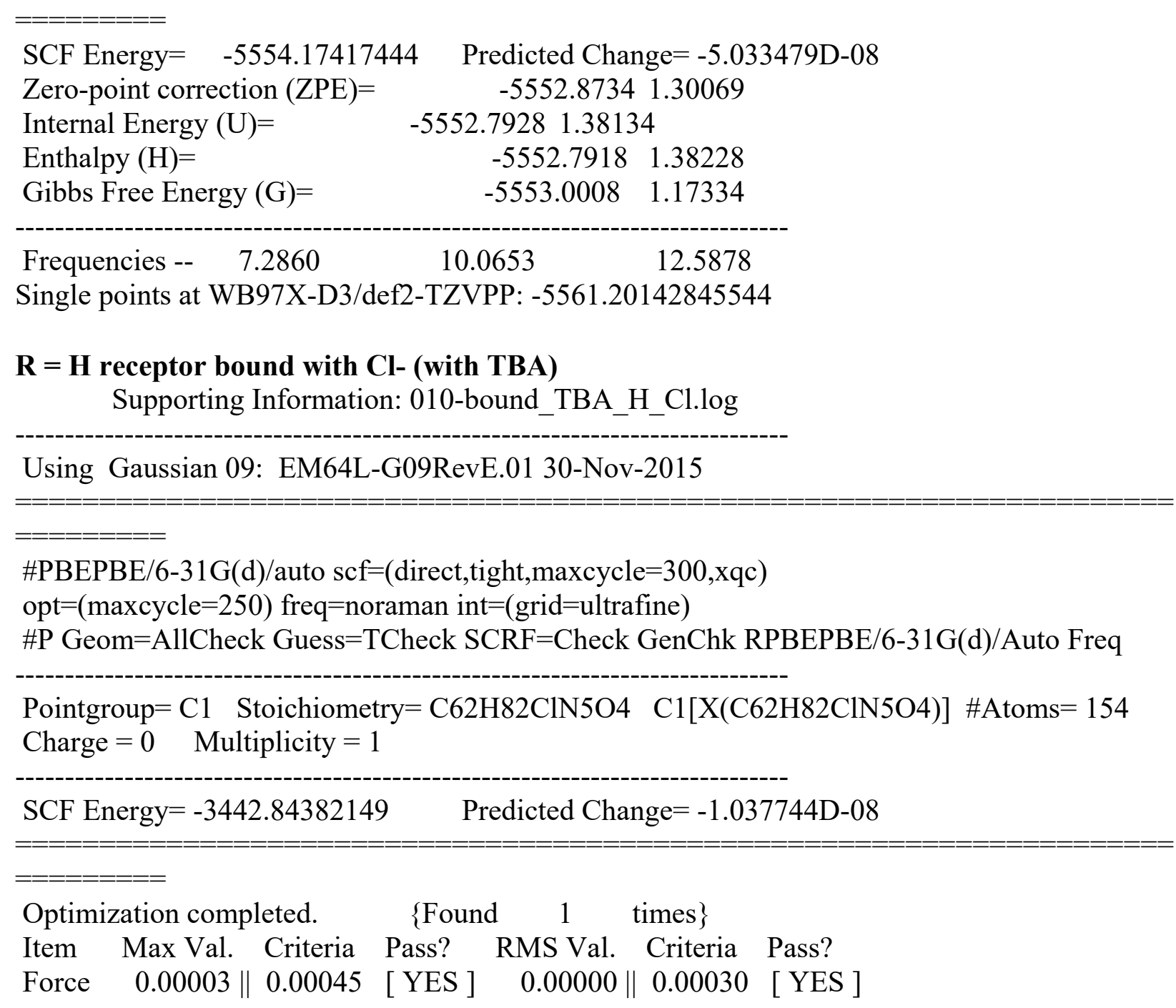


Displ $0.00361 \| 0.00180 \quad$ [NO ] $\quad 0.00361 \| 0.00180$ [ Y YES ]

\begin{tabular}{|c|c|c|c|}
\hline \multirow{2}{*}{$\begin{array}{l}\text { Atomic } \\
\text { Type }\end{array}$} & \multicolumn{3}{|c|}{ Coordinates (Angstroms) } \\
\hline & $\mathrm{X}$ & $\mathrm{Y} \quad \mathrm{Z}$ & \\
\hline $\mathrm{N}$ & 2.142465 & 0.975036 & -2.472644 \\
\hline $\mathrm{C}$ & 0.832186 & 0.683292 & -3.225428 \\
\hline $\mathrm{C}$ & -0.025744 & -0.478180 & -2.724814 \\
\hline $\mathrm{C}$ & -1.314733 & -0.547650 & -3.571251 \\
\hline $\mathrm{C}$ & -2.190312 & -1.752629 & -3.207881 \\
\hline $\mathrm{C}$ & 1.799036 & 1.627951 & -1.121142 \\
\hline $\mathrm{C}$ & 2.981018 & 2.105147 & -0.270211 \\
\hline $\mathrm{C}$ & 2.469590 & 3.019515 & 0.864565 \\
\hline $\mathrm{C}$ & 3.591025 & 3.477035 & 1.805510 \\
\hline $\mathrm{C}$ & 2.927675 & -0.310574 & -2.204361 \\
\hline $\mathrm{C}$ & 3.329956 & -1.131534 & -3.433270 \\
\hline $\mathrm{C}$ & 3.984332 & -2.458628 & -3.001011 \\
\hline $\mathrm{C}$ & 4.431464 & -3.304746 & -4.199209 \\
\hline $\mathrm{C}$ & 2.987049 & 1.914094 & -3.334866 \\
\hline $\mathrm{C}$ & 2.374177 & 3.284104 & -3.637741 \\
\hline $\mathrm{C}$ & 3.370532 & 4.172738 & -4.407397 \\
\hline $\mathrm{C}$ & 2.737461 & 5.505841 & -4.828473 \\
\hline $\mathrm{H}$ & 0.253469 & 1.619527 & -3.178709 \\
\hline $\mathrm{H}$ & 1.123357 & 0.518927 & -4.277219 \\
\hline $\mathrm{H}$ & -0.290541 & -0.355813 & -1.657973 \\
\hline $\mathrm{H}$ & 0.513604 & -1.438749 & -2.820271 \\
\hline $\mathrm{H}$ & -1.048465 & -0.593550 & -4.646713 \\
\hline $\mathrm{H}$ & -1.890391 & 0.386601 & -3.430675 \\
\hline $\mathrm{H}$ & -3.089854 & -1.788699 & -3.845084 \\
\hline $\mathrm{H}$ & -1.643046 & -2.702893 & -3.340041 \\
\hline $\mathrm{H}$ & -2.527646 & -1.704967 & -2.159326 \\
\hline $\mathrm{H}$ & 1.134513 & 2.471032 & -1.363848 \\
\hline $\mathrm{H}$ & 1.189047 & 0.894279 & -0.563296 \\
\hline $\mathrm{H}$ & 3.522218 & 1.249488 & 0.172250 \\
\hline $\mathrm{H}$ & 3.712550 & 2.681821 & -0.867802 \\
\hline $\mathrm{H}$ & 1.693347 & 2.487702 & 1.443459 \\
\hline $\mathrm{H}$ & 1.976179 & 3.901223 & 0.412358 \\
\hline $\mathrm{H}$ & 3.196490 & 4.171312 & 2.566095 \\
\hline $\mathrm{H}$ & 4.033522 & 2.622510 & 2.344567 \\
\hline $\mathrm{H}$ & 4.393384 & 3.999583 & 1.252649 \\
\hline $\mathrm{H}$ & 3.822384 & -0.004932 & -1.639077 \\
\hline $\mathrm{H}$ & 2.298937 & -0.907024 & -1.523947 \\
\hline $\mathrm{H}$ & 2.449420 & -1.355931 & -4.063267 \\
\hline $\mathrm{H}$ & 4.045307 & -0.572983 & -4.064969 \\
\hline $\mathrm{H}$ & 3.270661 & -3.030811 & -2.379104 \\
\hline $\mathrm{H}$ & 4.851271 & -2.246053 & -2.347629 \\
\hline $\mathrm{H}$ & 4.900960 & -4.244755 & -3.862959 \\
\hline $\mathrm{H}$ & 3.576940 & -3.571618 & -4.846238 \\
\hline
\end{tabular}




\begin{tabular}{|c|c|c|c|}
\hline $\mathrm{H}$ & 5.169260 & -2.766680 & -4.821146 \\
\hline $\mathrm{H}$ & 3.177970 & 1.372460 & -4.274703 \\
\hline $\mathrm{H}$ & 3.952303 & 2.024451 & -2.815332 \\
\hline $\mathrm{H}$ & 1.453635 & 3.172205 & -4.238357 \\
\hline $\mathrm{H}$ & 2.081090 & 3.806094 & -2.710192 \\
\hline $\mathrm{H}$ & 3.741047 & 3.636429 & -5.302696 \\
\hline $\mathrm{H}$ & 4.255950 & 4.362057 & -3.770648 \\
\hline $\mathrm{H}$ & 3.483904 & 6.171297 & -5.293366 \\
\hline $\mathrm{H}$ & 1.928220 & 5.343571 & -5.562727 \\
\hline $\mathrm{H}$ & 2.297052 & 6.030605 & -3.962685 \\
\hline $\mathrm{O}$ & -0.321473 & 6.399636 & -2.824478 \\
\hline $\mathrm{O}$ & 3.733743 & 0.493473 & 3.736672 \\
\hline $\mathrm{N}$ & -2.727720 & 1.763559 & -0.754642 \\
\hline $\mathrm{N}$ & 2.822959 & -0.895369 & 2.102510 \\
\hline $\mathrm{C}$ & -0.948831 & -3.633863 & 0.283420 \\
\hline $\mathrm{C}$ & -1.138879 & -6.386093 & -0.256591 \\
\hline $\mathrm{C}$ & 0.120152 & -5.804972 & -0.070867 \\
\hline $\mathrm{C}$ & 0.223121 & -4.417551 & 0.206325 \\
\hline $\mathrm{C}$ & -2.200514 & 2.943335 & -1.311716 \\
\hline $\mathrm{C}$ & -2.642865 & 3.488009 & -2.535307 \\
\hline $\mathrm{C}$ & -2.043449 & 4.641475 & -3.065402 \\
\hline $\mathrm{C}$ & -0.986225 & 5.272731 & -2.386360 \\
\hline $\mathrm{C}$ & -0.549005 & 4.741081 & -1.157188 \\
\hline $\mathrm{C}$ & -1.149935 & 3.597247 & -0.626243 \\
\hline $\mathrm{C}$ & -0.835046 & 7.034376 & -3.991594 \\
\hline $\mathrm{C}$ & 1.493309 & -3.809753 & 0.419582 \\
\hline $\mathrm{C}$ & 2.595887 & -3.301837 & 0.630717 \\
\hline $\mathrm{C}$ & 3.868993 & -2.729071 & 0.894795 \\
\hline $\mathrm{C}$ & 3.988927 & -1.522246 & 1.655091 \\
\hline $\mathrm{C}$ & 5.287774 & -1.013734 & 1.885309 \\
\hline $\mathrm{C}$ & 6.410509 & -1.676480 & 1.387228 \\
\hline $\mathrm{C}$ & 6.323333 & -2.871689 & 0.636374 \\
\hline $\mathrm{C}$ & 5.036207 & -3.371844 & 0.408429 \\
\hline $\mathrm{C}$ & 8.363125 & -2.595560 & -0.819514 \\
\hline $\mathrm{C}$ & 7.287032 & -4.852281 & -0.671140 \\
\hline $\mathrm{C}$ & 2.737955 & 0.066938 & 3.133235 \\
\hline $\mathrm{C}$ & -4.035465 & 1.322391 & -0.922528 \\
\hline $\mathrm{C}$ & -2.217754 & -4.222665 & 0.086158 \\
\hline $\mathrm{C}$ & -2.303002 & -5.613393 & -0.180603 \\
\hline $\mathrm{C}$ & 7.598093 & -3.563816 & 0.117162 \\
\hline $\mathrm{N}$ & 1.438736 & 0.465451 & 3.360904 \\
\hline $\mathrm{N}$ & -4.275326 & 0.114242 & -0.253473 \\
\hline $\mathrm{O}$ & -4.888410 & 1.921176 & -1.586926 \\
\hline $\mathrm{C}$ & -3.386284 & -3.412563 & 0.134479 \\
\hline $\mathrm{C}$ & 8.505265 & -3.936864 & 1.316346 \\
\hline $\mathrm{C}$ & 0.999968 & 1.431603 & 4.295257 \\
\hline $\mathrm{C}$ & -5.523436 & -0.513437 & -0.139865 \\
\hline $\mathrm{C}$ & -4.392528 & -2.703295 & 0.142767 \\
\hline
\end{tabular}




\begin{tabular}{|c|c|c|c|}
\hline $\mathrm{C}$ & -0.356202 & 1.828612 & 4.216042 \\
\hline $\mathrm{C}$ & 1.815582 & 1.999730 & 5.292309 \\
\hline $\mathrm{C}$ & -5.575005 & -1.918107 & 0.109887 \\
\hline $\mathrm{C}$ & -6.741442 & 0.191640 & -0.230594 \\
\hline $\mathrm{C}$ & -0.877154 & 2.765658 & 5.104801 \\
\hline $\mathrm{C}$ & 1.286937 & 2.944591 & 6.186050 \\
\hline $\mathrm{C}$ & -6.831981 & -2.552598 & 0.284199 \\
\hline $\mathrm{C}$ & -7.958481 & -0.470199 & -0.067640 \\
\hline $\mathrm{C}$ & -0.059947 & 3.335441 & 6.100916 \\
\hline $\mathrm{C}$ & -8.042051 & -1.856081 & 0.201092 \\
\hline $\mathrm{O}$ & -0.672635 & 4.251580 & 6.926110 \\
\hline $\mathrm{C}$ & -9.414069 & -2.532369 & 0.384937 \\
\hline $\mathrm{C}$ & 0.126164 & 4.829664 & 7.950557 \\
\hline $\mathrm{C}$ & -9.289662 & -4.043780 & 0.664591 \\
\hline $\mathrm{C}$ & -10.152359 & -1.877233 & 1.579043 \\
\hline $\mathrm{C}$ & -10.255896 & -2.344867 & -0.901941 \\
\hline $\mathrm{H}$ & -2.116569 & 1.265878 & -0.080987 \\
\hline $\mathrm{H}$ & 1.945068 & -1.311637 & 1.772250 \\
\hline $\mathrm{H}$ & -0.875896 & -2.562261 & 0.502348 \\
\hline $\mathrm{H}$ & 1.029684 & -6.410761 & -0.126441 \\
\hline $\mathrm{H}$ & -3.478930 & 3.018344 & -3.055596 \\
\hline $\mathrm{H}$ & -2.418964 & 5.037236 & -4.012853 \\
\hline $\mathrm{H}$ & 0.245026 & 5.261464 & -0.611490 \\
\hline $\mathrm{H}$ & -0.816355 & 3.202553 & 0.339872 \\
\hline $\mathrm{H}$ & -0.222887 & 7.936753 & -4.138001 \\
\hline $\mathrm{H}$ & -0.746868 & 6.386819 & -4.886577 \\
\hline $\mathrm{H}$ & -1.895106 & 7.325731 & -3.862824 \\
\hline $\mathrm{H}$ & 5.394920 & -0.112522 & 2.488382 \\
\hline $\mathrm{H}$ & 4.888055 & -4.296807 & -0.154937 \\
\hline $\mathrm{H}$ & 6.763352 & -5.597941 & -0.047870 \\
\hline $\mathrm{H}$ & 6.665530 & -4.649523 & -1.562038 \\
\hline $\mathrm{H}$ & 7.393451 & -1.240850 & 1.600217 \\
\hline $\mathrm{H}$ & 8.636876 & -1.658705 & -0.304946 \\
\hline $\mathrm{H}$ & 7.749705 & -2.330987 & -1.699520 \\
\hline $\mathrm{H}$ & 9.295606 & -3.065352 & -1.181664 \\
\hline $\mathrm{H}$ & 8.228234 & -5.311408 & -1.020138 \\
\hline $\mathrm{H}$ & -3.286083 & -6.069718 & -0.330763 \\
\hline $\mathrm{H}$ & 7.991591 & -4.639619 & 1.994723 \\
\hline $\mathrm{H}$ & 8.790312 & -3.048929 & 1.905698 \\
\hline $\mathrm{H}$ & 9.434595 & -4.417707 & 0.960611 \\
\hline $\mathrm{H}$ & -0.998365 & 1.389564 & 3.444023 \\
\hline $\mathrm{H}$ & 2.861978 & 1.702386 & 5.361448 \\
\hline $\mathrm{H}$ & -6.716133 & 1.260259 & -0.443753 \\
\hline $\mathrm{H}$ & -1.925875 & 3.071985 & 5.044476 \\
\hline $\mathrm{H}$ & 1.946952 & 3.364947 & 6.949469 \\
\hline $\mathrm{H}$ & -6.817235 & -3.627670 & 0.479875 \\
\hline $\mathrm{H}$ & -8.876318 & 0.123676 & -0.143925 \\
\hline $\mathrm{H}$ & -0.535846 & 5.518701 & 8.496372 \\
\hline
\end{tabular}




$\begin{array}{cccc}\mathrm{H} & 0.513843 & 4.064001 & 8.651165 \\ \mathrm{H} & 0.981225 & 5.398426 & 7.534354 \\ \mathrm{H} & -10.294992 & -4.482362 & 0.789879 \\ \mathrm{H} & -8.720675 & -4.244022 & 1.589386 \\ \mathrm{H} & -8.795260 & -4.575174 & -0.167572 \\ \mathrm{H} & -9.582934 & -2.009760 & 2.515287 \\ \mathrm{H} & -11.148617 & -2.336631 & 1.713517 \\ \mathrm{H} & -10.299591 & -0.794654 & 1.425086 \\ \mathrm{H} & -11.252417 & -2.807110 & -0.779657 \\ \mathrm{H} & -9.761481 & -2.816823 & -1.768757 \\ \mathrm{H} & -10.407276 & -1.278381 & -1.140615 \\ \mathrm{H} & -3.468918 & -0.400791 & 0.117435 \\ \mathrm{H} & 0.717550 & 0.172680 & 2.675860 \\ \mathrm{H} & -1.214371 & -7.458836 & -0.463577 \\ \mathrm{Cl} & -0.652866 & -0.071874 & 0.937154\end{array}$

Statistical Thermodynamic Analysis

Temperature $=298.150$ Kelvin $\quad$ Pressure $=1.00000$ Atm

\begin{tabular}{|c|c|}
\hline & \\
\hline $\begin{array}{l}\text { SCF Energy }=-3442.8438214 \\
\text { Zero-point correction }(\mathrm{ZPE})=\end{array}$ & $9 \quad$ Predicted Change $=-1.037744 \mathrm{D}-08$ \\
\hline Zero-point correction (ZPE)= & $-3441.5443 \quad 1.29944$ \\
\hline Internal Energy $(U)=$ & $-3441.4635 \quad 1.38031$ \\
\hline Enthalpy $(H)=$ & $\begin{array}{lll}-3441.4625 & 1.38125\end{array}$ \\
\hline Gibbs Free Energy $(G)=$ & $-3441.6723 \quad 1.17142$ \\
\hline $\begin{array}{l}\text { Frequencies -- } 7.0387 \\
\text { Single points at WB97X-D3/def2 }\end{array}$ & $\begin{array}{cc}9.3185 & 11.1074 \\
\text { 2-TZVPP: } & -3447.19432821083\end{array}$ \\
\hline \multicolumn{2}{|c|}{$\begin{aligned} \mathbf{R}= & \mathbf{H} \text { receptor bound with } \mathbf{S e H}-(\text { with } \mathbf{T B A}) \\
& \text { Supporting Information: } 010 \text {-bound TBA H SeH.log }\end{aligned}$} \\
\hline
\end{tabular}

\#PBEPBE/6-31G(d)/auto scf=(direct,tight,maxcycle=300,xqc) $\mathrm{opt}=($ maxcycle $=250)$ freq $=$ noraman int $=($ grid $=$ ultrafine $)$ \#P Geom=AllCheck Guess=TCheck SCRF=Check GenChk RPBEPBE/6-31G(d)/Auto Freq

Pointgroup= $\mathrm{C} 1 \quad$ Stoichiometry $=\mathrm{C} 62 \mathrm{H} 83 \mathrm{~N} 5 \mathrm{O} 4 \mathrm{Se} \quad \mathrm{C} 1[\mathrm{X}(\mathrm{C} 62 \mathrm{H} 83 \mathrm{~N} 5 \mathrm{O} 4 \mathrm{Se})]$ \#Atoms= 155 Charge $=0 \quad$ Multiplicity $=1$

SCF Energy $=-5382.43263274 \quad$ Predicted Change $=-1.258933 \mathrm{D}-08$

$========$

Optimization completed. $\quad$ FFound 2 times\}

Item Max Val. Criteria Pass? RMS Val. Criteria Pass? 
Force $\quad 0.00003 \| 0.00045$ [YES ] $0.00000 \| 0.00030$ [ [YES ] Displ $0.00166 \| 0.00180$ [YES ] $0.00166 \| 0.00180$ [Y $0.0 S$ ]

\begin{tabular}{|c|c|c|c|}
\hline \multirow{2}{*}{$\begin{array}{l}\text { Atomic } \\
\text { Type }\end{array}$} & \multicolumn{3}{|c|}{ Coordinates (Angstroms) } \\
\hline & $\mathrm{X}$ & $\mathrm{Y} \quad \mathrm{Z}$ & \\
\hline $\mathrm{N}$ & -3.051866 & 2.500334 & 0.359564 \\
\hline $\mathrm{C}$ & -1.855717 & 3.278813 & 0.934170 \\
\hline $\mathrm{C}$ & -0.749238 & 2.476197 & 1.621607 \\
\hline $\mathrm{C}$ & 0.330495 & 3.461287 & 2.121334 \\
\hline $\mathrm{C}$ & 1.463838 & 2.760183 & 2.878857 \\
\hline $\mathrm{C}$ & -2.560882 & 1.695821 & -0.847443 \\
\hline $\mathrm{C}$ & -3.579141 & 0.833809 & -1.592475 \\
\hline $\mathrm{C}$ & -2.861057 & 0.222025 & -2.817012 \\
\hline $\mathrm{C}$ & -3.738510 & -0.769333 & -3.586630 \\
\hline $\mathrm{C}$ & -3.635266 & 1.539399 & 1.394618 \\
\hline $\mathrm{C}$ & -4.071594 & 2.156367 & 2.725770 \\
\hline $\mathrm{C}$ & -4.655833 & 1.068664 & 3.650081 \\
\hline $\mathrm{C}$ & -5.067171 & 1.625424 & 5.018436 \\
\hline $\mathrm{C}$ & -4.129244 & 3.504965 & -0.063096 \\
\hline $\mathrm{C}$ & -3.797064 & 4.368884 & -1.283761 \\
\hline $\mathrm{C}$ & -4.776911 & 5.549211 & -1.424711 \\
\hline $\mathrm{C}$ & -4.499025 & 6.363790 & -2.695775 \\
\hline $\mathrm{H}$ & -1.429407 & 3.835735 & 0.084694 \\
\hline $\mathrm{H}$ & -2.288535 & 4.009342 & 1.638382 \\
\hline $\mathrm{H}$ & -0.281260 & 1.743783 & 0.936749 \\
\hline $\mathrm{H}$ & -1.143192 & 1.908101 & 2.484646 \\
\hline $\mathrm{H}$ & -0.141512 & 4.220630 & 2.776994 \\
\hline $\mathrm{H}$ & 0.748838 & 4.009614 & 1.256290 \\
\hline $\mathrm{H}$ & 2.200739 & 3.495442 & 3.243446 \\
\hline $\mathrm{H}$ & 1.083338 & 2.199652 & 3.750906 \\
\hline $\mathrm{H}$ & 2.000106 & 2.046906 & 2.231222 \\
\hline $\mathrm{H}$ & -2.124624 & 2.442580 & -1.527530 \\
\hline $\mathrm{H}$ & -1.723675 & 1.056726 & -0.510600 \\
\hline $\mathrm{H}$ & -3.947783 & 0.010644 & -0.953846 \\
\hline $\mathrm{H}$ & -4.461370 & 1.415236 & -1.925762 \\
\hline $\mathrm{H}$ & -1.931923 & -0.260590 & -2.457742 \\
\hline $\mathrm{H}$ & -2.540550 & 1.041094 & -3.491368 \\
\hline $\mathrm{H}$ & -3.188114 & -1.199213 & -4.440042 \\
\hline $\mathrm{H}$ & -4.058961 & -1.604080 & -2.939985 \\
\hline $\mathrm{H}$ & -4.646687 & -0.279880 & -3.983646 \\
\hline $\mathrm{H}$ & -4.492059 & 1.053162 & 0.901772 \\
\hline $\mathrm{H}$ & -2.871690 & 0.761436 & 1.557099 \\
\hline $\mathrm{H}$ & -3.214060 & 2.632710 & 3.235341 \\
\hline $\mathrm{H}$ & -4.835114 & 2.941945 & 2.573362 \\
\hline $\mathrm{H}$ & -3.909984 & 0.261859 & 3.777235 \\
\hline $\mathrm{H}$ & -5.531428 & 0.603068 & 3.157359 \\
\hline $\mathrm{H}$ & -5.487524 & 0.831149 & 5.657528 \\
\hline
\end{tabular}




\begin{tabular}{|c|c|c|c|}
\hline $\mathrm{H}$ & -4.202246 & 2.060013 & 5.550082 \\
\hline $\mathrm{H}$ & -5.832673 & 2.415720 & 4.919349 \\
\hline $\mathrm{H}$ & -4.311740 & 4.141345 & 0.817709 \\
\hline $\mathrm{H}$ & -5.043214 & 2.916057 & -0.245444 \\
\hline $\mathrm{H}$ & -2.772006 & 4.775095 & -1.222000 \\
\hline $\mathrm{H}$ & -3.838075 & 3.756331 & -2.202714 \\
\hline $\mathrm{H}$ & -4.690670 & 6.200730 & -0.533833 \\
\hline $\mathrm{H}$ & -5.819394 & 5.176112 & -1.437936 \\
\hline $\mathrm{H}$ & -5.146342 & 7.255261 & -2.745342 \\
\hline $\mathrm{H}$ & -3.446814 & 6.697742 & -2.736202 \\
\hline $\mathrm{H}$ & -4.688704 & 5.761459 & -3.601676 \\
\hline $\mathrm{O}$ & -0.605306 & 6.649015 & -3.400751 \\
\hline $\mathrm{O}$ & -2.037570 & -4.778940 & -2.090457 \\
\hline $\mathrm{N}$ & 2.393385 & 2.518402 & -1.079581 \\
\hline $\mathrm{N}$ & -2.137189 & -2.887270 & -0.721909 \\
\hline $\mathrm{C}$ & 0.633679 & -1.191366 & 2.789313 \\
\hline $\mathrm{C}$ & 0.487968 & -1.372604 & 5.583147 \\
\hline $\mathrm{C}$ & -0.674418 & -1.535909 & 4.820995 \\
\hline $\mathrm{C}$ & -0.609180 & -1.454408 & 3.406074 \\
\hline $\mathrm{C}$ & 1.744859 & 3.610052 & -1.669835 \\
\hline $\mathrm{C}$ & 2.259488 & 4.921243 & -1.727237 \\
\hline $\mathrm{C}$ & 1.501398 & 5.955125 & -2.301362 \\
\hline $\mathrm{C}$ & 0.224730 & 5.702310 & -2.832313 \\
\hline $\mathrm{C}$ & -0.280934 & 4.387117 & -2.798913 \\
\hline $\mathrm{C}$ & 0.466588 & 3.358573 & -2.226137 \\
\hline $\mathrm{C}$ & -0.084526 & 7.969159 & -3.519986 \\
\hline $\mathrm{C}$ & -1.757825 & -1.684189 & 2.594529 \\
\hline $\mathrm{C}$ & -2.717446 & -1.979941 & 1.878558 \\
\hline $\mathrm{C}$ & -3.760362 & -2.449407 & 1.034318 \\
\hline $\mathrm{C}$ & -3.439319 & -3.002961 & -0.247438 \\
\hline $\mathrm{C}$ & -4.475500 & -3.626199 & -0.978613 \\
\hline $\mathrm{C}$ & -5.778695 & -3.649426 & -0.481561 \\
\hline $\mathrm{C}$ & -6.136340 & -3.060148 & 0.755224 \\
\hline $\mathrm{C}$ & -5.102632 & -2.472774 & 1.493748 \\
\hline $\mathrm{C}$ & -8.502129 & -2.380916 & 0.209159 \\
\hline $\mathrm{C}$ & -7.780553 & -2.406111 & 2.607113 \\
\hline $\mathrm{C}$ & -1.479449 & -3.798296 & -1.585078 \\
\hline $\mathrm{C}$ & 3.704542 & 2.439894 & -0.635524 \\
\hline $\mathrm{C}$ & 1.805914 & -1.024250 & 3.558359 \\
\hline $\mathrm{C}$ & 1.722127 & -1.124788 & 4.970321 \\
\hline $\mathrm{C}$ & -7.597711 & -3.099287 & 1.241729 \\
\hline $\mathrm{N}$ & -0.164939 & -3.430392 & -1.755256 \\
\hline $\mathrm{N}$ & 3.963824 & 1.189382 & -0.052701 \\
\hline $\mathrm{O}$ & 4.537081 & 3.349065 & -0.725629 \\
\hline $\mathrm{C}$ & 3.031082 & -0.734266 & 2.895375 \\
\hline $\mathrm{C}$ & -8.054831 & -4.572841 & 1.383023 \\
\hline $\mathrm{C}$ & 0.862664 & -4.105528 & -2.438065 \\
\hline $\mathrm{C}$ & 5.204123 & 0.801611 & 0.474627 \\
\hline
\end{tabular}




\begin{tabular}{|c|c|c|c|}
\hline $\mathrm{C}$ & 4.053761 & -0.450632 & 2.272516 \\
\hline $\mathrm{C}$ & 2.148382 & -3.516884 & -2.368395 \\
\hline $\mathrm{C}$ & 0.689873 & -5.299272 & -3.160275 \\
\hline $\mathrm{C}$ & 5.247408 & -0.070445 & 1.602618 \\
\hline $\mathrm{C}$ & 6.424513 & 1.233370 & -0.082240 \\
\hline $\mathrm{C}$ & 3.232254 & -4.110460 & -3.008223 \\
\hline $\mathrm{C}$ & 1.787409 & -5.895755 & -3.801425 \\
\hline $\mathrm{C}$ & 6.500698 & -0.510806 & 2.099747 \\
\hline $\mathrm{C}$ & 7.639672 & 0.797071 & 0.445930 \\
\hline $\mathrm{C}$ & 3.062415 & -5.308370 & -3.731700 \\
\hline $\mathrm{C}$ & 7.714356 & -0.094816 & 1.541207 \\
\hline $\mathrm{O}$ & 4.199301 & -5.812934 & -4.324864 \\
\hline $\mathrm{C}$ & 9.083879 & -0.562174 & 2.070412 \\
\hline $\mathrm{C}$ & 4.059145 & -7.023379 & -5.056476 \\
\hline $\mathrm{C}$ & 8.952106 & -1.524246 & 3.268381 \\
\hline $\mathrm{C}$ & 9.845636 & -1.297585 & 0.939388 \\
\hline $\mathrm{C}$ & 9.906829 & 0.668949 & 2.525329 \\
\hline $\mathrm{H}$ & 1.817896 & 1.655114 & -1.007615 \\
\hline $\mathrm{H}$ & -1.559535 & -2.147517 & -0.301300 \\
\hline $\mathrm{H}$ & 0.700230 & -1.103849 & 1.700855 \\
\hline $\mathrm{H}$ & -1.633755 & -1.747137 & 5.303380 \\
\hline $\mathrm{H}$ & 3.255309 & 5.116627 & -1.326253 \\
\hline $\mathrm{H}$ & 1.928641 & 6.961200 & -2.325199 \\
\hline $\mathrm{H}$ & -1.254016 & 4.186871 & -3.260495 \\
\hline $\mathrm{H}$ & 0.078676 & 2.333901 & -2.171552 \\
\hline $\mathrm{H}$ & -0.866487 & 8.557688 & -4.022780 \\
\hline $\mathrm{H}$ & 0.130823 & 8.418053 & -2.530242 \\
\hline $\mathrm{H}$ & 0.838818 & 7.989321 & -4.130470 \\
\hline $\mathrm{H}$ & -4.226580 & -4.102568 & -1.928038 \\
\hline $\mathrm{H}$ & -5.296580 & -2.042056 & 2.479829 \\
\hline $\mathrm{H}$ & -7.188031 & -2.896534 & 3.399164 \\
\hline $\mathrm{H}$ & -7.490788 & -1.340198 & 2.568344 \\
\hline $\mathrm{H}$ & -6.547790 & -4.139815 & -1.089120 \\
\hline $\mathrm{H}$ & -8.436511 & -2.848760 & -0.787744 \\
\hline $\mathrm{H}$ & -8.214204 & -1.320049 & 0.102467 \\
\hline $\mathrm{H}$ & -9.559057 & -2.419358 & 0.529853 \\
\hline $\mathrm{H}$ & -8.841455 & -2.451161 & 2.908623 \\
\hline $\mathrm{H}$ & 2.628059 & -0.999156 & 5.570667 \\
\hline $\mathrm{H}$ & -7.436748 & -5.107478 & 2.124708 \\
\hline $\mathrm{H}$ & -7.978521 & -5.117928 & 0.427083 \\
\hline $\mathrm{H}$ & -9.108402 & -4.618967 & 1.713723 \\
\hline $\mathrm{H}$ & 2.265257 & -2.582937 & -1.802819 \\
\hline $\mathrm{H}$ & -0.300884 & -5.753627 & -3.211405 \\
\hline $\mathrm{H}$ & 6.402397 & 1.922798 & -0.926991 \\
\hline $\mathrm{H}$ & 4.228697 & -3.661334 & -2.957005 \\
\hline $\mathrm{H}$ & 1.627309 & -6.824986 & -4.354621 \\
\hline $\mathrm{H}$ & 6.482421 & -1.186862 & 2.958179 \\
\hline $\mathrm{H}$ & 8.561885 & 1.155699 & -0.024884 \\
\hline
\end{tabular}




$\begin{array}{cccc}\mathrm{H} & 5.061688 & -7.259406 & -5.444298 \\ \mathrm{H} & 3.711630 & -7.855823 & -4.412948 \\ \mathrm{H} & 3.356384 & -6.912365 & -5.906063 \\ \mathrm{H} & 9.955867 & -1.832352 & 3.609295 \\ \mathrm{H} & 8.395484 & -2.439230 & 3.000058 \\ \mathrm{H} & 8.441630 & -1.046877 & 4.123274 \\ \mathrm{H} & 9.289634 & -2.190740 & 0.605539 \\ \mathrm{H} & 10.839924 & -1.623638 & 1.295337 \\ \mathrm{H} & 9.999579 & -0.648861 & 0.060274 \\ \mathrm{H} & 10.900873 & 0.352148 & 2.890203 \\ \mathrm{H} & 9.394804 & 1.205332 & 3.343046 \\ \mathrm{H} & 10.064258 & 1.384553 & 1.700475 \\ \mathrm{H} & 3.173346 & 0.537231 & 0.051678 \\ \mathrm{H} & 0.131690 & -2.535625 & -1.321494 \\ \mathrm{H} & 0.789294 & -0.304345 & -2.266746 \\ \mathrm{Se} & 0.523300 & -0.282997 & -0.800234 \\ \mathrm{H} & 0.432385 & -1.446100 & 6.674403\end{array}$

Statistical Thermodynamic Analysis

Temperature $=298.150$ Kelvin Pressure $=1.00000$ Atm

\begin{tabular}{|c|c|}
\hline-5382.43263274 & Predicted Change $=-1.25893$ \\
\hline Zero-point correction $(\mathrm{ZPE})=$ & $-5381.1233 \quad 1.30932$ \\
\hline Internal Energy $(U)=$ & -5381.04261 .38995 \\
\hline Enthalpy $(H)=$ & $\begin{array}{lll}-5381.0417 & 1.39089\end{array}$ \\
\hline Gibbs Free Energy $(G)=$ & $-5381.2484 \quad 1.18421$ \\
\hline $\begin{array}{l}\text { Frequencies -- } 7.4957 \\
\text { Single points at WB97X-D3/def2 }\end{array}$ & $\begin{array}{cc}\text { 10.9116 } & 12.5747 \\
\text { 2-TZVPP: }-5389.15578709255\end{array}$ \\
\hline \multicolumn{2}{|c|}{$\mathbf{R}=\mathbf{H}$ receptor bound with $\mathbf{S H}-$ (with TBA) } \\
\hline
\end{tabular}

Using Gaussian 09: EM64L-G09RevE.01 30-Nov-2015
\#PBEPBE/6-31G(d)/auto scf=(direct,tight,maxcycle=300,xqc) opt $=($ maxcycle $=250)$ freq $=$ noraman int $=($ grid $=$ ultrafine $)$ \#P Geom=AllCheck Guess=TCheck SCRF=Check GenChk RPBEPBE/6-31G(d)/Auto Freq
Pointgroup $=\mathrm{C} 1 \quad$ Stoichiometry $=\mathrm{C} 62 \mathrm{H} 83 \mathrm{~N} 5 \mathrm{O} 4 \mathrm{~S} \quad \mathrm{C} 1[\mathrm{X}(\mathrm{C} 62 \mathrm{H} 83 \mathrm{~N} 5 \mathrm{O} 4 \mathrm{~S})]$ \#Atoms $=155$ Charge $=0 \quad$ Multiplicity $=1$

SCF Energy $=-3381.40719741 \quad$ Predicted Change $=-8.385035 \mathrm{D}-08$

$========$
Optimization completed. $\quad$ Found $1 \quad$ times




\begin{tabular}{|c|c|c|c|c|c|c|}
\hline & & & & & Cri & \\
\hline & & 0.0 & $E S$ & & & \\
\hline & 0.00867 & 0.00180 & [ NO ] & 0.00867 & 0.00180 & [NO ] \\
\hline
\end{tabular}

\begin{tabular}{|c|c|c|c|}
\hline \multirow{2}{*}{$\begin{array}{l}\text { Atomic } \\
\text { Type }\end{array}$} & \multicolumn{3}{|c|}{ Coordinates (Angstroms) } \\
\hline & $\mathrm{X}$ & $\begin{array}{ll}\mathrm{Y} & \mathrm{Z}\end{array}$ & \\
\hline $\mathrm{N}$ & 2.218290 & 2.268672 & -1.476090 \\
\hline $\mathrm{C}$ & 0.930594 & 2.467769 & -2.290028 \\
\hline $\mathrm{C}$ & 0.028462 & 1.249996 & -2.489747 \\
\hline $\mathrm{C}$ & -1.189836 & 1.665146 & -3.342153 \\
\hline $\mathrm{C}$ & -2.115739 & 0.484747 & -3.657600 \\
\hline $\mathrm{C}$ & 1.834873 & 2.048611 & -0.001736 \\
\hline $\mathrm{C}$ & 2.982109 & 1.917130 & 1.003650 \\
\hline $\mathrm{C}$ & 2.403071 & 1.937251 & 2.435589 \\
\hline $\mathrm{C}$ & 3.478873 & 1.757469 & 3.513775 \\
\hline $\mathrm{C}$ & 3.004349 & 1.050548 & -1.967662 \\
\hline $\mathrm{C}$ & 3.376558 & 1.034154 & -3.453919 \\
\hline $\mathrm{C}$ & 4.083248 & -0.286178 & -3.818495 \\
\hline $\mathrm{C}$ & 4.495397 & -0.336201 & -5.294780 \\
\hline $\mathrm{C}$ & 3.082824 & 3.521193 & -1.634044 \\
\hline $\mathrm{C}$ & 2.439069 & 4.847452 & -1.218509 \\
\hline $\mathrm{C}$ & 3.478700 & 5.985205 & -1.219970 \\
\hline $\mathrm{C}$ & 2.830866 & 7.350037 & -0.949950 \\
\hline $\mathrm{H}$ & 0.371507 & 3.258588 & -1.765453 \\
\hline $\mathrm{H}$ & 1.254582 & 2.872463 & -3.264400 \\
\hline $\mathrm{H}$ & -0.319681 & 0.850779 & -1.518990 \\
\hline $\mathrm{H}$ & 0.563959 & 0.433224 & -3.007905 \\
\hline $\mathrm{H}$ & -0.836483 & 2.123555 & -4.287975 \\
\hline $\mathrm{H}$ & -1.756891 & 2.449034 & -2.805607 \\
\hline $\mathrm{H}$ & -2.957684 & 0.807949 & -4.292610 \\
\hline $\mathrm{H}$ & -1.578130 & -0.319411 & -4.190603 \\
\hline $\mathrm{H}$ & -2.541420 & 0.049758 & -2.738381 \\
\hline $\mathrm{H}$ & 1.190891 & 2.902007 & 0.258915 \\
\hline $\mathrm{H}$ & 1.191150 & 1.148682 & 0.035265 \\
\hline $\mathrm{H}$ & 3.536164 & 0.972439 & 0.856647 \\
\hline $\mathrm{H}$ & 3.710857 & 2.745524 & 0.913225 \\
\hline $\mathrm{H}$ & 1.642141 & 1.140672 & 2.526809 \\
\hline $\mathrm{H}$ & 1.873727 & 2.897687 & 2.592217 \\
\hline $\mathrm{H}$ & 3.035300 & 1.844473 & 4.519850 \\
\hline $\mathrm{H}$ & 3.947791 & 0.760954 & 3.451224 \\
\hline $\mathrm{H}$ & 4.270539 & 2.524242 & 3.426480 \\
\hline $\mathrm{H}$ & 3.911996 & 1.007270 & -1.344904 \\
\hline $\mathrm{H}$ & 2.390024 & 0.172554 & -1.711052 \\
\hline $\mathrm{H}$ & 2.475958 & 1.141281 & -4.085800 \\
\hline $\mathrm{H}$ & 4.049183 & 1.875035 & -3.705704 \\
\hline $\mathrm{H}$ & 3.412588 & -1.133716 & -3.583986 \\
\hline $\mathrm{H}$ & 4.973877 & -0.416812 & -3.175409 \\
\hline
\end{tabular}




\begin{tabular}{|c|c|c|c|}
\hline $\mathrm{H}$ & 5.004184 & -1.286684 & -5.528929 \\
\hline $\mathrm{H}$ & 3.618350 & -0.254529 & -5.961191 \\
\hline $\mathrm{H}$ & 5.188870 & 0.485410 & -5.549161 \\
\hline $\mathrm{H}$ & 3.367687 & 3.562497 & -2.696980 \\
\hline $\mathrm{H}$ & 3.999097 & 3.332212 & -1.052570 \\
\hline $\mathrm{H}$ & 1.618876 & 5.110241 & -1.910435 \\
\hline $\mathrm{H}$ & 1.989216 & 4.781742 & -0.212435 \\
\hline $\mathrm{H}$ & 4.009453 & 6.010061 & -2.191761 \\
\hline $\mathrm{H}$ & 4.247735 & 5.774948 & -0.452139 \\
\hline $\mathrm{H}$ & 3.594170 & 8.140722 & -0.858269 \\
\hline $\mathrm{H}$ & 2.150859 & 7.633361 & -1.773117 \\
\hline $\mathrm{H}$ & 2.234626 & 7.333818 & -0.020904 \\
\hline $\mathrm{O}$ & -0.406327 & 7.119438 & 0.894557 \\
\hline $\mathrm{O}$ & 3.649691 & -1.836276 & 3.539970 \\
\hline $\mathrm{N}$ & -2.756589 & 2.072882 & 0.137837 \\
\hline $\mathrm{N}$ & 2.833286 & -1.917568 & 1.359024 \\
\hline $\mathrm{C}$ & -0.856639 & -2.984554 & -1.804841 \\
\hline $\mathrm{C}$ & -0.957337 & -4.709014 & -4.022191 \\
\hline $\mathrm{C}$ & 0.281561 & -4.367029 & -3.470266 \\
\hline $\mathrm{C}$ & 0.340826 & -3.499706 & -2.348771 \\
\hline $\mathrm{C}$ & -2.250913 & 3.372960 & 0.305784 \\
\hline $\mathrm{C}$ & -2.737803 & 4.498125 & -0.392384 \\
\hline $\mathrm{C}$ & -2.151203 & 5.761358 & -0.214758 \\
\hline $\mathrm{C}$ & -1.062607 & 5.928256 & 0.659483 \\
\hline $\mathrm{C}$ & -0.581427 & 4.812257 & 1.372026 \\
\hline $\mathrm{C}$ & -1.171057 & 3.557694 & 1.201996 \\
\hline $\mathrm{C}$ & -0.939209 & 8.281127 & 0.265997 \\
\hline $\mathrm{C}$ & 1.595592 & -3.154506 & -1.770130 \\
\hline $\mathrm{C}$ & 2.696206 & -2.895367 & -1.279054 \\
\hline $\mathrm{C}$ & 3.953136 & -2.611756 & -0.680328 \\
\hline $\mathrm{C}$ & 4.026055 & -2.130272 & 0.666036 \\
\hline $\mathrm{C}$ & 5.306074 & -1.878318 & 1.209772 \\
\hline $\mathrm{C}$ & 6.456218 & -2.101028 & 0.451212 \\
\hline $\mathrm{C}$ & 6.415933 & -2.580934 & -0.878755 \\
\hline $\mathrm{C}$ & 5.146857 & -2.826541 & -1.415340 \\
\hline $\mathrm{C}$ & 8.495078 & -1.479656 & -1.783742 \\
\hline $\mathrm{C}$ & 7.460885 & -3.352180 & -3.087004 \\
\hline $\mathrm{C}$ & 2.684849 & -1.843996 & 2.761665 \\
\hline $\mathrm{C}$ & -4.066605 & 1.760729 & -0.207681 \\
\hline $\mathrm{C}$ & -2.105717 & -3.316690 & -2.376249 \\
\hline $\mathrm{C}$ & -2.144728 & -4.194327 & -3.490240 \\
\hline $\mathrm{C}$ & 7.720220 & -2.815862 & -1.664460 \\
\hline $\mathrm{N}$ & 1.358734 & -1.777181 & 3.128688 \\
\hline $\mathrm{N}$ & -4.275268 & 0.375970 & -0.252474 \\
\hline $\mathrm{O}$ & -4.941123 & 2.599509 & -0.453344 \\
\hline $\mathrm{C}$ & -3.299208 & -2.742632 & -1.856568 \\
\hline $\mathrm{C}$ & 8.595903 & -3.848104 & -0.910809 \\
\hline $\mathrm{C}$ & 0.838289 & -1.747792 & 4.441907 \\
\hline
\end{tabular}




\begin{tabular}{|c|c|c|c|}
\hline $\mathrm{C}$ & -5.507529 & -0.249016 & -0.485537 \\
\hline $\mathrm{C}$ & -4.327725 & -2.206023 & -1.445515 \\
\hline $\mathrm{C}$ & -0.569542 & -1.799770 & 4.576173 \\
\hline $\mathrm{C}$ & 1.626178 & -1.674662 & 5.606585 \\
\hline $\mathrm{C}$ & -5.526794 & -1.568964 & -1.029620 \\
\hline $\mathrm{C}$ & -6.741077 & 0.362876 & -0.180890 \\
\hline $\mathrm{C}$ & -1.168838 & -1.767674 & 5.832886 \\
\hline $\mathrm{C}$ & 1.019580 & -1.650351 & 6.872209 \\
\hline $\mathrm{C}$ & -6.766659 & -2.232778 & -1.214445 \\
\hline $\mathrm{C}$ & -7.941534 & -0.315769 & -0.391501 \\
\hline $\mathrm{C}$ & -0.379375 & -1.692659 & 6.997063 \\
\hline $\mathrm{C}$ & -7.991748 & -1.632915 & -0.903990 \\
\hline $\mathrm{O}$ & -1.069832 & -1.670490 & 8.187665 \\
\hline $\mathrm{C}$ & -9.346060 & -2.340410 & -1.101574 \\
\hline $\mathrm{C}$ & -0.296297 & -1.607076 & 9.379186 \\
\hline $\mathrm{C}$ & -9.185952 & -3.763328 & -1.674097 \\
\hline $\mathrm{C}$ & -10.072979 & -2.445513 & 0.262513 \\
\hline $\mathrm{C}$ & -10.217405 & -1.517948 & -2.083497 \\
\hline $\mathrm{H}$ & -2.123490 & 1.289933 & 0.417111 \\
\hline $\mathrm{H}$ & 1.979329 & -2.059587 & 0.808632 \\
\hline $\mathrm{H}$ & -0.826748 & -2.331106 & -0.926378 \\
\hline $\mathrm{H}$ & 1.210146 & -4.768516 & -3.887121 \\
\hline $\mathrm{H}$ & -3.596286 & 4.380078 & -1.055306 \\
\hline $\mathrm{H}$ & -2.560462 & 6.609616 & -0.770001 \\
\hline $\mathrm{H}$ & 0.236520 & 4.955445 & 2.086655 \\
\hline $\mathrm{H}$ & -0.805128 & 2.699292 & 1.775759 \\
\hline $\mathrm{H}$ & -0.319809 & 9.121979 & 0.612038 \\
\hline $\mathrm{H}$ & -0.880234 & 8.216033 & -0.838624 \\
\hline $\mathrm{H}$ & -1.992285 & 8.455549 & 0.559097 \\
\hline $\mathrm{H}$ & 5.375291 & -1.540757 & 2.243651 \\
\hline $\mathrm{H}$ & 5.035758 & -3.211128 & -2.432414 \\
\hline $\mathrm{H}$ & 6.931328 & -4.320712 & -3.069707 \\
\hline $\mathrm{H}$ & 6.866252 & -2.644212 & -3.692303 \\
\hline $\mathrm{H}$ & 7.423630 & -1.894899 & 0.923352 \\
\hline $\mathrm{H}$ & 8.735156 & -1.055805 & -0.793749 \\
\hline $\mathrm{H}$ & 7.903727 & -0.728856 & -2.337956 \\
\hline $\mathrm{H}$ & 9.446819 & -1.632515 & -2.324252 \\
\hline $\mathrm{H}$ & 8.422160 & -3.507074 & -3.606996 \\
\hline $\mathrm{H}$ & -3.112068 & -4.454044 & -3.930544 \\
\hline $\mathrm{H}$ & 8.075151 & -4.817217 & -0.824004 \\
\hline $\mathrm{H}$ & 8.844093 & -3.507694 & 0.108721 \\
\hline $\mathrm{H}$ & 9.545660 & -4.013944 & -1.451021 \\
\hline $\mathrm{H}$ & -1.193326 & -1.872027 & 3.678012 \\
\hline $\mathrm{H}$ & 2.712052 & -1.645046 & 5.515042 \\
\hline $\mathrm{H}$ & -6.739948 & 1.380376 & 0.210432 \\
\hline $\mathrm{H}$ & -2.257433 & -1.807749 & 5.935279 \\
\hline $\mathrm{H}$ & 1.661001 & -1.597155 & 7.755908 \\
\hline $\mathrm{H}$ & -6.727357 & -3.244065 & -1.626910 \\
\hline
\end{tabular}




$\begin{array}{cccc}\mathrm{H} & -8.872810 & 0.201405 & -0.133954 \\ \mathrm{H} & -1.019832 & -1.602957 & 10.208262 \\ \mathrm{H} & 0.372452 & -2.484284 & 9.482536 \\ \mathrm{H} & 0.315229 & -0.684210 & 9.424221 \\ \mathrm{H} & -10.179560 & -4.228814 & -1.795854 \\ \mathrm{H} & -8.594033 & -4.410731 & -1.003594 \\ \mathrm{H} & -8.698412 & -3.753967 & -2.664821 \\ \mathrm{H} & -9.482112 & -3.042105 & 0.978987 \\ \mathrm{H} & -11.057027 & -2.932973 & 0.137019 \\ \mathrm{H} & -10.244543 & -1.452722 & 0.712276 \\ \mathrm{H} & -11.201692 & -2.001640 & -2.221143 \\ \mathrm{H} & -9.731039 & -1.439727 & -3.071373 \\ \mathrm{H} & -10.395104 & -0.493625 & -1.714114 \\ \mathrm{H} & -3.455785 & -0.236372 & -0.138089 \\ \mathrm{H} & 0.660697 & -1.615458 & 2.374576 \\ \mathrm{H} & -1.044437 & 0.021236 & 2.165767 \\ \mathrm{H} & -0.998192 & -5.385304 & -4.882588 \\ \mathrm{~S} & -0.788758 & -0.463026 & 0.917487\end{array}$

Statistical Thermodynamic Analysis

Temperature $=298.150$ Kelvin $\quad$ Pressure $=1.00000$ Atm

\begin{tabular}{|c|c|}
\hline SCF Energy $=\quad-3381.4071974$ & Predicted Change $=-8.385035 \mathrm{D}-08$ \\
\hline Zero-point correction $(\mathrm{ZPE})=$ & $-3380.1011 \quad 1.30607$ \\
\hline Internal Energy $(U)=$ & -3380.01931 .38781 \\
\hline Enthalpy $(\mathrm{H})=$ & $-3380.0184 \quad 1.38876$ \\
\hline Gibbs Free Energy $(\mathrm{G})=$ & $-3380.2301 \quad 1.17708$ \\
\hline Frequencies -- & 11.9605 \\
\hline
\end{tabular}

$R=H$ receptor bound with $B r-($ no $T B A)$

Supporting Information: 010-bound_noTBA_H_Br.log

Using Gaussian 09: EM64L-G09RevE.01 30-Nov-2015
$======$
\#PBEPBE/6-31G(d)/auto scf=(direct,tight, $\operatorname{maxcycle}=300, \mathrm{xqc})$
$\mathrm{opt}=($ maxcycle $=250)$ freq $=$ noraman $\mathrm{int}=($ grid $=$ ultrafine $)$
\#P Geom=AllCheck Guess=TCheck SCRF=Check GenChk RPBEPBE/6-31G(d)/Auto Freq
Pointgroup= C1 Stoichiometry $=\mathrm{C} 46 \mathrm{H} 46 \mathrm{BrN} 4 \mathrm{O} 4(1-) \quad \mathrm{C} 1[\mathrm{X}(\mathrm{C} 46 \mathrm{H} 46 \mathrm{BrN} 4 \mathrm{O} 4)]$ \#Atoms= 101
Charge $=-1 \quad$ Multiplicity $=1$

SCF Energy $=-4869.09302852 \quad$ Predicted Change $=-8.093322 \mathrm{D}-09$ 


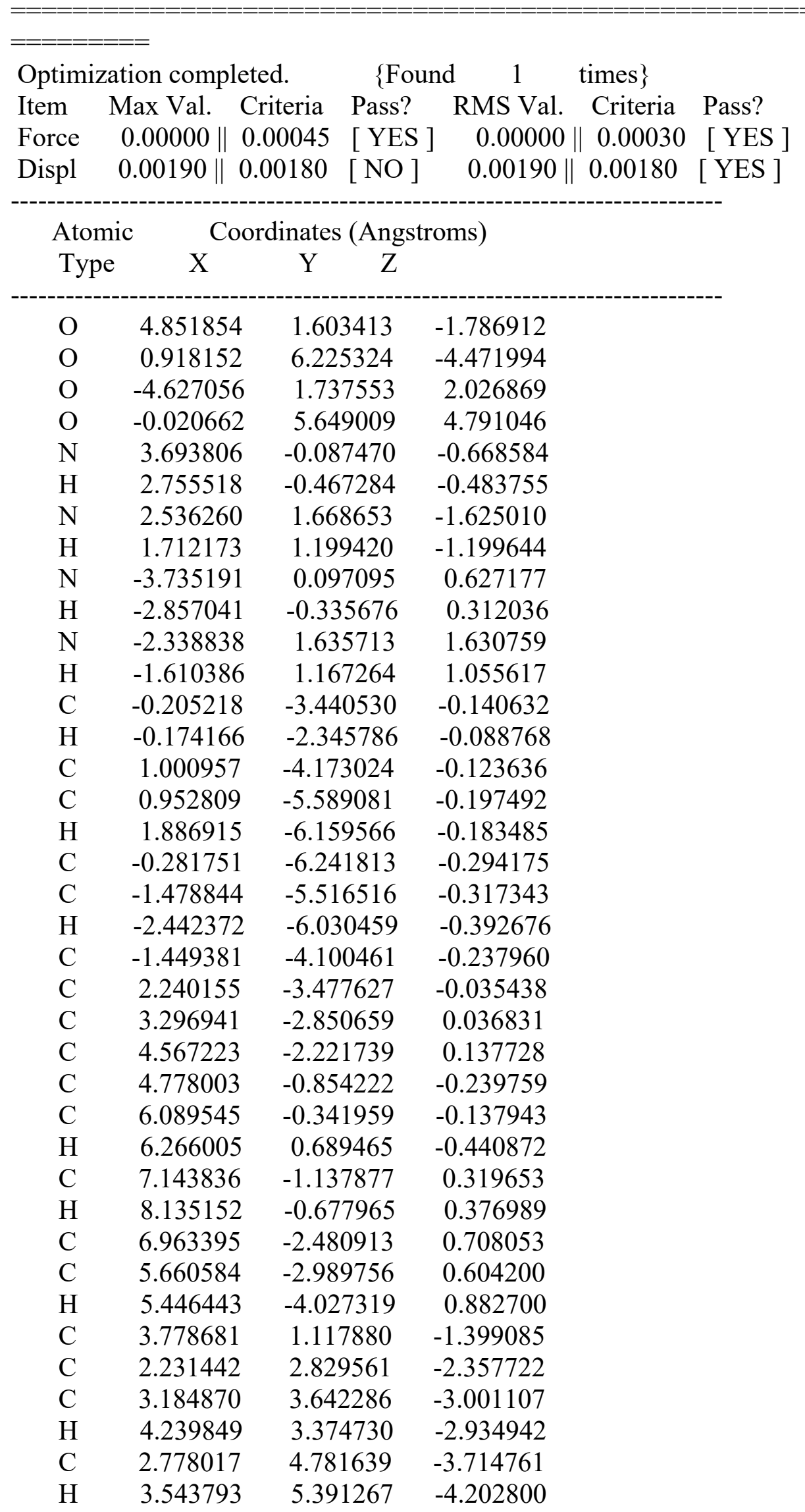




\begin{tabular}{|c|c|c|c|}
\hline $\mathrm{C}$ & 1.419552 & 5.126637 & -3.795581 \\
\hline $\mathrm{C}$ & 0.465123 & 4.315964 & -3.151424 \\
\hline $\mathrm{H}$ & -0.592234 & 4.591129 & -3.215886 \\
\hline$C$ & 0.862309 & 3.184594 & -2.443930 \\
\hline $\mathrm{H}$ & 0.120706 & 2.551023 & -1.941780 \\
\hline $\mathrm{C}$ & 1.865198 & 7.057206 & -5.12039 \\
\hline $\mathrm{H}$ & 1.286612 & 7.866367 & -5.593264 \\
\hline $\mathrm{H}$ & 2.431864 & 6.510959 & -5.90188 \\
\hline $\mathrm{H}$ & 2.589176 & 7.498008 & -4.405177 \\
\hline $\mathrm{C}$ & -2.645898 & -3.329378 & -0.24923 \\
\hline $\mathrm{C}$ & -3.658821 & -2.630041 & -0.24642 \\
\hline $\mathrm{C}$ & -4.868595 & -1.885464 & -0.23413 \\
\hline $\mathrm{C}$ & -4.918096 & -0.533458 & 0.24171 \\
\hline $\mathrm{C}$ & -6.175833 & 0.104827 & 0.27729 \\
\hline $\mathrm{H}$ & -6.228625 & 1.123357 & 0.660903 \\
\hline $\mathrm{C}$ & -7.332289 & -0.554958 & -0.14955 \\
\hline $\mathrm{H}$ & -8.276061 & -0.003352 & -0.10025 \\
\hline $\mathrm{C}$ & -7.310302 & -1.876424 & -0.64077 \\
\hline $\mathrm{C}$ & -6.060149 & -2.512328 & -0.668777 \\
\hline $\mathrm{H}$ & -5.969902 & -3.543331 & -1.027779 \\
\hline $\mathrm{C}$ & -3.642851 & 1.216857 & 1.48137 \\
\hline $\mathrm{C}$ & -1.855047 & 2.667579 & 2.45356 \\
\hline $\mathrm{C}$ & -2.658862 & 0207 & 3.28579 \\
\hline $\mathrm{H}$ & -3.736235 & 3.302272 & 3.303280 \\
\hline $\mathrm{C}$ & -2.076164 & 2922 & 4.078302 \\
\hline $\mathrm{H}$ & -2.727968 & 5.078958 & 4.71399 \\
\hline $\mathrm{C}$ & -0.689265 & 9697 & 4.05040 \\
\hline $\mathrm{C}$ & 0.115528 & 3.888949 & 3.217029 \\
\hline $\mathrm{H}$ & 1.195664 & & 3.19781 \\
\hline $\mathrm{C}$ & -0.456240 & 2.892236 & 2.43110 \\
\hline $\mathrm{H}$ & 0.166152 & 2.265583 & 1.780377 \\
\hline $\mathrm{C}$ & -0.814533 & 7410 & 5.633205 \\
\hline $\mathrm{H}$ & -0.116829 & 7.158502 & 6.13190 \\
\hline $\mathrm{H}$ & -1.351273 & 5.875060 & 6.40214 \\
\hline $\mathrm{H}$ & -1.559730 & 7.054827 & 5.05903 \\
\hline $\mathrm{C}$ & 8.107639 & -3.377688 & 1.22132 \\
\hline $\mathrm{C}$ & -8.569679 & -2.622118 & -1.12580 \\
\hline $\mathrm{C}$ & 7.781487 & & 2.65522 \\
\hline $\mathrm{H}$ & 6.835526 & -4.431193 & 2.68685 \\
\hline $\mathrm{H}$ & 8.584755 & -4.524205 & 3.03337 \\
\hline $\mathrm{H}$ & 7.682440 & -3.009724 & 3.34687 \\
\hline $\mathrm{C}$ & 9.458116 & -2.633557 & 1.26212 \\
\hline $\mathrm{H}$ & 9.762588 & -2.283879 & 0.26000 \\
\hline $\mathrm{H}$ & 9.422483 & -1.758965 & 1.93517 \\
\hline $\mathrm{H}$ & 10.246495 & -3.311696 & 1.6347 \\
\hline $\mathrm{C}$ & 8.261798 & -4.605607 & 0.28970 \\
\hline $\mathrm{H}$ & 8.512185 & -4.289554 & -0.73800 \\
\hline $\mathrm{H}$ & 9.067820 & -5.270158 & 0.65345 \\
\hline
\end{tabular}




$\begin{array}{llll}\mathrm{H} & 7.332179 & -5.197720 & 0.240613 \\ \mathrm{C} & -8.390666 & -3.030693 & -2.609179 \\ \mathrm{H} & -7.509189 & -3.679259 & -2.748649 \\ \mathrm{H} & -9.278538 & -3.583335 & -2.969514 \\ \mathrm{H} & -8.255235 & -2.140611 & -3.247980 \\ \mathrm{C} & -8.780747 & -3.895157 & -0.268949 \\ \mathrm{H} & -8.929658 & -3.633100 & 0.793079 \\ \mathrm{H} & -9.670564 & -4.453444 & -0.615605 \\ \mathrm{H} & -7.912486 & -4.573259 & -0.327782 \\ \mathrm{C} & -9.840053 & -1.754091 & -1.016764 \\ \mathrm{H} & -10.040422 & -1.451730 & 0.026137 \\ \mathrm{H} & -9.764111 & -0.839777 & -1.631150 \\ \mathrm{H} & -10.714621 & -2.326585 & -1.374012 \\ \mathrm{H} & -0.311436 & -7.335576 & -0.353517 \\ \mathrm{Br} & -0.066501 & 0.159205 & -0.268291\end{array}$

Statistical Thermodynamic Analysis

Temperature $=298.150$ Kelvin $\quad$ Pressure $=1.00000$ Atm

\begin{tabular}{|c|c|c|}
\hline \multicolumn{3}{|l|}{$========$} \\
\hline $\begin{array}{l}\mathrm{SCF} \text { Energy }=-4869.09302852 \\
\text { Zero-point correction }(\mathrm{ZPE})=\end{array}$ & Predicted Chan & $\begin{array}{l}\text { ige }=-8.0933 \\
080262\end{array}$ \\
\hline Internal Energy $(U)=$ & $-4868.2349 \quad 0.8581$ & 2.00202 \\
\hline Enthalpy $(\mathrm{H})=$ & -4868.2339 & 0.85907 \\
\hline Gibbs Free Energy $(\mathrm{G})=$ & -4868.3896 & 0.70335 \\
\hline Frequencies -- 2.5551 & 5.6876 & 10.4082 \\
\hline Single points at WB97X-D3/def2 & 2-TZVPP: -4875.04C & 04734945 \\
\hline $\begin{array}{r}\mathbf{R}=\mathbf{H} \text { receptor bound with } \mathbf{C l}- \\
\text { Supporting Information: }\end{array}$ & $\begin{array}{l}\text { (no TBA) } \\
010 \text {-bound noTBA }\end{array}$ & $\mathrm{H}$ Cl.log \\
\hline
\end{tabular}

Using Gaussian 09: EM64L-G09RevE.01 30-Nov-2015

\#PBEPBE/6-31G(d)/auto scf=(direct,tight,maxcycle=300,xqc) $\mathrm{opt}=($ maxcycle $=250)$ freq $=$ noraman int $=($ grid $=$ ultrafine $)$ \#P Geom=AllCheck Guess=TCheck SCRF=Check GenChk RPBEPBE/6-31G(d)/Auto Freq

Pointgroup= $\mathrm{C} 1 \quad$ Stoichiometry $=\mathrm{C} 46 \mathrm{H} 46 \mathrm{ClN} 4 \mathrm{O} 4(1-) \quad \mathrm{C} 1[\mathrm{X}(\mathrm{C} 46 \mathrm{H} 46 \mathrm{ClN} 4 \mathrm{O} 4)]$ \#Atoms= 101 Charge $=-1 \quad$ Multiplicity $=1$

SCF Energy $=-2757.77090989 \quad$ Predicted Change $=-1.094038$ D -09

$========$

Optimization completed. $\quad$ FFound 2 times\} Item Max Val. Criteria Pass? RMS Val. Criteria Pass? 
Force $\quad 0.00000 \| 0.00045$ [YES ] $0.00000 \| 0.00030$ [ [YES ] Displ $0.00119 \| 0.00180$ [YES ] $0.00119 \| 0.00180$ [Y 0.0

\begin{tabular}{|c|c|c|c|}
\hline \multirow{2}{*}{$\begin{array}{l}\text { Atomic } \\
\text { Type }\end{array}$} & \multicolumn{3}{|c|}{ Coordinates (Angstroms) } \\
\hline & $\mathrm{X}$ & $\begin{array}{ll}Y & Z\end{array}$ & \\
\hline $\mathrm{Cl}$ & 0.000000 & 0.030714 & -0.000001 \\
\hline $\mathrm{O}$ & -4.586524 & -1.760131 & -1.753352 \\
\hline $\mathrm{O}$ & -0.218958 & -6.130595 & -4.117610 \\
\hline $\mathrm{O}$ & 4.586523 & -1.760128 & 1.753361 \\
\hline $\mathrm{O}$ & 0.218966 & -6.130604 & 4.117605 \\
\hline $\mathrm{N}$ & -3.636801 & 0.053980 & -0.638323 \\
\hline $\mathrm{H}$ & -2.747031 & 0.522689 & -0.425363 \\
\hline $\mathrm{N}$ & -2.281817 & -1.613530 & -1.494384 \\
\hline $\mathrm{H}$ & -1.518855 & -1.071697 & -1.036276 \\
\hline $\mathrm{N}$ & 3.636801 & 0.053973 & 0.638315 \\
\hline $\mathrm{H}$ & 2.747030 & 0.522678 & 0.425348 \\
\hline $\mathrm{N}$ & 2.281818 & -1.613542 & 1.494370 \\
\hline $\mathrm{H}$ & 1.518857 & -1.071716 & 1.036252 \\
\hline $\mathrm{C}$ & -0.000003 & 3.521575 & -0.000006 \\
\hline $\mathrm{H}$ & -0.000004 & 2.424487 & -0.000007 \\
\hline $\mathrm{C}$ & -1.225818 & 4.221455 & 0.032933 \\
\hline $\mathrm{C}$ & -1.216961 & 5.640146 & 0.035367 \\
\hline $\mathrm{H}$ & -2.167167 & 6.182966 & 0.061146 \\
\hline $\mathrm{C}$ & 0.000001 & 6.330789 & -0.000003 \\
\hline $\mathrm{C}$ & 1.216960 & 5.640144 & -0.035374 \\
\hline $\mathrm{H}$ & 2.167168 & 6.182962 & -0.061151 \\
\hline $\mathrm{C}$ & 1.225814 & 4.221452 & -0.032943 \\
\hline $\mathrm{C}$ & -2.445073 & 3.487830 & 0.060059 \\
\hline $\mathrm{C}$ & -3.479973 & 2.821878 & 0.081057 \\
\hline $\mathrm{C}$ & -4.710837 & 2.113842 & 0.107354 \\
\hline $\mathrm{C}$ & -4.799928 & 0.734732 & -0.279286 \\
\hline $\mathrm{C}$ & -6.075357 & 0.130132 & -0.256455 \\
\hline $\mathrm{H}$ & -6.158547 & -0.910756 & -0.566829 \\
\hline $\mathrm{C}$ & -7.208859 & 0.849104 & 0.134924 \\
\hline $\mathrm{H}$ & -8.166865 & 0.320222 & 0.132583 \\
\hline $\mathrm{C}$ & -7.148656 & 2.200851 & 0.530333 \\
\hline $\mathrm{C}$ & -5.881461 & 2.801857 & 0.504580 \\
\hline $\mathrm{H}$ & -5.759066 & 3.851419 & 0.793636 \\
\hline $\mathrm{C}$ & -3.577852 & -1.171391 & -1.335442 \\
\hline $\mathrm{C}$ & -1.860329 & -2.771621 & -2.174128 \\
\hline $\mathrm{C}$ & -2.720777 & -3.658215 & -2.851851 \\
\hline $\mathrm{H}$ & -3.792217 & -3.457554 & -2.854306 \\
\hline $\mathrm{C}$ & -2.204142 & -4.786440 & -3.509670 \\
\hline $\mathrm{H}$ & -2.901763 & -5.452900 & -4.024923 \\
\hline $\mathrm{C}$ & -0.825264 & -5.049389 & -3.501885 \\
\hline $\mathrm{C}$ & 0.037189 & -4.166556 & -2.824659 \\
\hline $\mathrm{H}$ & 1.110967 & -4.377920 & -2.817229 \\
\hline
\end{tabular}




\begin{tabular}{|c|c|c|c|}
\hline $\mathrm{C}$ & -0.470438 & -3.046027 & -2.172069 \\
\hline $\mathrm{H}$ & 0.205966 & -2.362801 & -1.646078 \\
\hline $\mathrm{C}$ & -1.074195 & -7.037512 & -4.792303 \\
\hline $\mathrm{H}$ & -0.420897 & -7.821842 & -5.206042 \\
\hline $\mathrm{H}$ & -1.626331 & -6.549885 & -5.621588 \\
\hline $\mathrm{H}$ & -1.810685 & -7.502512 & -4.105772 \\
\hline $\mathrm{C}$ & 2.445068 & 3.487824 & -0.060067 \\
\hline $\mathrm{C}$ & 3.479967 & 2.821870 & -0.081063 \\
\hline $\mathrm{C}$ & 4.710833 & 2.113838 & -0.107356 \\
\hline $\mathrm{C}$ & 4.799927 & 0.734729 & 0.279286 \\
\hline $\mathrm{C}$ & 6.075358 & 0.130133 & 0.256461 \\
\hline $\mathrm{H}$ & 6.158551 & -0.910754 & 0.566836 \\
\hline $\mathrm{C}$ & 7.208859 & 0.849110 & -0.134913 \\
\hline $\mathrm{H}$ & 8.166867 & 0.320230 & -0.132566 \\
\hline $\mathrm{C}$ & 7.148654 & 2.200857 & -0.530323 \\
\hline $\mathrm{C}$ & 5.881456 & 2.801857 & -0.504576 \\
\hline $\mathrm{H}$ & 5.759059 & 3.851419 & -0.793633 \\
\hline $\mathrm{C}$ & 3.577852 & -1.171396 & 1.335439 \\
\hline $\mathrm{C}$ & 1.860331 & -2.771632 & 2.174117 \\
\hline $\mathrm{C}$ & 2.720780 & -3.658217 & 2.851851 \\
\hline $\mathrm{H}$ & 3.792219 & -3.457549 & 2.854312 \\
\hline $\mathrm{C}$ & 2.204146 & -4.786441 & 3.509672 \\
\hline $\mathrm{H}$ & 2.901767 & -5.452894 & 4.024935 \\
\hline $\mathrm{C}$ & 0.825270 & -5.049398 & 3.501879 \\
\hline $\mathrm{C}$ & -0.037183 & -4.166573 & 2.824642 \\
\hline $\mathrm{H}$ & -1.110960 & -4.377944 & 2.817205 \\
\hline $\mathrm{C}$ & 0.470442 & -3.046045 & 2.172049 \\
\hline $\mathrm{H}$ & -0.205962 & -2.362826 & 1.646049 \\
\hline $\mathrm{C}$ & 1.074203 & -7.037512 & 4.792309 \\
\hline $\mathrm{H}$ & 0.420907 & -7.821844 & 5.206048 \\
\hline $\mathrm{H}$ & 1.626330 & -6.549877 & 5.621596 \\
\hline $\mathrm{H}$ & 1.810700 & -7.502513 & 4.105787 \\
\hline $\mathrm{C}$ & -8.382944 & 3.012212 & 0.972156 \\
\hline $\mathrm{C}$ & 8.382941 & 3.012222 & -0.972141 \\
\hline $\mathrm{C}$ & -8.181132 & 3.512843 & 2.424046 \\
\hline $\mathrm{H}$ & -7.280217 & 4.143089 & 2.514866 \\
\hline $\mathrm{H}$ & -9.049933 & 4.113874 & 2.752061 \\
\hline $\mathrm{H}$ & -8.066431 & 2.663390 & 3.119707 \\
\hline $\mathrm{C}$ & -9.677611 & 2.174908 & 0.928288 \\
\hline $\mathrm{H}$ & -9.893292 & 1.809020 & -0.090939 \\
\hline $\mathrm{H}$ & -9.622692 & 1.302077 & 1.602317 \\
\hline $\mathrm{H}$ & -10.533522 & 2.794370 & 1.250750 \\
\hline $\mathrm{C}$ & -8.565614 & 4.231404 & 0.034258 \\
\hline $\mathrm{H}$ & -8.729747 & 3.904248 & -1.007285 \\
\hline $\mathrm{H}$ & -9.437169 & 4.835780 & 0.348521 \\
\hline $\mathrm{H}$ & -7.678745 & 4.887595 & 0.043072 \\
\hline $\mathrm{C}$ & 8.181124 & 3.512865 & -2.424027 \\
\hline $\mathrm{H}$ & 7.280208 & 4.143110 & -2.514839 \\
\hline
\end{tabular}




$\begin{array}{cccc}\mathrm{H} & 9.049923 & 4.113901 & -2.752038 \\ \mathrm{H} & 8.066422 & 2.663419 & -3.119695 \\ \mathrm{C} & 8.565614 & 4.231405 & -0.034233 \\ \mathrm{H} & 8.729748 & 3.904239 & 1.007307 \\ \mathrm{H} & 9.437169 & 4.835782 & -0.348493 \\ \mathrm{H} & 7.678746 & 4.887598 & -0.043040 \\ \mathrm{C} & 9.677608 & 2.174917 & -0.928285 \\ \mathrm{H} & 9.893287 & 1.809013 & 0.090937 \\ \mathrm{H} & 9.622689 & 1.302095 & -1.602327 \\ \mathrm{H} & 10.533519 & 2.794384 & -1.250737 \\ \mathrm{H} & 0.000002 & 7.426591 & -0.000002\end{array}$

Statistical Thermodynamic Analysis

Temperature $=298.150$ Kelvin $\quad$ Pressure $=1.00000$ Atm

\begin{tabular}{|c|c|c|}
\hline-2757.77090989 & Predicted Char & $\mathrm{nge}=-1.0940$ \\
\hline Zero-point correction $(\mathrm{ZPE})=$ & -2756.9685 & 0.80232 \\
\hline Internal Energy $(U)=$ & -2756.91310 .8577 & \\
\hline Enthalpy $(H)=$ & -2756.9121 & 0.85872 \\
\hline Gibbs Free Energy $(\mathrm{G})=$ & -2757.0673 & 0.70354 \\
\hline Frequencies -- $\quad 3.5510$ & 6.7914 & 11.2703 \\
\hline Single points at WB97X-D3/def2 & 2-TZVPP: -2761.03 & 3250327914 \\
\hline
\end{tabular}

Using Gaussian 09: EM64L-G09RevE.01 30-Nov-2015
\#PBEPBE/6-31G(d)/auto scf=(direct,tight,maxcycle=300,xqc) $\mathrm{opt}=($ maxcycle $=250)$ freq $=$ noraman int $=($ grid $=$ ultrafine $)$ \#P Geom=AllCheck Guess=TCheck SCRF=Check GenChk RPBEPBE/6-31G(d)/Auto Freq Pointgroup= $\mathrm{C} 1$ Stoichiometry $=\mathrm{C} 46 \mathrm{H} 47 \mathrm{~N} 4 \mathrm{O} 4 \mathrm{Se}(1-) \quad \mathrm{C} 1[\mathrm{X}(\mathrm{C} 46 \mathrm{H} 47 \mathrm{~N} 4 \mathrm{O} 4 \mathrm{Se})]$ \#Atoms= 102
Charge $=-1 \quad$ Multiplicity $=1$

SCF Energy $=-4697.35597517 \quad$ Predicted Change $=-9.057321 \mathrm{D}-09$

\begin{tabular}{|c|c|c|c|c|}
\hline \multicolumn{5}{|c|}{$========$} \\
\hline Optim & zation completed. & $\{$ Found & times\} & \\
\hline Item & Max Val. Criteria & Pass? & RMS Val. & Pass? \\
\hline Force & $0.00001 \| 0.00045$ & [YES ] & $0.00000 \| 0.00030$ & [YES ] \\
\hline Displ & $0.00249 \| 0.00180$ & {$[\mathrm{NO}]$} & $0.00249 \| 0.00180$ & [YES ] \\
\hline
\end{tabular}




\begin{tabular}{|c|c|c|c|}
\hline \multirow{2}{*}{$\begin{array}{l}\text { Atomic } \\
\text { Type }\end{array}$} & \multicolumn{3}{|c|}{ Coordinates (Angstroms) } \\
\hline & $\mathrm{X}$ & $\begin{array}{ll}Y & Z\end{array}$ & \\
\hline $\mathrm{O}$ & 5.016111 & 1.580003 & -1.320966 \\
\hline $\mathrm{O}$ & 1.405686 & 6.724901 & -3.395773 \\
\hline $\mathrm{O}$ & -4.731950 & 1.882903 & 1.638475 \\
\hline $\mathrm{O}$ & -0.246059 & 6.420156 & 3.483285 \\
\hline $\mathrm{N}$ & 3.759159 & -0.210863 & -0.498474 \\
\hline $\mathrm{H}$ & 2.796717 & -0.573964 & -0.414669 \\
\hline $\mathrm{N}$ & 2.707529 & 1.740121 & -1.163840 \\
\hline $\mathrm{H}$ & 1.843449 & 1.238069 & -0.848495 \\
\hline $\mathrm{N}$ & -3.810328 & 0.070455 & 0.486556 \\
\hline $\mathrm{H}$ & -2.916661 & -0.381280 & 0.239477 \\
\hline $\mathrm{N}$ & -2.457942 & 1.811244 & 1.172992 \\
\hline $\mathrm{H}$ & -1.723375 & 1.283956 & 0.643720 \\
\hline $\mathrm{C}$ & -0.275171 & -3.584680 & -0.117268 \\
\hline $\mathrm{H}$ & -0.237007 & -2.486758 & -0.133712 \\
\hline $\mathrm{C}$ & 0.926066 & -4.322970 & -0.084934 \\
\hline $\mathrm{C}$ & 0.867938 & -5.740976 & -0.086883 \\
\hline $\mathrm{H}$ & 1.798463 & -6.317024 & -0.061457 \\
\hline $\mathrm{C}$ & -0.372673 & -6.388465 & -0.123485 \\
\hline $\mathrm{C}$ & -1.566006 & -5.656832 & -0.153340 \\
\hline $\mathrm{H}$ & -2.534228 & -6.167037 & -0.177097 \\
\hline $\mathrm{C}$ & -1.524845 & -4.238856 & -0.147780 \\
\hline $\mathrm{C}$ & 2.169571 & -3.633051 & -0.039101 \\
\hline $\mathrm{C}$ & 3.234027 & -3.017335 & 0.015137 \\
\hline $\mathrm{C}$ & 4.531031 & -2.448650 & 0.123916 \\
\hline $\mathrm{C}$ & 4.803333 & -1.065377 & -0.140564 \\
\hline $\mathrm{C}$ & 6.140854 & -0.629889 & -0.000597 \\
\hline $\mathrm{H}$ & 6.366981 & 0.414125 & -0.211858 \\
\hline $\mathrm{C}$ & 7.157633 & -1.511361 & 0.376543 \\
\hline $\mathrm{H}$ & 8.169470 & -1.104091 & 0.465903 \\
\hline $\mathrm{C}$ & 6.917204 & -2.874403 & 0.642625 \\
\hline $\mathrm{C}$ & 5.590629 & -3.306431 & 0.507680 \\
\hline $\mathrm{H}$ & 5.325451 & -4.352007 & 0.698789 \\
\hline $\mathrm{C}$ & 3.914157 & 1.092662 & -1.025059 \\
\hline $\mathrm{C}$ & 2.481421 & 3.004417 & -1.738487 \\
\hline $\mathrm{C}$ & 3.498163 & 3.894669 & -2.137325 \\
\hline $\mathrm{H}$ & 4.539815 & 3.599328 & -2.009758 \\
\hline $\mathrm{C}$ & 3.171525 & 5.141377 & -2.695600 \\
\hline $\mathrm{H}$ & 3.986073 & 5.807010 & -2.995223 \\
\hline $\mathrm{C}$ & 1.830075 & 5.521741 & -2.859515 \\
\hline $\mathrm{C}$ & 0.811662 & 4.635035 & -2.461027 \\
\hline $\mathrm{H}$ & -0.232645 & 4.936364 & -2.588770 \\
\hline $\mathrm{C}$ & 1.130166 & 3.395289 & -1.913124 \\
\hline $\mathrm{H}$ & 0.336886 & 2.700865 & -1.611976 \\
\hline $\mathrm{C}$ & 2.417758 & 7.636342 & -3.788591 \\
\hline $\mathrm{H}$ & 1.894107 & 8.523636 & -4.177941 \\
\hline
\end{tabular}




\begin{tabular}{|c|c|c|c|}
\hline $\mathrm{H}$ & 3.066882 & 7.218866 & -4.585271 \\
\hline $\mathrm{H}$ & 3.058721 & 7.937723 & -2.935248 \\
\hline $\mathrm{C}$ & -2.712631 & -3.455728 & -0.165151 \\
\hline $\mathrm{C}$ & -3.710917 & -2.735893 & -0.167423 \\
\hline $\mathrm{C}$ & -4.925767 & -2.000983 & -0.180865 \\
\hline $\mathrm{C}$ & -4.985680 & -0.612580 & 0.173972 \\
\hline $\mathrm{C}$ & -6.254891 & 0.007532 & 0.165623 \\
\hline $\mathrm{H}$ & -6.319392 & 1.055964 & 0.454207 \\
\hline $\mathrm{C}$ & -7.407151 & -0.703115 & -0.181855 \\
\hline $\mathrm{H}$ & -8.357236 & -0.160207 & -0.169966 \\
\hline $\mathrm{C}$ & -7.374815 & -2.064599 & -0.546445 \\
\hline $\mathrm{C}$ & -6.115602 & -2.681286 & -0.535084 \\
\hline $\mathrm{H}$ & -6.013149 & -3.738978 & -0.801403 \\
\hline $\mathrm{C}$ & -3.742621 & 1.319553 & 1.145746 \\
\hline $\mathrm{C}$ & -2.002184 & 2.991238 & 1.786888 \\
\hline $\mathrm{C}$ & -2.817737 & 3.891663 & 2.499335 \\
\hline $\mathrm{H}$ & -3.884056 & 3.682569 & 2.590900 \\
\hline $\mathrm{C}$ & -2.260001 & 5.043687 & 3.078301 \\
\hline $\mathrm{H}$ & -2.919856 & 5.723154 & 3.625356 \\
\hline $\mathrm{C}$ & -0.887977 & 5.314706 & 2.952450 \\
\hline $\mathrm{C}$ & -0.071390 & 4.415930 & 2.239075 \\
\hline $\mathrm{H}$ & 0.996203 & 4.634029 & 2.137315 \\
\hline $\mathrm{C}$ & -0.617914 & 3.270393 & 1.668103 \\
\hline $\mathrm{H}$ & 0.008604 & 2.560582 & 1.113241 \\
\hline $\mathrm{C}$ & -1.053918 & 7.342439 & 4.194151 \\
\hline $\mathrm{H}$ & -0.377441 & 8.144079 & 4.530150 \\
\hline $\mathrm{H}$ & -1.534806 & 6.878128 & 5.079370 \\
\hline $\mathrm{H}$ & -1.846403 & 7.780252 & 3.553529 \\
\hline $\mathrm{C}$ & 8.019688 & -3.867480 & 1.060572 \\
\hline $\mathrm{C}$ & -8.629822 & -2.868883 & -0.939962 \\
\hline $\mathrm{C}$ & 7.682173 & -4.462276 & 2.450399 \\
\hline $\mathrm{H}$ & 6.708663 & -4.981297 & 2.444543 \\
\hline $\mathrm{H}$ & 8.453935 & -5.192486 & 2.758343 \\
\hline $\mathrm{H}$ & 7.633240 & -3.668219 & 3.215782 \\
\hline $\mathrm{C}$ & 9.405563 & -3.196085 & 1.148831 \\
\hline $\mathrm{H}$ & 9.717209 & -2.773217 & 0.177616 \\
\hline $\mathrm{H}$ & 9.419373 & -2.384362 & 1.897240 \\
\hline $\mathrm{H}$ & 10.163106 & -3.941998 & 1.448931 \\
\hline $\mathrm{C}$ & 8.104665 & -5.014659 & 0.023267 \\
\hline $\mathrm{H}$ & 8.362111 & -4.622065 & -0.975909 \\
\hline $\mathrm{H}$ & 8.879262 & -5.747675 & 0.317167 \\
\hline $\mathrm{H}$ & 7.146022 & -5.553409 & -0.065859 \\
\hline $\mathrm{C}$ & -8.469151 & -3.405686 & -2.384041 \\
\hline $\mathrm{H}$ & -7.578800 & -4.049881 & -2.481028 \\
\hline $\mathrm{H}$ & -9.353174 & -4.002414 & -2.677438 \\
\hline $\mathrm{H}$ & -8.360074 & -2.574186 & -3.101974 \\
\hline $\mathrm{C}$ & -8.806279 & -4.063537 & 0.030153 \\
\hline $\mathrm{H}$ & -8.940835 & -3.710218 & 1.067378 \\
\hline
\end{tabular}




$\begin{array}{lllc}\mathrm{H} & -9.693194 & -4.662737 & -0.249116 \\ \mathrm{H} & -7.928742 & -4.732050 & 0.015608 \\ \mathrm{C} & -9.911871 & -2.013008 & -0.885272 \\ \mathrm{H} & -10.098329 & -1.620879 & 0.129947 \\ \mathrm{H} & -9.861441 & -1.156673 & -1.580499 \\ \mathrm{H} & -10.783374 & -2.627800 & -1.173056 \\ \mathrm{H} & -0.410293 & -7.483629 & -0.126787 \\ \mathrm{H} & 0.306753 & -0.122294 & 1.090483 \\ \mathrm{Se} & -0.054763 & 0.056121 & -0.337212\end{array}$

Statistical Thermodynamic Analysis

Temperature $=298.150$ Kelvin $\quad$ Pressure $=1.00000$ Atm

\begin{tabular}{|c|c|c|}
\hline$-4697.3559751^{\prime}$ & Predicted Chan & $\operatorname{ge}=-9.057321 \mathrm{D}-09$ \\
\hline Zero-point correction $(\mathrm{ZPE})=$ & -4696.5440 & 0.81196 \\
\hline Internal Energy $(U)=$ & -4696.4886 0.8673 & \\
\hline Enthalpy $(H)=$ & -4696.4877 & 0.86826 \\
\hline Gibbs Free Energy $(G)=$ & -4696.6405 & 0.71540 \\
\hline Frequencies -- $\quad 6.6328$ & 8.4370 & 13.8920 \\
\hline
\end{tabular}

$\mathrm{R}=\mathrm{H}$ receptor bound with $\mathrm{SH}-($ no $\mathrm{TBA})$

Single Supporting Information: 010-bound_noTBA_H_SH.log

Using Gaussian 09: EM64L-G09RevE.01 30-Nov-2015

\#PBEPBE/6-31G(d)/auto scf=(direct,tight,maxcycle=300,xqc)

$\mathrm{opt}=($ maxcycle $=250)$ freq $=$ noraman int $=($ grid $=$ ultrafine $)$

\#P Geom=AllCheck Guess=TCheck SCRF=Check GenChk RPBEPBE/6-31G(d)/Auto Freq

Pointgroup $=\mathrm{C} 1 \quad$ Stoichiometry $=\mathrm{C} 46 \mathrm{H} 47 \mathrm{~N} 4 \mathrm{O} 4 \mathrm{~S}(1-) \quad \mathrm{C} 1[\mathrm{X}(\mathrm{C} 46 \mathrm{H} 47 \mathrm{~N} 4 \mathrm{O} 4 \mathrm{~S})] \quad$ \#Atoms $=102$

Charge $=-1 \quad$ Multiplicity $=1$

SCF Energy $=-2696.33963685 \quad$ Predicted Change $=-8.840506 \mathrm{D}-09$

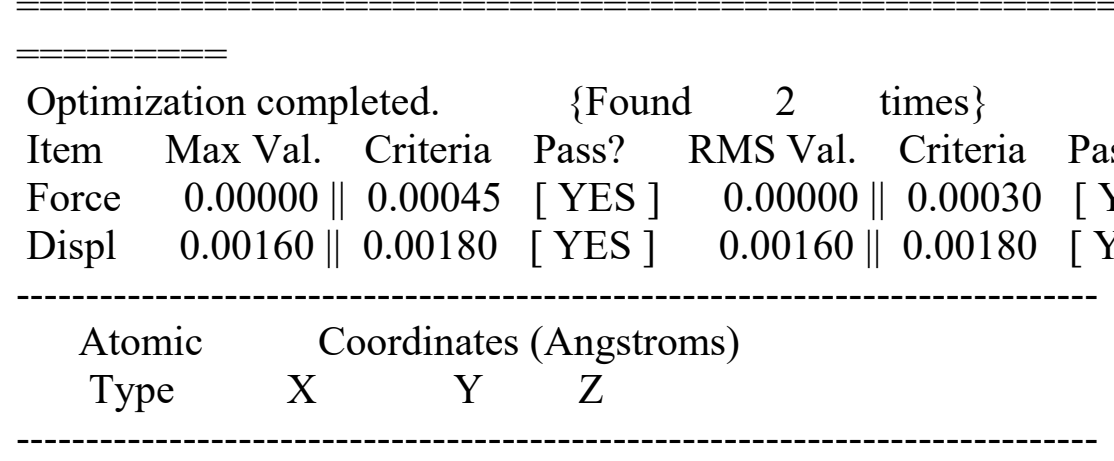




\begin{tabular}{|c|c|c|c|}
\hline $\mathrm{O}$ & -4.706813 & 1.818361 & 1.621452 \\
\hline $\mathrm{O}$ & -0.410334 & 6.394168 & 3.678090 \\
\hline $\mathrm{O}$ & 4.833402 & 1.681009 & -1.477573 \\
\hline $\mathrm{O}$ & 0.923850 & 6.539013 & -3.636760 \\
\hline $\mathrm{N}$ & -3.748978 & -0.015919 & 0.546742 \\
\hline $\mathrm{H}$ & -2.852293 & -0.487215 & 0.360604 \\
\hline $\mathrm{N}$ & -2.408626 & 1.718832 & 1.286121 \\
\hline $\mathrm{H}$ & -1.646923 & 1.182388 & 0.801265 \\
\hline $\mathrm{N}$ & 3.724395 & -0.143661 & -0.541557 \\
\hline $\mathrm{H}$ & 2.797195 & -0.574521 & -0.419240 \\
\hline $\mathrm{N}$ & 2.517076 & 1.680757 & -1.303395 \\
\hline $\mathrm{H}$ & 1.692262 & 1.157421 & -0.914408 \\
\hline $\mathrm{C}$ & -0.112080 & -3.558355 & -0.058458 \\
\hline $\mathrm{H}$ & -0.090518 & -2.458807 & -0.020487 \\
\hline $\mathrm{C}$ & -1.350167 & -4.234147 & -0.120201 \\
\hline $\mathrm{C}$ & -1.368084 & -5.651831 & -0.177332 \\
\hline $\mathrm{H}$ & -2.328352 & -6.175277 & -0.224833 \\
\hline $\mathrm{C}$ & -0.164349 & -6.365971 & -0.169135 \\
\hline $\mathrm{C}$ & 1.065067 & -5.699598 & -0.108520 \\
\hline $\mathrm{H}$ & 2.005226 & -6.260360 & -0.105086 \\
\hline $\mathrm{C}$ & 1.099943 & -4.282111 & -0.055331 \\
\hline $\mathrm{C}$ & -2.557349 & -3.480461 & -0.125966 \\
\hline $\mathrm{C}$ & -3.585567 & -2.803872 & -0.131216 \\
\hline $\mathrm{C}$ & -4.817945 & -2.098583 & -0.148746 \\
\hline $\mathrm{C}$ & -4.909863 & -0.713190 & 0.214381 \\
\hline $\mathrm{C}$ & -6.190162 & -0.117020 & 0.191253 \\
\hline $\mathrm{H}$ & -6.277294 & 0.928722 & 0.483367 \\
\hline $\mathrm{C}$ & -7.323375 & -0.848740 & -0.175588 \\
\hline $\mathrm{H}$ & -8.283970 & -0.324480 & -0.173741 \\
\hline $\mathrm{C}$ & -7.260285 & -2.207791 & -0.544838 \\
\hline $\mathrm{C}$ & -5.989443 & -2.800290 & -0.520366 \\
\hline $\mathrm{H}$ & -5.863260 & -3.854311 & -0.791083 \\
\hline $\mathrm{C}$ & -3.696832 & 1.238503 & 1.193403 \\
\hline $\mathrm{C}$ & -2.001600 & 2.912719 & 1.911210 \\
\hline $\mathrm{C}$ & -2.861838 & 3.779617 & 2.615509 \\
\hline $\mathrm{H}$ & -3.921691 & 3.534061 & 2.682025 \\
\hline $\mathrm{C}$ & -2.361829 & 4.946512 & 3.216104 \\
\hline $\mathrm{H}$ & -3.059951 & 5.594480 & 3.753869 \\
\hline $\mathrm{C}$ & -0.999136 & 5.270798 & 3.124022 \\
\hline $\mathrm{C}$ & -0.136498 & 4.409682 & 2.420031 \\
\hline $\mathrm{H}$ & 0.924099 & 4.668437 & 2.343644 \\
\hline $\mathrm{C}$ & -0.628132 & 3.250218 & 1.825411 \\
\hline $\mathrm{H}$ & 0.049895 & 2.587416 & 1.276624 \\
\hline $\mathrm{C}$ & -1.267258 & 7.277917 & 4.380582 \\
\hline $\mathrm{H}$ & -0.628900 & 8.101222 & 4.738115 \\
\hline $\mathrm{H}$ & -1.748090 & 6.786726 & 5.251160 \\
\hline $\mathrm{H}$ & -2.062541 & 7.691796 & 3.727669 \\
\hline $\mathrm{C}$ & 2.334681 & -3.576658 & 0.001958 \\
\hline
\end{tabular}




\begin{tabular}{|c|c|c|c|}
\hline $\mathrm{C}$ & 3.387379 & -2.941263 & 0.055512 \\
\hline $\mathrm{C}$ & 4.651148 & -2.298951 & 0.141425 \\
\hline $\mathrm{C}$ & 4.830399 & -0.910349 & -0.172929 \\
\hline $\mathrm{C}$ & 6.136968 & -0.383351 & -0.069277 \\
\hline $\mathrm{H}$ & 6.291046 & 0.665249 & -0.319957 \\
\hline $\mathrm{C}$ & 7.213073 & -1.184287 & 0.324110 \\
\hline $\mathrm{H}$ & 8.197621 & -0.710325 & 0.384933 \\
\hline $\mathrm{C}$ & 7.064222 & -2.549363 & 0.642217 \\
\hline $\mathrm{C}$ & 5.767231 & -3.072530 & 0.540323 \\
\hline $\mathrm{H}$ & 5.574152 & -4.126018 & 0.770433 \\
\hline $\mathrm{C}$ & 3.771818 & 1.133881 & -1.140681 \\
\hline $\mathrm{C}$ & 2.215037 & 2.917302 & -1.905498 \\
\hline $\mathrm{C}$ & 3.162831 & 3.760886 & -2.520514 \\
\hline $\mathrm{H}$ & 4.210764 & 3.461587 & -2.528975 \\
\hline $\mathrm{C}$ & 2.763826 & 4.973328 & -3.106305 \\
\hline $\mathrm{H}$ & 3.528201 & 5.601210 & -3.573134 \\
\hline $\mathrm{C}$ & 1.416553 & 5.367159 & -3.089293 \\
\hline $\mathrm{C}$ & 0.466763 & 4.529912 & -2.474453 \\
\hline $\mathrm{H}$ & -0.581707 & 4.843226 & -2.455216 \\
\hline $\mathrm{C}$ & 0.858343 & 3.326187 & -1.893667 \\
\hline $\mathrm{H}$ & 0.113881 & 2.680766 & -1.413333 \\
\hline $\mathrm{C}$ & 1.869959 & 7.400944 & -4.245907 \\
\hline $\mathrm{H}$ & 1.299783 & 8.269680 & -4.611076 \\
\hline $\mathrm{H}$ & 2.382647 & 6.916981 & -5.102239 \\
\hline $\mathrm{H}$ & 2.639460 & 7.747491 & -3.526369 \\
\hline $\mathrm{C}$ & -8.494382 & -3.034341 & -0.957869 \\
\hline $\mathrm{C}$ & 8.233529 & -3.452579 & 1.082043 \\
\hline $\mathrm{C}$ & -8.305039 & -3.561895 & -2.401896 \\
\hline $\mathrm{H}$ & -7.401419 & -4.188666 & -2.490041 \\
\hline $\mathrm{H}$ & -9.173667 & -4.174127 & -2.709043 \\
\hline $\mathrm{H}$ & -8.202762 & -2.725400 & -3.115019 \\
\hline $\mathrm{C}$ & -9.793205 & -2.203379 & -0.916468 \\
\hline $\mathrm{H}$ & -10.000062 & -1.818942 & 0.097737 \\
\hline $\mathrm{H}$ & -9.750228 & -1.343442 & -1.607731 \\
\hline $\mathrm{H}$ & -10.649040 & -2.833527 & -1.217756 \\
\hline $\mathrm{C}$ & -8.660429 & -4.236248 & 0.005097 \\
\hline $\mathrm{H}$ & -8.815353 & -3.889956 & 1.041843 \\
\hline $\mathrm{H}$ & -9.531860 & -4.851279 & -0.288171 \\
\hline $\mathrm{H}$ & -7.769993 & -4.887624 & -0.000504 \\
\hline $\mathrm{C}$ & 7.950152 & -4.015029 & 2.497259 \\
\hline $\mathrm{H}$ & 7.013140 & -4.596827 & 2.523096 \\
\hline $\mathrm{H}$ & 8.771650 & -4.680641 & 2.822520 \\
\hline $\mathrm{H}$ & 7.856366 & -3.197352 & 3.232973 \\
\hline $\mathrm{C}$ & 8.382308 & -4.630392 & 0.087035 \\
\hline $\mathrm{H}$ & 8.603621 & -4.260111 & -0.929191 \\
\hline $\mathrm{H}$ & 9.205863 & -5.300151 & 0.398251 \\
\hline $\mathrm{H}$ & 7.459951 & -5.233072 & 0.030047 \\
\hline $\mathrm{C}$ & 9.573686 & -2.690341 & 1.127412 \\
\hline
\end{tabular}




$\begin{array}{llll}\mathrm{H} & 9.846780 & -2.284929 & 0.137321 \\ \mathrm{H} & 9.542646 & -1.851956 & 1.845271 \\ \mathrm{H} & 10.381291 & -3.373990 & 1.444765 \\ \mathrm{H} & -0.184668 & -7.460795 & -0.211553 \\ \mathrm{~S} & -0.064745 & 0.002876 & -0.217987 \\ \mathrm{H} & -0.515394 & -0.037134 & -1.503679\end{array}$

Statistical Thermodynamic Analysis

Temperature $=298.150$ Kelvin $\quad$ Pressure $=1.00000$ Atm

\begin{tabular}{|c|c|}
\hline SCF Energy $=\quad-2696.33963685$ & $5 \quad$ Predicted Change $=-8.840506 \mathrm{D}-09$ \\
\hline Zero-point correction $(\mathrm{ZPE})=$ & -2695.53030 .80927 \\
\hline Internal Energy $(\mathrm{U})=$ & -2695.47430 .86533 \\
\hline Enthalpy $(\mathrm{H})=$ & $-2695.4733 \quad 0.86628$ \\
\hline Gibbs Free Energy $(\mathrm{G})=$ & $-2695.6282 \quad 0.71134$ \\
\hline $\begin{array}{l}\text { Frequencies -- } \quad 6.2078 \\
\text { TZVPP: }-2699.58669936948\end{array}$ & 13.2702 points at WB97X-D3/def2- \\
\hline \multicolumn{2}{|c|}{$\begin{array}{l}\mathbf{R}=\mathbf{N M e}_{2} \text { receptor unbound (from crystal structure) } \\
\quad \text { Supporting Information: } 000 \text {-unbound_crystal_structure_NMe2.log }\end{array}$} \\
\hline \multicolumn{2}{|c|}{ Using Gaussian 09: EM64L-G09RevE.01 30-Nov-2015 } \\
\hline \multicolumn{2}{|c|}{$=======$} \\
\hline \multicolumn{2}{|c|}{$\begin{array}{l}\text { \#PBEPBE } / 6-31 \mathrm{G}(\mathrm{d}) / \text { auto } \mathrm{scf}=(\text { direct,tight, } \operatorname{maxcycle}=300, \mathrm{xqc}) \\
\text { opt }=(\text { maxcycle}=250) \text { freq=noraman int }=(\text { grid= }=\text { ultrafine }) \\
\text { \#P Geom=AllCheck Guess=TCheck SCRF=Check GenChk RPBEPBE } / 6-31 \mathrm{G}(\mathrm{d}) / \text { Auto Frec }\end{array}$} \\
\hline \multicolumn{2}{|c|}{$\begin{array}{l}\text { Pointgroup }=\mathrm{C} 1 \quad \text { Stoichiometry }=\mathrm{C} 48 \mathrm{H} 51 \mathrm{~N} 5 \mathrm{O} 4 \mathrm{C} 1[\mathrm{X}(\mathrm{C} 48 \mathrm{H} 51 \mathrm{~N} 5 \mathrm{O} 4)] \text { \#Atoms }=108 \\
\text { Charge }=0 \quad \text { Multiplicity }=1\end{array}$} \\
\hline SCF Energy= -2431.42582624 & Predicted Change $=-7.281382 \mathrm{D}-08$ \\
\hline
\end{tabular}

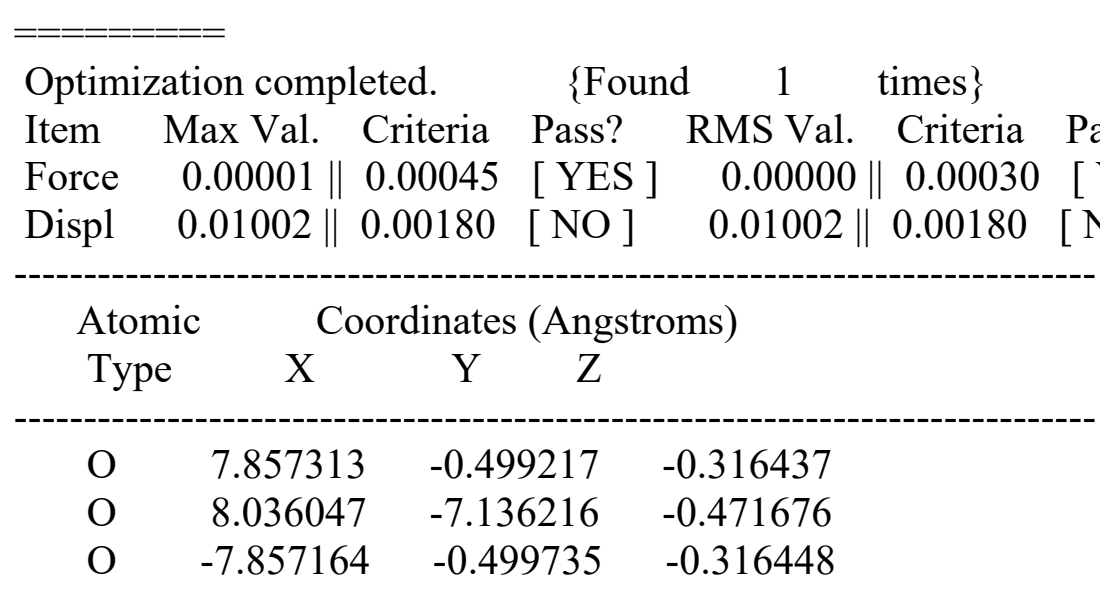




\begin{tabular}{|c|c|c|c|}
\hline $\mathrm{O}$ & -8.035089 & -7.136762 & -0.471417 \\
\hline $\mathrm{N}$ & -0.000147 & -2.667451 & 1.620347 \\
\hline $\mathrm{N}$ & 5.702542 & 0.370879 & -0.174869 \\
\hline $\mathrm{N}$ & 6.008222 & -1.909652 & -0.285836 \\
\hline $\mathrm{N}$ & -5.702502 & 0.370631 & -0.174915 \\
\hline $\mathrm{N}$ & -6.007902 & -1.909943 & -0.285789 \\
\hline $\mathrm{C}$ & -0.000083 & 1.405150 & 0.409478 \\
\hline $\mathrm{H}$ & -0.000066 & 2.448556 & 0.087677 \\
\hline $\mathrm{C}$ & 1.217435 & 0.722433 & 0.619245 \\
\hline $\mathrm{C}$ & 1.213682 & -0.630379 & 1.029998 \\
\hline $\mathrm{H}$ & 2.174424 & -1.118815 & 1.204242 \\
\hline $\mathrm{C}$ & -0.000126 & -1.333772 & 1.234238 \\
\hline $\mathrm{C}$ & -1.213912 & -0.630355 & 1.029951 \\
\hline $\mathrm{H}$ & -2.174670 & -1.118773 & 1.204154 \\
\hline $\mathrm{C}$ & -1.217623 & 0.722458 & 0.619200 \\
\hline $\mathrm{C}$ & 2.458243 & 1.392065 & 0.413327 \\
\hline $\mathrm{C}$ & 3.514900 & 2.000617 & 0.233355 \\
\hline $\mathrm{C}$ & 4.790264 & 2.592944 & 0.025528 \\
\hline $\mathrm{C}$ & 5.933664 & 1.749677 & -0.168249 \\
\hline $\mathrm{C}$ & 7.189899 & 2.349876 & -0.353152 \\
\hline $\mathrm{H}$ & 8.062854 & 1.711298 & -0.490765 \\
\hline $\mathrm{C}$ & 7.308473 & 3.745599 & -0.353921 \\
\hline $\mathrm{H}$ & 8.307013 & 4.165973 & -0.502203 \\
\hline $\mathrm{C}$ & 6.204605 & 4.604064 & -0.175507 \\
\hline $\mathrm{C}$ & 4.953059 & 3.994600 & 0.013328 \\
\hline $\mathrm{H}$ & 4.056167 & 4.604106 & 0.163450 \\
\hline $\mathrm{C}$ & 6.635630 & -0.665223 & -0.264923 \\
\hline $\mathrm{C}$ & 6.605245 & -3.188004 & -0.337177 \\
\hline $\mathrm{C}$ & 5.742276 & -4.305476 & -0.393473 \\
\hline $\mathrm{H}$ & 4.654754 & -4.153873 & -0.403439 \\
\hline $\mathrm{C}$ & 6.247660 & -5.602490 & -0.437689 \\
\hline $\mathrm{H}$ & 5.577829 & -6.466132 & -0.483188 \\
\hline $\mathrm{C}$ & 7.639140 & -5.821624 & -0.425725 \\
\hline $\mathrm{C}$ & 8.503454 & -4.714413 & -0.369752 \\
\hline $\mathrm{H}$ & 9.587944 & -4.849555 & -0.360768 \\
\hline $\mathrm{C}$ & 7.995033 & -3.407629 & -0.326141 \\
\hline $\mathrm{H}$ & 8.669073 & -2.551616 & -0.288338 \\
\hline $\mathrm{C}$ & 9.437549 & -7.388451 & -0.463933 \\
\hline $\mathrm{H}$ & 9.545613 & -8.482402 & -0.505789 \\
\hline $\mathrm{H}$ & 9.917073 & -7.008758 & 0.459311 \\
\hline $\mathrm{H}$ & 9.939756 & -6.937635 & -1.341968 \\
\hline $\mathrm{C}$ & -2.458408 & 1.392120 & 0.413238 \\
\hline $\mathrm{C}$ & -3.515063 & 2.000666 & 0.233232 \\
\hline $\mathrm{C}$ & -4.790504 & 2.592819 & 0.025389 \\
\hline $\mathrm{C}$ & -5.933798 & 1.749399 & -0.168353 \\
\hline $\mathrm{C}$ & -7.190109 & 2.349431 & -0.353277 \\
\hline $\mathrm{H}$ & -8.062983 & 1.710736 & -0.490863 \\
\hline $\mathrm{C}$ & -7.308860 & 3.745138 & -0.354102 \\
\hline
\end{tabular}




\begin{tabular}{|c|c|c|c|}
\hline $\mathrm{H}$ & -8.307453 & 4.165379 & -0.502398 \\
\hline $\mathrm{C}$ & -6.205102 & 4.603751 & -0.175724 \\
\hline $\mathrm{C}$ & -4.953477 & 3.994454 & 0.013133 \\
\hline $\mathrm{H}$ & -4.056661 & 4.604080 & 0.163227 \\
\hline $\mathrm{C}$ & -6.635462 & -0.665590 & -0.264923 \\
\hline $\mathrm{C}$ & -6.604770 & -3.188370 & -0.337077 \\
\hline $\mathrm{C}$ & -7.994531 & -3.408164 & -0.326038 \\
\hline $\mathrm{H}$ & -8.668675 & -2.552232 & -0.288273 \\
\hline $\mathrm{C}$ & -8.502792 & -4.715012 & -0.369596 \\
\hline $\mathrm{H}$ & -9.587266 & -4.850286 & -0.360611 \\
\hline $\mathrm{C}$ & -7.638343 & -5.822120 & -0.425519 \\
\hline $\mathrm{C}$ & -6.246890 & -5.602817 & -0.437487 \\
\hline $\mathrm{H}$ & -5.576954 & -6.466379 & -0.482946 \\
\hline $\mathrm{C}$ & -5.741664 & -4.305740 & -0.393322 \\
\hline $\mathrm{H}$ & -4.654161 & -4.154005 & -0.403290 \\
\hline $\mathrm{C}$ & -9.436561 & -7.389168 & -0.463678 \\
\hline $\mathrm{H}$ & -9.544491 & -8.483134 & -0.505493 \\
\hline $\mathrm{H}$ & -9.938814 & -6.938446 & -1.341735 \\
\hline $\mathrm{H}$ & -9.916140 & -7.009498 & 0.459547 \\
\hline $\mathrm{H}$ & 4.716418 & 0.134156 & -0.034102 \\
\hline $\mathrm{H}$ & 4.990354 & -1.920431 & -0.339076 \\
\hline $\mathrm{H}$ & -4.716348 & 0.134037 & -0.034141 \\
\hline $\mathrm{H}$ & -4.990033 & -1.920601 & -0.339017 \\
\hline $\mathrm{C}$ & -6.322507 & 6.141143 & -0.177250 \\
\hline $\mathrm{C}$ & -7.773285 & 6.614518 & -0.401216 \\
\hline $\mathrm{H}$ & -8.171422 & 6.273644 & -1.373030 \\
\hline $\mathrm{H}$ & -8.449699 & 6.257164 & 0.394937 \\
\hline $\mathrm{H}$ & -7.807434 & 7.717791 & -0.396252 \\
\hline $\mathrm{C}$ & -5.442248 & 6.723641 & -1.311145 \\
\hline $\mathrm{H}$ & -4.380872 & 6.447812 & -1.189156 \\
\hline $\mathrm{H}$ & -5.774622 & 6.356488 & -2.297628 \\
\hline $\mathrm{H}$ & -5.504104 & 7.827015 & -1.315839 \\
\hline $\mathrm{C}$ & -5.836253 & 6.696448 & 1.184744 \\
\hline $\mathrm{H}$ & -6.455078 & 6.309408 & 2.012799 \\
\hline $\mathrm{H}$ & -4.788025 & 6.419636 & 1.389786 \\
\hline $\mathrm{H}$ & -5.900328 & 7.799673 & 1.193697 \\
\hline $\mathrm{C}$ & 6.321816 & 6.141470 & -0.176971 \\
\hline $\mathrm{C}$ & 5.441521 & 6.723900 & -1.310874 \\
\hline $\mathrm{H}$ & 4.380176 & 6.447936 & -1.188929 \\
\hline $\mathrm{H}$ & 5.503242 & 7.827281 & -1.315529 \\
\hline $\mathrm{H}$ & 5.773973 & 6.356821 & -2.297359 \\
\hline $\mathrm{C}$ & 7.772541 & 6.615038 & -0.400869 \\
\hline $\mathrm{H}$ & 8.448969 & 6.257759 & 0.395305 \\
\hline $\mathrm{H}$ & 8.170762 & 6.274232 & -1.372673 \\
\hline $\mathrm{H}$ & 7.806546 & 7.718315 & -0.395886 \\
\hline $\mathrm{C}$ & 5.835444 & 6.696662 & 1.185027 \\
\hline $\mathrm{H}$ & 4.787247 & 6.419704 & 1.390026 \\
\hline $\mathrm{H}$ & 6.454295 & 6.309676 & 2.013089 \\
\hline
\end{tabular}




$\begin{array}{lrrr}\mathrm{H} & 5.899372 & 7.799895 & 1.194022 \\ \mathrm{C} & 1.254195 & -3.313908 & 1.971760 \\ \mathrm{H} & 1.948107 & -3.342239 & 1.108645 \\ \mathrm{H} & 1.774222 & -2.807945 & 2.811173 \\ \mathrm{H} & 1.054080 & -4.353275 & 2.269087 \\ \mathrm{C} & -1.254513 & -3.313878 & 1.971726 \\ \mathrm{H} & -1.948397 & -3.342207 & 1.108589 \\ \mathrm{H} & -1.054428 & -4.353245 & 2.269074 \\ \mathrm{H} & -1.774556 & -2.807894 & 2.811117\end{array}$

Statistical Thermodynamic Analysis

Temperature $=298.150$ Kelvin $\quad$ Pressure $=1.00000$ Atm

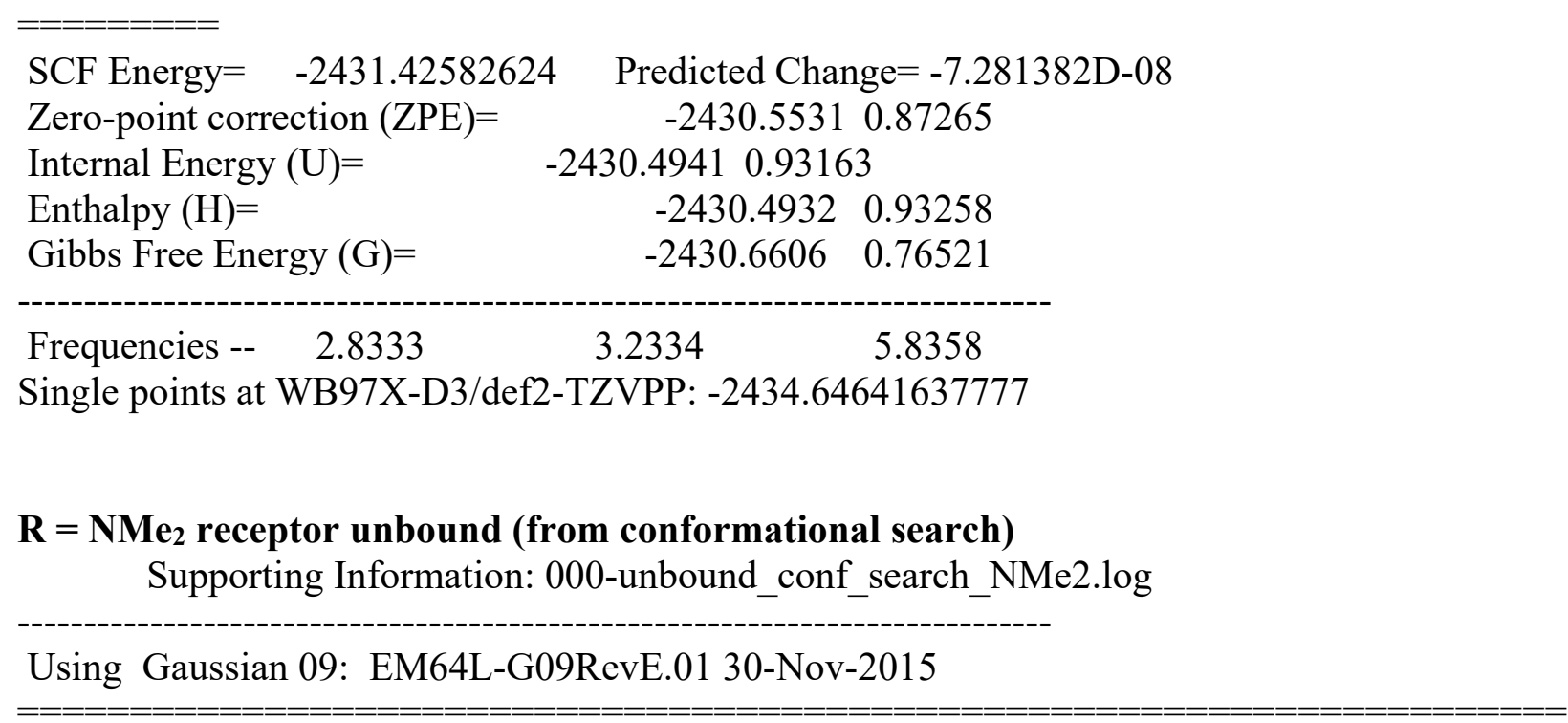

\begin{tabular}{|c|c|}
\hline $\begin{array}{l}\# \mathrm{PBEPBE} / 6-31 \mathrm{G}(\mathrm{d}) / \text { auto } \mathrm{scf}=(\mathrm{d} \\
\mathrm{opt}=(\mathrm{maxcycle}=250) \text { freq }=\text { noran } \\
\# \mathrm{P} \text { Geom }=\text { AllCheck Guess }=\mathrm{TCh}\end{array}$ & $\begin{array}{l}\text { ct,tight,maxcycle=300,xqc) } \\
\text { int }=(\text { grid=ultrafine) } \\
\text { k SCRF=Check GenChk RPBEPBE/6-31G(d)/Auto Freq }\end{array}$ \\
\hline $\begin{array}{l}\text { Pointgroup }=\mathrm{C} 1 \quad \text { Stoichiometry }= \\
\text { Charge }=0 \quad \text { Multiplicity }=1\end{array}$ & $48 \mathrm{H} 51 \mathrm{~N} 5 \mathrm{O} 4 \mathrm{C} 1[\mathrm{X}(\mathrm{C} 48 \mathrm{H} 51 \mathrm{~N} 5 \mathrm{O} 4)] \#$ Atoms= 108 \\
\hline SCF Energy= -2431.43503471 & Predicted Change $=-1.844268 \mathrm{D}-08$ \\
\hline
\end{tabular}

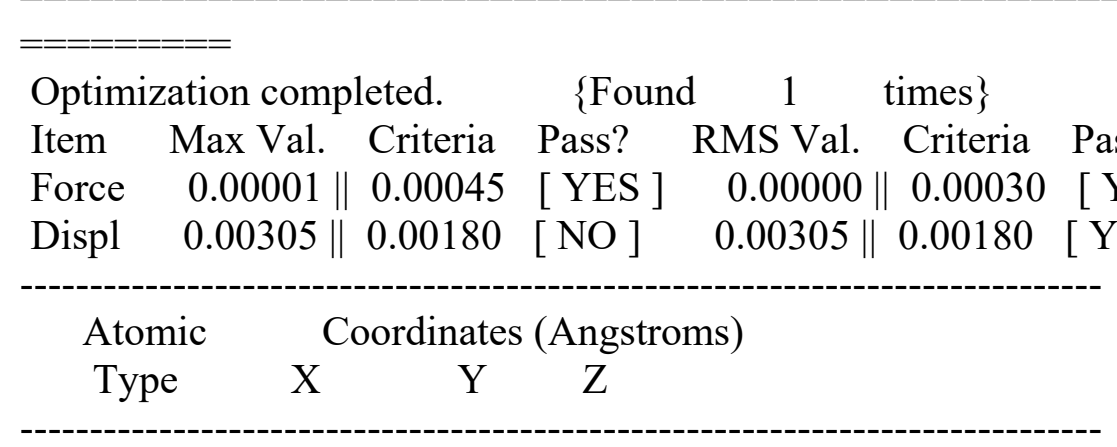




\begin{tabular}{|c|c|c|c|}
\hline $\mathrm{C}$ & -0.003664 & 1.459170 & -0.052604 \\
\hline $\mathrm{C}$ & -1.211220 & 2.178037 & 0.077618 \\
\hline $\mathrm{N}$ & -3.355671 & -1.807647 & 1.120043 \\
\hline $\mathrm{C}$ & -1.207456 & 3.590920 & 0.061699 \\
\hline $\mathrm{N}$ & -1.686236 & -3.294819 & 1.801721 \\
\hline $\mathrm{O}$ & -3.791120 & -4.087173 & 1.356498 \\
\hline $\mathrm{C}$ & -0.000108 & 4.318044 & -0.094792 \\
\hline $\mathrm{N}$ & 3.353452 & -1.857453 & -1.063646 \\
\hline $\mathrm{O}$ & 1.987659 & 0.417642 & 3.867513 \\
\hline $\mathrm{C}$ & 1.207456 & 3.583851 & -0.211509 \\
\hline $\mathrm{N}$ & 1.686198 & -3.372772 & -1.685935 \\
\hline $\mathrm{O}$ & 3.791077 & -4.144438 & -1.205531 \\
\hline $\mathrm{C}$ & 1.206773 & 2.170933 & -0.194701 \\
\hline $\mathrm{O}$ & -1.983051 & 0.252410 & -3.909027 \\
\hline $\mathrm{C}$ & -2.439909 & 1.471044 & 0.225292 \\
\hline $\mathrm{C}$ & -3.505602 & 0.861696 & 0.331626 \\
\hline $\mathrm{C}$ & -4.693932 & 0.086343 & 0.432000 \\
\hline $\mathrm{C}$ & -4.619002 & -1.295566 & 0.805008 \\
\hline $\mathrm{C}$ & -5.807971 & -2.044136 & 0.851275 \\
\hline $\mathrm{C}$ & -7.035838 & -1.440424 & 0.552826 \\
\hline $\mathrm{C}$ & -7.147098 & -0.081073 & 0.196574 \\
\hline $\mathrm{C}$ & -5.953245 & 0.656922 & 0.142657 \\
\hline $\mathrm{C}$ & -8.491183 & 0.599676 & -0.131483 \\
\hline $\mathrm{C}$ & -8.454480 & 1.140662 & -1.582695 \\
\hline $\mathrm{C}$ & -8.724687 & 1.777465 & 0.847290 \\
\hline $\mathrm{C}$ & -9.682128 & -0.372670 & -0.009096 \\
\hline $\mathrm{C}$ & -3.022315 & -3.123678 & 1.432293 \\
\hline $\mathrm{C}$ & -0.757049 & -2.329432 & 2.291240 \\
\hline $\mathrm{C}$ & -1.136690 & -1.338598 & 3.224545 \\
\hline $\mathrm{C}$ & -0.199186 & -0.428720 & 3.710626 \\
\hline $\mathrm{C}$ & 1.150265 & -0.505139 & 3.306595 \\
\hline $\mathrm{C}$ & 1.540812 & -1.499312 & 2.388020 \\
\hline $\mathrm{C}$ & 0.585536 & -2.390608 & 1.879019 \\
\hline $\mathrm{C}$ & 3.364099 & 0.373474 & 3.484663 \\
\hline $\mathrm{C}$ & 2.435052 & 1.457183 & -0.309188 \\
\hline $\mathrm{C}$ & 3.500503 & 0.843215 & -0.387657 \\
\hline $\mathrm{C}$ & 4.689060 & 0.064388 & -0.451579 \\
\hline $\mathrm{C}$ & 4.615630 & -1.331965 & -0.766600 \\
\hline $\mathrm{C}$ & 5.804949 & -2.081373 & -0.777499 \\
\hline $\mathrm{C}$ & 7.031598 & -1.465502 & -0.499614 \\
\hline $\mathrm{C}$ & 7.141351 & -0.092515 & -0.199652 \\
\hline $\mathrm{C}$ & 5.947286 & 0.646883 & -0.181650 \\
\hline $\mathrm{C}$ & 8.484093 & 0.601580 & 0.105107 \\
\hline $\mathrm{C}$ & 9.674793 & -0.376265 & 0.034911 \\
\hline $\mathrm{C}$ & 8.724554 & 1.732925 & -0.925382 \\
\hline $\mathrm{C}$ & 8.439326 & 1.208682 & 1.529690 \\
\hline $\mathrm{C}$ & 3.021626 & -3.185540 & -1.322052 \\
\hline $\mathrm{C}$ & 0.757874 & -2.428433 & -2.216788 \\
\hline
\end{tabular}




\begin{tabular}{|c|c|c|c|}
\hline $\mathrm{C}$ & 1.138697 & -1.479342 & -3.192063 \\
\hline $\mathrm{C}$ & 0.202480 & -0.589848 & -3.716795 \\
\hline $\mathrm{C}$ & -1.146997 & -0.646762 & -3.309475 \\
\hline $\mathrm{C}$ & -1.538776 & -1.599925 & -2.348859 \\
\hline $\mathrm{C}$ & -0.584694 & -2.469942 & -1.802122 \\
\hline $\mathrm{C}$ & -3.358895 & 0.228930 & -3.522199 \\
\hline $\mathrm{H}$ & -0.005326 & 0.366766 & -0.037160 \\
\hline $\mathrm{H}$ & -2.161725 & 4.107575 & 0.175949 \\
\hline $\mathrm{H}$ & -2.594919 & -1.121823 & 1.097695 \\
\hline $\mathrm{H}$ & -1.470860 & -4.281060 & 1.948234 \\
\hline $\mathrm{H}$ & 2.165566 & 4.094967 & -0.318380 \\
\hline $\mathrm{H}$ & 2.592188 & -1.171917 & -1.071794 \\
\hline $\mathrm{H}$ & 1.472295 & -4.364313 & -1.793859 \\
\hline $\mathrm{H}$ & -5.750391 & -3.100321 & 1.116657 \\
\hline $\mathrm{H}$ & -7.931386 & -2.065855 & 0.605654 \\
\hline $\mathrm{H}$ & -5.968878 & 1.716063 & -0.134836 \\
\hline $\mathrm{H}$ & -9.405994 & 1.646207 & -1.828476 \\
\hline $\mathrm{H}$ & -8.305020 & 0.320171 & -2.305990 \\
\hline $\mathrm{H}$ & -7.639681 & 1.870797 & -1.727071 \\
\hline $\mathrm{H}$ & -8.771128 & 1.420278 & 1.890670 \\
\hline $\mathrm{H}$ & -9.677816 & 2.286304 & 0.614921 \\
\hline $\mathrm{H}$ & -7.918962 & 2.528736 & 0.784280 \\
\hline $\mathrm{H}$ & -9.779514 & -0.775383 & 1.014283 \\
\hline $\mathrm{H}$ & -9.592331 & -1.222451 & -0.708272 \\
\hline $\mathrm{H}$ & -10.619914 & 0.157885 & -0.248975 \\
\hline $\mathrm{H}$ & -2.171488 & -1.293162 & 3.577778 \\
\hline $\mathrm{H}$ & -0.486440 & 0.343424 & 4.430295 \\
\hline $\mathrm{H}$ & 2.575362 & -1.577581 & 2.045895 \\
\hline $\mathrm{H}$ & 0.890194 & -3.144114 & 1.144657 \\
\hline $\mathrm{H}$ & 3.854418 & 1.175449 & 4.055810 \\
\hline $\mathrm{H}$ & 3.489199 & 0.560730 & 2.402408 \\
\hline $\mathrm{H}$ & 3.826750 & -0.597119 & 3.747206 \\
\hline $\mathrm{H}$ & 5.748514 & -3.147682 & -0.998996 \\
\hline $\mathrm{H}$ & 7.927359 & -2.092410 & -0.522873 \\
\hline $\mathrm{H}$ & 5.961820 & 1.716833 & 0.050824 \\
\hline $\mathrm{H}$ & 10.611454 & 0.163795 & 0.257356 \\
\hline $\mathrm{H}$ & 9.779059 & -0.824488 & -0.968673 \\
\hline $\mathrm{H}$ & 9.579113 & -1.193834 & 0.770773 \\
\hline $\mathrm{H}$ & 9.676502 & 2.251687 & -0.710780 \\
\hline $\mathrm{H}$ & 7.918725 & 2.486358 & -0.902067 \\
\hline $\mathrm{H}$ & 8.777292 & 1.327980 & -1.950861 \\
\hline $\mathrm{H}$ & 9.390119 & 1.723737 & 1.757854 \\
\hline $\mathrm{H}$ & 8.284148 & 0.422461 & 2.288947 \\
\hline $\mathrm{H}$ & 7.624894 & 1.945790 & 1.635455 \\
\hline $\mathrm{H}$ & 2.173580 & -1.450484 & -3.546829 \\
\hline $\mathrm{H}$ & 0.490699 & 0.150388 & -4.468866 \\
\hline $\mathrm{H}$ & -2.573362 & -1.661755 & -2.003513 \\
\hline $\mathrm{H}$ & -0.890275 & -3.190731 & -1.035999 \\
\hline
\end{tabular}




$\begin{array}{llll}\mathrm{H} & -3.825003 & -0.750533 & -3.742270 \\ \mathrm{H} & -3.847768 & 1.007401 & -4.126119 \\ \mathrm{H} & -3.481246 & 0.462620 & -2.448678 \\ \mathrm{~N} & -0.000967 & 5.707238 & -0.138149 \\ \mathrm{C} & -1.224124 & 6.430377 & 0.173423 \\ \mathrm{H} & -1.600189 & 6.218343 & 1.196404 \\ \mathrm{H} & -1.033251 & 7.510535 & 0.093654 \\ \mathrm{H} & -2.028007 & 6.179916 & -0.543796 \\ \mathrm{C} & 1.264922 & 6.423186 & -0.104716 \\ \mathrm{H} & 1.067215 & 7.504551 & -0.137726 \\ \mathrm{H} & 1.857338 & 6.205885 & 0.808927 \\ \mathrm{H} & 1.888211 & 6.171108 & -0.982959\end{array}$

Statistical Thermodynamic Analysis

Temperature $=298.150$ Kelvin Pressure $=1.00000 \mathrm{Atm}$

\begin{tabular}{|c|c|}
\hline SCF Energy $=\quad-2431.43503471$ & Predicted Change $=-1.844268 \mathrm{D}-0$ \\
\hline Zero-point correction $(\mathrm{ZPE})=$ & -2430.56150 .87344 \\
\hline Internal Energy $(U)=$ & -2430.50330 .93172 \\
\hline Enthalpy $(\mathrm{H})=$ & $-2430.5023 \quad 0.93267$ \\
\hline Gibbs Free Energy $(\mathrm{G})=$ & $-2430.6610 \quad 0.77399$ \\
\hline Frequencies -- 6.9738 & 15.5866 \\
\hline \multicolumn{2}{|c|}{ Single points at WB97X-D3/def2-TZVPP: -2434.67496132812 } \\
\hline
\end{tabular}

Using Gaussian 09: EM64L-G09RevE.01 30-Nov-2015
\#PBEPBE/6-31G(d)/auto scf=(direct,tight, $\operatorname{maxcycle}=300, \mathrm{xqc})$
$\mathrm{opt}=($ maxcycle $=250)$ freq $=$ noraman $\mathrm{int}=($ grid $=$ ultrafine $)$
\#P Geom=AllCheck Guess=TCheck SCRF=Check GenChk RPBEPBE/6-31G(d)/Auto Freq
Pointgroup $=\mathrm{C} 1$ Stoichiometry $=\mathrm{C} 64 \mathrm{H} 87 \mathrm{BrN6O} 4 \mathrm{C} 1[\mathrm{X}(\mathrm{C} 64 \mathrm{H} 87 \mathrm{BrN6O})]$ \#Atoms $=162$
Charge $=0 \quad$ Multiplicity $=1$

$\mathrm{SCF}$ Energy $=-5687.96910396 \quad$ Predicted Change $=-2.316832 \mathrm{D}-08$

\begin{tabular}{|c|c|c|c|c|c|c|}
\hline \multicolumn{3}{|c|}{ Optimization completed. } & $\{$ Foun & 1 & times $\}$ & \multirow[b]{2}{*}{ Pass? } \\
\hline Item & Max Val. & Criteria & Pass? & RMS Val. & Criteria & \\
\hline Force & 0.00000 & 0.00045 & [ YES ] & 0.00000 & || 0.00030 & {$[\mathrm{YES}]$} \\
\hline Displ & 0.00290 & 0.00180 & {$[\mathrm{NO}]$} & $0.00290 \|$ & 0.00180 & {$[\mathrm{YES}]$} \\
\hline
\end{tabular}




\begin{tabular}{|c|c|c|c|}
\hline \multirow{2}{*}{$\begin{array}{l}\text { Atomic } \\
\text { Type }\end{array}$} & \multicolumn{3}{|c|}{ Coordinates (Angstroms) } \\
\hline & $\mathrm{X}$ & $\begin{array}{ll}Y & Z\end{array}$ & \\
\hline $\mathrm{N}$ & 2.463237 & 1.027474 & -2.528245 \\
\hline $\mathrm{C}$ & 1.157769 & 0.816541 & -3.318434 \\
\hline $\mathrm{C}$ & 0.160374 & -0.205396 & -2.771538 \\
\hline $\mathrm{C}$ & -1.091693 & -0.212515 & -3.674882 \\
\hline $\mathrm{C}$ & -2.113611 & -1.273216 & -3.248509 \\
\hline $\mathrm{C}$ & 2.124422 & 1.771310 & -1.231194 \\
\hline $\mathrm{C}$ & 3.287240 & 2.171920 & -0.320863 \\
\hline $\mathrm{C}$ & 2.736431 & 3.122809 & 0.765669 \\
\hline $\mathrm{C}$ & 3.793211 & 3.540297 & 1.794758 \\
\hline $\mathrm{C}$ & 3.117980 & -0.308708 & -2.179068 \\
\hline $\mathrm{C}$ & 3.479846 & -1.208790 & -3.363510 \\
\hline $\mathrm{C}$ & 4.080477 & -2.537757 & -2.864759 \\
\hline $\mathrm{C}$ & 4.464309 & -3.473134 & -4.017720 \\
\hline $\mathrm{C}$ & 3.420842 & 1.856450 & -3.388745 \\
\hline $\mathrm{C}$ & 3.041222 & 3.327430 & -3.585577 \\
\hline $\mathrm{C}$ & 3.898262 & 3.981740 & -4.685968 \\
\hline $\mathrm{C}$ & 3.639954 & 5.491716 & -4.781373 \\
\hline $\mathrm{H}$ & 0.682440 & 1.809033 & -3.369163 \\
\hline $\mathrm{H}$ & 1.469466 & 0.537186 & -4.339148 \\
\hline $\mathrm{H}$ & -0.141051 & 0.024469 & -1.731911 \\
\hline $\mathrm{H}$ & 0.598564 & -1.220810 & -2.763641 \\
\hline $\mathrm{H}$ & -0.788065 & -0.390232 & -4.726519 \\
\hline $\mathrm{H}$ & -1.563072 & 0.788298 & -3.650186 \\
\hline $\mathrm{H}$ & -2.984670 & -1.270839 & -3.925185 \\
\hline $\mathrm{H}$ & -1.674712 & -2.286530 & -3.262906 \\
\hline $\mathrm{H}$ & -2.485041 & -1.087925 & -2.227129 \\
\hline $\mathrm{H}$ & 1.568925 & 2.663986 & -1.555739 \\
\hline $\mathrm{H}$ & 1.409809 & 1.142256 & -0.670673 \\
\hline $\mathrm{H}$ & 3.736059 & 1.288864 & 0.168040 \\
\hline $\mathrm{H}$ & 4.094969 & 2.687697 & -0.875355 \\
\hline $\mathrm{H}$ & 1.884900 & 2.628794 & 1.269211 \\
\hline $\mathrm{H}$ & 2.322865 & 4.024529 & 0.271129 \\
\hline $\mathrm{H}$ & 3.368795 & 4.262669 & 2.511799 \\
\hline $\mathrm{H}$ & 4.153339 & 2.677796 & 2.380319 \\
\hline $\mathrm{H}$ & 4.662916 & 4.018314 & 1.307544 \\
\hline $\mathrm{H}$ & 4.019740 & -0.059410 & -1.597413 \\
\hline $\mathrm{H}$ & 2.419706 & -0.819883 & -1.496726 \\
\hline $\mathrm{H}$ & 2.585532 & -1.428882 & -3.975382 \\
\hline $\mathrm{H}$ & 4.212410 & -0.713264 & -4.027630 \\
\hline $\mathrm{H}$ & 3.353024 & -3.036837 & -2.197517 \\
\hline $\mathrm{H}$ & 4.969256 & -2.326322 & -2.240620 \\
\hline $\mathrm{H}$ & 4.899652 & -4.411736 & -3.634913 \\
\hline $\mathrm{H}$ & 3.584651 & -3.738741 & -4.630665 \\
\hline $\mathrm{H}$ & 5.210740 & -3.006605 & -4.685445 \\
\hline $\mathrm{H}$ & 3.477552 & 1.341995 & -4.361382 \\
\hline
\end{tabular}




\begin{tabular}{|c|c|c|c|}
\hline $\mathrm{H}$ & 4.411627 & 1.776708 & -2.911889 \\
\hline $\mathrm{H}$ & 1.976305 & 3.438434 & -3.856573 \\
\hline $\mathrm{H}$ & 3.182286 & 3.884493 & -2.642119 \\
\hline $\mathrm{H}$ & 3.675627 & 3.497369 & -5.656224 \\
\hline $\mathrm{H}$ & 4.972163 & 3.798420 & -4.486769 \\
\hline $\mathrm{H}$ & 4.173614 & 5.933451 & -5.639475 \\
\hline $\mathrm{H}$ & 2.562602 & 5.707307 & -4.892538 \\
\hline $\mathrm{H}$ & 3.984255 & 6.009633 & -3.868982 \\
\hline $\mathrm{O}$ & -0.029547 & 6.275380 & -3.925812 \\
\hline $\mathrm{O}$ & 3.640051 & 0.739952 & 4.117617 \\
\hline $\mathrm{N}$ & -2.731978 & 2.269557 & -1.072352 \\
\hline $\mathrm{N}$ & 2.790990 & -0.474050 & 2.316513 \\
\hline $\mathrm{C}$ & -0.963410 & -3.087570 & 0.280847 \\
\hline $\mathrm{C}$ & -1.194237 & -5.865825 & -0.349136 \\
\hline $\mathrm{C}$ & 0.074161 & -5.273100 & -0.125535 \\
\hline $\mathrm{C}$ & 0.184569 & -3.900888 & 0.197488 \\
\hline $\mathrm{C}$ & -2.155214 & 3.308440 & -1.818418 \\
\hline $\mathrm{C}$ & -2.725673 & 3.864583 & -2.981858 \\
\hline $\mathrm{C}$ & -2.045249 & 4.859413 & -3.701757 \\
\hline $\mathrm{C}$ & -0.786905 & 5.319117 & -3.277106 \\
\hline $\mathrm{C}$ & -0.226239 & 4.785376 & -2.099255 \\
\hline $\mathrm{C}$ & -0.900994 & 3.797605 & -1.379907 \\
\hline $\mathrm{C}$ & -0.634294 & 6.922804 & -5.041339 \\
\hline $\mathrm{C}$ & 1.462293 & -3.328323 & 0.466399 \\
\hline $\mathrm{C}$ & 2.555758 & -2.840982 & 0.758921 \\
\hline $\mathrm{C}$ & 3.829773 & -2.327056 & 1.123287 \\
\hline $\mathrm{C}$ & 3.950968 & -1.154778 & 1.935068 \\
\hline $\mathrm{C}$ & 5.252168 & -0.728818 & 2.288372 \\
\hline $\mathrm{C}$ & 6.376140 & -1.433436 & 1.855609 \\
\hline $\mathrm{C}$ & 6.287655 & -2.592702 & 1.050805 \\
\hline $\mathrm{C}$ & 4.998962 & -3.012429 & 0.702305 \\
\hline $\mathrm{C}$ & 8.443662 & -2.378069 & -0.237083 \\
\hline $\mathrm{C}$ & 7.250477 & -4.580670 & -0.247511 \\
\hline $\mathrm{C}$ & 2.678411 & 0.423180 & 3.403265 \\
\hline $\mathrm{C}$ & -4.045899 & 1.827181 & -1.136475 \\
\hline $\mathrm{C}$ & -2.229281 & -3.662535 & 0.043243 \\
\hline $\mathrm{C}$ & -2.341667 & -5.035843 & -0.270837 \\
\hline $\mathrm{C}$ & 7.562585 & -3.334154 & 0.605293 \\
\hline $\mathrm{N}$ & 1.392177 & 0.893290 & 3.550394 \\
\hline $\mathrm{N}$ & -4.254461 & 0.701238 & -0.325921 \\
\hline $\mathrm{O}$ & -4.928192 & 2.353840 & -1.823431 \\
\hline $\mathrm{C}$ & -3.390961 & -2.840528 & 0.108849 \\
\hline $\mathrm{C}$ & 8.355907 & -3.790656 & 1.855309 \\
\hline $\mathrm{C}$ & 0.932217 & 1.851730 & 4.481284 \\
\hline $\mathrm{C}$ & -5.498938 & 0.088267 & -0.118880 \\
\hline $\mathrm{C}$ & -4.378755 & -2.107778 & 0.155908 \\
\hline $\mathrm{C}$ & -0.361473 & 2.381986 & 4.262721 \\
\hline $\mathrm{C}$ & 1.666808 & 2.283914 & 5.600518 \\
\hline
\end{tabular}




\begin{tabular}{|c|c|c|c|}
\hline $\mathrm{C}$ & -5.552096 & -1.306938 & 0.172521 \\
\hline $\mathrm{C}$ & -6.709795 & 0.808958 & -0.159816 \\
\hline $\mathrm{C}$ & -0.903578 & 3.314437 & 5.143106 \\
\hline $\mathrm{C}$ & 1.117877 & 3.227365 & 6.483517 \\
\hline $\mathrm{C}$ & -6.804854 & -1.917058 & 0.439317 \\
\hline $\mathrm{C}$ & -7.924638 & 0.171194 & 0.091181 \\
\hline $\mathrm{C}$ & -0.168900 & 3.747594 & 6.264686 \\
\hline $\mathrm{C}$ & -8.008657 & -1.205038 & 0.405498 \\
\hline $\mathrm{O}$ & -0.796391 & 4.672356 & 7.069663 \\
\hline $\mathrm{C}$ & -9.376176 & -1.855725 & 0.689200 \\
\hline $\mathrm{C}$ & -0.081605 & 5.117987 & 8.214857 \\
\hline $\mathrm{C}$ & -9.255435 & -3.360272 & 1.005368 \\
\hline $\mathrm{C}$ & -10.034198 & -1.156264 & 1.904900 \\
\hline $\mathrm{C}$ & -10.290180 & -1.692857 & -0.550906 \\
\hline $\mathrm{H}$ & -2.103134 & 1.825123 & -0.381385 \\
\hline $\mathrm{H}$ & 1.925013 & -0.761294 & 1.845251 \\
\hline $\mathrm{H}$ & -0.874254 & -2.023892 & 0.520699 \\
\hline $\mathrm{H}$ & 0.988613 & -5.866498 & -0.178896 \\
\hline $\mathrm{H}$ & -3.709279 & 3.522035 & -3.305953 \\
\hline $\mathrm{H}$ & -2.517386 & 5.266188 & -4.600016 \\
\hline $\mathrm{H}$ & 0.731569 & 5.182111 & -1.745271 \\
\hline $\mathrm{H}$ & -0.468418 & 3.378244 & -0.463795 \\
\hline $\mathrm{H}$ & 0.089974 & 7.678738 & -5.380004 \\
\hline $\mathrm{H}$ & -0.836854 & 6.215472 & -5.869769 \\
\hline $\mathrm{H}$ & -1.580768 & 7.421918 & -4.757682 \\
\hline $\mathrm{H}$ & 5.356852 & 0.142725 & 2.934028 \\
\hline $\mathrm{H}$ & 4.849166 & -3.910742 & 0.097810 \\
\hline $\mathrm{H}$ & 6.642614 & -5.315514 & 0.308650 \\
\hline $\mathrm{H}$ & 6.712004 & -4.320083 & -1.176661 \\
\hline $\mathrm{H}$ & 7.359696 & -1.060061 & 2.162996 \\
\hline $\mathrm{H}$ & 8.722251 & -1.473172 & 0.329397 \\
\hline $\mathrm{H}$ & 7.912438 & -2.054194 & -1.149997 \\
\hline $\mathrm{H}$ & 9.377045 & -2.883439 & -0.545124 \\
\hline $\mathrm{H}$ & 8.192180 & -5.078085 & -0.537960 \\
\hline $\mathrm{H}$ & -3.338620 & -5.440200 & -0.454136 \\
\hline $\mathrm{H}$ & 7.757516 & -4.486129 & 2.468640 \\
\hline $\mathrm{H}$ & 8.638730 & -2.937459 & 2.494762 \\
\hline $\mathrm{H}$ & 9.284812 & -4.308036 & 1.553715 \\
\hline $\mathrm{H}$ & -0.928948 & 2.056554 & 3.382509 \\
\hline $\mathrm{H}$ & 2.667055 & 1.884932 & 5.771069 \\
\hline $\mathrm{H}$ & -6.683043 & 1.871213 & -0.403757 \\
\hline $\mathrm{H}$ & -1.903660 & 3.725995 & 4.976611 \\
\hline $\mathrm{H}$ & 1.712924 & 3.543364 & 7.344425 \\
\hline $\mathrm{H}$ & -6.793177 & -2.986108 & 0.665771 \\
\hline $\mathrm{H}$ & -8.837781 & 0.775678 & 0.050963 \\
\hline $\mathrm{H}$ & -0.742487 & 5.837132 & 8.721946 \\
\hline $\mathrm{H}$ & 0.148977 & 4.283676 & 8.906467 \\
\hline $\mathrm{H}$ & 0.864605 & 5.624290 & 7.938650 \\
\hline
\end{tabular}




$\begin{array}{lccl}\mathrm{H} & -10.257397 & -3.780174 & 1.201905 \\ \mathrm{H} & -8.635651 & -3.542436 & 1.900775 \\ \mathrm{H} & -8.817664 & -3.922262 & 0.161637 \\ \mathrm{H} & -9.411659 & -1.269674 & 2.809300 \\ \mathrm{H} & -11.026337 & -1.597528 & 2.111591 \\ \mathrm{H} & -10.176851 & -0.076619 & 1.727895 \\ \mathrm{H} & -11.283874 & -2.136246 & -0.356612 \\ \mathrm{H} & -9.854141 & -2.196882 & -1.430908 \\ \mathrm{H} & -10.440987 & -0.631568 & -0.811895 \\ \mathrm{H} & -3.430818 & 0.219703 & 0.053951 \\ \mathrm{H} & 0.712559 & 0.667106 & 2.804643 \\ \mathrm{~N} & -1.311310 & -7.224585 & -0.629539 \\ \mathrm{C} & -0.112702 & -8.001620 & -0.898096 \\ \mathrm{H} & 0.463521 & -7.620145 & -1.768238 \\ \mathrm{H} & 0.562121 & -8.008395 & -0.021635 \\ \mathrm{H} & -0.398024 & -9.043680 & -1.104722 \\ \mathrm{C} & -2.598710 & -7.758342 & -1.045202 \\ \mathrm{H} & -2.498687 & -8.839005 & -1.224615 \\ \mathrm{H} & -3.359814 & -7.620775 & -0.254757 \\ \mathrm{H} & -2.982886 & -7.285255 & -1.973823 \\ \mathrm{Br} & -0.575858 & 0.569644 & 0.854006 \\ ----------------------------------------------------------------------\end{array}$

Statistical Thermodynamic Analysis

Temperature $=298.150$ Kelvin Pressure $=1.00000$ Atm

\begin{tabular}{|c|c|}
\hline SCF Energy $=\quad-5687.96910396$ & Predicted Change $=-2.31683$ \\
\hline Zero-point correction $(\mathrm{ZPE})=$ & -5686.59701 .37206 \\
\hline Internal Energy $(U)=$ & -5686.51181 .45722 \\
\hline Enthalpy $(\mathrm{H})=$ & $-5686.5109 \quad 1.45817$ \\
\hline Gibbs Free Energy $(G)=$ & $-5686.7296 \quad 1.23946$ \\
\hline Frequencies -- 6.6243 & $8.4198 \quad 11.5589$ \\
\hline \multicolumn{2}{|c|}{ Single points at WB97X-D3/def2-TZVPP: -5695.18505649016} \\
\hline
\end{tabular}

Using Gaussian 09: EM64L-G09RevE.01 30-Nov-2015

\footnotetext{
\#PBEPBE/6-31G(d)/auto scf $=($ direct,tight, $m a x c y c l e=300, x q c)$ $\mathrm{opt}=($ maxcycle $=250)$ freq $=$ noraman int $=($ grid $=$ ultrafine $)$ \#P Geom=AllCheck Guess=TCheck SCRF=Check GenChk RPBEPBE/6-31G(d)/Auto Freq Pointgroup $=\mathrm{C} 1$ Stoichiometry $=\mathrm{C} 64 \mathrm{H} 87 \mathrm{ClN} 6 \mathrm{O} 4 \mathrm{C} 1 \mathrm{X}(\mathrm{C} 64 \mathrm{H} 87 \mathrm{ClN} 6 \mathrm{O} 4)]$ \#Atoms= 162
} 
Charge $=0 \quad$ Multiplicity $=1$

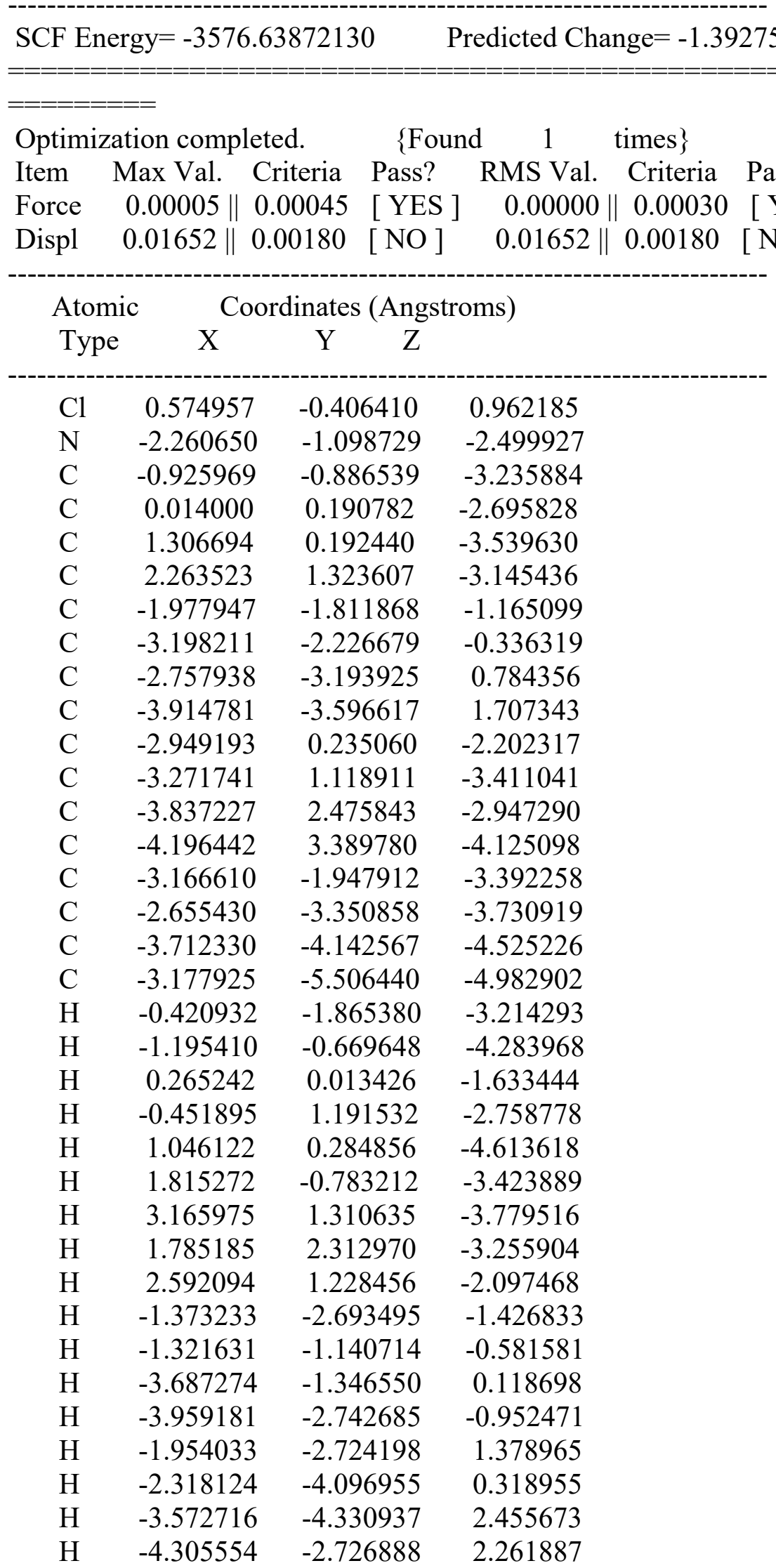




\begin{tabular}{|c|c|c|c|}
\hline $\mathrm{H}$ & -4.744910 & -4.054012 & 1.137932 \\
\hline $\mathrm{H}$ & -3.871719 & -0.017184 & -1.655701 \\
\hline $\mathrm{H}$ & -2.285922 & 0.762575 & -1.498183 \\
\hline $\mathrm{H}$ & -2.366601 & 1.298505 & -4.020009 \\
\hline $\mathrm{H}$ & -4.013884 & 0.632343 & -4.070896 \\
\hline $\mathrm{H}$ & -3.097298 & 2.974231 & -2.293412 \\
\hline $\mathrm{H}$ & -4.730939 & 2.306277 & -2.317815 \\
\hline $\mathrm{H}$ & -4.605035 & 4.349975 & -3.766955 \\
\hline $\mathrm{H}$ & -3.310919 & 3.614880 & -4.745825 \\
\hline $\mathrm{H}$ & -4.956825 & 2.926459 & -4.779236 \\
\hline $\mathrm{H}$ & -3.310857 & -1.367728 & -4.317269 \\
\hline $\mathrm{H}$ & -4.140560 & -2.000599 & -2.879948 \\
\hline $\mathrm{H}$ & -1.727199 & -3.291290 & -4.327152 \\
\hline $\mathrm{H}$ & -2.404453 & -3.917392 & -2.817264 \\
\hline $\mathrm{H}$ & -4.040515 & -3.556035 & -5.405465 \\
\hline $\mathrm{H}$ & -4.610763 & -4.283804 & -3.894226 \\
\hline $\mathrm{H}$ & -3.970263 & -6.102420 & -5.465813 \\
\hline $\mathrm{H}$ & -2.357575 & -5.383810 & -5.712509 \\
\hline $\mathrm{H}$ & -2.778536 & -6.085541 & -4.132016 \\
\hline $\mathrm{O}$ & -0.203631 & -6.681631 & -3.013458 \\
\hline $\mathrm{O}$ & -3.860055 & -0.670928 & 3.744293 \\
\hline $\mathrm{N}$ & 2.527543 & -2.300491 & -0.797100 \\
\hline $\mathrm{N}$ & -2.848551 & 0.674808 & 2.133784 \\
\hline $\mathrm{C}$ & 1.102822 & 3.154491 & 0.377800 \\
\hline $\mathrm{C}$ & 1.498739 & 5.954767 & -0.055265 \\
\hline $\mathrm{C}$ & 0.197214 & 5.421056 & 0.117971 \\
\hline $\mathrm{C}$ & 0.003890 & 4.036579 & 0.331404 \\
\hline $\mathrm{C}$ & 1.919678 & -3.421041 & -1.391602 \\
\hline $\mathrm{C}$ & 2.330260 & -3.964210 & -2.627005 \\
\hline $\mathrm{C}$ & 1.649327 & -5.053646 & -3.193107 \\
\hline $\mathrm{C}$ & 0.541404 & -5.621103 & -2.539230 \\
\hline $\mathrm{C}$ & 0.135201 & -5.091837 & -1.298443 \\
\hline $\mathrm{C}$ & 0.816443 & -4.012116 & -0.731728 \\
\hline $\mathrm{C}$ & 0.266929 & -7.317042 & -4.197931 \\
\hline $\mathrm{C}$ & -1.312579 & 3.523686 & 0.522475 \\
\hline $\mathrm{C}$ & -2.449432 & 3.088821 & 0.714459 \\
\hline $\mathrm{C}$ & -3.760914 & 2.598706 & 0.958968 \\
\hline $\mathrm{C}$ & -3.967213 & 1.387171 & 1.692676 \\
\hline $\mathrm{C}$ & -5.298383 & 0.960880 & 1.903523 \\
\hline $\mathrm{C}$ & -6.371286 & 1.706959 & 1.413156 \\
\hline $\mathrm{C}$ & -6.198899 & 2.909676 & 0.689769 \\
\hline $\mathrm{C}$ & -4.879446 & 3.327589 & 0.480253 \\
\hline $\mathrm{C}$ & -8.236134 & 2.803070 & -0.791062 \\
\hline $\mathrm{C}$ & -7.020320 & 4.981993 & -0.573125 \\
\hline $\mathrm{C}$ & -2.834927 & -0.307664 & 3.148503 \\
\hline $\mathrm{C}$ & 3.860956 & -1.939957 & -0.955603 \\
\hline $\mathrm{C}$ & 2.403342 & 3.676201 & 0.211769 \\
\hline $\mathrm{C}$ & 2.597610 & 5.061243 & 0.006120 \\
\hline
\end{tabular}




\begin{tabular}{|c|c|c|c|}
\hline $\mathrm{C}$ & -7.421037 & 3.695860 & 0.177724 \\
\hline $\mathrm{N}$ & -1.568260 & -0.803171 & 3.370167 \\
\hline $\mathrm{N}$ & 4.178851 & -0.771666 & -0.249967 \\
\hline $\mathrm{O}$ & 4.672851 & -2.571679 & -1.641041 \\
\hline $\mathrm{C}$ & 3.521040 & 2.793608 & 0.238125 \\
\hline $\mathrm{C}$ & -8.315590 & 4.095361 & 1.377852 \\
\hline $\mathrm{C}$ & -1.200625 & -1.806803 & 4.295571 \\
\hline $\mathrm{C}$ & 5.465363 & -0.230663 & -0.117501 \\
\hline $\mathrm{C}$ & 4.477993 & 2.019234 & 0.228514 \\
\hline $\mathrm{C}$ & 0.126801 & -2.291846 & 4.219414 \\
\hline $\mathrm{C}$ & -2.058545 & -2.331388 & 5.280958 \\
\hline $\mathrm{C}$ & 5.607296 & 1.159552 & 0.174118 \\
\hline $\mathrm{C}$ & 6.634469 & -1.011485 & -0.228008 \\
\hline $\mathrm{C}$ & 0.578842 & -3.271034 & 5.100365 \\
\hline $\mathrm{C}$ & -1.599097 & -3.318689 & 6.167167 \\
\hline $\mathrm{C}$ & 6.902738 & 1.703699 & 0.369518 \\
\hline $\mathrm{C}$ & 7.892122 & -0.436691 & -0.043688 \\
\hline $\mathrm{C}$ & -0.280365 & -3.796305 & 6.085483 \\
\hline $\mathrm{C}$ & 8.064946 & 0.931839 & 0.267154 \\
\hline $\mathrm{O}$ & 0.265723 & -4.759685 & 6.904170 \\
\hline $\mathrm{C}$ & 9.477677 & 1.510721 & 0.473997 \\
\hline $\mathrm{C}$ & -0.575818 & -5.294520 & 7.917545 \\
\hline $\mathrm{C}$ & 9.451855 & 3.017534 & 0.801049 \\
\hline $\mathrm{C}$ & 10.167675 & 0.771914 & 1.647947 \\
\hline $\mathrm{C}$ & 10.310114 & 1.309068 & -0.816846 \\
\hline $\mathrm{H}$ & 1.951355 & -1.785641 & -0.105258 \\
\hline $\mathrm{H}$ & -1.943197 & 1.040814 & 1.818764 \\
\hline $\mathrm{H}$ & 0.953244 & 2.084999 & 0.557300 \\
\hline $\mathrm{H}$ & -0.681726 & 6.067810 & 0.104774 \\
\hline $\mathrm{H}$ & 3.204211 & -3.545355 & -3.128013 \\
\hline $\mathrm{H}$ & 2.001913 & -5.450559 & -4.148883 \\
\hline $\mathrm{H}$ & -0.701285 & -5.564510 & -0.773006 \\
\hline $\mathrm{H}$ & 0.504952 & -3.618601 & 0.242199 \\
\hline $\mathrm{H}$ & -0.411672 & -8.164720 & -4.375338 \\
\hline $\mathrm{H}$ & 0.234357 & -6.636818 & -5.072261 \\
\hline $\mathrm{H}$ & 1.300759 & -7.692990 & -4.074724 \\
\hline $\mathrm{H}$ & -5.469614 & 0.056492 & 2.486780 \\
\hline $\mathrm{H}$ & -4.665923 & 4.252446 & -0.061815 \\
\hline $\mathrm{H}$ & -6.454745 & 5.674796 & 0.074125 \\
\hline $\mathrm{H}$ & -6.405534 & 4.763630 & -1.464982 \\
\hline $\mathrm{H}$ & -7.382476 & 1.332663 & 1.610246 \\
\hline $\mathrm{H}$ & -8.574812 & 1.873247 & -0.303144 \\
\hline $\mathrm{H}$ & -7.630650 & 2.521325 & -1.671196 \\
\hline $\mathrm{H}$ & -9.132460 & 3.340705 & -1.150300 \\
\hline $\mathrm{H}$ & -7.926876 & 5.510453 & -0.915907 \\
\hline $\mathrm{H}$ & 3.620749 & 5.423091 & -0.108899 \\
\hline $\mathrm{H}$ & -7.765670 & 4.746682 & 2.078808 \\
\hline $\mathrm{H}$ & -8.662171 & 3.212693 & 1.941513 \\
\hline
\end{tabular}




$\begin{array}{lccc}\mathrm{H} & -9.209054 & 4.642953 & 1.026591 \\ \mathrm{H} & 0.800934 & -1.888628 & 3.455165 \\ \mathrm{H} & -3.083710 & -1.966927 & 5.346838 \\ \mathrm{H} & 6.539320 & -2.069253 & -0.473383 \\ \mathrm{H} & 1.605331 & -3.645561 & 5.042190 \\ \mathrm{H} & -2.290569 & -3.703281 & 6.921588 \\ \mathrm{H} & 8.769361 & -1.086990 & -0.136539 \\ \mathrm{H} & 0.035992 & -6.030996 & 8.460069 \\ \mathrm{H} & -0.917337 & -4.512321 & 8.623981 \\ \mathrm{H} & -1.463654 & -5.801869 & 7.490579 \\ \mathrm{H} & 10.483386 & 3.384912 & 0.941875 \\ \mathrm{H} & 8.893561 & 3.225382 & 1.730682 \\ \mathrm{H} & 8.996406 & 3.606121 & -0.014739 \\ \mathrm{H} & 9.604684 & 0.911975 & 2.586993 \\ \mathrm{H} & 11.191420 & 1.160662 & 1.798321 \\ \mathrm{H} & 10.244113 & -0.312613 & 1.460213 \\ \mathrm{H} & 11.334284 & 1.701384 & -0.678977 \\ \mathrm{H} & 9.850185 & 1.838803 & -1.669166 \\ \mathrm{H} & 10.392351 & 0.242915 & -1.088511 \\ \mathrm{H} & 3.407781 & -0.216158 & 0.137362 \\ \mathrm{H} & -0.827048 & -0.556657 & 2.688075 \\ \mathrm{~N} & 1.692078 & 7.315917 & -0.288668 \\ \mathrm{C} & 0.574857 & 8.228347 & -0.098546 \\ \mathrm{H} & -0.247763 & 7.997399 & -0.800379 \\ \mathrm{H} & 0.161860 & 8.200061 & 0.932386 \\ \mathrm{H} & 0.910003 & 9.254845 & -0.308983 \\ \mathrm{C} & 3.040418 & 7.859003 & -0.214250 \\ \mathrm{H} & 3.002061 & 8.941416 & -0.407206 \\ \mathrm{H} & 3.519096 & 7.697014 & 0.774917 \\ \mathrm{H} & 3.691317 & 7.407082 & -0.985036 \\ -----------------------------------------------------------------\end{array}$

Statistical Thermodynamic Analysis

Temperature $=298.150$ Kelvin Pressure $=1.00000$ Atm
$========$
SCF Energy $=\quad-3576.63872130 \quad$ Predicted Change $=-1.392755 D-07$
Zero-point correction $(\mathrm{ZPE})=\quad-3575.26801 .37068$
Internal Energy $(\mathrm{U})=\quad-3575.18251 .45616$
Enthalpy $(\mathrm{H})=$
Gibbs Free Energy $(\mathrm{G})=\quad-3575.4020 \quad 1.23667$
Frequencies -- $\quad 5.8002 \quad 7.7233 \quad 10.4807$
Single points at WB97X-D3/def2-TZVPP: -3581.17748940541

$\mathbf{R}=\mathrm{NMe}_{2}$ receptor bound with $\mathrm{SeH}$ - (with $\mathrm{TBA}$ )

Supporting Information: 010-bound_TBA_NMe2_SeH.log
} 
Using Gaussian 09: EM64L-G09RevE.01 30-Nov-2015

\#PBEPBE/6-31G(d)/auto scf=(direct,tight, maxcycle $=300, \mathrm{xqc})$

$\mathrm{opt}=($ maxcycle $=250)$ freq $=$ noraman int $=($ grid $=$ ultrafine $)$

\#P Geom=AllCheck Guess=TCheck SCRF=Check GenChk RPBEPBE/6-31G(d)/Auto Freq

Pointgroup $=\mathrm{C} 1 \quad$ Stoichiometry $=\mathrm{C} 64 \mathrm{H} 88 \mathrm{~N} 6 \mathrm{O} 4 \mathrm{Se} \quad \mathrm{C} 1[\mathrm{X}(\mathrm{C} 64 \mathrm{H} 88 \mathrm{~N} 6 \mathrm{O} 4 \mathrm{Se})]$ \#Atoms $=163$

Charge $=0 \quad$ Multiplicity $=1$

SCF Energy $=-5516.22786030 \quad$ Predicted Change $=-4.114237 \mathrm{D}-09$

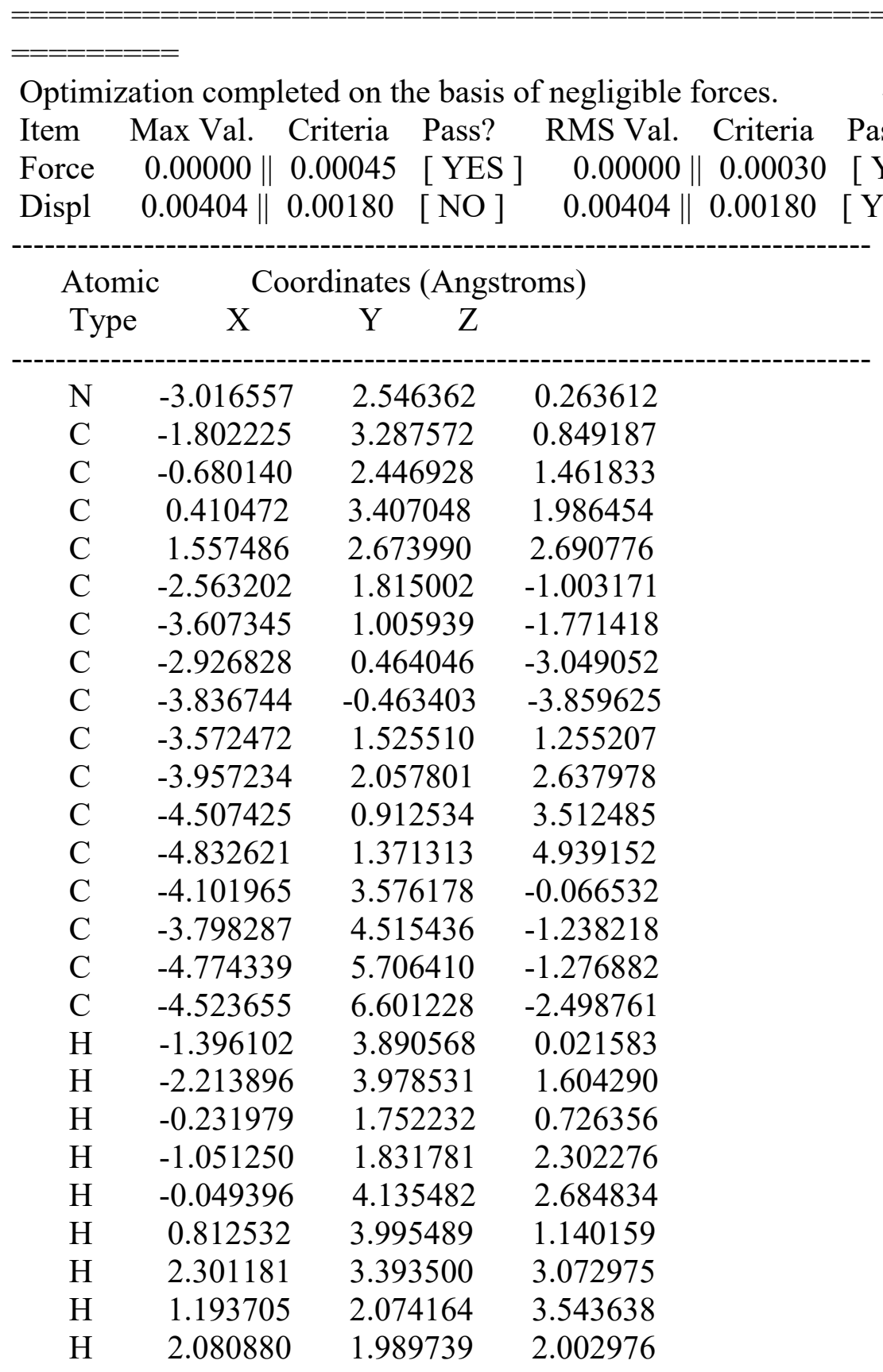




\begin{tabular}{|c|c|c|c|}
\hline $\mathrm{H}$ & -2.140022 & 2.599914 & -1.647727 \\
\hline $\mathrm{H}$ & -1.722475 & 1.151131 & -0.728039 \\
\hline $\mathrm{H}$ & -3.966101 & 0.148350 & -1.173756 \\
\hline $\mathrm{H}$ & -4.492952 & 1.612356 & -2.046161 \\
\hline $\mathrm{H}$ & -1.997099 & -0.053043 & -2.743312 \\
\hline $\mathrm{H}$ & -2.609392 & 1.320138 & -3.677371 \\
\hline $\mathrm{H}$ & -3.311847 & -0.846155 & -4.750753 \\
\hline $\mathrm{H}$ & -4.157106 & -1.332856 & -3.260387 \\
\hline $\mathrm{H}$ & -4.745025 & 0.064019 & -4.204523 \\
\hline $\mathrm{H}$ & -4.449405 & 1.076407 & 0.762356 \\
\hline $\mathrm{H}$ & -2.810627 & 0.733945 & 1.341214 \\
\hline $\mathrm{H}$ & -3.079108 & 2.499615 & 3.143327 \\
\hline $\mathrm{H}$ & -4.723199 & 2.852527 & 2.565251 \\
\hline $\mathrm{H}$ & -3.768033 & 0.090510 & 3.539311 \\
\hline $\mathrm{H}$ & -5.415846 & 0.494040 & 3.037229 \\
\hline $\mathrm{H}$ & -5.239648 & 0.539447 & 5.538074 \\
\hline $\mathrm{H}$ & -3.929186 & 1.744257 & 5.453894 \\
\hline $\mathrm{H}$ & -5.581171 & 2.183776 & 4.943157 \\
\hline $\mathrm{H}$ & -4.260496 & 4.155231 & 0.857492 \\
\hline $\mathrm{H}$ & -5.021677 & 3.000763 & -0.262725 \\
\hline $\mathrm{H}$ & -2.769646 & 4.912758 & -1.178639 \\
\hline $\mathrm{H}$ & -3.867175 & 3.963054 & -2.192993 \\
\hline $\mathrm{H}$ & -4.661709 & 6.298107 & -0.347972 \\
\hline $\mathrm{H}$ & -5.819065 & 5.339546 & -1.287668 \\
\hline $\mathrm{H}$ & -5.166009 & 7.497317 & -2.473409 \\
\hline $\mathrm{H}$ & -3.470534 & 6.931772 & -2.544143 \\
\hline $\mathrm{H}$ & -4.739999 & 6.060468 & -3.436954 \\
\hline $\mathrm{O}$ & -0.621738 & 6.889600 & -3.272984 \\
\hline $\mathrm{O}$ & -2.075885 & -4.608648 & -2.694678 \\
\hline $\mathrm{N}$ & 2.397083 & 2.593821 & -1.303450 \\
\hline $\mathrm{N}$ & -2.165511 & -2.804044 & -1.212829 \\
\hline $\mathrm{C}$ & 0.607889 & -1.268725 & 2.360658 \\
\hline $\mathrm{C}$ & 0.407479 & -1.403467 & 5.204215 \\
\hline $\mathrm{C}$ & -0.738796 & -1.569069 & 4.385399 \\
\hline $\mathrm{C}$ & -0.637152 & -1.514133 & 2.975818 \\
\hline $\mathrm{C}$ & 1.744408 & 3.726308 & -1.804428 \\
\hline $\mathrm{C}$ & 2.266642 & 5.035782 & -1.783946 \\
\hline $\mathrm{C}$ & 1.503444 & 6.110596 & -2.269538 \\
\hline $\mathrm{C}$ & 0.214109 & 5.901597 & -2.788693 \\
\hline $\mathrm{C}$ & -0.299595 & 4.589906 & -2.834051 \\
\hline $\mathrm{C}$ & 0.452626 & 3.520638 & -2.348620 \\
\hline $\mathrm{C}$ & -0.093929 & 8.211455 & -3.314099 \\
\hline $\mathrm{C}$ & -1.782056 & -1.742430 & 2.156898 \\
\hline $\mathrm{C}$ & -2.737822 & -2.017252 & 1.427906 \\
\hline $\mathrm{C}$ & -3.782138 & -2.451959 & 0.567054 \\
\hline $\mathrm{C}$ & -3.466376 & -2.943398 & -0.740982 \\
\hline $\mathrm{C}$ & -4.505786 & -3.528949 & -1.498215 \\
\hline $\mathrm{C}$ & -5.807593 & -3.574647 & -0.998527 \\
\hline
\end{tabular}




\begin{tabular}{|c|c|c|c|}
\hline C & -6.160188 & -3.045109 & 0.266236 \\
\hline $\mathrm{C}$ & -5.123029 & -2.495278 & 1.028641 \\
\hline $\mathrm{C}$ & -8.526687 & -2.337029 & -0.239741 \\
\hline $\mathrm{C}$ & -7.797245 & -2.475030 & 2.151818 \\
\hline $\mathrm{C}$ & -1.514883 & -3.658985 & -2.135535 \\
\hline $\mathrm{C}$ & 3.706241 & 2.485387 & -0.858849 \\
\hline $\mathrm{C}$ & 1.753581 & -1.089122 & 3.163246 \\
\hline $\mathrm{C}$ & 1.653108 & -1.156335 & 4.570667 \\
\hline $\mathrm{C}$ & -7.620144 & -3.104383 & 0.755019 \\
\hline $\mathrm{N}$ & -0.202443 & -3.278759 & -2.296239 \\
\hline $\mathrm{N}$ & 3.956988 & 1.204245 & -0.343844 \\
\hline $\mathrm{O}$ & 4.543859 & 3.393726 & -0.897112 \\
\hline $\mathrm{C}$ & 2.996118 & -0.802006 & 2.527141 \\
\hline $\mathrm{C}$ & -8.079730 & -4.582024 & 0.828990 \\
\hline $\mathrm{C}$ & 0.817320 & -3.906779 & -3.033235 \\
\hline $\mathrm{C}$ & 5.193416 & 0.783252 & 0.168018 \\
\hline $\mathrm{C}$ & 4.027272 & -0.519122 & 1.918366 \\
\hline $\mathrm{C}$ & 2.101637 & -3.316780 & -2.948615 \\
\hline $\mathrm{C}$ & 0.638549 & -5.056522 & -3.822485 \\
\hline $\mathrm{C}$ & 5.227054 & -0.130611 & 1.262515 \\
\hline $\mathrm{C}$ & 6.417381 & 1.222467 & -0.374262 \\
\hline $\mathrm{C}$ & 3.177844 & -3.866041 & -3.639012 \\
\hline $\mathrm{C}$ & 1.728436 & -5.608294 & -4.514771 \\
\hline $\mathrm{C}$ & 6.475752 & -0.603353 & 1.740057 \\
\hline $\mathrm{C}$ & 7.628541 & 0.753699 & 0.135211 \\
\hline $\mathrm{C}$ & 3.001855 & -5.019695 & -4.429693 \\
\hline $\mathrm{C}$ & 7.693651 & -0.179677 & 1.195791 \\
\hline $\mathrm{O}$ & 4.131749 & -5.482550 & -5.069247 \\
\hline $\mathrm{C}$ & 9.058542 & -0.681720 & 1.705154 \\
\hline $\mathrm{C}$ & 3.985052 & -6.648435 & -5.868419 \\
\hline $\mathrm{C}$ & 8.917937 & -1.684872 & 2.867913 \\
\hline $\mathrm{C}$ & 9.810126 & -1.384441 & 0.546919 \\
\hline $\mathrm{C}$ & 9.896468 & 0.522569 & 2.202607 \\
\hline $\mathrm{H}$ & 1.817321 & 1.730614 & -1.278049 \\
\hline $\mathrm{H}$ & -1.586373 & -2.087757 & -0.754796 \\
\hline $\mathrm{H}$ & 0.692803 & -1.192174 & 1.274100 \\
\hline $\mathrm{H}$ & -1.716991 & -1.772898 & 4.824856 \\
\hline $\mathrm{H}$ & 3.271945 & 5.197761 & -1.391732 \\
\hline $\mathrm{H}$ & 1.937027 & 7.113650 & -2.233958 \\
\hline $\mathrm{H}$ & -1.284224 & 4.426922 & -3.285839 \\
\hline $\mathrm{H}$ & 0.057125 & 2.497432 & -2.352958 \\
\hline $\mathrm{H}$ & -0.882027 & 8.837496 & -3.758745 \\
\hline $\mathrm{H}$ & 0.146061 & 8.591210 & -2.301281 \\
\hline $\mathrm{H}$ & 0.816346 & 8.267581 & -3.941810 \\
\hline $\mathrm{H}$ & -4.260646 & -3.957785 & -2.470963 \\
\hline $\mathrm{H}$ & -5.313160 & -2.110711 & 2.034484 \\
\hline $\mathrm{H}$ & -7.203887 & -3.003736 & 2.918246 \\
\hline $\mathrm{H}$ & -7.503832 & -1.409510 & 2.161748 \\
\hline
\end{tabular}




$\begin{array}{lccc}\text { H } & -6.579363 & -4.033837 & -1.626760 \\ \mathrm{H} & -8.464983 & -2.757919 & -1.257584 \\ \mathrm{H} & -8.236837 & -1.272952 & -0.297914 \\ \mathrm{H} & -9.582756 & -2.388475 & 0.082188 \\ \mathrm{H} & -8.857485 & -2.530929 & 2.454024 \\ \mathrm{H} & 2.560189 & -1.004560 & 5.158029 \\ \mathrm{H} & -7.460393 & -5.151656 & 1.543107 \\ \mathrm{H} & -8.006592 & -5.081873 & -0.151596 \\ \mathrm{H} & -9.132557 & -4.642043 & 1.160018 \\ \mathrm{H} & 2.223500 & -2.417132 & -2.330881 \\ \mathrm{H} & -0.351037 & -5.511901 & -3.885442 \\ \mathrm{H} & 6.401619 & 1.943324 & -1.192682 \\ \mathrm{H} & 4.173049 & -3.415553 & -3.576439 \\ \mathrm{H} & 1.563512 & -6.503807 & -5.119782 \\ \mathrm{H} & 6.451055 & -1.311144 & 2.572367 \\ \mathrm{H} & 8.554282 & 1.119808 & -0.322815 \\ \mathrm{H} & 4.982410 & -6.856642 & -6.284637 \\ \mathrm{H} & 3.650593 & -7.518982 & -5.269874 \\ \mathrm{H} & 3.268864 & -6.491350 & -6.699397 \\ \mathrm{H} & 9.918786 & -2.015945 & 3.195815 \\ \mathrm{H} & 8.350866 & -2.583390 & 2.567772 \\ \mathrm{H} & 8.413632 & -1.233013 & 3.740185 \\ \mathrm{H} & 9.243176 & -2.258385 & 0.182084 \\ \mathrm{H} & 10.801295 & -1.734552 & 0.888600 \\ \mathrm{H} & 9.969676 & -0.706022 & -0.308418 \\ \mathrm{H} & 10.887710 & 0.181803 & 2.553406 \\ \mathrm{H} & 9.391974 & 1.034722 & 3.040339 \\ \mathrm{H} & 10.060068 & 1.265864 & 1.403874 \\ \mathrm{H} & 3.160565 & 0.555869 & -0.265542 \\ \mathrm{H} & 0.098053 & -2.412175 & -1.810492 \\ \mathrm{~N} & 0.313698 & -1.487709 & 6.589834 \\ \mathrm{C} & -0.998288 & -1.543936 & 7.212346 \\ \mathrm{H} & -1.630886 & -0.664559 & 6.963153 \\ \mathrm{H} & -1.548074 & -2.453596 & 6.905195 \\ \mathrm{H} & -0.877724 & -1.581868 & 8.304915 \\ \mathrm{C} & 1.464419 & -1.134497 & 7.406572 \\ \mathrm{H} & 1.204463 & -1.260618 & 8.467858 \\ \mathrm{H} & 2.322552 & -1.798124 & 7.192106 \\ \mathrm{H} & 1.797188 & -0.086374 & 7.251343 \\ \mathrm{H} & 0.719988 & -0.126099 & -2.622769 \\ \mathrm{Se} & 0.498845 & -0.199878 & -1.150210 \\ ----------------------------------------------------------------- \\ -\end{array}$

Statistical Thermodynamic Analysis

Temperature $=298.150$ Kelvin Pressure $=1.00000$ Atm

$========$
SCF Energy $=\quad-5516.22786030$

Zero-point correction $(\mathrm{ZPE})=$
Predicted Change $=-4.114237 \mathrm{D}-09$

$-5514.84751 .38029$ 
Internal Energy $(\mathrm{U})=$

Enthalpy $(\mathrm{H})=$

Gibbs Free Energy $(\mathrm{G})=$
$-5514.76221 .46563$

$-5514.7612 \quad 1.46657$

$-5514.9794 \quad 1.24836$

$\begin{array}{llll}\text { Frequencies -- } & 5.8121 & 7.7882 & 11.3389\end{array}$

Single points at WB97X-D3/def2-TZVPP: -5523.14036080218

$\mathrm{NMe}_{2}$ hat receptor bound with $\mathrm{SH}$ - (with TBA)

Supporting Information: 010-bound_TBA_NMe2_SH.log

Using Gaussian 09: EM64L-G09RevE.01 30-Nov-2015

\#PBEPBE/6-31G(d)/auto scf=(direct,tight,maxcycle=300,xqc)

$\mathrm{opt}=($ maxcycle $=250)$ freq $=$ noraman int $=($ grid $=$ ultrafine $)$

\#P Geom=AllCheck Guess=TCheck SCRF=Check GenChk RPBEPBE/6-31G(d)/Auto Freq

Pointgroup=C1 Stoichiometry= C64H88N6O4S C1[X(C64H88N6O4S) $]$ \#Atoms $=163$

Charge $=0 \quad$ Multiplicity $=1$

SCF Energy $=-3515.20230275 \quad$ Predicted Change $=-1.233681 \mathrm{D}-07$

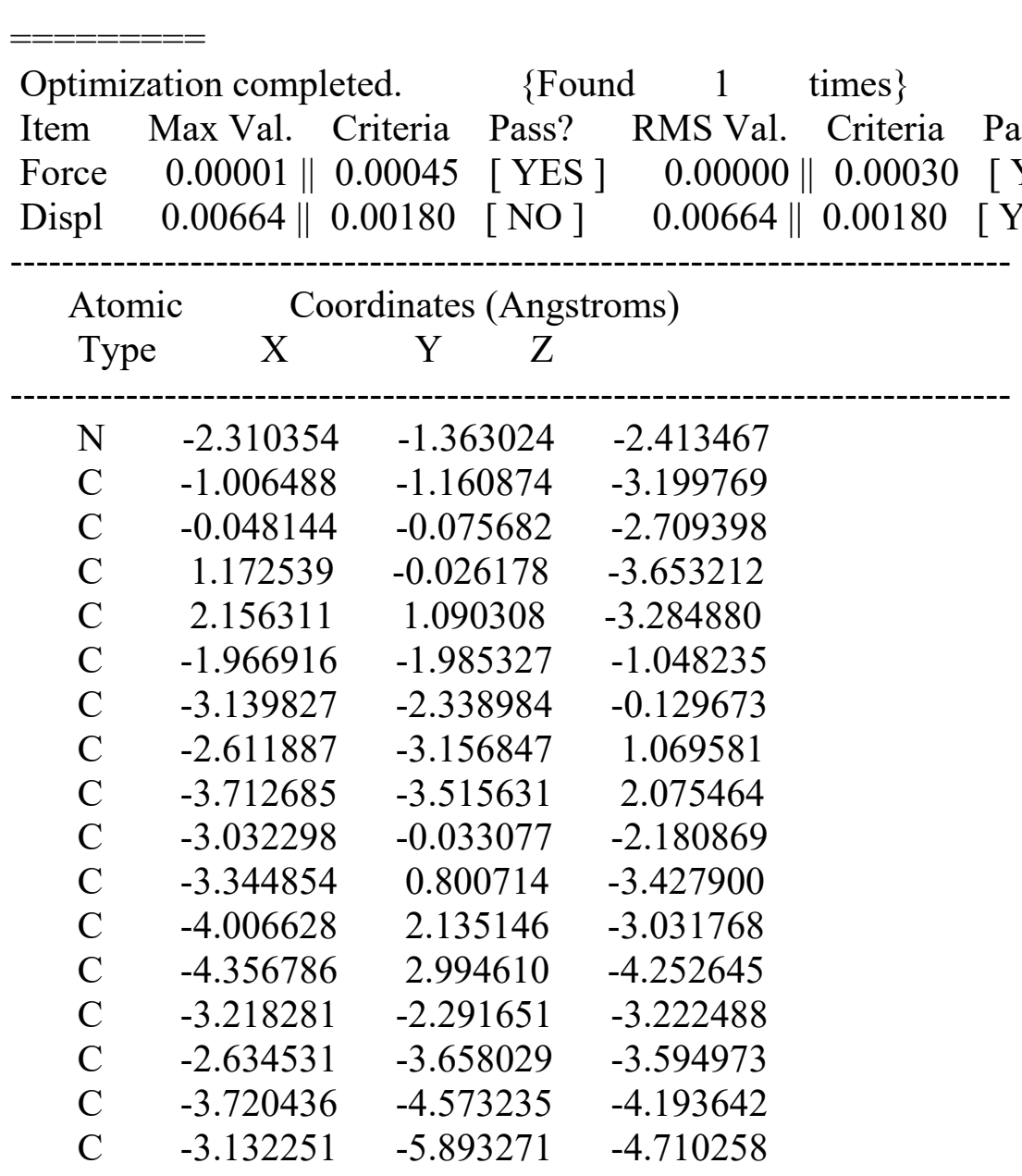




\begin{tabular}{|c|c|c|c|}
\hline $\mathrm{H}$ & -0.498264 & -2.137825 & -3.180425 \\
\hline $\mathrm{H}$ & -1.316156 & -0.961406 & -4.240244 \\
\hline $\mathrm{H}$ & 0.290953 & -0.282407 & -1.677402 \\
\hline $\mathrm{H}$ & -0.533275 & 0.917636 & -2.701516 \\
\hline $\mathrm{H}$ & 0.823402 & 0.115096 & -4.696297 \\
\hline $\mathrm{H}$ & 1.691478 & -1.002964 & -3.625573 \\
\hline $\mathrm{H}$ & 2.999193 & 1.117432 & -3.995915 \\
\hline $\mathrm{H}$ & 1.667878 & 2.080670 & -3.298656 \\
\hline $\mathrm{H}$ & 2.576765 & 0.940883 & -2.276915 \\
\hline $\mathrm{H}$ & -1.375688 & -2.883790 & -1.281156 \\
\hline $\mathrm{H}$ & -1.280523 & -1.284382 & -0.534736 \\
\hline $\mathrm{H}$ & -3.637206 & -1.428757 & 0.251157 \\
\hline $\mathrm{H}$ & -3.908358 & -2.943001 & -0.649394 \\
\hline $\mathrm{H}$ & -1.814633 & -2.580430 & 1.573572 \\
\hline $\mathrm{H}$ & -2.138041 & -4.082864 & 0.688316 \\
\hline $\mathrm{H}$ & -3.309225 & -4.157375 & 2.876664 \\
\hline $\mathrm{H}$ & -4.125364 & -2.614173 & 2.559137 \\
\hline $\mathrm{H}$ & -4.541104 & -4.063381 & 1.589618 \\
\hline $\mathrm{H}$ & -3.961896 & -0.285602 & -1.646364 \\
\hline $\mathrm{H}$ & -2.398100 & 0.536184 & -1.482337 \\
\hline $\mathrm{H}$ & -2.422281 & 1.016593 & -3.996913 \\
\hline $\mathrm{H}$ & -4.025704 & 0.258929 & -4.110523 \\
\hline $\mathrm{H}$ & -3.327499 & 2.691481 & -2.359280 \\
\hline $\mathrm{H}$ & -4.919830 & 1.931903 & -2.441233 \\
\hline $\mathrm{H}$ & -4.835475 & 3.939531 & -3.944140 \\
\hline $\mathrm{H}$ & -3.454888 & 3.252376 & -4.835881 \\
\hline $\mathrm{H}$ & -5.056342 & 2.471096 & -4.928896 \\
\hline $\mathrm{H}$ & -3.483039 & -1.738533 & -4.137164 \\
\hline $\mathrm{H}$ & -4.138099 & -2.408498 & -2.627904 \\
\hline $\mathrm{H}$ & -1.819376 & -3.539644 & -4.331237 \\
\hline $\mathrm{H}$ & -2.191836 & -4.162699 & -2.718489 \\
\hline $\mathrm{H}$ & -4.242869 & -4.049611 & -5.018050 \\
\hline $\mathrm{H}$ & -4.487067 & -4.778955 & -3.422189 \\
\hline $\mathrm{H}$ & -3.928441 & -6.578006 & -5.047141 \\
\hline $\mathrm{H}$ & -2.457285 & -5.715715 & -5.566513 \\
\hline $\mathrm{H}$ & -2.544053 & -6.403253 & -3.927393 \\
\hline $\mathrm{O}$ & 0.072452 & -6.844214 & -3.040502 \\
\hline $\mathrm{O}$ & -3.712667 & -0.496454 & 4.062640 \\
\hline $\mathrm{N}$ & 2.622758 & -2.309900 & -0.919076 \\
\hline $\mathrm{N}$ & -2.854032 & 0.680938 & 2.244779 \\
\hline $\mathrm{C}$ & 0.921701 & 3.057470 & 0.108776 \\
\hline $\mathrm{C}$ & 1.147271 & 5.731643 & -0.881572 \\
\hline $\mathrm{C}$ & -0.118398 & 5.172605 & -0.575249 \\
\hline $\mathrm{C}$ & -0.228721 & 3.849912 & -0.086428 \\
\hline $\mathrm{C}$ & 2.068669 & -3.469101 & -1.486137 \\
\hline $\mathrm{C}$ & 2.543815 & -4.064766 & -2.673726 \\
\hline $\mathrm{C}$ & 1.906980 & -5.193213 & -3.214591 \\
\hline $\mathrm{C}$ & 0.779389 & -5.749265 & -2.585077 \\
\hline
\end{tabular}




\begin{tabular}{|c|c|c|c|}
\hline $\mathrm{C}$ & 0.309942 & -5.170582 & -1.389529 \\
\hline $\mathrm{C}$ & 0.948759 & -4.053444 & -0.847012 \\
\hline $\mathrm{C}$ & 0.587231 & -7.507043 & -4.190975 \\
\hline $\mathrm{C}$ & -1.514604 & 3.318233 & 0.224034 \\
\hline $\mathrm{C}$ & -2.633626 & 2.885731 & 0.507730 \\
\hline $\mathrm{C}$ & -3.911449 & 2.380968 & 0.871567 \\
\hline $\mathrm{C}$ & -4.026190 & 1.269971 & 1.767418 \\
\hline $\mathrm{C}$ & -5.323662 & 0.819459 & 2.100437 \\
\hline $\mathrm{C}$ & -6.451654 & 1.448426 & 1.571238 \\
\hline $\mathrm{C}$ & -6.370201 & 2.550863 & 0.689075 \\
\hline $\mathrm{C}$ & -5.083599 & 2.992664 & 0.359009 \\
\hline $\mathrm{C}$ & -8.462887 & 2.165155 & -0.662041 \\
\hline $\mathrm{C}$ & -7.347574 & 4.404724 & -0.784882 \\
\hline $\mathrm{C}$ & -2.734131 & -0.124874 & 3.398162 \\
\hline $\mathrm{C}$ & 3.949730 & -1.911872 & -1.037148 \\
\hline $\mathrm{C}$ & 2.187522 & 3.601562 & -0.198087 \\
\hline $\mathrm{C}$ & 2.296339 & 4.926442 & -0.678456 \\
\hline $\mathrm{C}$ & -7.650965 & 3.210611 & 0.142859 \\
\hline $\mathrm{N}$ & -1.418865 & -0.434575 & 3.667236 \\
\hline $\mathrm{N}$ & 4.200333 & -0.729106 & -0.329974 \\
\hline $\mathrm{O}$ & 4.804807 & -2.520435 & -1.691128 \\
\hline $\mathrm{C}$ & 3.355352 & 2.799235 & -0.050250 \\
\hline $\mathrm{C}$ & -8.510428 & 3.723800 & 1.325299 \\
\hline $\mathrm{C}$ & -0.924637 & -1.165953 & 4.770345 \\
\hline $\mathrm{C}$ & 5.455918 & -0.123367 & -0.185807 \\
\hline $\mathrm{C}$ & 4.356666 & 2.088049 & 0.027648 \\
\hline $\mathrm{C}$ & 0.481448 & -1.265220 & 4.897016 \\
\hline $\mathrm{C}$ & -1.736158 & -1.787544 & 5.738683 \\
\hline $\mathrm{C}$ & 5.526371 & 1.282124 & 0.054532 \\
\hline $\mathrm{C}$ & 6.662383 & -0.851052 & -0.242847 \\
\hline $\mathrm{C}$ & 1.055297 & -1.971356 & 5.951333 \\
\hline $\mathrm{C}$ & -1.154605 & -2.491989 & 6.804539 \\
\hline $\mathrm{C}$ & 6.789112 & 1.893401 & 0.262725 \\
\hline $\mathrm{C}$ & 7.887221 & -0.211576 & -0.050309 \\
\hline $\mathrm{C}$ & 0.242135 & -2.593110 & 6.918935 \\
\hline $\mathrm{C}$ & 7.987953 & 1.173293 & 0.217266 \\
\hline $\mathrm{O}$ & 0.909040 & -3.261050 & 7.921420 \\
\hline $\mathrm{C}$ & 9.366611 & 1.824654 & 0.438741 \\
\hline $\mathrm{C}$ & 0.111582 & -3.886227 & 8.918723 \\
\hline $\mathrm{C}$ & 9.262826 & 3.339080 & 0.710682 \\
\hline $\mathrm{C}$ & 10.057987 & 1.160199 & 1.655561 \\
\hline $\mathrm{C}$ & 10.242066 & 1.617751 & -0.822463 \\
\hline $\mathrm{H}$ & 2.007845 & -1.774706 & -0.264606 \\
\hline $\mathrm{H}$ & -1.986383 & 1.058163 & 1.848552 \\
\hline $\mathrm{H}$ & 0.844617 & 2.044153 & 0.513095 \\
\hline $\mathrm{H}$ & -1.033943 & 5.753948 & -0.696753 \\
\hline $\mathrm{H}$ & 3.431458 & -3.651813 & -3.155317 \\
\hline $\mathrm{H}$ & 2.308274 & -5.628294 & -4.133862 \\
\hline
\end{tabular}




\begin{tabular}{|c|c|c|c|}
\hline $\mathrm{H}$ & -0.539675 & -5.634889 & -0.876803 \\
\hline $\mathrm{H}$ & 0.590759 & -3.621931 & 0.094028 \\
\hline $\mathrm{H}$ & -0.075919 & -8.367503 & -4.365202 \\
\hline $\mathrm{H}$ & 0.575456 & -6.851548 & -5.084374 \\
\hline $\mathrm{H}$ & 1.620637 & -7.867439 & -4.024786 \\
\hline $\mathrm{H}$ & -5.423134 & -0.005693 & 2.805347 \\
\hline $\mathrm{H}$ & -4.940898 & 3.847926 & -0.306461 \\
\hline $\mathrm{H}$ & -6.788851 & 5.199313 & -0.260199 \\
\hline $\mathrm{H}$ & -6.762970 & 4.099540 & -1.671594 \\
\hline $\mathrm{H}$ & -7.433955 & 1.062011 & 1.866393 \\
\hline $\mathrm{H}$ & -8.733048 & 1.292717 & -0.043045 \\
\hline $\mathrm{H}$ & -7.883607 & 1.797789 & -1.528071 \\
\hline $\mathrm{H}$ & -9.399746 & 2.612126 & -1.041498 \\
\hline $\mathrm{H}$ & -8.293318 & 4.844674 & -1.146237 \\
\hline $\mathrm{H}$ & 3.294459 & 5.309595 & -0.897135 \\
\hline $\mathrm{H}$ & -7.962631 & 4.483163 & 1.909459 \\
\hline $\mathrm{H}$ & -8.788910 & 2.907652 & 2.013177 \\
\hline $\mathrm{H}$ & -9.443905 & 4.182798 & 0.951874 \\
\hline $\mathrm{H}$ & 1.123942 & -0.775261 & 4.156584 \\
\hline $\mathrm{H}$ & -2.820286 & -1.709973 & 5.654982 \\
\hline $\mathrm{H}$ & 6.621236 & -1.920107 & -0.452613 \\
\hline $\mathrm{H}$ & 2.142515 & -2.047502 & 6.048006 \\
\hline $\mathrm{H}$ & -1.813878 & -2.959361 & 7.540841 \\
\hline $\mathrm{H}$ & 6.789996 & 2.969957 & 0.450987 \\
\hline $\mathrm{H}$ & 8.796024 & -0.822062 & -0.099699 \\
\hline $\mathrm{H}$ & 0.818474 & -4.353325 & 9.620950 \\
\hline $\mathrm{H}$ & -0.514321 & -3.152797 & 9.464500 \\
\hline $\mathrm{H}$ & -0.546374 & -4.667875 & 8.489845 \\
\hline $\mathrm{H}$ & 10.272196 & 3.759074 & 0.864581 \\
\hline $\mathrm{H}$ & 8.670325 & 3.552800 & 1.617511 \\
\hline $\mathrm{H}$ & 8.803220 & 3.876911 & -0.137179 \\
\hline $\mathrm{H}$ & 9.464109 & 1.306432 & 2.574424 \\
\hline $\mathrm{H}$ & 11.058494 & 1.601278 & 1.817698 \\
\hline $\mathrm{H}$ & 10.188929 & 0.074531 & 1.508961 \\
\hline $\mathrm{H}$ & 11.243200 & 2.061749 & -0.672592 \\
\hline $\mathrm{H}$ & 9.781709 & 2.095958 & -1.704511 \\
\hline $\mathrm{H}$ & 10.380135 & 0.547922 & -1.053994 \\
\hline $\mathrm{H}$ & 3.397042 & -0.242770 & 0.091621 \\
\hline $\mathrm{H}$ & -0.713990 & -0.215001 & 2.933854 \\
\hline $\mathrm{N}$ & 1.258473 & 7.028617 & -1.379578 \\
\hline $\mathrm{C}$ & 0.092584 & 7.898282 & -1.347731 \\
\hline $\mathrm{H}$ & -0.722083 & 7.491401 & -1.975200 \\
\hline $\mathrm{H}$ & -0.308632 & 8.047771 & -0.322724 \\
\hline $\mathrm{H}$ & 0.366403 & 8.881260 & -1.758753 \\
\hline $\mathrm{C}$ & 2.574817 & 7.645702 & -1.453335 \\
\hline $\mathrm{H}$ & 2.470236 & 8.667598 & -1.846983 \\
\hline $\mathrm{H}$ & 3.082204 & 7.701346 & -0.466992 \\
\hline $\mathrm{H}$ & 3.234560 & 7.089279 & -2.144180 \\
\hline
\end{tabular}




$\begin{array}{llll}\mathrm{S} & 0.718604 & -0.522344 & 1.094279 \\ \mathrm{H} & 0.885189 & -1.628130 & 1.874053\end{array}$

Statistical Thermodynamic Analysis

Temperature $=298.150$ Kelvin $\quad$ Pressure $=1.00000$ Atm

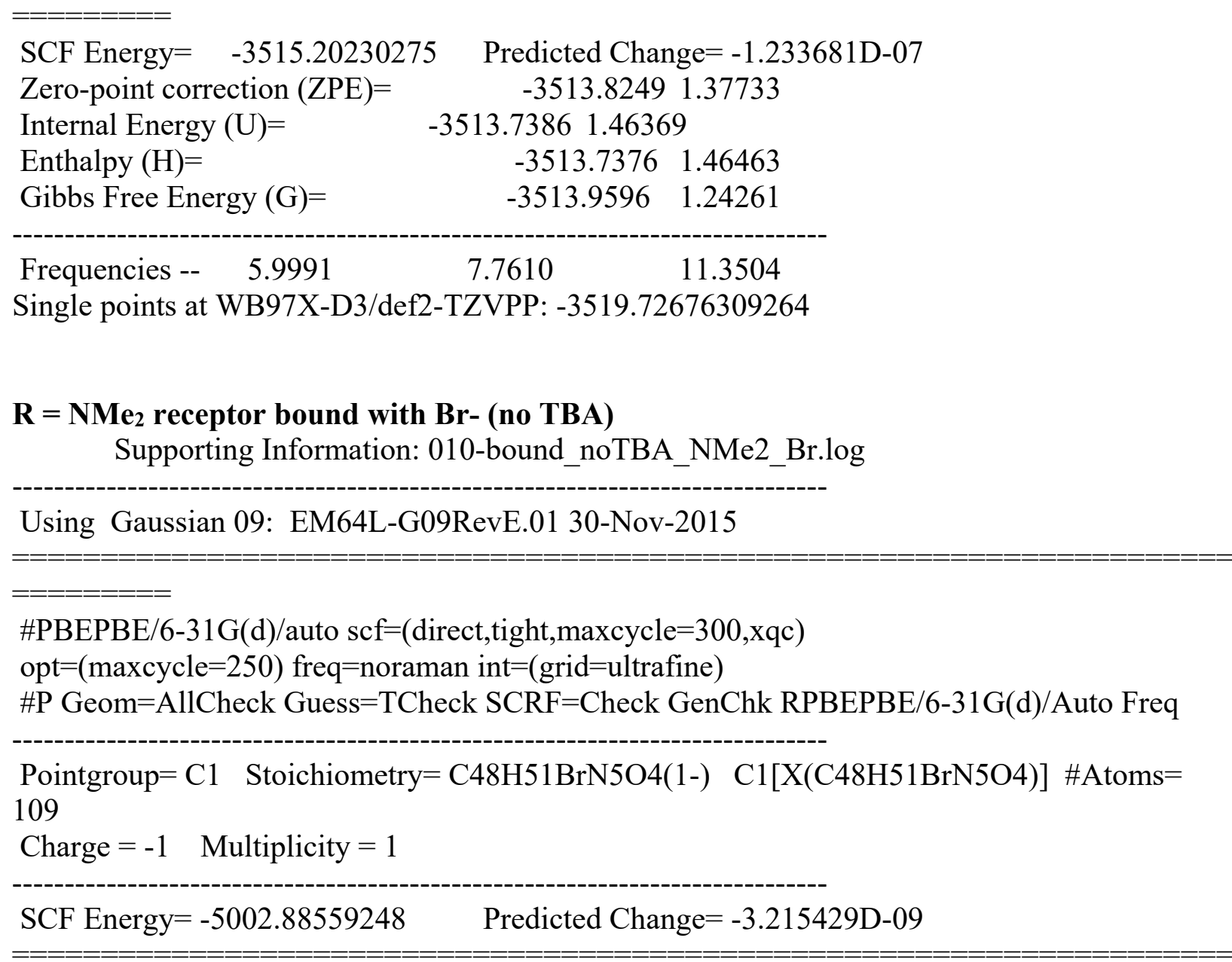

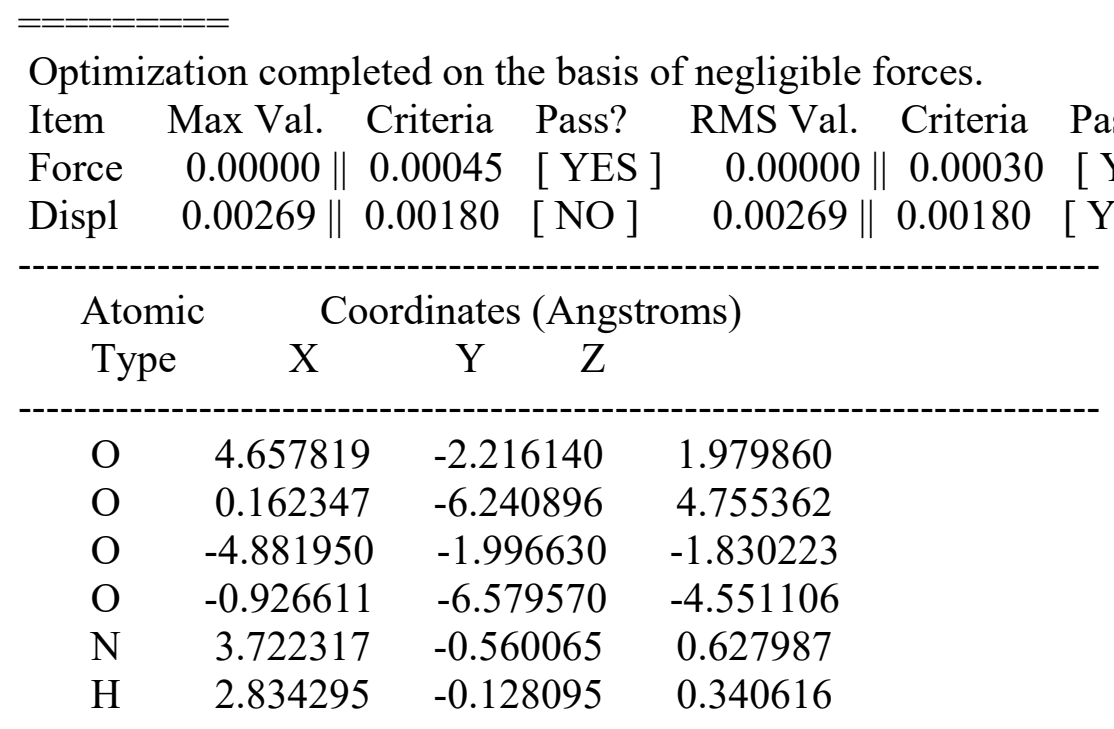




\begin{tabular}{|c|c|c|c|}
\hline $\mathrm{N}$ & 2.361789 & -2.132832 & 1.630605 \\
\hline $\mathrm{H}$ & 1.615490 & -1.661511 & 1.080915 \\
\hline $\mathrm{N}$ & -3.734288 & -0.338677 & -0.654578 \\
\hline $\mathrm{H}$ & -2.797861 & 0.033702 & -0.447450 \\
\hline $\mathrm{N}$ & -2.569947 & -2.082270 & -1.624997 \\
\hline $\mathrm{H}$ & -1.751812 & -1.632639 & -1.167417 \\
\hline $\mathrm{C}$ & 0.156275 & 2.969775 & -0.112534 \\
\hline $\mathrm{H}$ & 0.120690 & 1.875434 & -0.093382 \\
\hline $\mathrm{C}$ & 1.393159 & 3.641411 & -0.180128 \\
\hline $\mathrm{C}$ & 1.438045 & 5.055364 & -0.204787 \\
\hline $\mathrm{H}$ & 2.416643 & 5.537139 & -0.246110 \\
\hline $\mathrm{C}$ & 0.247570 & 5.821201 & -0.169994 \\
\hline $\mathrm{C}$ & -0.988050 & 5.135191 & -0.083358 \\
\hline $\mathrm{H}$ & -1.932579 & 5.680191 & -0.034830 \\
\hline $\mathrm{C}$ & -1.034568 & 3.721090 & -0.063364 \\
\hline $\mathrm{C}$ & 2.594525 & 2.875798 & -0.208735 \\
\hline $\mathrm{C}$ & 3.607844 & 2.177245 & -0.222342 \\
\hline $\mathrm{C}$ & 4.823173 & 1.440969 & -0.235285 \\
\hline $\mathrm{C}$ & 4.892492 & 0.084735 & 0.225720 \\
\hline $\mathrm{C}$ & 6.155672 & -0.543954 & 0.229987 \\
\hline $\mathrm{H}$ & 6.223527 & -1.566168 & 0.601058 \\
\hline $\mathrm{C}$ & 7.299260 & 0.129260 & -0.210494 \\
\hline $\mathrm{H}$ & 8.247858 & -0.415637 & -0.184647 \\
\hline $\mathrm{C}$ & 7.257884 & 1.455816 & -0.685768 \\
\hline $\mathrm{C}$ & 6.002107 & 2.081470 & -0.684246 \\
\hline $\mathrm{H}$ & 5.896877 & 3.115217 & -1.031237 \\
\hline $\mathrm{C}$ & 3.657741 & -1.695815 & 1.463176 \\
\hline $\mathrm{C}$ & 1.908220 & -3.187188 & 2.442235 \\
\hline $\mathrm{C}$ & 2.739183 & -3.993977 & 3.243435 \\
\hline $\mathrm{H}$ & 3.814128 & -3.810575 & 3.243775 \\
\hline $\mathrm{C}$ & 2.186492 & -5.020481 & 4.026973 \\
\hline $\mathrm{H}$ & 2.859237 & -5.628919 & 4.638123 \\
\hline $\mathrm{C}$ & 0.802684 & -5.257300 & 4.021107 \\
\hline $\mathrm{C}$ & -0.029325 & -4.452537 & 3.219022 \\
\hline $\mathrm{H}$ & -1.107022 & -4.643385 & 3.216819 \\
\hline $\mathrm{C}$ & 0.512559 & -3.432381 & 2.441953 \\
\hline $\mathrm{H}$ & -0.131797 & -2.802761 & 1.815855 \\
\hline $\mathrm{C}$ & 0.984499 & -7.064496 & 5.564432 \\
\hline $\mathrm{H}$ & 0.307041 & -7.776177 & 6.062165 \\
\hline $\mathrm{H}$ & 1.527870 & -6.480320 & 6.335030 \\
\hline $\mathrm{H}$ & 1.726707 & -7.628700 & 4.963664 \\
\hline $\mathrm{C}$ & -2.282863 & 3.038525 & 0.014944 \\
\hline $\mathrm{C}$ & -3.342480 & 2.415526 & 0.078773 \\
\hline $\mathrm{C}$ & -4.614036 & 1.786935 & 0.166766 \\
\hline $\mathrm{C}$ & -4.822382 & 0.423846 & -0.227404 \\
\hline $\mathrm{C}$ & -6.134268 & -0.089926 & -0.142405 \\
\hline $\mathrm{H}$ & -6.308180 & -1.117617 & -0.459408 \\
\hline $\mathrm{C}$ & -7.192547 & 0.700204 & 0.316443 \\
\hline
\end{tabular}




\begin{tabular}{|c|c|c|c|}
\hline $\mathrm{H}$ & -8.184291 & 0.239737 & 0.360505 \\
\hline $\mathrm{C}$ & -7.014529 & 2.038159 & 0.722855 \\
\hline $\mathrm{C}$ & -5.710934 & 2.548605 & 0.634884 \\
\hline $\mathrm{H}$ & -5.499393 & 3.582935 & 0.927235 \\
\hline $\mathrm{C}$ & -3.812971 & -1.527532 & -1.411488 \\
\hline $\mathrm{C}$ & -2.258504 & -3.227040 & -2.379636 \\
\hline $\mathrm{C}$ & -3.204283 & -4.015893 & -3.062906 \\
\hline $\mathrm{H}$ & -4.258401 & -3.741672 & -3.011661 \\
\hline $\mathrm{C}$ & -2.791099 & -5.140264 & -3.796474 \\
\hline $\mathrm{H}$ & -3.551022 & -5.731409 & -4.315467 \\
\hline $\mathrm{C}$ & -1.433869 & -5.493929 & -3.857698 \\
\hline $\mathrm{C}$ & -0.487117 & -4.707284 & -3.173687 \\
\hline $\mathrm{H}$ & 0.569303 & -4.989127 & -3.223160 \\
\hline $\mathrm{C}$ & -0.890478 & -3.590723 & -2.446392 \\
\hline $\mathrm{H}$ & -0.155143 & -2.975560 & -1.913011 \\
\hline $\mathrm{C}$ & -1.866030 & -7.386467 & -5.240516 \\
\hline $\mathrm{H}$ & -1.283948 & -8.188084 & -5.721946 \\
\hline $\mathrm{H}$ & -2.411799 & -6.815659 & -6.019376 \\
\hline $\mathrm{H}$ & -2.608590 & -7.839514 & -4.552439 \\
\hline $\mathrm{N}$ & 0.290596 & 7.223360 & -0.231081 \\
\hline $\mathrm{C}$ & 8.502112 & 2.217242 & -1.185202 \\
\hline $\mathrm{C}$ & -8.162555 & 2.928670 & 1.238699 \\
\hline $\mathrm{C}$ & 8.293658 & 2.638899 & -2.661033 \\
\hline $\mathrm{H}$ & 7.403758 & 3.280279 & -2.778461 \\
\hline $\mathrm{H}$ & 9.169978 & 3.203372 & -3.031346 \\
\hline $\mathrm{H}$ & 8.155135 & 1.753903 & -3.306196 \\
\hline $\mathrm{C}$ & 9.781706 & 1.359488 & -1.107007 \\
\hline $\mathrm{H}$ & 10.002375 & 1.048136 & -0.070871 \\
\hline $\mathrm{H}$ & 9.703007 & 0.450868 & -1.729417 \\
\hline $\mathrm{H}$ & 10.645109 & 1.943247 & -1.473218 \\
\hline $\mathrm{C}$ & 8.717393 & 3.483653 & -0.319602 \\
\hline $\mathrm{H}$ & 8.887310 & 3.212425 & 0.736974 \\
\hline $\mathrm{H}$ & 9.596046 & 4.053330 & -0.676245 \\
\hline $\mathrm{H}$ & 7.842177 & 4.154354 & -0.356309 \\
\hline $\mathrm{C}$ & -7.846914 & 3.398040 & 2.680706 \\
\hline $\mathrm{H}$ & -6.900895 & 3.963825 & 2.726020 \\
\hline $\mathrm{H}$ & -8.652723 & 4.053579 & 3.061047 \\
\hline $\mathrm{H}$ & -7.753122 & 2.534989 & 3.362614 \\
\hline $\mathrm{C}$ & -8.310345 & 4.167791 & 0.320993 \\
\hline $\mathrm{H}$ & -8.553379 & 3.864029 & -0.712173 \\
\hline $\mathrm{H}$ & -9.118968 & 4.828064 & 0.686935 \\
\hline $\mathrm{H}$ & -7.380262 & 4.760167 & 0.285517 \\
\hline $\mathrm{C}$ & -9.513304 & 2.184235 & 1.260966 \\
\hline $\mathrm{H}$ & -9.810679 & 1.846843 & 0.252531 \\
\hline $\mathrm{H}$ & -9.482099 & 1.301349 & 1.923309 \\
\hline $\mathrm{H}$ & -10.304391 & 2.857678 & 1.636470 \\
\hline $\mathrm{C}$ & -0.909718 & 7.965661 & 0.120262 \\
\hline $\mathrm{H}$ & -1.273348 & 7.753798 & 1.149972 \\
\hline
\end{tabular}




$\begin{array}{lrrr}\mathrm{H} & -0.700141 & 9.043727 & 0.040501 \\ \mathrm{H} & -1.731853 & 7.735647 & -0.581336 \\ \mathrm{C} & 1.566110 & 7.884553 & -0.005000 \\ \mathrm{H} & 2.016427 & 7.647610 & 0.984154 \\ \mathrm{H} & 2.297019 & 7.602106 & -0.784147 \\ \mathrm{H} & 1.420745 & 8.974195 & -0.068921 \\ \mathrm{Br} & 0.015876 & -0.643202 & -0.161412\end{array}$

Statistical Thermodynamic Analysis

Temperature $=298.150$ Kelvin Pressure $=1.00000$ Atm

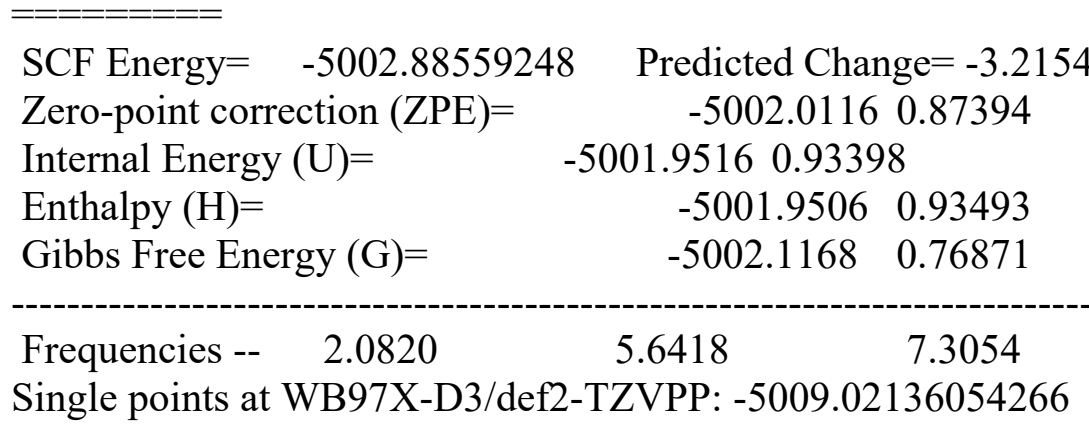

$\mathbf{R}=\mathrm{NMe}_{2}$ receptor bound with $\mathrm{Cl}-($ no $\mathrm{TBA})$

Supporting Information: 010-bound_noTBA_NMe2_Cl_new.log

Using Gaussian 09: EM64L-G09RevE.01 30-Nov-2015

\#PBEPBE/6-31G(d)/auto scf=(direct,tight,maxcycle=300,xqc)

$\mathrm{opt}=($ maxcycle $=250)$ freq $=$ noraman int $=($ grid $=$ ultrafine $)$

\#P Geom=AllCheck Guess=TCheck SCRF=Check GenChk RPBEPBE/6-31G(d)/Auto Freq

Pointgroup $=\mathrm{C} 1$ Stoichiometry $=\mathrm{C} 48 \mathrm{H} 51 \mathrm{ClN} 5 \mathrm{O} 4(1-) \quad \mathrm{C} 1[\mathrm{X}(\mathrm{C} 48 \mathrm{H} 51 \mathrm{ClN} 5 \mathrm{O} 4)]$ \#Atoms= 109 Charge $=-1 \quad$ Multiplicity $=1$

SCF Energy $=-2891.56343839 \quad$ Predicted Change $=-1.032293 \mathrm{D}-08$

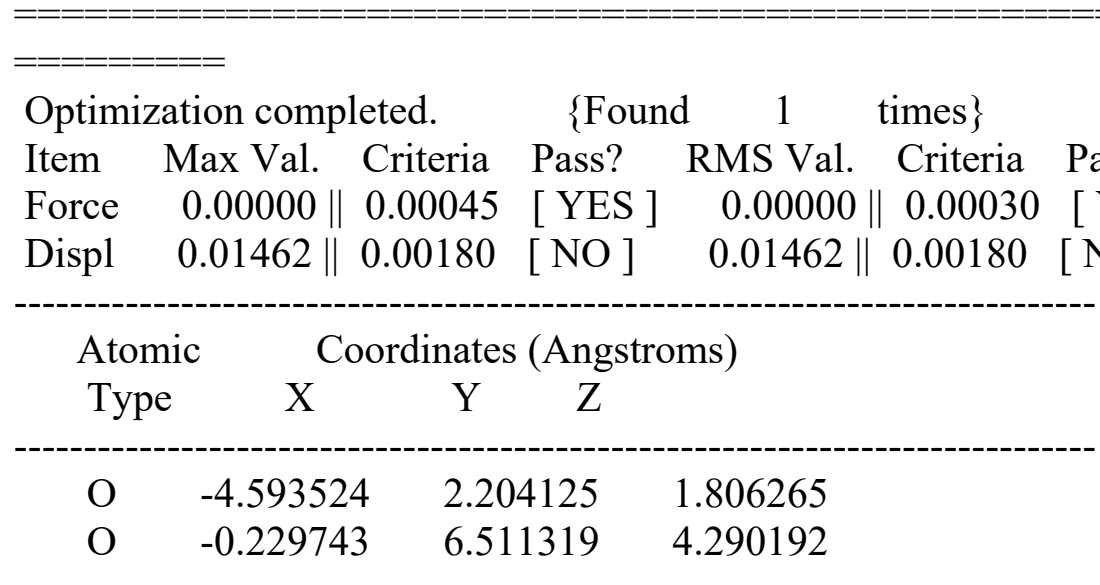




\begin{tabular}{|c|c|c|c|}
\hline $\mathrm{O}$ & 4.612363 & 2.220329 & -1.783767 \\
\hline $\mathrm{O}$ & 0.286816 & 6.614742 & -4.180000 \\
\hline $\mathrm{N}$ & -3.643071 & 0.425914 & 0.636232 \\
\hline $\mathrm{H}$ & -2.753720 & -0.038090 & 0.411994 \\
\hline $\mathrm{N}$ & -2.289167 & 2.072030 & 1.534425 \\
\hline $\mathrm{H}$ & -1.526383 & 1.549547 & 1.053378 \\
\hline $\mathrm{N}$ & 3.645700 & 0.419848 & -0.662042 \\
\hline $\mathrm{H}$ & 2.752405 & -0.043186 & -0.451862 \\
\hline $\mathrm{N}$ & 2.306027 & 2.095756 & -1.525887 \\
\hline $\mathrm{H}$ & 1.537719 & 1.566146 & -1.061739 \\
\hline $\mathrm{C}$ & -0.011234 & -3.008221 & -0.068857 \\
\hline $\mathrm{H}$ & -0.008072 & -1.912444 & -0.069414 \\
\hline $\mathrm{C}$ & -1.228098 & -3.718894 & -0.097018 \\
\hline $\mathrm{C}$ & -1.232062 & -5.133873 & -0.090423 \\
\hline $\mathrm{H}$ & -2.197038 & -5.643936 & -0.101624 \\
\hline $\mathrm{C}$ & -0.019715 & -5.864425 & -0.063113 \\
\hline $\mathrm{C}$ & 1.196176 & -5.140901 & -0.015217 \\
\hline $\mathrm{H}$ & 2.157291 & -5.656603 & 0.027270 \\
\hline $\mathrm{C}$ & 1.201095 & -3.725926 & -0.025785 \\
\hline $\mathrm{C}$ & -2.452808 & -2.991686 & -0.118849 \\
\hline $\mathrm{C}$ & -3.488382 & -2.326759 & -0.134317 \\
\hline $\mathrm{C}$ & -4.718609 & -1.616767 & -0.152951 \\
\hline $\mathrm{C}$ & -4.806644 & -0.245463 & 0.260402 \\
\hline $\mathrm{C}$ & -6.080326 & 0.362659 & 0.245278 \\
\hline $\mathrm{H}$ & -6.162310 & 1.397294 & 0.576320 \\
\hline $\mathrm{C}$ & -7.214421 & -0.345608 & -0.163967 \\
\hline $\mathrm{H}$ & -8.171266 & 0.185309 & -0.154353 \\
\hline $\mathrm{C}$ & -7.155347 & -1.689207 & -0.585959 \\
\hline $\mathrm{C}$ & -5.889356 & -2.293602 & -0.567943 \\
\hline $\mathrm{H}$ & -5.768403 & -3.337501 & -0.877542 \\
\hline $\mathrm{C}$ & -3.584613 & 1.630724 & 1.367728 \\
\hline $\mathrm{C}$ & -1.868689 & 3.209638 & 2.248101 \\
\hline $\mathrm{C}$ & -2.728736 & 4.070032 & 2.959432 \\
\hline $\mathrm{H}$ & -3.799294 & 3.864650 & 2.961445 \\
\hline $\mathrm{C}$ & -2.212900 & 5.178609 & 3.650520 \\
\hline $\mathrm{H}$ & -2.910224 & 5.824896 & 4.191289 \\
\hline $\mathrm{C}$ & -0.835254 & 5.447757 & 3.643080 \\
\hline $\mathrm{C}$ & 0.026758 & 4.591267 & 2.932295 \\
\hline $\mathrm{H}$ & 1.099622 & 4.807425 & 2.925594 \\
\hline $\mathrm{C}$ & -0.479955 & 3.490253 & 2.246563 \\
\hline $\mathrm{H}$ & 0.195621 & 2.827215 & 1.694209 \\
\hline $\mathrm{C}$ & -1.084915 & 7.391396 & 4.999347 \\
\hline $\mathrm{H}$ & -0.432788 & 8.164832 & 5.434951 \\
\hline $\mathrm{H}$ & -1.629970 & 6.874138 & 5.815303 \\
\hline $\mathrm{H}$ & -1.827736 & 7.875286 & 4.332916 \\
\hline $\mathrm{C}$ & 2.429622 & -3.006162 & 0.014247 \\
\hline $\mathrm{C}$ & 3.469310 & -2.348294 & 0.046790 \\
\hline $\mathrm{C}$ & 4.704142 & -1.647308 & 0.085180 \\
\hline
\end{tabular}




\begin{tabular}{|c|c|c|c|}
\hline $\mathrm{C}$ & 4.803261 & -0.267768 & -0.297211 \\
\hline $\mathrm{C}$ & 6.081098 & 0.330867 & -0.263912 \\
\hline $\mathrm{H}$ & 6.171430 & 1.371983 & -0.571631 \\
\hline $\mathrm{C}$ & 7.208637 & -0.394179 & 0.133909 \\
\hline $\mathrm{H}$ & 8.169170 & 0.130102 & 0.139637 \\
\hline $\mathrm{C}$ & 7.138575 & -1.746322 & 0.525917 \\
\hline $\mathrm{C}$ & 5.868565 & -2.341301 & 0.489604 \\
\hline $\mathrm{H}$ & 5.739238 & -3.390956 & 0.775435 \\
\hline $\mathrm{C}$ & 3.597968 & 1.642524 & -1.364069 \\
\hline $\mathrm{C}$ & 1.895898 & 3.253282 & -2.213106 \\
\hline $\mathrm{C}$ & 2.764692 & 4.126395 & -2.897802 \\
\hline $\mathrm{H}$ & 3.834079 & 3.915005 & -2.899263 \\
\hline $\mathrm{C}$ & 2.258926 & 5.254987 & -3.563479 \\
\hline $\mathrm{H}$ & 2.962853 & 5.910674 & -4.083999 \\
\hline $\mathrm{C}$ & 0.882791 & 5.531759 & -3.556712 \\
\hline $\mathrm{C}$ & 0.012060 & 4.662548 & -2.872504 \\
\hline $\mathrm{H}$ & -1.059608 & 4.884583 & -2.866258 \\
\hline $\mathrm{C}$ & 0.508760 & 3.541689 & -2.212064 \\
\hline $\mathrm{H}$ & -0.173560 & 2.868781 & -1.680311 \\
\hline $\mathrm{C}$ & 1.150664 & 7.507171 & -4.862719 \\
\hline $\mathrm{H}$ & 0.505206 & 8.294937 & -5.282281 \\
\hline $\mathrm{H}$ & 1.697026 & 7.007179 & -5.688501 \\
\hline $\mathrm{H}$ & 1.892727 & 7.970101 & -4.180734 \\
\hline $\mathrm{N}$ & -0.022757 & -7.268564 & -0.094237 \\
\hline $\mathrm{C}$ & -8.389763 & -2.489210 & -1.047775 \\
\hline $\mathrm{C}$ & 8.365499 & -2.564755 & 0.975211 \\
\hline $\mathrm{C}$ & -8.183657 & -2.963022 & -2.508028 \\
\hline $\mathrm{H}$ & -7.283618 & -3.593240 & -2.607295 \\
\hline $\mathrm{H}$ & -9.052498 & -3.555867 & -2.850674 \\
\hline $\mathrm{H}$ & -8.064393 & -2.100865 & -3.187082 \\
\hline $\mathrm{C}$ & -9.682812 & -1.650017 & -0.993192 \\
\hline $\mathrm{H}$ & -9.901670 & -1.302932 & 0.031916 \\
\hline $\mathrm{H}$ & -9.623094 & -0.764643 & -1.650229 \\
\hline $\mathrm{H}$ & -10.538884 & -2.261227 & -1.330762 \\
\hline $\mathrm{C}$ & -8.578860 & -3.725370 & -0.133676 \\
\hline $\mathrm{H}$ & -8.746607 & -3.417254 & 0.913097 \\
\hline $\mathrm{H}$ & -9.450262 & -4.322215 & -0.462634 \\
\hline $\mathrm{H}$ & -7.692989 & -4.382759 & -0.151067 \\
\hline $\mathrm{C}$ & 8.150804 & -3.066782 & 2.424774 \\
\hline $\mathrm{H}$ & 7.245751 & -3.692147 & 2.508022 \\
\hline $\mathrm{H}$ & 9.014023 & -3.672997 & 2.758142 \\
\hline $\mathrm{H}$ & 8.035556 & -2.217804 & 3.120923 \\
\hline $\mathrm{C}$ & 8.548899 & -3.783369 & 0.036705 \\
\hline $\mathrm{H}$ & 8.722261 & -3.455257 & -1.003044 \\
\hline $\mathrm{H}$ & 9.415015 & -4.392881 & 0.356237 \\
\hline $\mathrm{H}$ & 7.658450 & -4.434776 & 0.037832 \\
\hline $\mathrm{C}$ & 9.664798 & -1.734164 & 0.942156 \\
\hline $\mathrm{H}$ & 9.889485 & -1.367507 & -0.074840 \\
\hline
\end{tabular}




$\begin{array}{cccc}\mathrm{H} & 9.609335 & -0.862203 & 1.617247 \\ \mathrm{H} & 10.515285 & -2.358507 & 1.269650 \\ \mathrm{C} & 1.204474 & -7.967470 & 0.253367 \\ \mathrm{H} & 1.577566 & -7.723194 & 1.272494 \\ \mathrm{H} & 1.025927 & -9.052731 & 0.199291 \\ \mathrm{H} & 2.008376 & -7.728280 & -0.465998 \\ \mathrm{C} & -1.273692 & -7.960627 & 0.173298 \\ \mathrm{H} & -1.709399 & -7.716211 & 1.167211 \\ \mathrm{H} & -2.028773 & -7.714956 & -0.595104 \\ \mathrm{H} & -1.098575 & -9.046852 & 0.127861 \\ \mathrm{Cl} & -0.000025 & 0.497313 & -0.020701\end{array}$

Statistical Thermodynamic Analysis

Temperature $=298.150$ Kelvin $\quad$ Pressure $=1.00000$ Atm

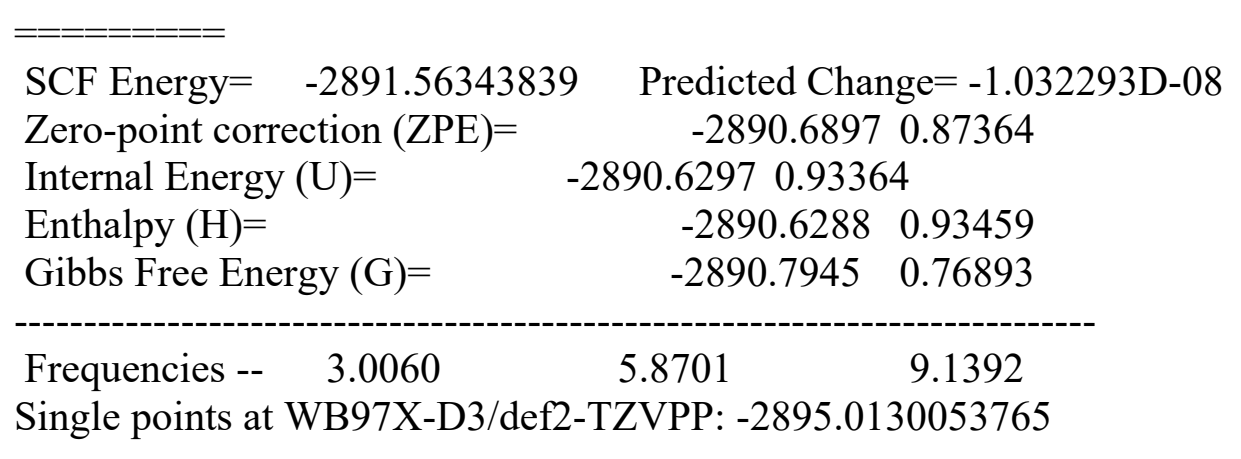

$\mathrm{R}=\mathrm{NMe}_{2}$ receptor bound with $\mathrm{SeH}-($ no $\mathrm{TBA})$

Supporting Information: 010-bound_noTBA_NMe2_SeH.log

Using Gaussian 09: EM64L-G09RevE.01 30-Nov-2015
\#PBEPBE/6-31G(d)/auto scf=(direct,tight,maxcycle=300,xqc)
opt $=($ maxcycle $=250)$ freq $=$ noraman int $=($ grid $=$ ultrafine $)$
\#P Geom=AllCheck Guess=TCheck SCRF=Check GenChk RPBEPBE/6-31G(d)/Auto Freq
Pointgroup $=\mathrm{C} 1$ Stoichiometry $=\mathrm{C} 48 \mathrm{H} 52 \mathrm{~N} 5 \mathrm{O} 4 \mathrm{Se}(1-) \quad \mathrm{C} 1[\mathrm{X}(\mathrm{C} 48 \mathrm{H} 52 \mathrm{~N} 5 \mathrm{O} 4 \mathrm{Se})]$ \#Atoms $=$
110
Charge $=-1 \quad$ Multiplicity $=1$

SCF Energy $=-4831.14847375 \quad$ Predicted Change $=-5.745491 \mathrm{D}-09$

\begin{tabular}{|c|c|c|c|c|}
\hline Optim & zation completed. & $\{$ Found & times $\}$ & \\
\hline Item & Max Val. Criteria & Pass? RMS Val. & Criteria & Pass? \\
\hline Force & $0.00000 \| 0.00045$ & [YES ] $\quad 0.00000$ & 0.00030 & [YES ] \\
\hline Displ & $0.00207 \| 0.00180$ & $0.00207 \mid$ & 0.00180 & [YES ] \\
\hline
\end{tabular}




\begin{tabular}{|c|c|c|c|}
\hline \multirow{2}{*}{$\begin{array}{l}\text { Atomic } \\
\text { Type }\end{array}$} & \multicolumn{3}{|c|}{ Coordinates (Angstroms) } \\
\hline & $\mathrm{X}$ & $\begin{array}{ll}Y & Z\end{array}$ & \\
\hline $\mathrm{O}$ & 5.131019 & 1.809374 & -1.322177 \\
\hline $\mathrm{O}$ & 1.758457 & 7.089439 & -3.453079 \\
\hline $\mathrm{O}$ & -4.630630 & 2.539495 & 1.621850 \\
\hline $\mathrm{O}$ & 0.000150 & 6.946287 & 3.418590 \\
\hline $\mathrm{N}$ & 3.796572 & 0.087799 & -0.475123 \\
\hline $\mathrm{H}$ & 2.819397 & -0.232534 & -0.388118 \\
\hline $\mathrm{N}$ & 2.832714 & 2.077110 & -1.161412 \\
\hline $\mathrm{H}$ & 1.946557 & 1.619682 & -0.838208 \\
\hline $\mathrm{N}$ & -3.770782 & 0.681681 & 0.495200 \\
\hline $\mathrm{H}$ & -2.893385 & 0.195110 & 0.256432 \\
\hline $\mathrm{N}$ & -2.359268 & 2.382143 & 1.164886 \\
\hline $\mathrm{H}$ & -1.641344 & 1.823793 & 0.644002 \\
\hline $\mathrm{C}$ & -0.370543 & -3.094353 & -0.048915 \\
\hline $\mathrm{H}$ & -0.287965 & -2.000562 & -0.073637 \\
\hline $\mathrm{C}$ & 0.787896 & -3.893890 & -0.019105 \\
\hline $\mathrm{C}$ & 0.681564 & -5.305272 & -0.020492 \\
\hline $\mathrm{H}$ & 1.603540 & -5.889534 & -0.006560 \\
\hline $\mathrm{C}$ & -0.584651 & -5.938665 & -0.041978 \\
\hline $\mathrm{C}$ & -1.741829 & -5.123548 & -0.084623 \\
\hline $\mathrm{H}$ & -2.740240 & -5.563635 & -0.117664 \\
\hline $\mathrm{C}$ & -1.635498 & -3.712682 & -0.080337 \\
\hline $\mathrm{C}$ & 2.064415 & -3.263749 & 0.016707 \\
\hline $\mathrm{C}$ & 3.152270 & -2.689866 & 0.063840 \\
\hline $\mathrm{C}$ & 4.472218 & -2.175128 & 0.169222 \\
\hline $\mathrm{C}$ & 4.803227 & -0.807116 & -0.107019 \\
\hline $\mathrm{C}$ & 6.157307 & -0.426482 & 0.033098 \\
\hline $\mathrm{H}$ & 6.427389 & 0.605211 & -0.186890 \\
\hline $\mathrm{C}$ & 7.135936 & -1.345928 & 0.420817 \\
\hline $\mathrm{H}$ & 8.163603 & -0.980158 & 0.509530 \\
\hline $\mathrm{C}$ & 6.837811 & -2.695156 & 0.698020 \\
\hline $\mathrm{C}$ & 5.494463 & -3.072429 & 0.563345 \\
\hline $\mathrm{H}$ & 5.185219 & -4.104135 & 0.763583 \\
\hline $\mathrm{C}$ & 4.008713 & 1.376326 & -1.017758 \\
\hline $\mathrm{C}$ & 2.664150 & 3.343264 & -1.751354 \\
\hline $\mathrm{C}$ & 3.720124 & 4.180121 & -2.163922 \\
\hline $\mathrm{H}$ & 4.747186 & 3.837959 & -2.035124 \\
\hline $\mathrm{C}$ & 3.450415 & 5.433653 & -2.737185 \\
\hline $\mathrm{H}$ & 4.294335 & 6.056605 & -3.047200 \\
\hline $\mathrm{C}$ & 2.127765 & 5.874339 & -2.902561 \\
\hline $\mathrm{C}$ & 1.070306 & 5.041322 & -2.490231 \\
\hline $\mathrm{H}$ & 0.040791 & 5.389488 & -2.619147 \\
\hline $\mathrm{C}$ & 1.332094 & 3.794940 & -1.927499 \\
\hline $\mathrm{H}$ & 0.508162 & 3.141920 & -1.616037 \\
\hline $\mathrm{C}$ & 2.810906 & 7.946581 & -3.861246 \\
\hline $\mathrm{H}$ & 2.328366 & 8.852394 & -4.261032 \\
\hline
\end{tabular}




\begin{tabular}{|c|c|c|c|}
\hline $\mathrm{H}$ & 3.436749 & 7.488248 & -4.654134 \\
\hline $\mathrm{H}$ & 3.468645 & 8.228815 & -3.014092 \\
\hline $\mathrm{C}$ & -2.798535 & -2.891571 & -0.110202 \\
\hline $\mathrm{C}$ & -3.769250 & -2.135164 & -0.123027 \\
\hline $\mathrm{C}$ & -4.957408 & -1.357683 & -0.148311 \\
\hline $\mathrm{C}$ & -4.969251 & 0.036404 & 0.188560 \\
\hline $\mathrm{C}$ & -6.215599 & 0.700936 & 0.169127 \\
\hline $\mathrm{H}$ & -6.243301 & 1.754686 & 0.443879 \\
\hline $\mathrm{C}$ & -7.392140 & 0.027194 & -0.171047 \\
\hline $\mathrm{H}$ & -8.322375 & 0.603616 & -0.167815 \\
\hline $\mathrm{C}$ & -7.407105 & -1.339156 & -0.517462 \\
\hline $\mathrm{C}$ & -6.170171 & -1.999490 & -0.496031 \\
\hline $\mathrm{H}$ & -6.104681 & -3.063547 & -0.748464 \\
\hline $\mathrm{C}$ & -3.660417 & 1.935321 & 1.139168 \\
\hline $\mathrm{C}$ & -1.865668 & 3.554445 & 1.763987 \\
\hline $\mathrm{C}$ & -2.651722 & 4.489792 & 2.464671 \\
\hline $\mathrm{H}$ & -3.724265 & 4.316521 & 2.558115 \\
\hline $\mathrm{C}$ & -2.057154 & 5.630410 & 3.029458 \\
\hline $\mathrm{H}$ & -2.694854 & 6.337639 & 3.567581 \\
\hline $\mathrm{C}$ & -0.677100 & 5.855384 & 2.901118 \\
\hline $\mathrm{C}$ & 0.110066 & 4.921770 & 2.199567 \\
\hline $\mathrm{H}$ & 1.184208 & 5.103862 & 2.095701 \\
\hline $\mathrm{C}$ & -0.473013 & 3.787382 & 1.642596 \\
\hline $\mathrm{H}$ & 0.130347 & 3.050713 & 1.096987 \\
\hline $\mathrm{C}$ & -0.777717 & 7.902288 & 4.118023 \\
\hline $\mathrm{H}$ & -0.075922 & 8.685721 & 4.445023 \\
\hline $\mathrm{H}$ & -1.273579 & 7.464113 & 5.008328 \\
\hline $\mathrm{H}$ & -1.555668 & 8.357817 & 3.471896 \\
\hline $\mathrm{N}$ & -0.690538 & -7.338855 & -0.008116 \\
\hline $\mathrm{C}$ & 7.896358 & -3.729902 & 1.128323 \\
\hline $\mathrm{C}$ & -8.688966 & -2.104536 & -0.901978 \\
\hline $\mathrm{C}$ & 7.530997 & -4.296353 & 2.522939 \\
\hline $\mathrm{H}$ & 6.536160 & -4.773242 & 2.519522 \\
\hline $\mathrm{H}$ & 8.270296 & -5.055718 & 2.840132 \\
\hline $\mathrm{H}$ & 7.514154 & -3.493382 & 3.280349 \\
\hline $\mathrm{C}$ & 9.309241 & -3.117055 & 1.213786 \\
\hline $\mathrm{H}$ & 9.640281 & -2.716642 & 0.239475 \\
\hline $\mathrm{H}$ & 9.355675 & -2.299610 & 1.954598 \\
\hline $\mathrm{H}$ & 10.034097 & -3.891399 & 1.522647 \\
\hline $\mathrm{C}$ & 7.935313 & -4.889760 & 0.102425 \\
\hline $\mathrm{H}$ & 8.211344 & -4.517946 & -0.899741 \\
\hline $\mathrm{H}$ & 8.677623 & -5.652018 & 0.405361 \\
\hline $\mathrm{H}$ & 6.954871 & -5.388193 & 0.015841 \\
\hline $\mathrm{C}$ & -8.546750 & -2.663699 & -2.339509 \\
\hline $\mathrm{H}$ & -7.678672 & -3.338763 & -2.428225 \\
\hline $\mathrm{H}$ & -9.450587 & -3.233388 & -2.626479 \\
\hline $\mathrm{H}$ & -8.409116 & -1.844936 & -3.067099 \\
\hline $\mathrm{C}$ & -8.906330 & -3.280922 & 0.082055 \\
\hline
\end{tabular}




$\begin{array}{cccc}\mathrm{H} & -9.028624 & -2.910847 & 1.114942 \\ \mathrm{H} & -9.813179 & -3.852961 & -0.190354 \\ \mathrm{H} & -8.051919 & -3.978874 & 0.075866 \\ \mathrm{C} & -9.941031 & -1.204839 & -0.857994 \\ \mathrm{H} & -10.113402 & -0.793980 & 0.152287 \\ \mathrm{H} & -9.861452 & -0.359317 & -1.563641 \\ \mathrm{H} & -10.833268 & -1.792871 & -1.138246 \\ \mathrm{C} & -1.970276 & -7.940812 & -0.347673 \\ \mathrm{H} & -2.330298 & -7.667267 & -1.364048 \\ \mathrm{H} & -1.875549 & -9.036659 & -0.295494 \\ \mathrm{H} & -2.748656 & -7.641276 & 0.377216 \\ \mathrm{C} & 0.501599 & -8.126314 & -0.280403 \\ \mathrm{H} & 0.952108 & -7.914739 & -1.275212 \\ \mathrm{H} & 1.276040 & -7.941993 & 0.485964 \\ \mathrm{H} & 0.242186 & -9.195579 & -0.236168 \\ \mathrm{Se} & -0.007107 & 0.537602 & -0.318386 \\ \mathrm{H} & 0.349583 & 0.365297 & 1.111213\end{array}$

Statistical Thermodynamic Analysis

Temperature $=298.150$ Kelvin Pressure $=1.00000$ Atm

\begin{tabular}{|c|c|}
\hline SCF Energy $=\quad-4831.1484737$ & Predicted Change $=-5.745491 \mathrm{D}-09$ \\
\hline Zero-point correction $(\mathrm{ZPE})=$ & -4830.26510 .88331 \\
\hline Internal Energy $(U)=$ & -4830.20520 .94320 \\
\hline Enthalpy $(\mathrm{H})=$ & $-4830.2043 \quad 0.94414$ \\
\hline Gibbs Free Energy $(\mathrm{G})=$ & $-4830.3674 \quad 0.78100$ \\
\hline Frequencies -- 5.8983 & $7.0241 \quad 13.2100$ \\
\hline \multicolumn{2}{|c|}{ Single points at WB97X-D3/def2-TZVPP: -4836.98270226589} \\
\hline
\end{tabular}

Using Gaussian 09: EM64L-G09RevE.01 30-Nov-2015

\#PBEPBE/6-31G(d)/auto scf=(direct,tight,maxcycle=300,xqc)

$\mathrm{opt}=($ maxcycle $=250)$ freq $=$ noraman int $=($ grid $=$ ultrafine $)$

\#P Geom=AllCheck Guess=TCheck SCRF=Check GenChk RPBEPBE/6-31G(d)/Auto Freq

Pointgroup= C1 Stoichiometry= C48H52N5O4S(1-) C1[X(C48H52N5O4S)] \#Atoms=110 Charge $=-1 \quad$ Multiplicity $=1$

SCF Energy $=-2830.13212278 \quad$ Predicted Change $=-3.000891 \mathrm{D}-08$ 
Optimization completed. $\quad$ Found 1 times Item Max Val. Criteria Pass? RMS Val. Criteria Pass? Force $0.00001 \| 0.00045$ [YES ] $0.00000 \| 0.00030 \quad$ [YES ] Displ $0.00251 \| 0.00180 \quad$ [NO ] $0.00251 \| 0.00180 \quad$ [YES ]

\begin{tabular}{|c|c|c|c|}
\hline \multirow{2}{*}{$\begin{array}{l}\text { Atomic } \\
\text { Type }\end{array}$} & \multicolumn{3}{|c|}{ Coordinates (Angstroms) } \\
\hline & $\mathrm{X}$ & $\mathrm{Y} \quad \mathrm{Z}$ & \\
\hline $\mathrm{O}$ & -4.670433 & 2.370318 & 1.628475 \\
\hline $\mathrm{O}$ & -0.316732 & 6.885163 & 3.695533 \\
\hline $\mathrm{O}$ & 4.907119 & 2.051055 & -1.483033 \\
\hline $\mathrm{O}$ & 1.129669 & 7.007947 & -3.648474 \\
\hline $\mathrm{N}$ & -3.738750 & 0.528841 & 0.543725 \\
\hline $\mathrm{H}$ & -2.849505 & 0.044532 & 0.355982 \\
\hline $\mathrm{N}$ & -2.374217 & 2.242387 & 1.289578 \\
\hline $\mathrm{H}$ & -1.619099 & 1.699784 & 0.800161 \\
\hline $\mathrm{N}$ & 3.752202 & 0.257929 & -0.542483 \\
\hline $\mathrm{H}$ & 2.815107 & -0.150960 & -0.421145 \\
\hline $\mathrm{N}$ & 2.591602 & 2.111142 & -1.308784 \\
\hline $\mathrm{H}$ & 1.752576 & 1.611878 & -0.917404 \\
\hline $\mathrm{C}$ & -0.158852 & -3.051892 & -0.079162 \\
\hline $\mathrm{H}$ & -0.116198 & -1.954240 & -0.048497 \\
\hline $\mathrm{C}$ & -1.399265 & -3.719040 & -0.130128 \\
\hline $\mathrm{C}$ & -1.452825 & -5.132785 & -0.168457 \\
\hline $\mathrm{H}$ & -2.435017 & -5.608327 & -0.196528 \\
\hline $\mathrm{C}$ & -0.266821 & -5.905608 & -0.165987 \\
\hline $\mathrm{C}$ & 0.973432 & -5.226434 & -0.099021 \\
\hline $\mathrm{H}$ & 1.916260 & -5.776178 & -0.076209 \\
\hline $\mathrm{C}$ & 1.027256 & -3.812662 & -0.063808 \\
\hline $\mathrm{C}$ & -2.599541 & -2.952092 & -0.135940 \\
\hline $\mathrm{C}$ & -3.616884 & -2.259366 & -0.141263 \\
\hline $\mathrm{C}$ & -4.838605 & -1.535207 & -0.159204 \\
\hline $\mathrm{C}$ & -4.910059 & -0.149683 & 0.207815 \\
\hline $\mathrm{C}$ & -6.180737 & 0.466533 & 0.184467 \\
\hline $\mathrm{H}$ & -6.251976 & 1.512607 & 0.479705 \\
\hline $\mathrm{C}$ & -7.324988 & -0.246167 & -0.186030 \\
\hline $\mathrm{H}$ & -8.277289 & 0.293038 & -0.183843 \\
\hline $\mathrm{C}$ & -7.282096 & -1.604731 & -0.559266 \\
\hline $\mathrm{C}$ & -6.020399 & -2.216948 & -0.534839 \\
\hline $\mathrm{H}$ & -5.910161 & -3.271936 & -0.808919 \\
\hline $\mathrm{C}$ & -3.668636 & 1.779121 & 1.196229 \\
\hline $\mathrm{C}$ & -1.952171 & 3.428905 & 1.918517 \\
\hline $\mathrm{C}$ & -2.800073 & 4.301187 & 2.631216 \\
\hline $\mathrm{H}$ & -3.862124 & 4.066350 & 2.701208 \\
\hline $\mathrm{C}$ & -2.285227 & 5.459858 & 3.235267 \\
\hline $\mathrm{H}$ & -2.974183 & 6.112298 & 3.779476 \\
\hline $\mathrm{C}$ & -0.919712 & 5.770511 & 3.138515 \\
\hline $\mathrm{C}$ & -0.069360 & 4.904043 & 2.426203 \\
\hline
\end{tabular}




\begin{tabular}{|c|c|c|c|}
\hline $\mathrm{H}$ & 0.993555 & 5.152109 & 2.346226 \\
\hline $\mathrm{C}$ & -0.575637 & 3.752746 & 1.827983 \\
\hline $\mathrm{H}$ & 0.092585 & 3.085475 & 1.272519 \\
\hline $\mathrm{C}$ & -1.161381 & 7.773743 & 4.406511 \\
\hline $\mathrm{H}$ & -0.513125 & 8.588698 & 4.765457 \\
\hline $\mathrm{H}$ & -1.643330 & 7.282871 & 5.276695 \\
\hline $\mathrm{H}$ & -1.955475 & 8.199097 & 3.759487 \\
\hline $\mathrm{C}$ & 2.281879 & -3.141129 & -0.001699 \\
\hline $\mathrm{C}$ & 3.348587 & -2.530079 & 0.056418 \\
\hline $\mathrm{C}$ & 4.626550 & -1.916802 & 0.148031 \\
\hline $\mathrm{C}$ & 4.839142 & -0.533316 & -0.167941 \\
\hline $\mathrm{C}$ & 6.156645 & -0.035628 & -0.059118 \\
\hline $\mathrm{H}$ & 6.335505 & 1.008651 & -0.311403 \\
\hline $\mathrm{C}$ & 7.212819 & -0.859576 & 0.341101 \\
\hline $\mathrm{H}$ & 8.207522 & -0.407809 & 0.405529 \\
\hline $\mathrm{C}$ & 7.031232 & -2.219955 & 0.661508 \\
\hline $\mathrm{C}$ & 5.723056 & -2.713965 & 0.554294 \\
\hline $\mathrm{H}$ & 5.505180 & -3.762366 & 0.785748 \\
\hline $\mathrm{C}$ & 3.831721 & 1.532107 & -1.144784 \\
\hline $\mathrm{C}$ & 2.322989 & 3.354435 & -1.912660 \\
\hline $\mathrm{C}$ & 3.292341 & 4.169543 & -2.532617 \\
\hline $\mathrm{H}$ & 4.331341 & 3.840672 & -2.543483 \\
\hline $\mathrm{C}$ & 2.926065 & 5.391583 & -3.119904 \\
\hline $\mathrm{H}$ & 3.706554 & 5.996457 & -3.590509 \\
\hline $\mathrm{C}$ & 1.590617 & 5.823596 & -3.099665 \\
\hline $\mathrm{C}$ & 0.619490 & 5.015014 & -2.479840 \\
\hline $\mathrm{H}$ & -0.419655 & 5.357936 & -2.458087 \\
\hline $\mathrm{C}$ & 0.978490 & 3.801930 & -1.897426 \\
\hline $\mathrm{H}$ & 0.217778 & 3.178669 & -1.413132 \\
\hline $\mathrm{C}$ & 2.097980 & 7.840416 & -4.263427 \\
\hline $\mathrm{H}$ & 1.551757 & 8.724016 & -4.629634 \\
\hline $\mathrm{H}$ & 2.593855 & 7.339212 & -5.119778 \\
\hline $\mathrm{H}$ & 2.879578 & 8.167071 & -3.547569 \\
\hline $\mathrm{C}$ & -8.528066 & -2.411071 & -0.976639 \\
\hline $\mathrm{C}$ & 8.177561 & -3.148549 & 1.108979 \\
\hline $\mathrm{C}$ & -8.345296 & -2.935876 & -2.422518 \\
\hline $\mathrm{H}$ & -7.450655 & -3.575184 & -2.512195 \\
\hline $\mathrm{H}$ & -9.222483 & -3.534181 & -2.732998 \\
\hline $\mathrm{H}$ & -8.229978 & -2.098170 & -3.132209 \\
\hline $\mathrm{C}$ & -9.814461 & -1.561113 & -0.933202 \\
\hline $\mathrm{H}$ & -10.016241 & -1.177303 & 0.082260 \\
\hline $\mathrm{H}$ & -9.757954 & -0.699310 & -1.621143 \\
\hline $\mathrm{H}$ & -10.679445 & -2.177234 & -1.237529 \\
\hline $\mathrm{C}$ & -8.713171 & -3.614136 & -0.018605 \\
\hline $\mathrm{H}$ & -8.864091 & -3.269481 & 1.019287 \\
\hline $\mathrm{H}$ & -9.593261 & -4.215236 & -0.315137 \\
\hline $\mathrm{H}$ & -7.832270 & -4.278353 & -0.025676 \\
\hline $\mathrm{C}$ & 7.873994 & -3.702667 & 2.523290 \\
\hline
\end{tabular}




\begin{tabular}{cccc}
$\mathrm{H}$ & 6.923708 & -4.262714 & 2.544652 \\
$\mathrm{H}$ & 8.678354 & -4.386407 & 2.853949 \\
$\mathrm{H}$ & 7.794757 & -2.882040 & 3.257422 \\
$\mathrm{C}$ & 8.305466 & -4.330784 & 0.116328 \\
$\mathrm{H}$ & 8.540855 & -3.966819 & -0.899017 \\
$\mathrm{H}$ & 9.111761 & -5.018895 & 0.432913 \\
$\mathrm{H}$ & 7.369753 & -4.912052 & 0.054639 \\
$\mathrm{C}$ & 9.534297 & -2.416617 & 1.160966 \\
$\mathrm{H}$ & 9.821997 & -2.018988 & 0.171864 \\
$\mathrm{H}$ & 9.517823 & -1.576483 & 1.877248 \\
$\mathrm{H}$ & 10.324622 & -3.117646 & 1.484027 \\
$\mathrm{~S}$ & -0.043117 & 0.519601 & -0.222572 \\
$\mathrm{H}$ & -0.494213 & 0.492980 & -1.508463 \\
$\mathrm{~N}$ & -0.318787 & -7.307380 & -0.240815 \\
$\mathrm{C}$ & -1.592218 & -7.963228 & 0.011598 \\
$\mathrm{H}$ & -2.016454 & -7.732317 & 1.013690 \\
$\mathrm{H}$ & -2.340586 & -7.669804 & -0.746576 \\
$\mathrm{H}$ & -1.454951 & -9.053125 & -0.065390 \\
$\mathrm{C}$ & 0.884129 & -8.058529 & 0.081998 \\
$\mathrm{H}$ & 1.694487 & -7.825390 & -0.632067 \\
$\mathrm{H}$ & 1.267391 & -7.858344 & 1.106981 \\
$\mathrm{H}$ & 0.668040 & -9.134843 & -0.004523 \\
\hline-----------------------------------------------------------------
\end{tabular}

Statistical Thermodynamic Analysis

Temperature $=298.150$ Kelvin Pressure $=1.00000$ Atm

\begin{tabular}{|c|c|}
\hline SCF Energy $=\quad-2830.13212278$ & 8 Predicted Change $=-3.000891 \mathrm{D}-08$ \\
\hline Zero-point correction $(\mathrm{ZPE})=$ & -2829.25150 .88060 \\
\hline Internal Energy $(U)=$ & -2829.19090 .94120 \\
\hline Enthalpy $(\mathrm{H})=$ & $-2829.1899 \quad 0.94214$ \\
\hline Gibbs Free Energy $(G)=$ & $-2829.3552 \quad 0.77688$ \\
\hline Frequencies -- 5.3769 & 12.1500 \\
\hline
\end{tabular}

